WHOI-89-37

\title{
Gulf Stream Recirculation Experiment - Part II
}

\author{
by
}

C. M. Wooding, W.B. Owens, M.E. Zemanovic, and J.R. Valdes

\author{
Woods Hole Oceanographic Institution \\ Woods Hole, Massachusetts 02543
}

September 1989

\section{Technical Report}

Funding was provided by the National Science Foundation under

Grant Numbers OCE 81-09145 and OCE 81-17467

Reproduction in whole or in part is permitted for any purpose of the United States Government. This report should be cited as:

Woods Hole Oceanog. Inst. Tech. Rept., WHOI-89-37.

Approved for publication; distribution unlimited.

Approved for Distribution:

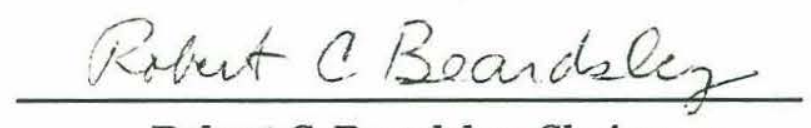

Robert C. Beardsley, Chairman Department of Physical Oceanography 


\title{
Gulf Stream Recirculation Experiment - Part II
}

\author{
Christine M. Wooding \\ W. Brechner Owens \\ Marguerite E. Zemanovic \\ and \\ James R. Valdes
}

June 13,1989 


\section{Abstract}

This report presents trajectories and time series of velocity, pressure, and temperature for twelve neutrally-buoyant floats launched during the Gulf Stream Recirculation EXperiment (GUSREX) and two from earlier experiments, that continued to operate after May 1982. These float data were obtained from Autonomous Listening Stations (ALSs) deployed from May 1982 to August 1985. 


\section{Table of Contents}

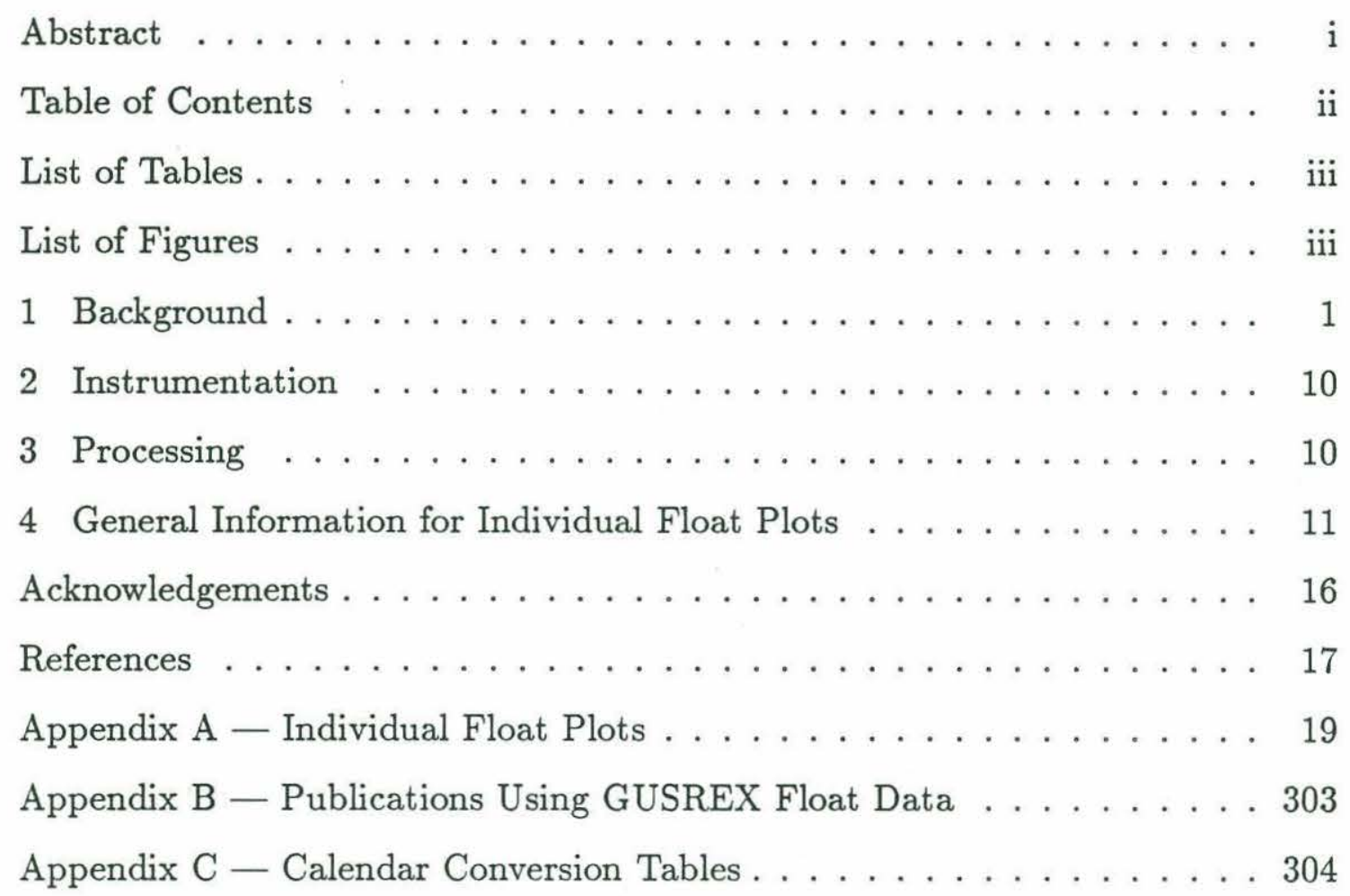




\section{List of Tables}

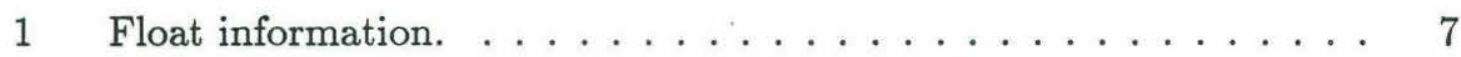

\section{List of Figures}

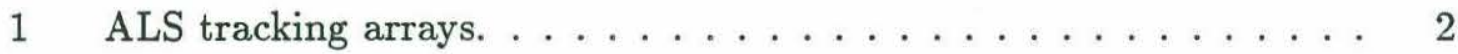

2700 -meter float trajectories reported here. . . . . . . . . 3

32000 -meter float trajectories reported here. . . . . . . . . 4

4 All 700 -meter GUSREX float trajectories. . . . . . . . . . 5 5

5 All 2000-meter GUSREX float trajectories. . . . . . . . . . 6

6 Total duration of floats reported here. . . . . . . . . . . . . 9

$7 \quad 700$-meter float duration - all GUSREX floats. . . . . . . . . 12

82000 -meter float duration - all GUSREX floats. . . . . . . . . 13

9700 -meter displacement diagram. . . . . . . . . . . . . . 14

102000 -meter displacement diagram. . . . . . . . . . . 15 


\section{Background}

Twelve neutrally-buoyant, SOund Fixing And Ranging (SOFAR) floats launched between April 1980 and August 1981 as part of the Gulf Stream Recirculation EXperiment (GUSREX) continued to work after the first two years of the experiment reported by Kennelly and McKee (1984). In addition, two floats, one launched in October 1976 over the Nares Abyssal Plain as part of POLYMODE and another launched in June 1979 as part of an earlier Gulf Stream experiment also were heard after May 1982. This report presents the trajectories and time series of temperature, pressure, and velocity for these fourteen floats. GUSREX part II coverage of the Northwest Atlantic Ocean lasted for 33 months and then was supplemented by an array of five ALSs deployed to track SOFAR floats launched in the close vicinity of $34^{\circ} \mathrm{N}, 70^{\circ} \mathrm{W}$ (Site L, Price et al., 1987) (Figure 1).

The GUSREX program, including the Site L coverage, lasted nearly five-and-a-half years. GUSREX was a joint program of Woods Hole Oceanographic Institution and the University of Rhode Island. It focused on the recirculation of the Gulf Stream, addressing such questions as the size and structure of the recirculation south of the Gulf Stream as proposed by Worthington (1976) and the interconnection of the Gulf Stream and the North Atlantic Current at the tail of the Grand Banks (Worthington, 1976; Clarke et al., 1980). As part of GUSREX, a total of forty-three floats was launched along $55^{\circ} \mathrm{W}$ from 24 to $44^{\circ} \mathrm{N}$ during two cruises in April-May 1980 and July-August 1981 (Kennelly and McKee, 1984). Except for one case, these floats were launched in pairs with one float ballasted to $700 \mathrm{~m}$ and the other to $2000 \mathrm{~m}$ (see Figures 2, 3, 4 and 5). Twelve of these floats, five shallow and seven deep, are presented in this report. Table I shows the duration and start and end locations for each float 


\section{GUSREX EXPERIMENT AUG 1981 - SEPT 1985}

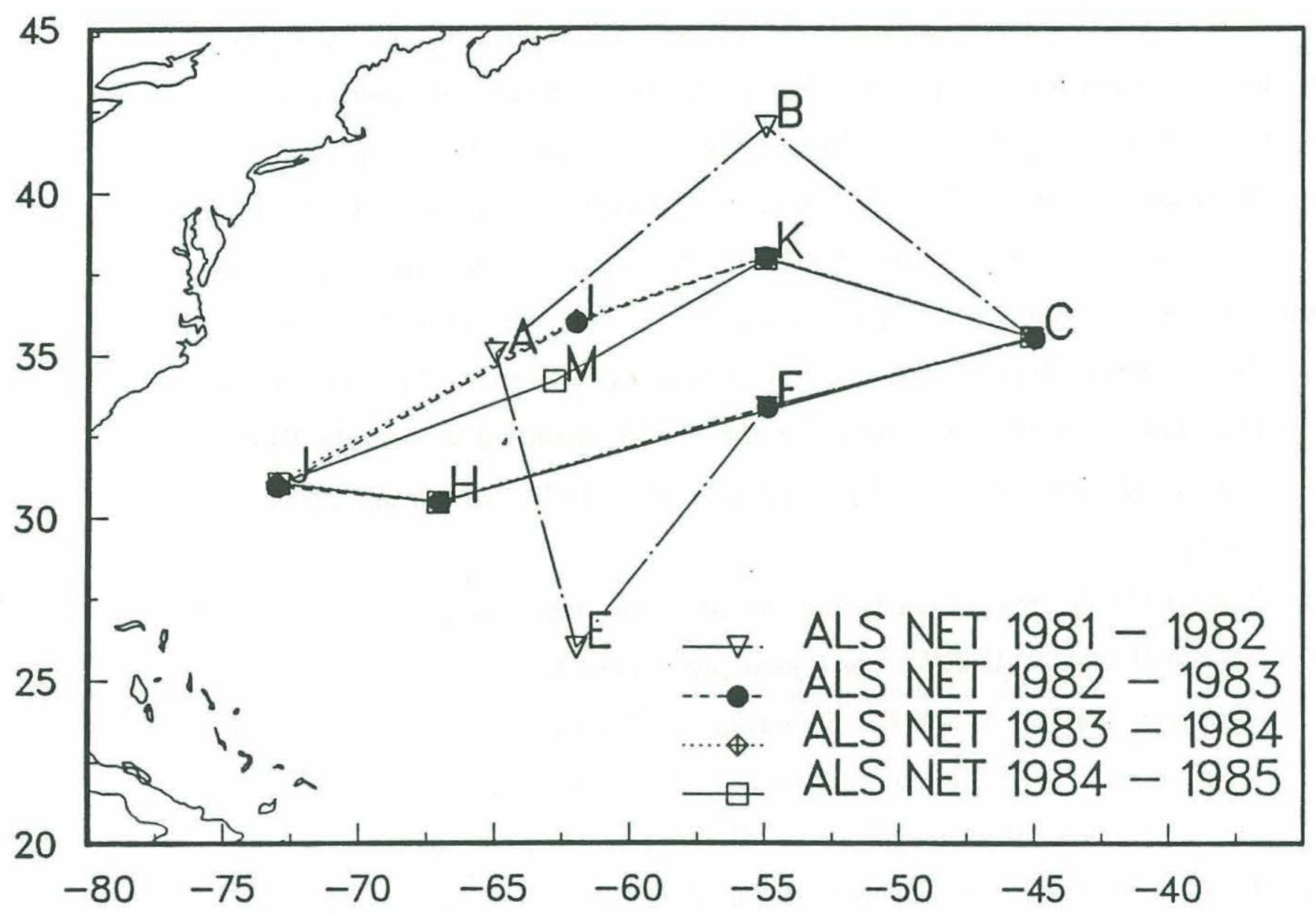

Figure 1: ALS tracking arrays for duration of GUSREX Site L Experiment. 


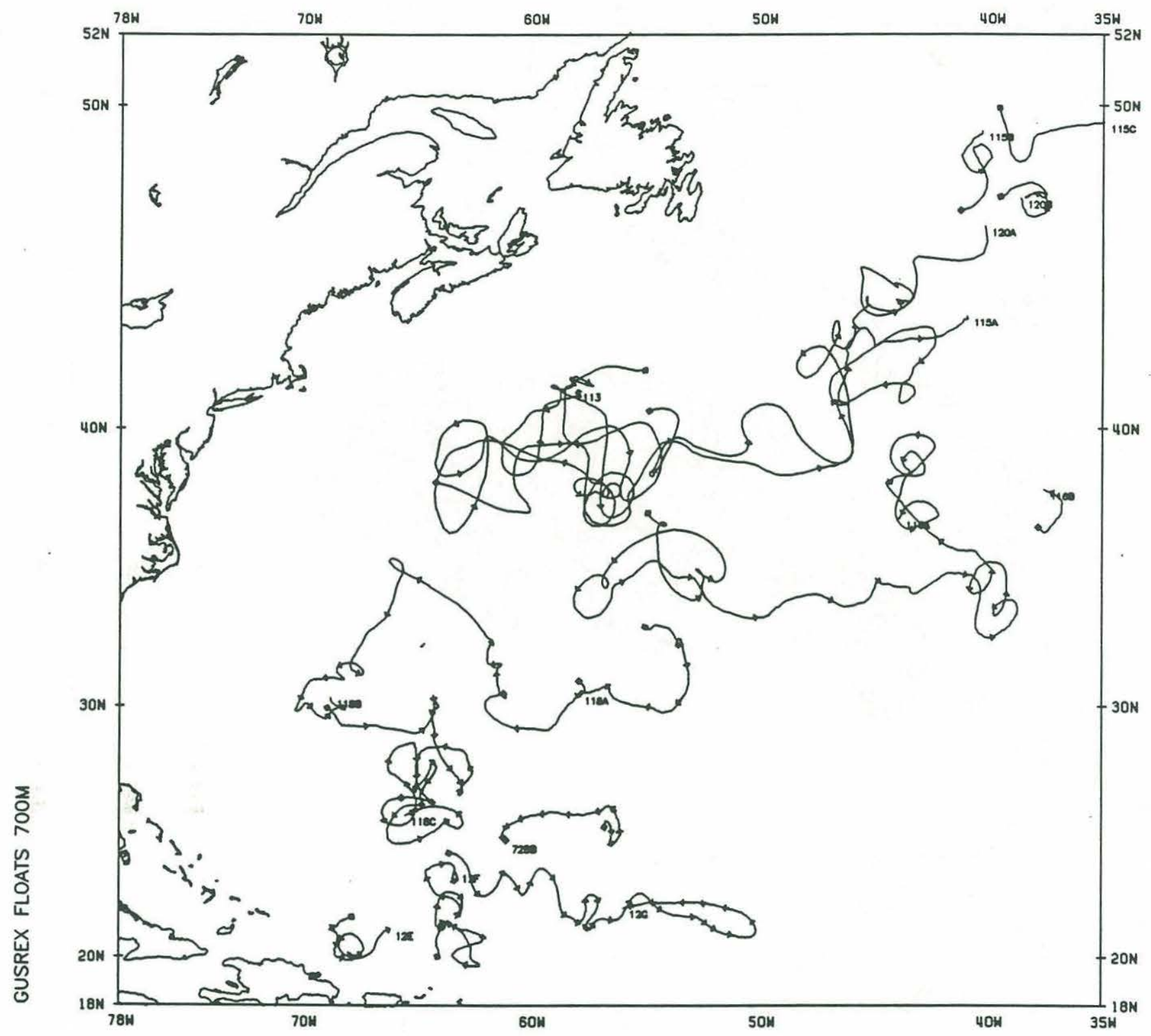

Figure 2: Composite 700-meter trajectories for floats covered in this report. Arrowheads mark every thirtieth day. 


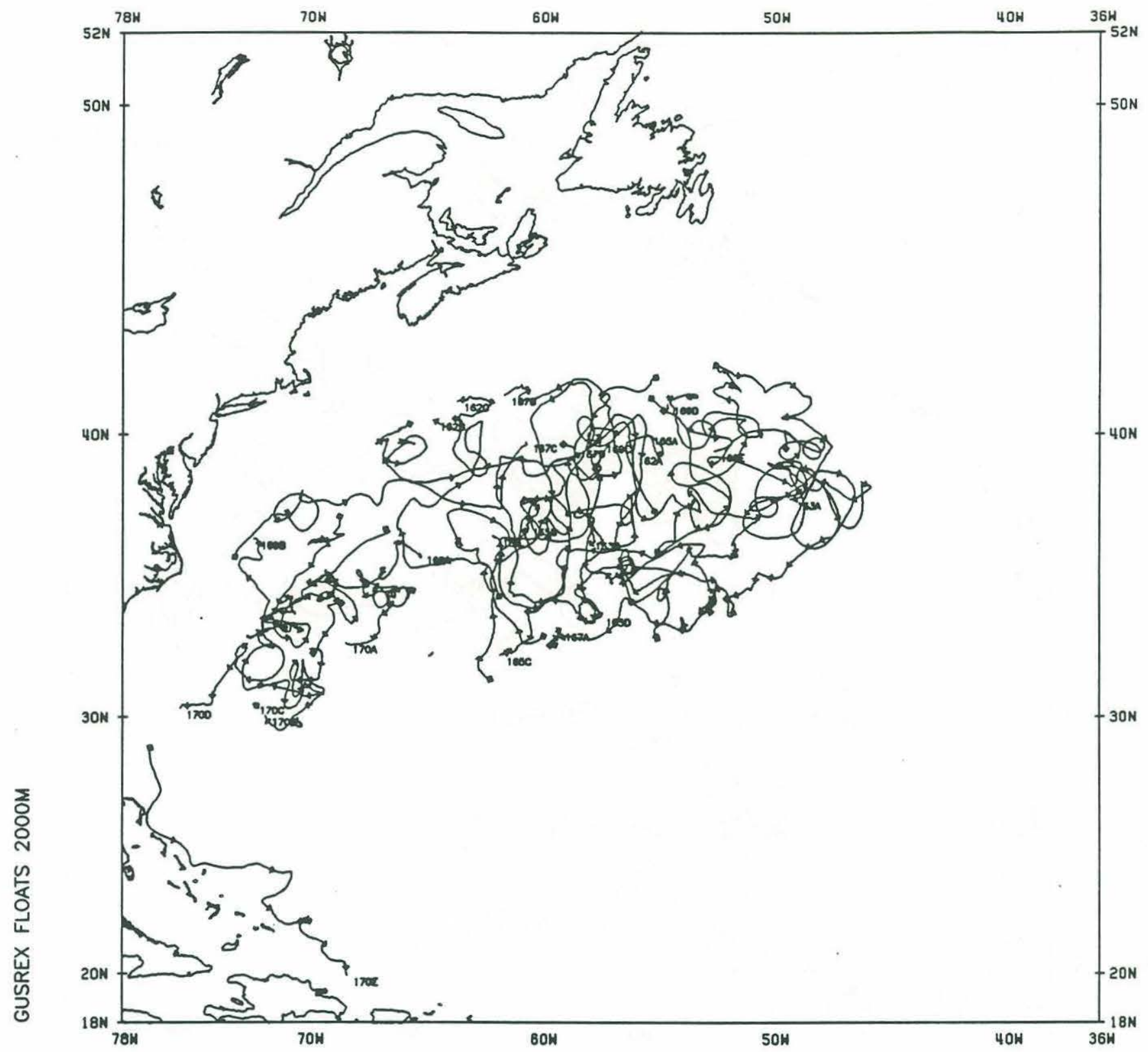

Figure 3: Composite 2000-meter trajectories for floats covered in this report. Arrowheads mark every thirtieth day. 


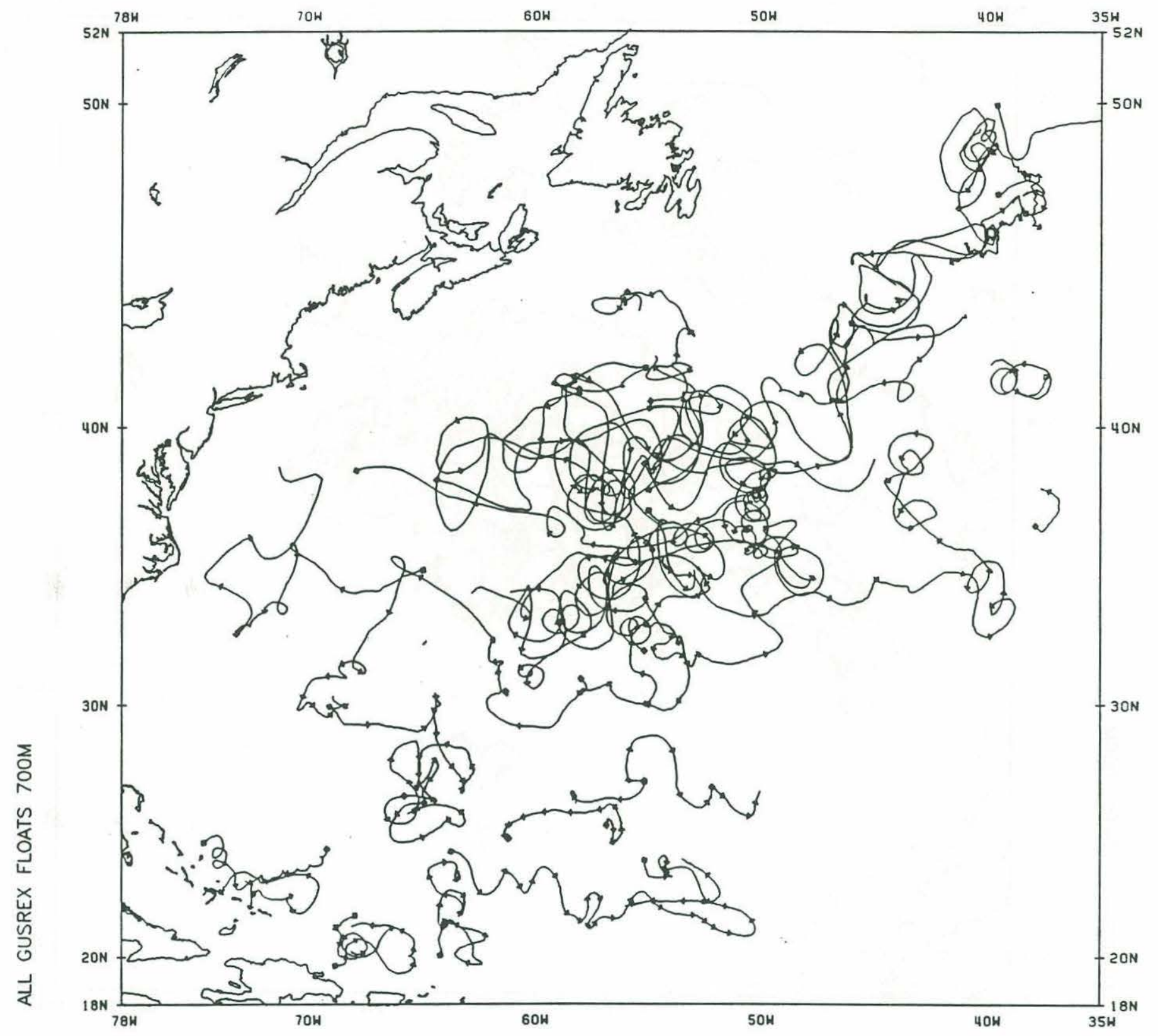

Figure 4: Composite 700-meter trajectories for floats covered in this report and earlier Gulf Stream floats. Arrowheads mark every thirtieth day. 


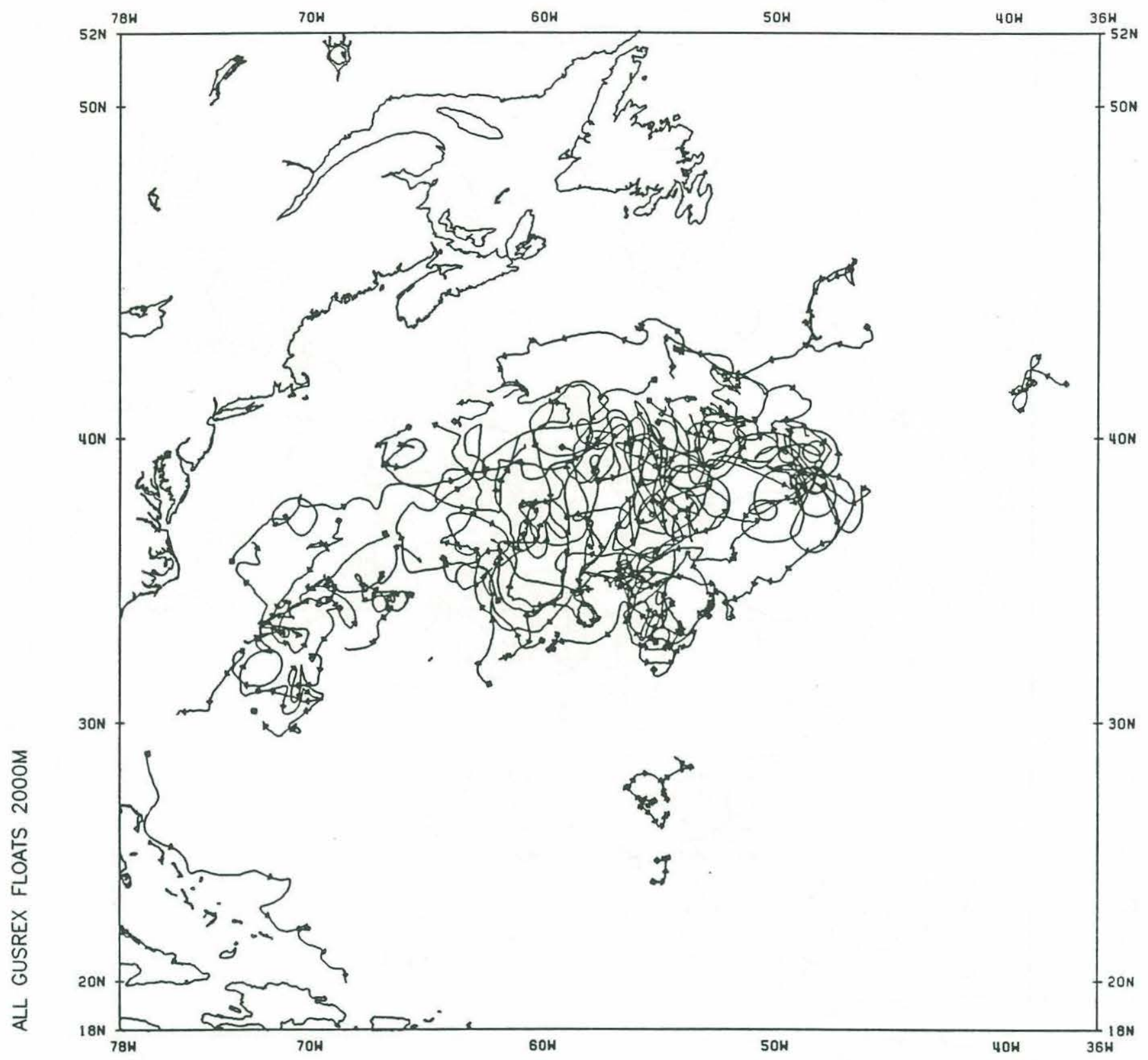

Figure 5: Composite 2000-meter trajectories for floats covered in this report and earlier Gulf Stream floats. Arrowheads mark every thirtieth day. 


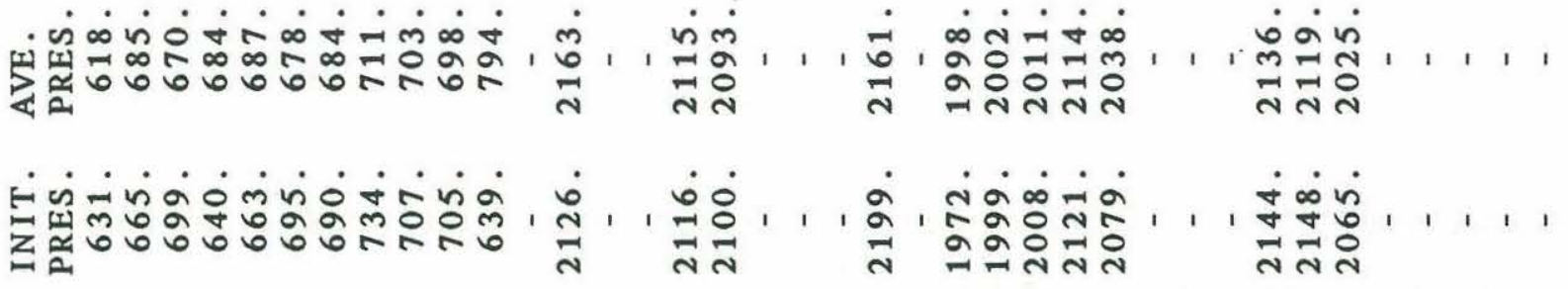

if

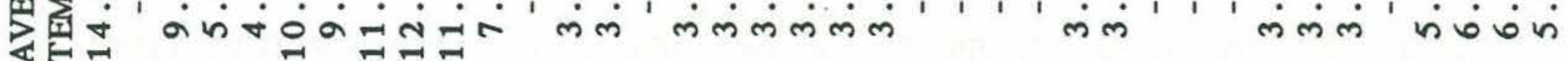

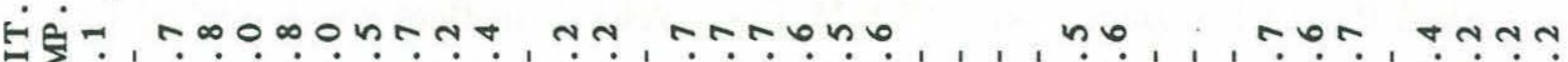

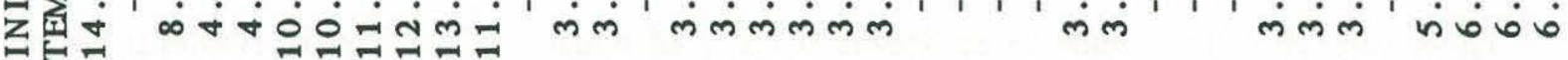

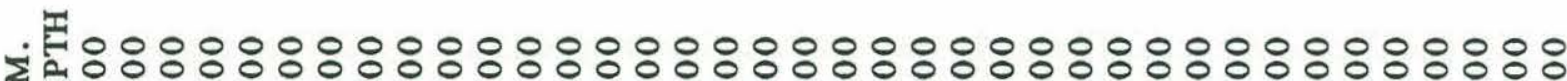

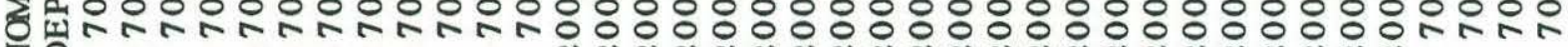
乙空

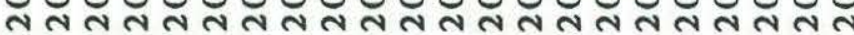

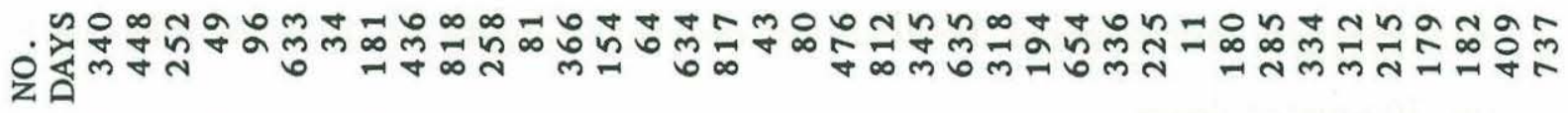

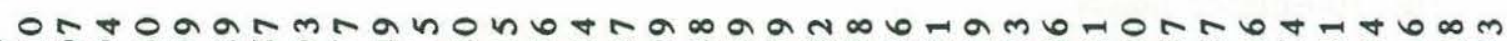
응

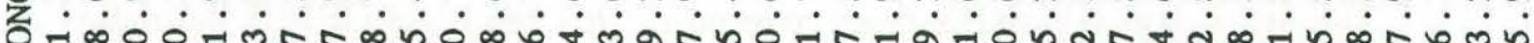
a

n F б

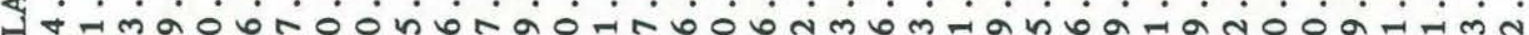
స

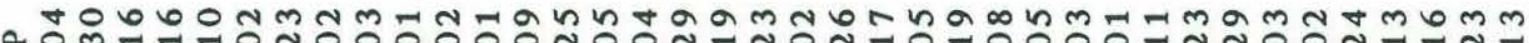

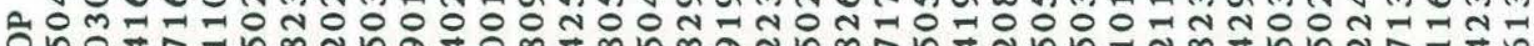

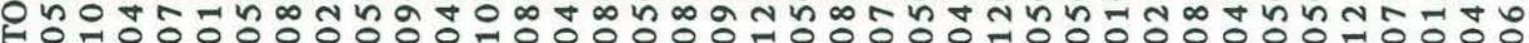
य

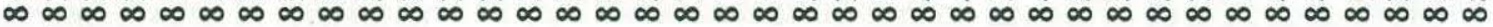

or ontomnanטمO

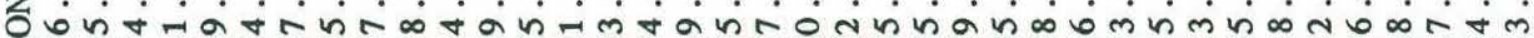

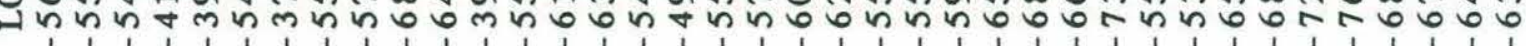
ખ సิ

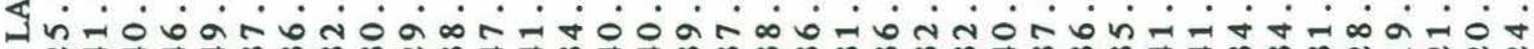
N $\forall \forall \forall$ ल

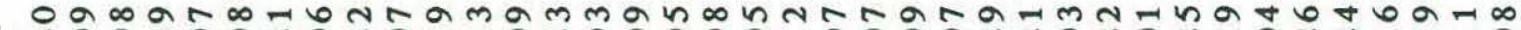
H,

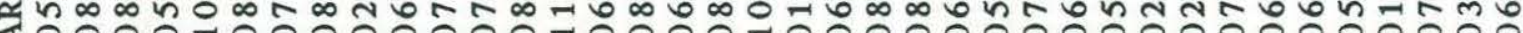

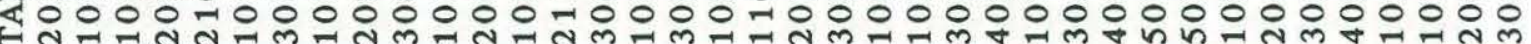

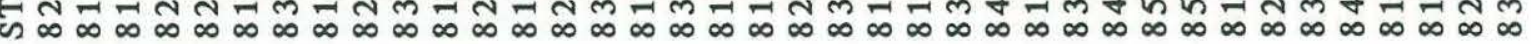

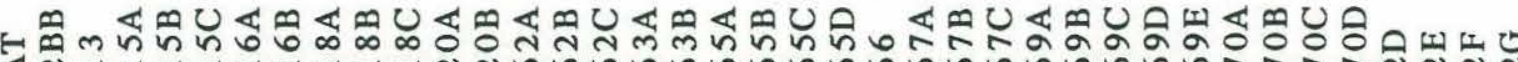
प

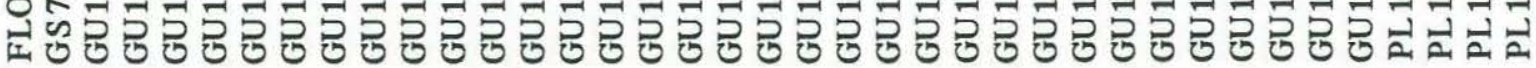

Table 1: Table of float information. 
segment. The initial and average values for temperature and pressure are shown (dash indicates absence of data).

Another float still operating in May 1982 was PL12 which was launched on October 4,1976 , at $24.4^{\circ} \mathrm{N}, 69.0^{\circ} \mathrm{W}$, as part of a pilot study for POLYMODE called Pre-LDE (Spain et al., 1980; McKee, 1986). This float was launched by Riser and Rossby (1983) in an isolated eddy of warm, saline Mediterranean water in the Nares Abyssal Plain. It was ballasted to approximately $900 \mathrm{~m}$ depth in order to track the "meddy". PL12 is the longest operating SOFAR float (Owens et al., 1988). In figures where the floats are divided by depth, PL12 is included with the 700-meter floats.

The other non-GUSREX float covered in this report is GS72B, launched on June 6,1979 , at $38.4^{\circ} \mathrm{N}, 67.9^{\circ} \mathrm{W}$, near but south of the axis of the Gulf Stream (Kennelly and McKee, 1984). This float had a nominal depth of $700 \mathrm{~m}$.

Figure 6 is a histogram of total durations. Excluding PL12 because it had such a different history, the average duration for all the Gulf Stream floats is 1.45 years (1.65 if floats with reused batteries aren't included). The average duration of the 13 floats reported here (without PL12) is 2.8 years, which could be lower than the actual value, since five floats (see plus signs on Figure 6) appeared to still be in operation when the last ALS array was retrieved. Of these, four were deep and one shallow, which agrees with earlier observations that the 700-meter floats were more prone to sudden failure (Kennelly and McKee, 1984). In addition, five other floats were fading at the end of their data records. For at least four of these, moving out of range appears to be a sufficient cause of the decreased signal power. GU120, on the other hand, looks like its lithium batteries, reused from an earlier experiment, were giving out. Alkaline-powered floats tend to die abruptly, as GU113 and GU116 did (Kennelly and McKee, 1984). 


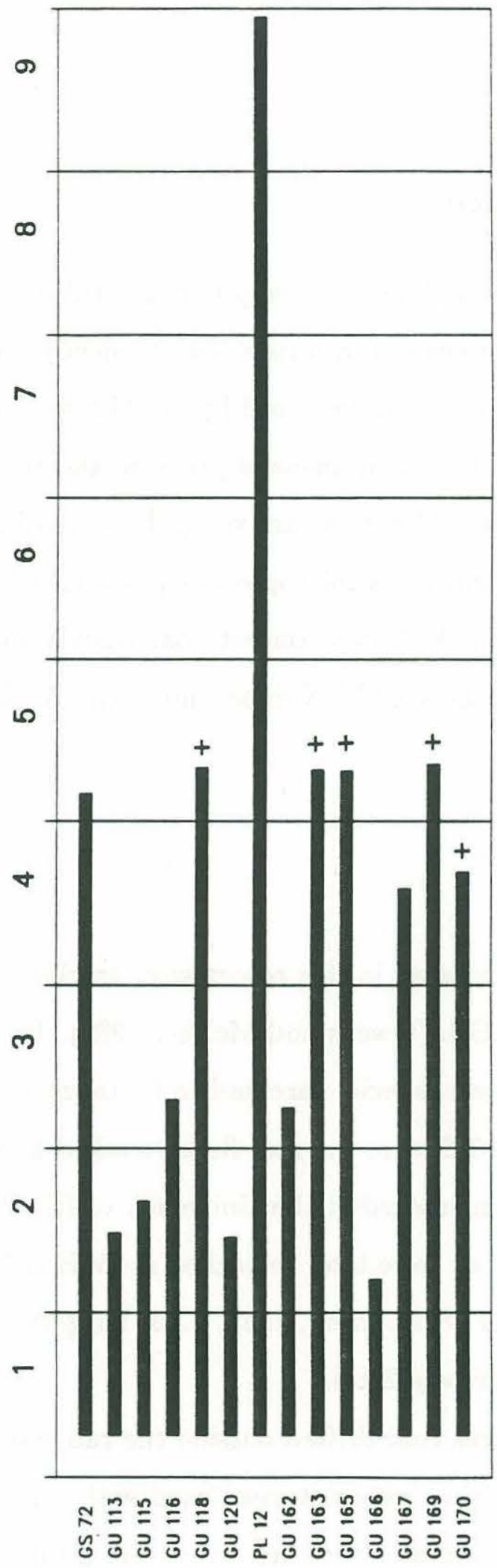

Figure 6: Total duration of floats covered in this report. 


\section{Instrumentation}

SOFAR floats are freely drifting, neutrally-buoyant subsurface instruments. Every eight hours each instrument transmits a low-frequency acoustic signal which, under optimal conditions, can be heard by an ALS at distances on the order of $2500 \mathrm{~km}$. Forty-eight-hour averages of pressure and temperature are transmitted on alternate days. The ALSs are vertical arrays of hydrophones deployed on a subsurface mooring. A microprocessor-controlled detection system records the times of arrival of the four strongest float signals during each ten-minute interval. During the GUSREX experiment, the ALSs were renewed annually.

\section{Processing}

In general, the floats discussed in this report were tracked using the methods which are standard at W.H.O.I. (Owens and McKee, 1989). Because of the long duration of some of these floats, special care had to be taken in calculating clock drifts. The data after May 1982 were the first floats tracked at W.H.O.I. The earlier data had initially been tracked at the University of Rhode Island (see Spain et al., 1980, for technique), but were later retracked at W.H.O.I. for consistency.

Analysis on Site L floats (Price et al., 1987, p.13) suggests that the precision of float positions is approximately $2 \mathrm{~km}$.

Temperatures or pressures that drifted outside the range of the sensors have been deleted, as have values that were not associated with a position.

Trajectories having gaps greater than ten days were broken into subfiles and labelled A, B, C, etc. This was necessary for all floats being tracked in May 1983, since the first setting ALSs ran out of tape before the next ALSs could be deployed. Gaps of less than ten days in position, temperature, or pressure were 
linearly interpolated to the eight-hour interval. See Figures 7 and 8 for times covered by each float. See Figures 9 and 10 for distance traveled by each float, by segment.

These interpolated series were then filtered using a seven point, one-day-half-width Gaussian filter. Finally, a cubic spline was fitted to the filtered positions to produce one location per day, and east and north components of velocity were calculated.

\section{General Information for Individual Float Plots}

A trajectory plot and a group of time series plots are presented for each float in Appendix A. The order of the time series plots is: "stick diagram" ( $u-v$ vectors), $u$ and $v$ velocity component overplot, and temperature and pressure overplot (where data are available). These plots were created with the objective of presenting the data for a particular float; thus the scales vary between floats. The time axis is consistent throughout, with 200 days per page. The time axis is annotated with the last four digits of the Julian day and with the calendar date. Data points are plotted at daily intervals.

The stick plots show a velocity vector for each day, with the stick length proportional to the speed in centimeters per second. North is toward the top of the page. The separate components on the $u$ and $v$ time series are plotted at the same scale.

Temperature and pressure are overplotted, temperature on a centigrade scale marked on the lefthand axis, pressure in decibars on the righthand axis. Pressure is plotted with deeper values at the bottom of the scale.

A trajectory for each float is plotted on a Mercator projection. Open circles denote the first float position, small dots mark the daily positions, large dots every 


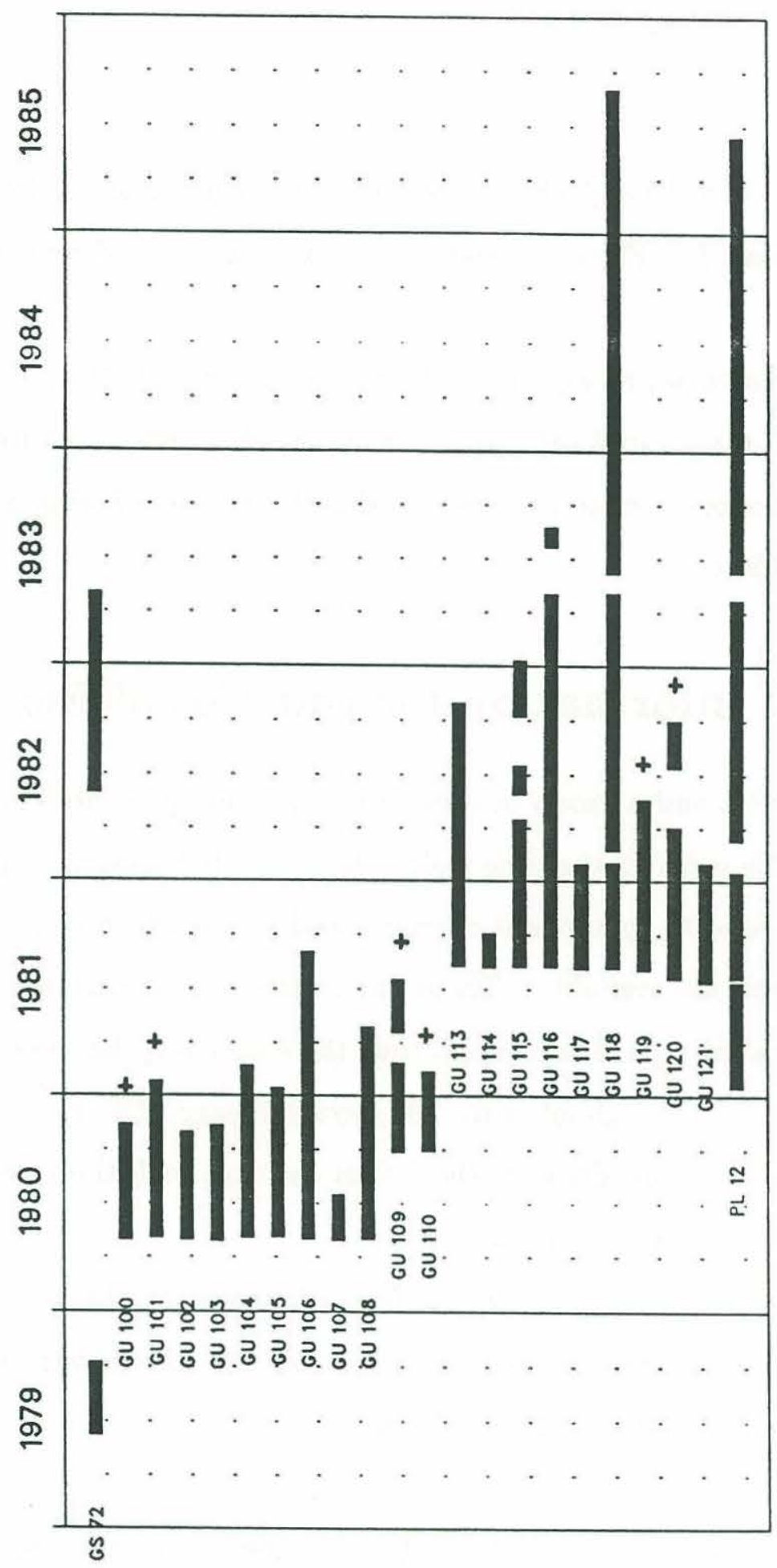

Figure 7: Float duration for all 700-meter GUSREX floats as a time line. + means reused from previous experiment. 


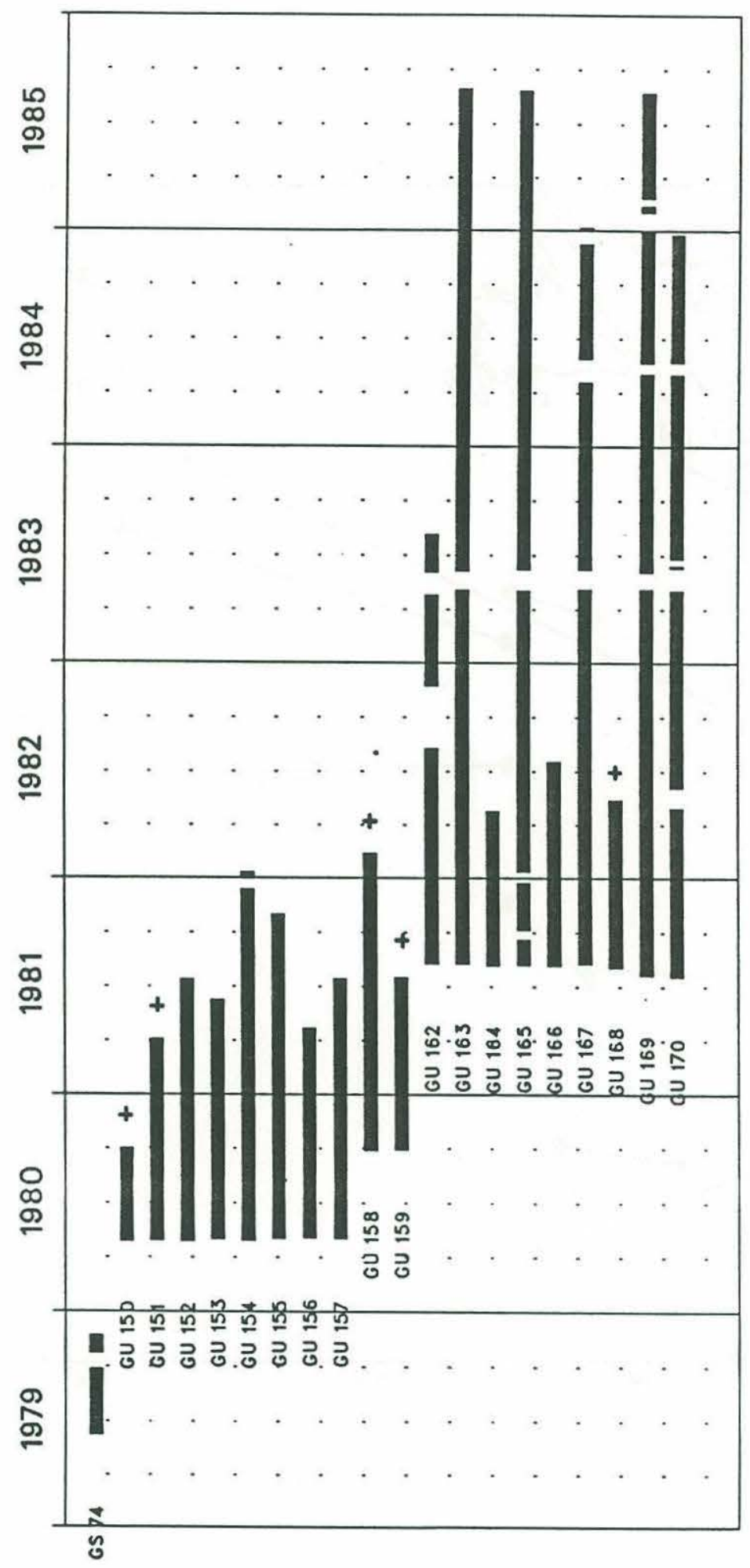

Figure 8: Float duration for all 2000-meter GUSREX floats as a time line. + means reused from previous experiment. 


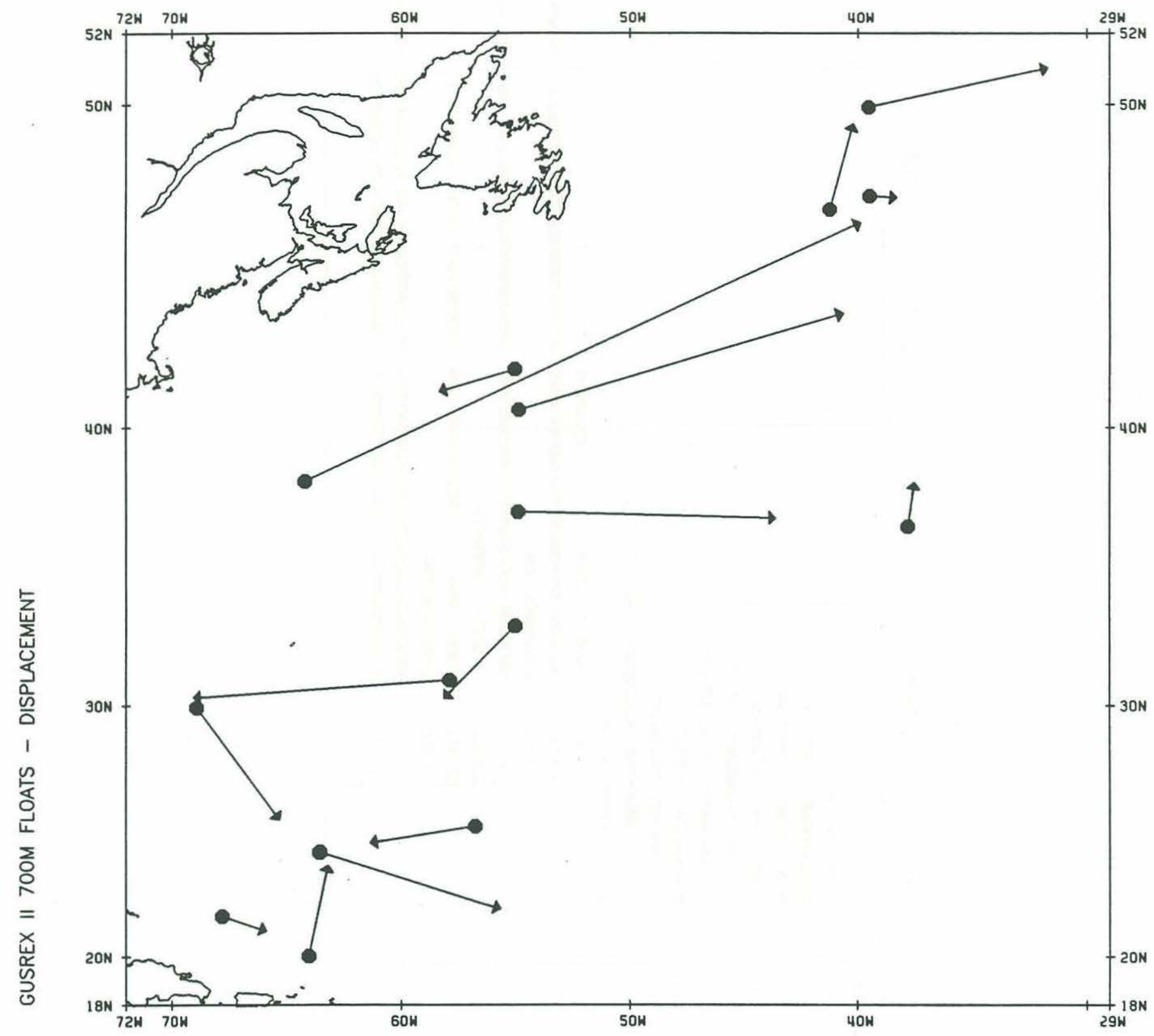

Figure 9: Total displacement of 700-meter floats covered in this report, by segment. Arrows mark final locations. 


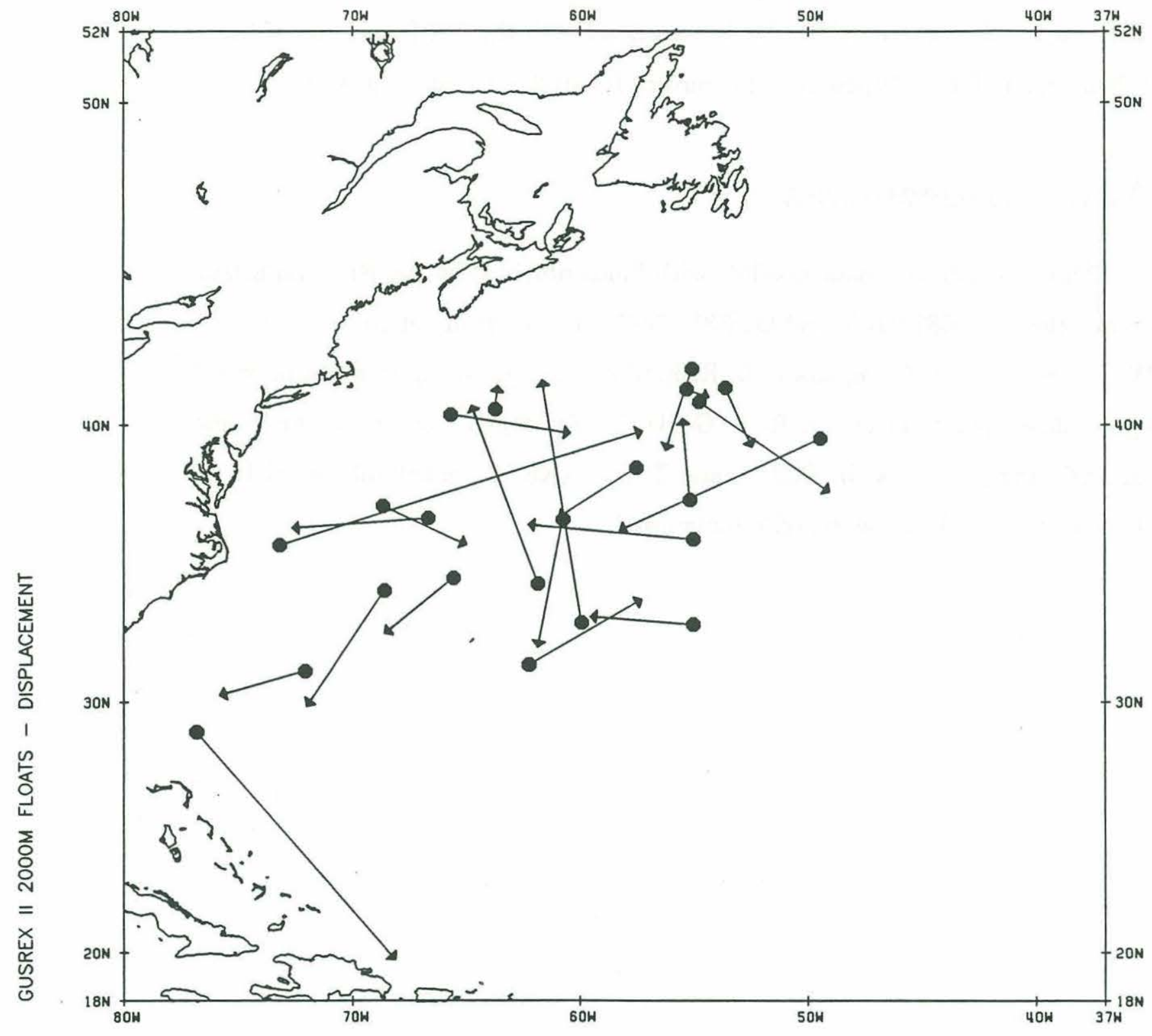

Figure 10: Total displacement of 2000-meter floats covered in this report, by segment. Arrows mark final locations. 
tenth day, and every twentieth day is annotated with the last four digits of the Julian day. Refer to Appendix C to convert Julian day to calendar date.

\section{Acknowledgements}

This research was made possible with funds provided by the National Science Foundation (OCE81-09145 and OCE81-17467). Principal investigators were W. B. Owens, J. F. Price, and P. L. Richardson. Technical support was provided by the float operations group. R. A. Goldsmith developed many of the programs for analyzing float data. B. Gaffron and T. K. McKęe made helpful editorial remarks and M. A. Lucas typed the manuscript. 


\section{References}

Clarke, R. A., H. W. Hill, R. F. Reiniger, and B. A. Warren, 1980. Current system south and east of the Grand Banks of Newfoundland. Journal of Physical Oceanography, 10, 25-65.

Kennelly, M. A., and T. K. McKee, 1984. Gulf Stream Recirculation Experiment (GUSREX) and Line Experiment SOFAR Float Data 1980-1982. Woods Hole Oceanographic Institution Technical Report WHOI-84-45, 444 pp.

McKee, T. K., 1986. A summary of historical SOFAR float data in the western North Atlantic 1972-81. Woods Hole Oceanographic Institution Technical Report WHOI-86-24, $722 \mathrm{pp}$.

Owens, W. B., and T. K. McKee, 1989. Programs and procedures used in processing data recorded by Autonomous Listening Stations (ALSs) for use in tracking freely drifting subsurface floats (SOFAR floats). Woods Hole Oceanographic Institution Technical Report, in press.

Owens, W. B., P. L. Richardson, W. J. Schmitz, Jr., H. T. Rossby, and D. C. Webb, 1988. Nine-year trajectory of a SOFAR float in the southwestern North Atlantic. Deep-Sea Research, 35, 1851-1857.

Price, J. F., T. K. McKee, W. B. Owens, and J. R. Valdes, 1987. Site L SOFAR Float Experiment, 1982-1985. Woods Hole Oceanographic Institution Technical Report WHOI-87-52, 289 pp.

Riser, S. C., and H. T. Rossby, 1983. Quasi-Lagrangian structure and variability of the subtropical Western North Atlantic circulation. Journal of Marine Research, 41, 127-162. 
Spain, D. L., R. M. O'Gara, and H. T. Rossby, 1980. SOFAR float data report of the POLYMODE Local Dynamics Experiment. University of Rhode Island Technical Report No. 80-1, 200 pp.

Worthington, L. V., 1976. On the North Atlantic circulation. The Johns Hopkins Oceanographic Studies, 6, 110 pp. 
APPENDIX A: Individual Float Plots 


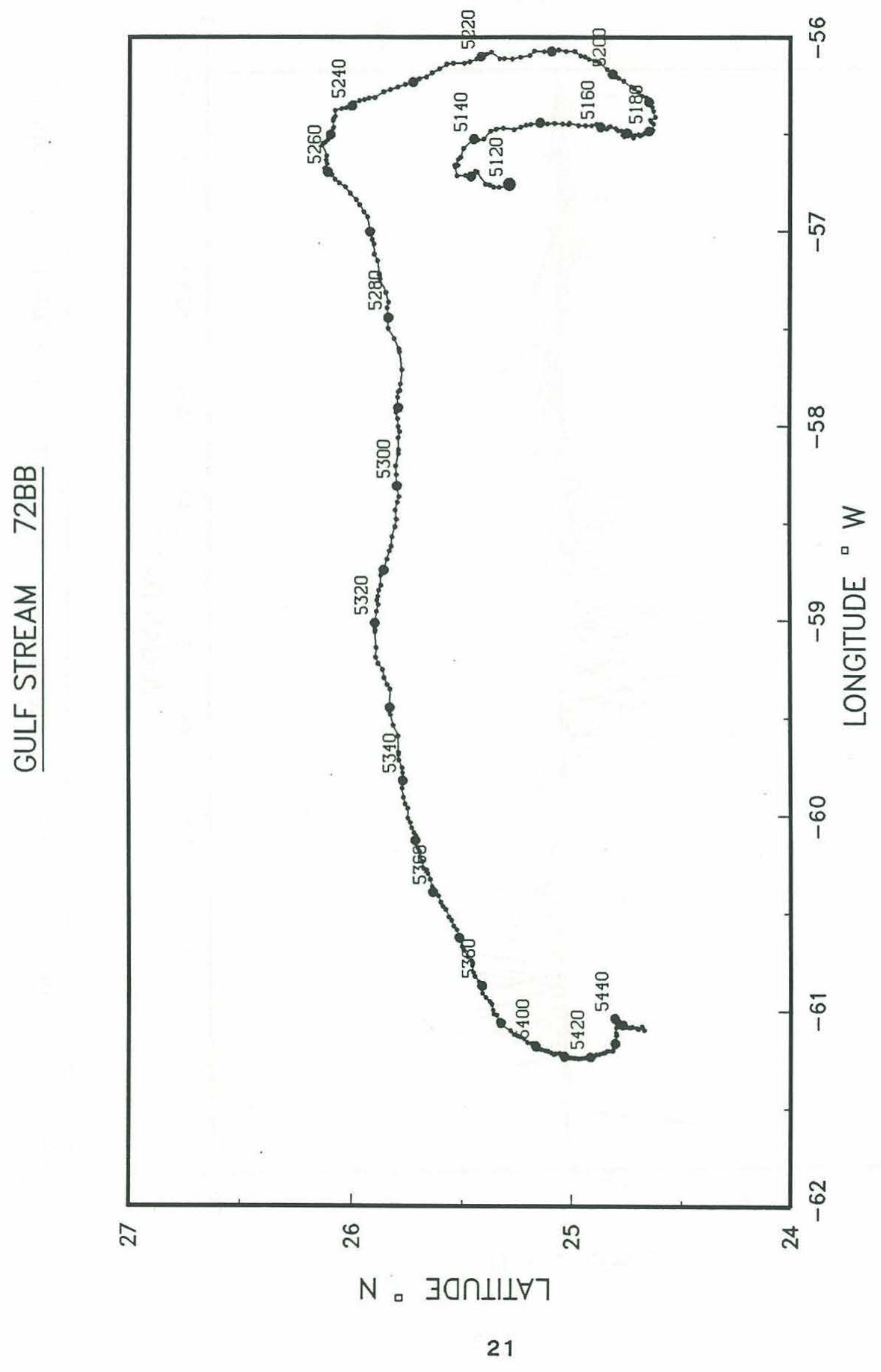




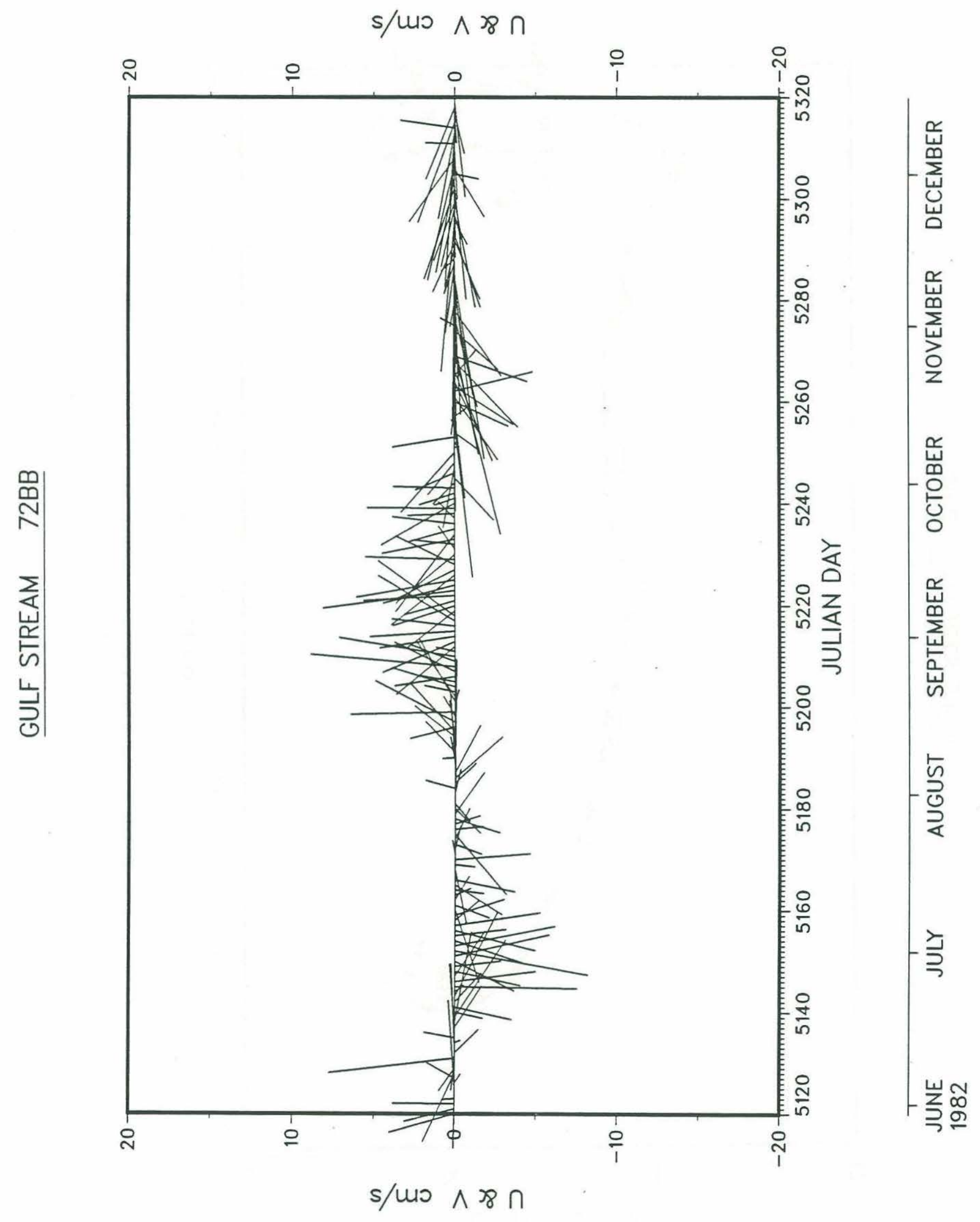




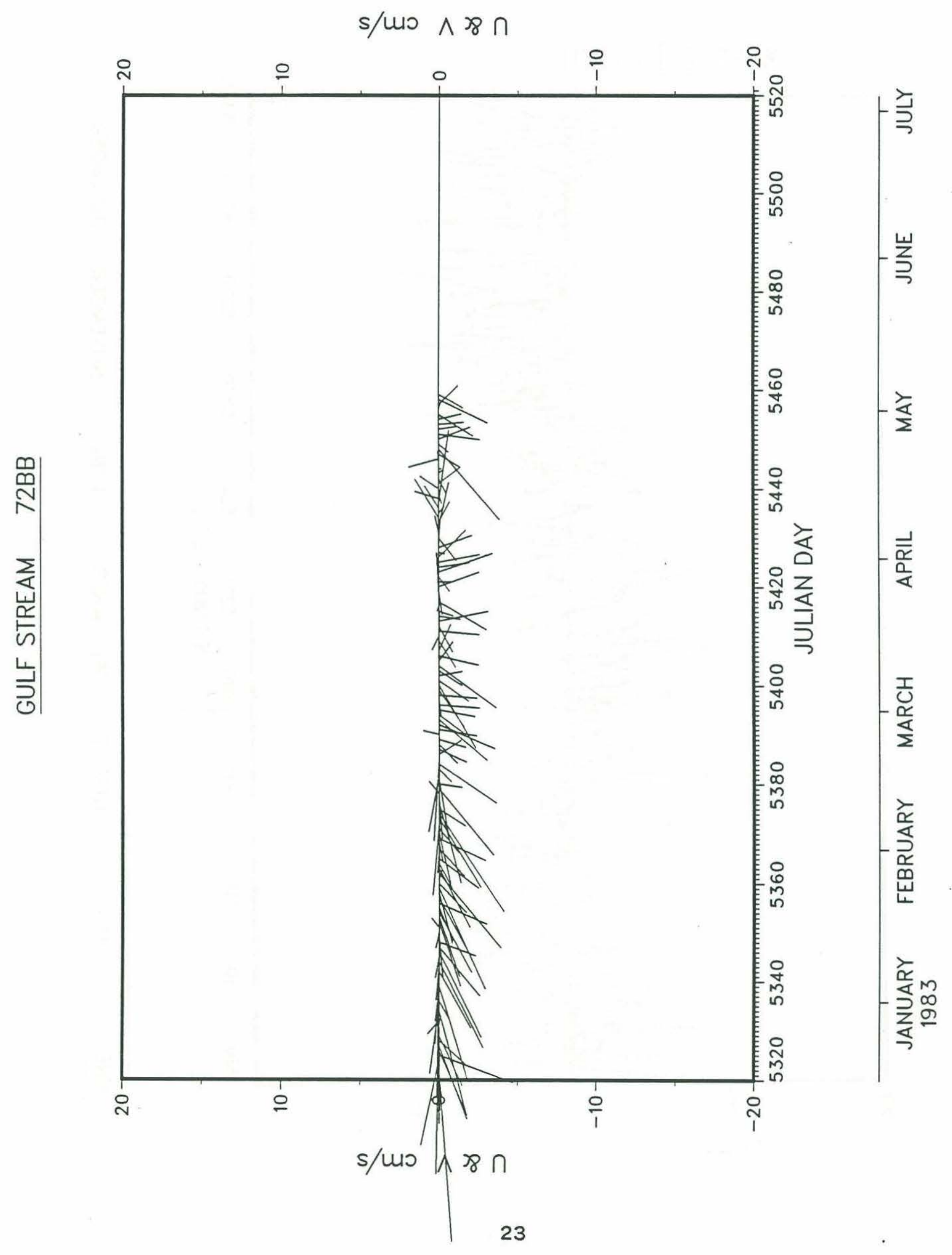




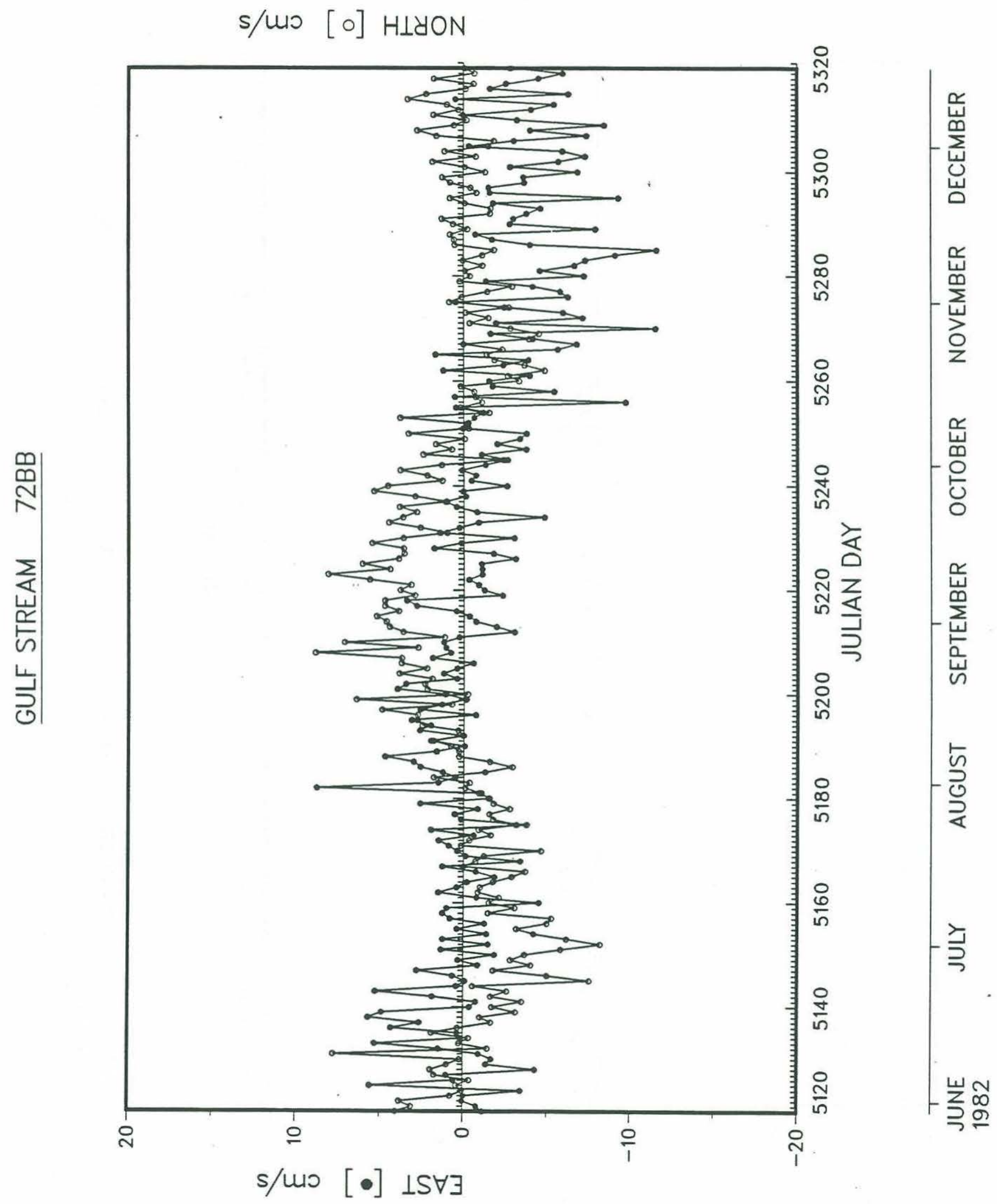




\section{s/Mo [o] HIYON}

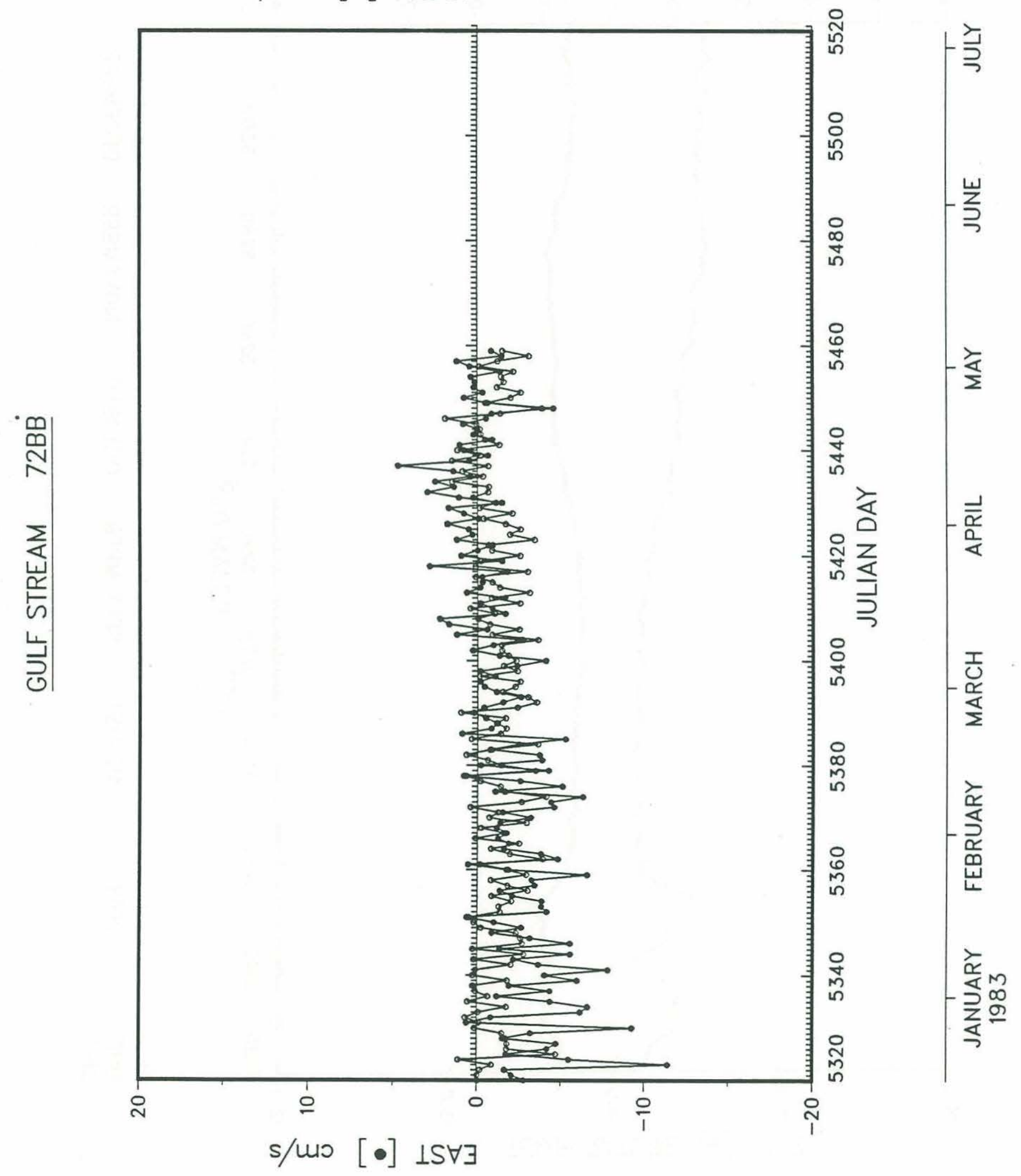




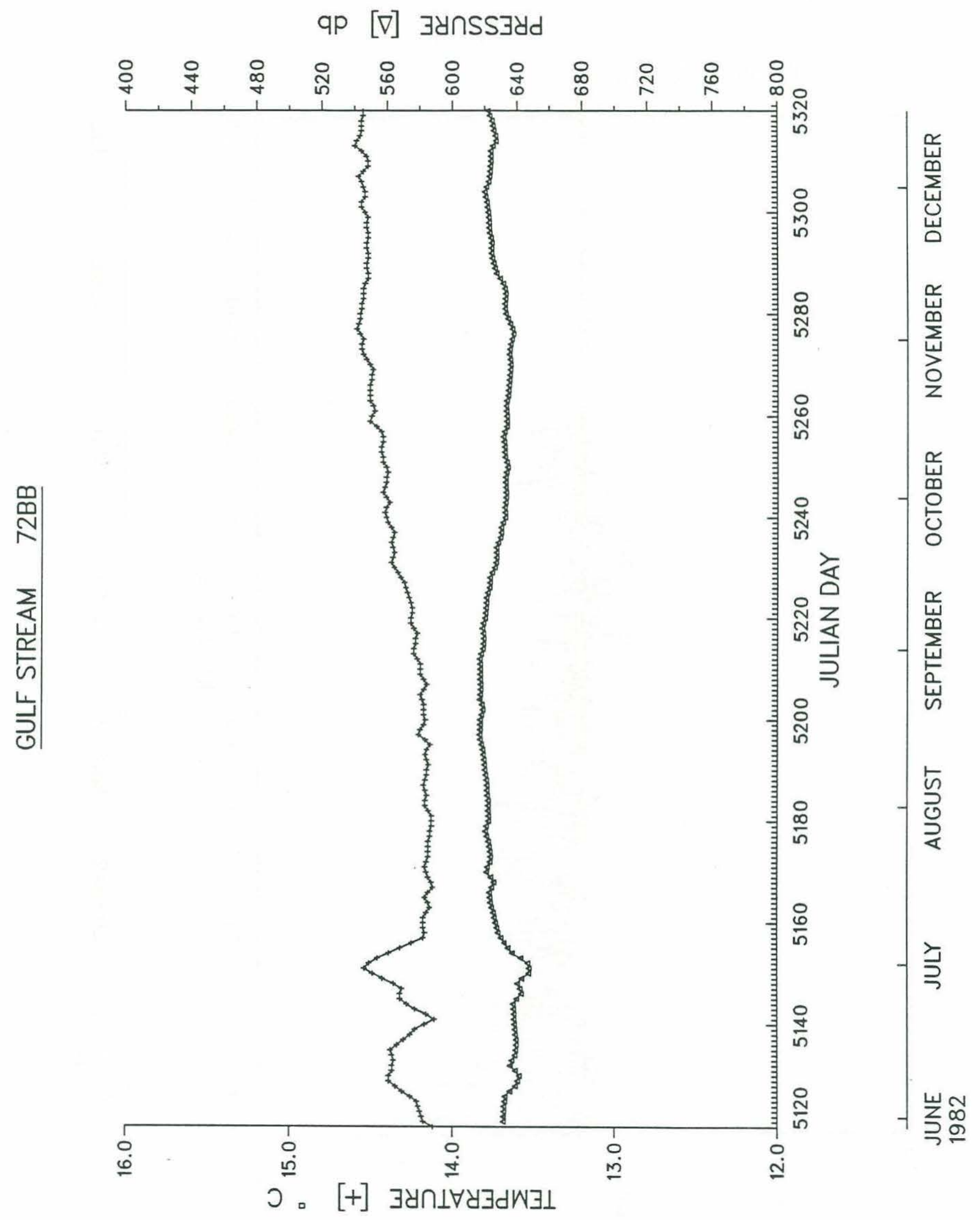




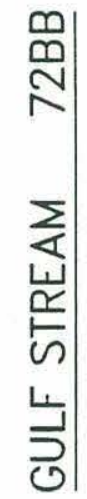

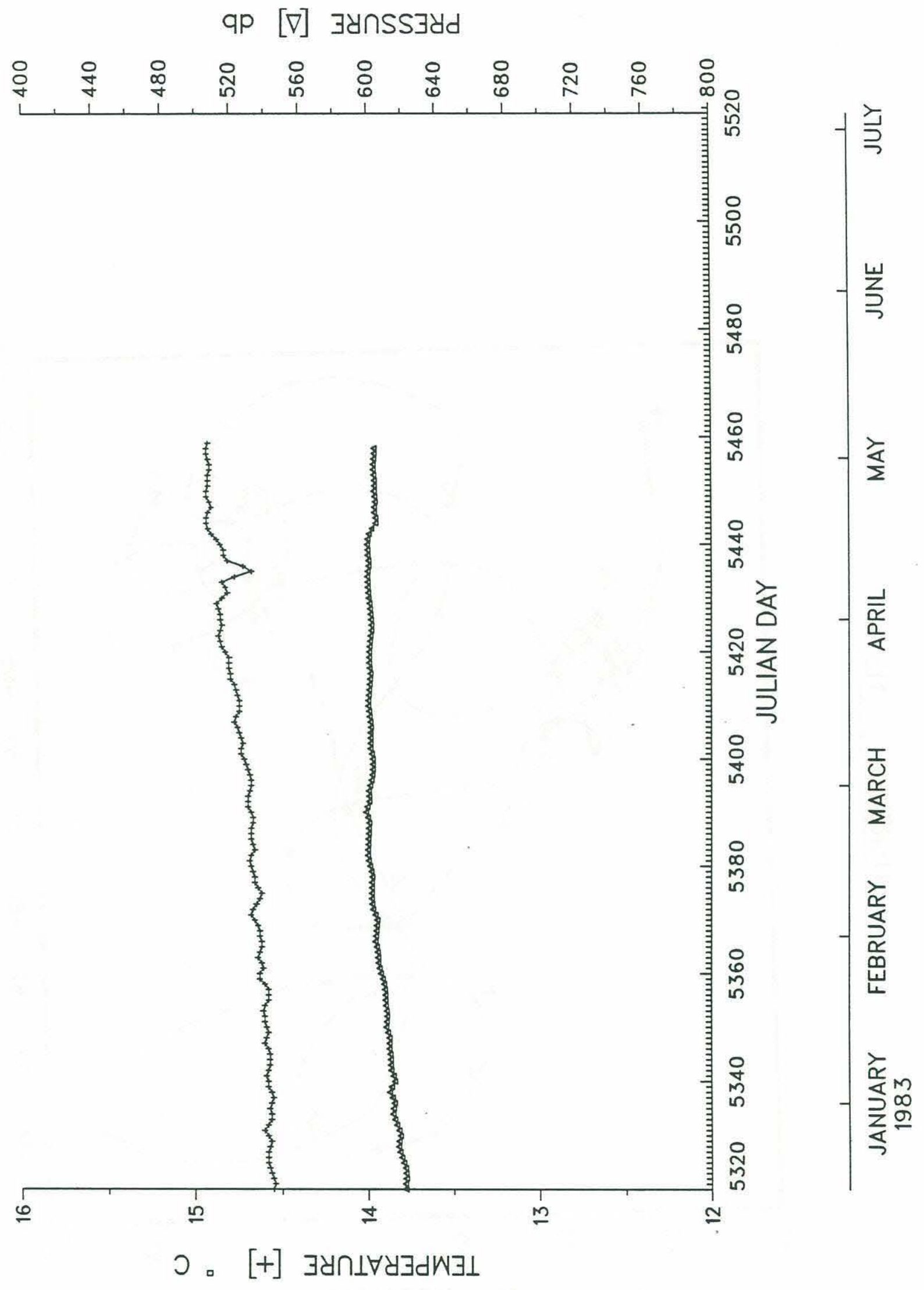




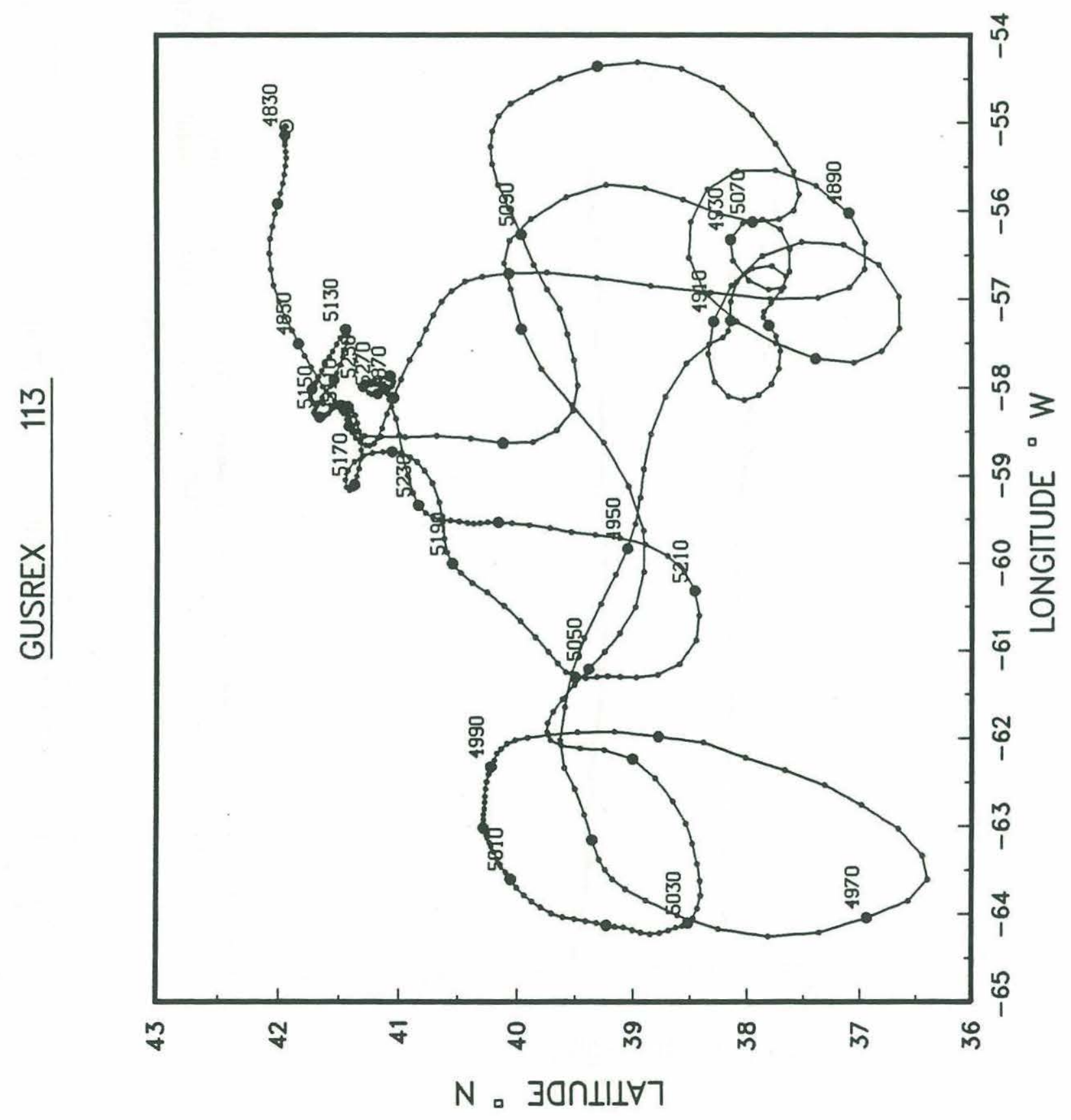




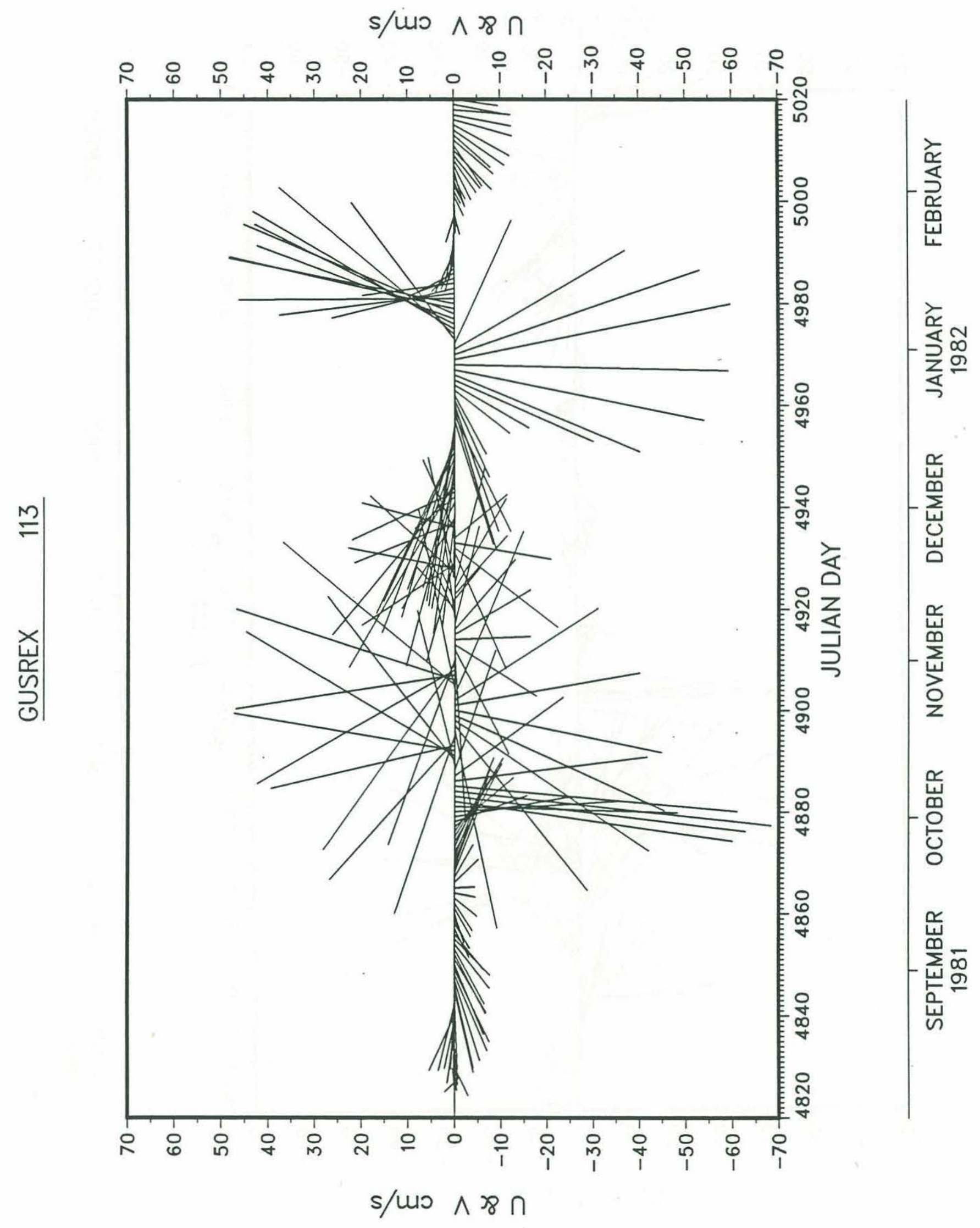




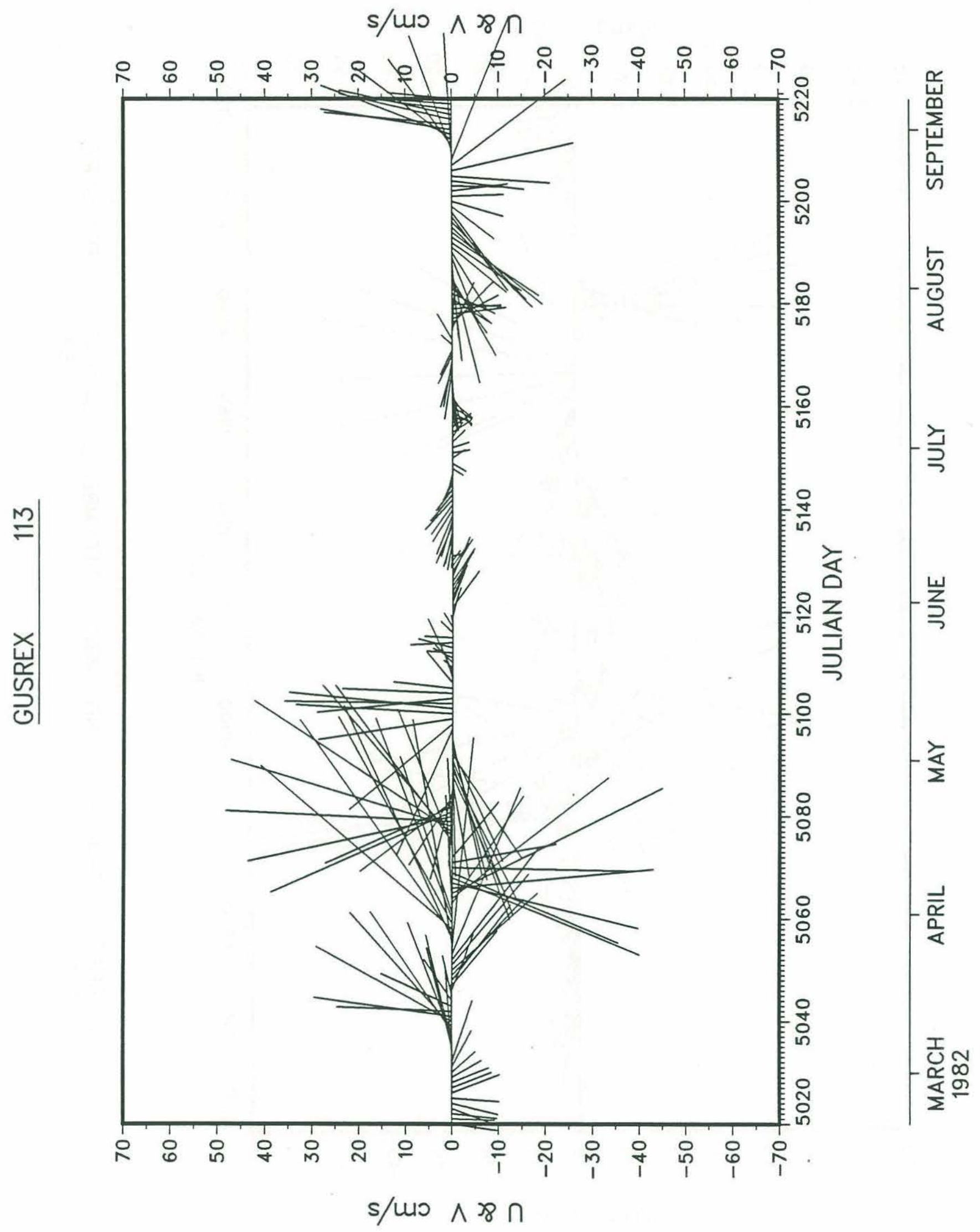




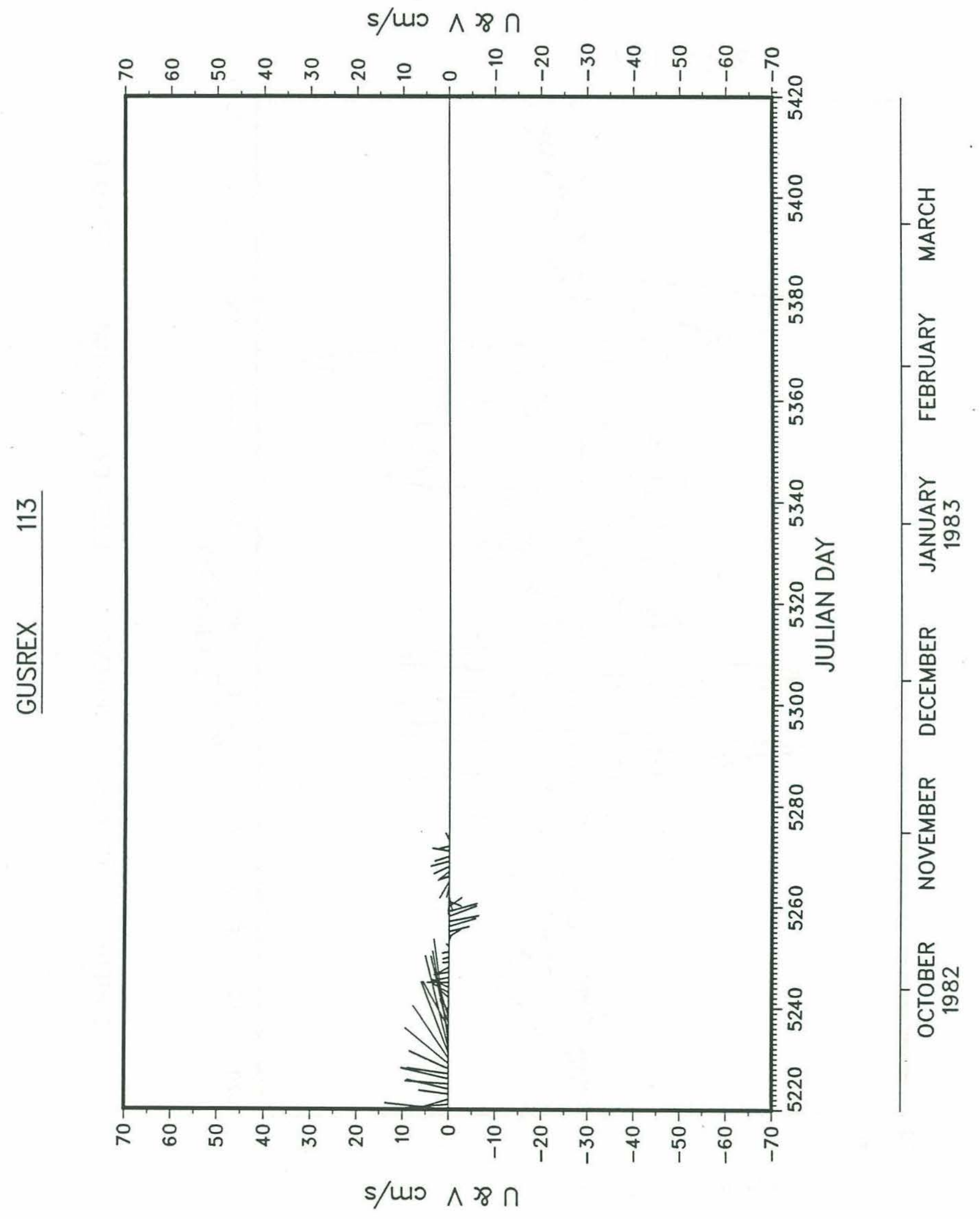




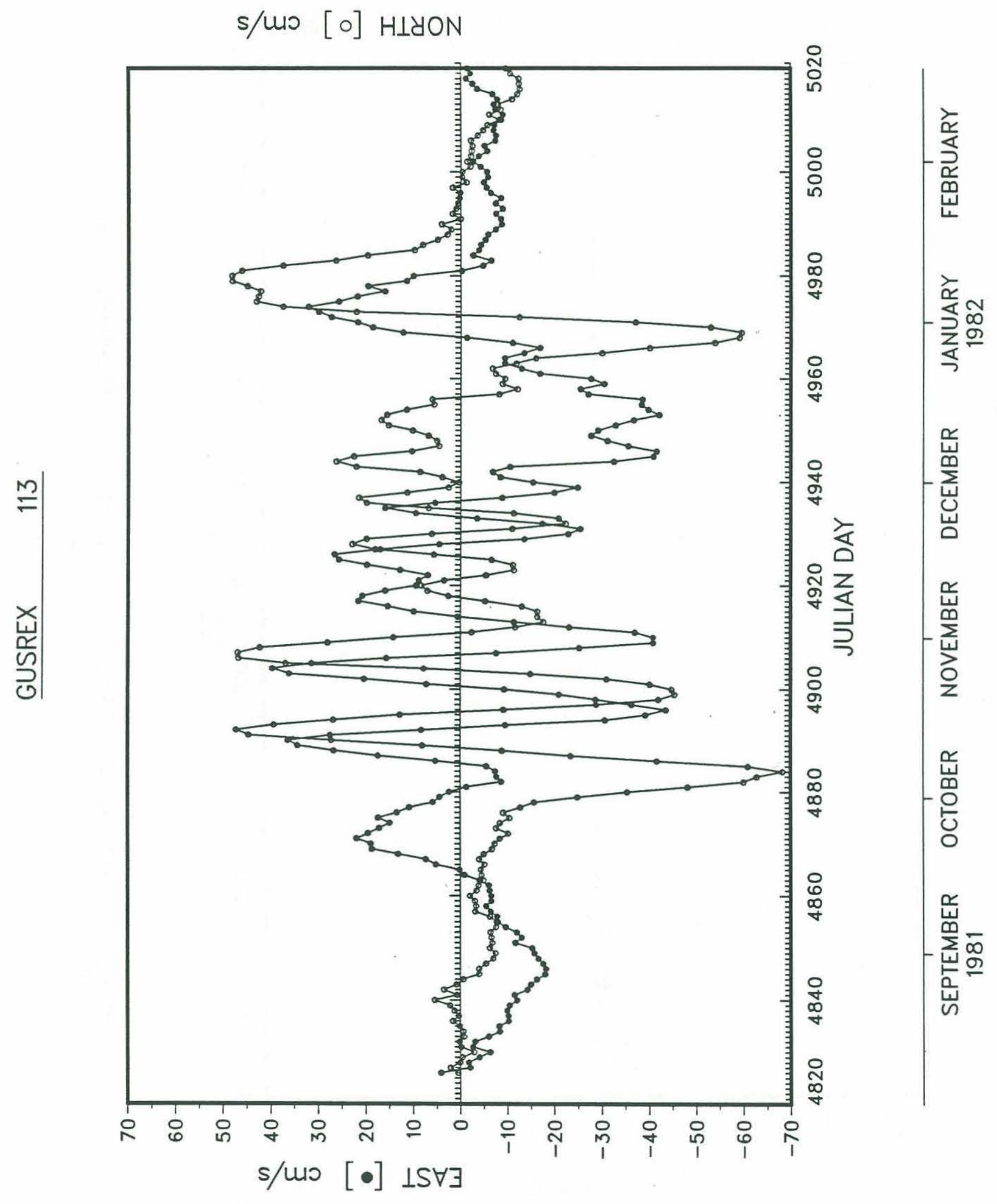




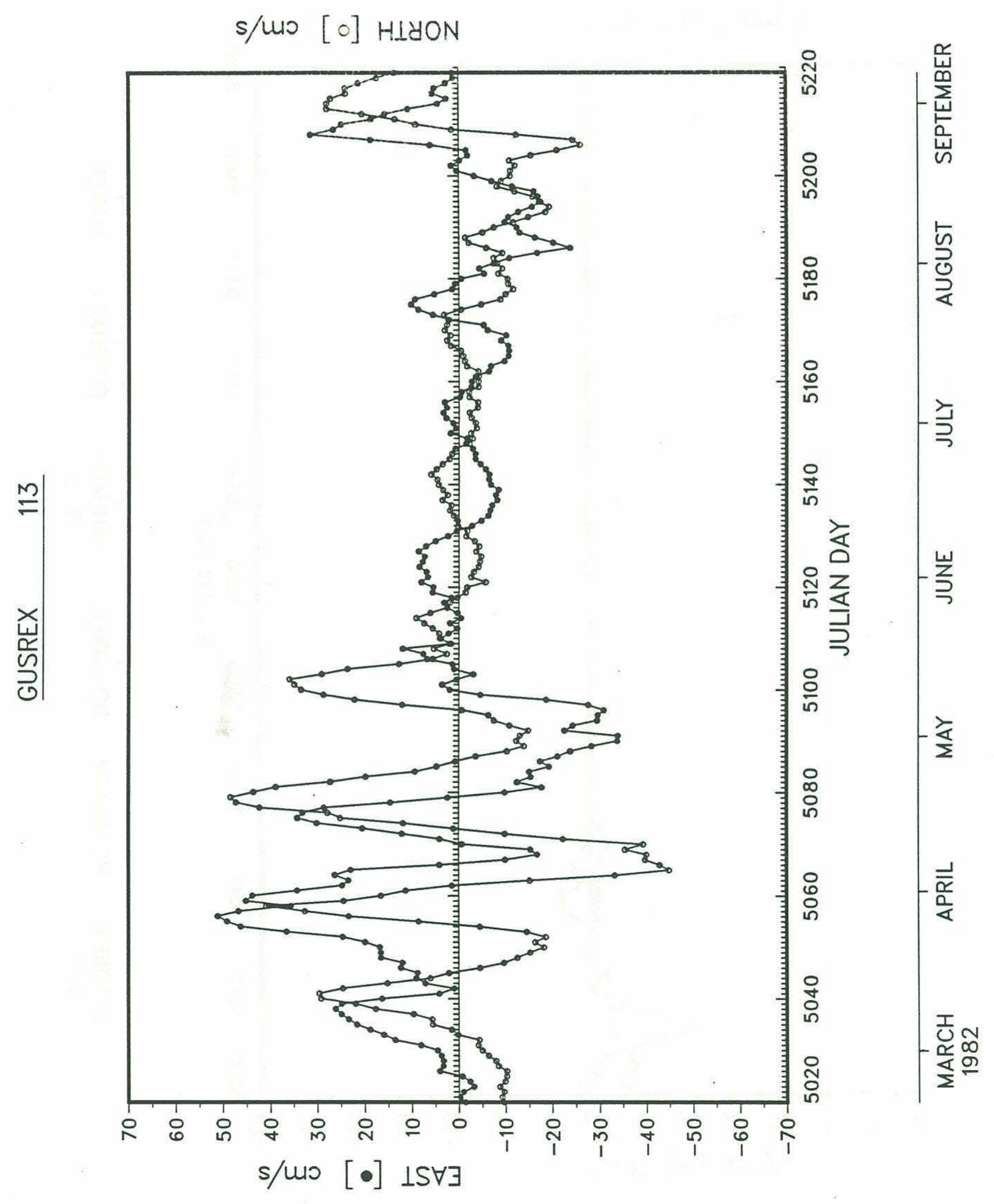




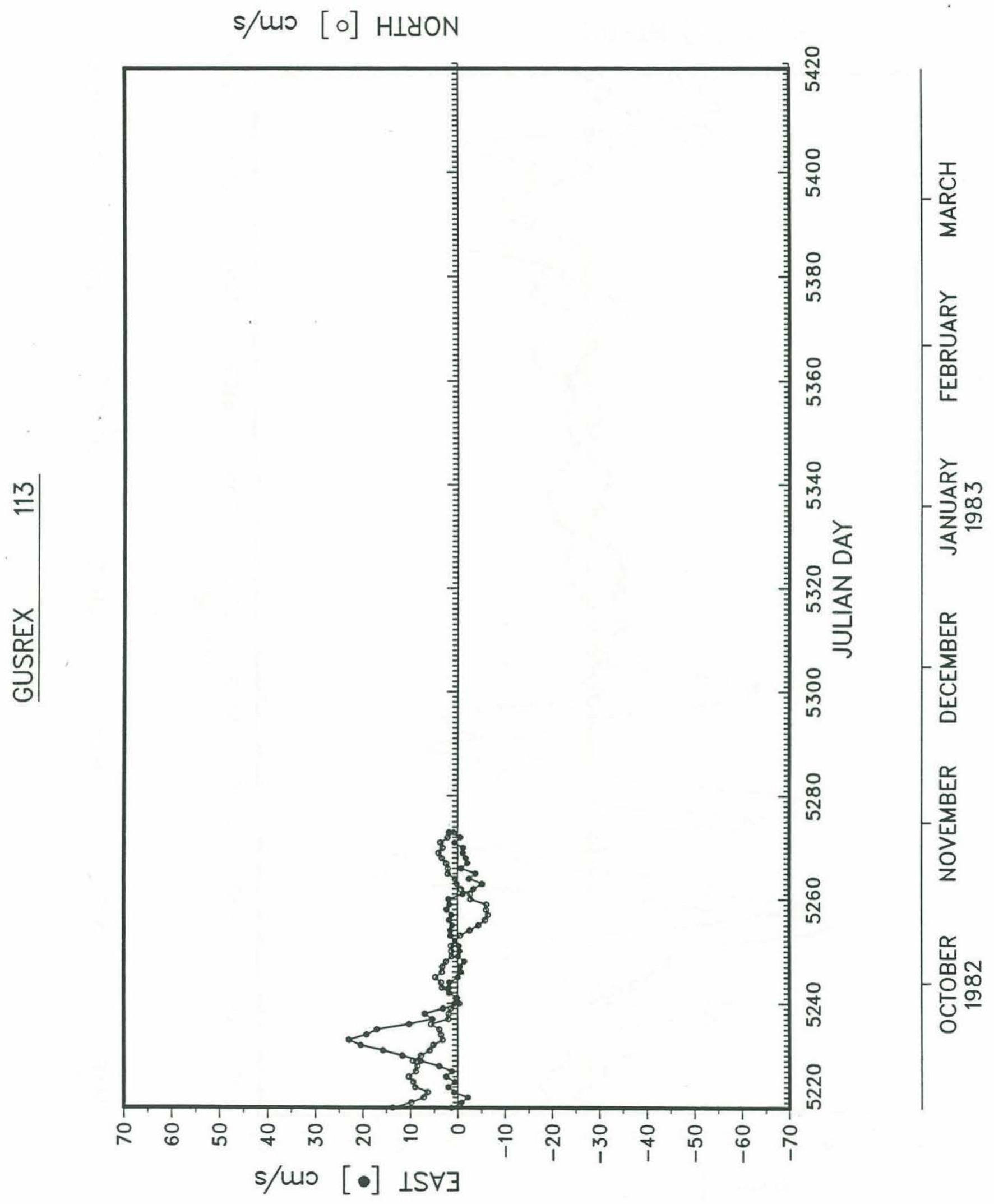




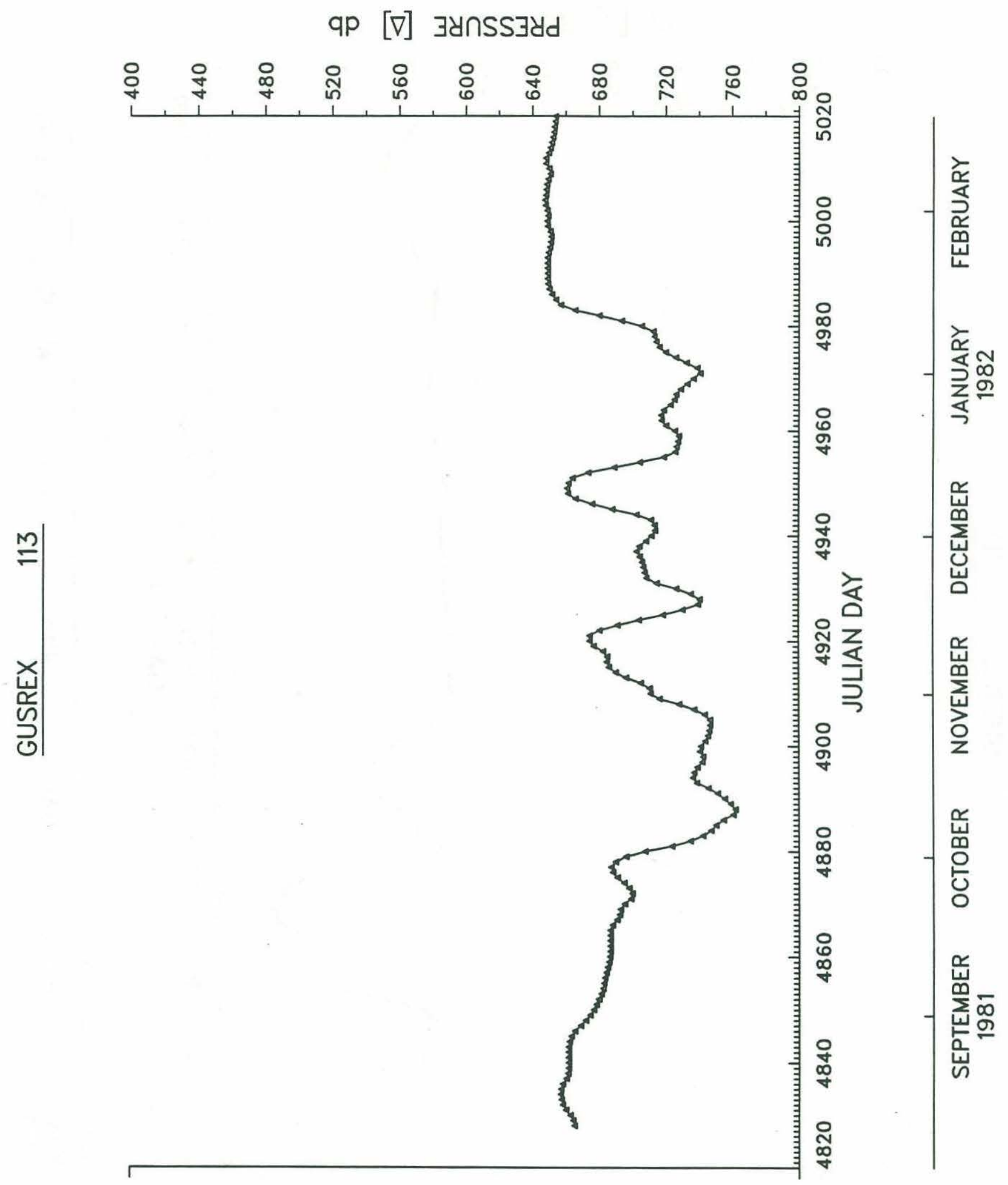




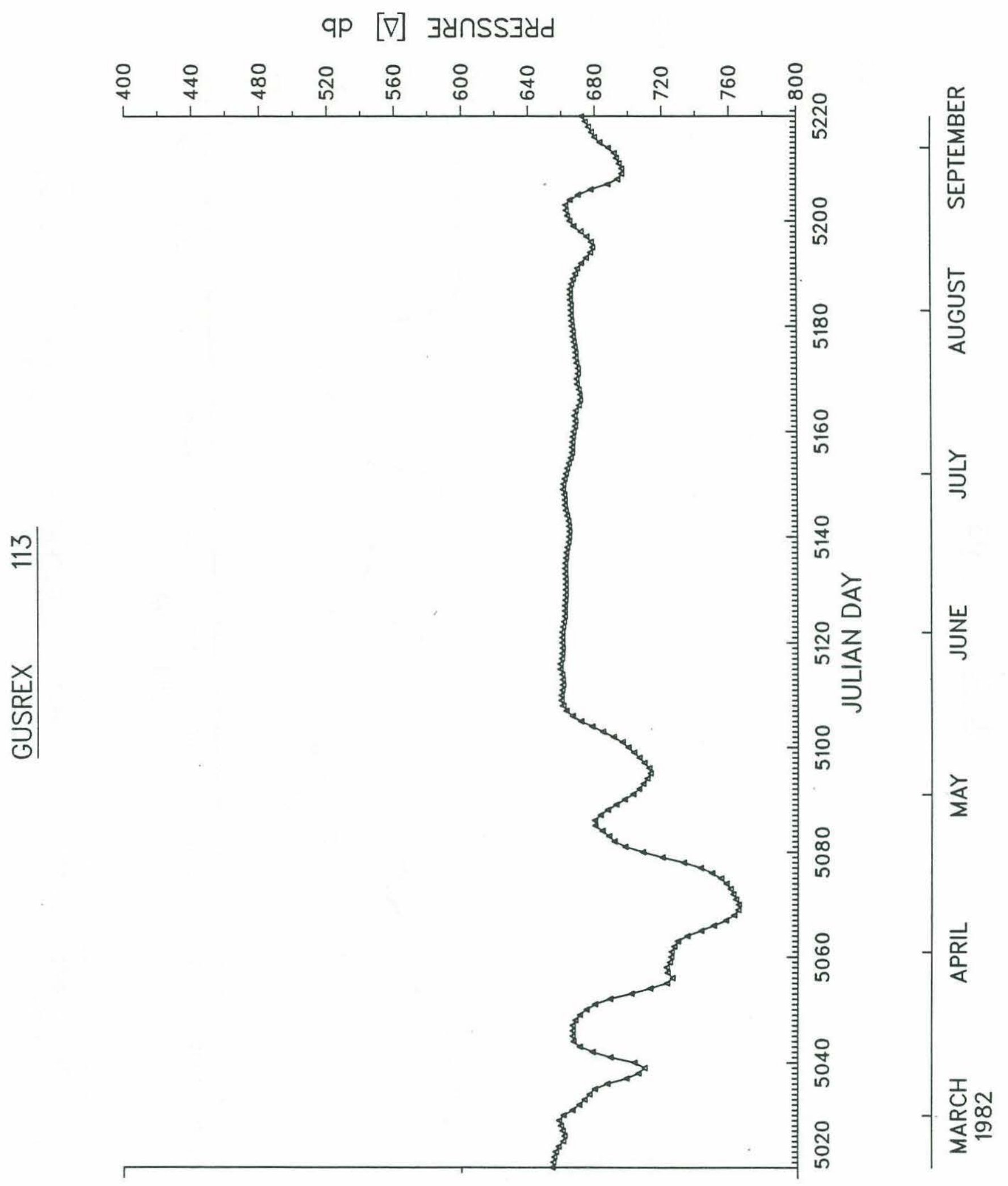




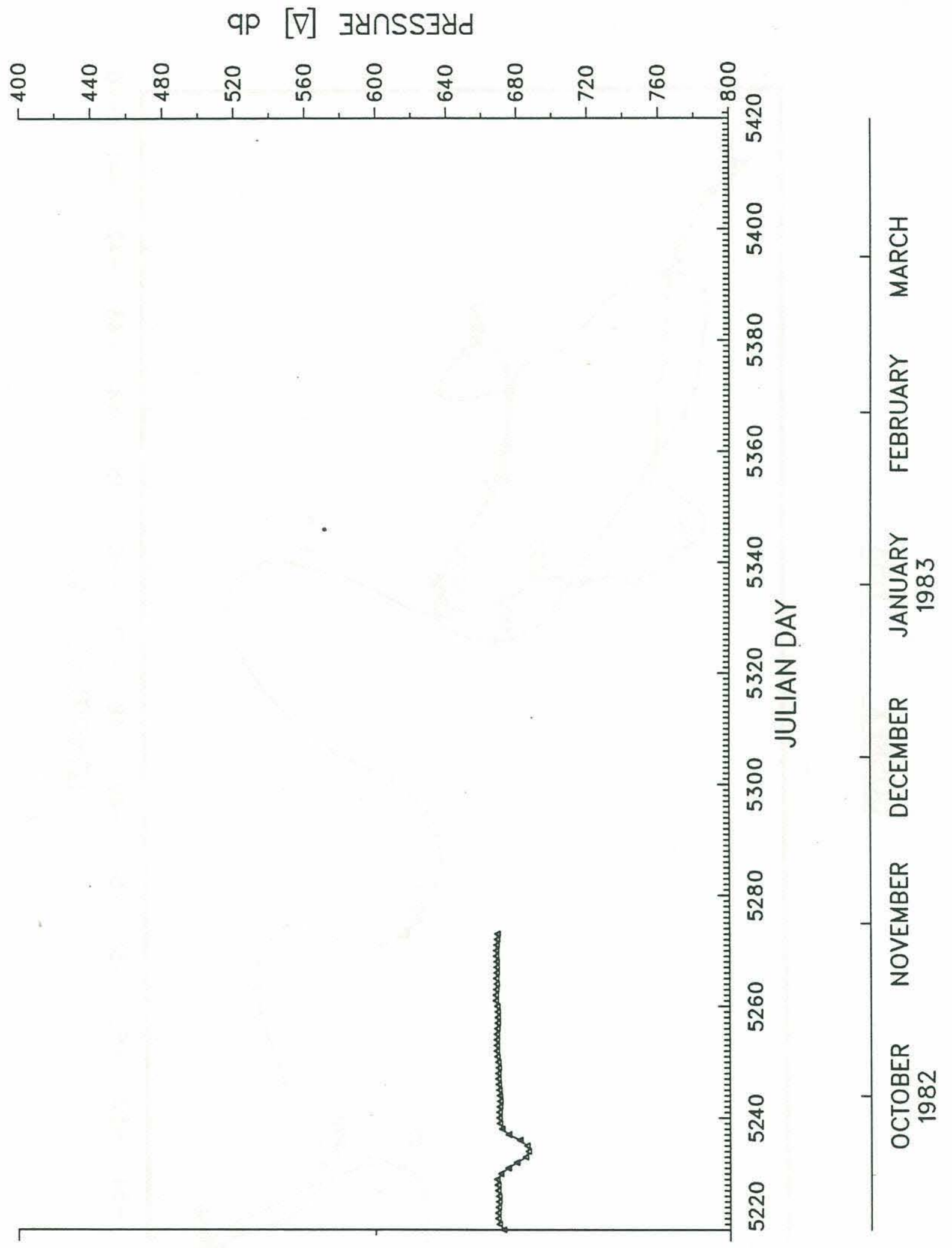




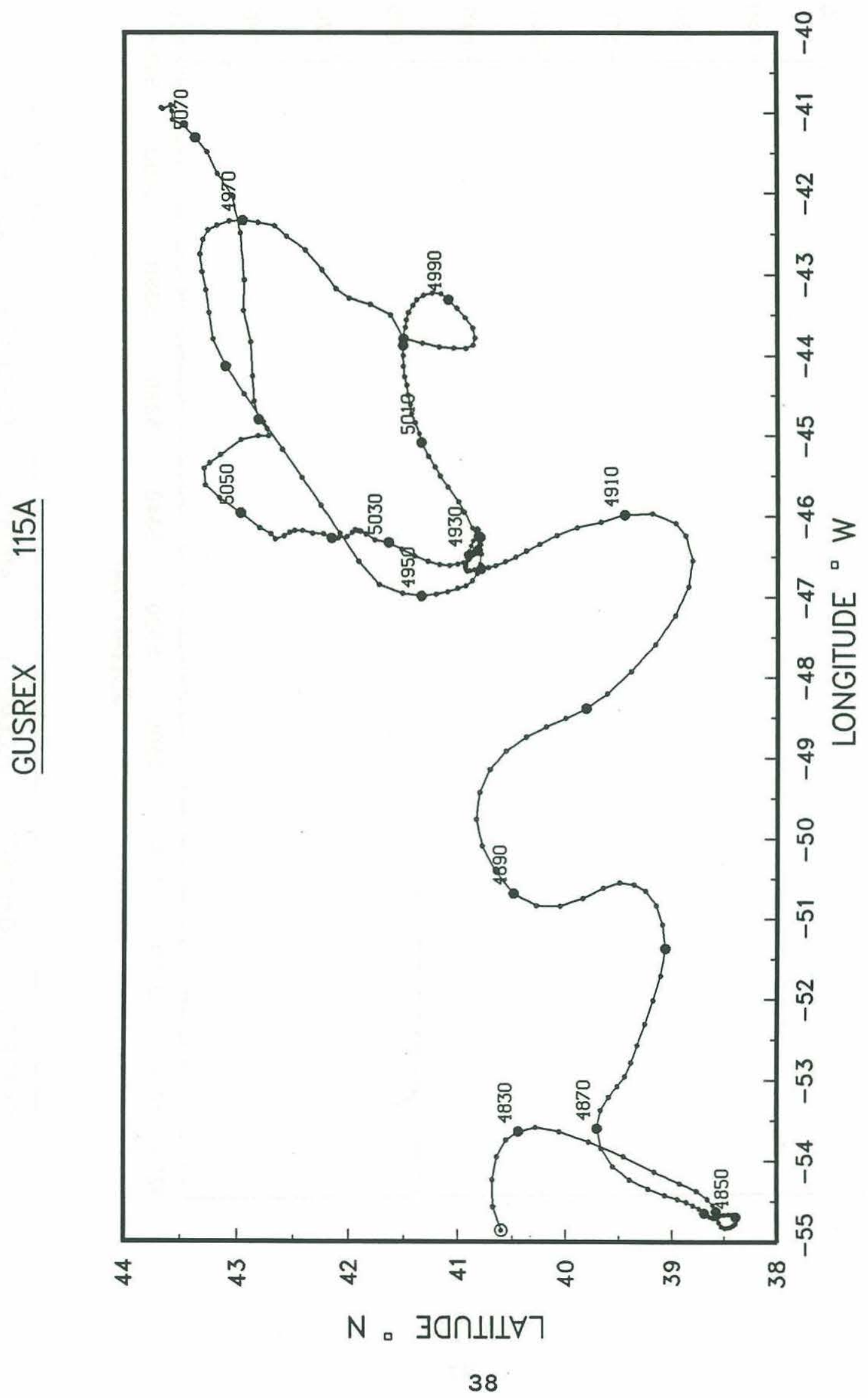




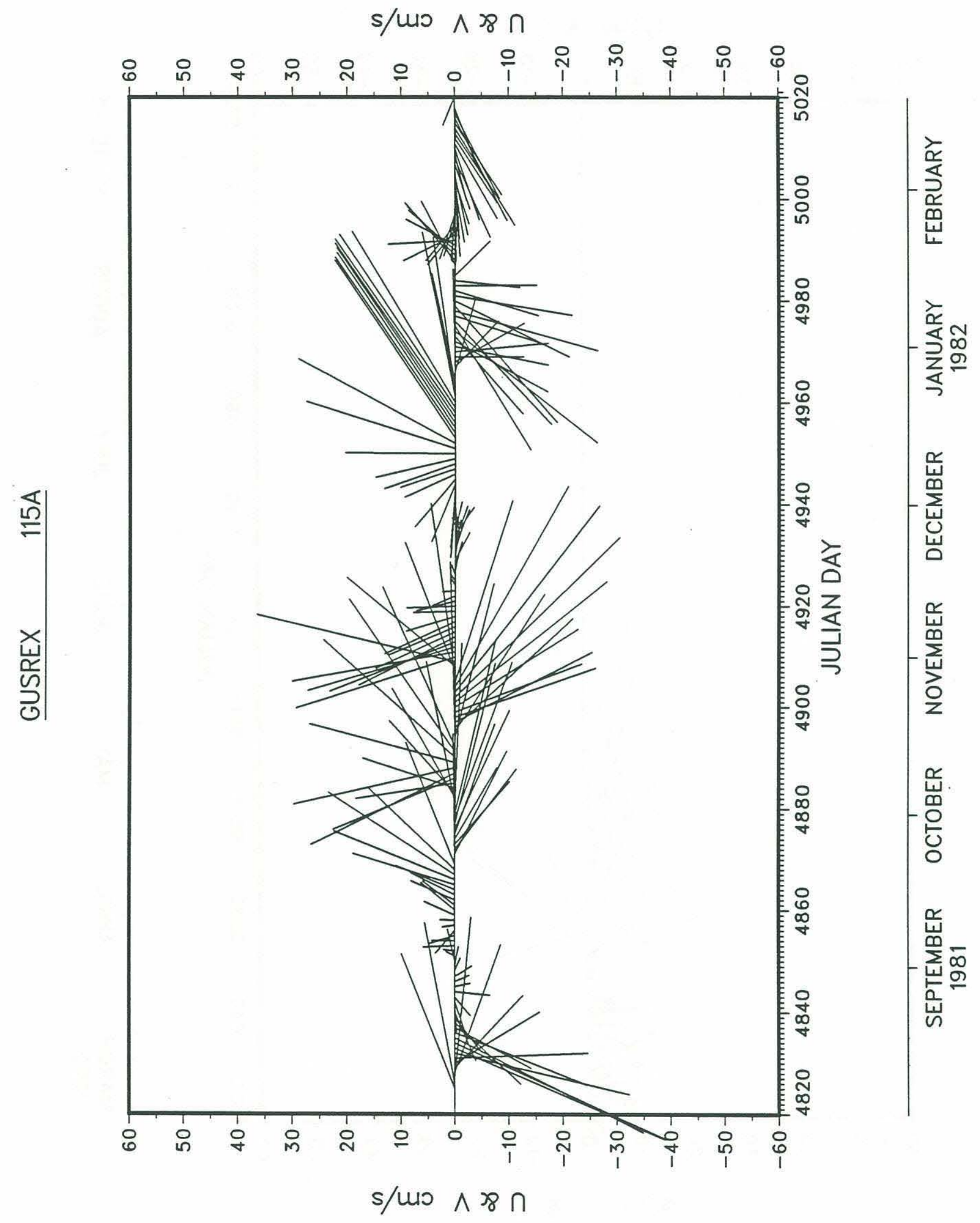




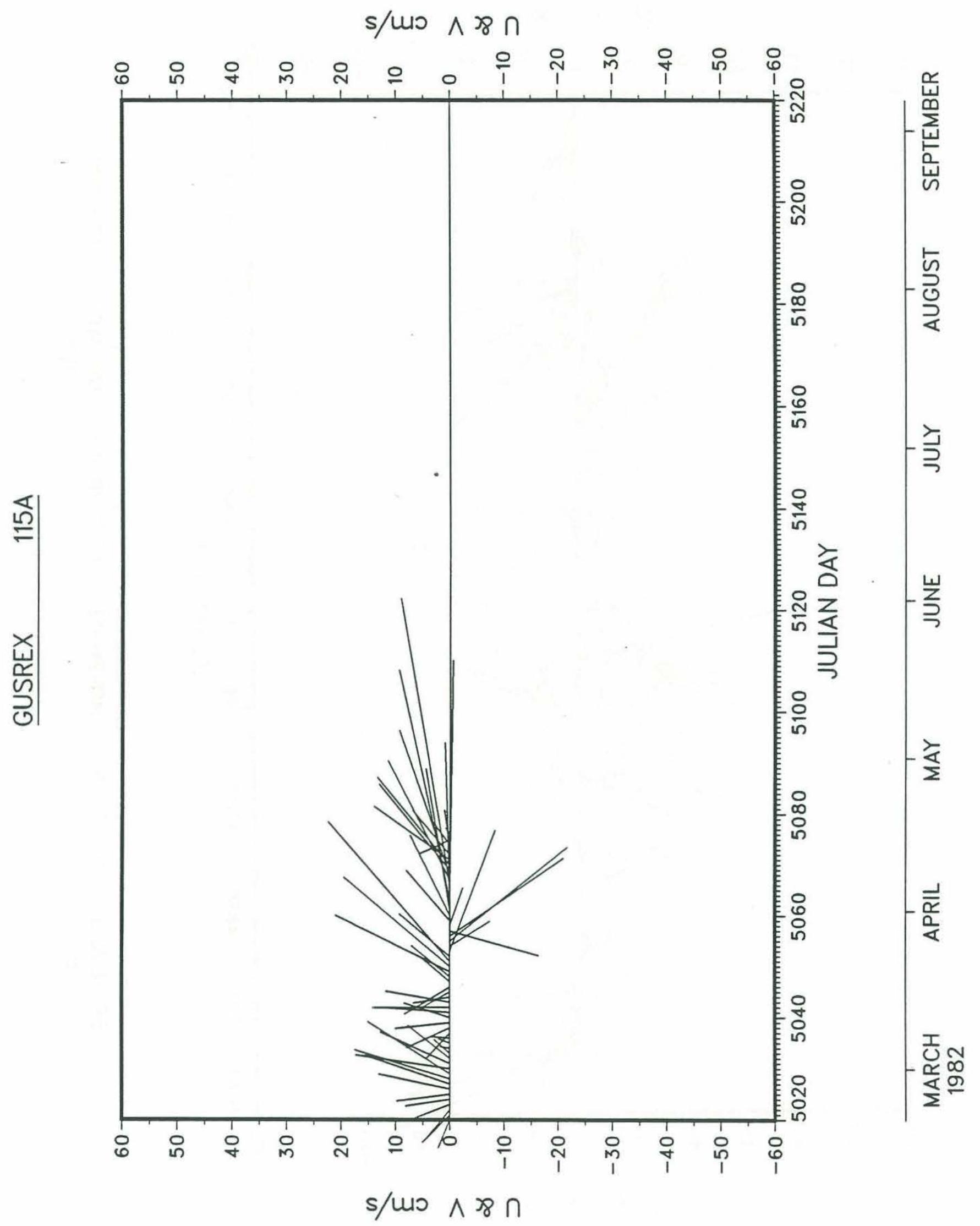




\section{s/uo [ o] HIVON}

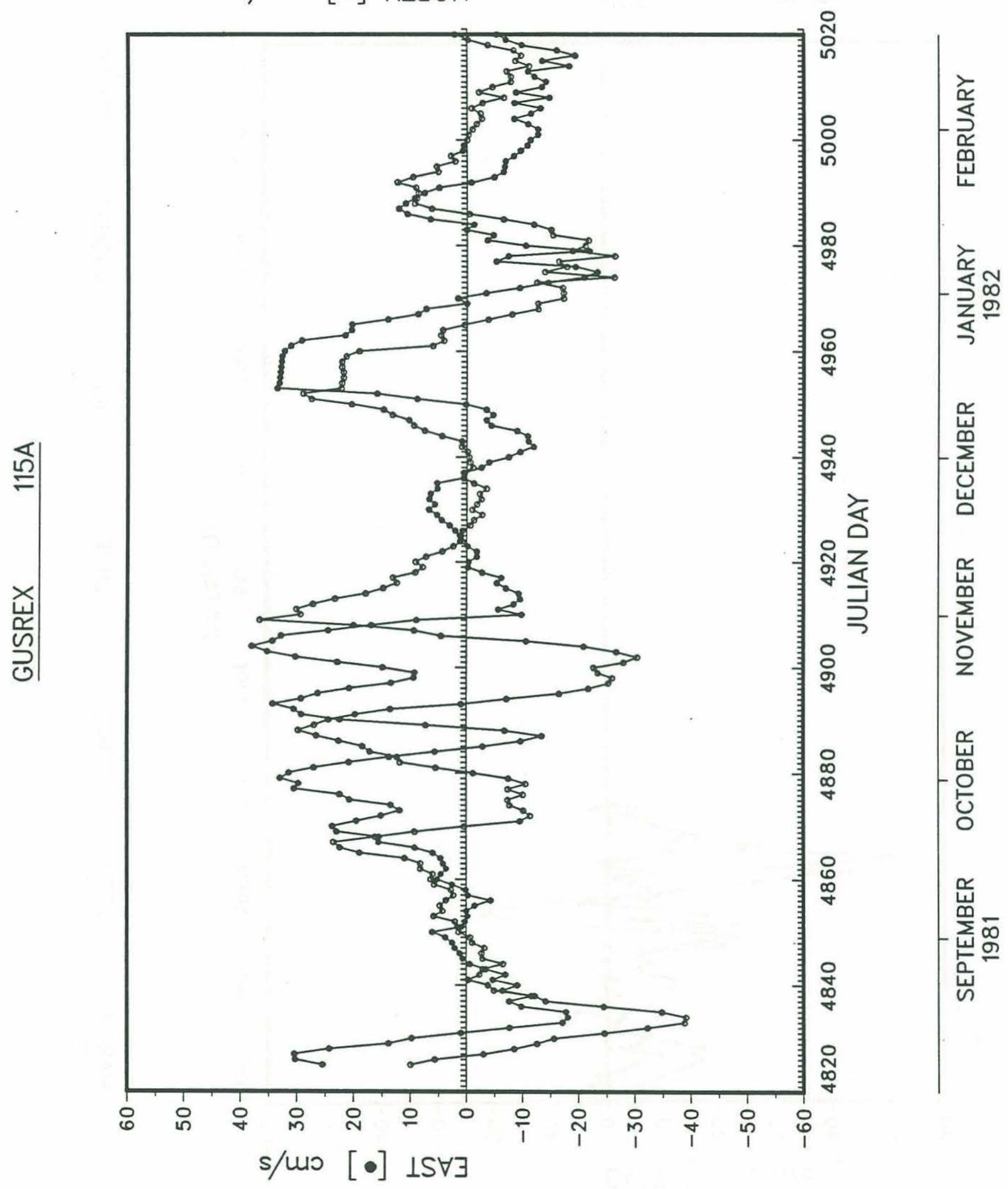




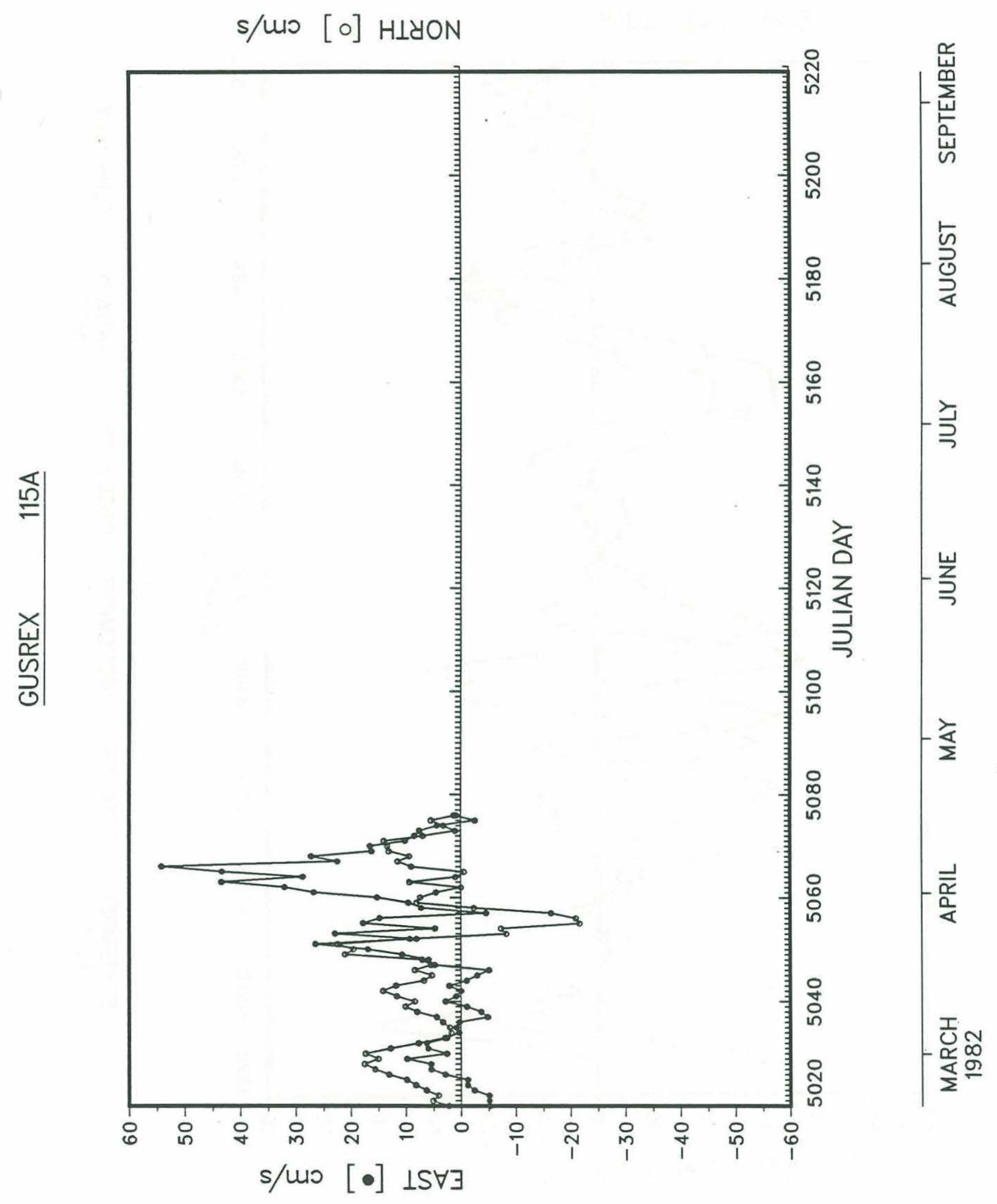



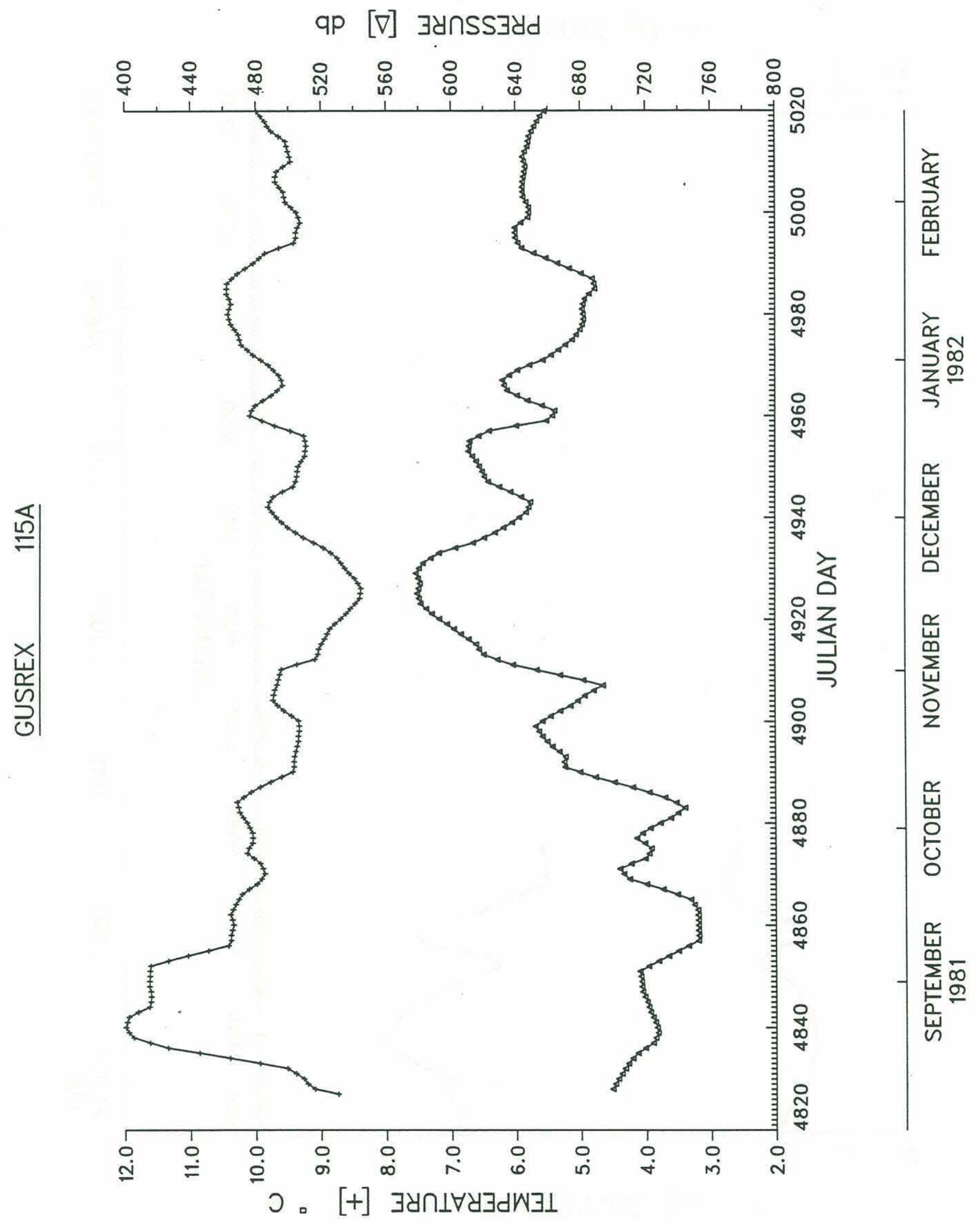


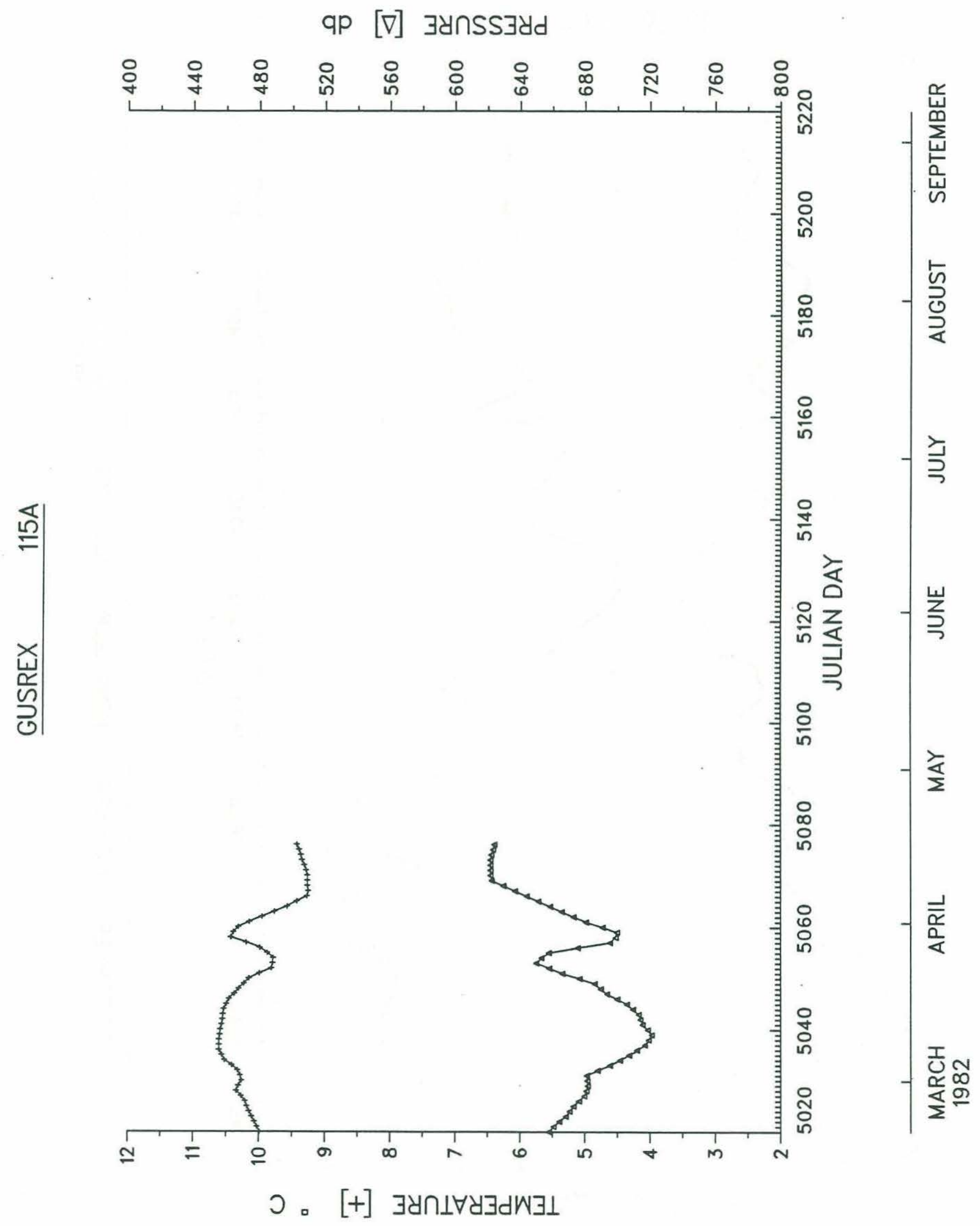




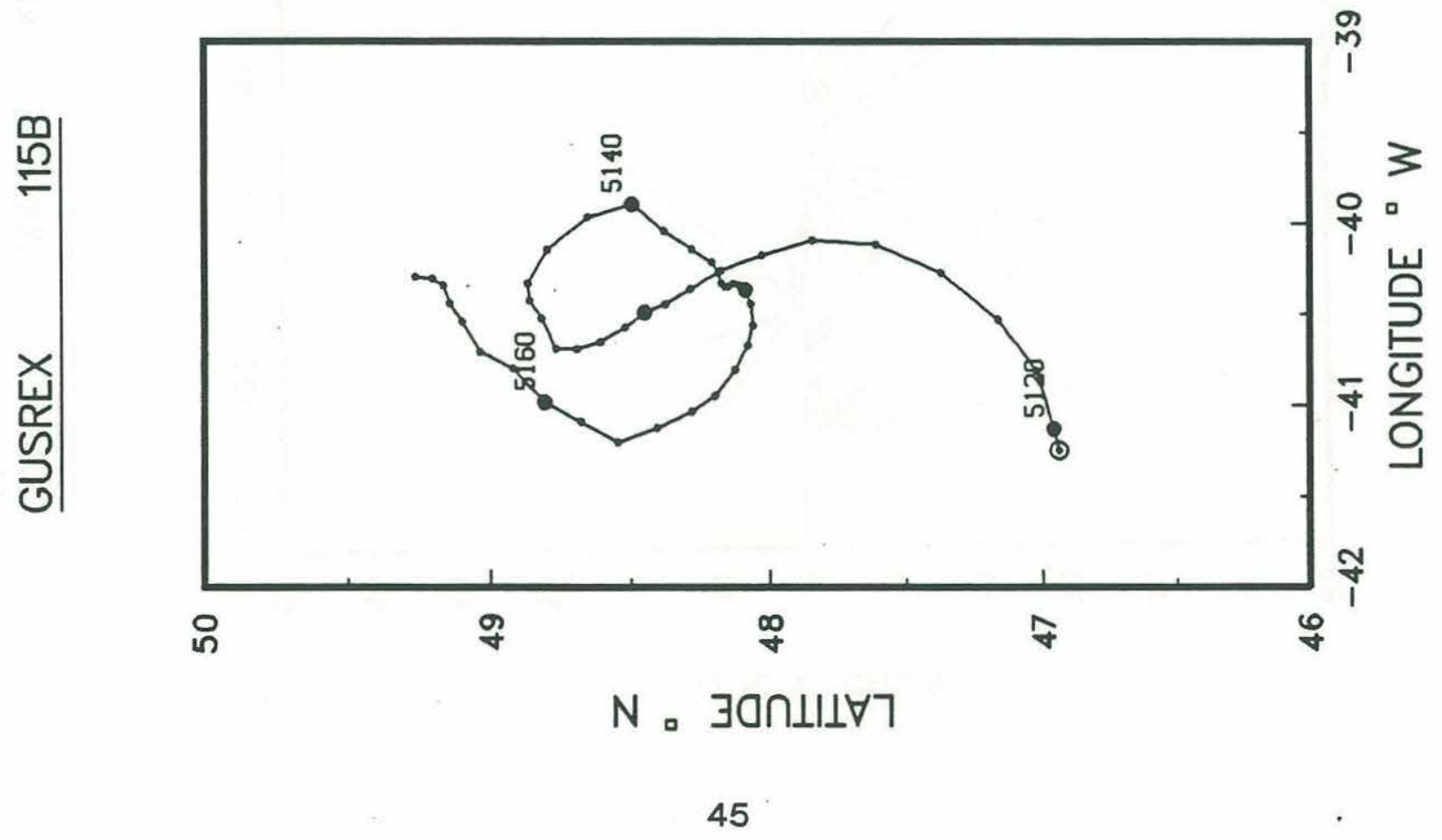




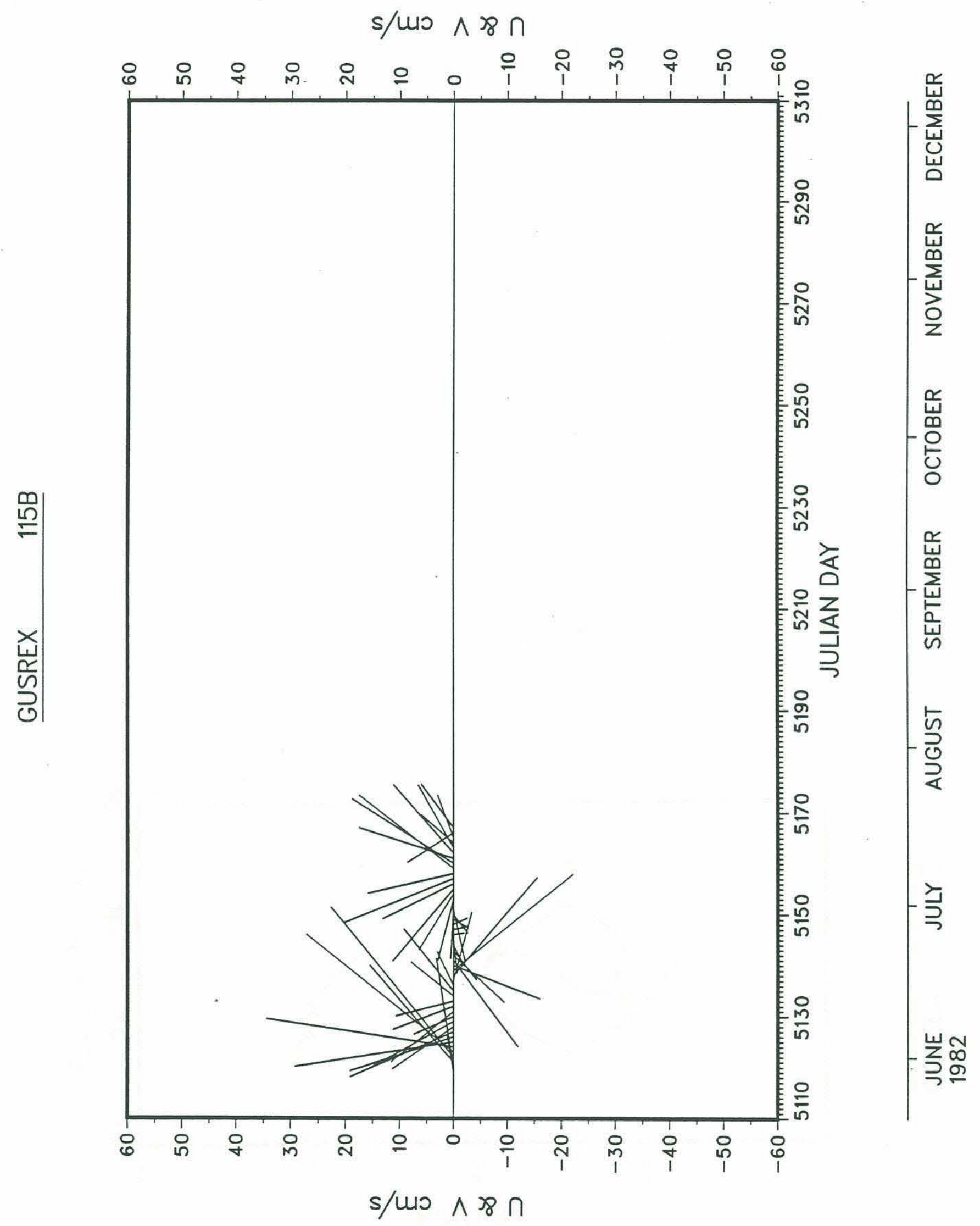




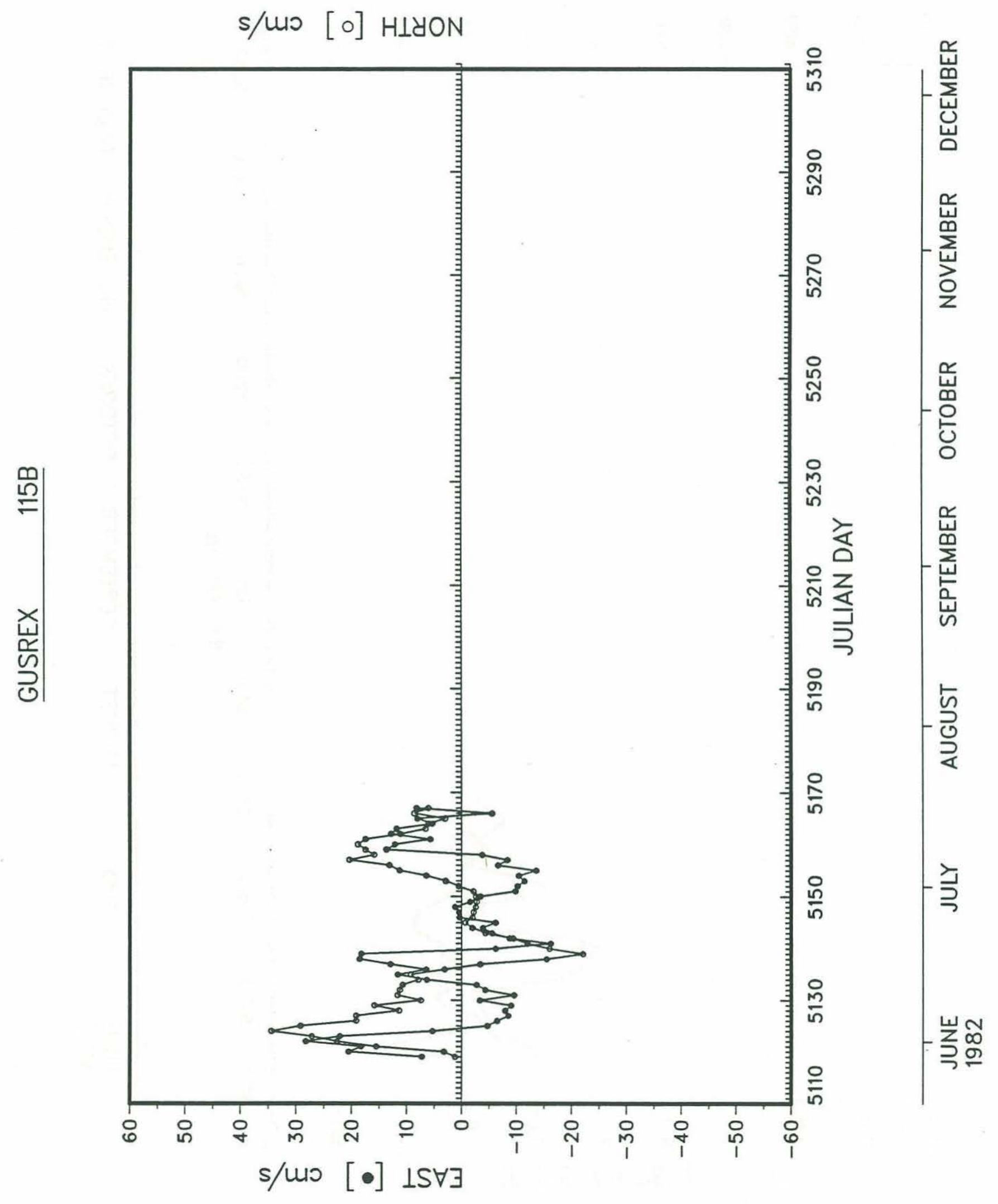




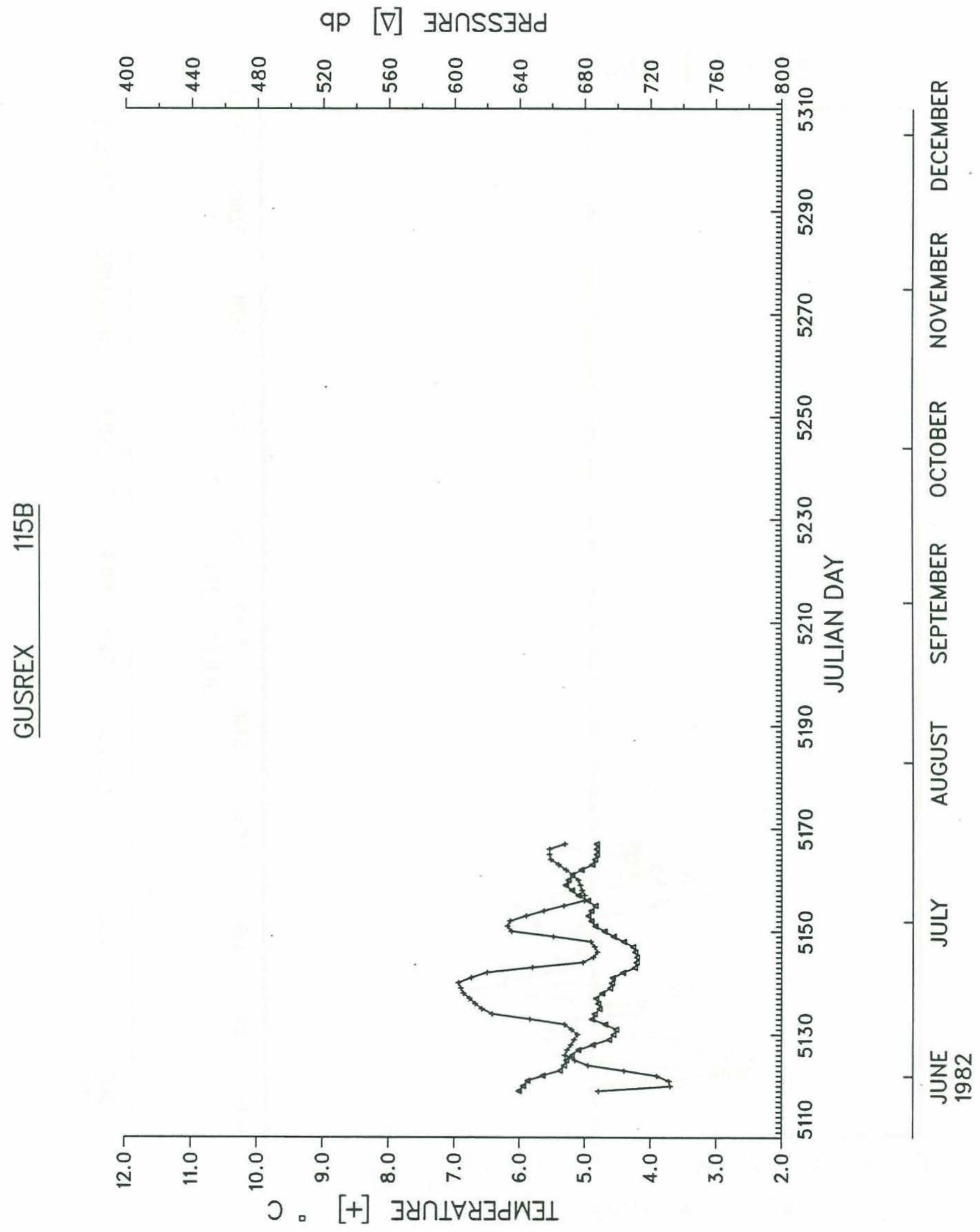




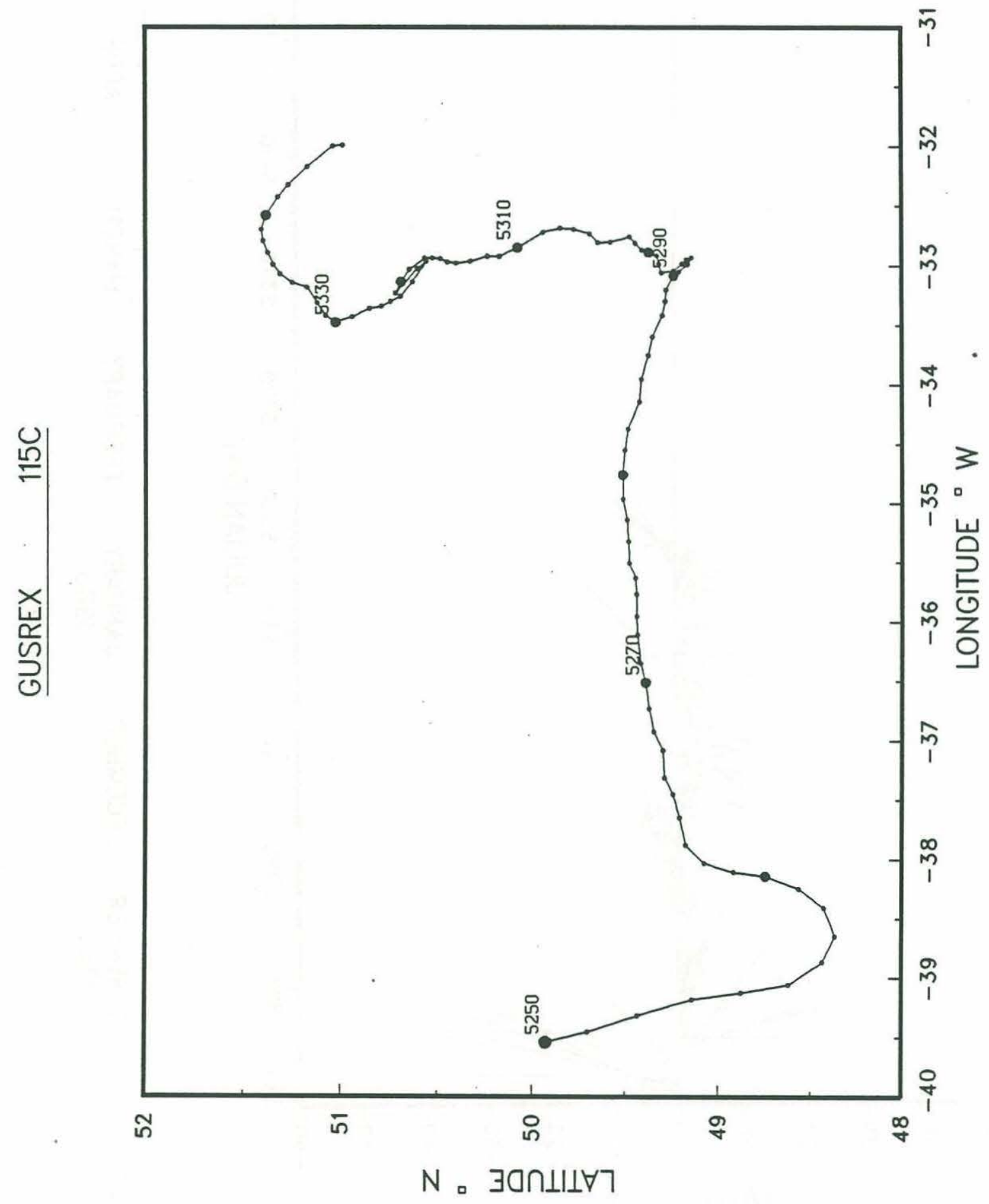




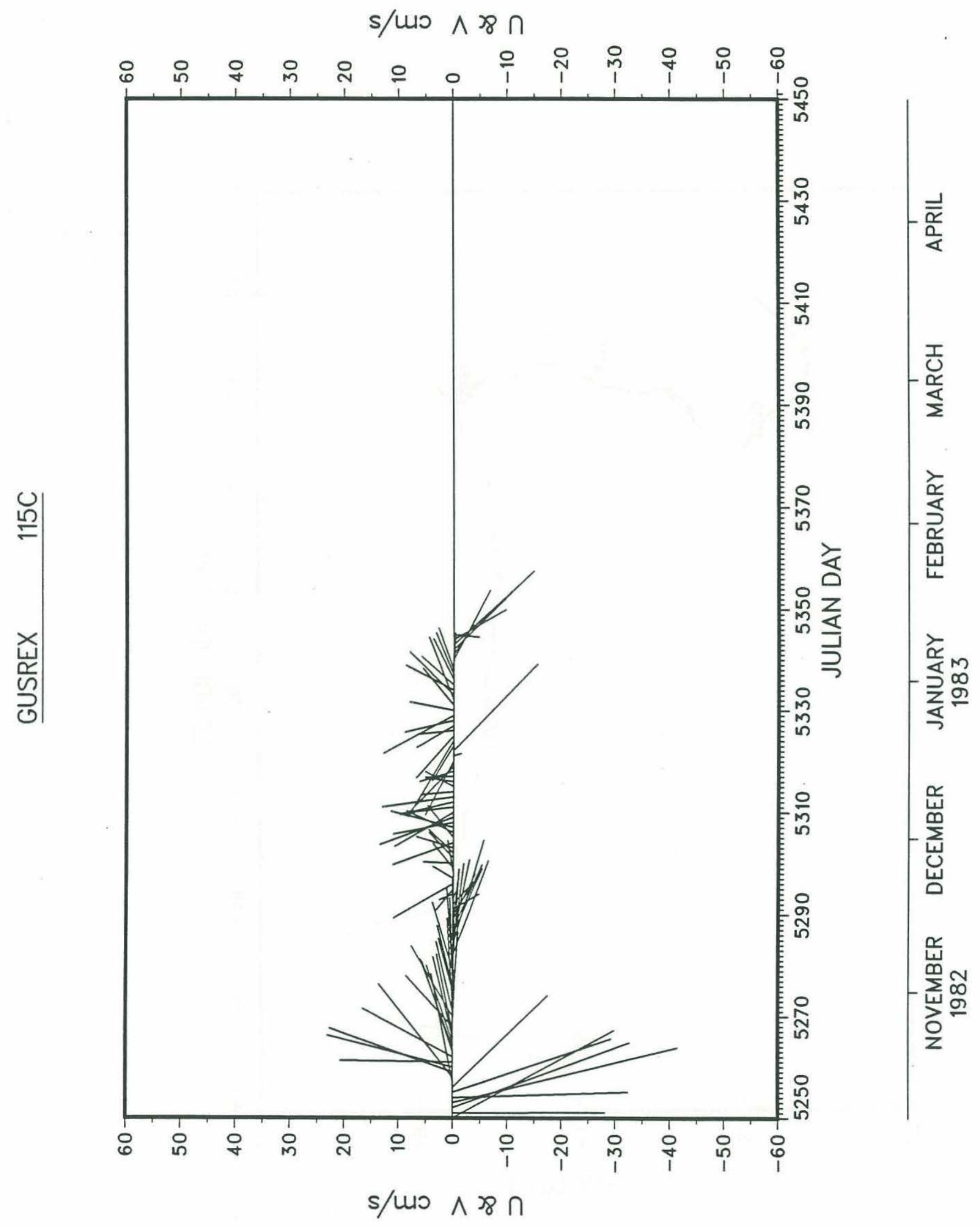




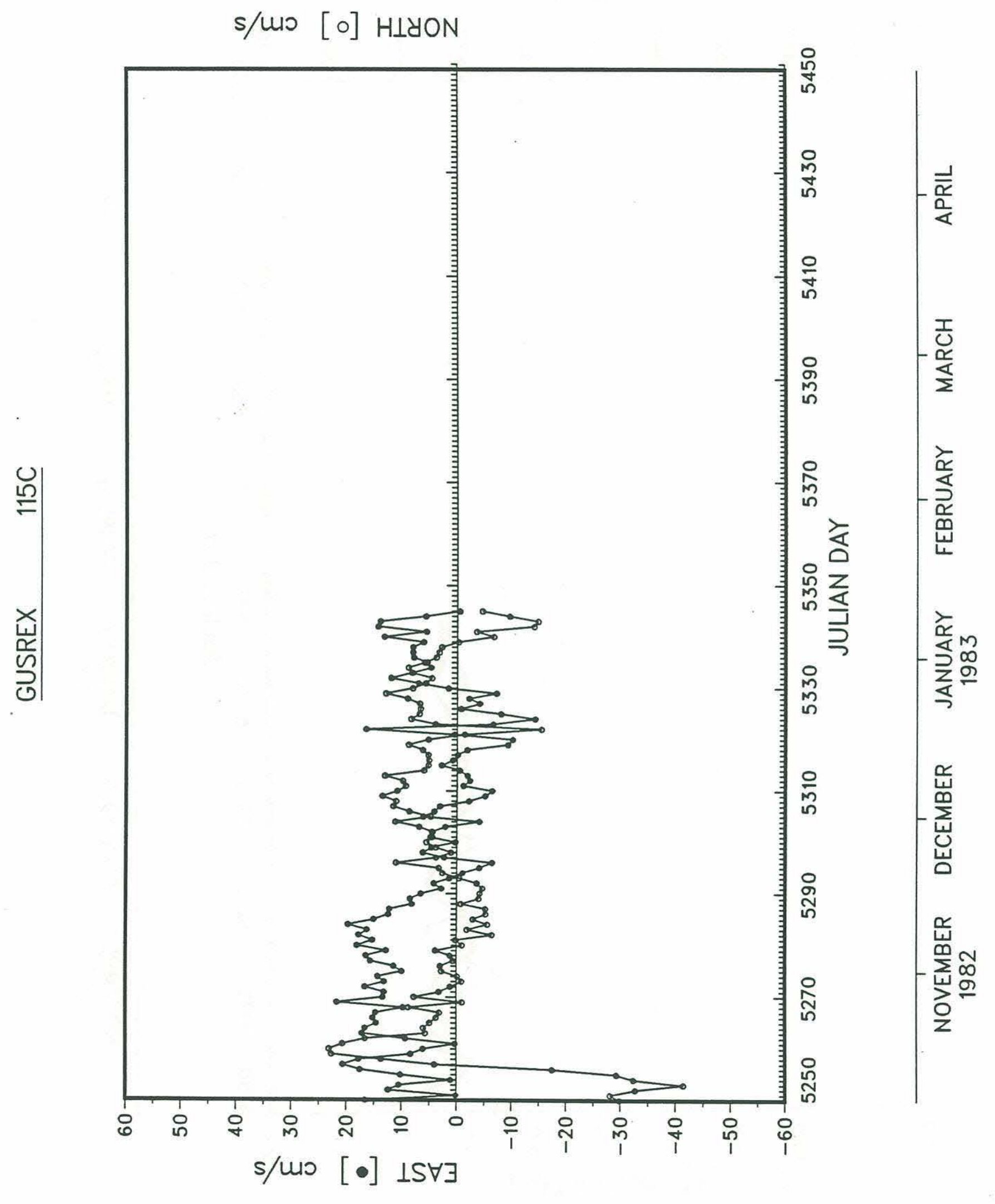




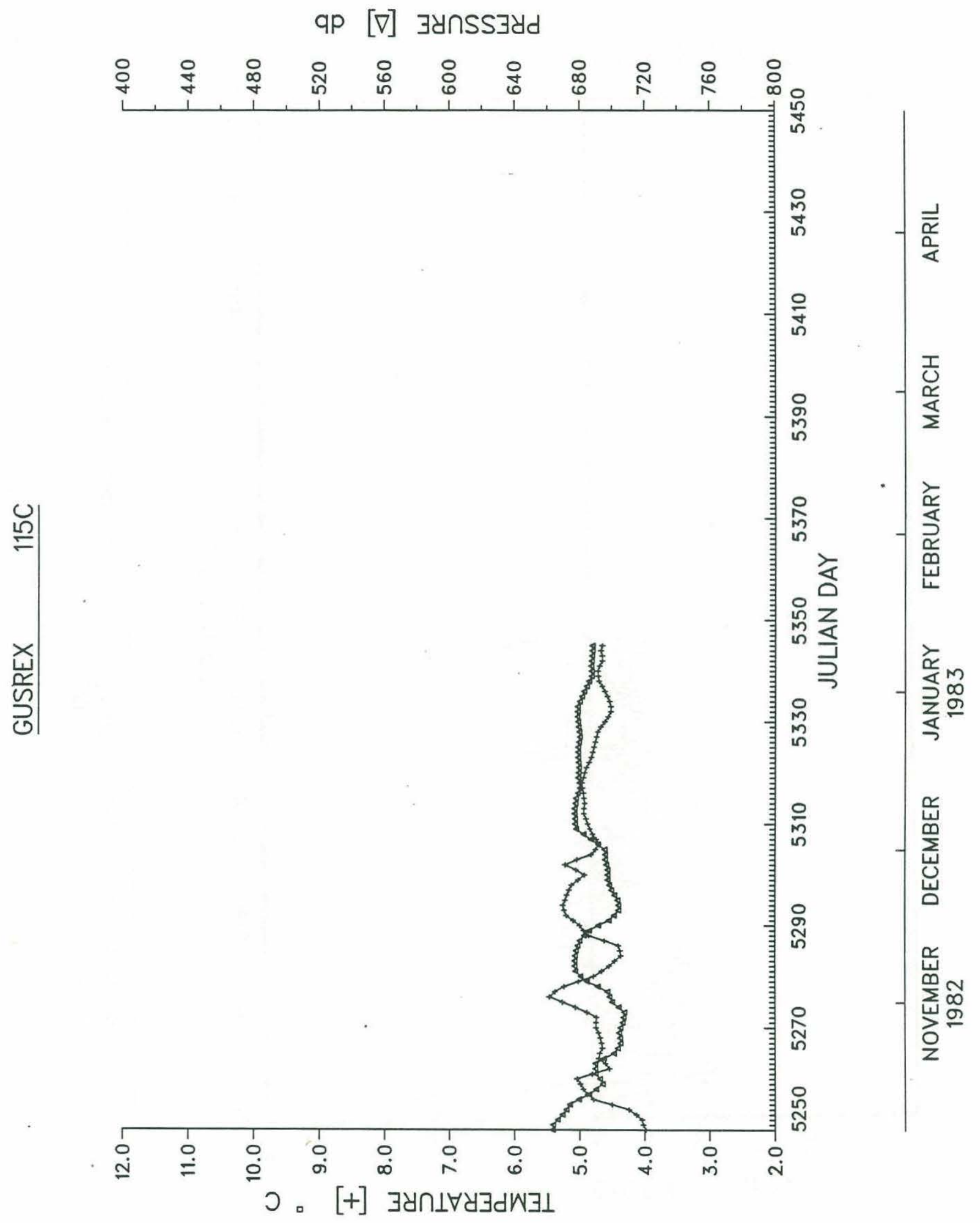




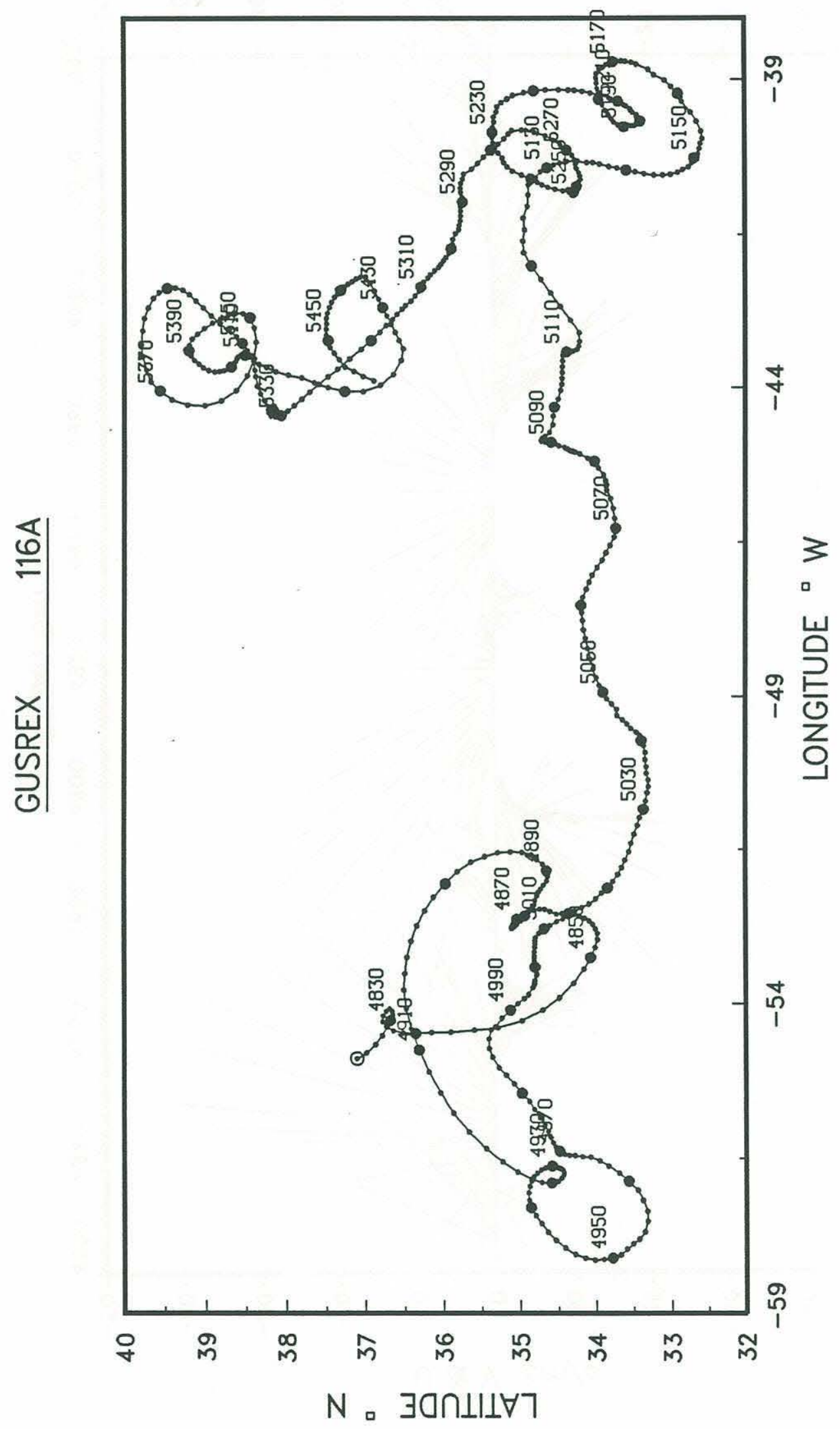




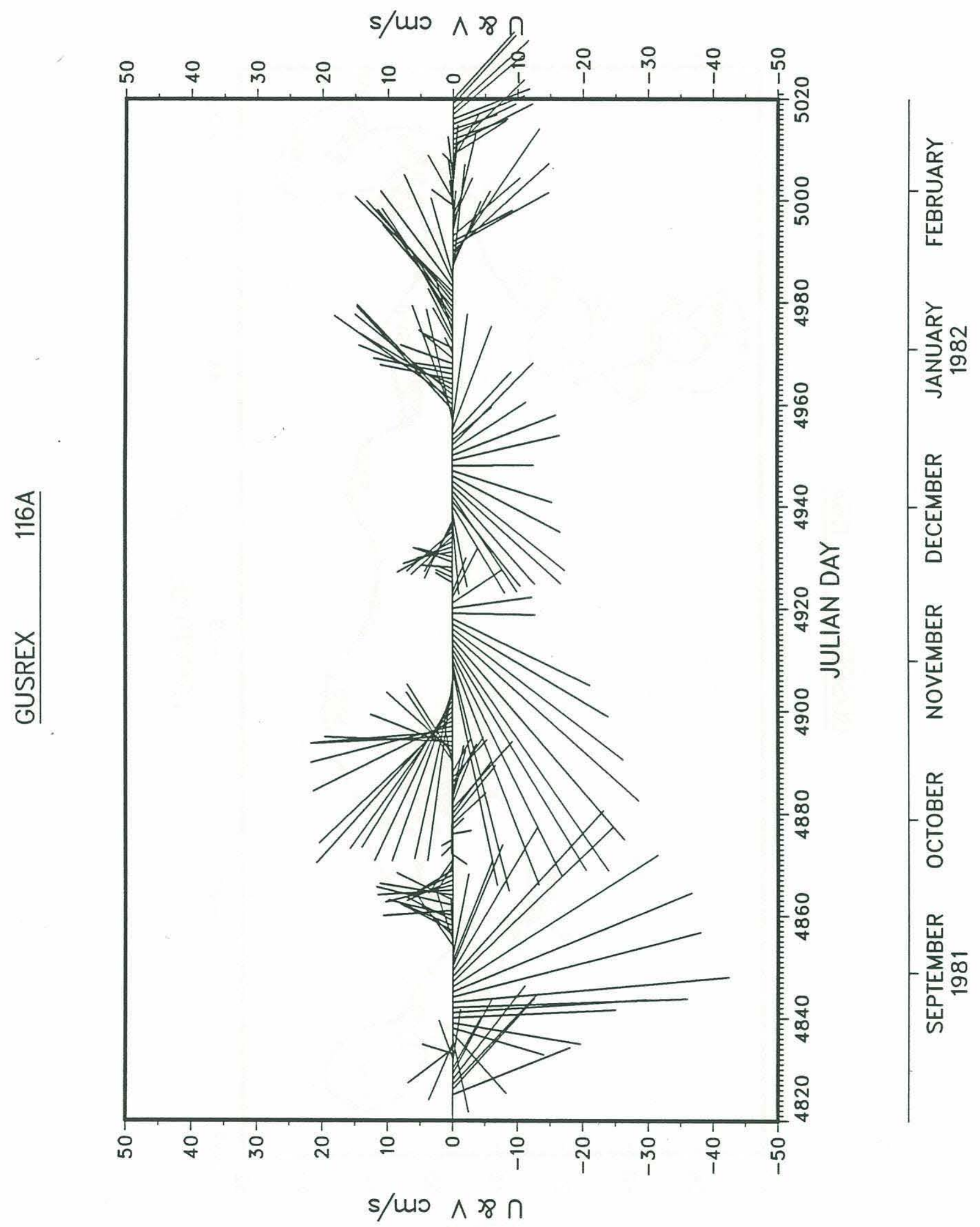




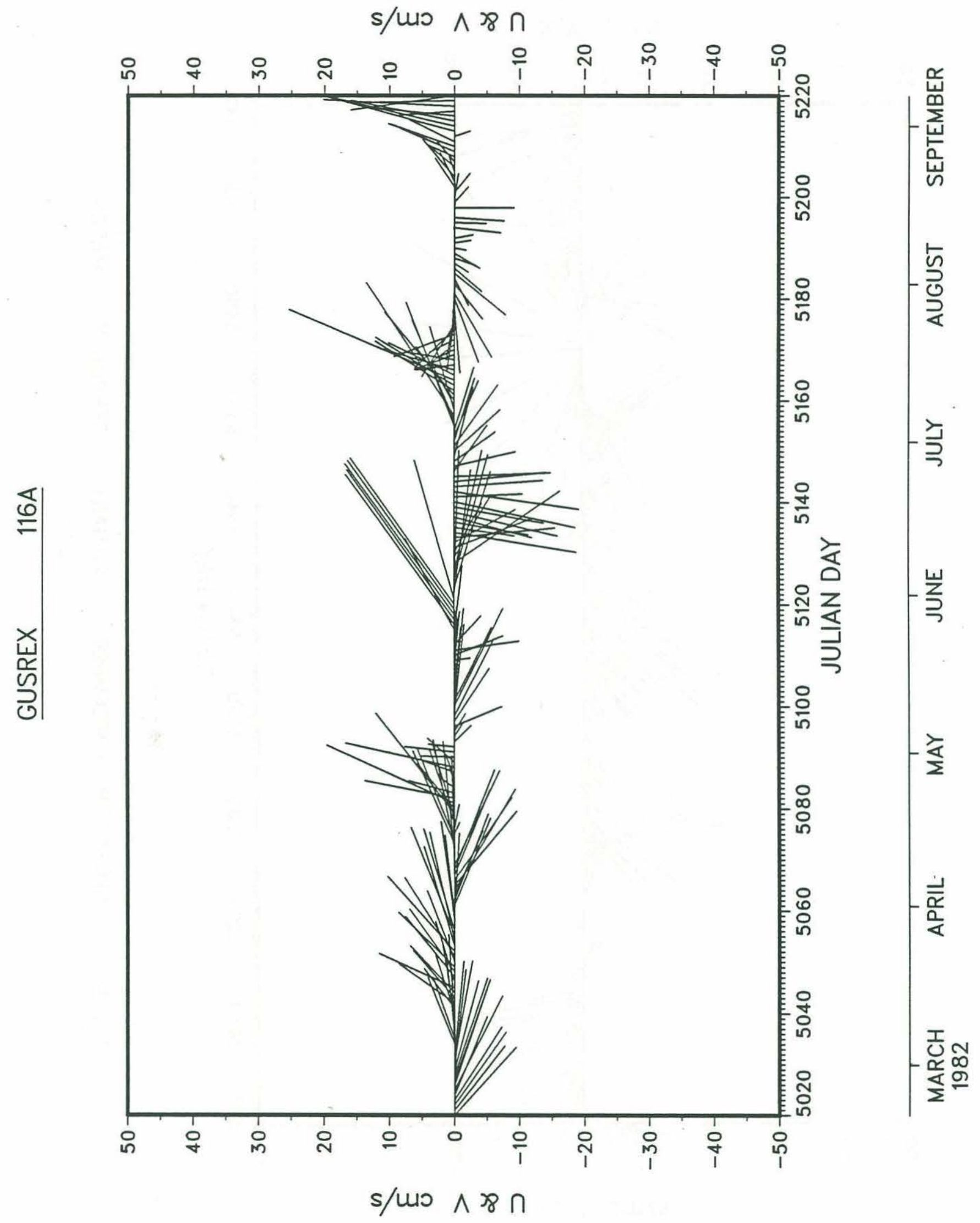




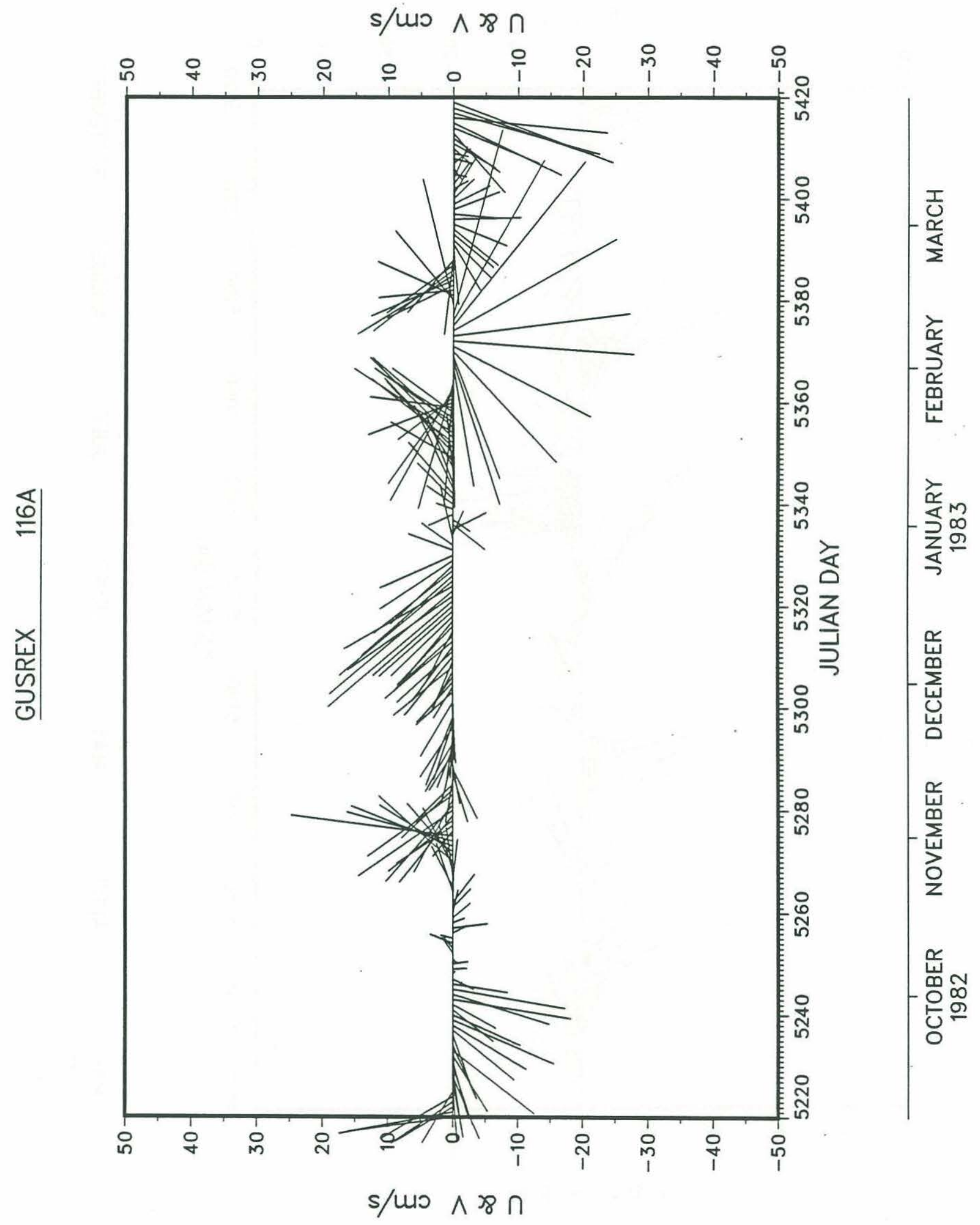




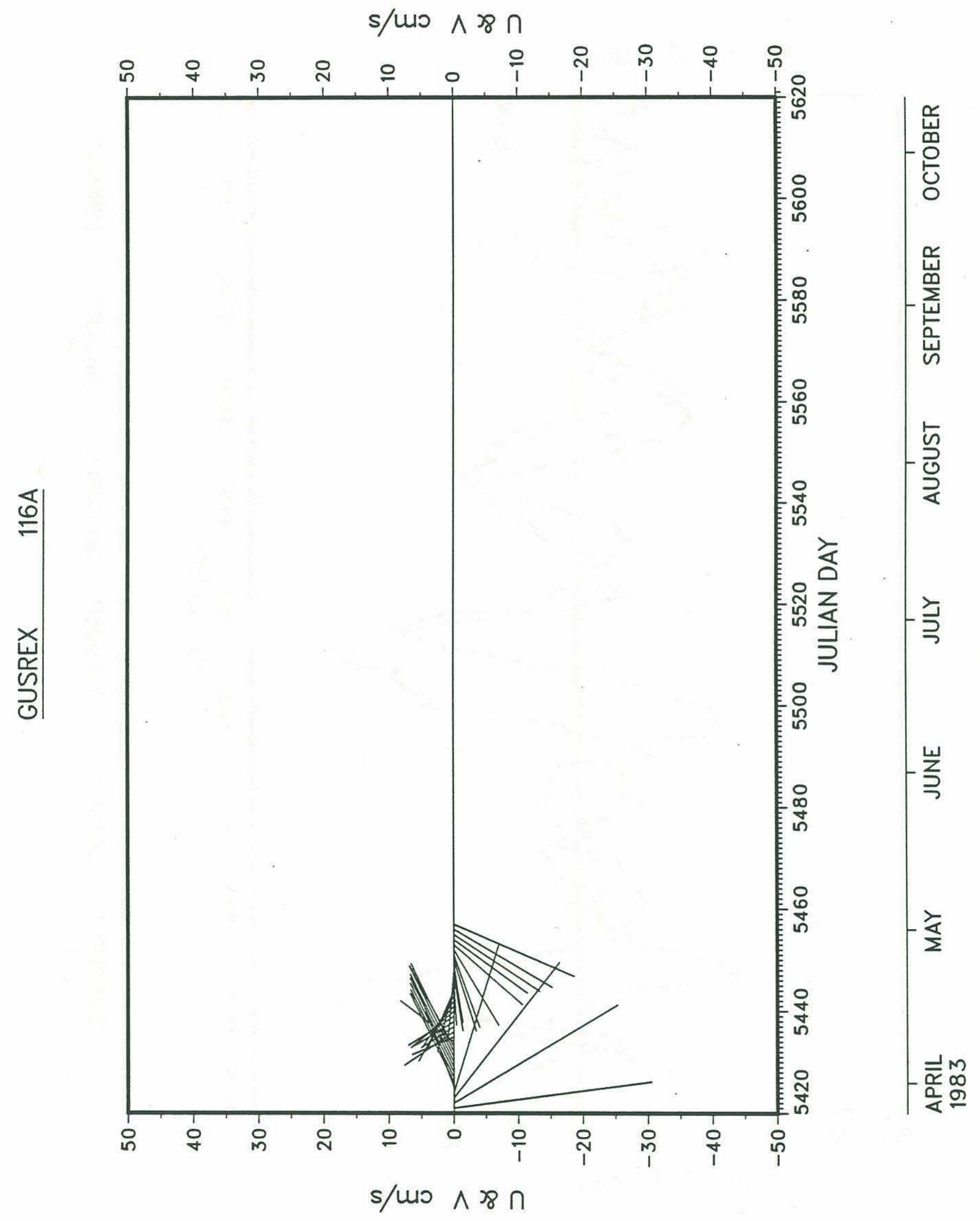


s/uo [o] HLYON

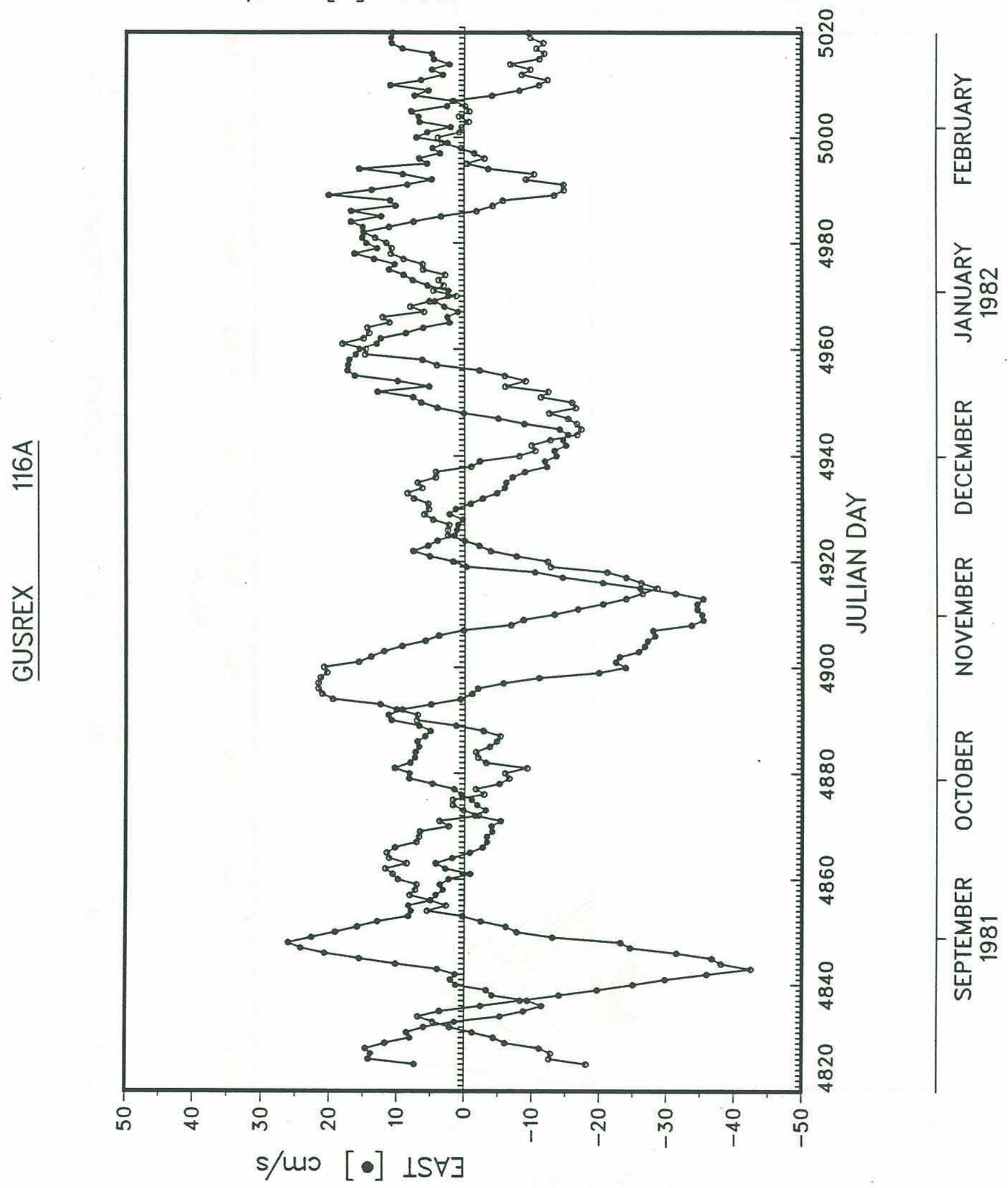




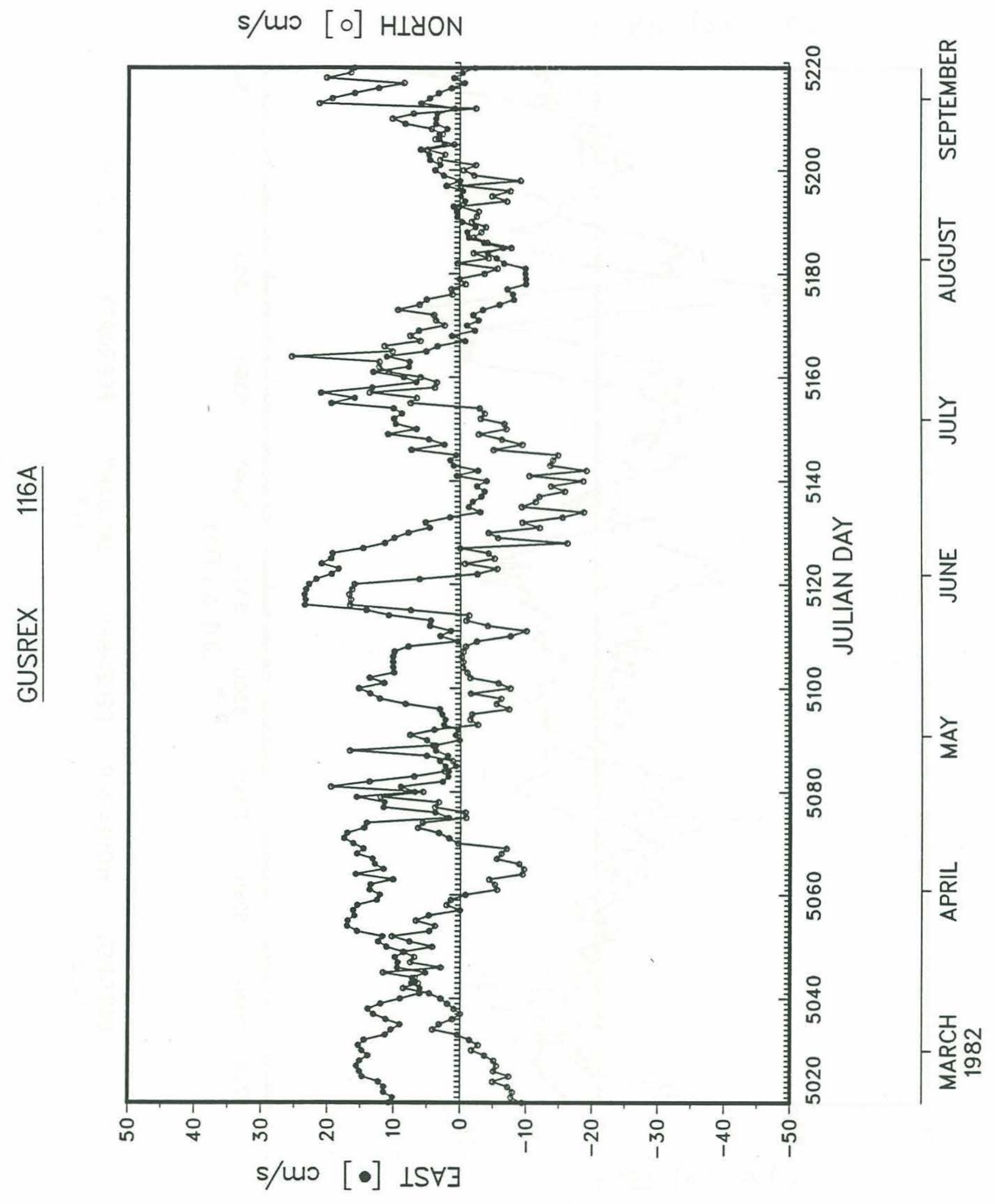




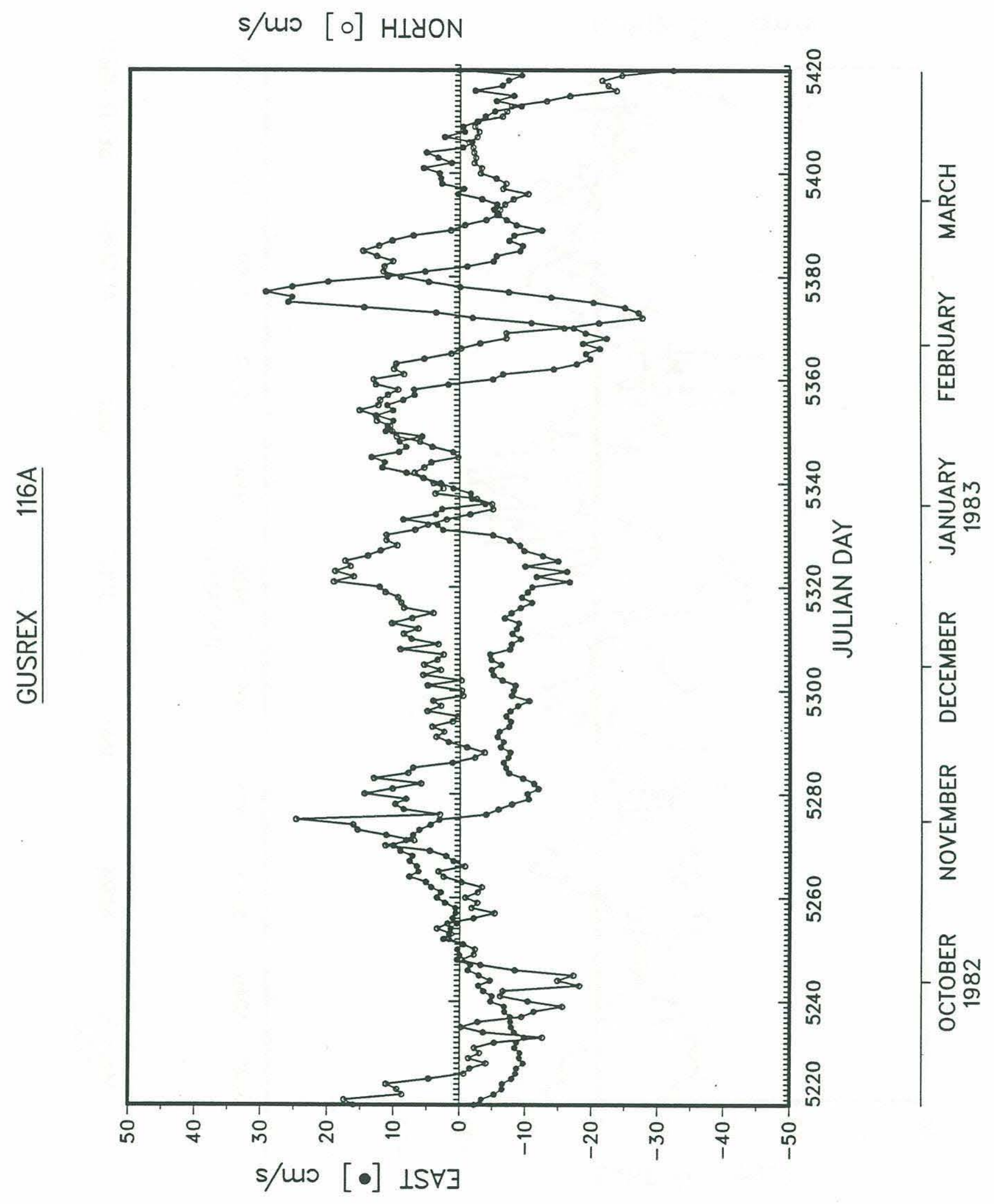


s/uo [o] HLYON

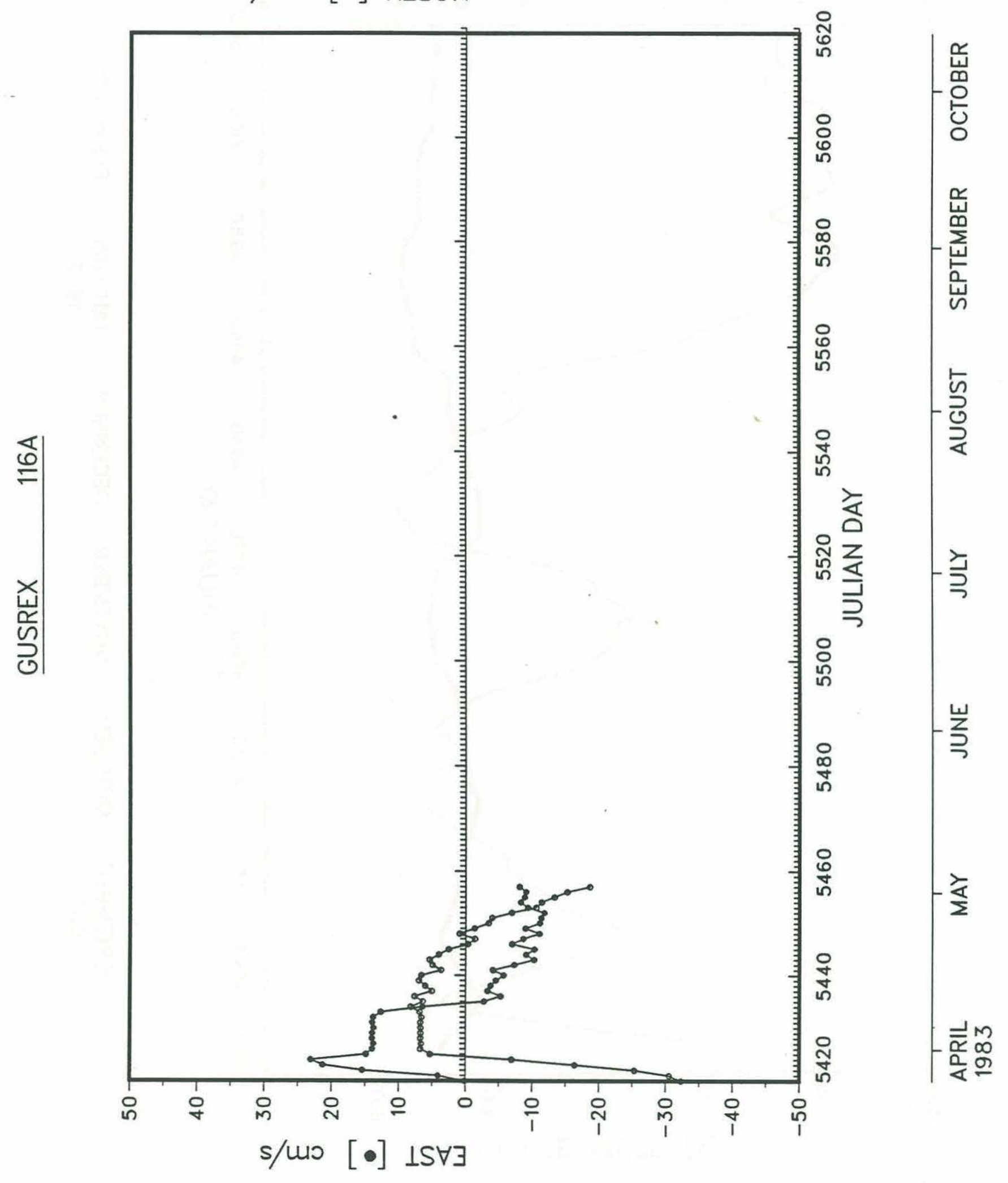




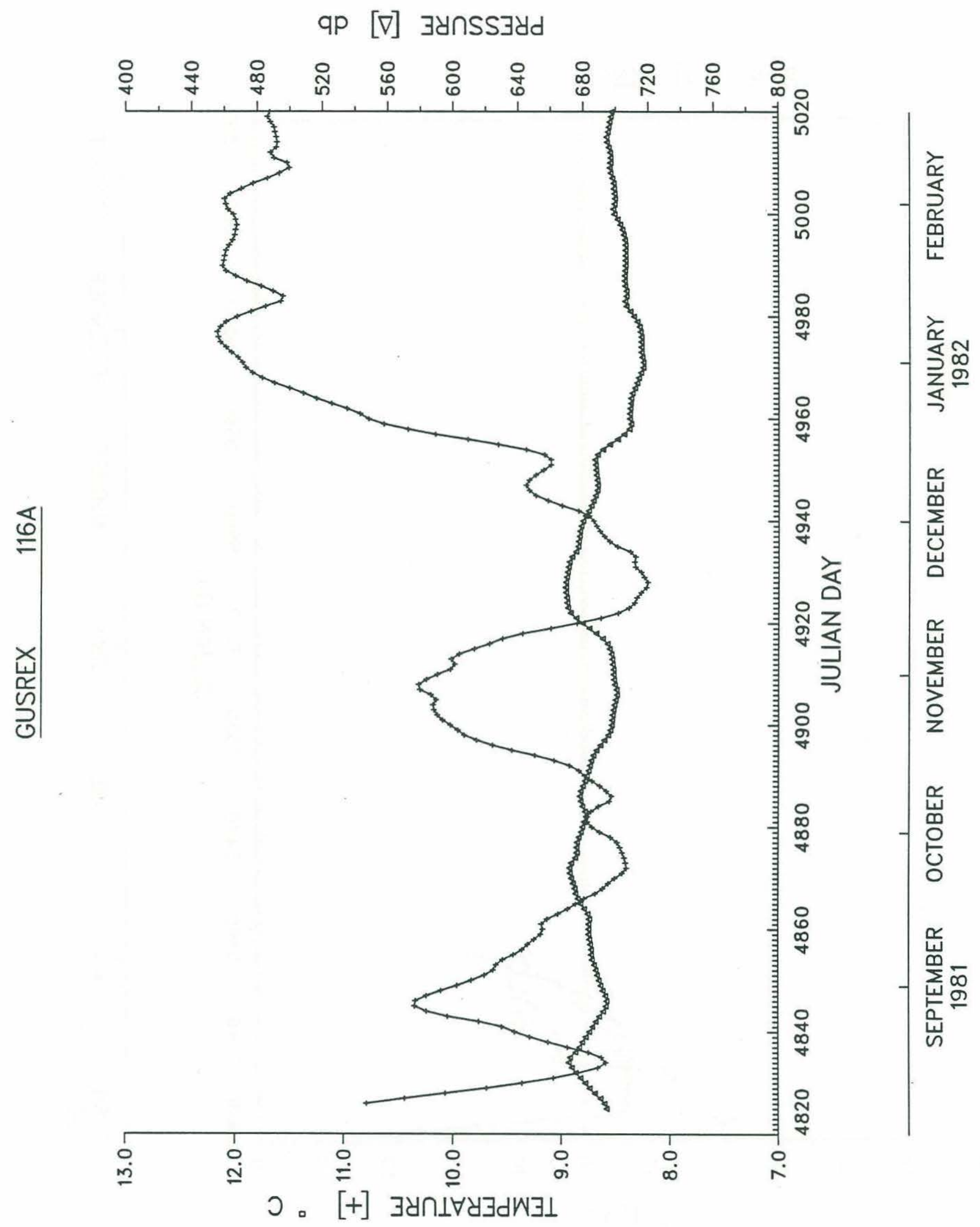




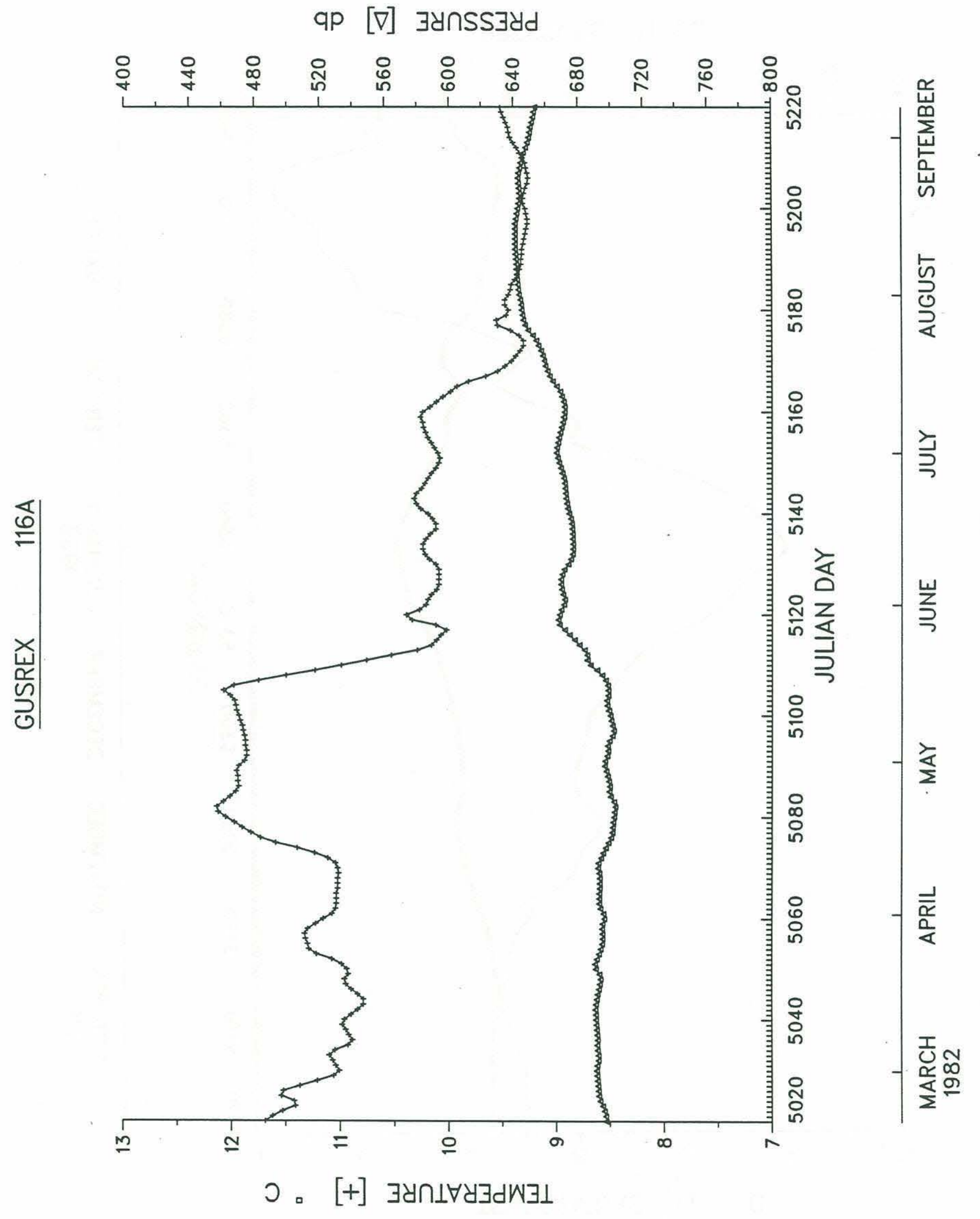




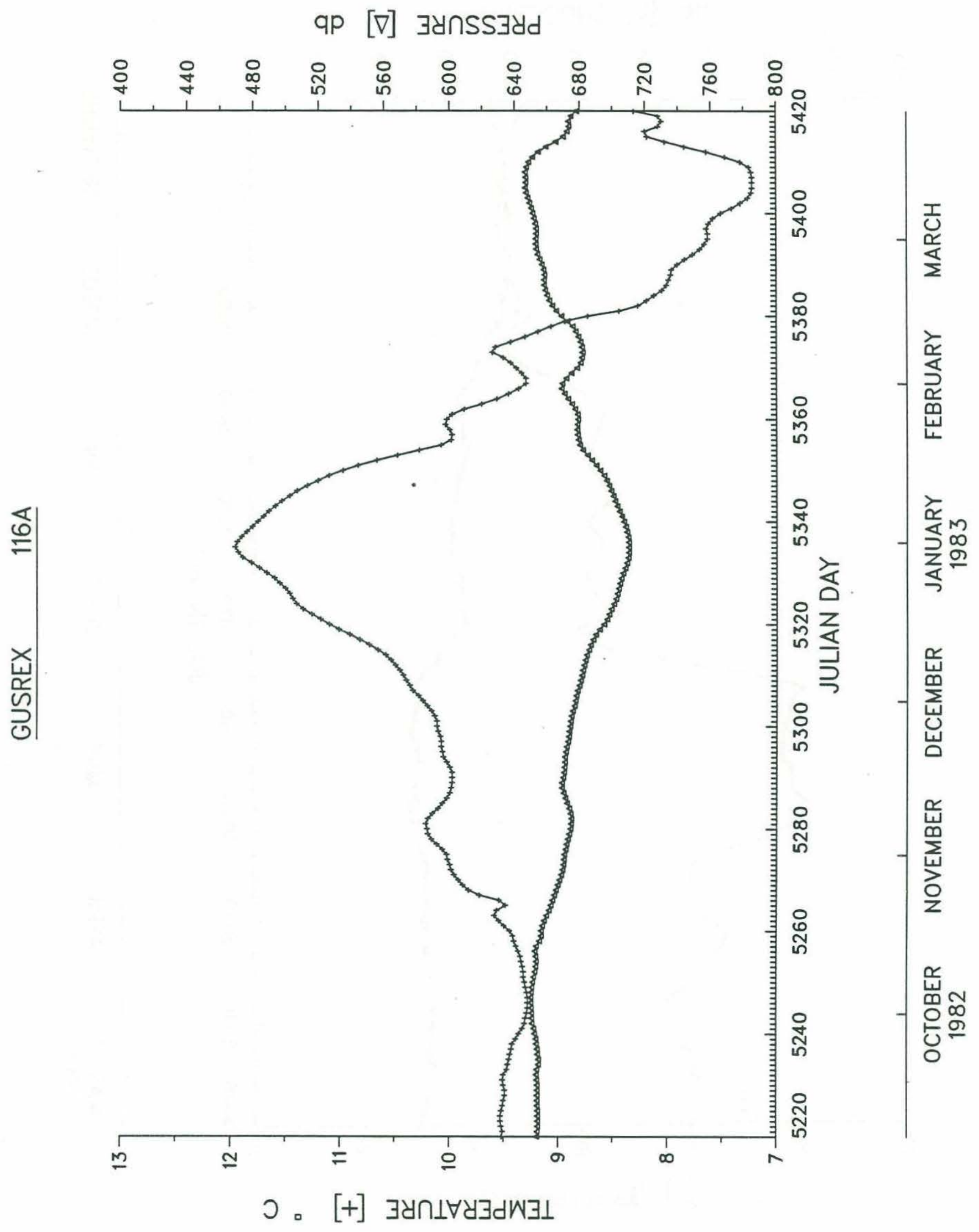




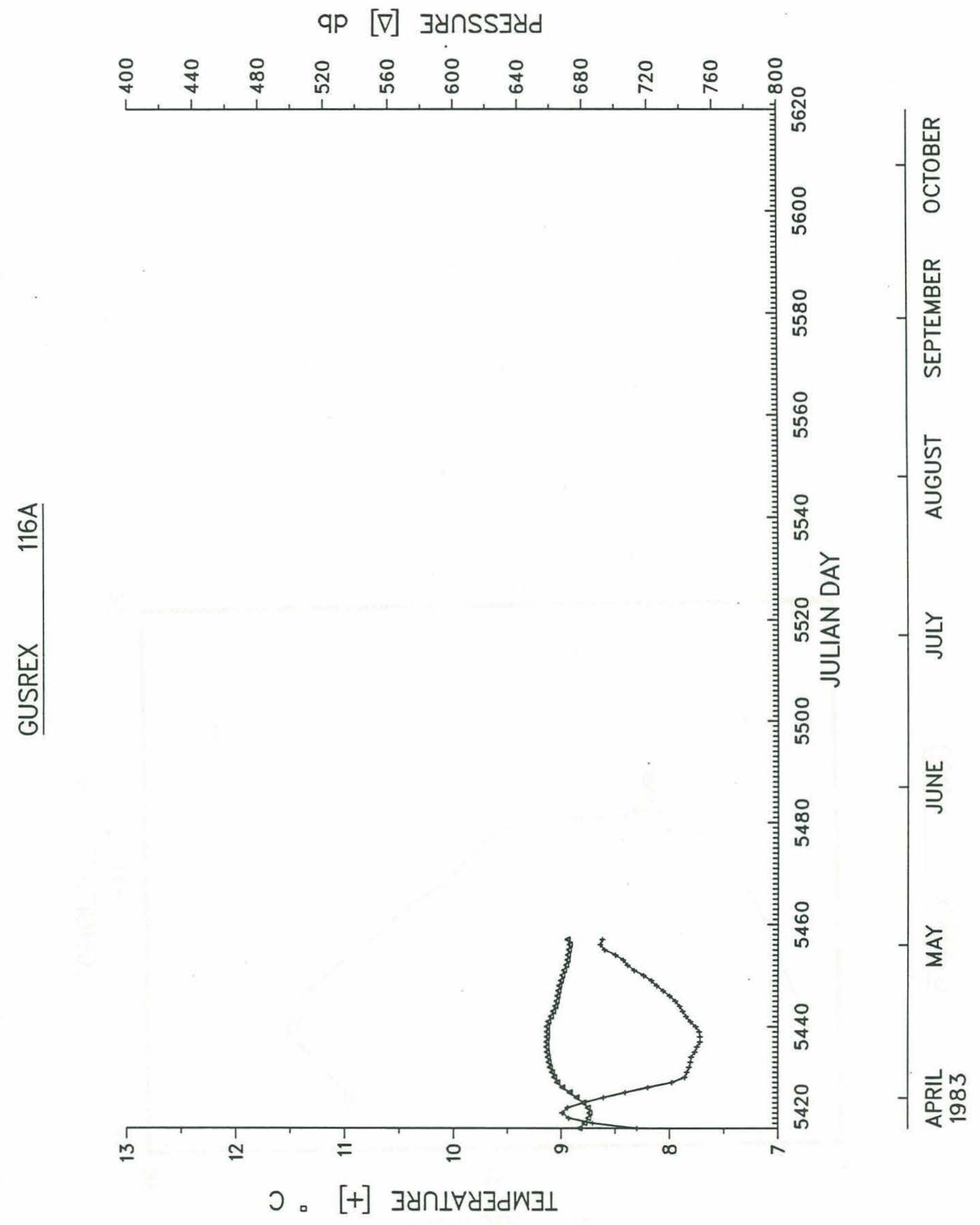




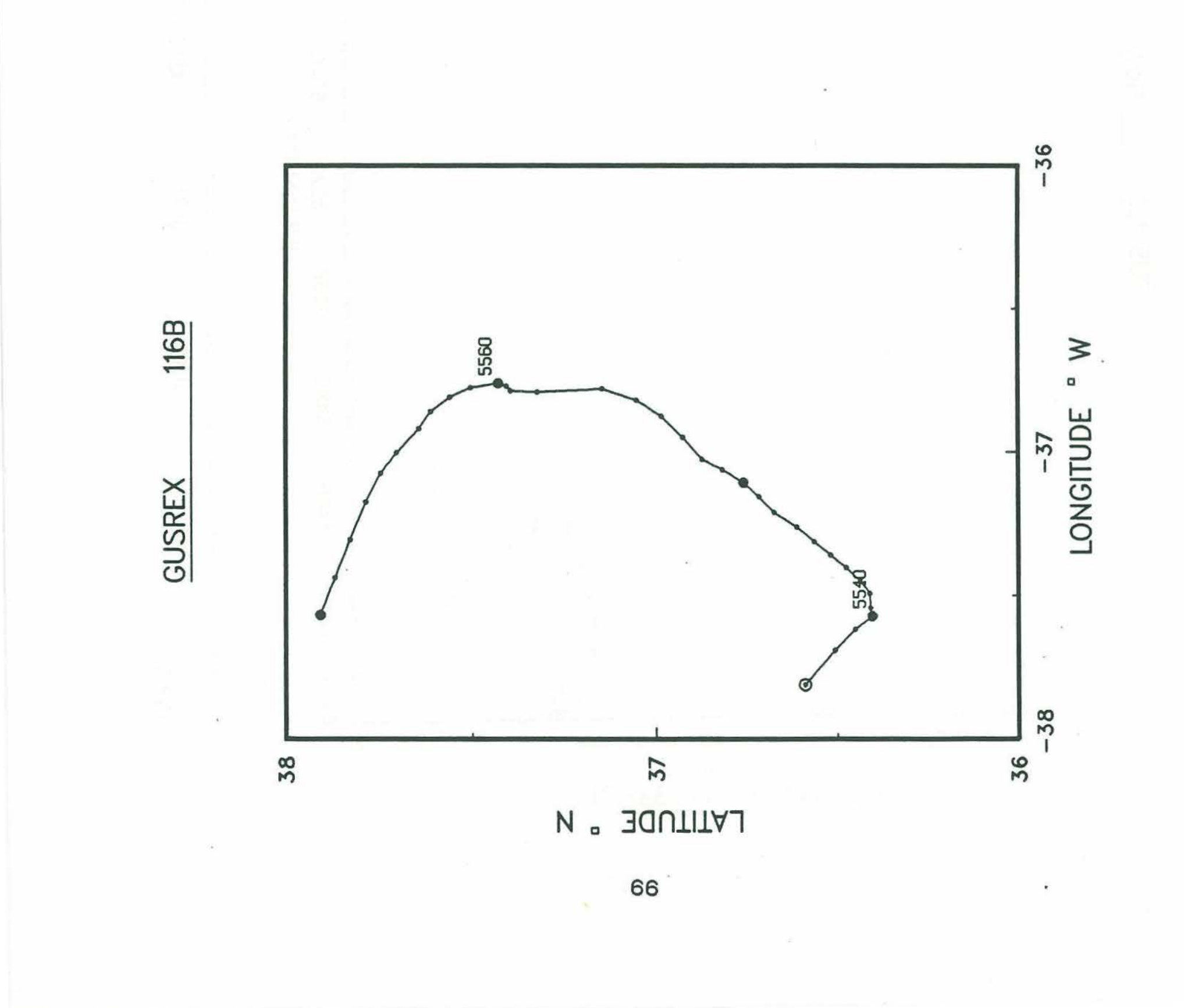




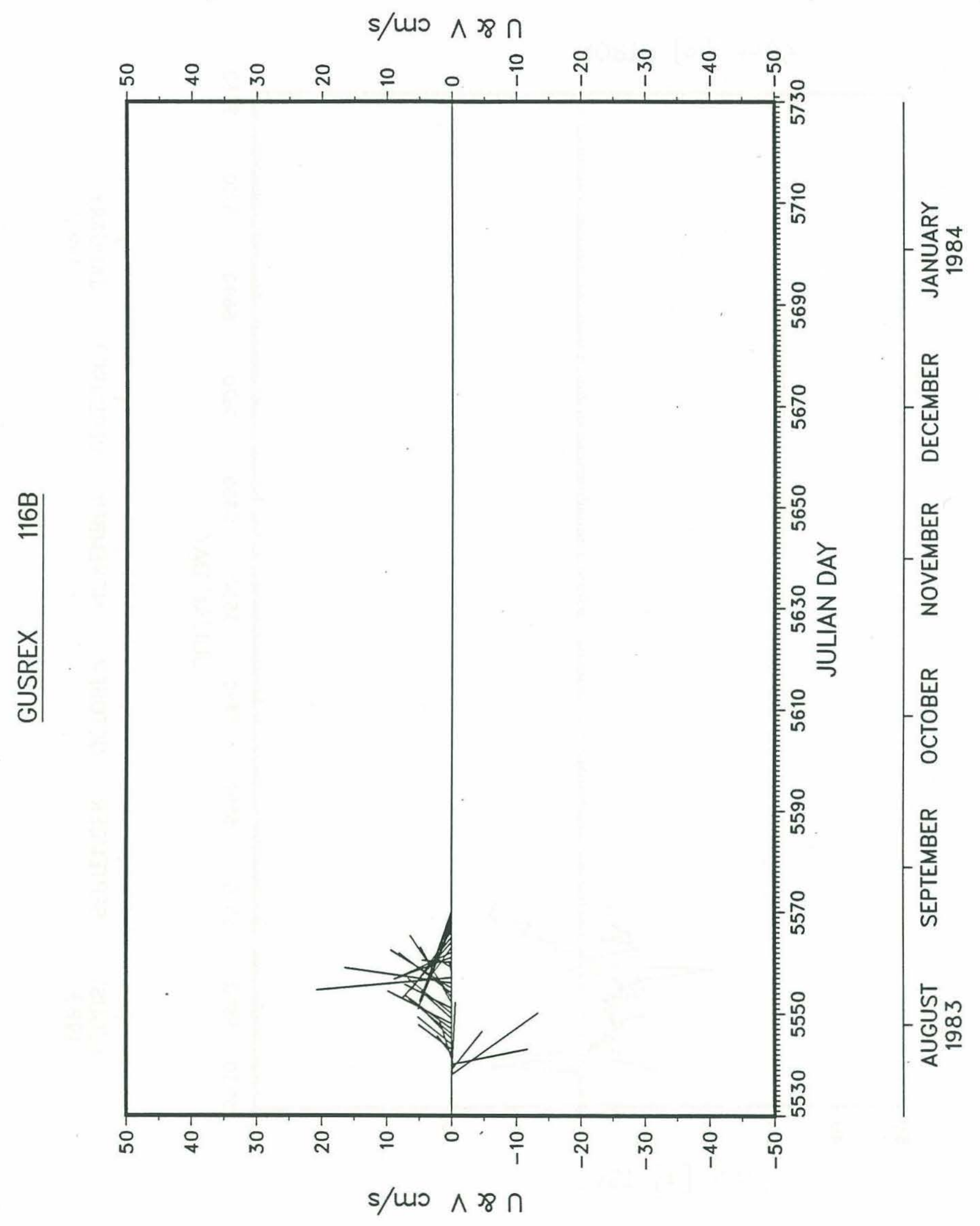




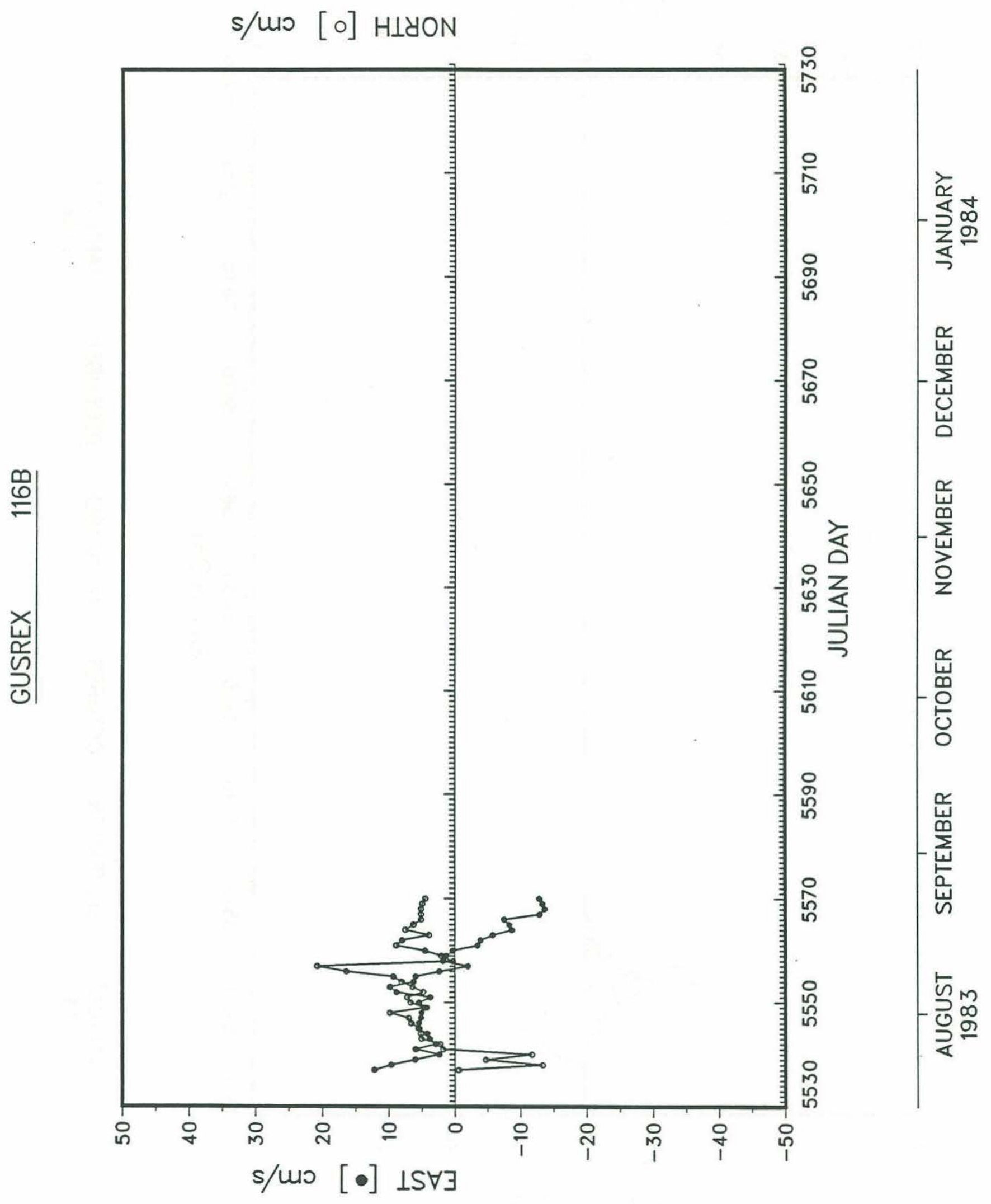




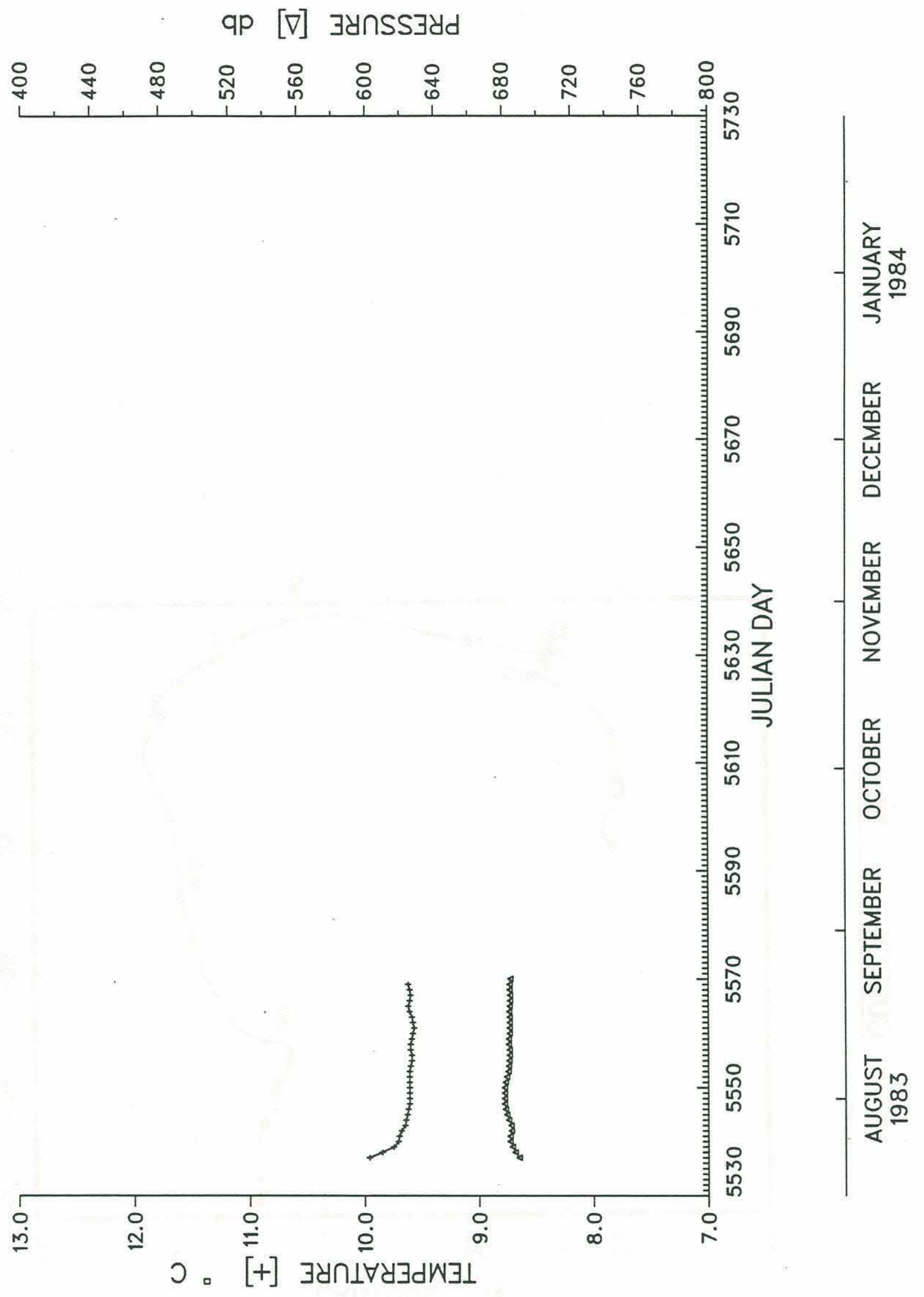




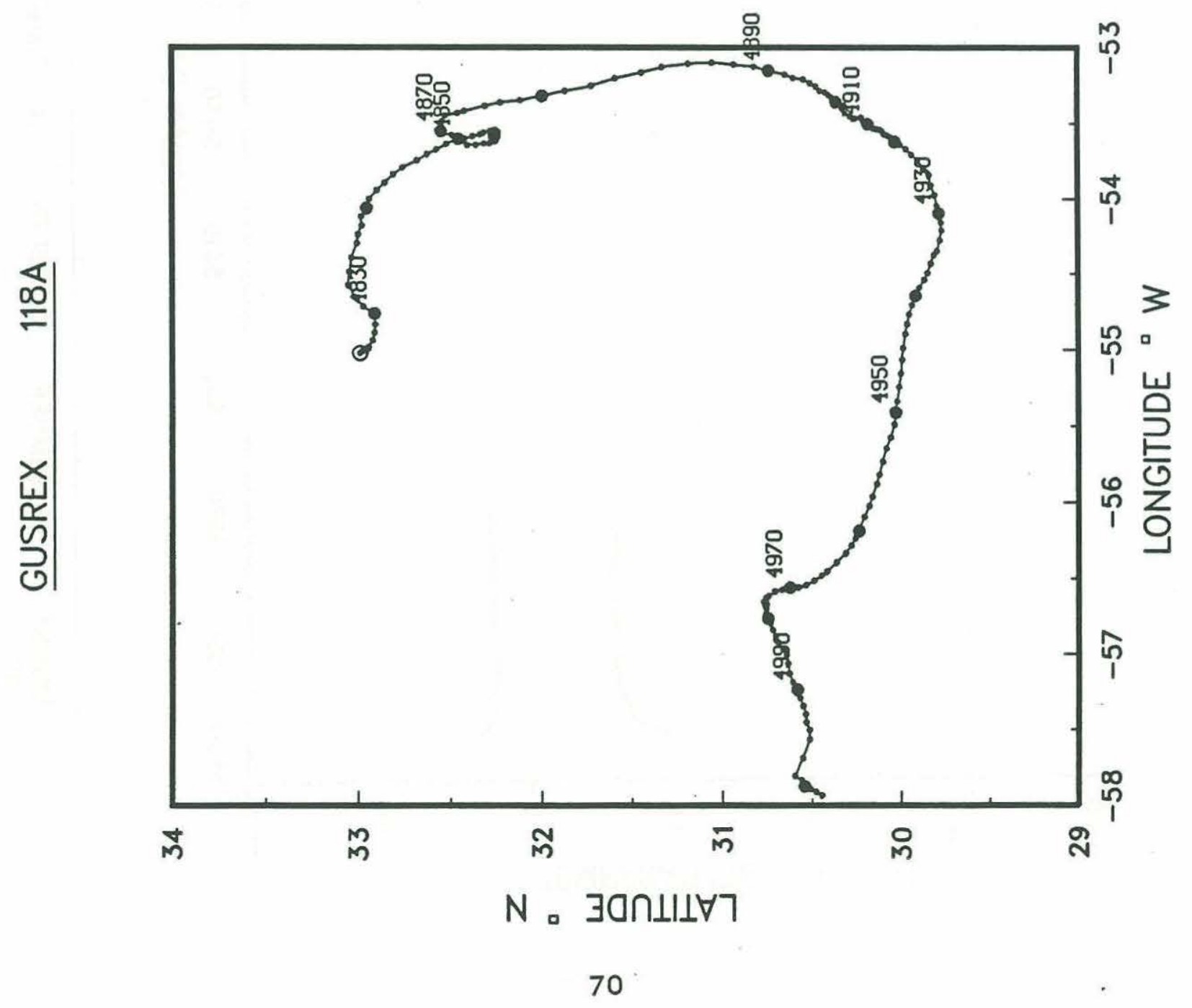




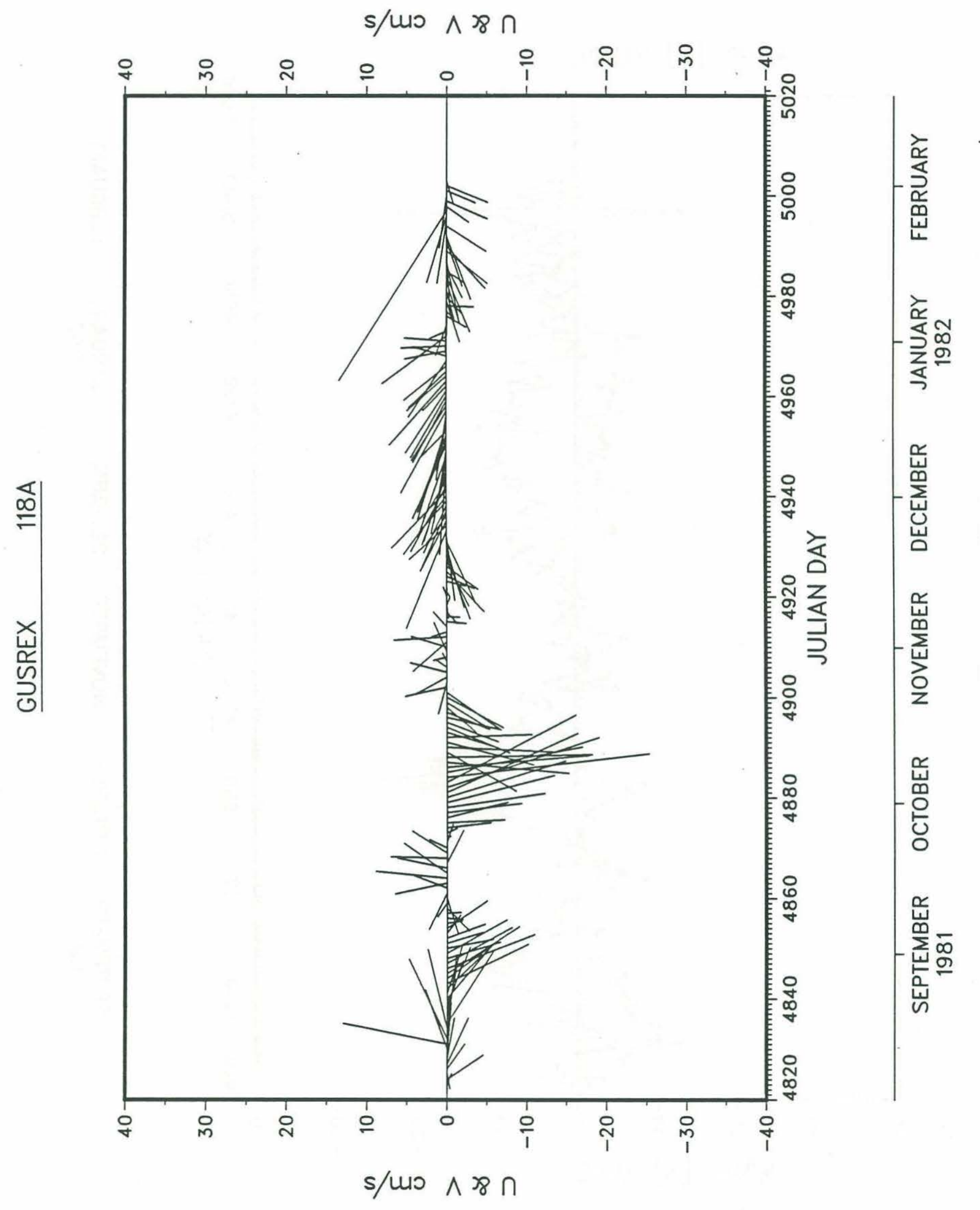




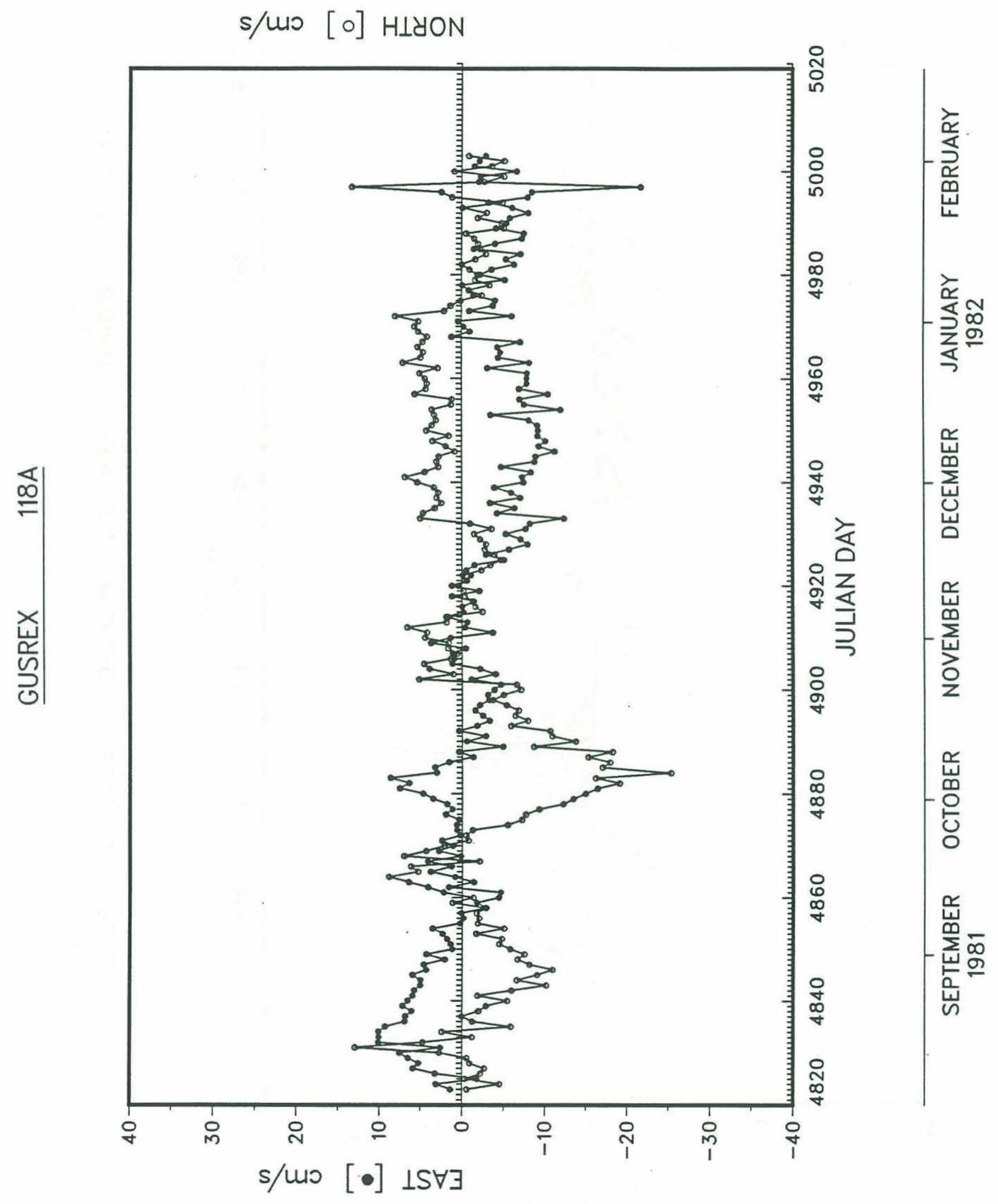




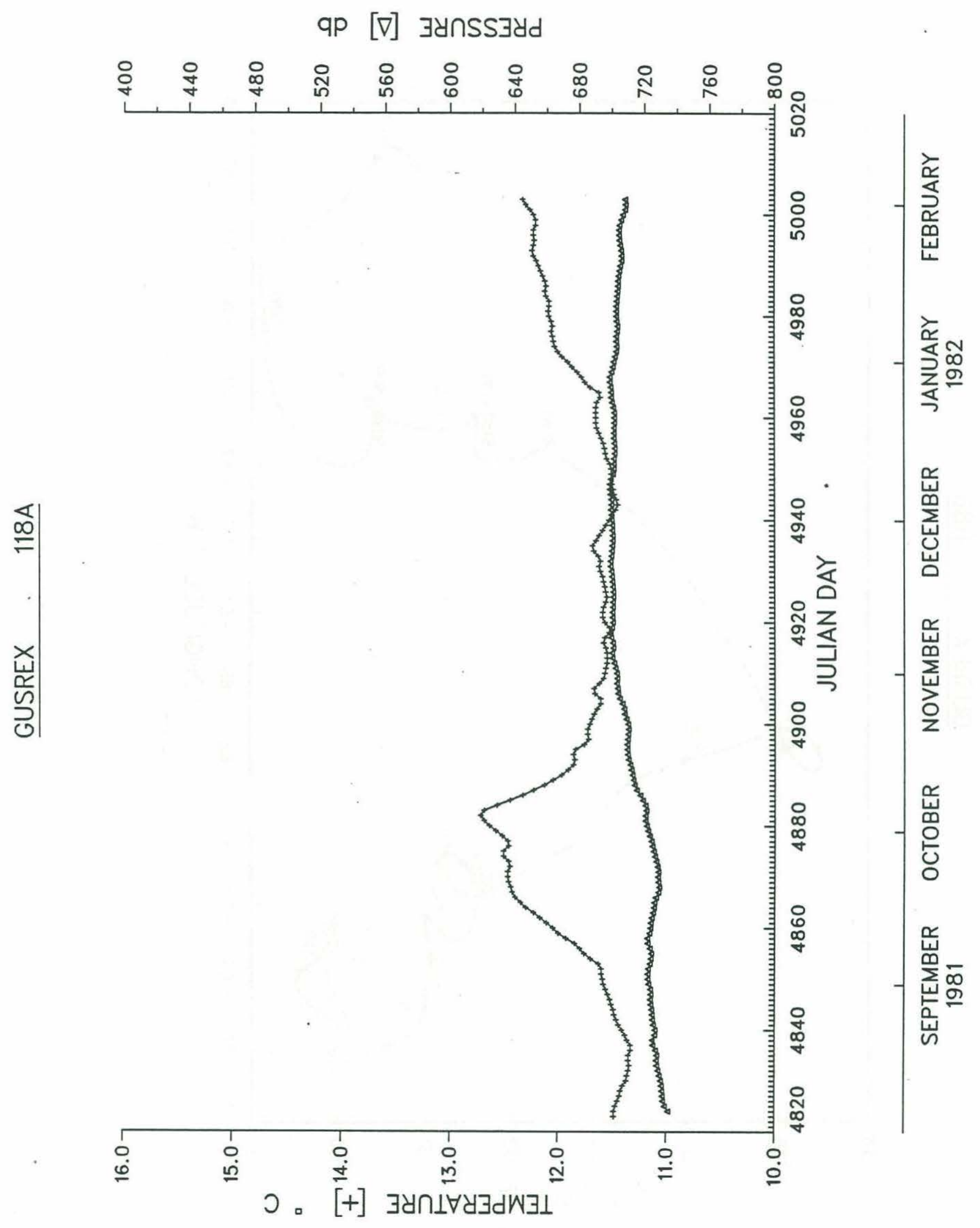




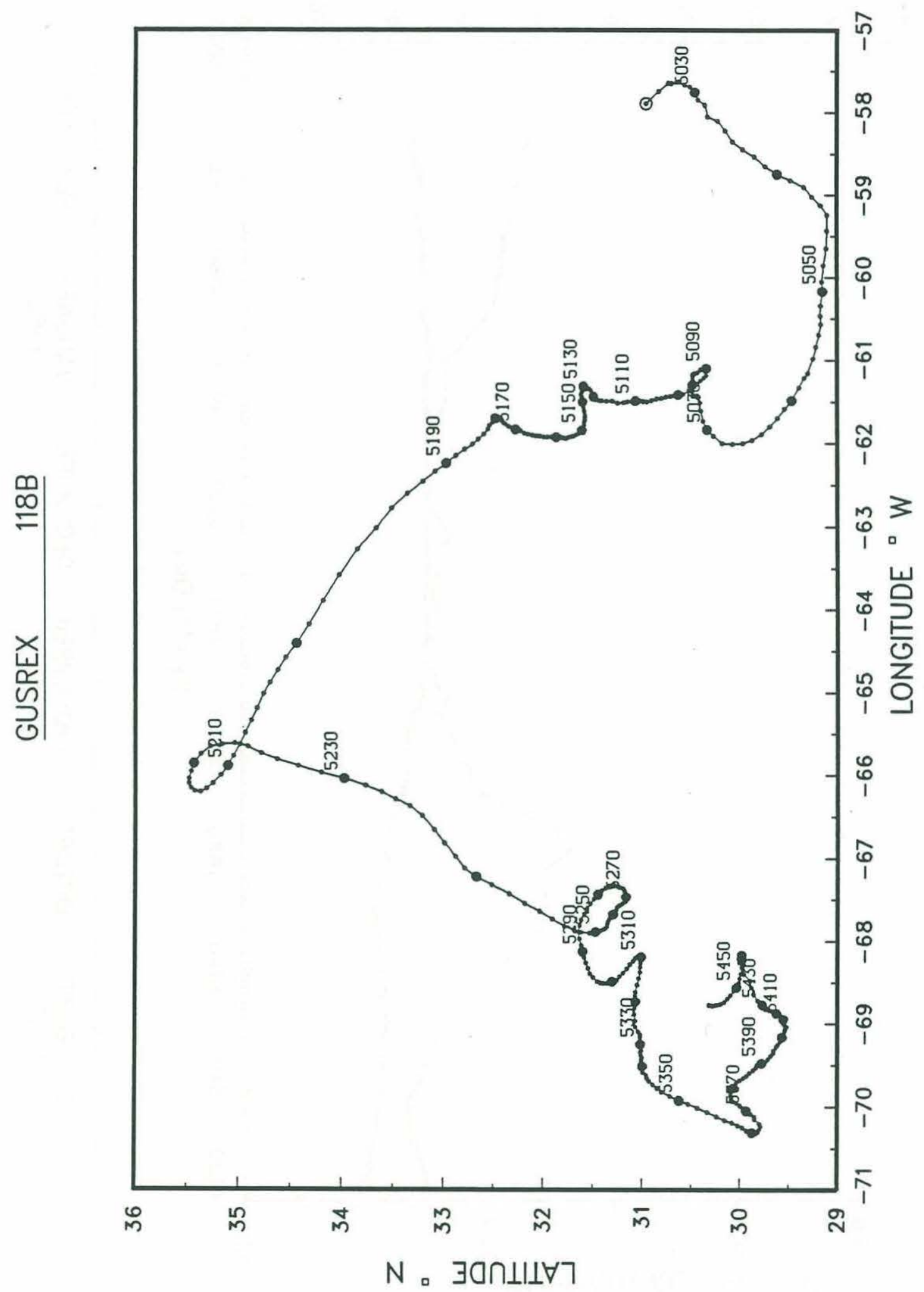




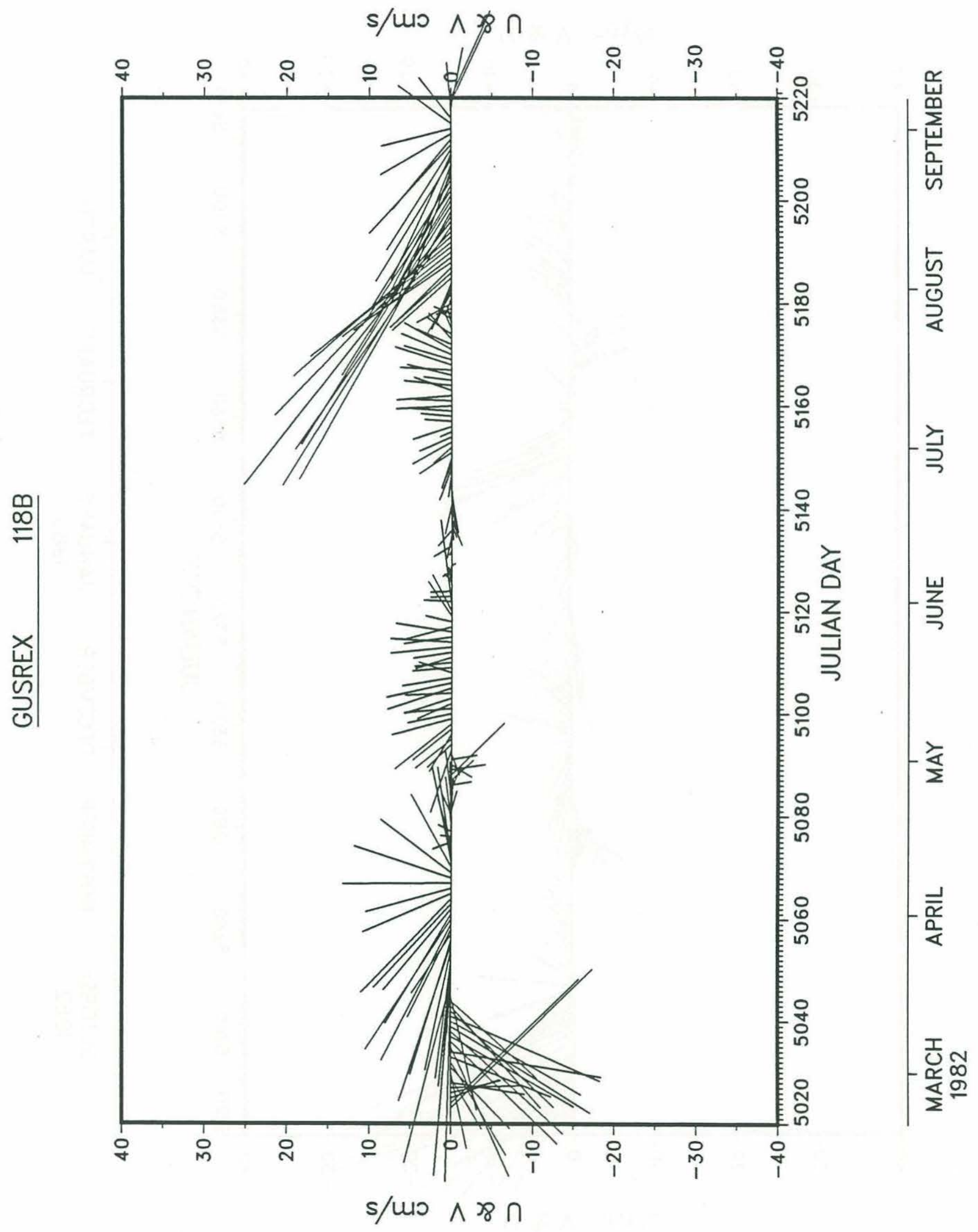




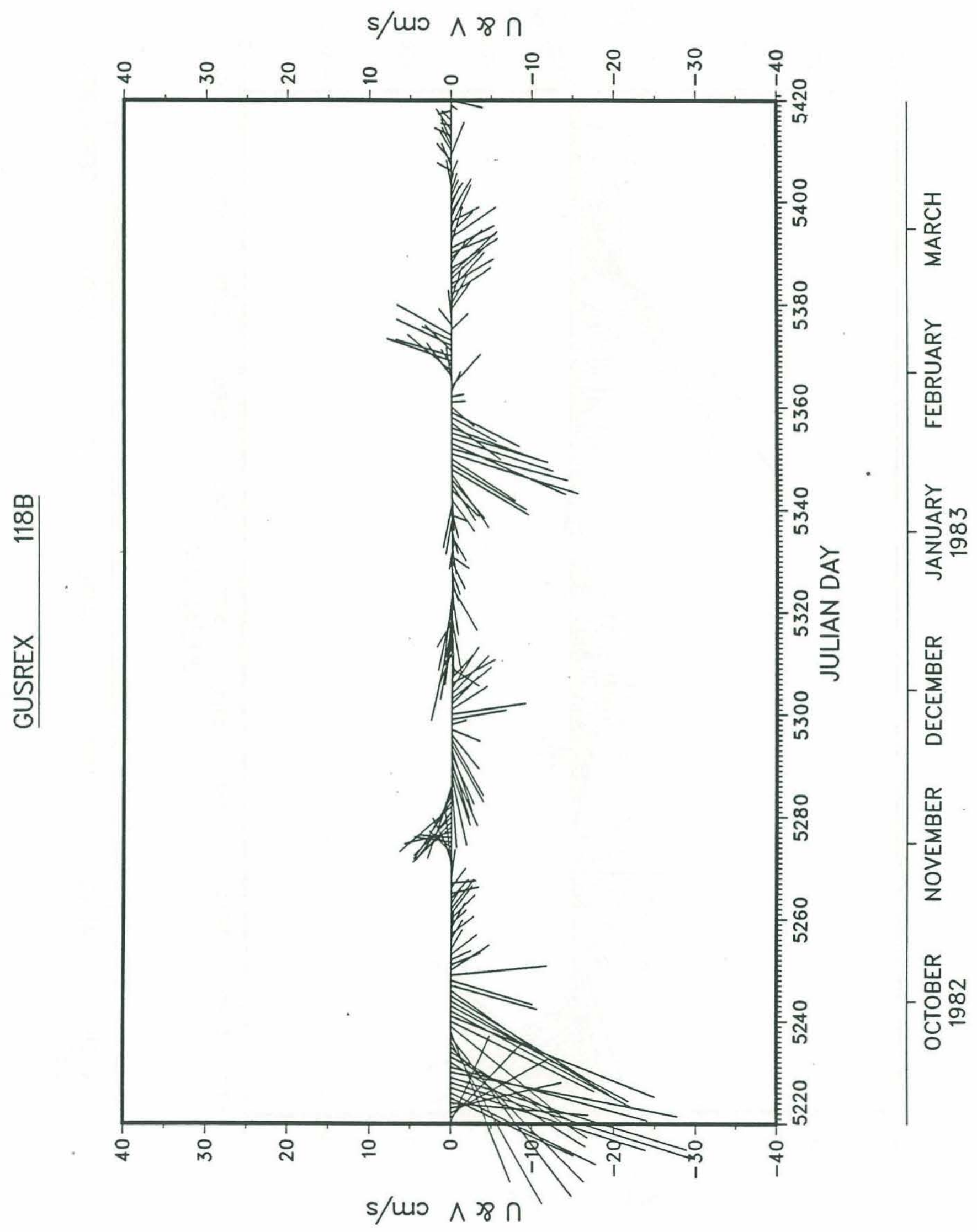




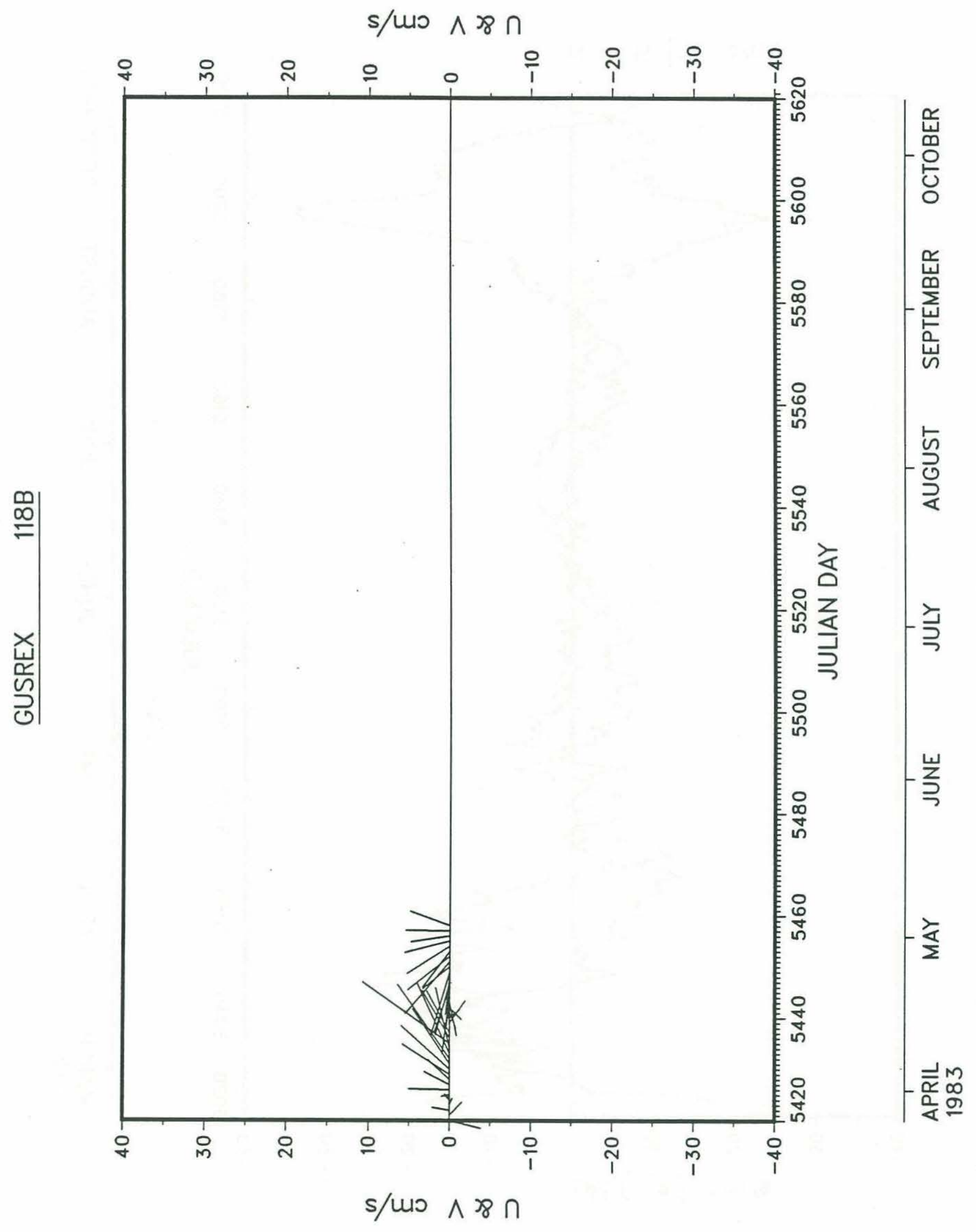




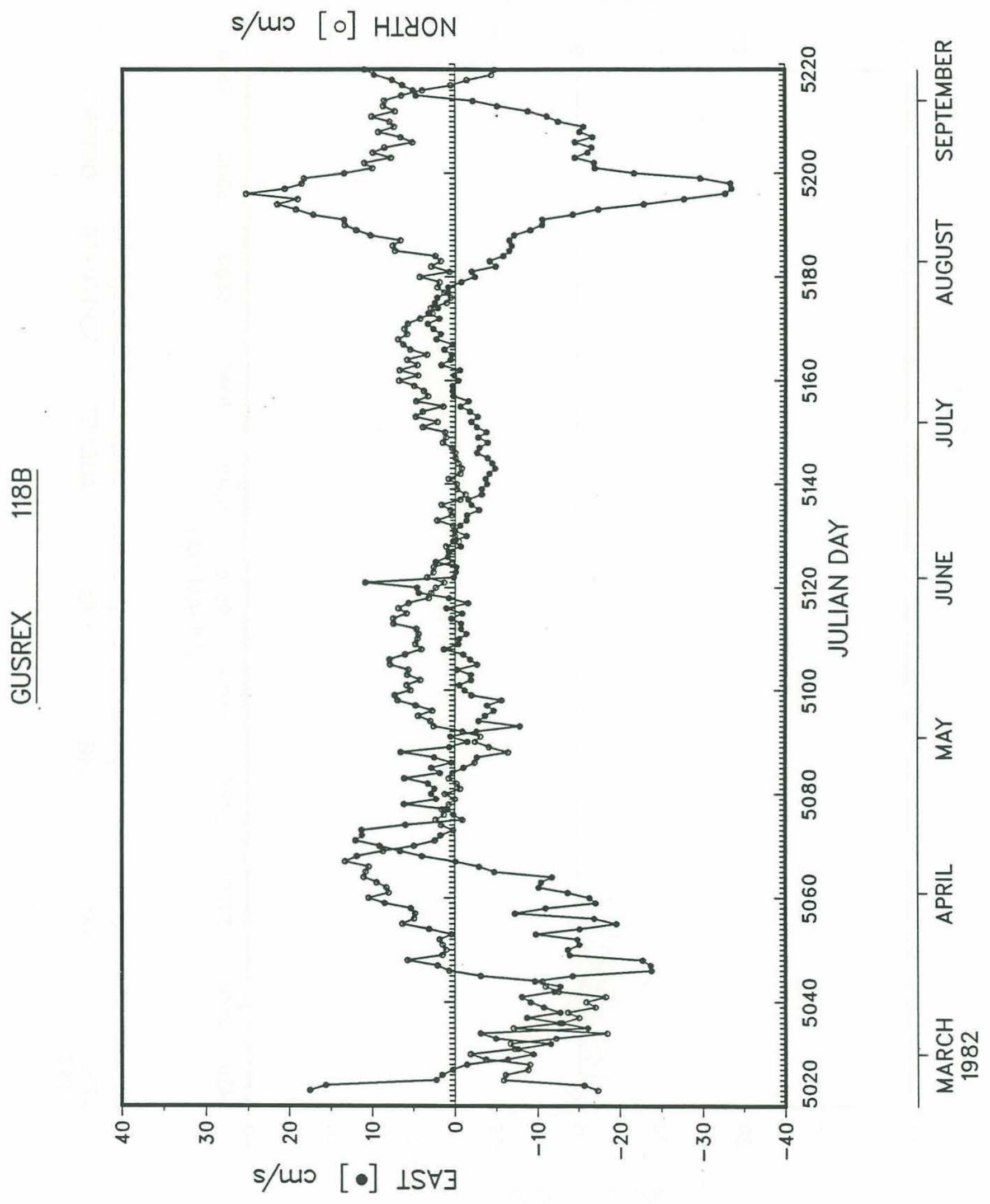


s/Mo [o] HLYON

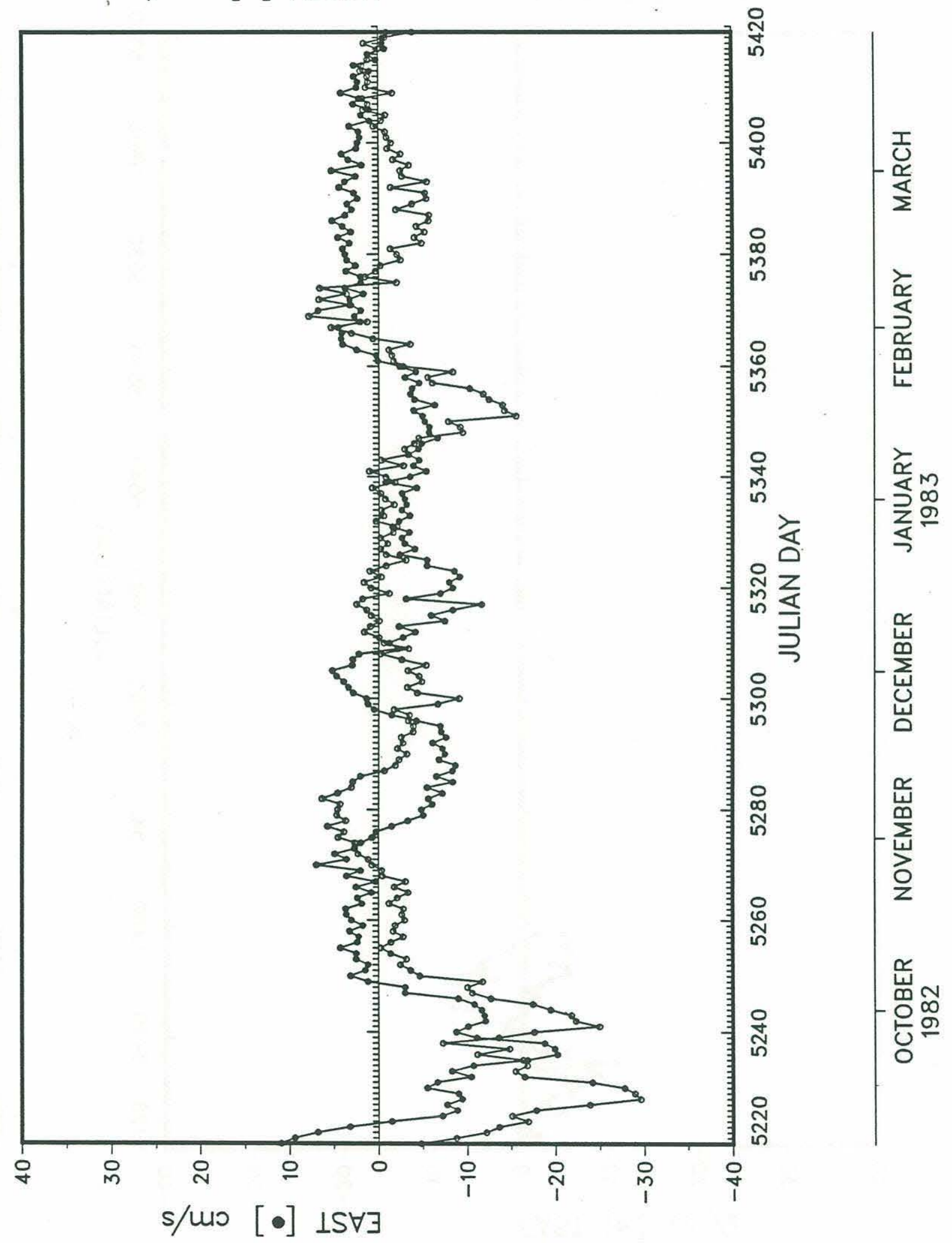




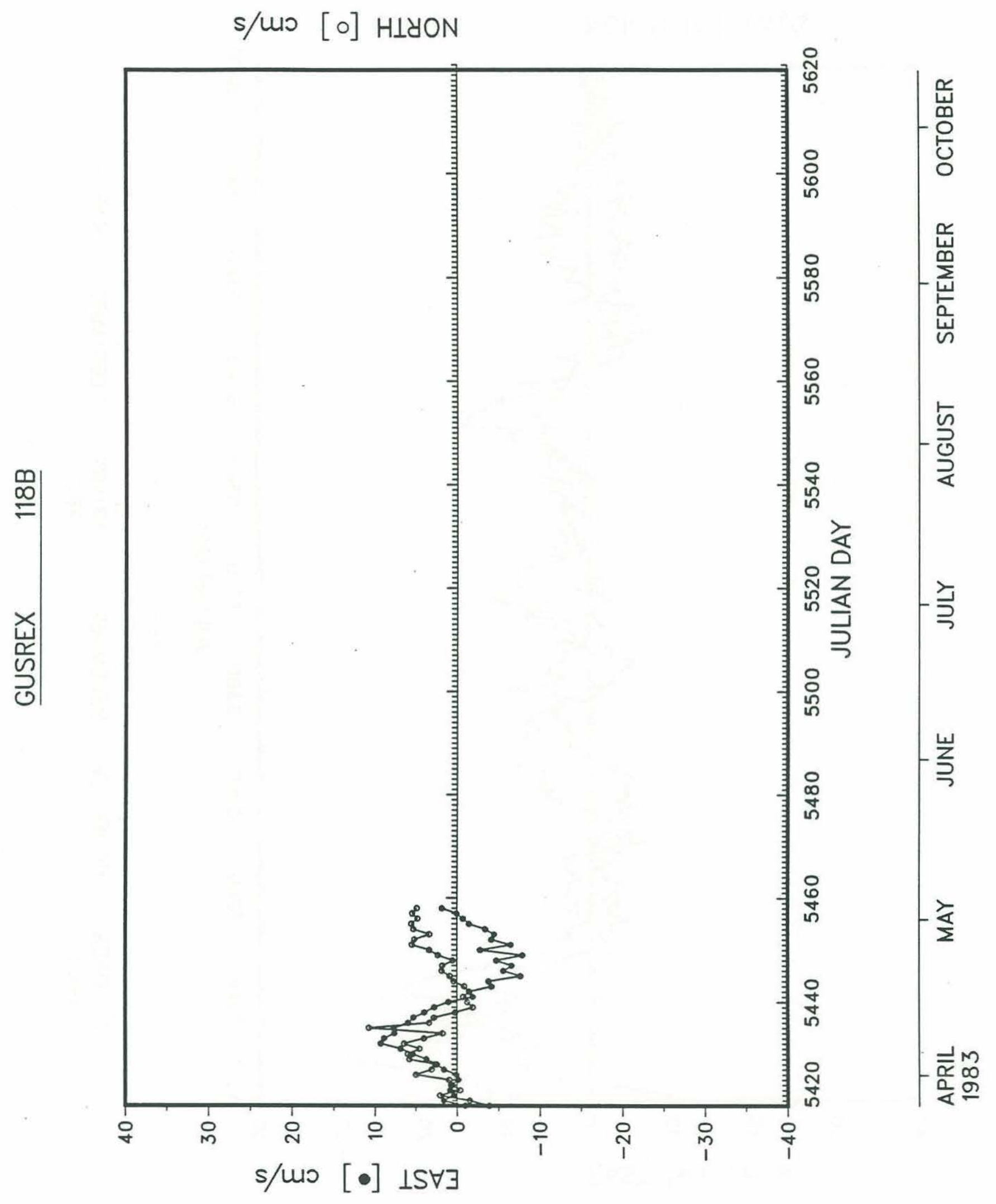




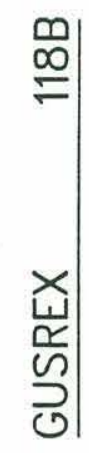

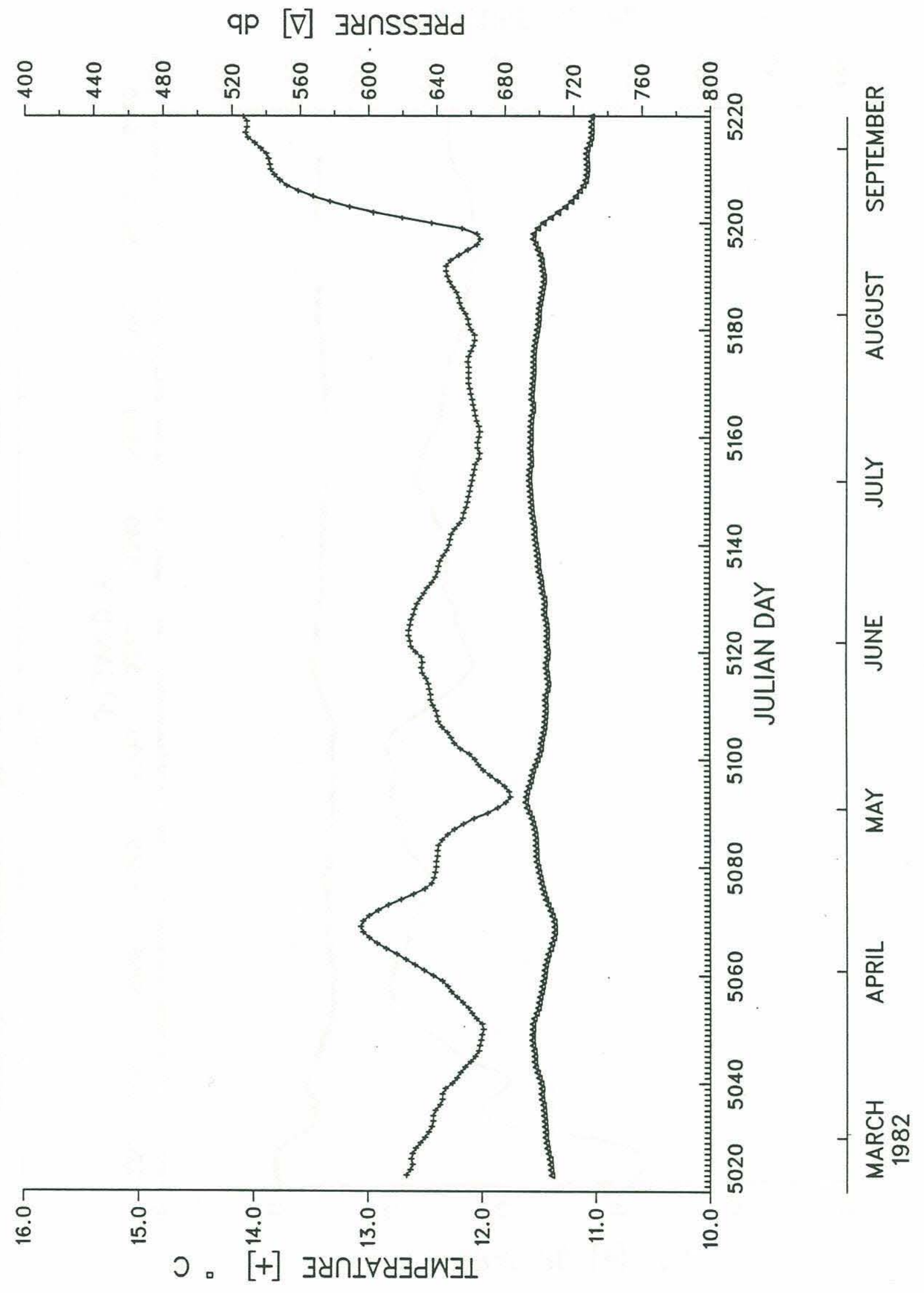




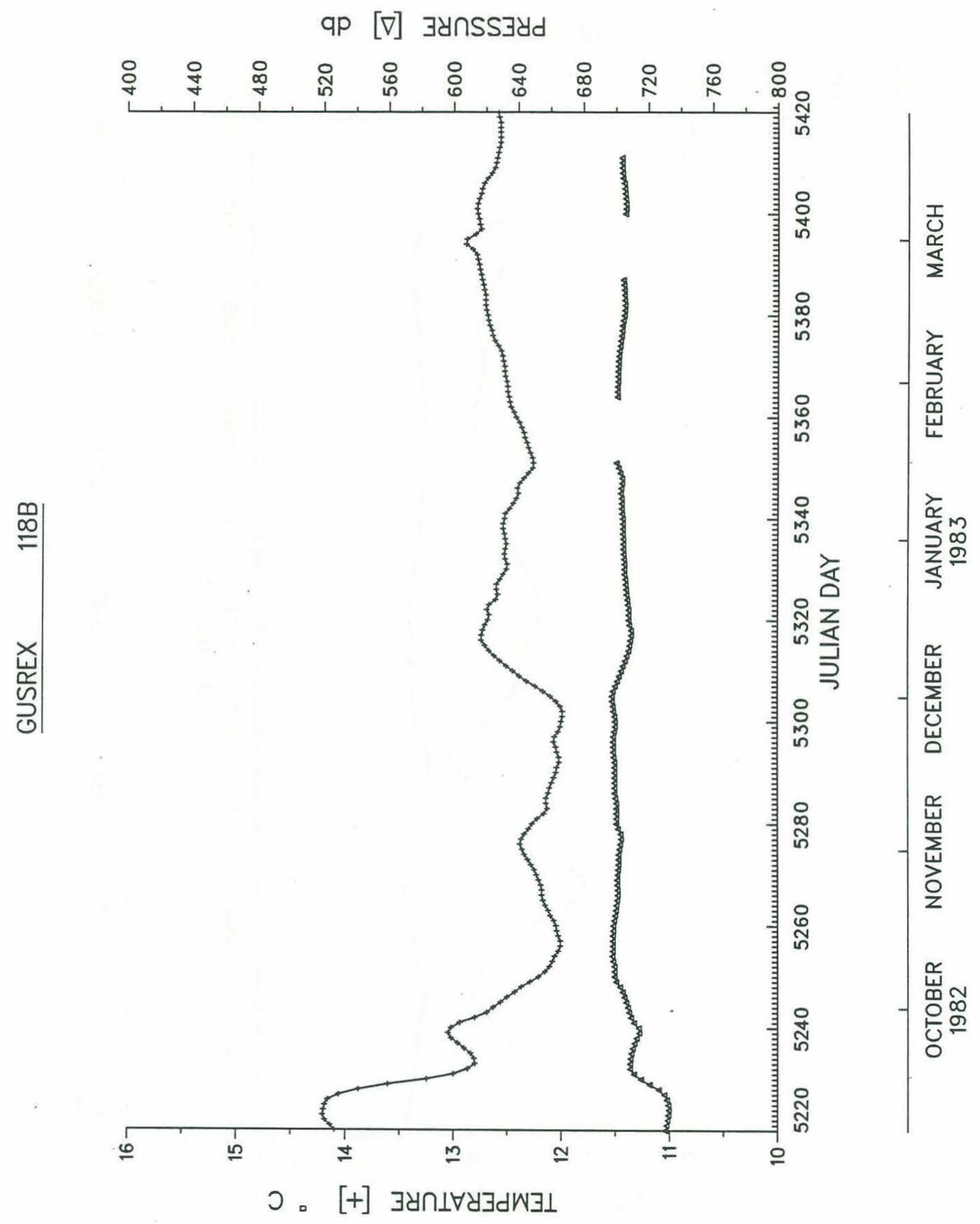




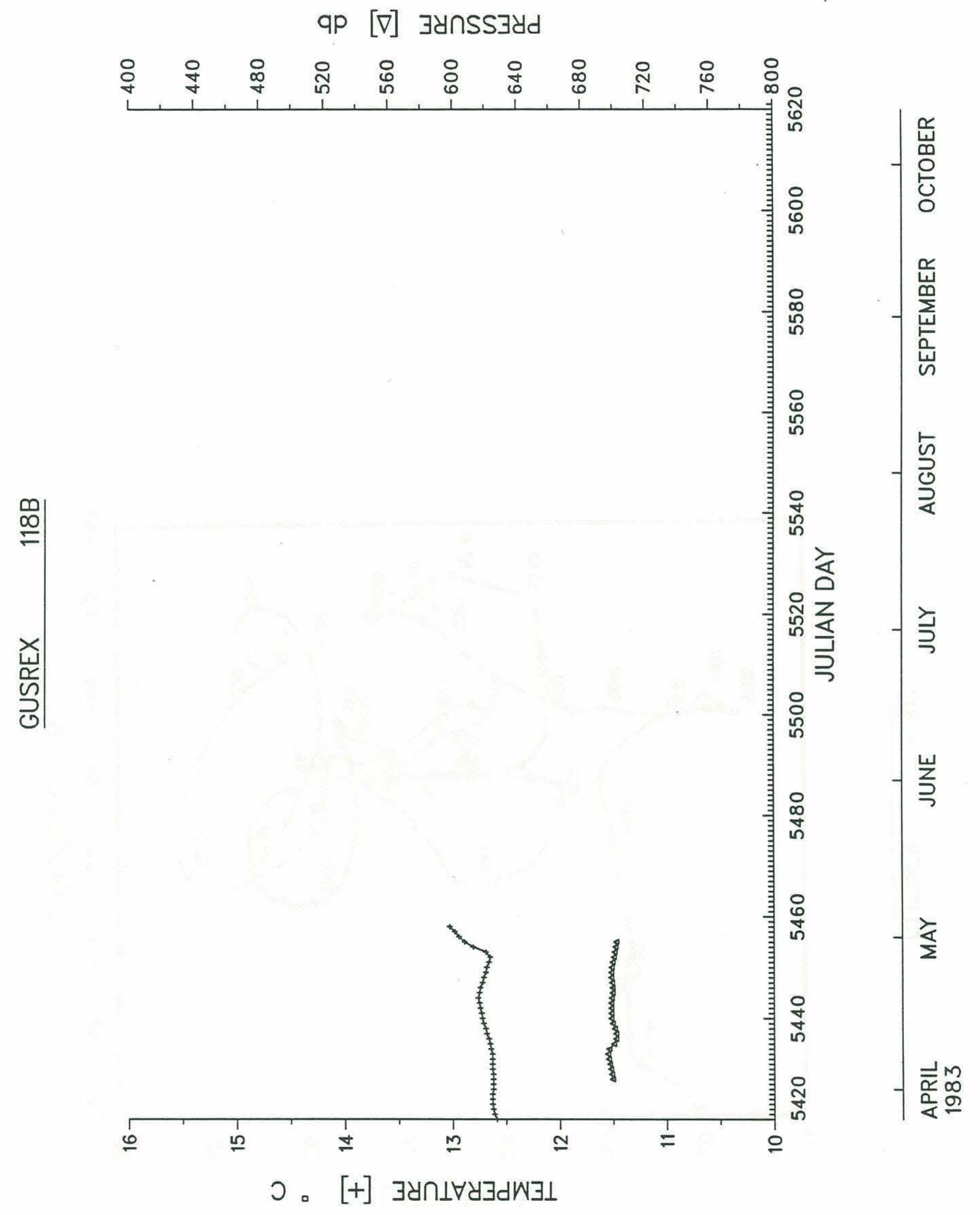




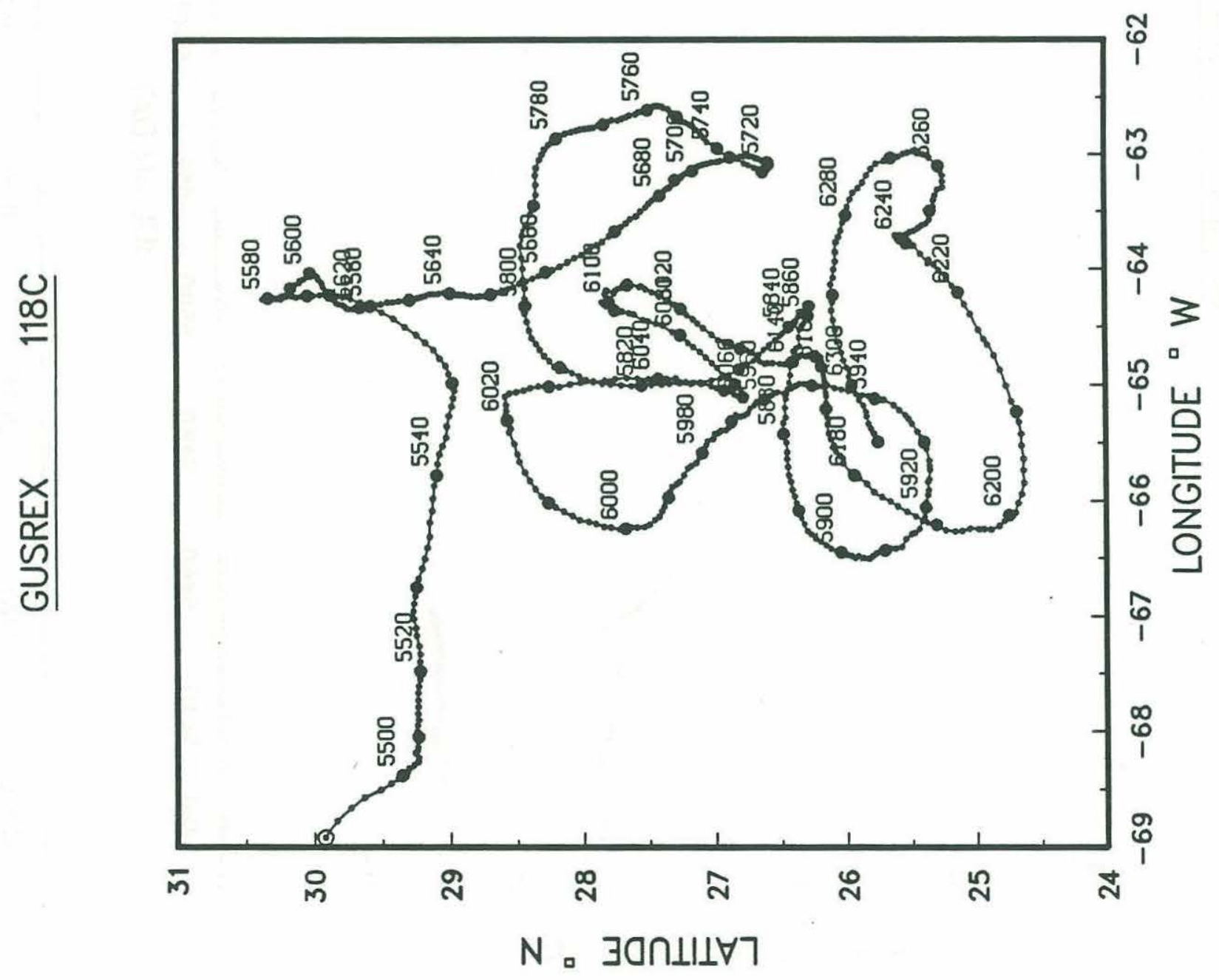




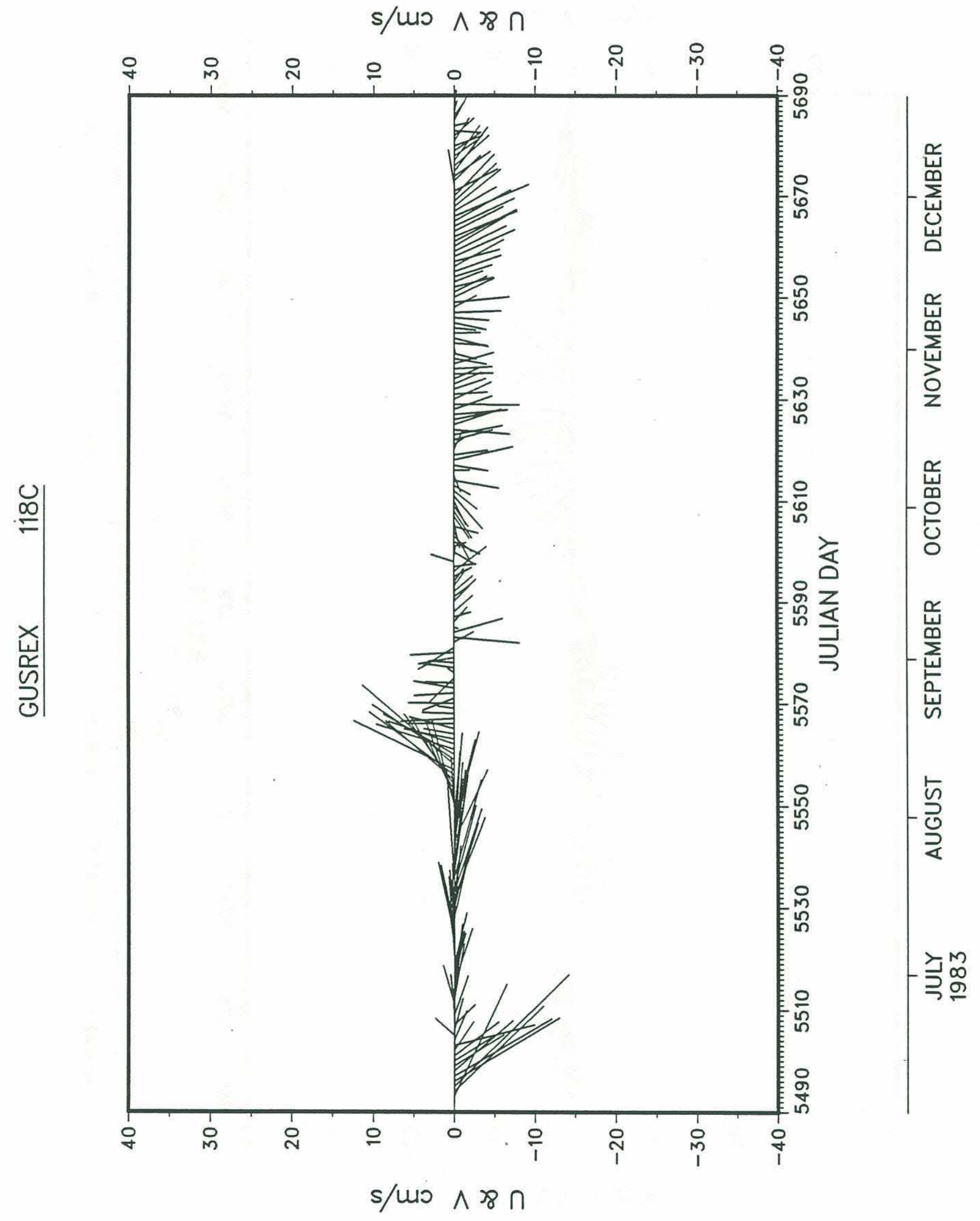




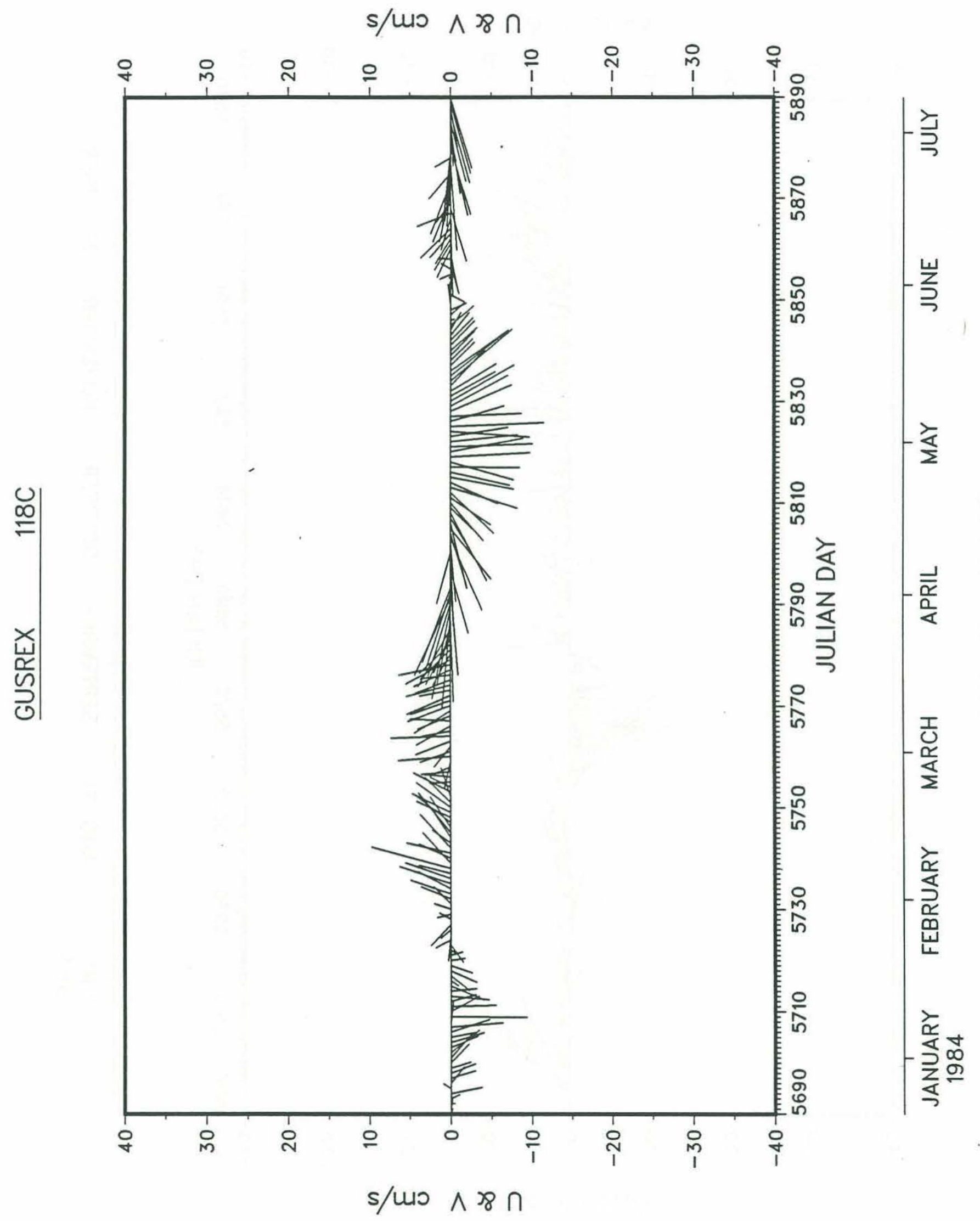




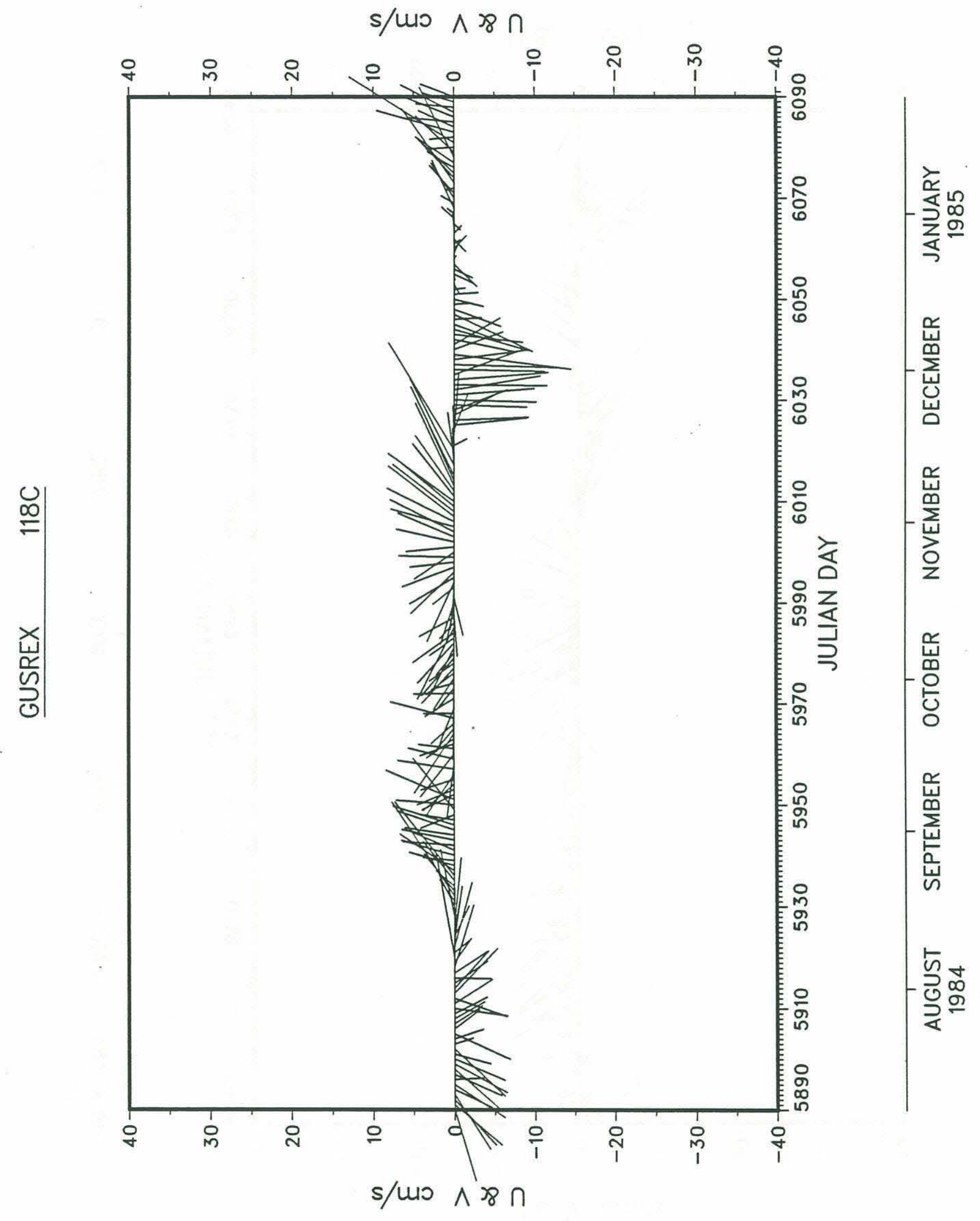




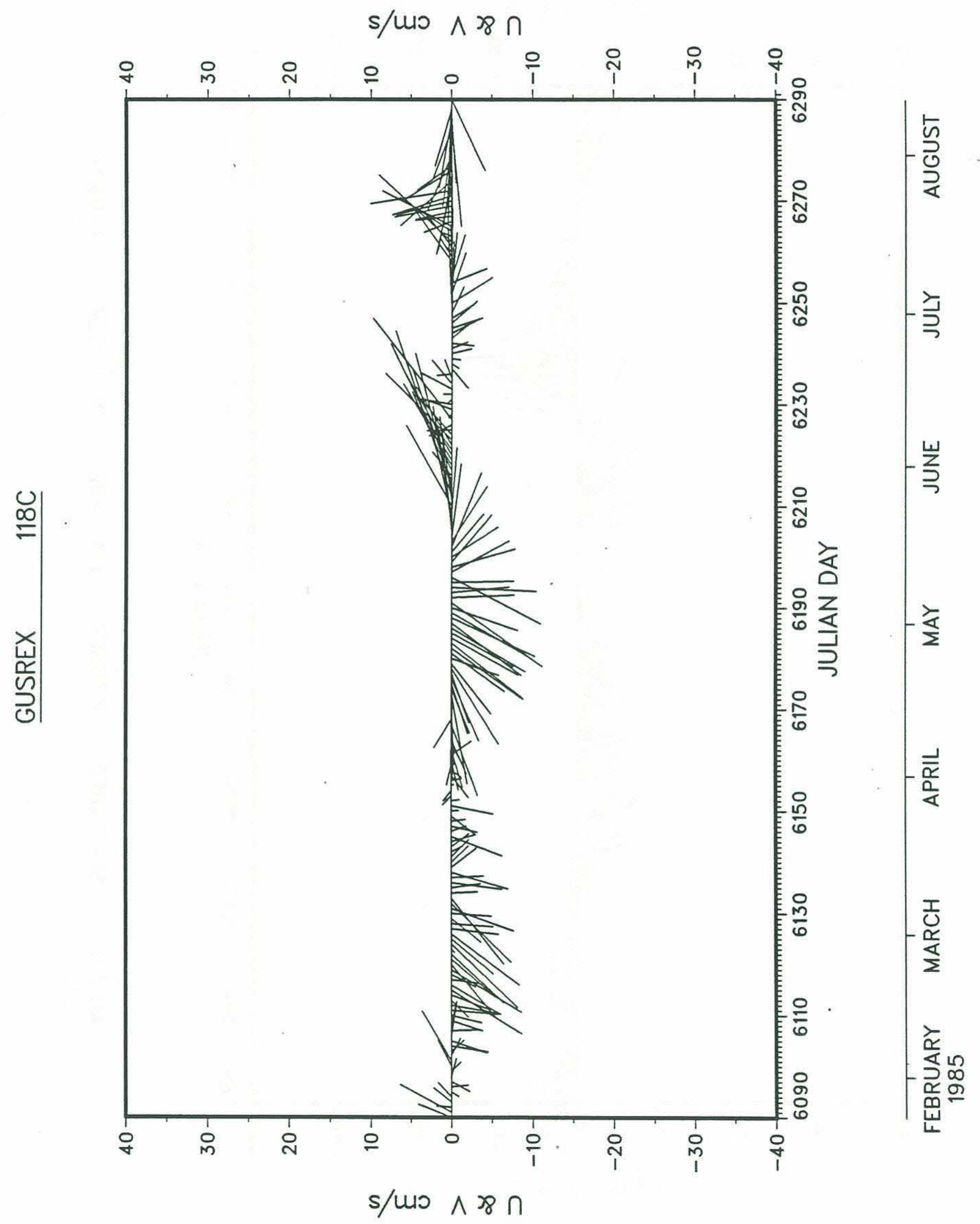




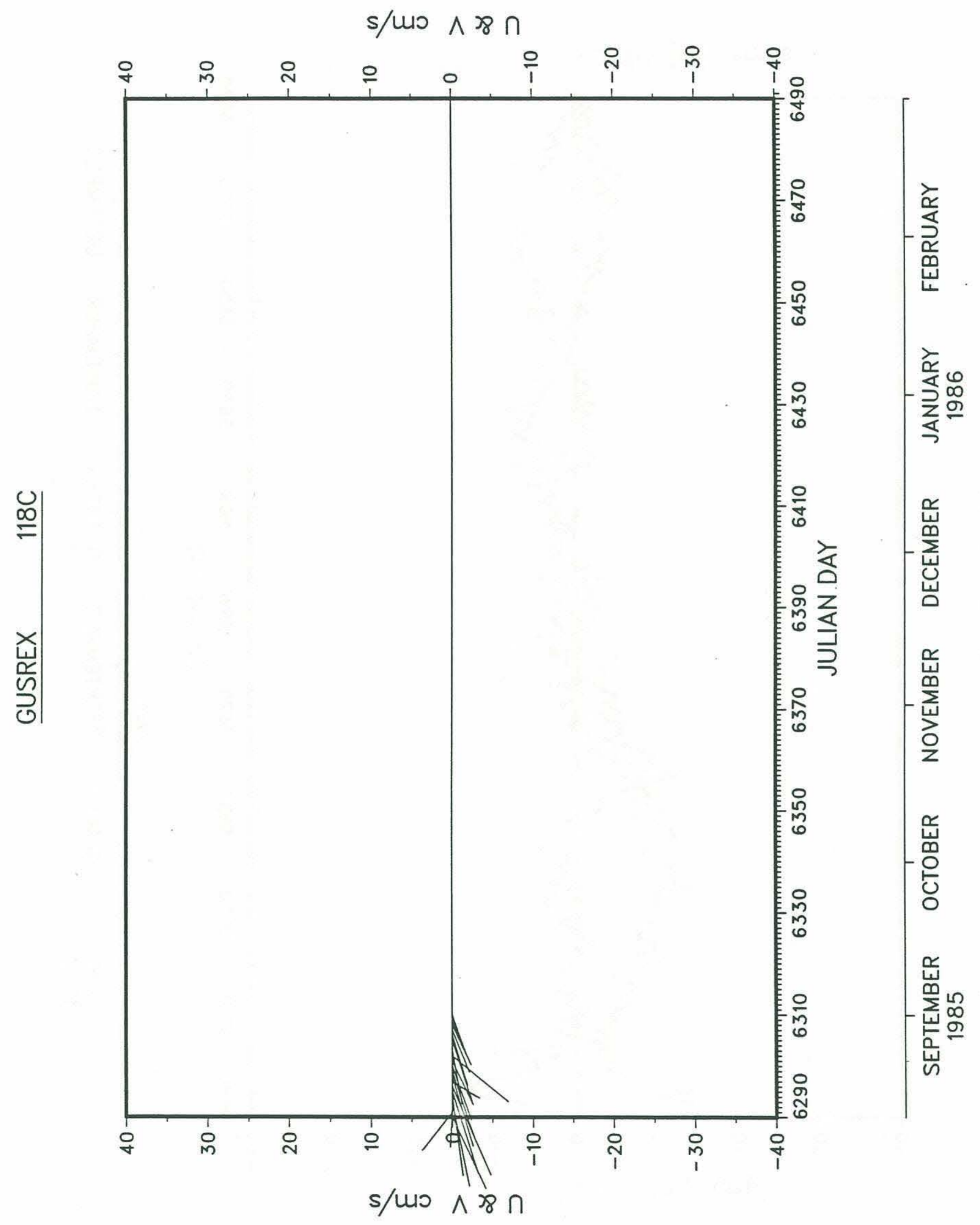




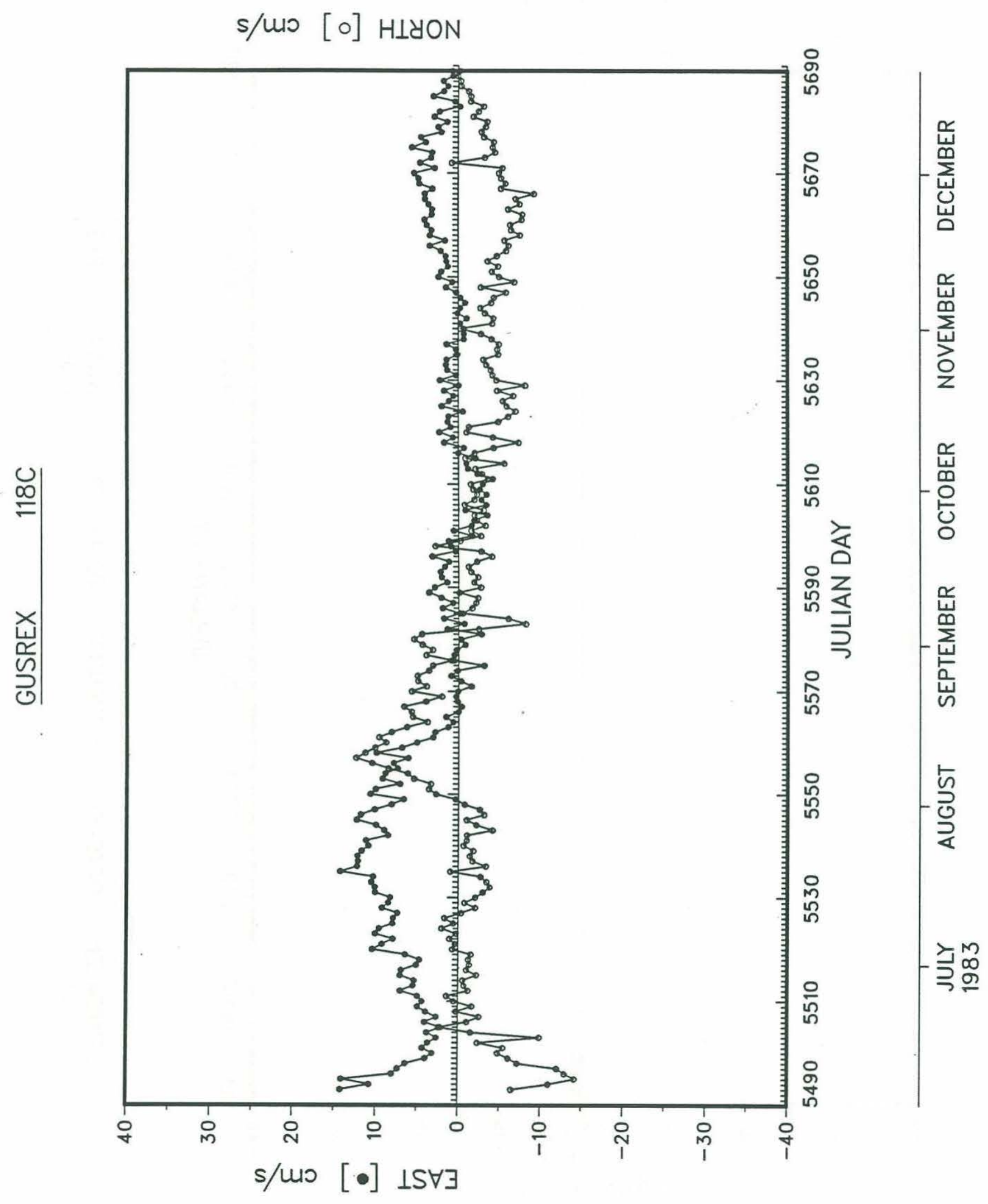




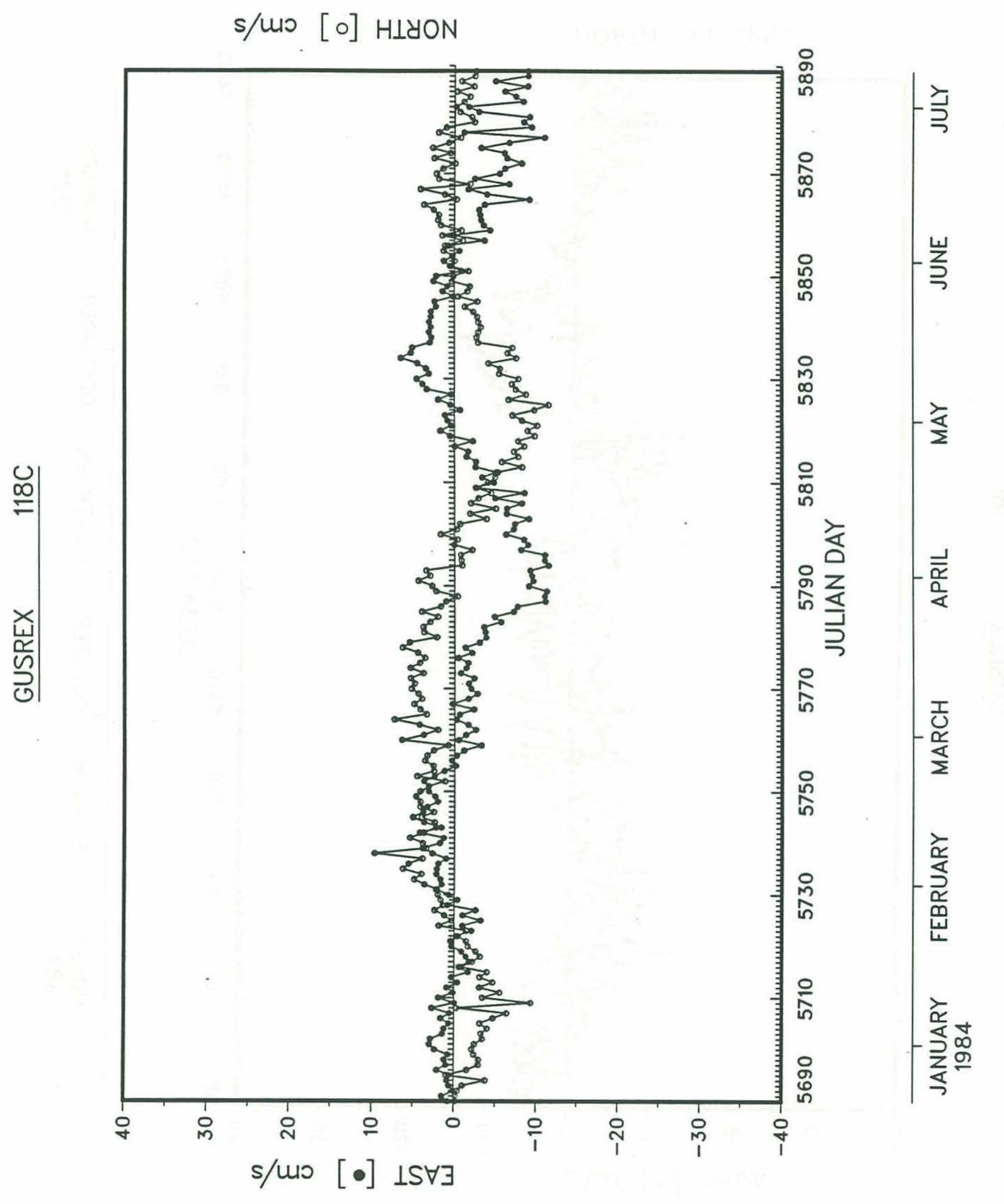




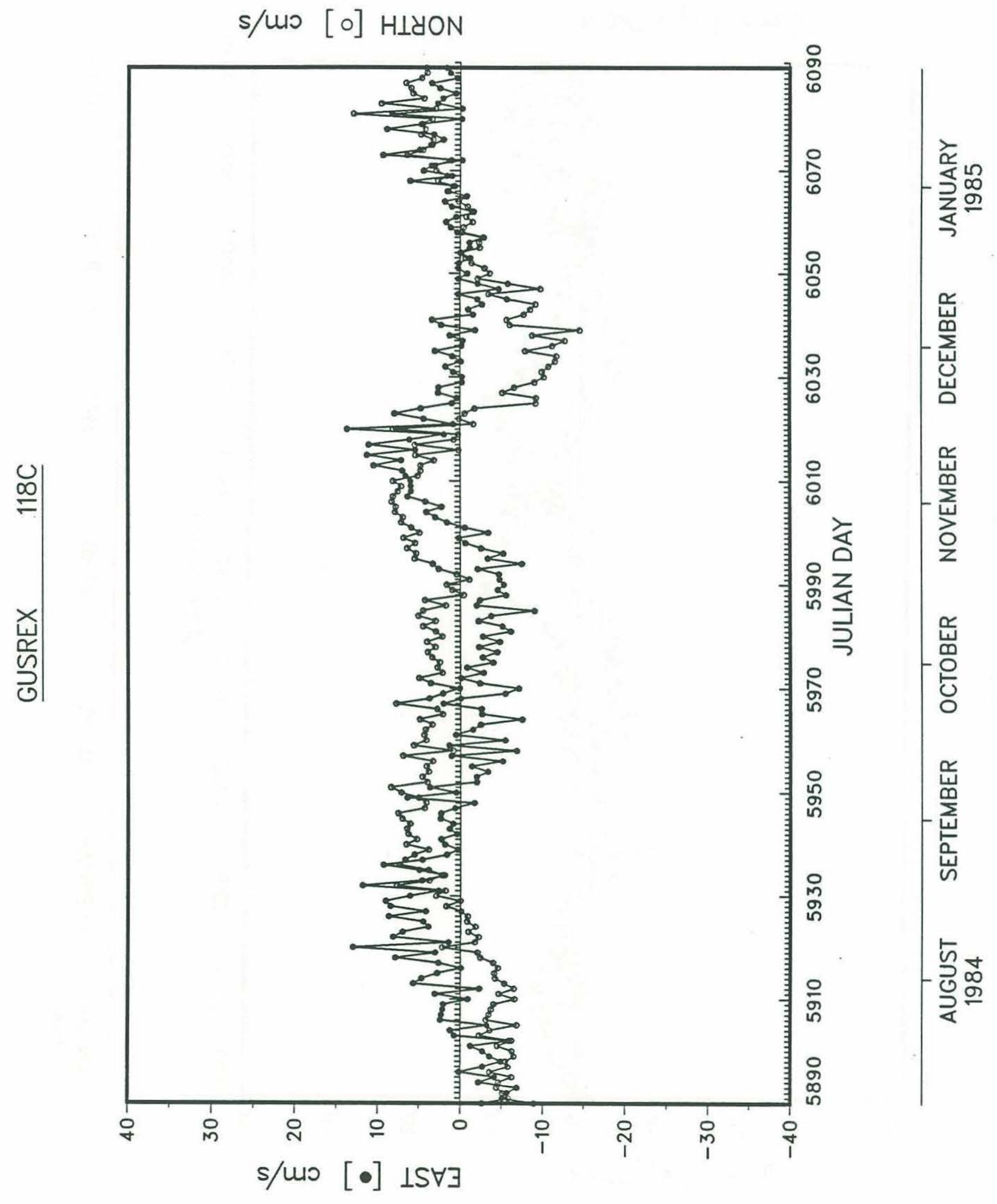


s/ur [o] HLYON

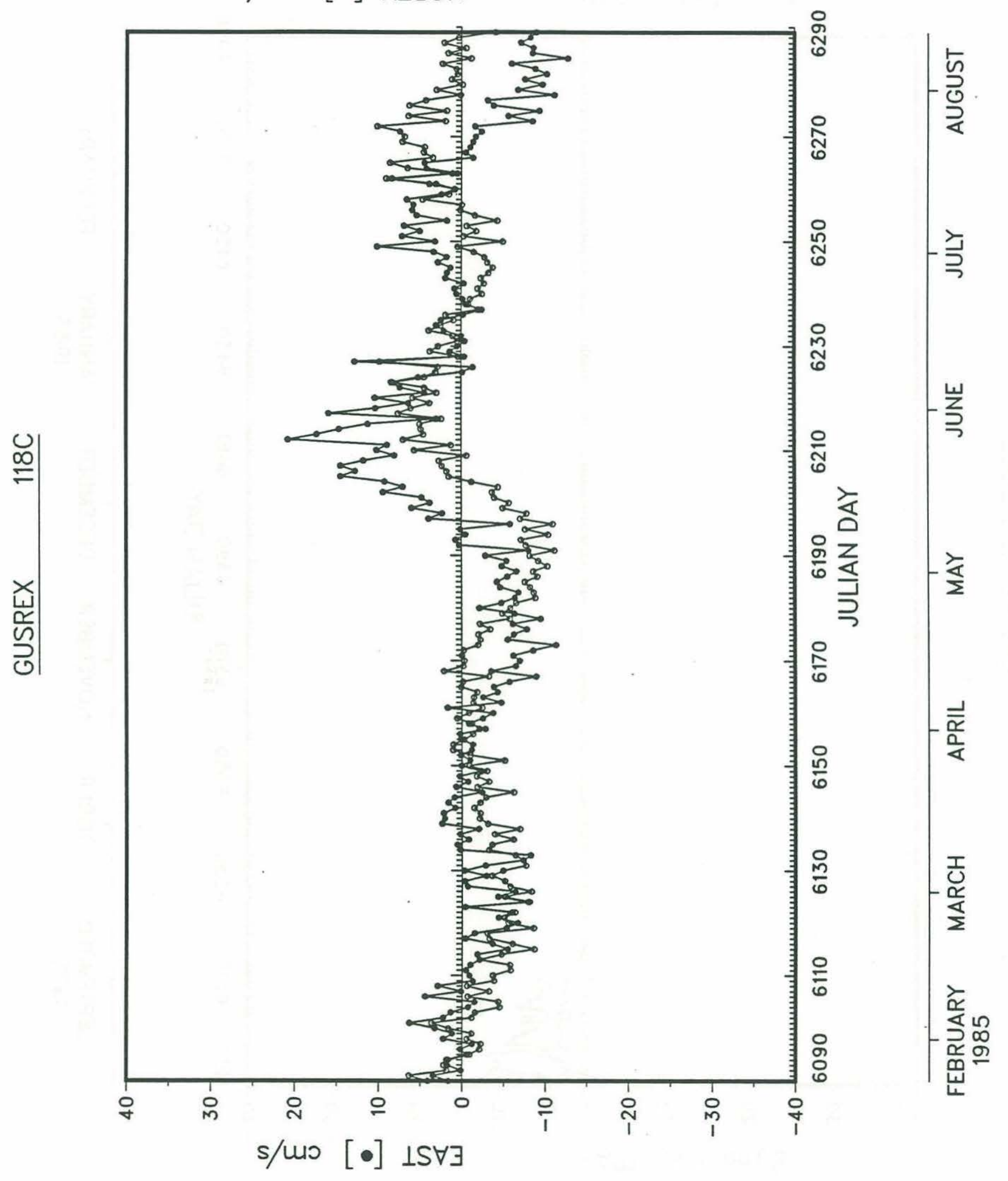




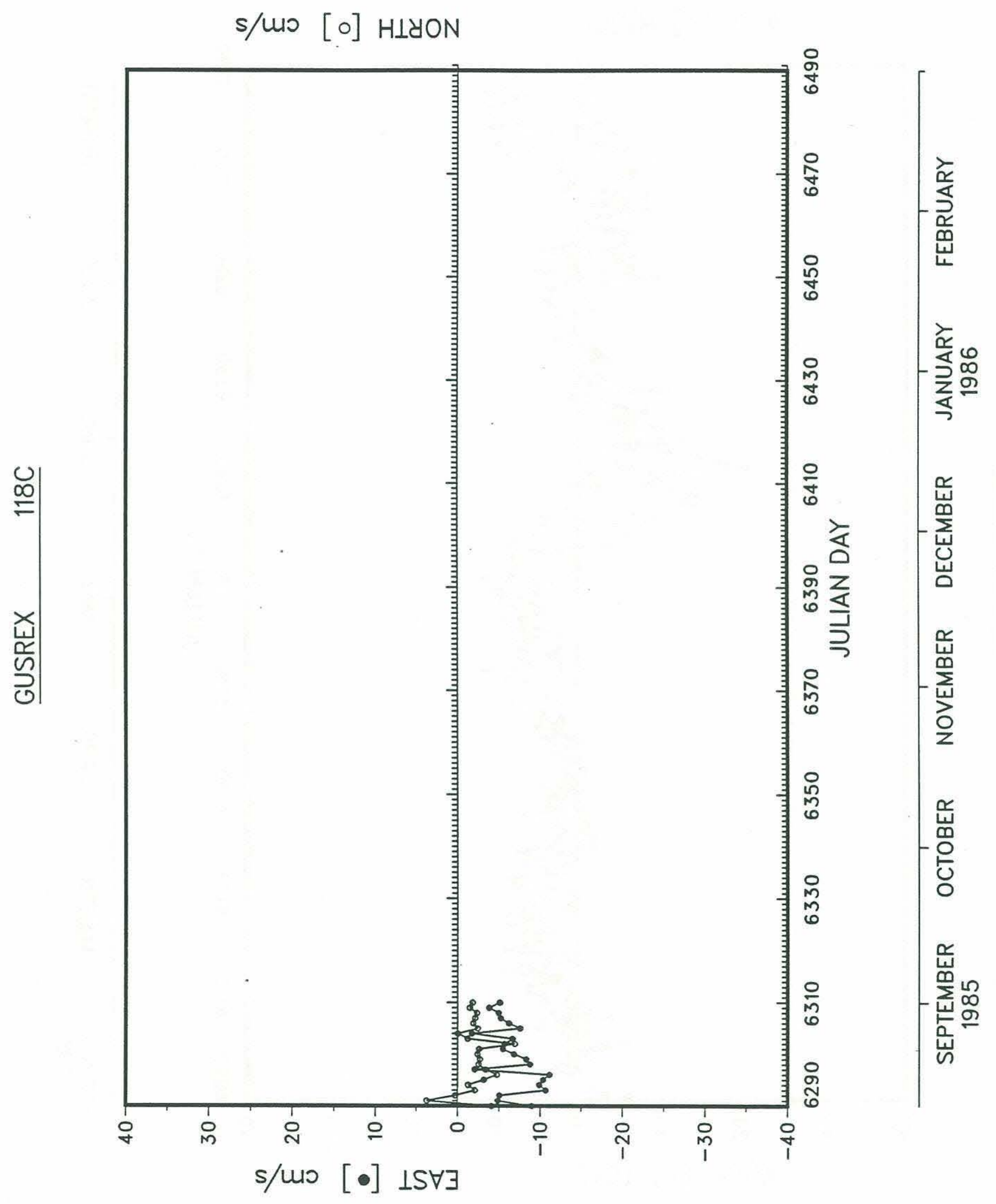




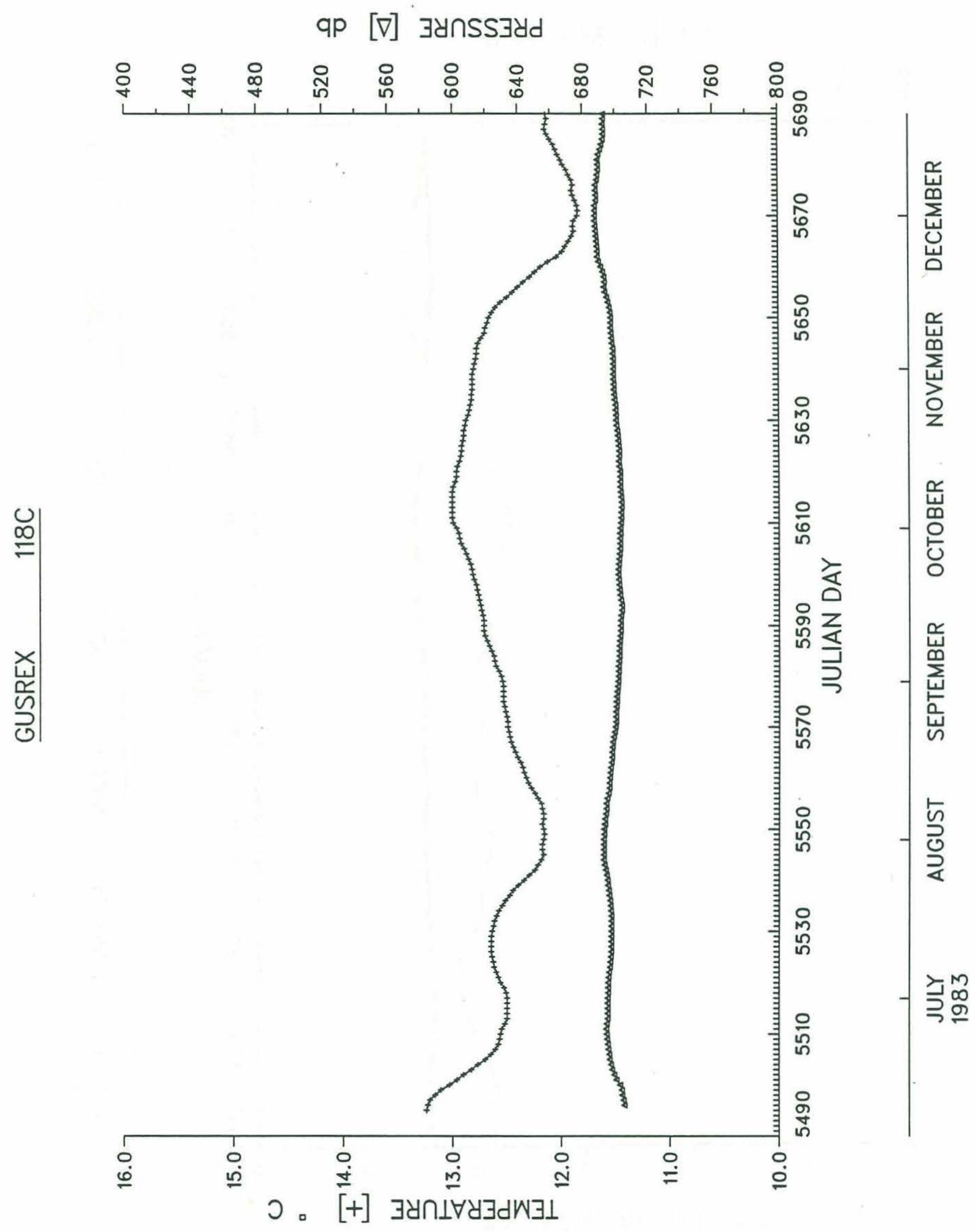




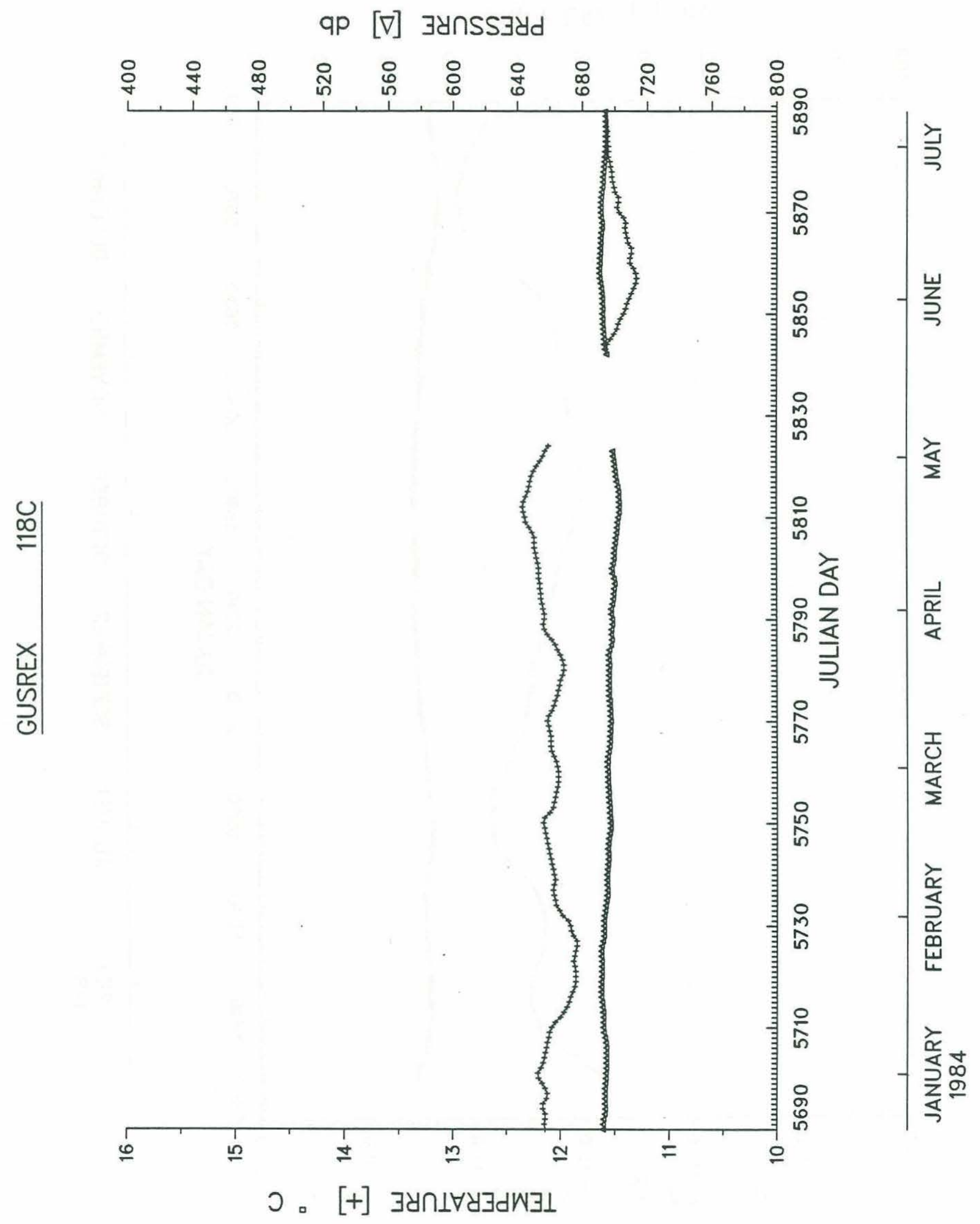




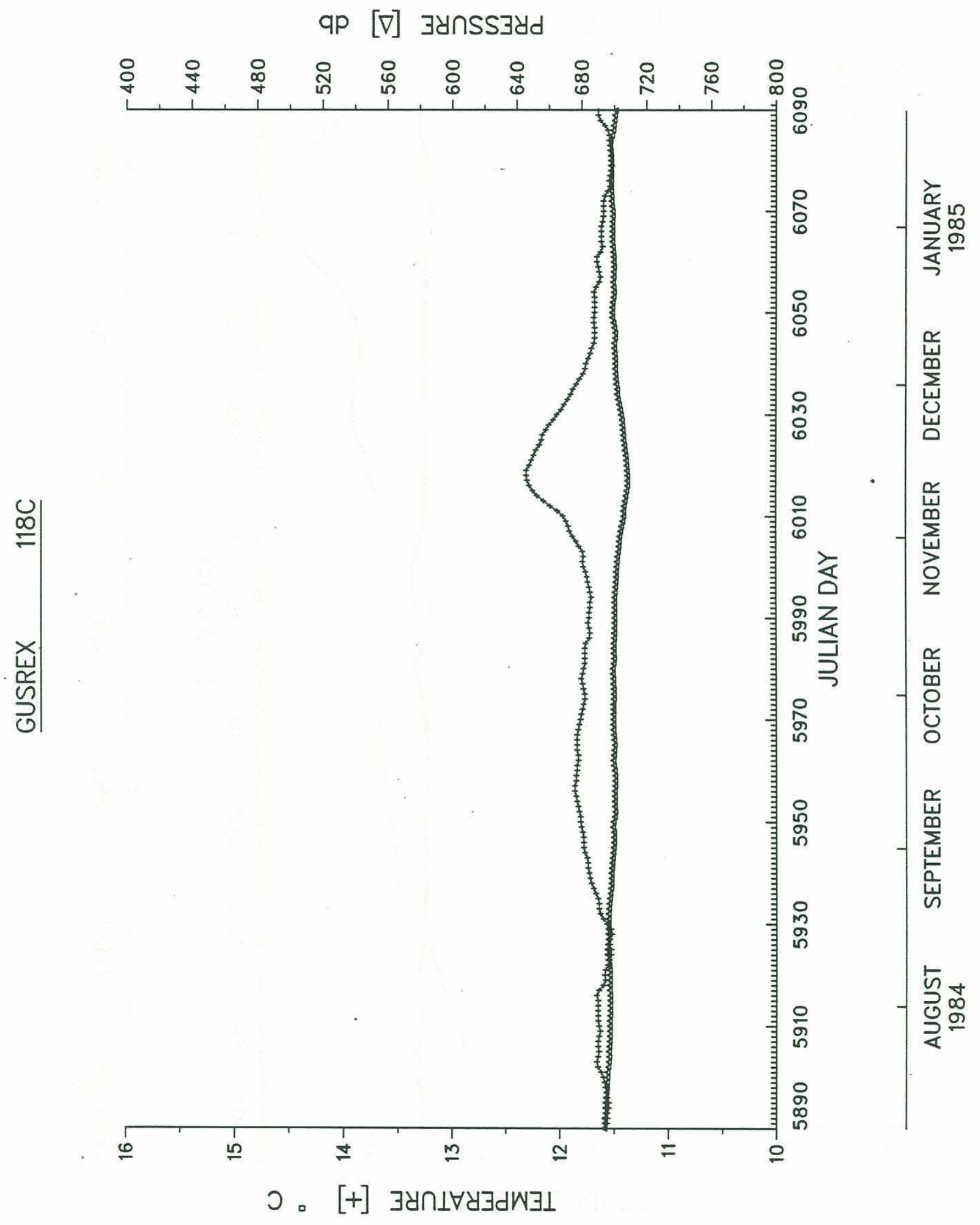




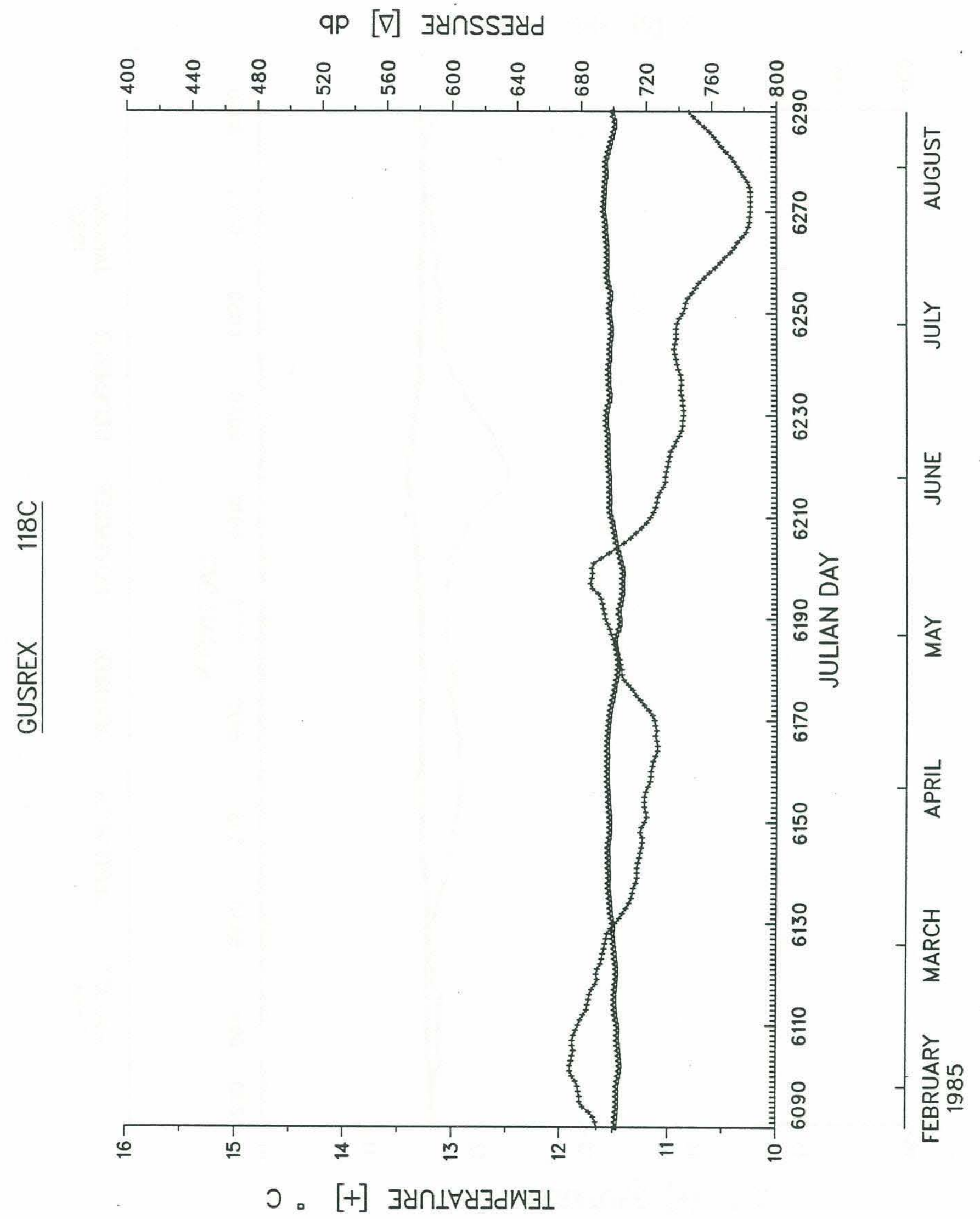




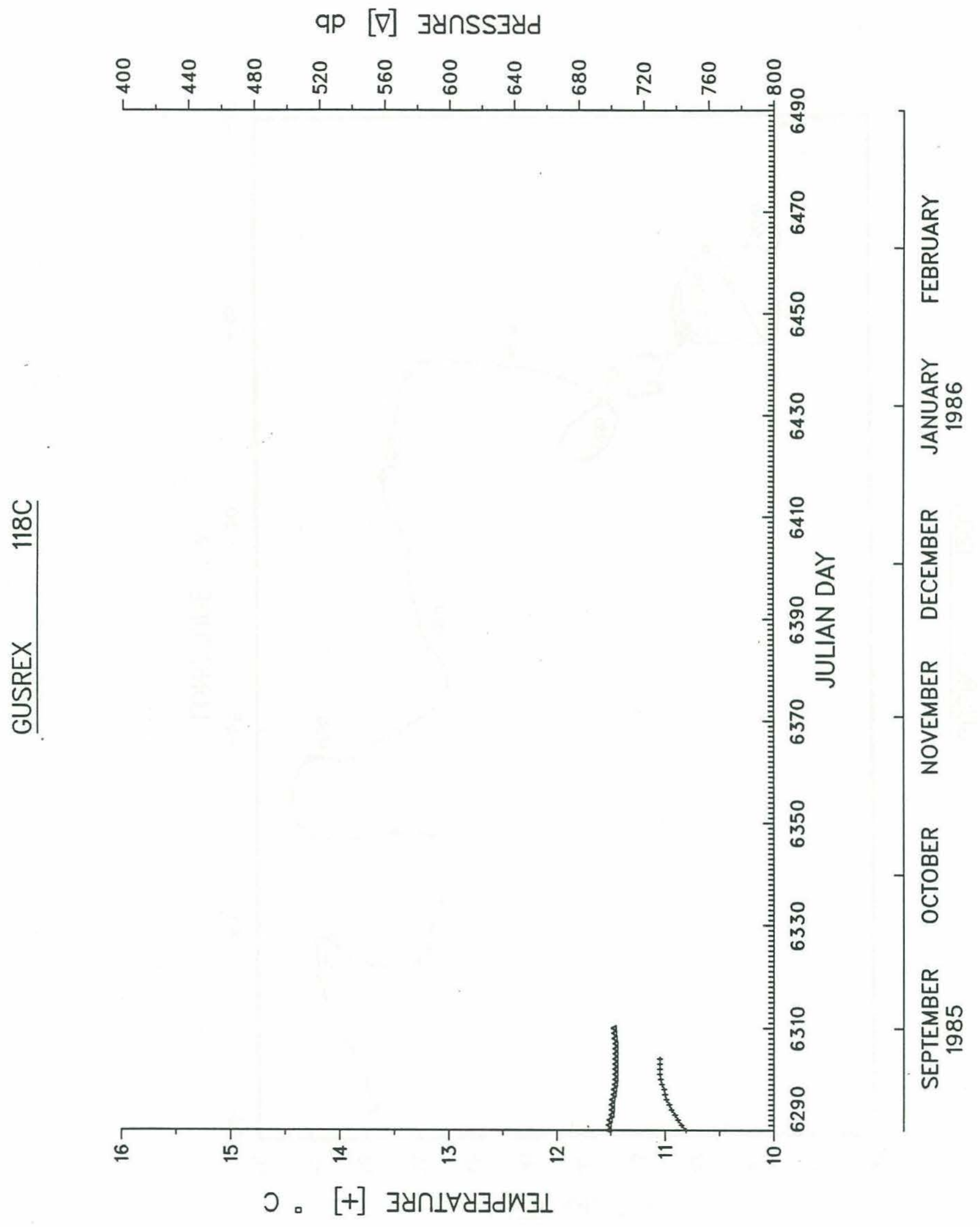




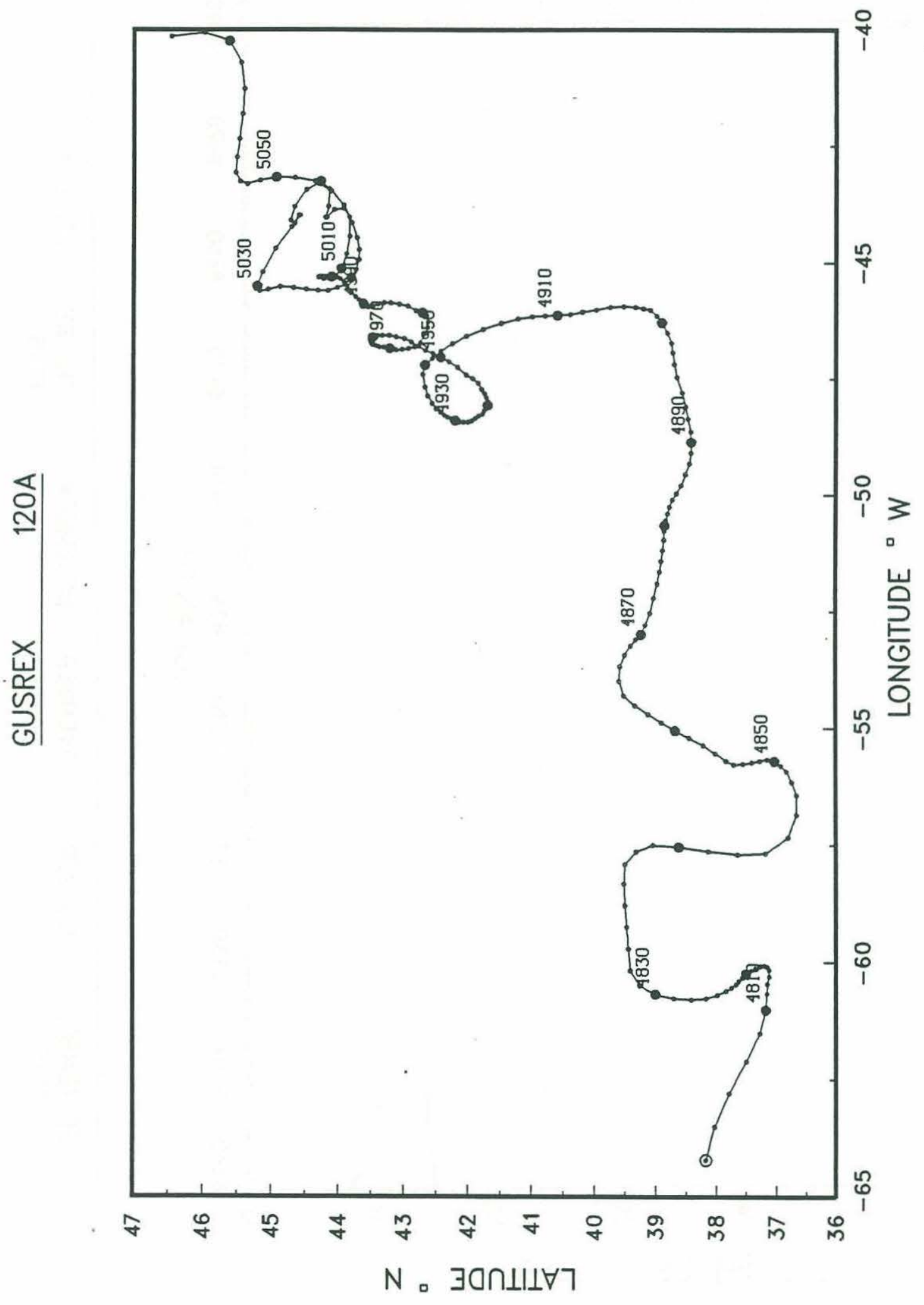




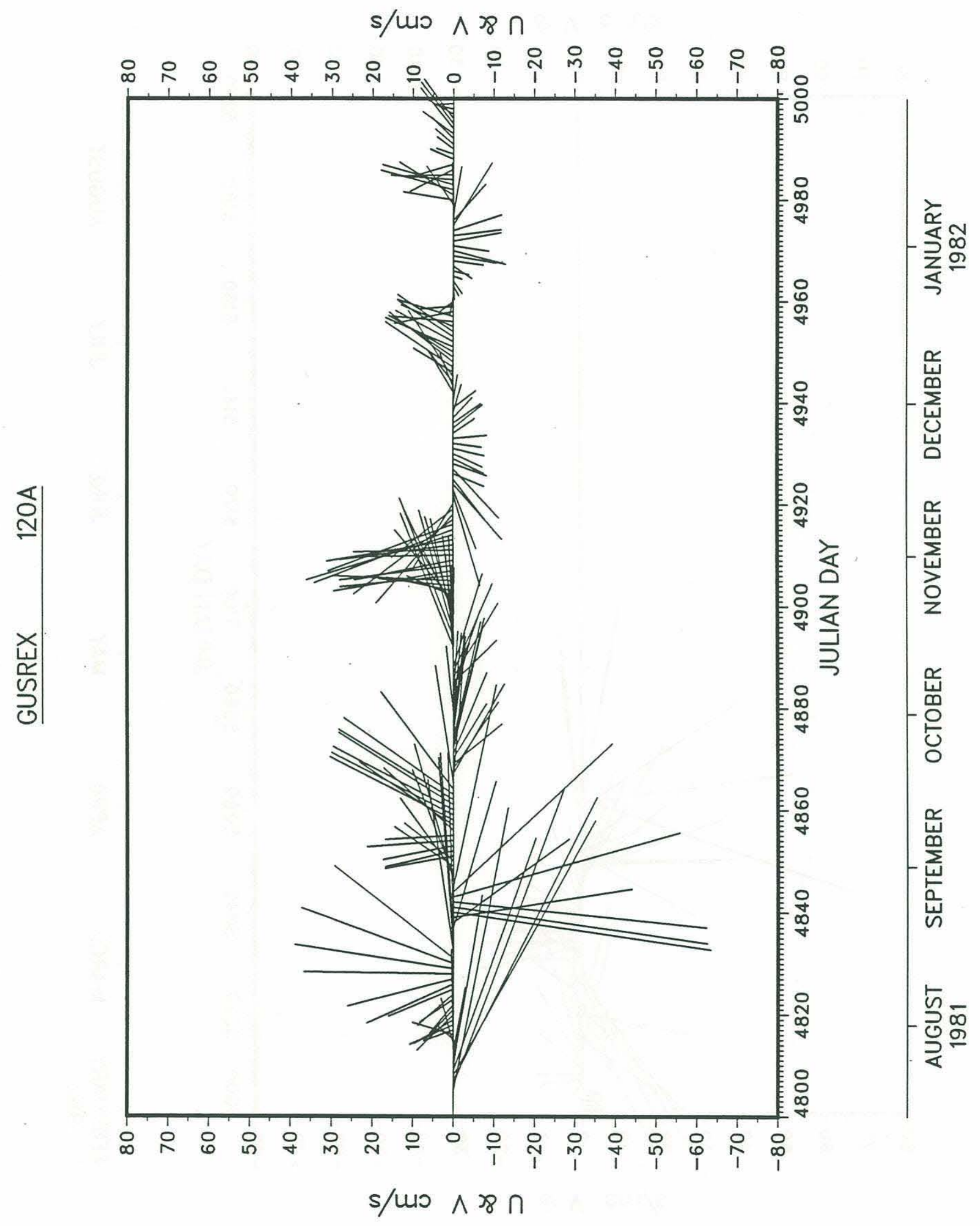




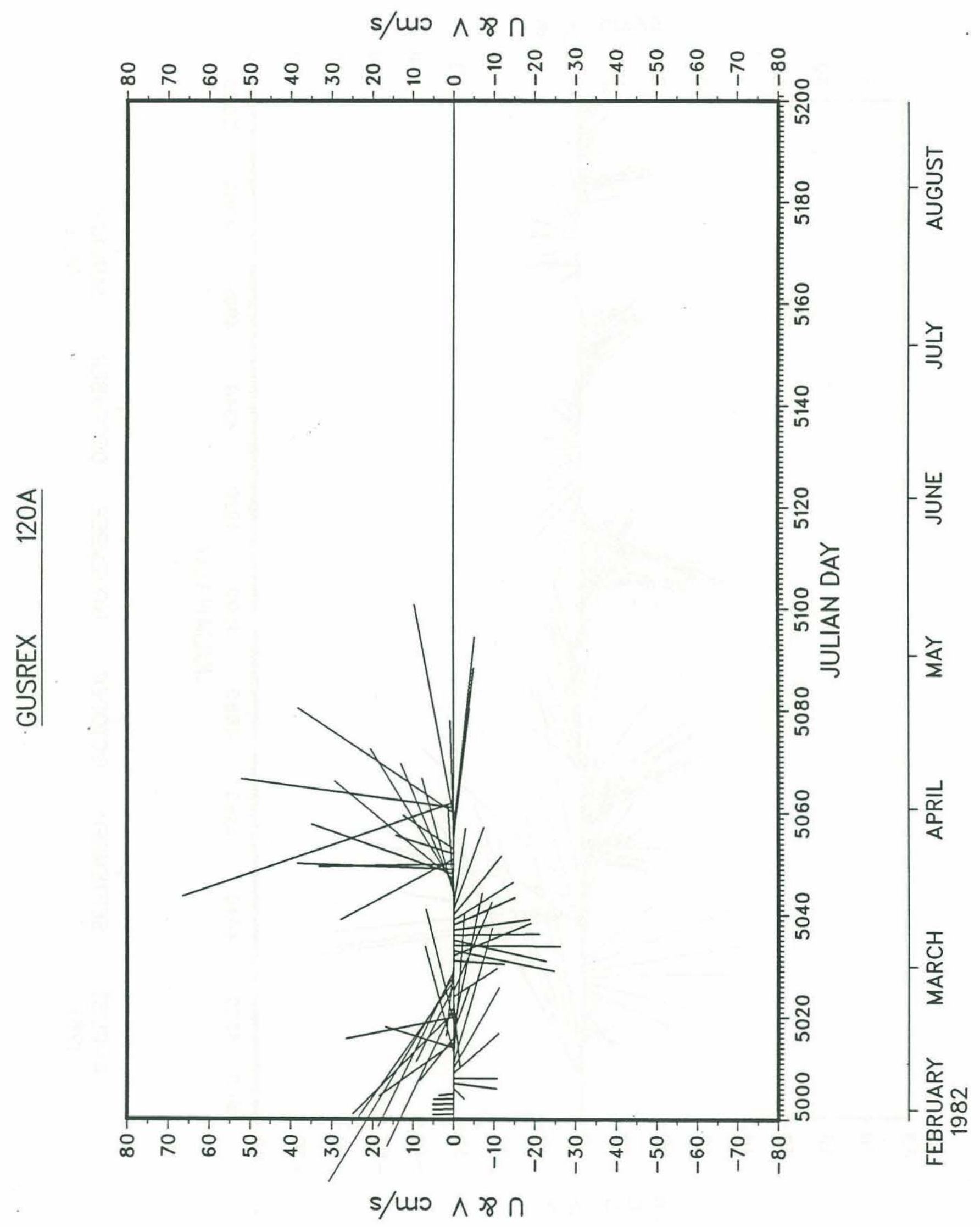




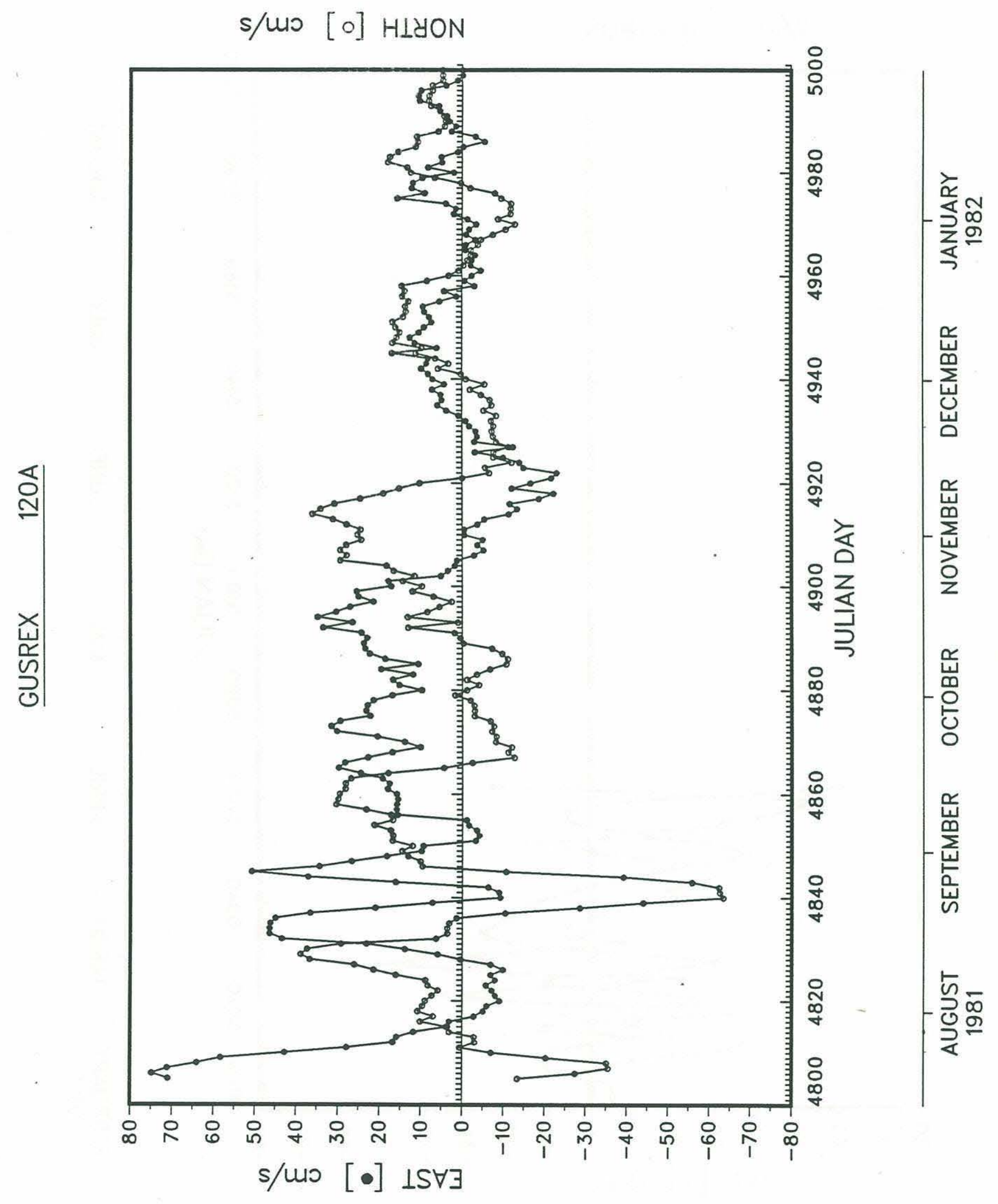




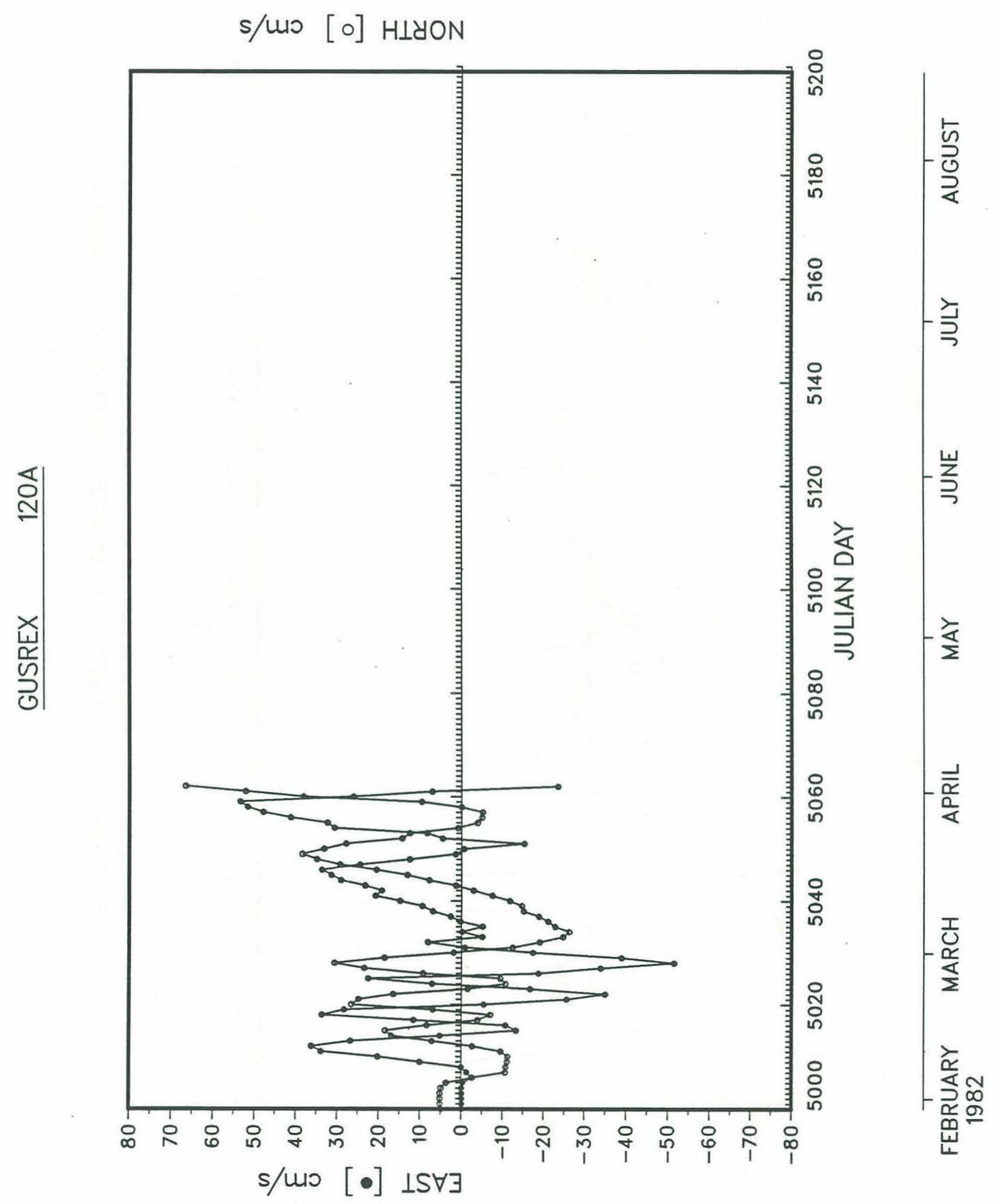




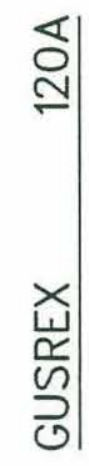

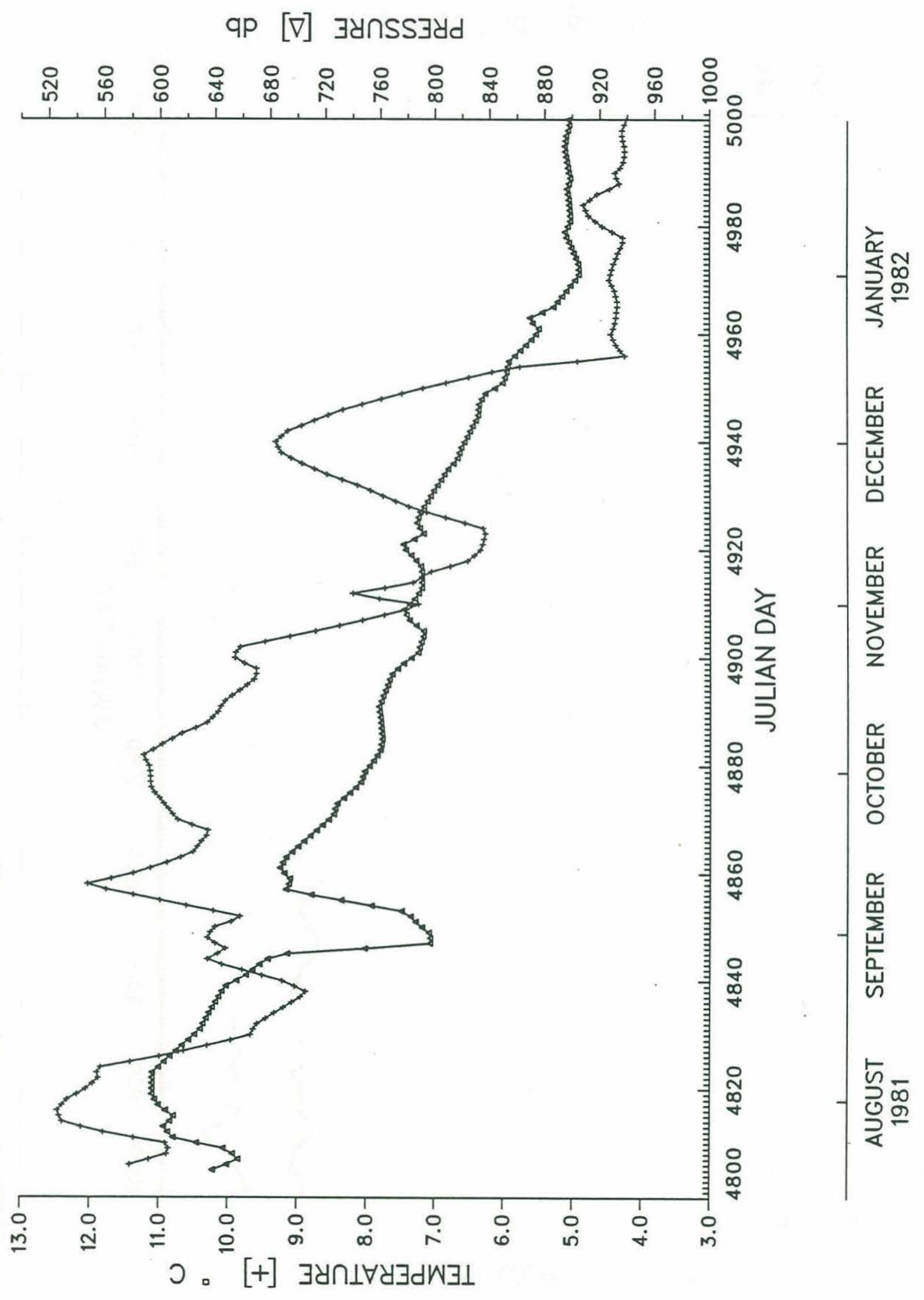




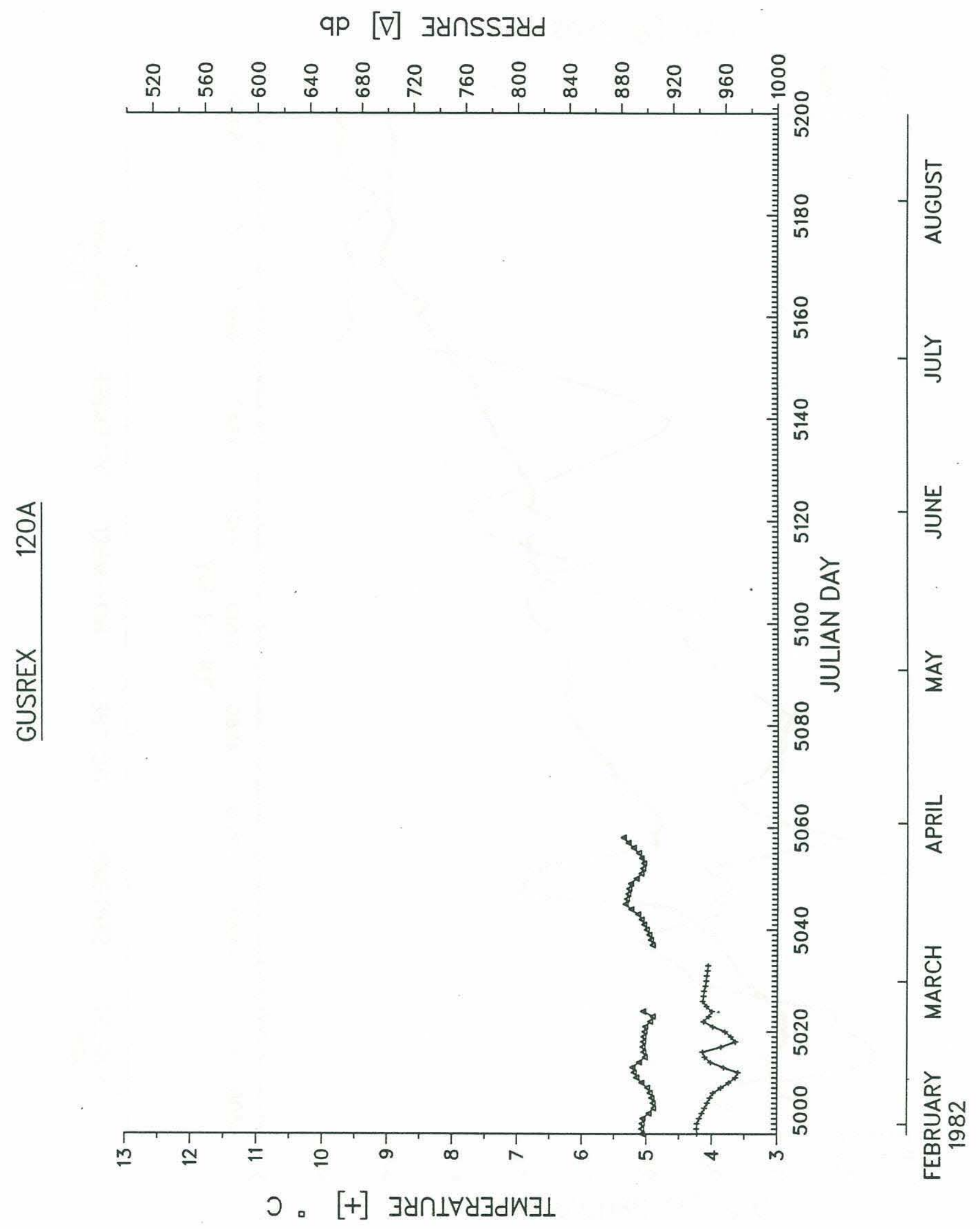




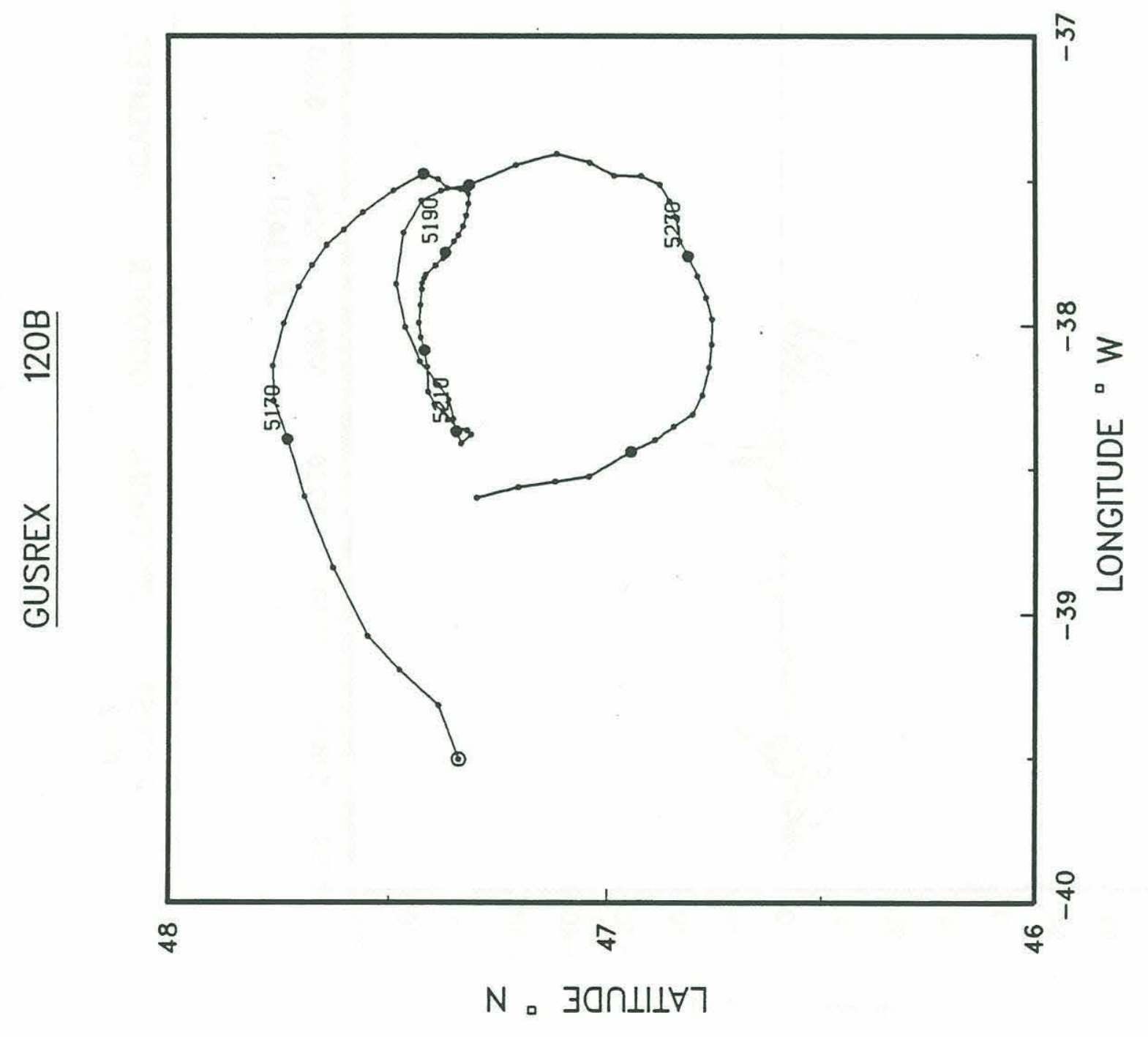




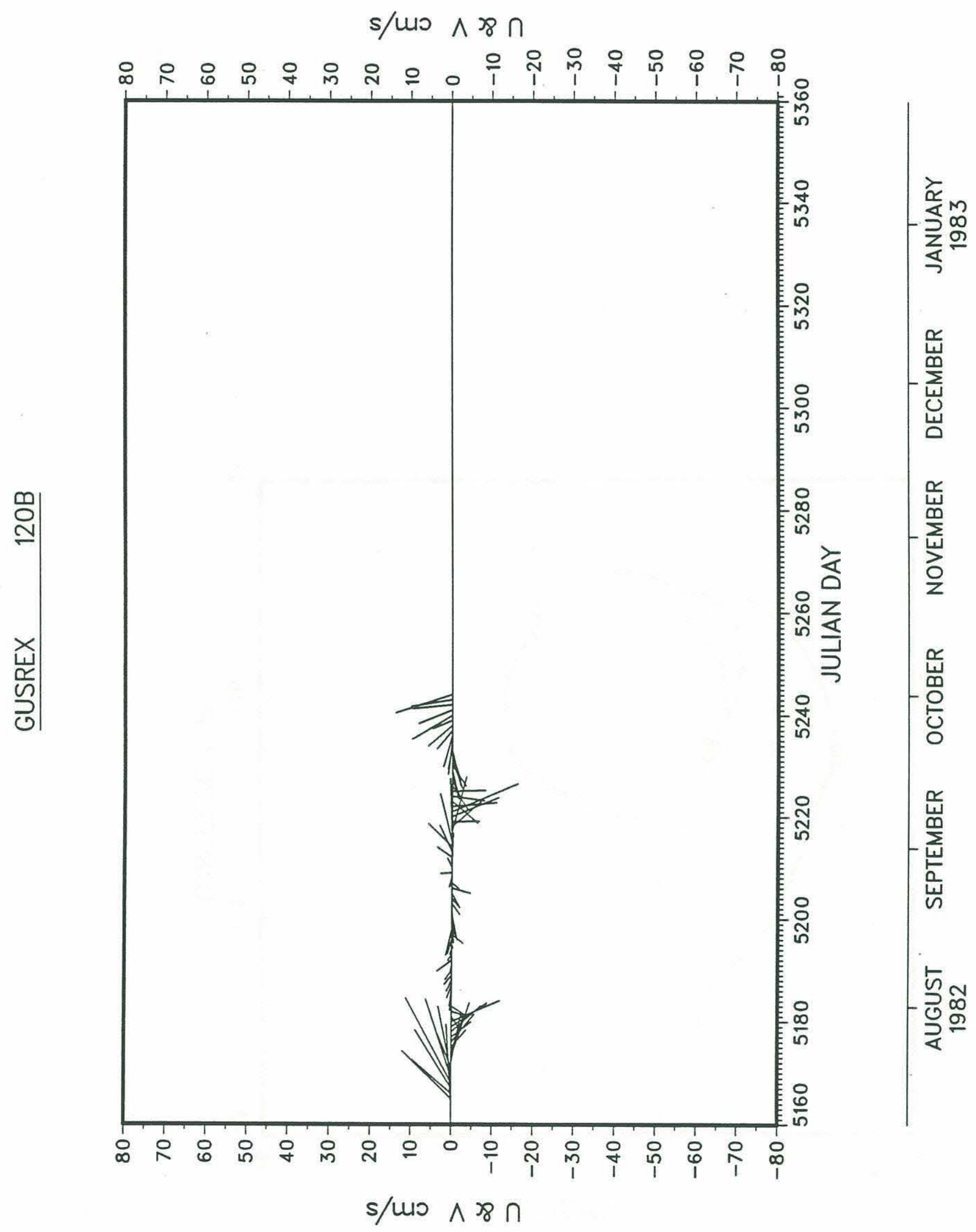




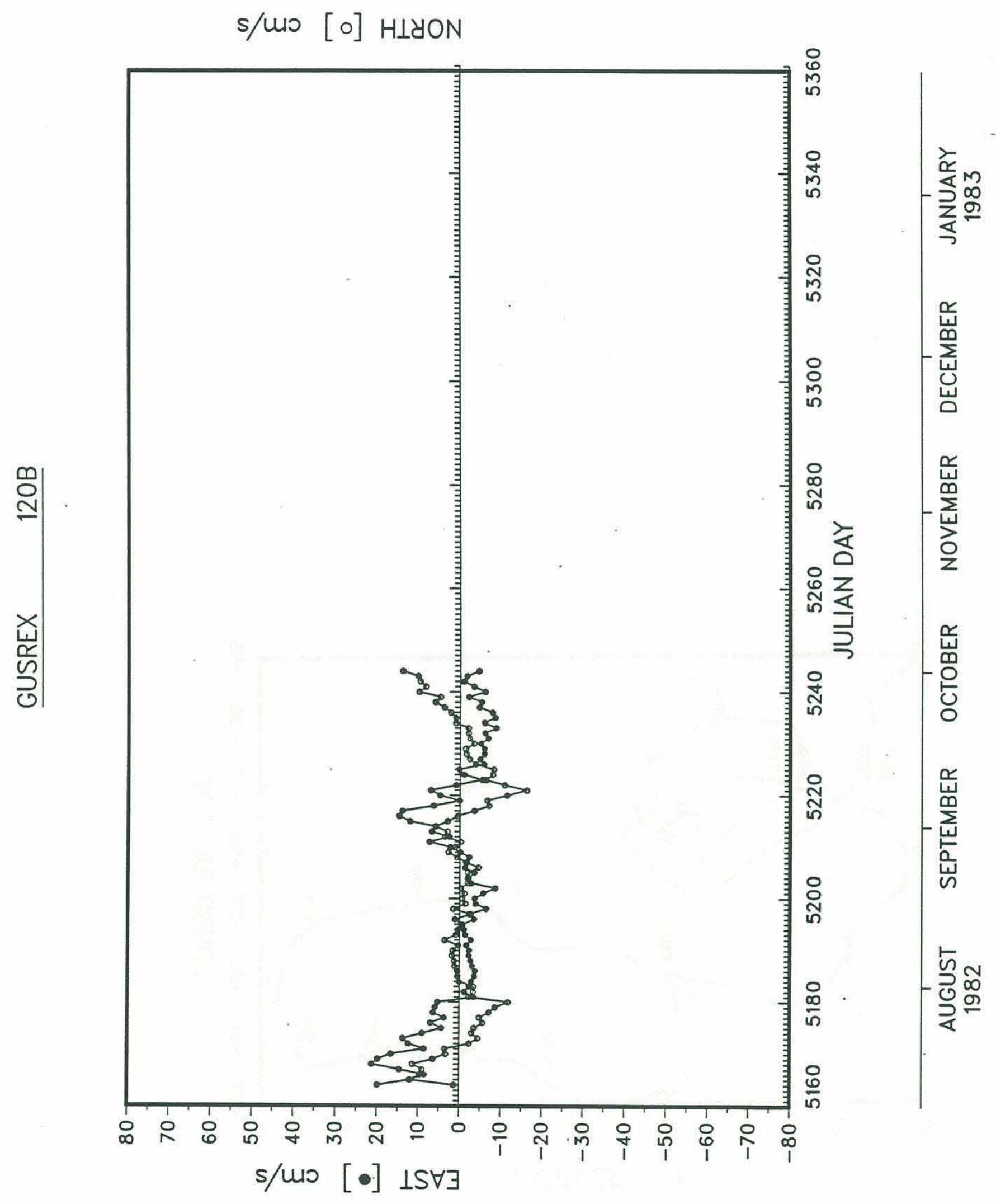




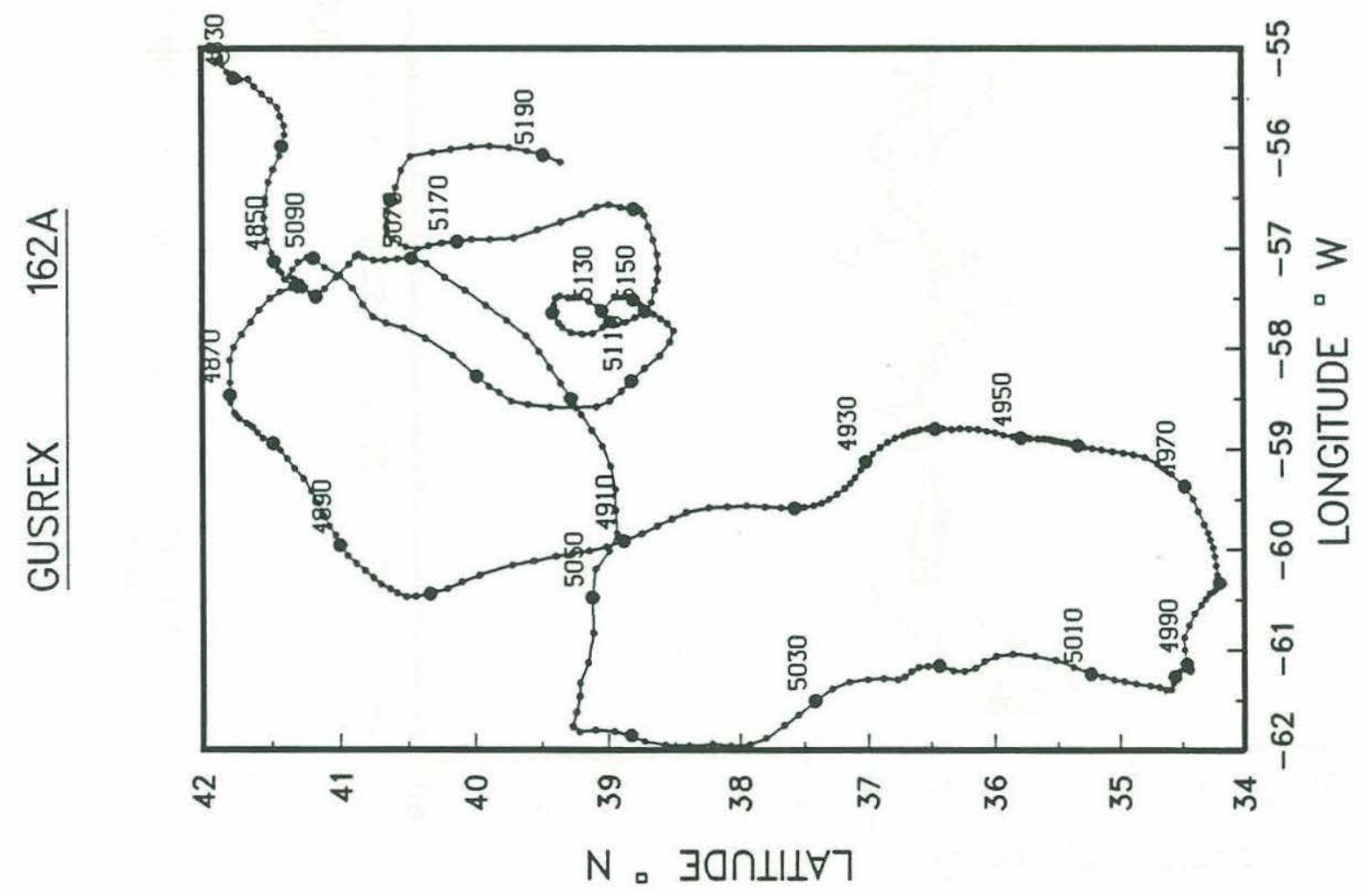




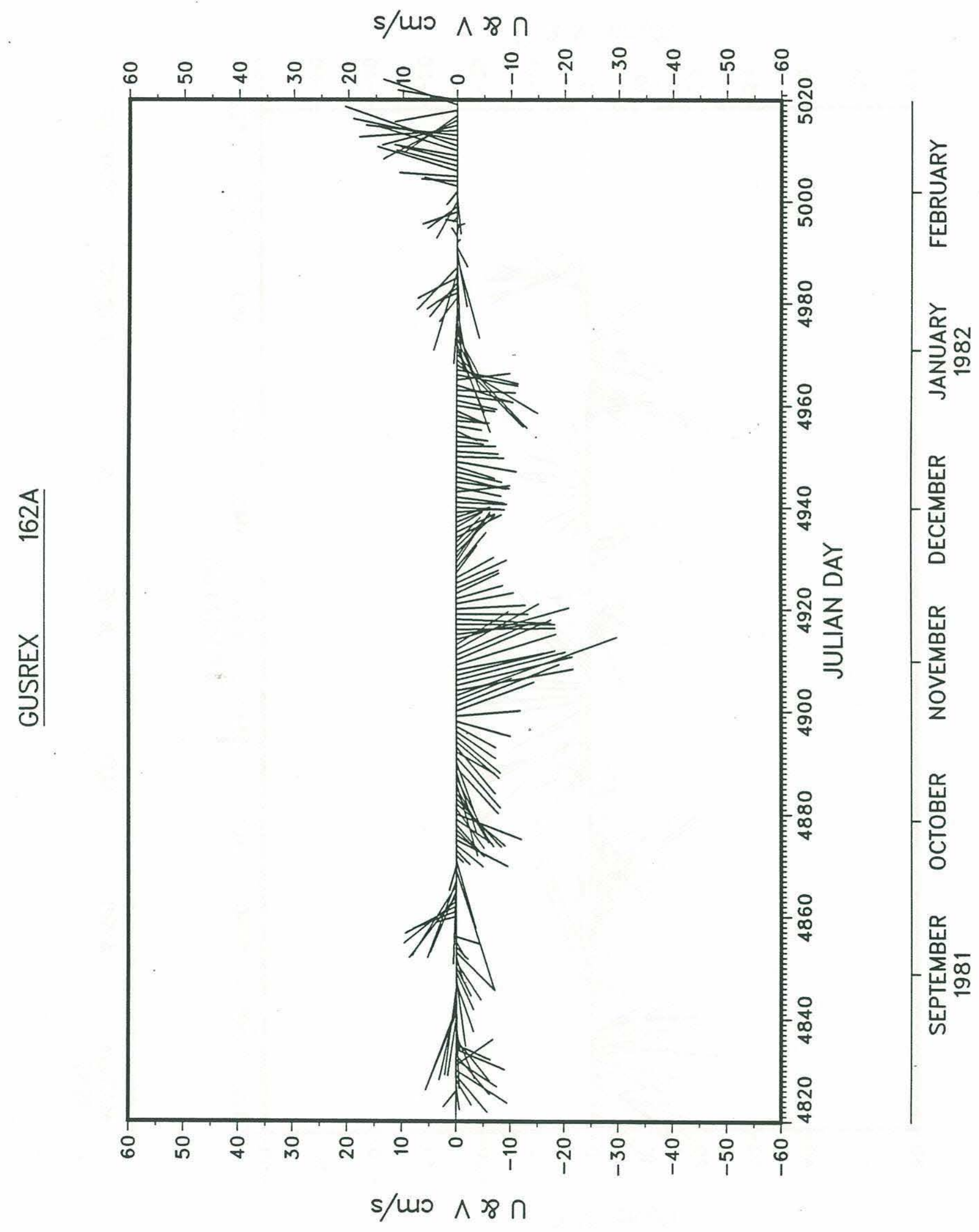




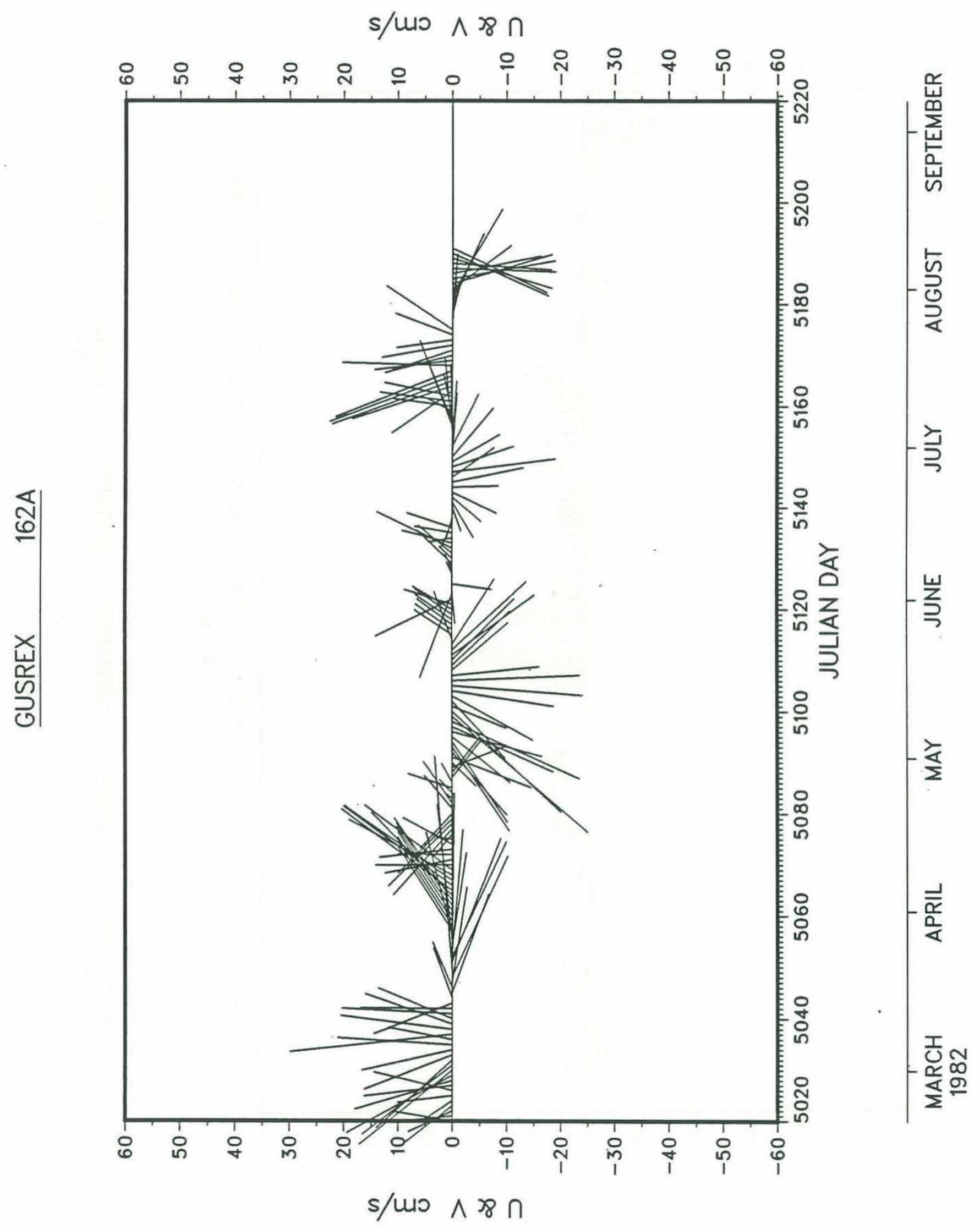


s/uo [0] HLYON

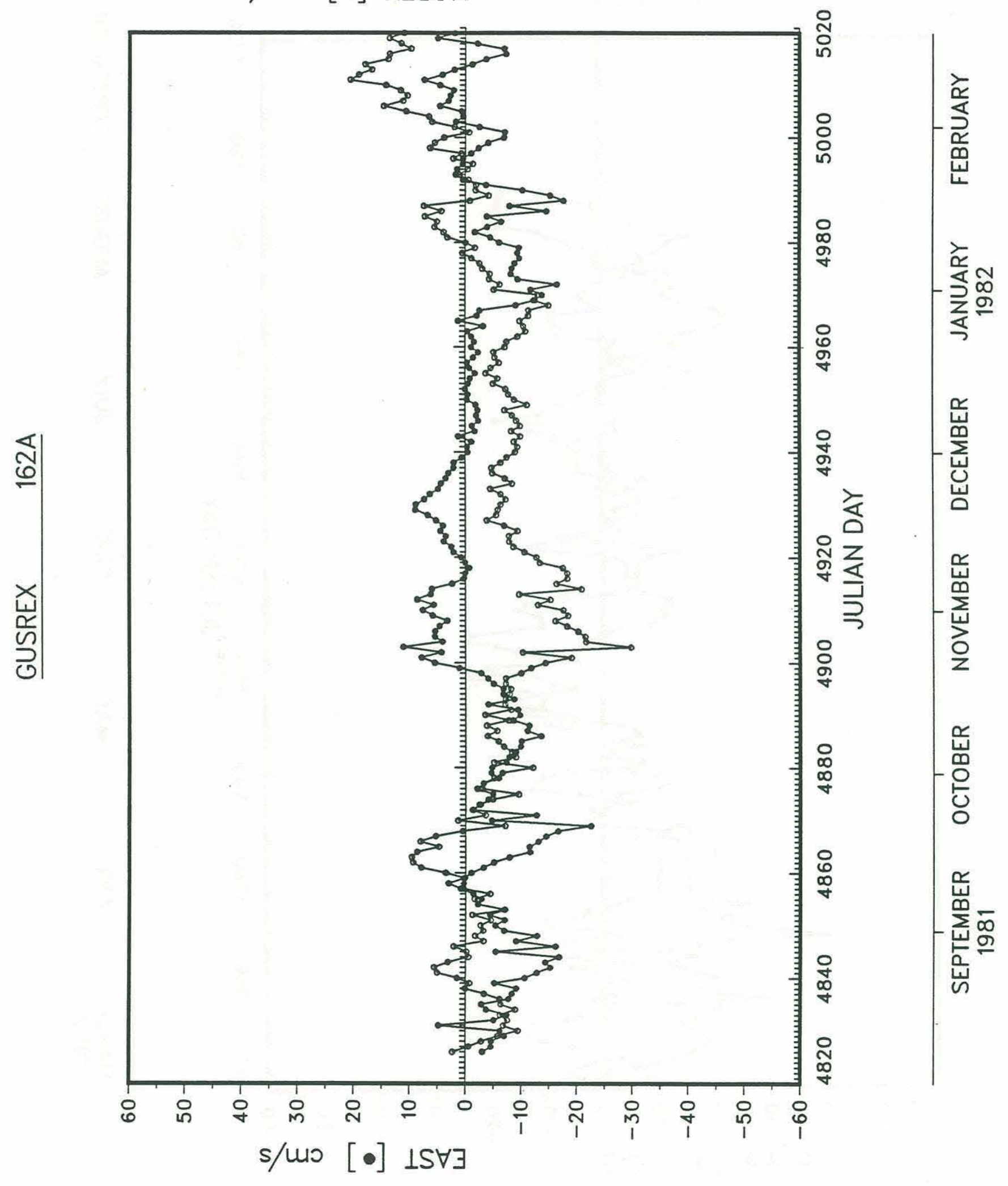




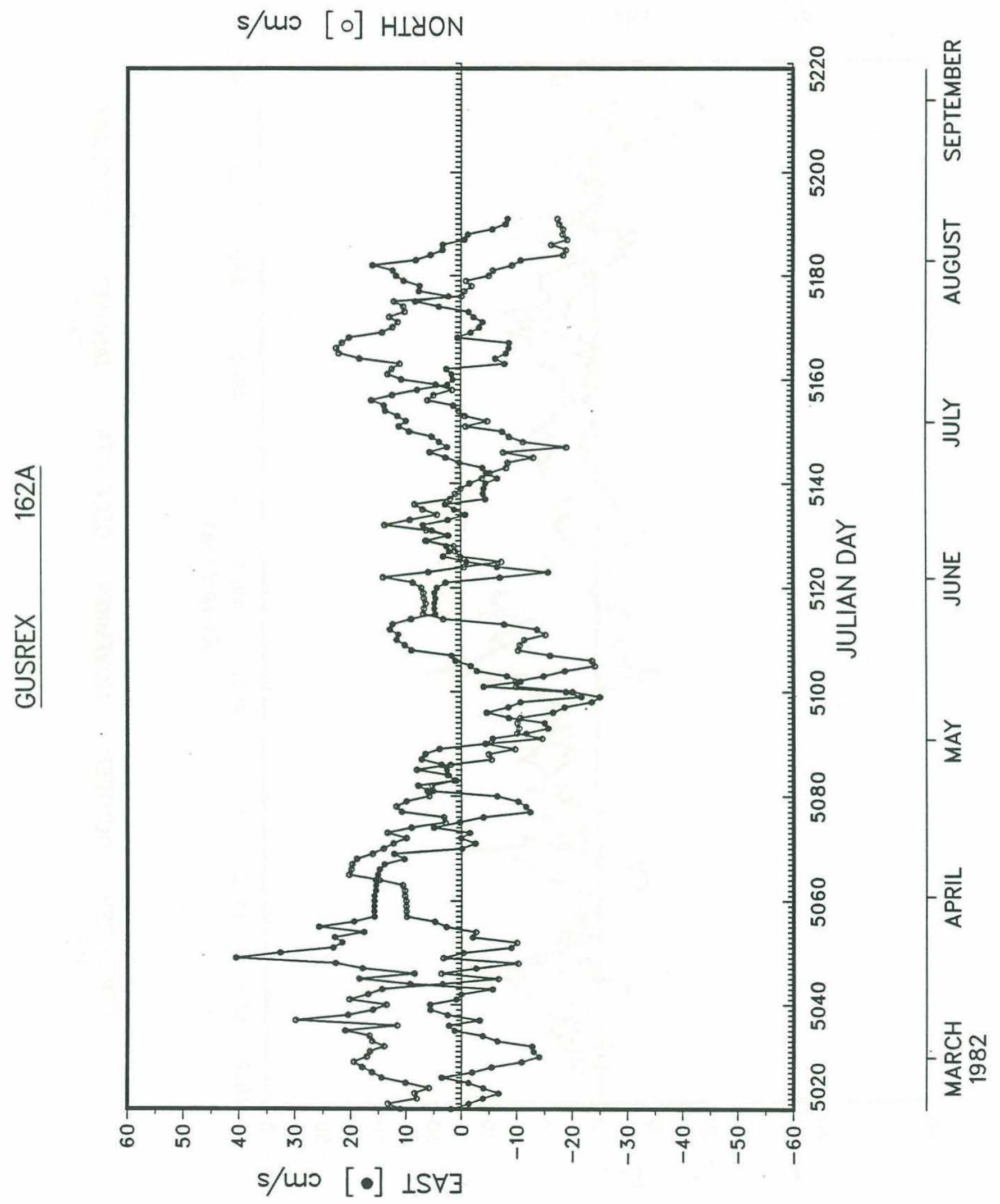




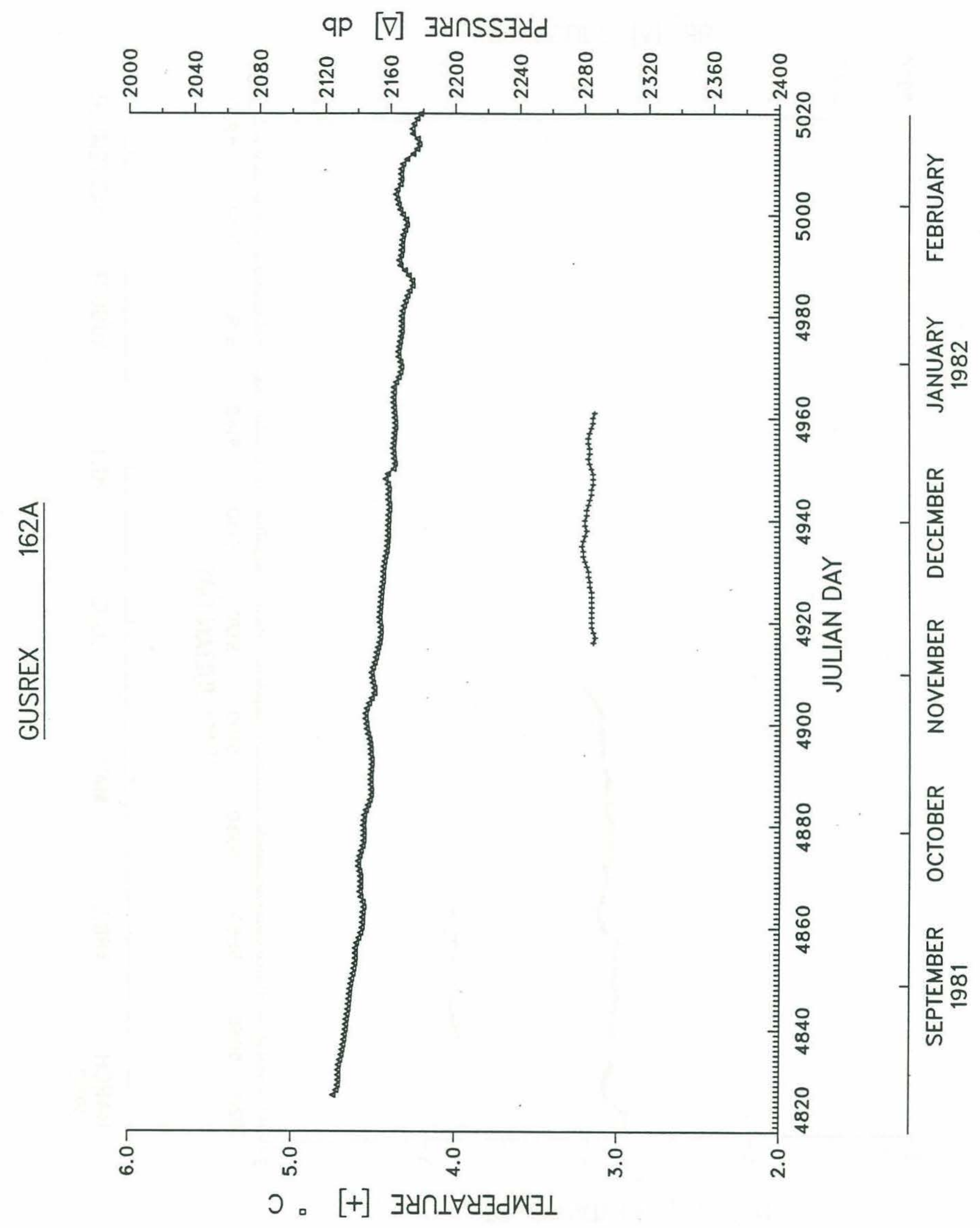




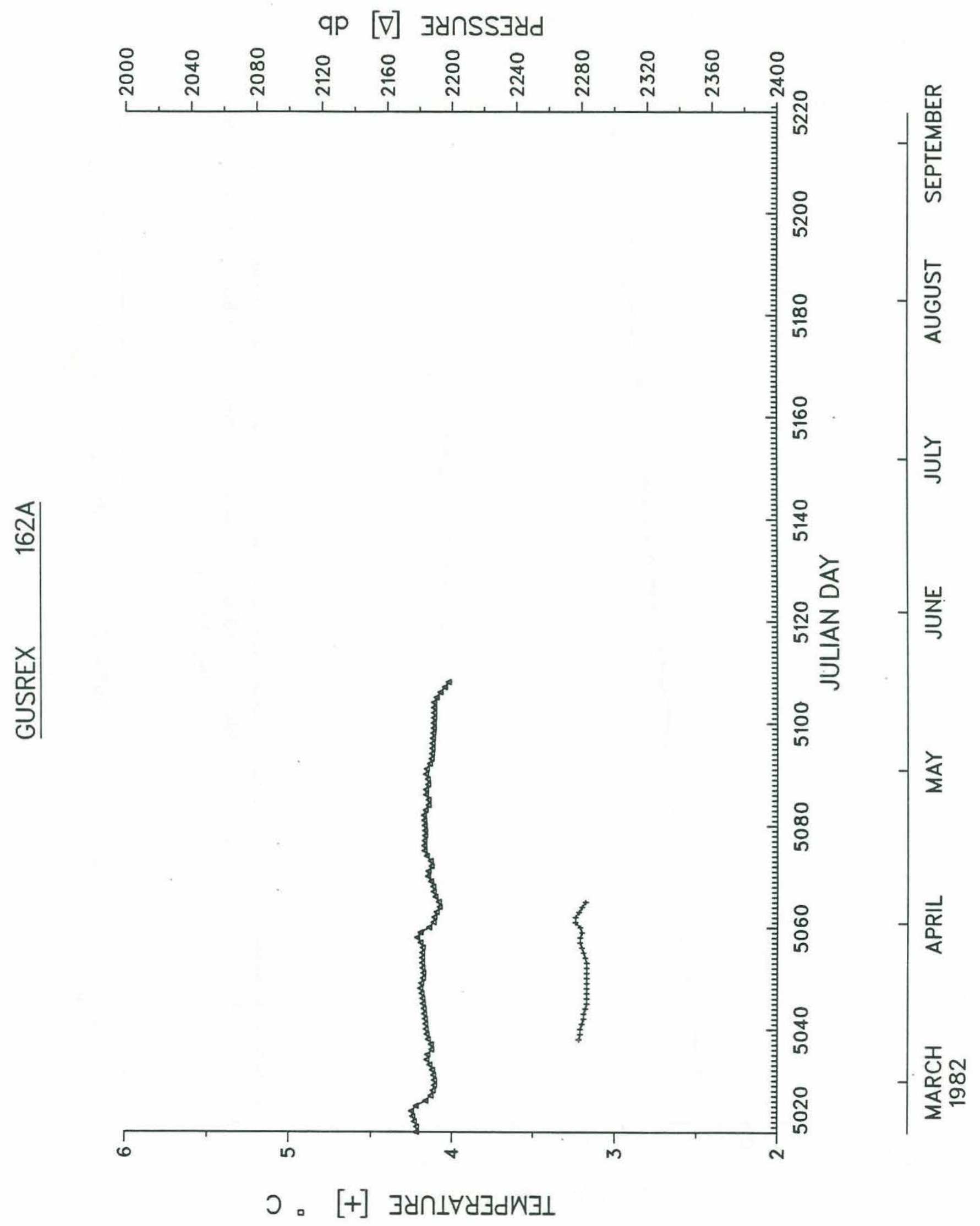




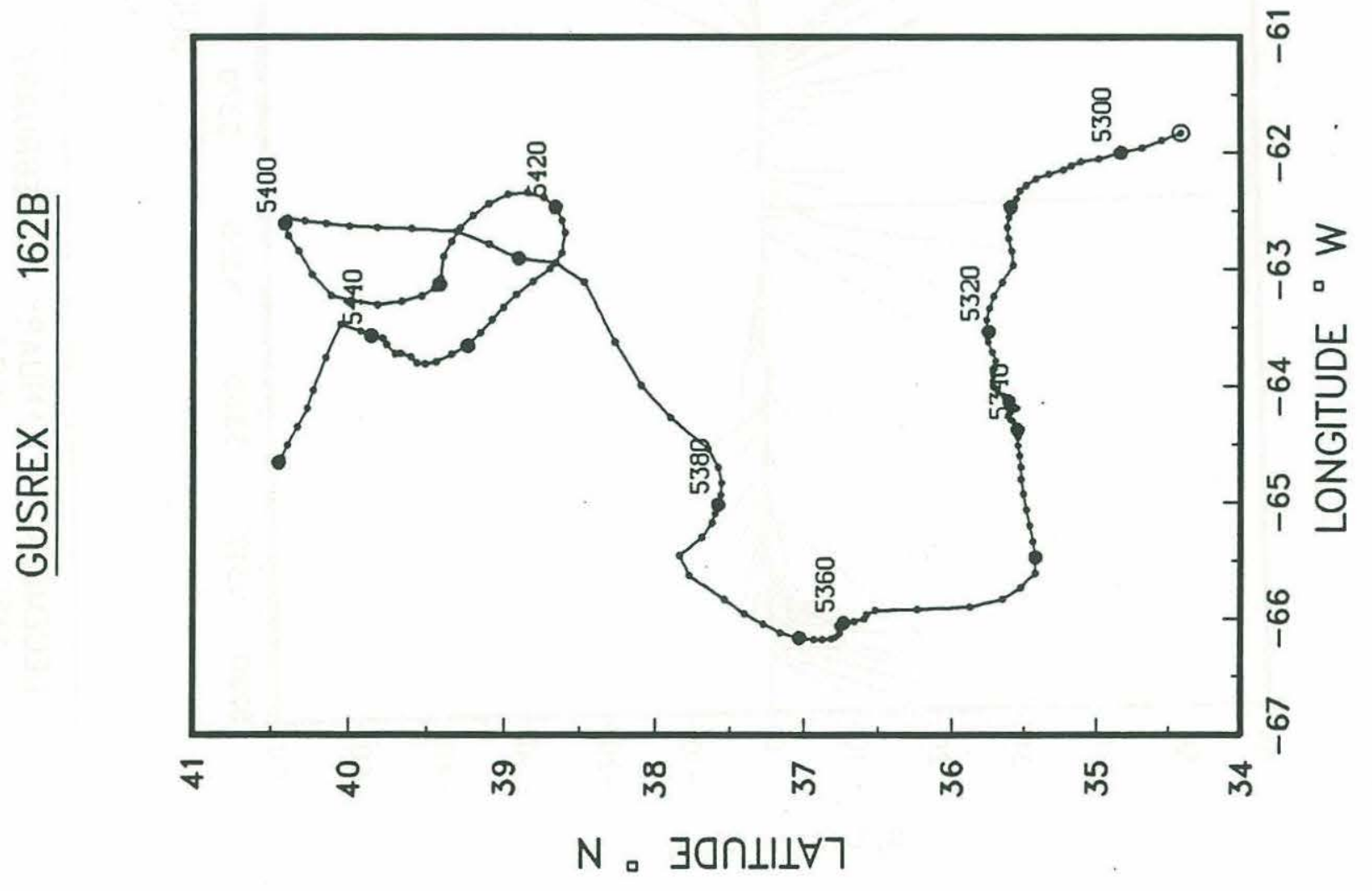




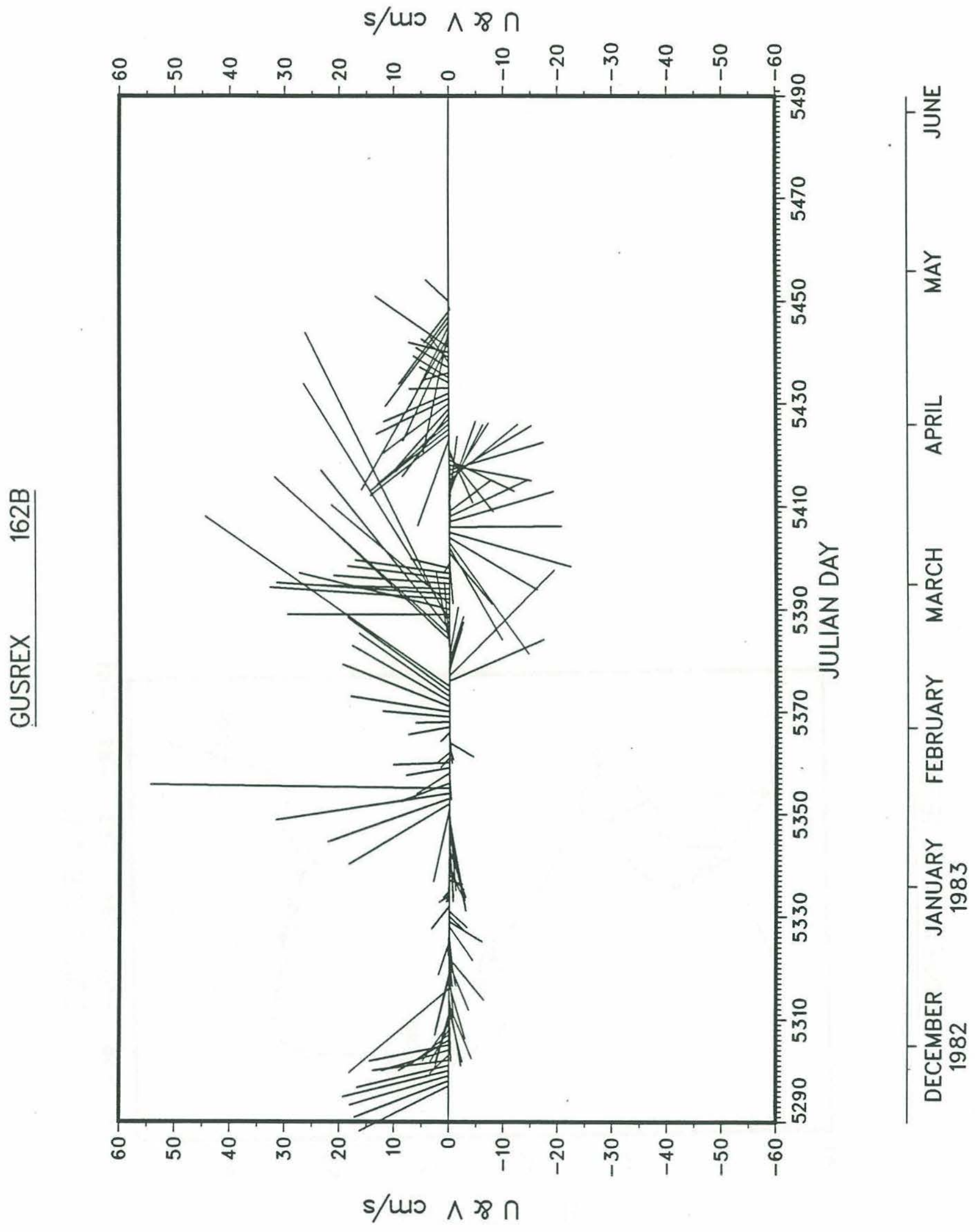




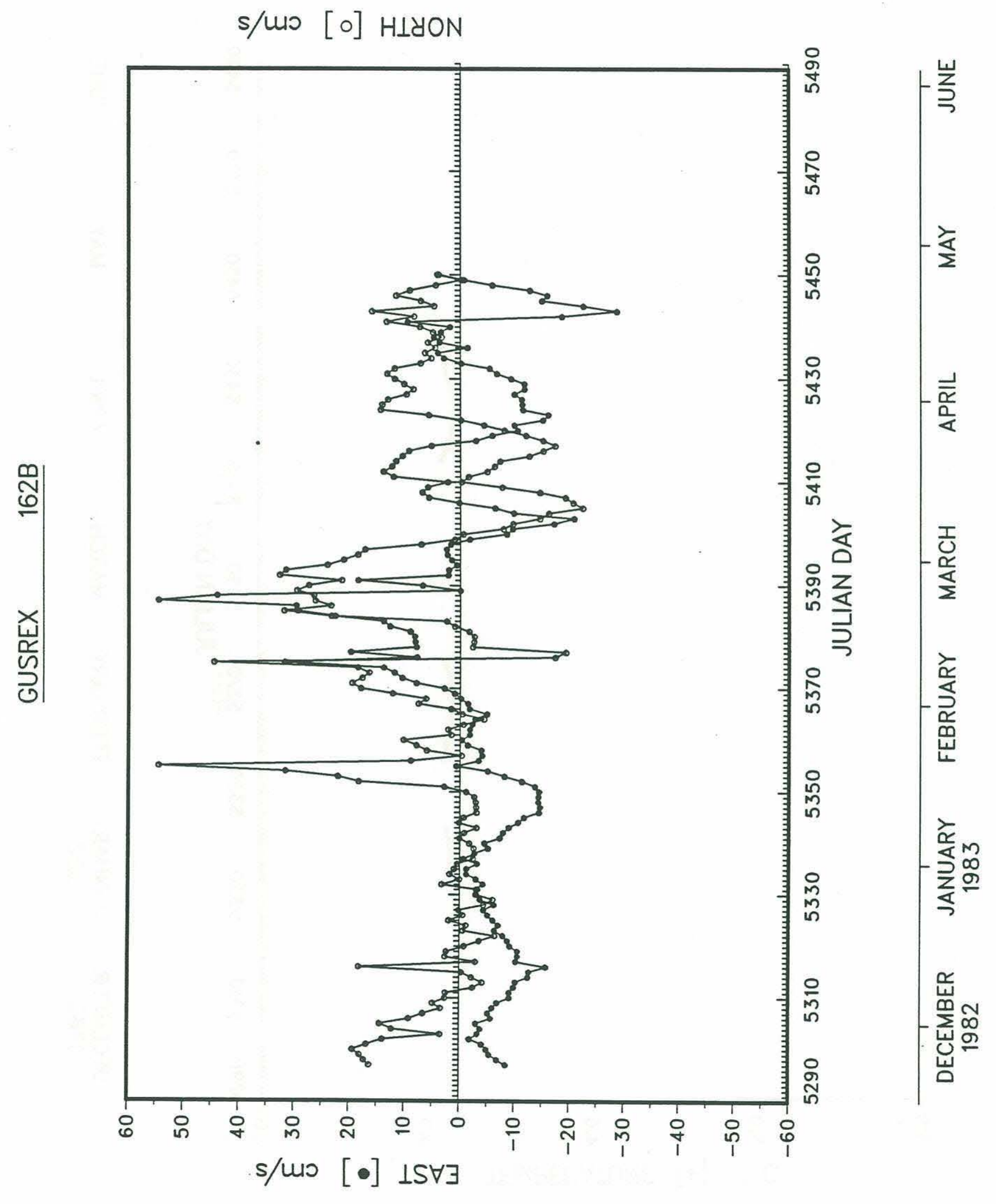




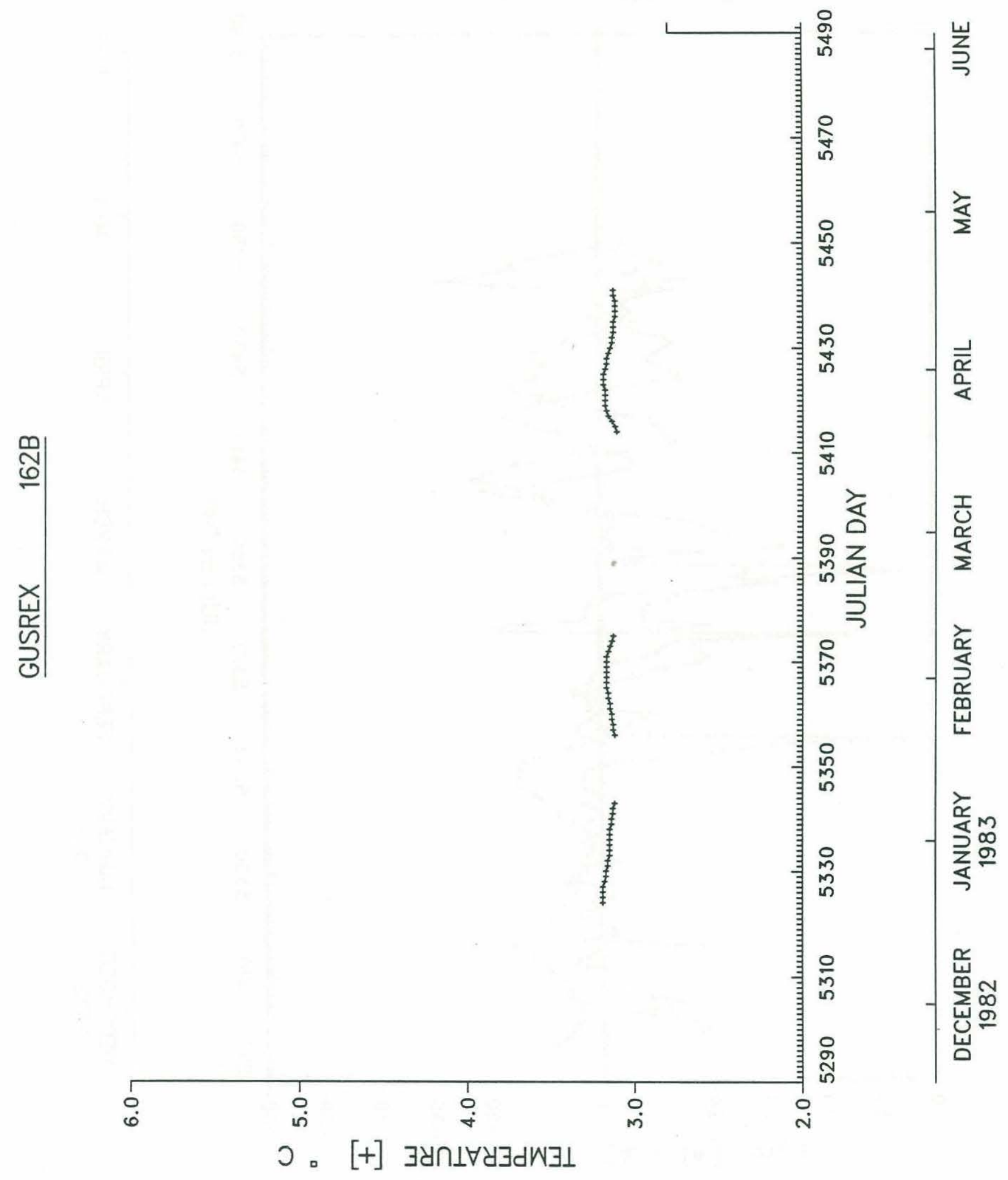




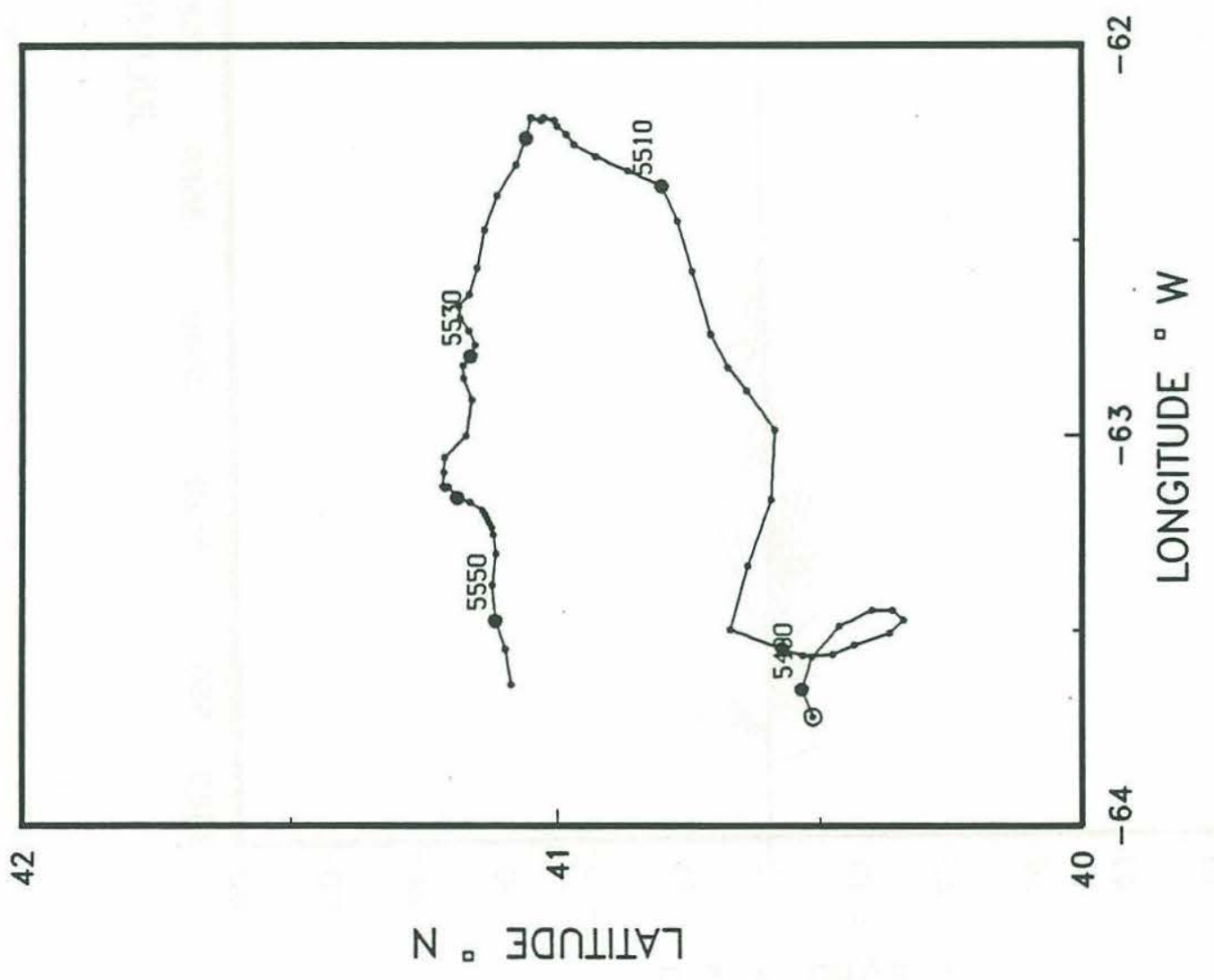

121 


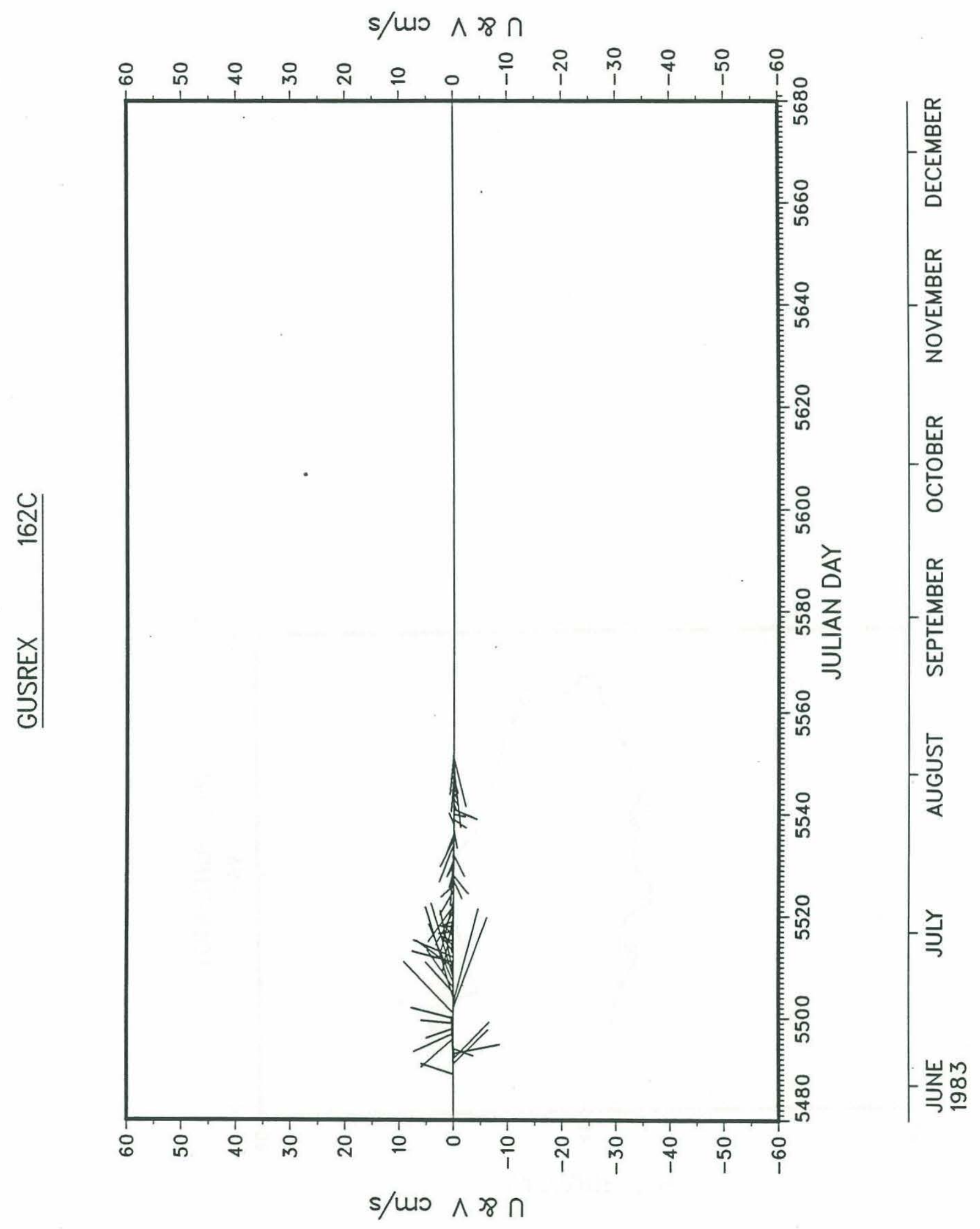




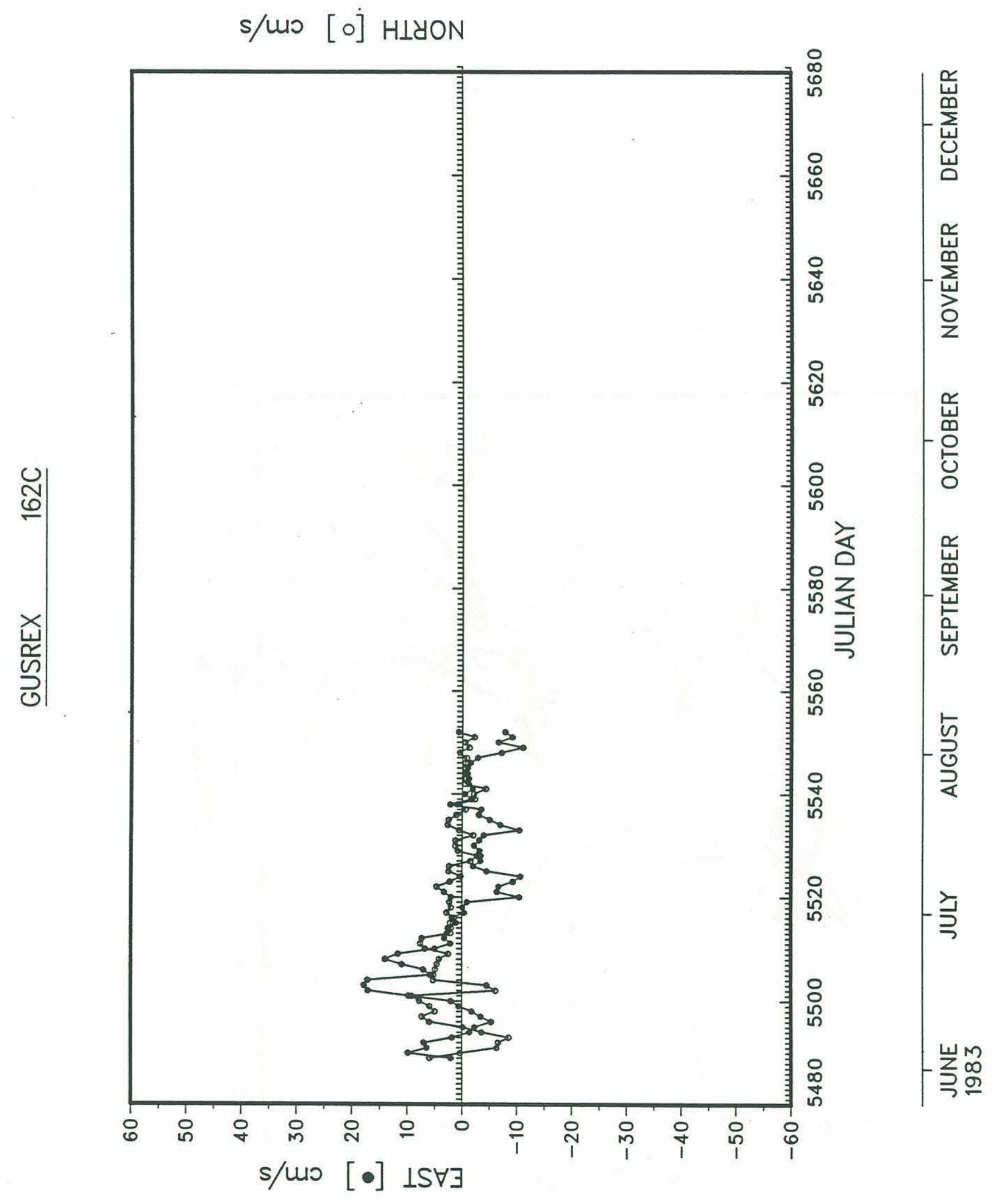




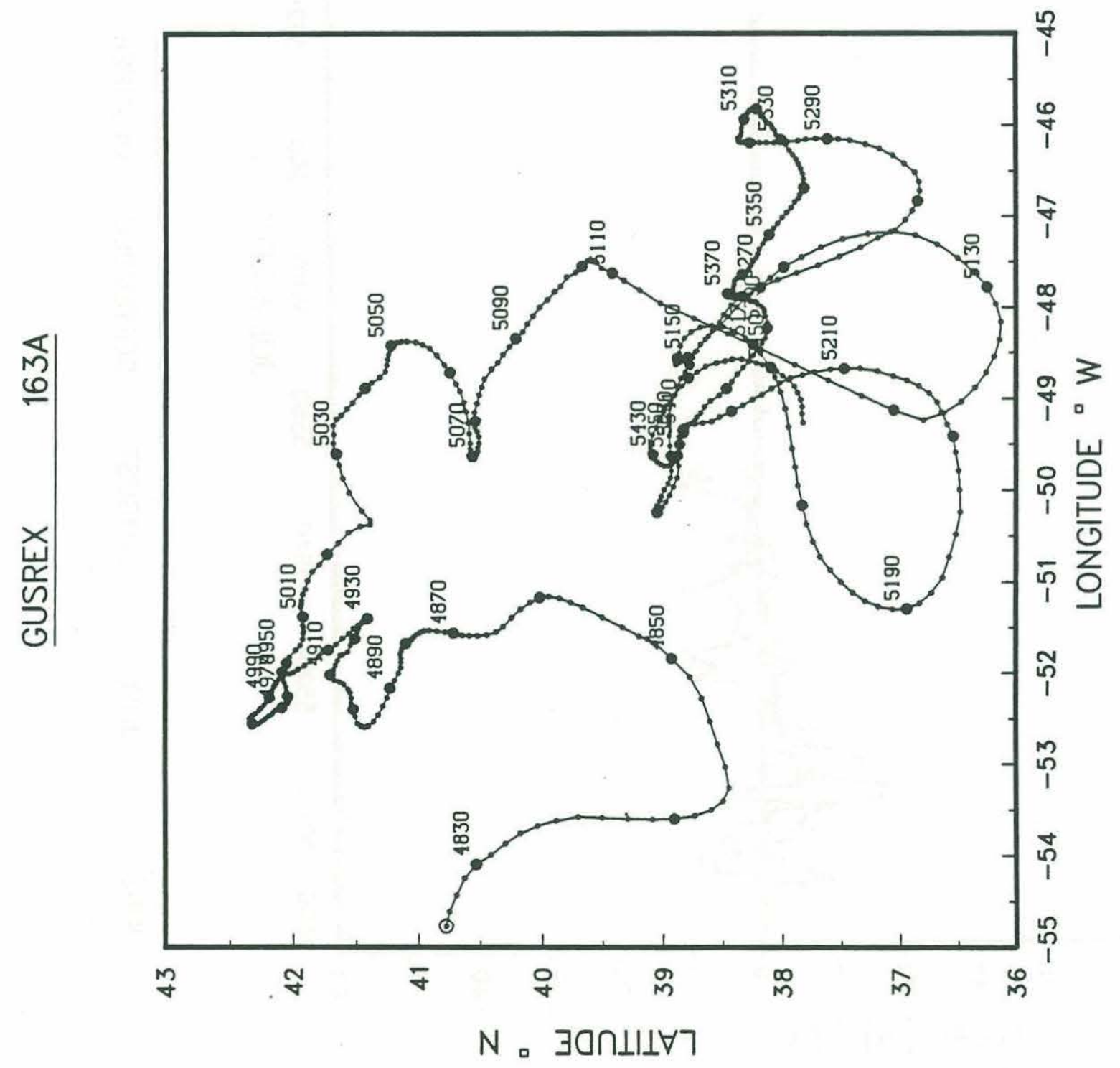




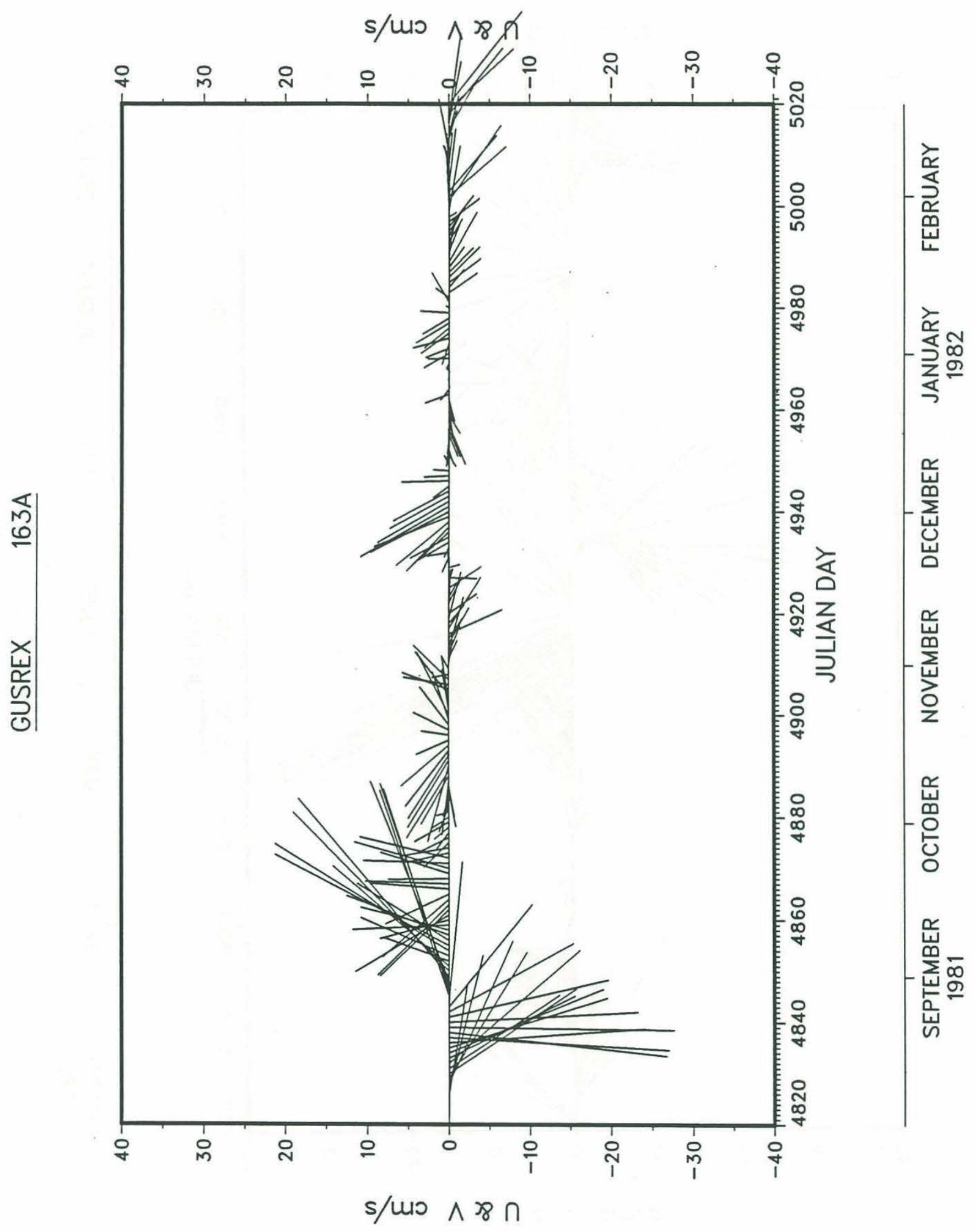




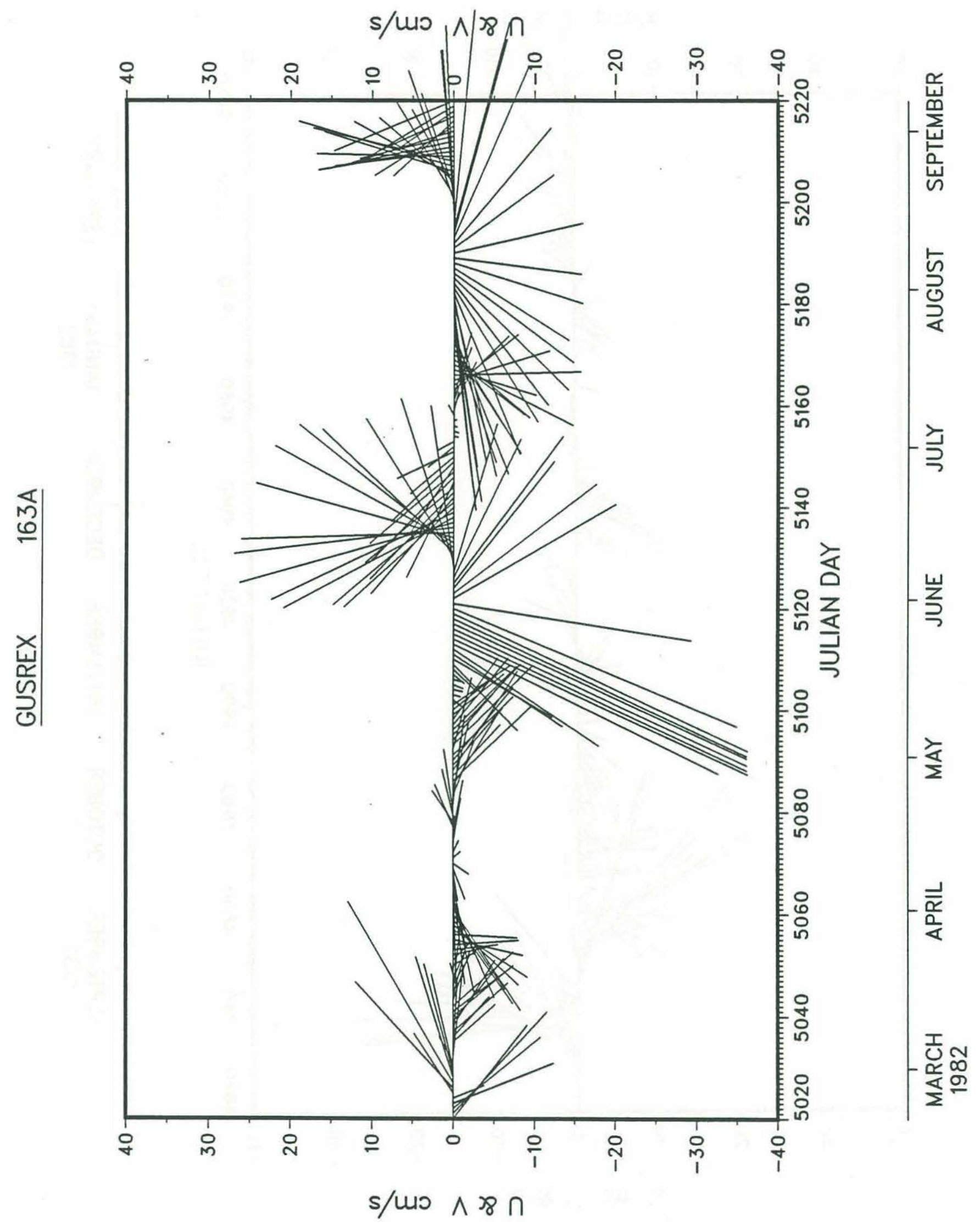




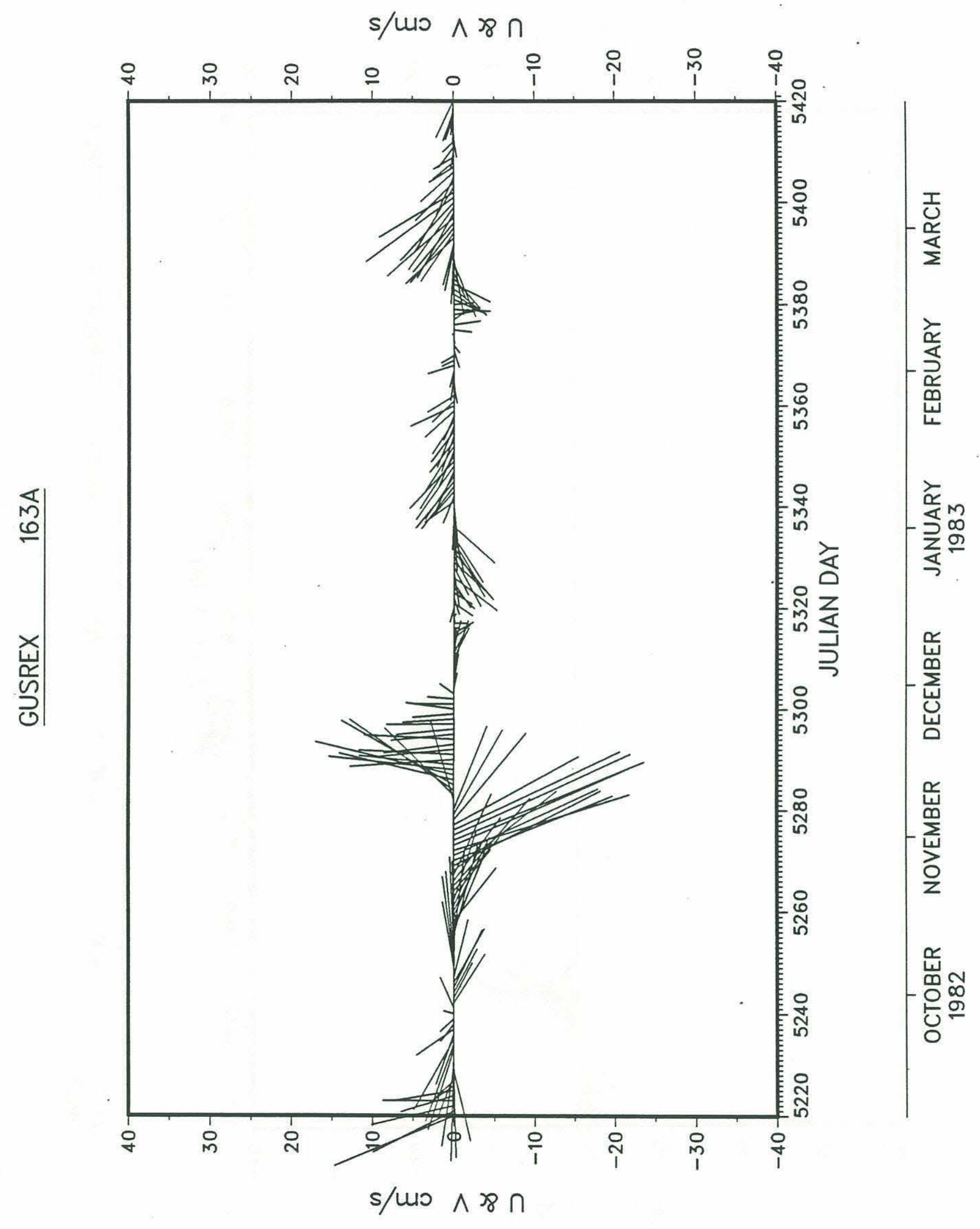




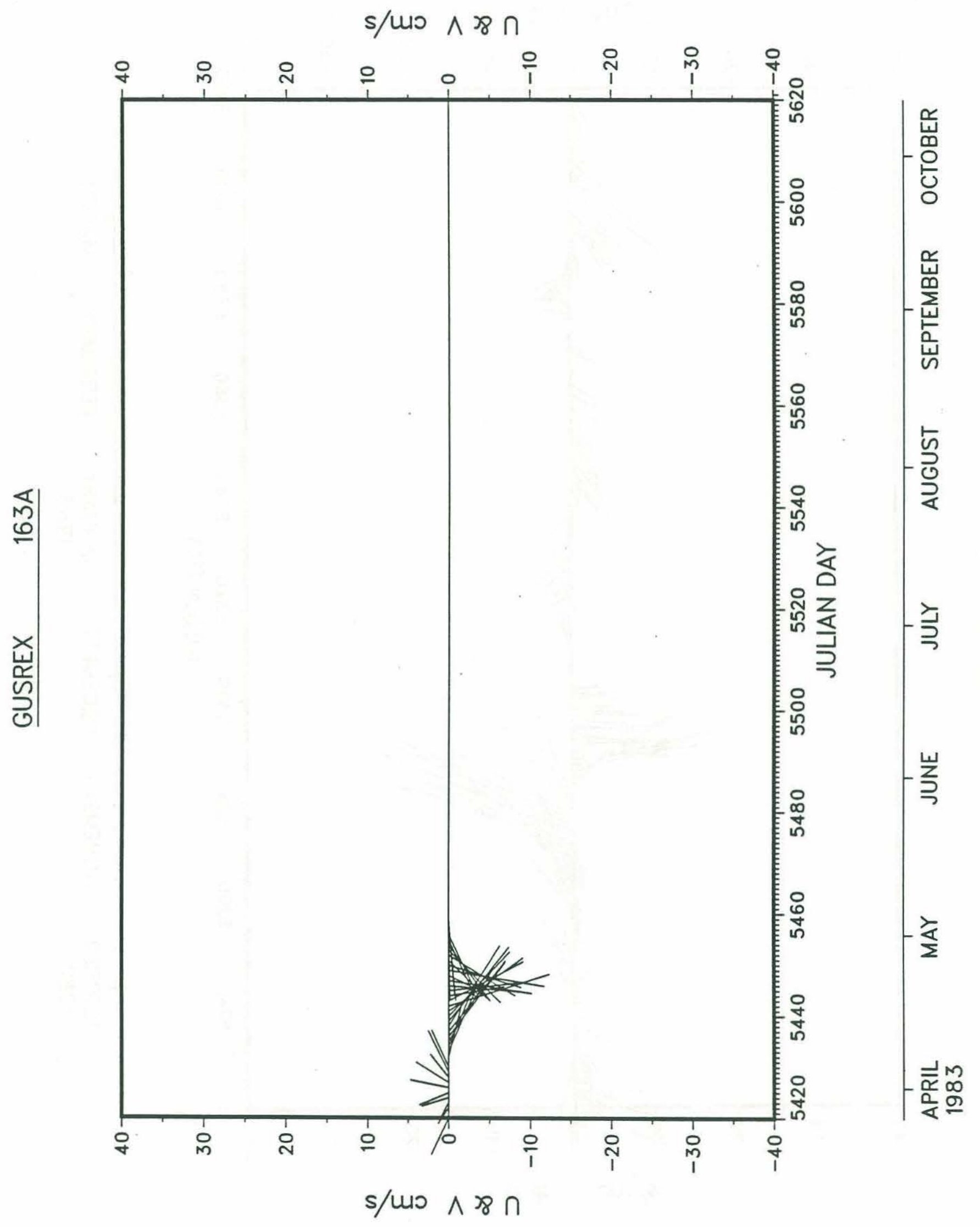




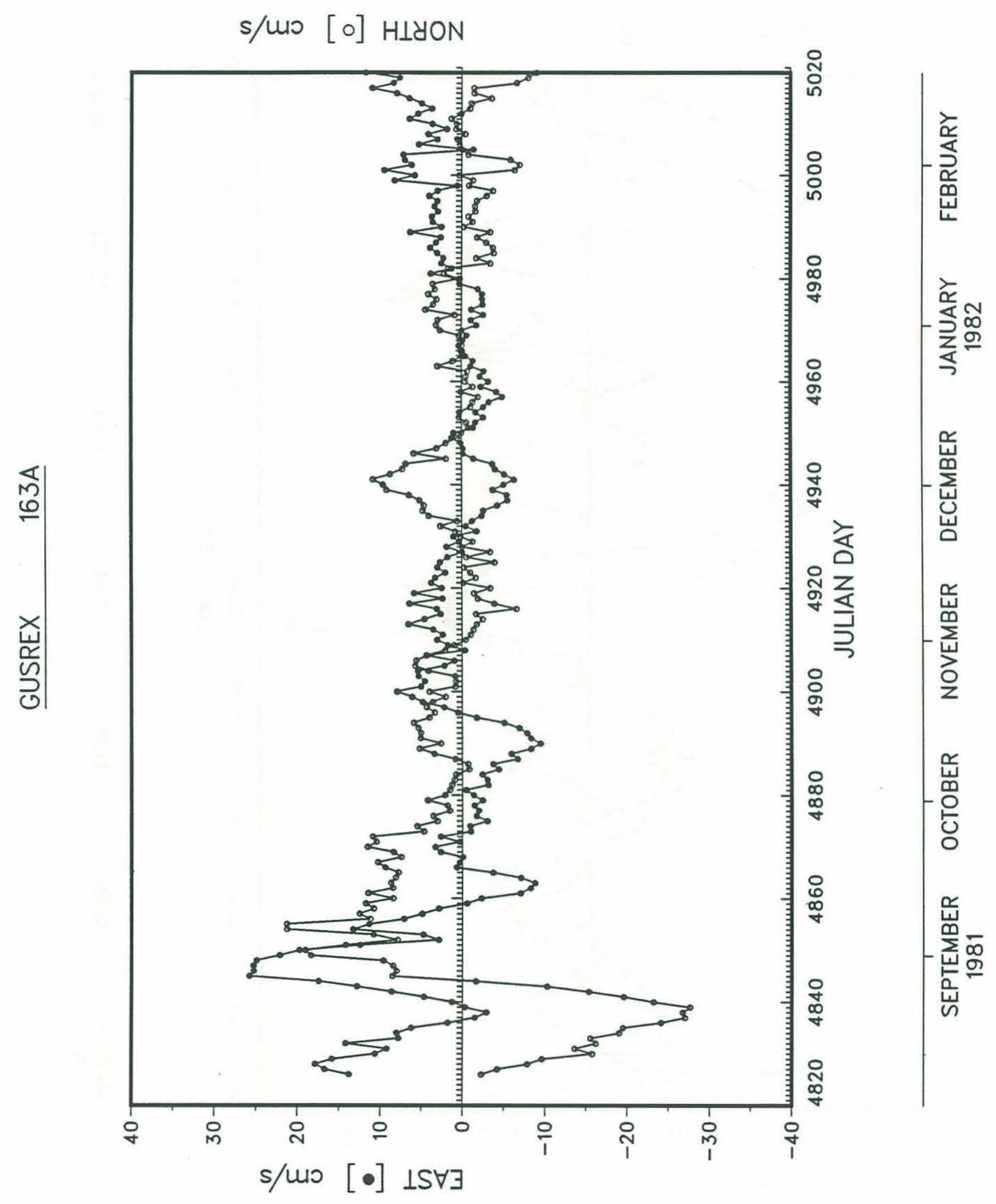




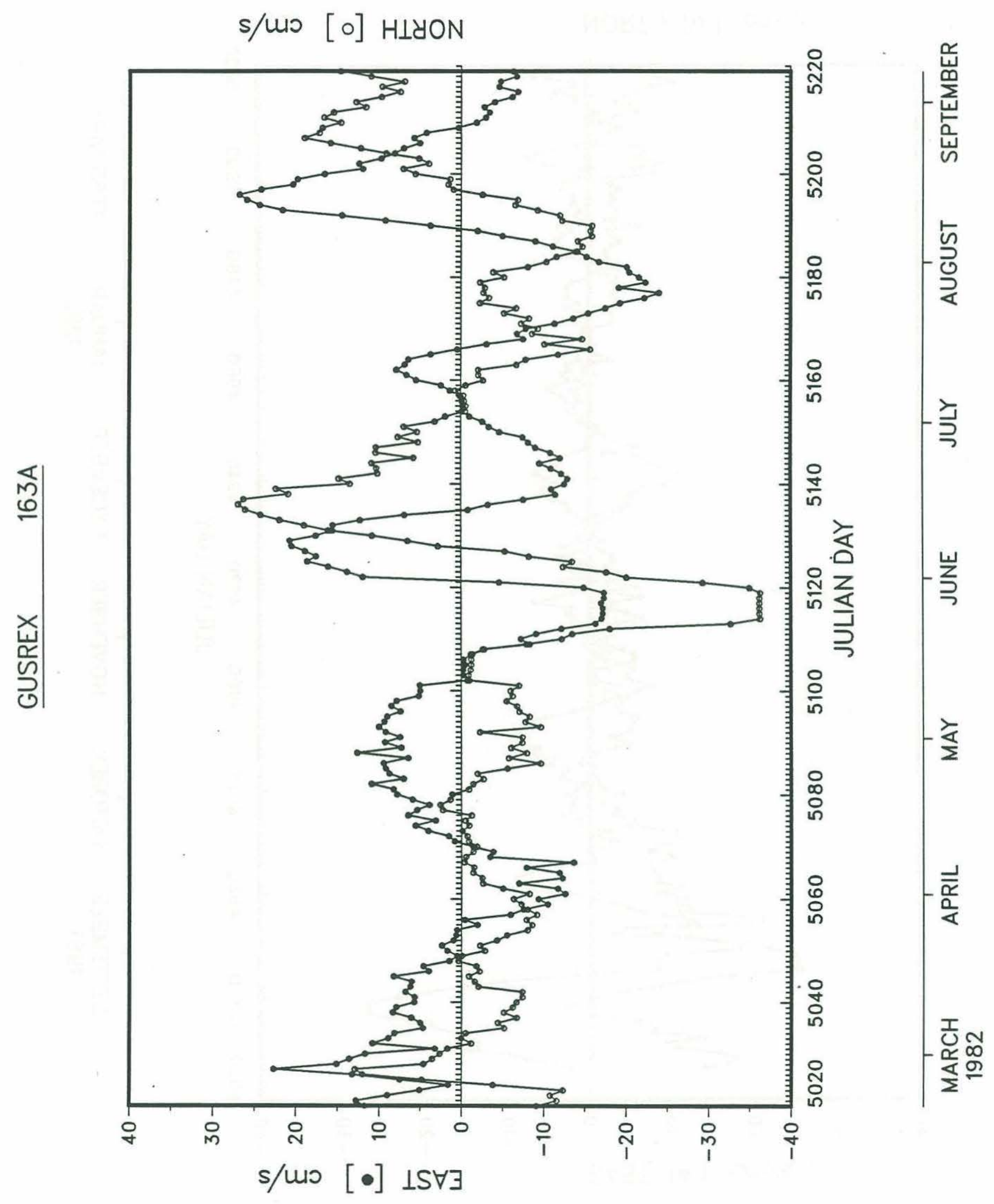




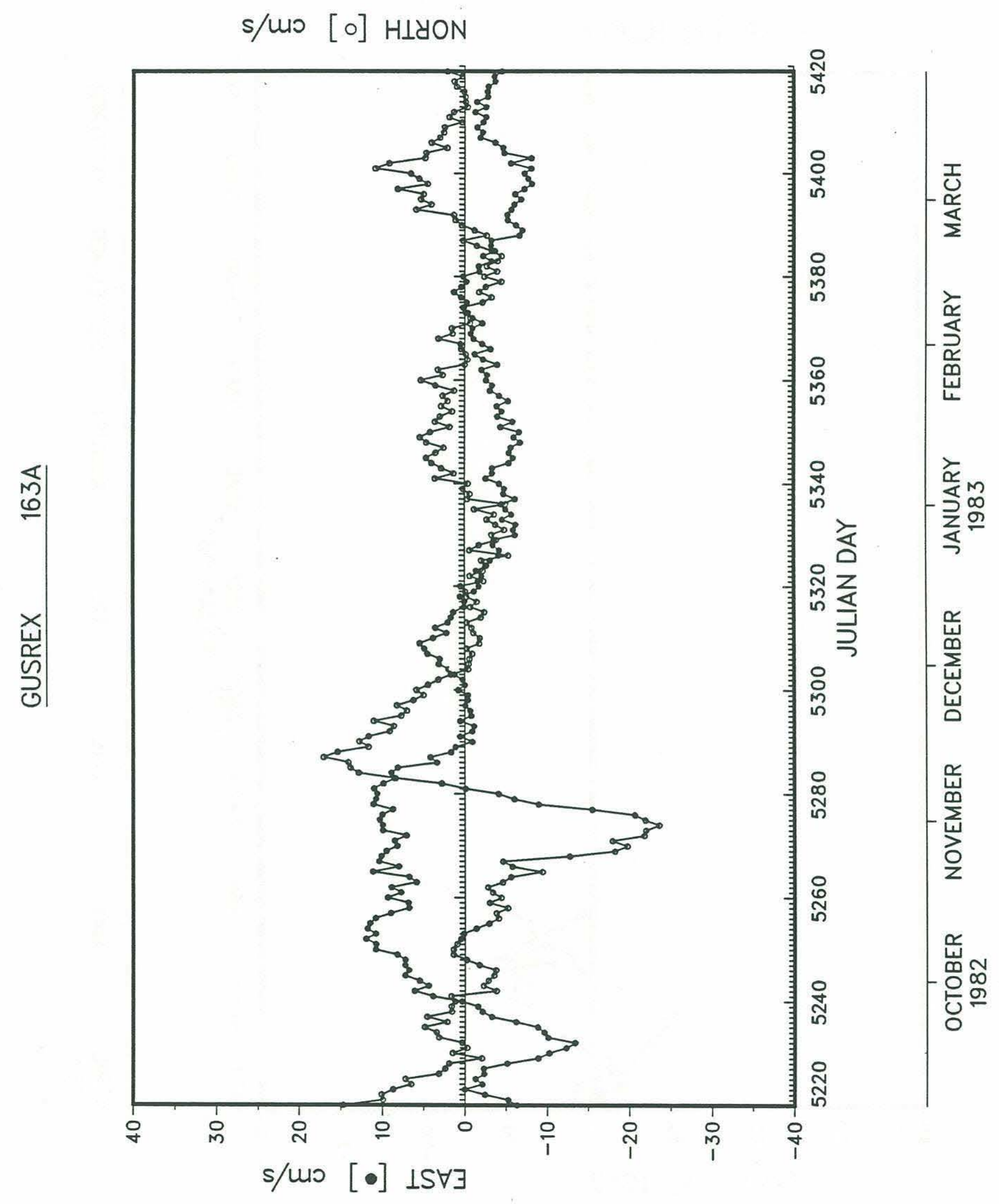




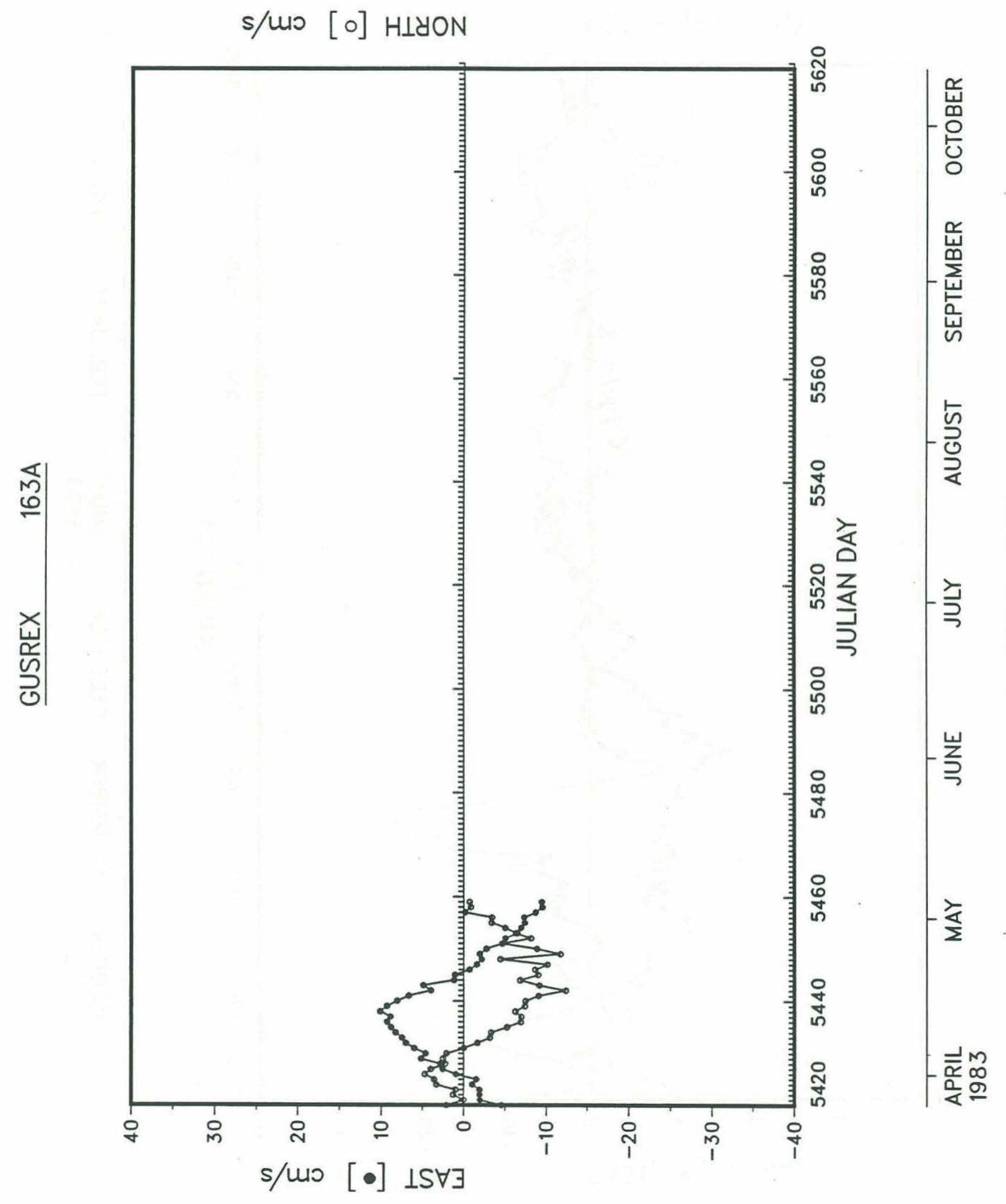




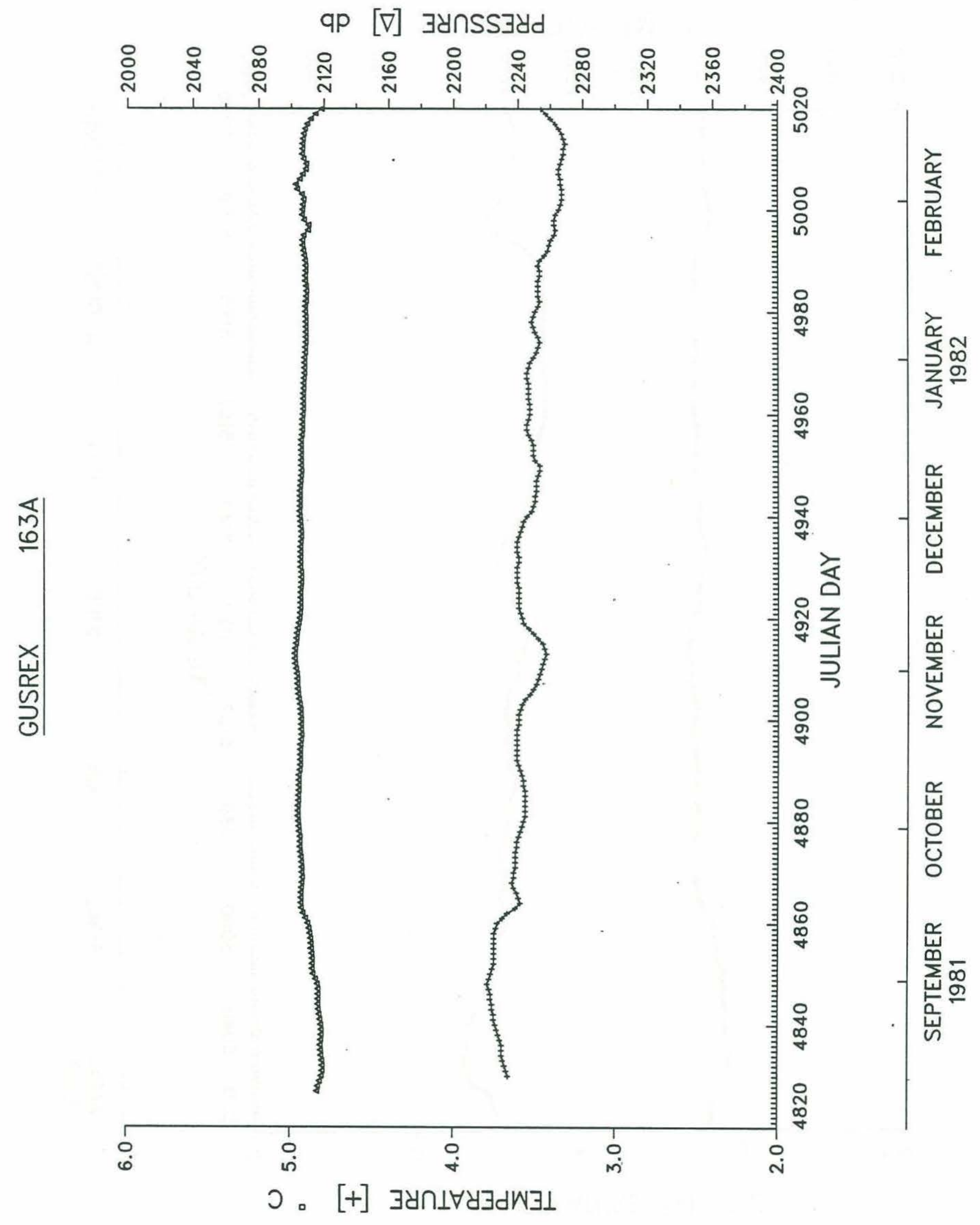




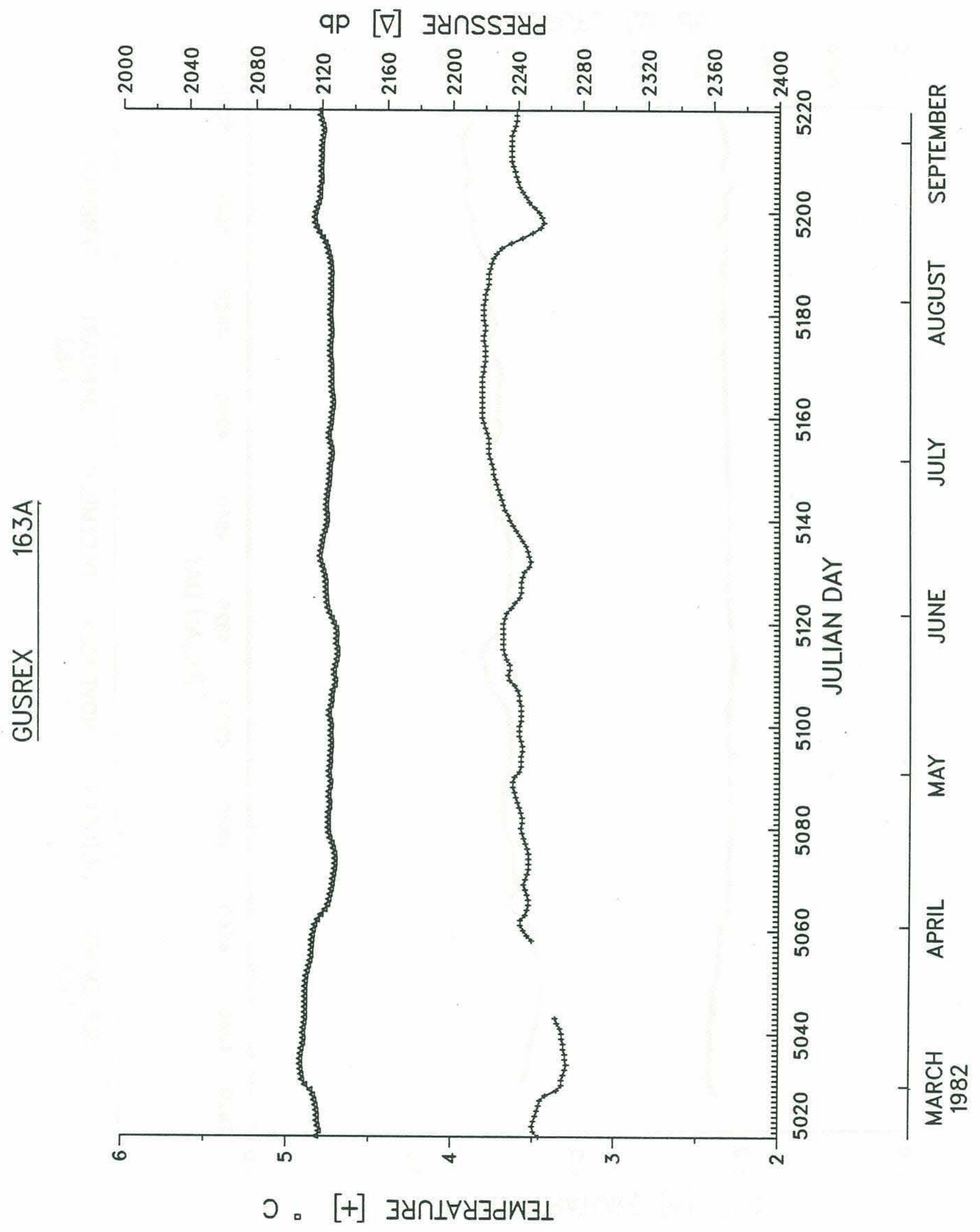




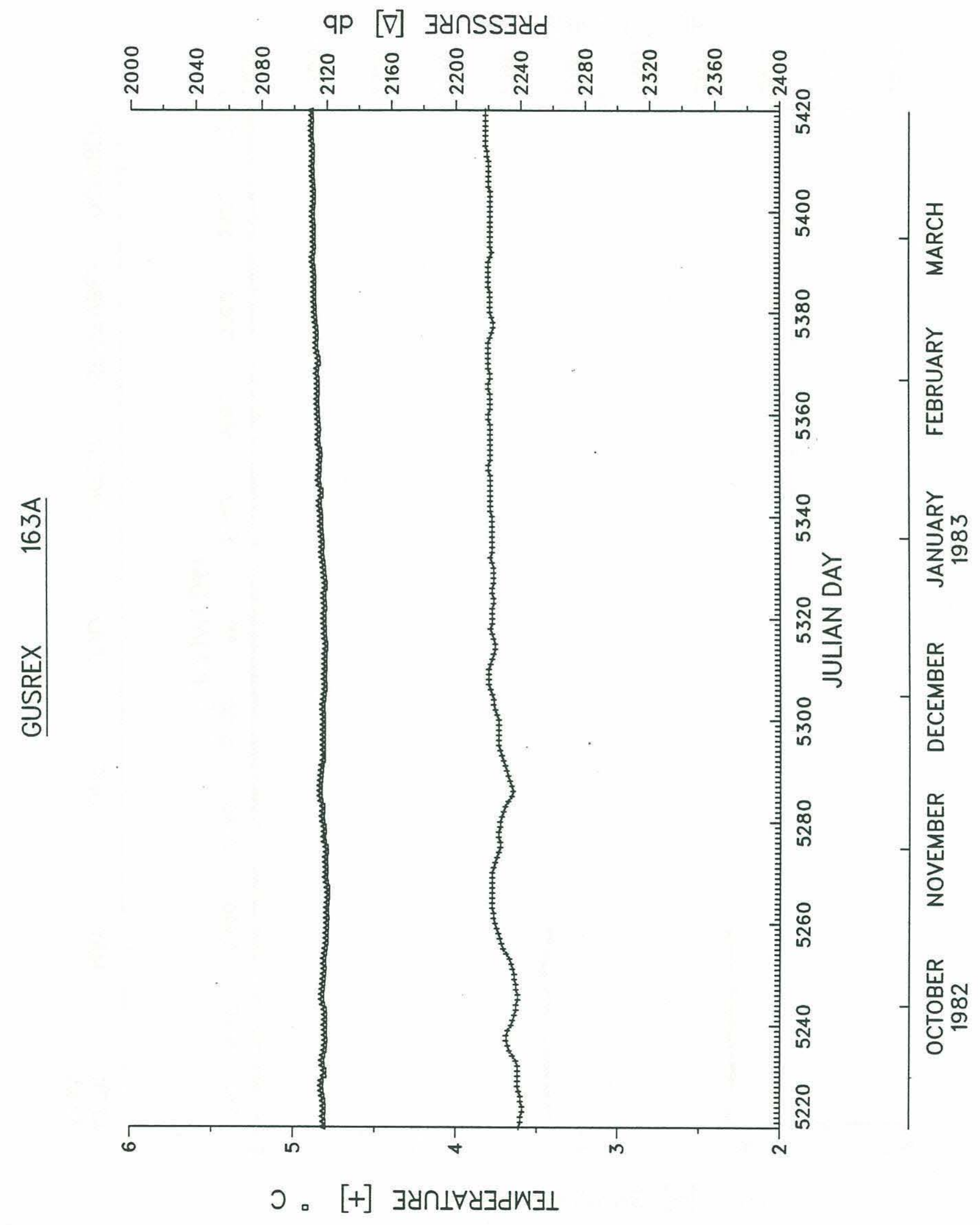




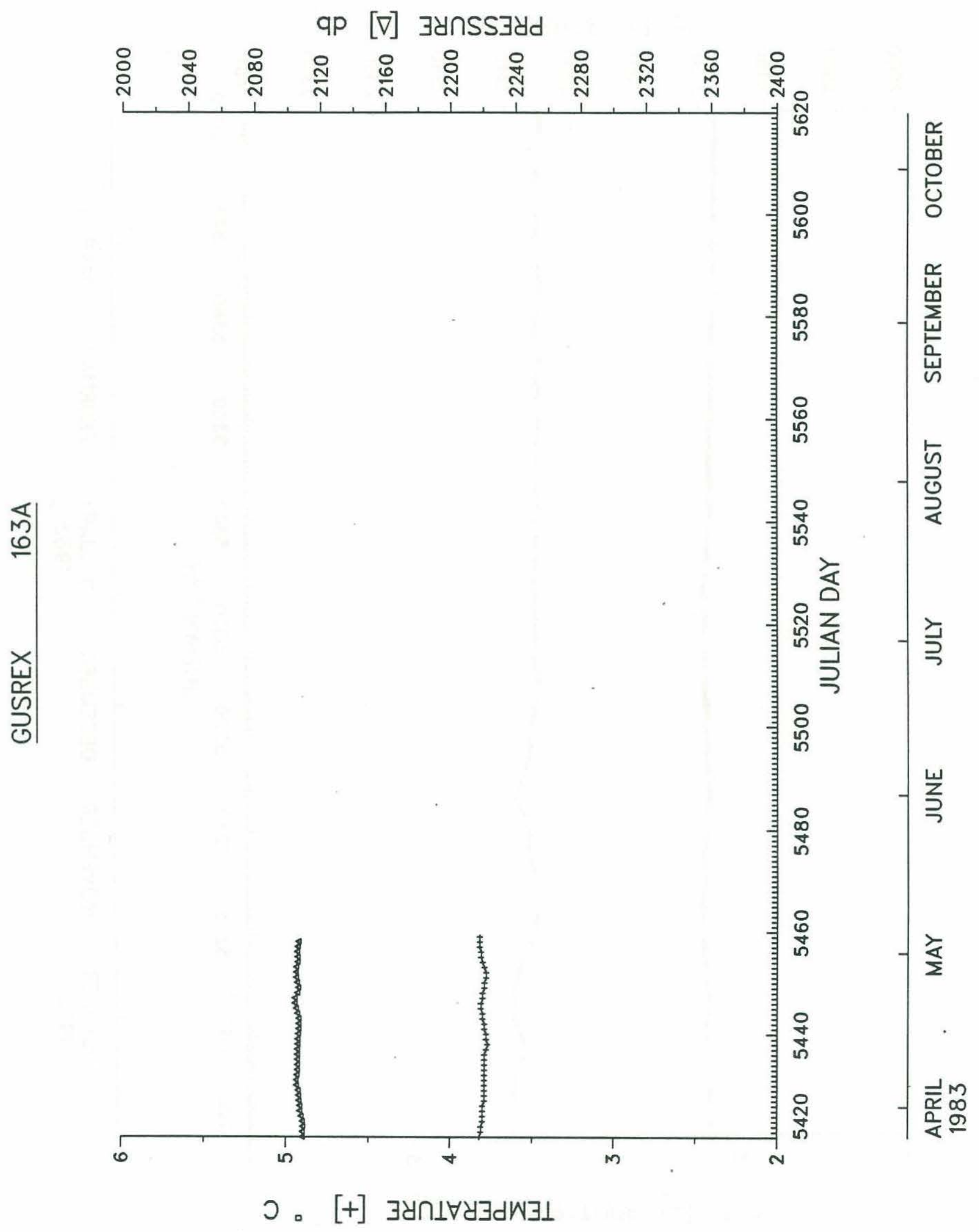




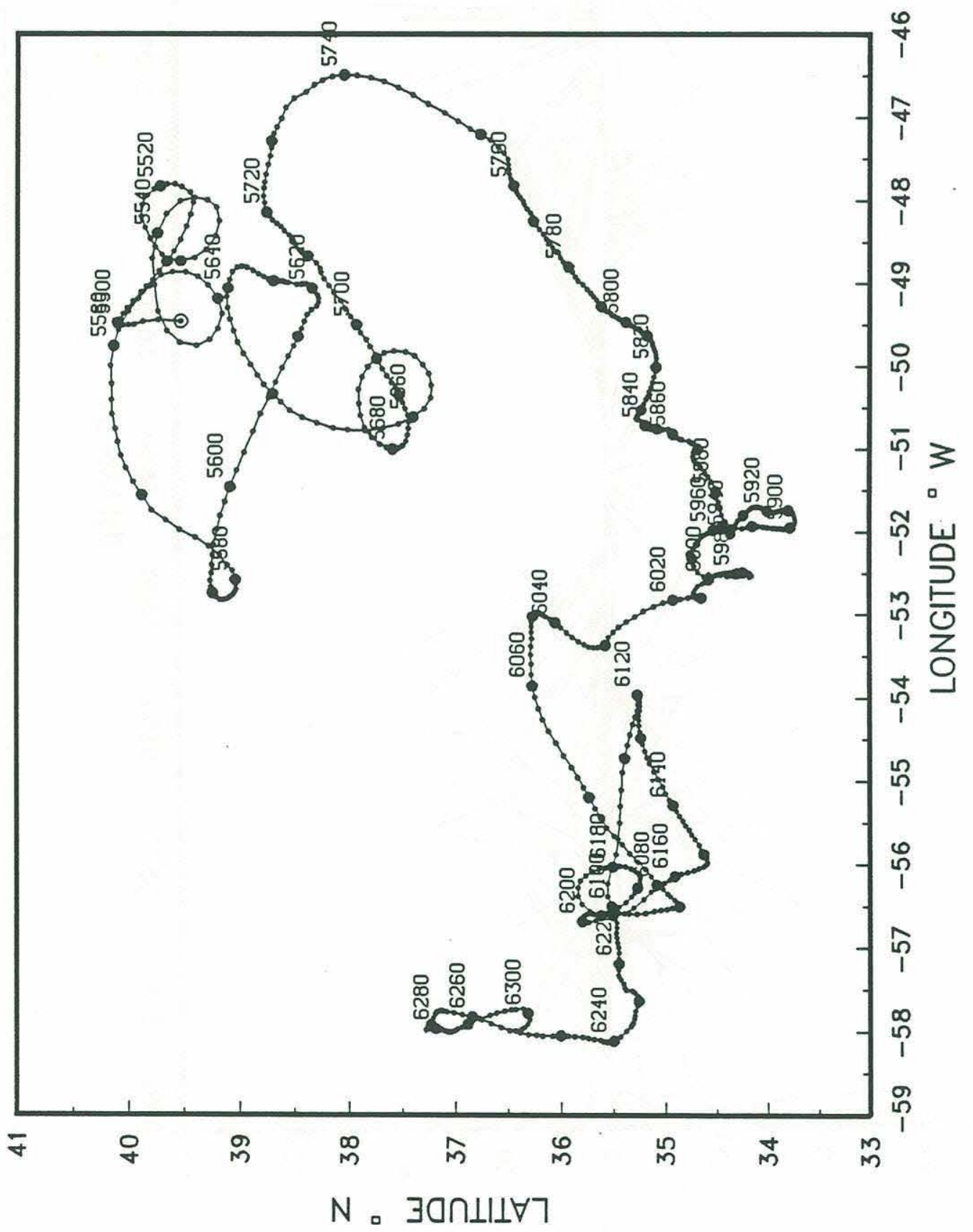




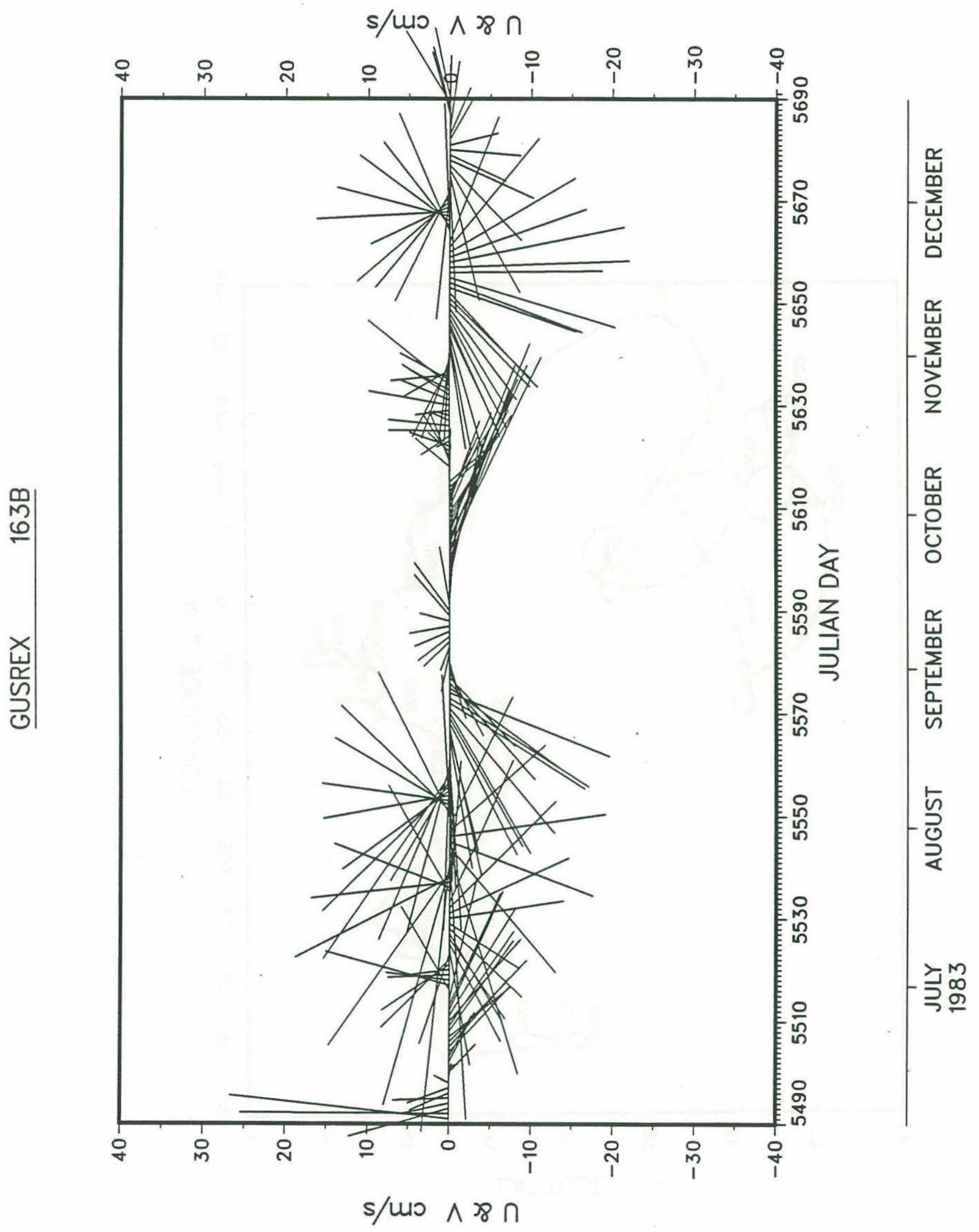




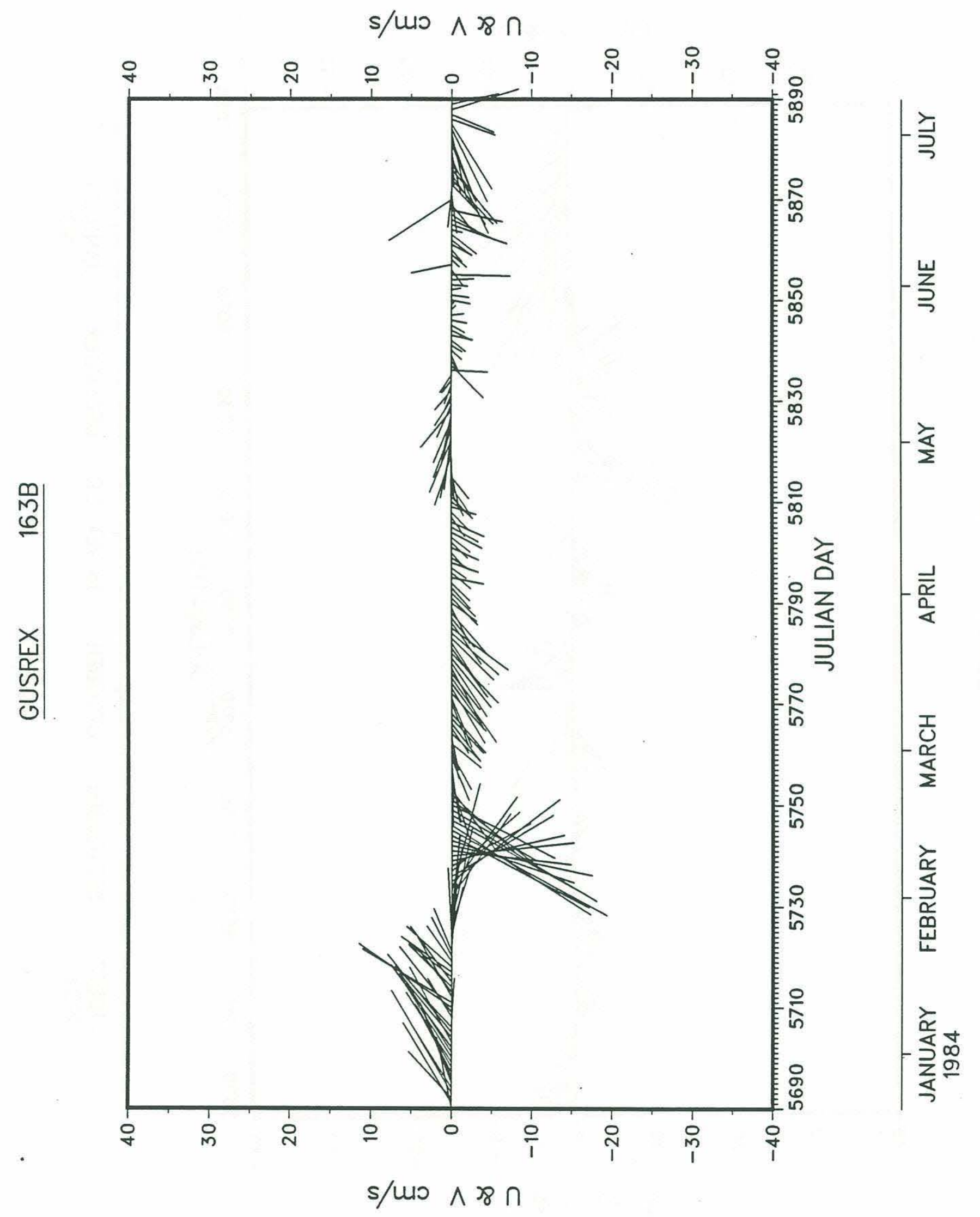




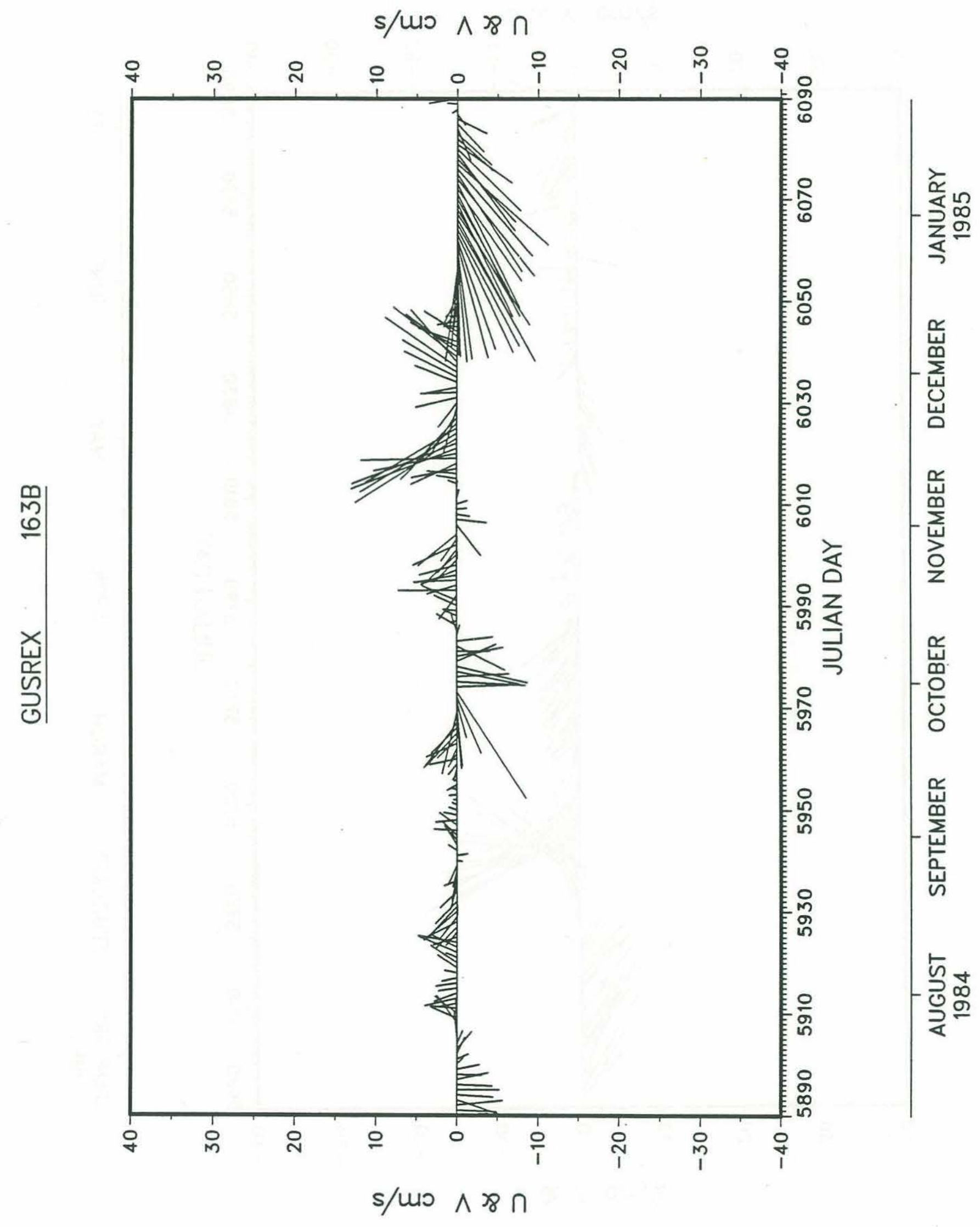




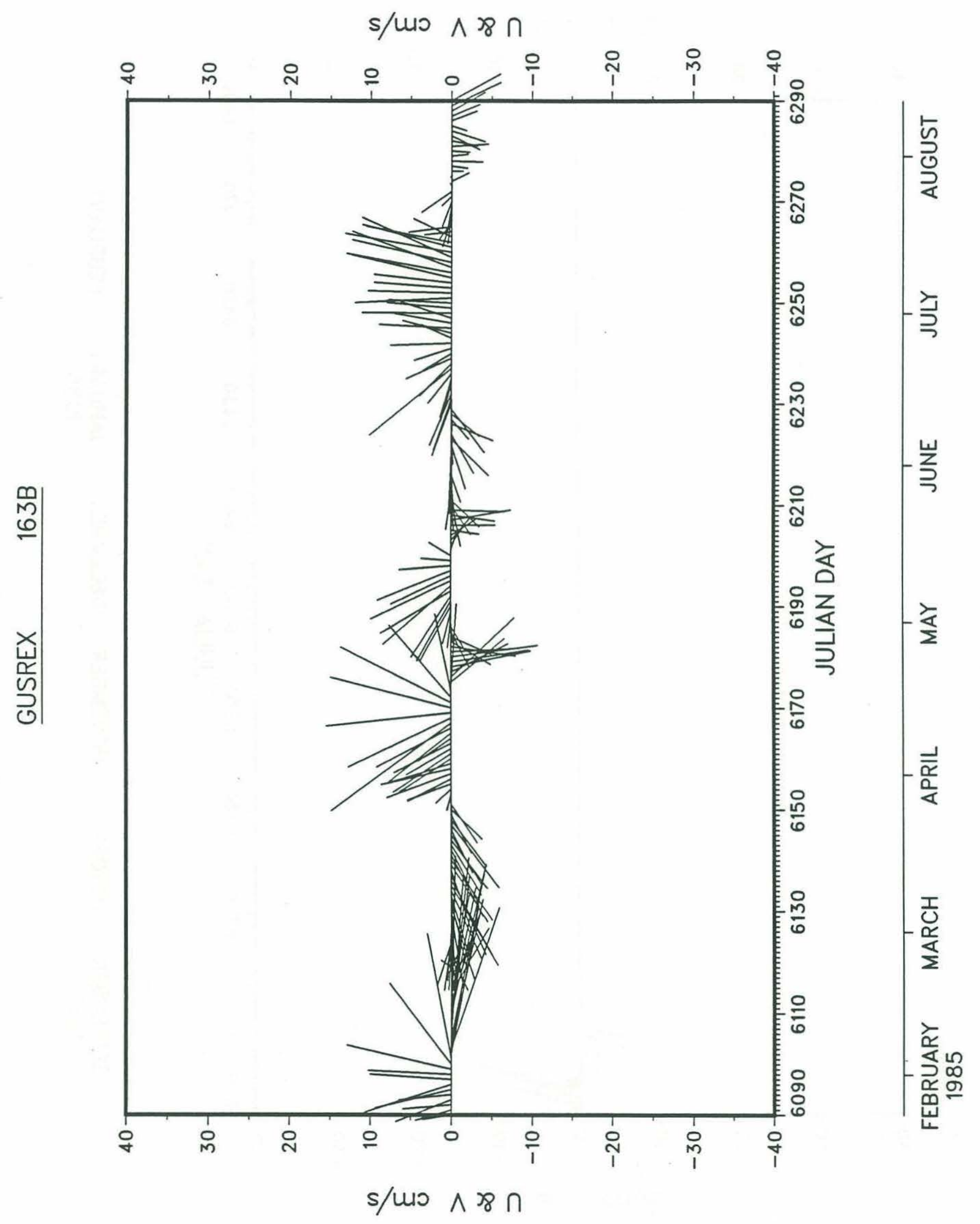




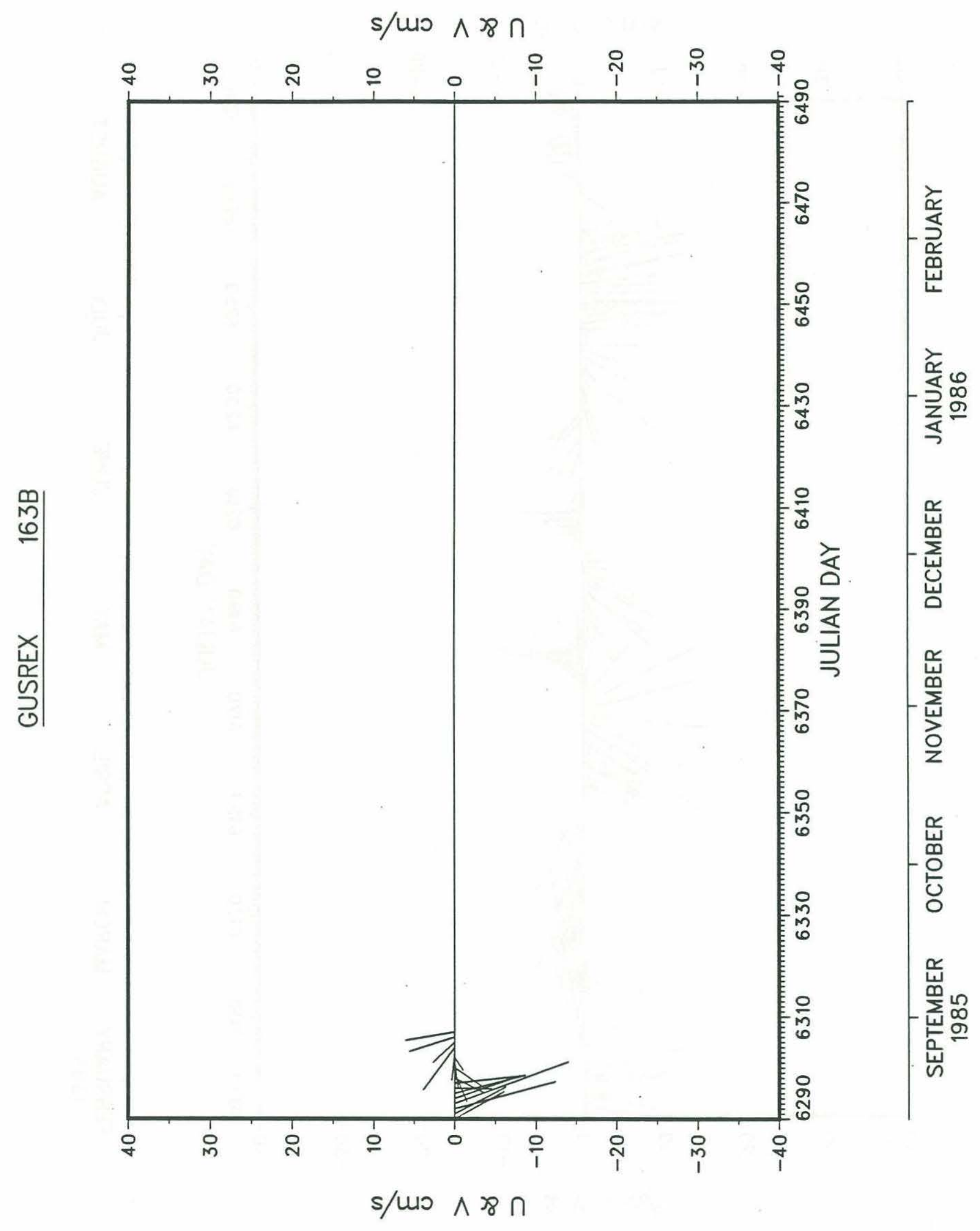


s/ur [o] HLYON

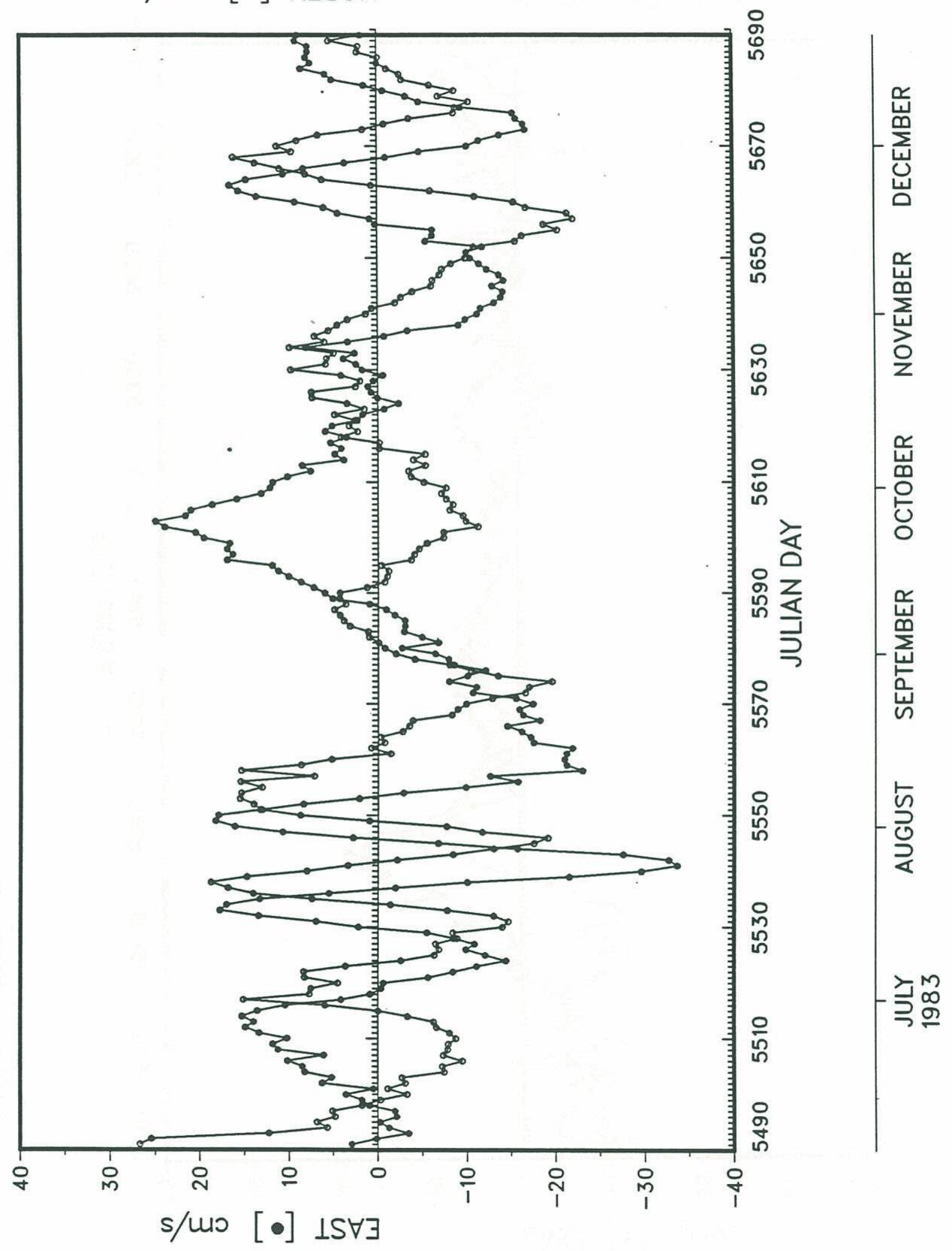




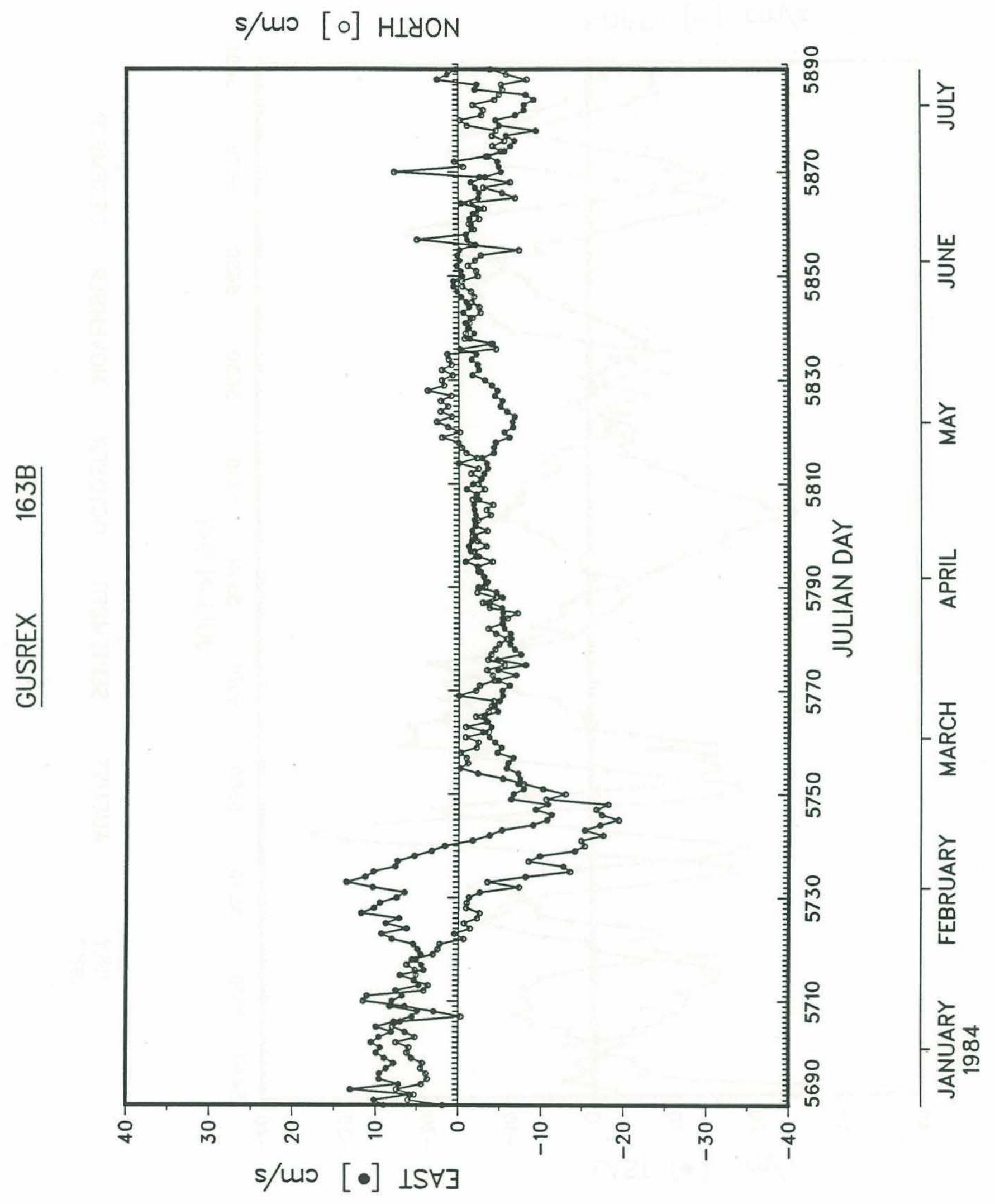




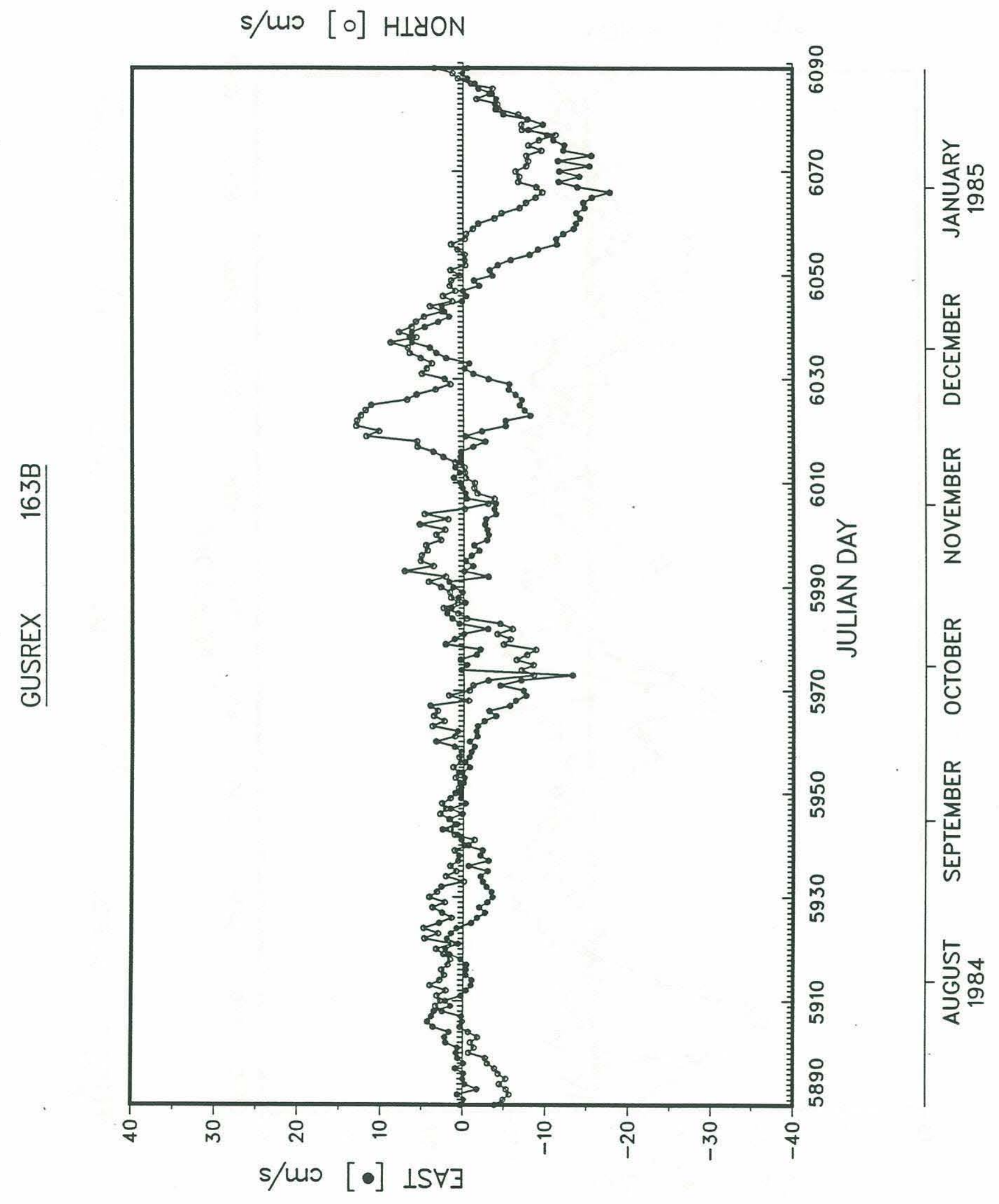




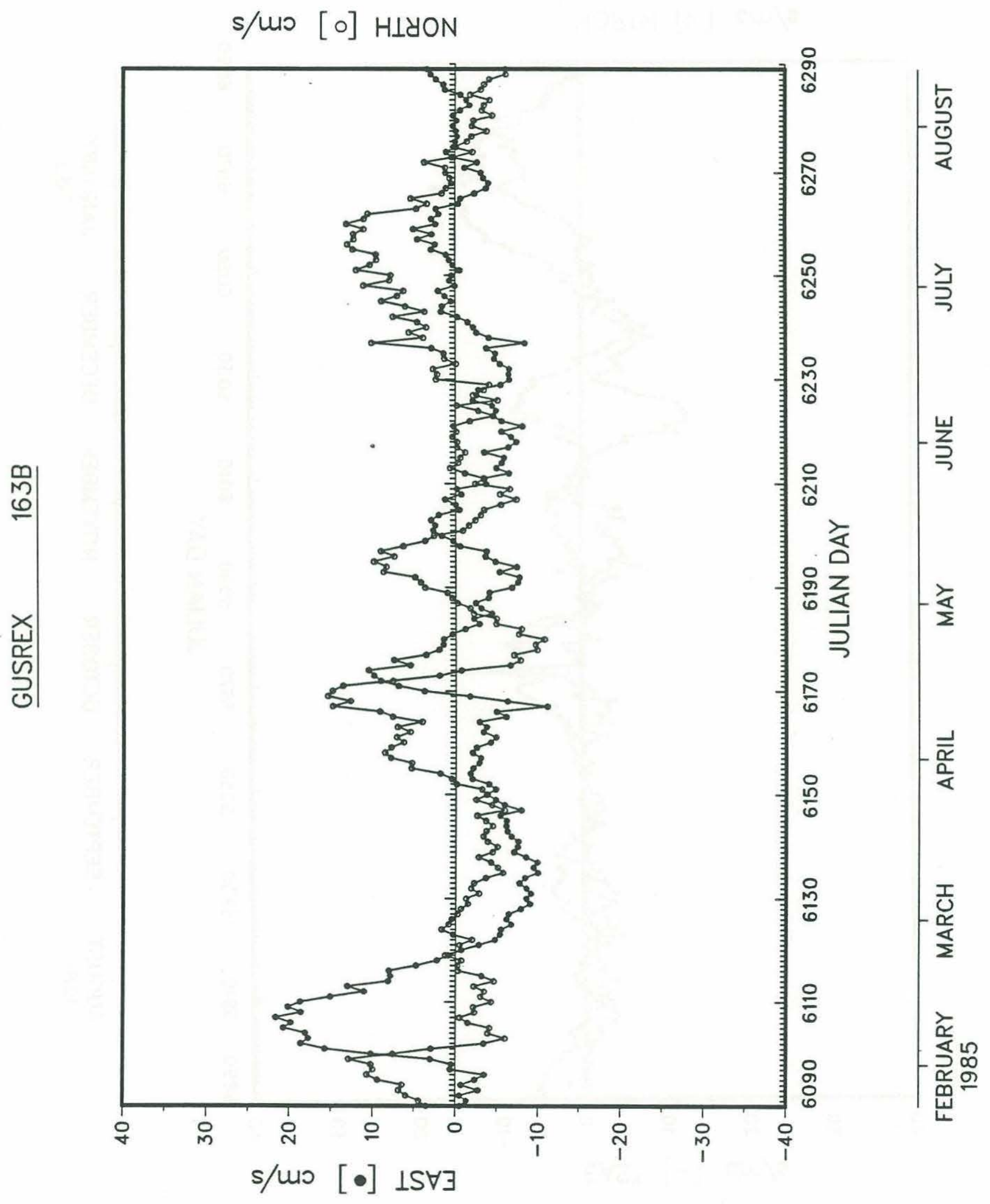




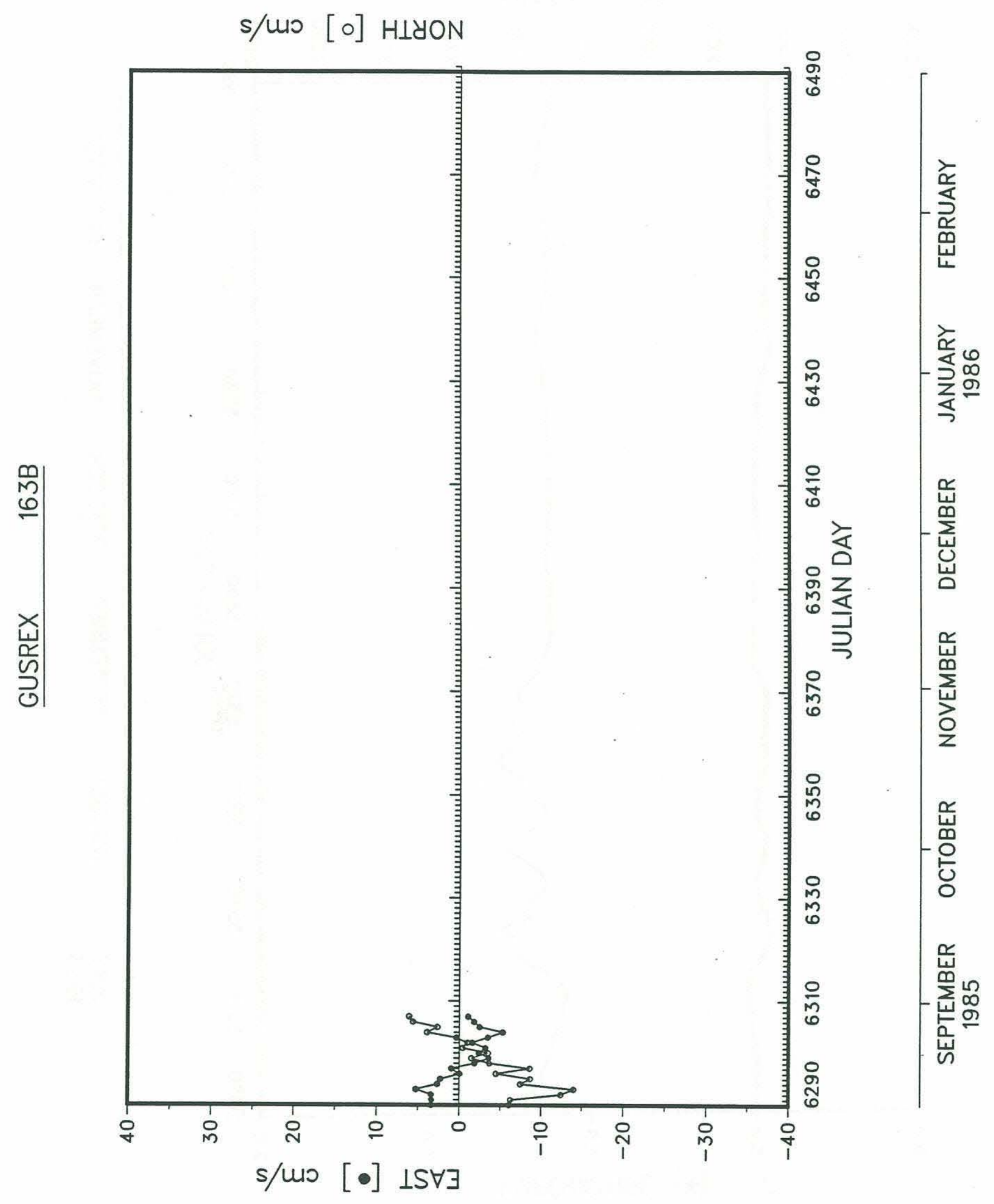




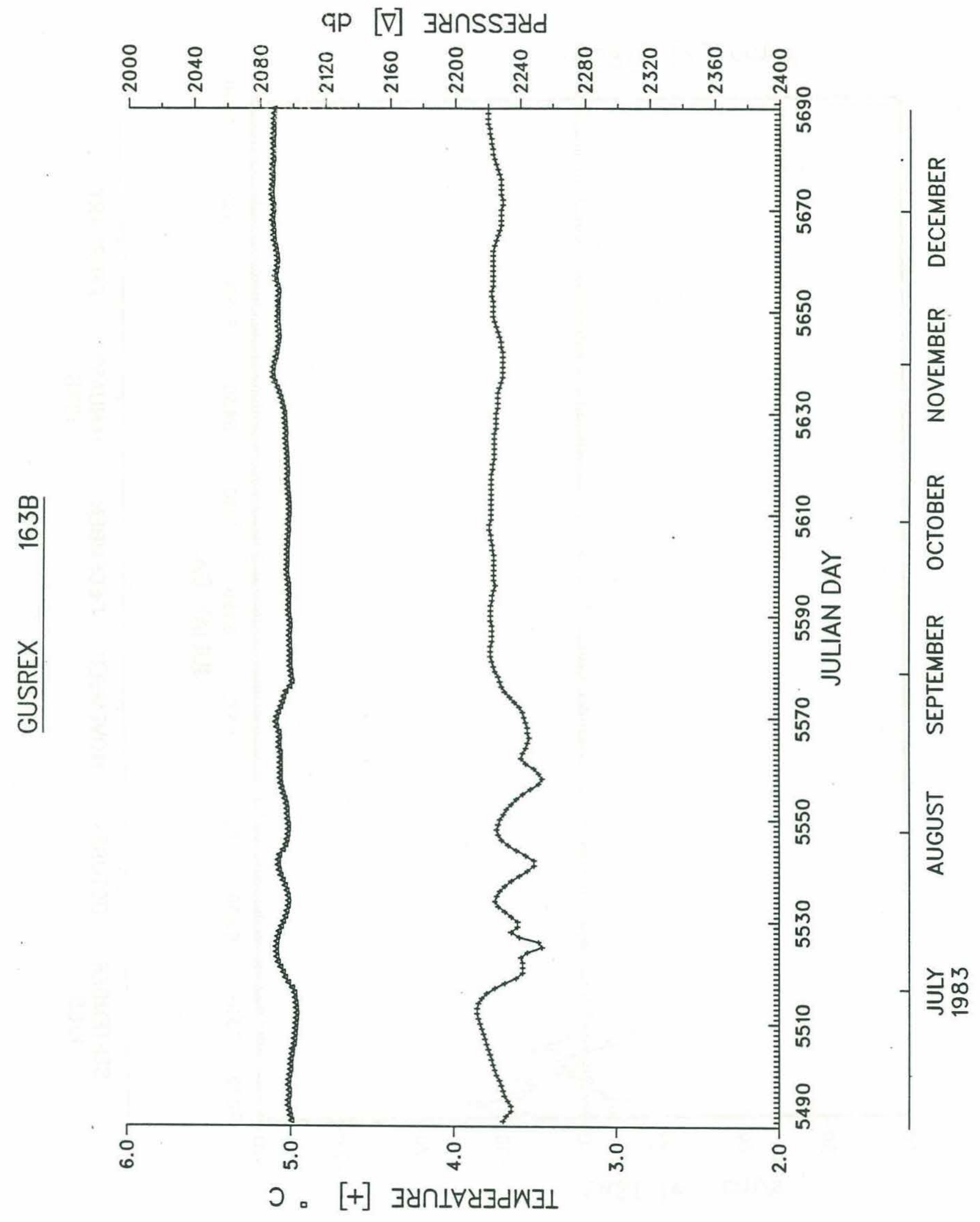




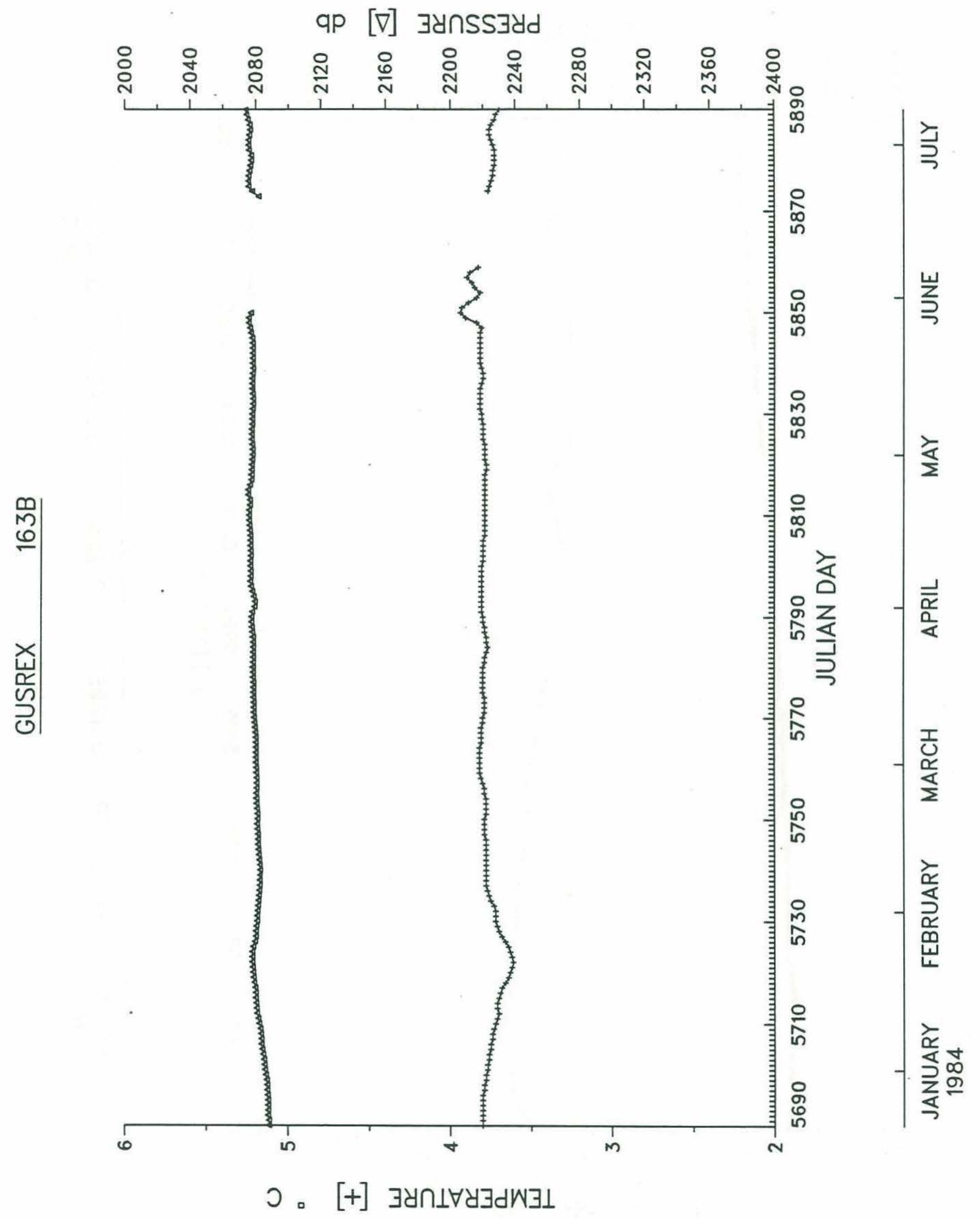




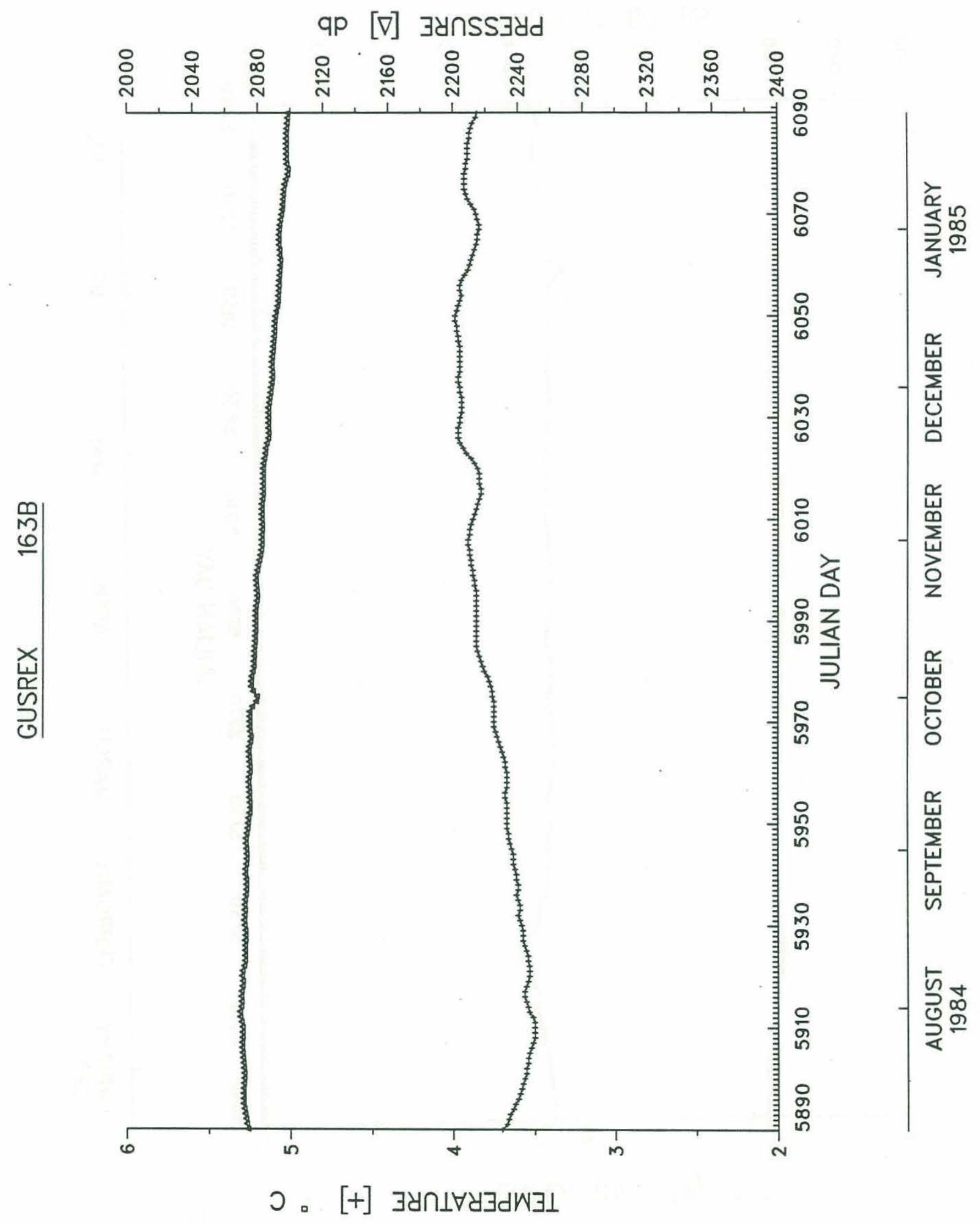




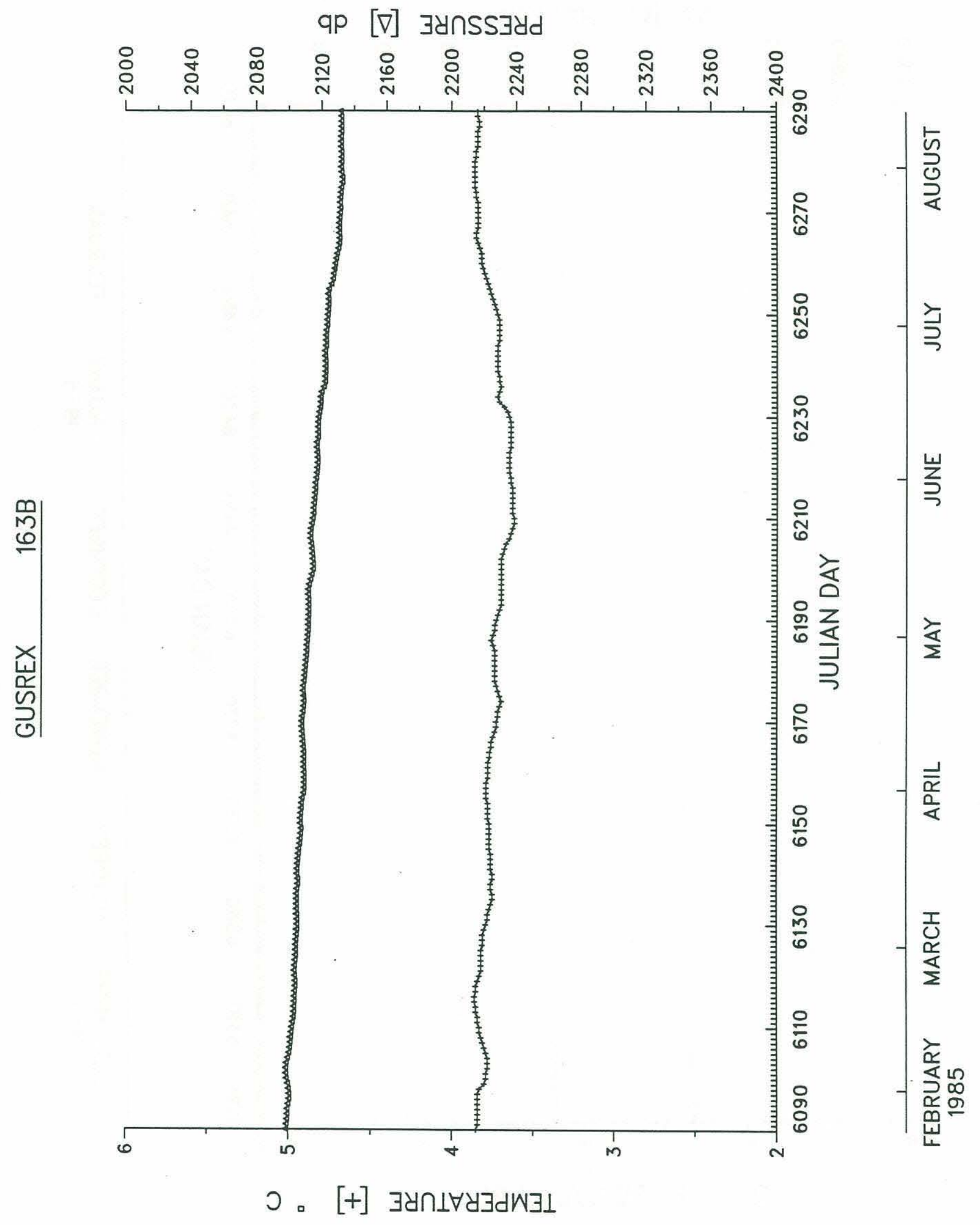




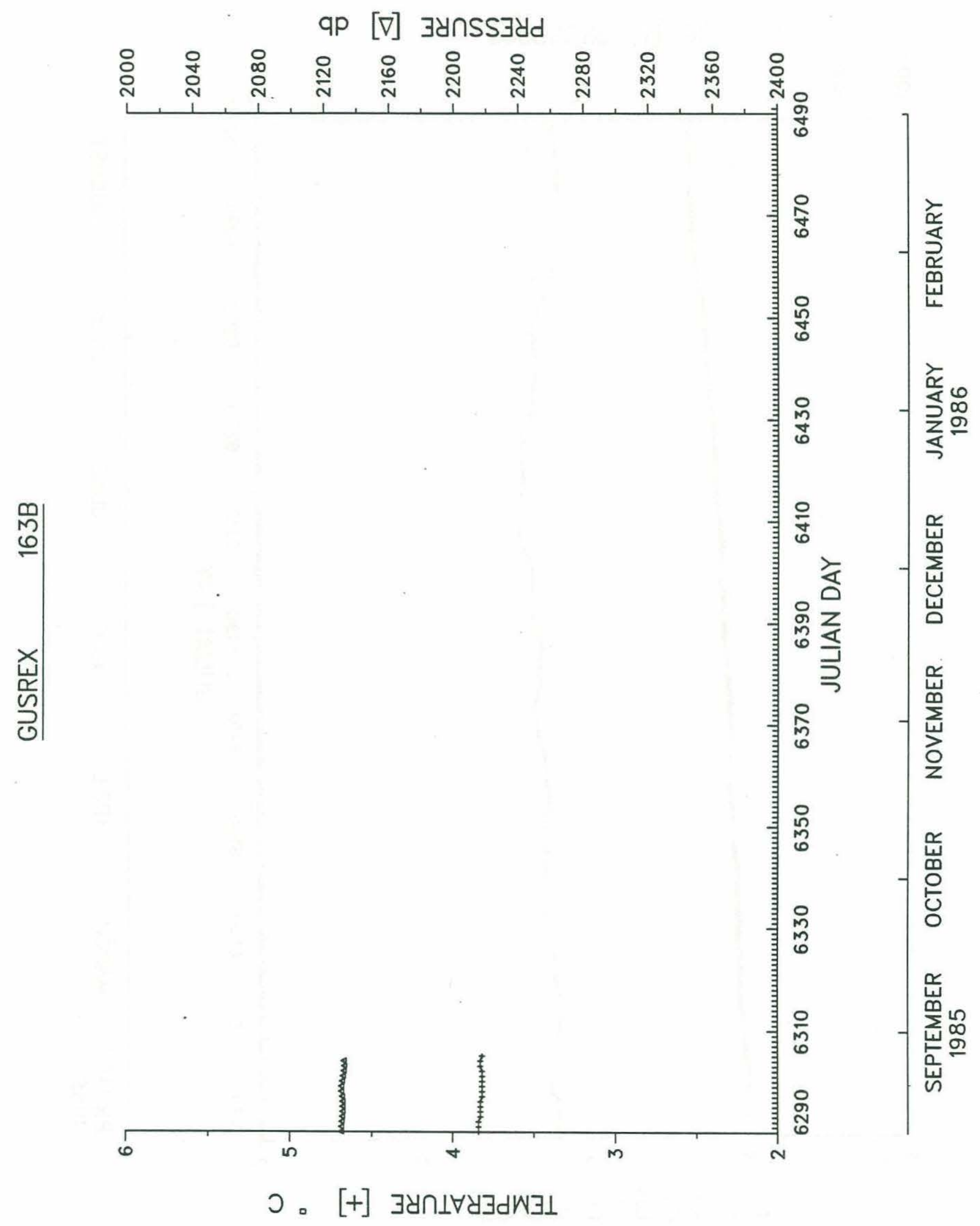




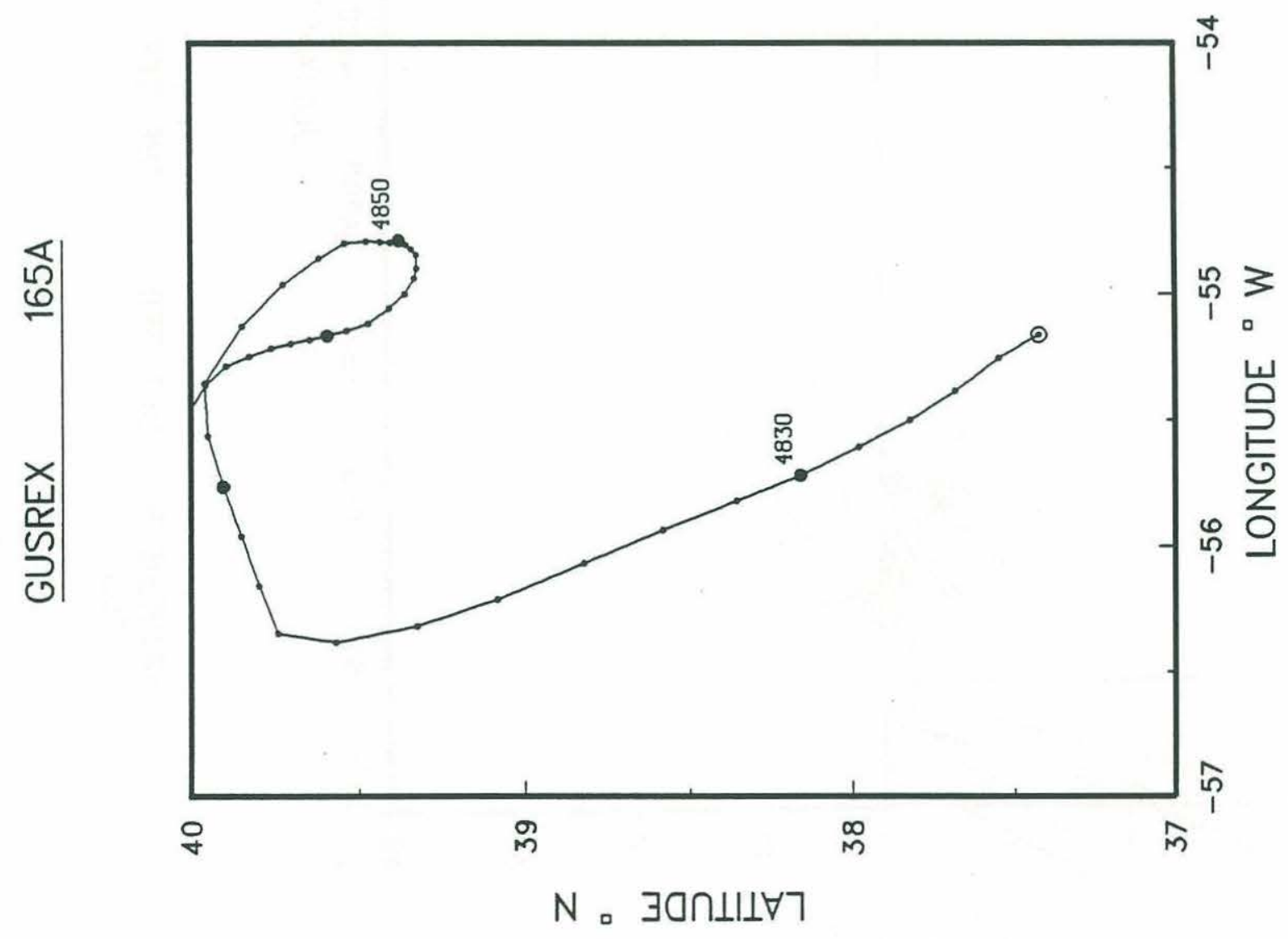




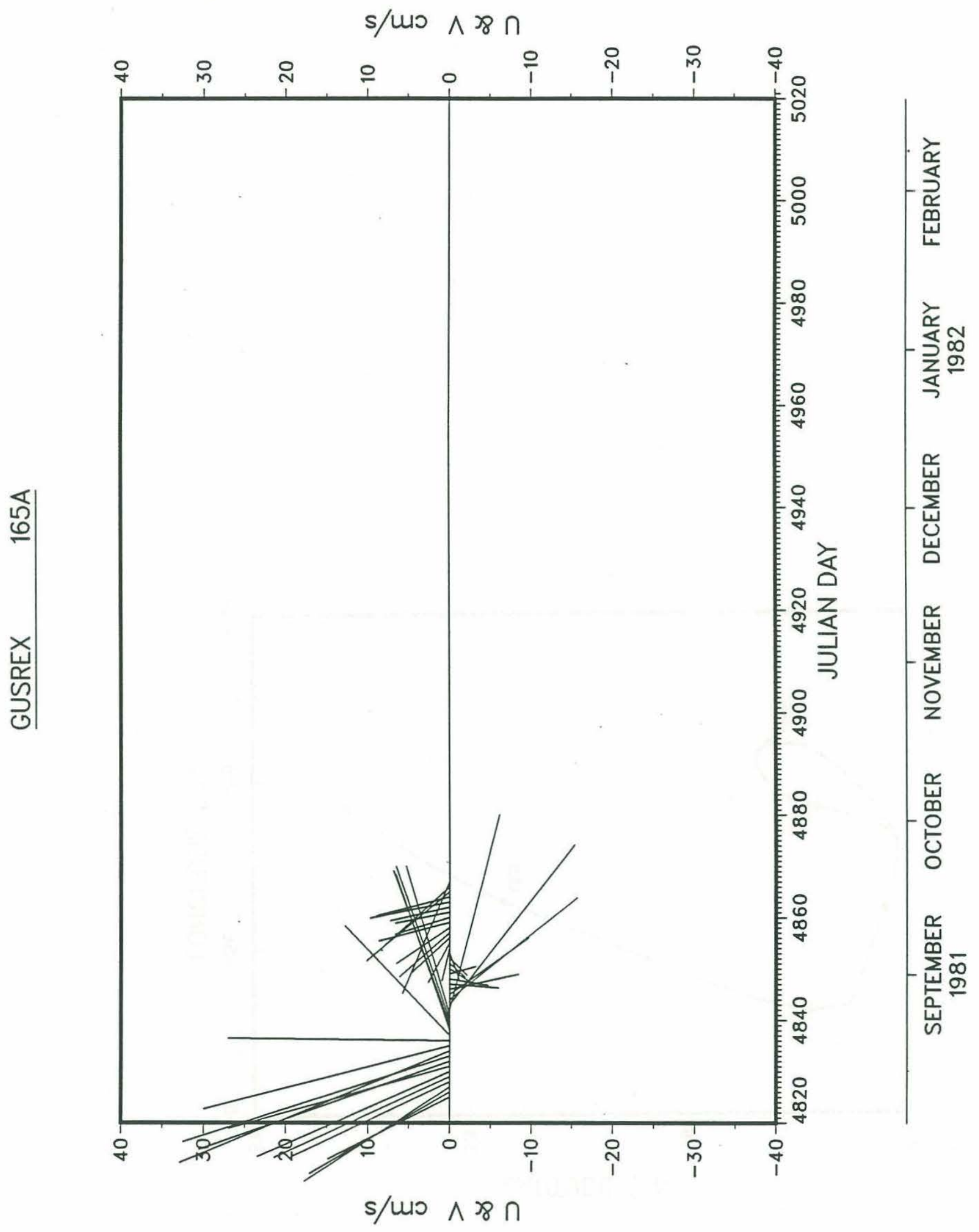




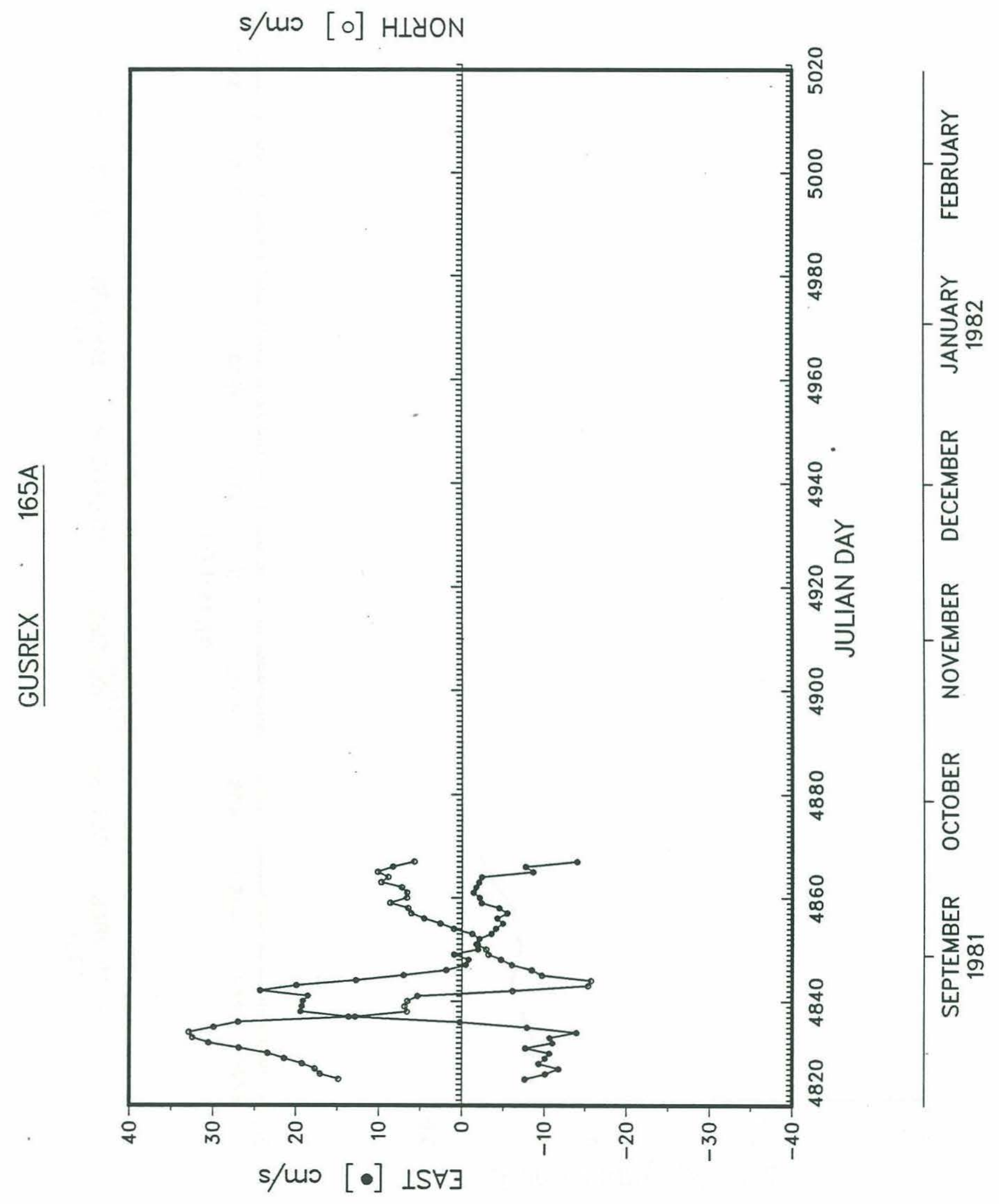



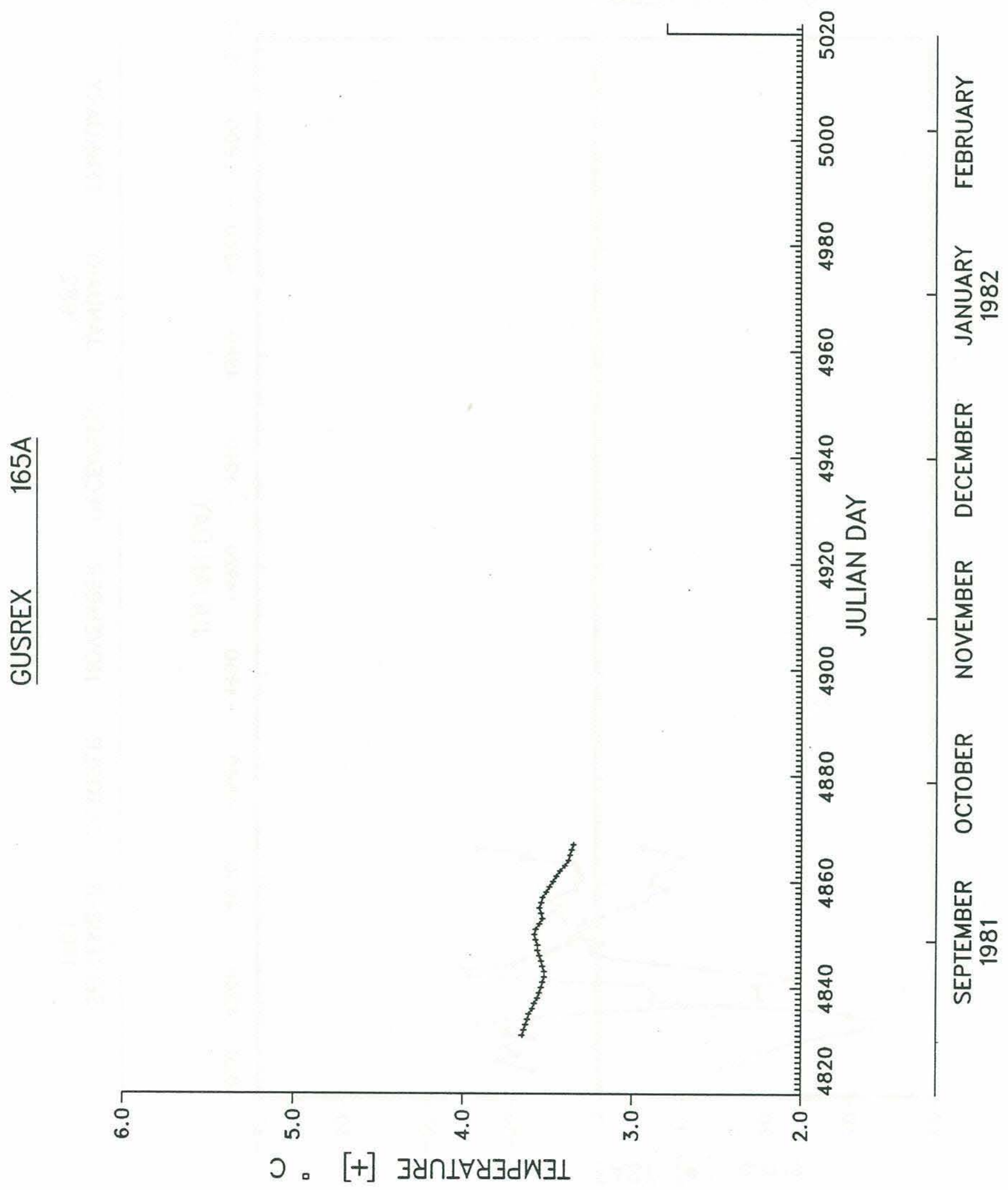


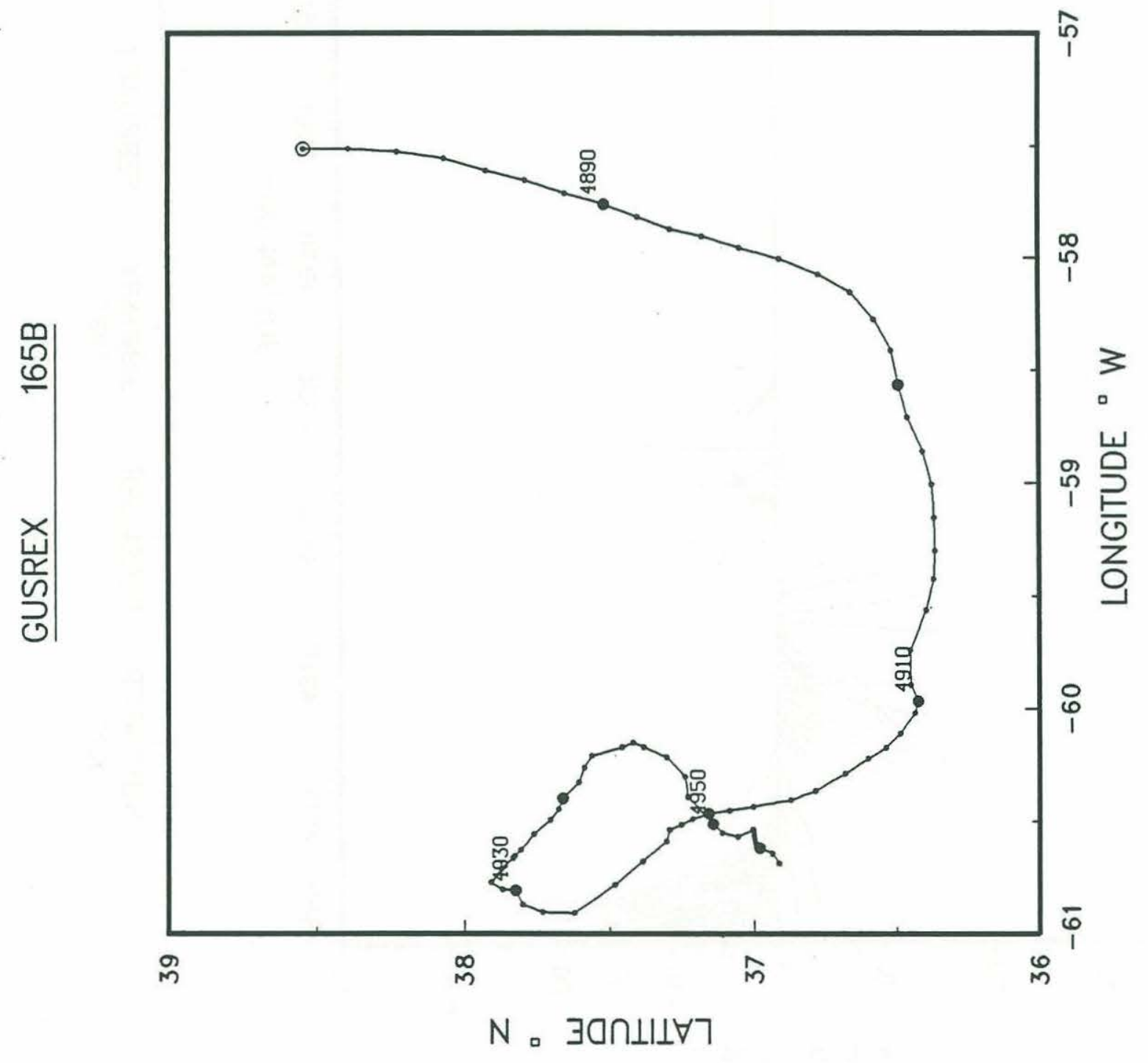




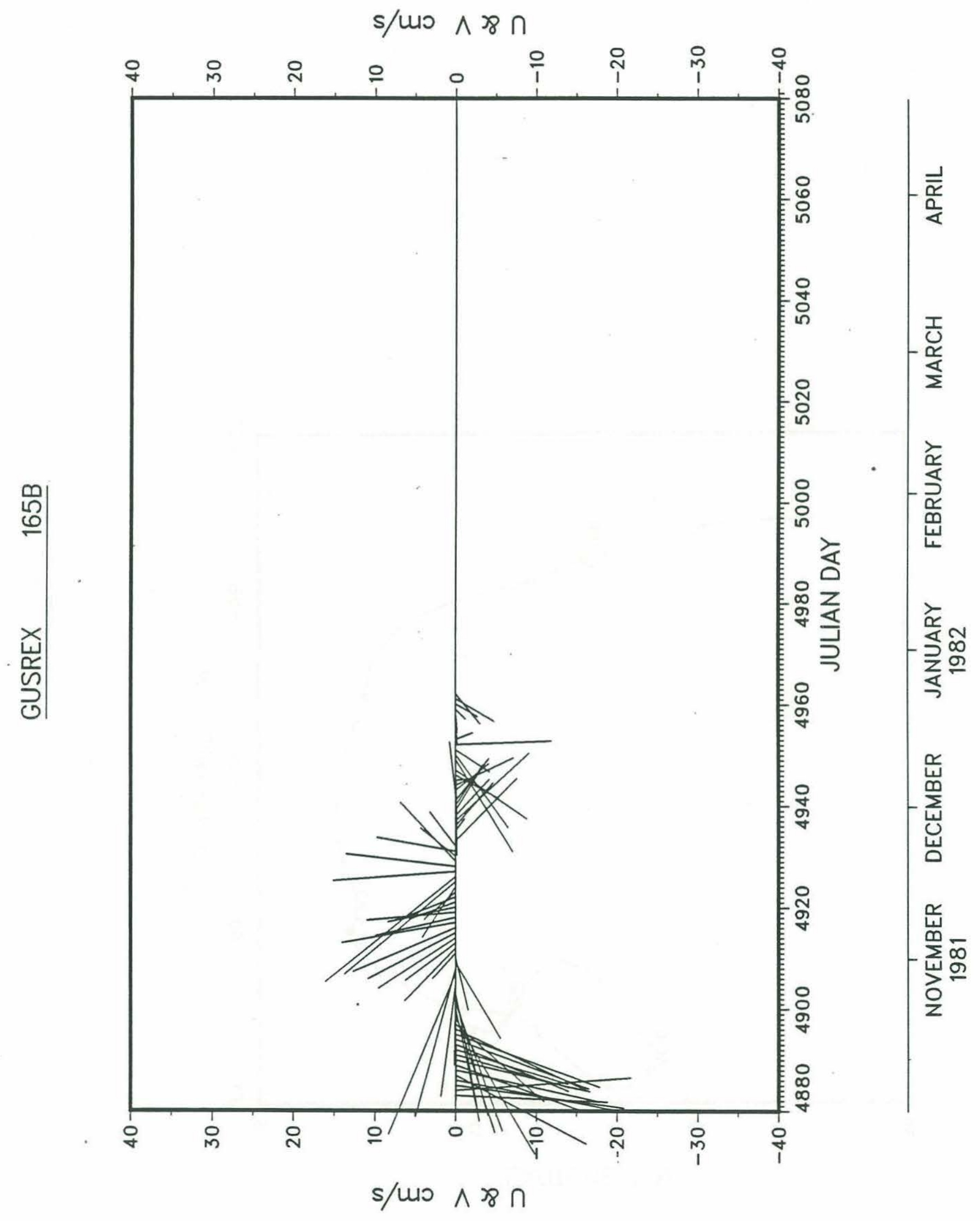




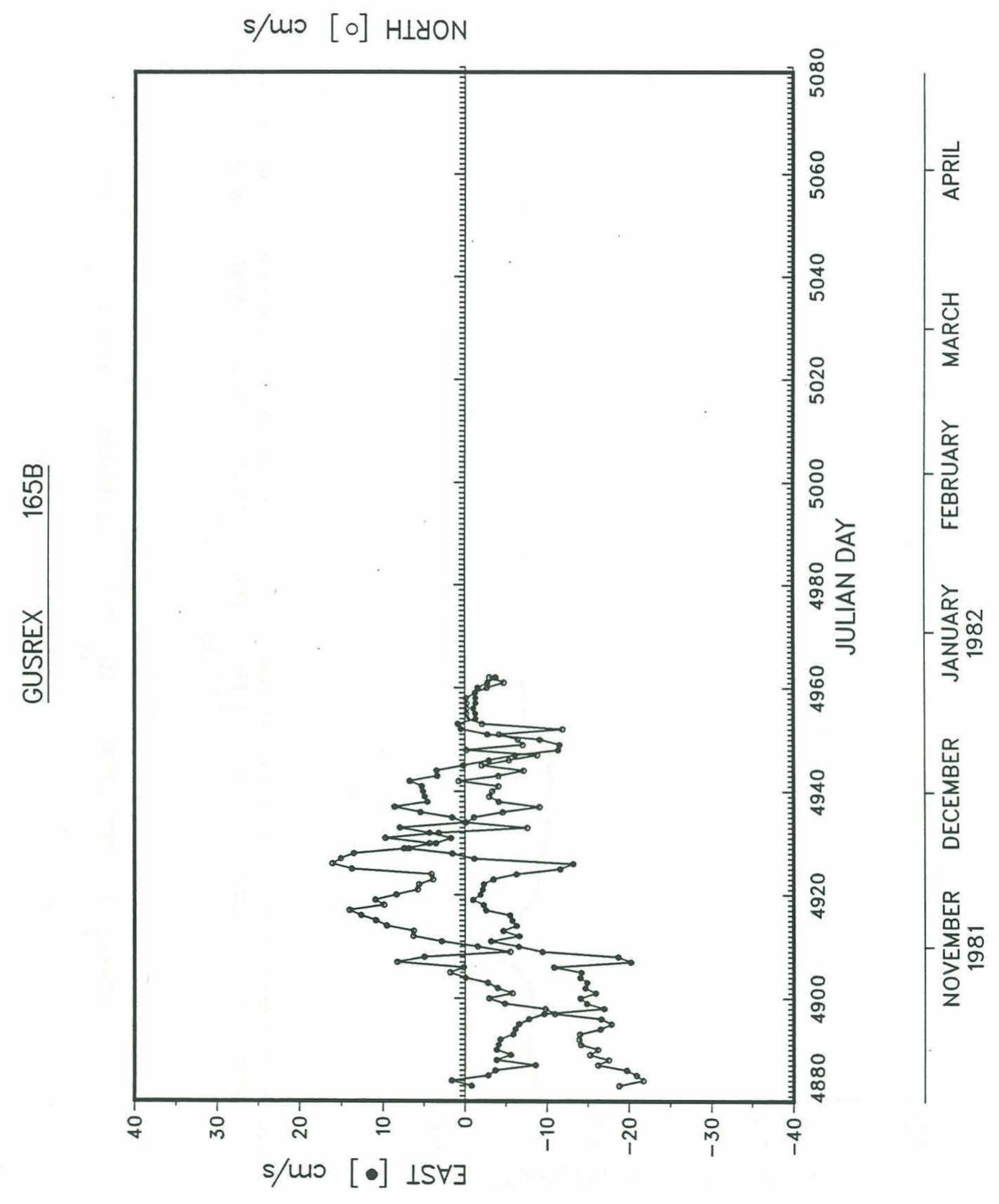




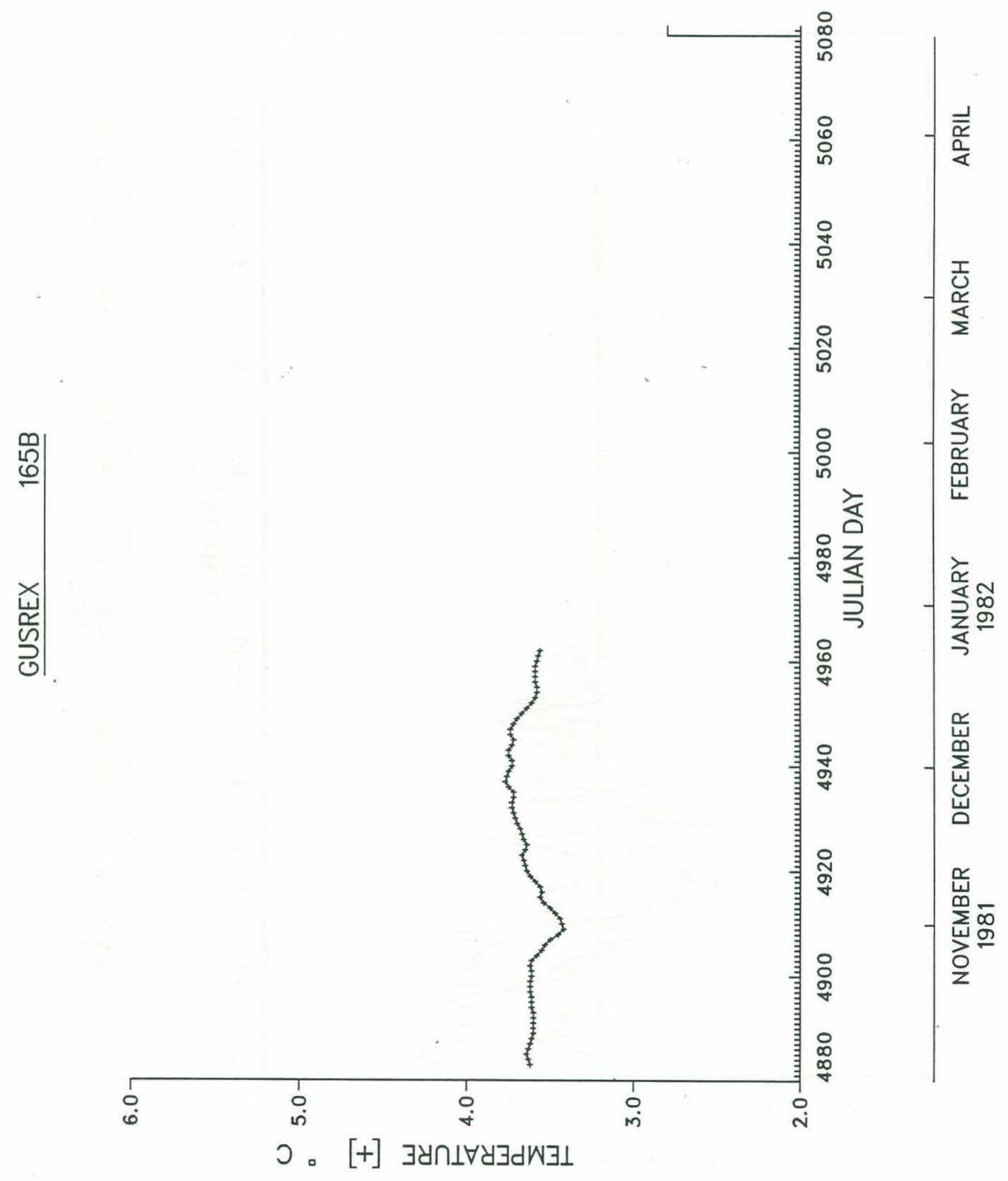




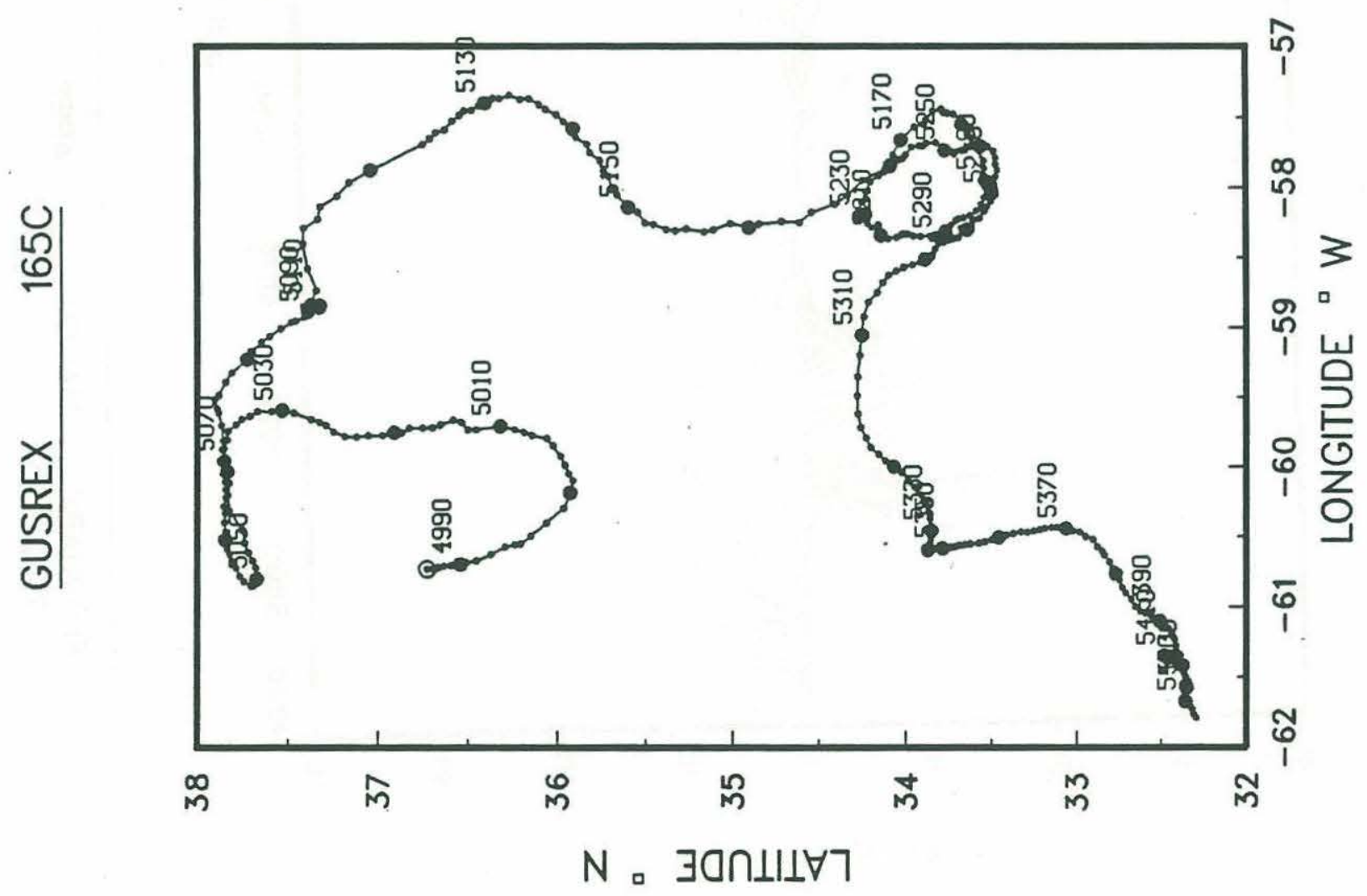




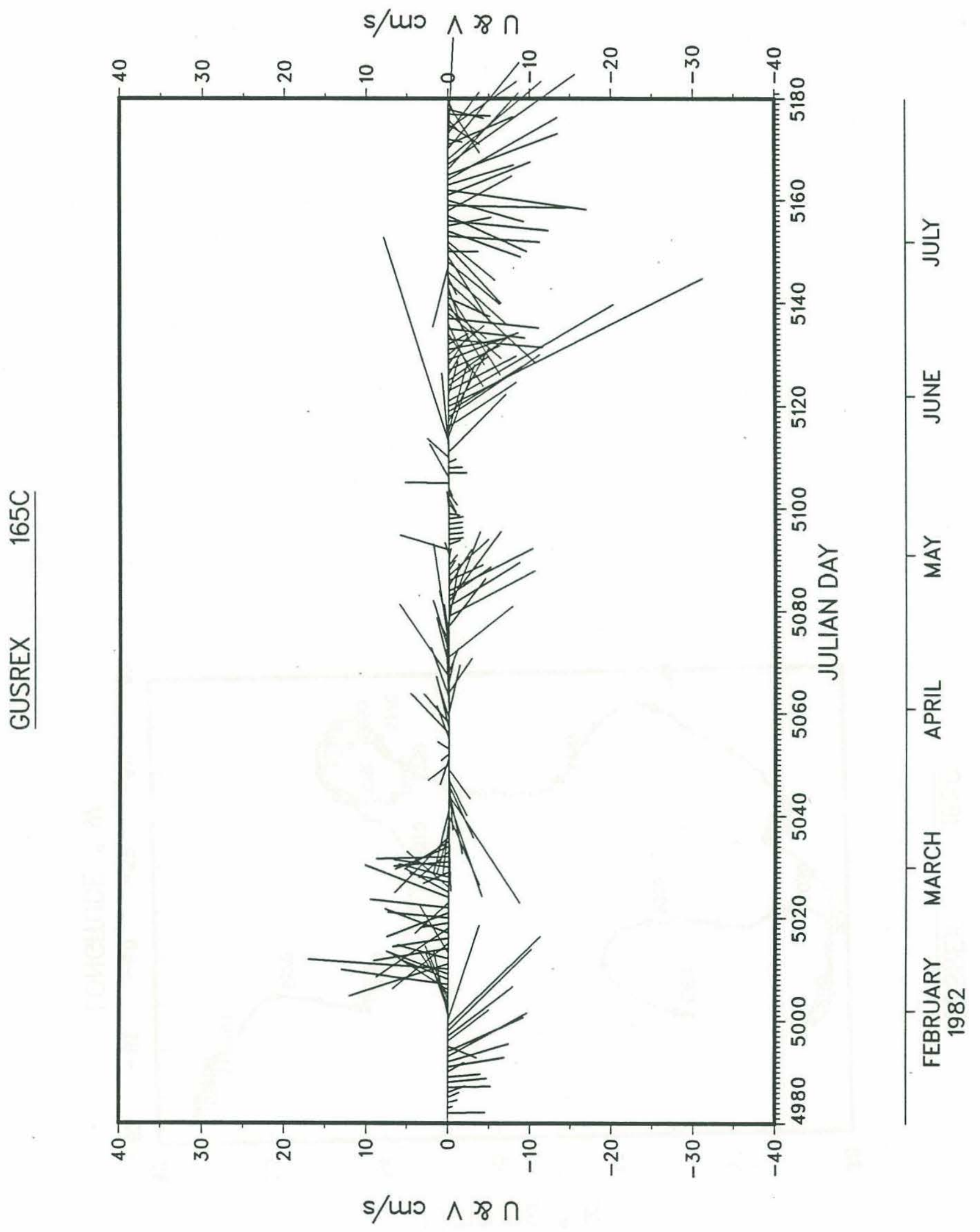




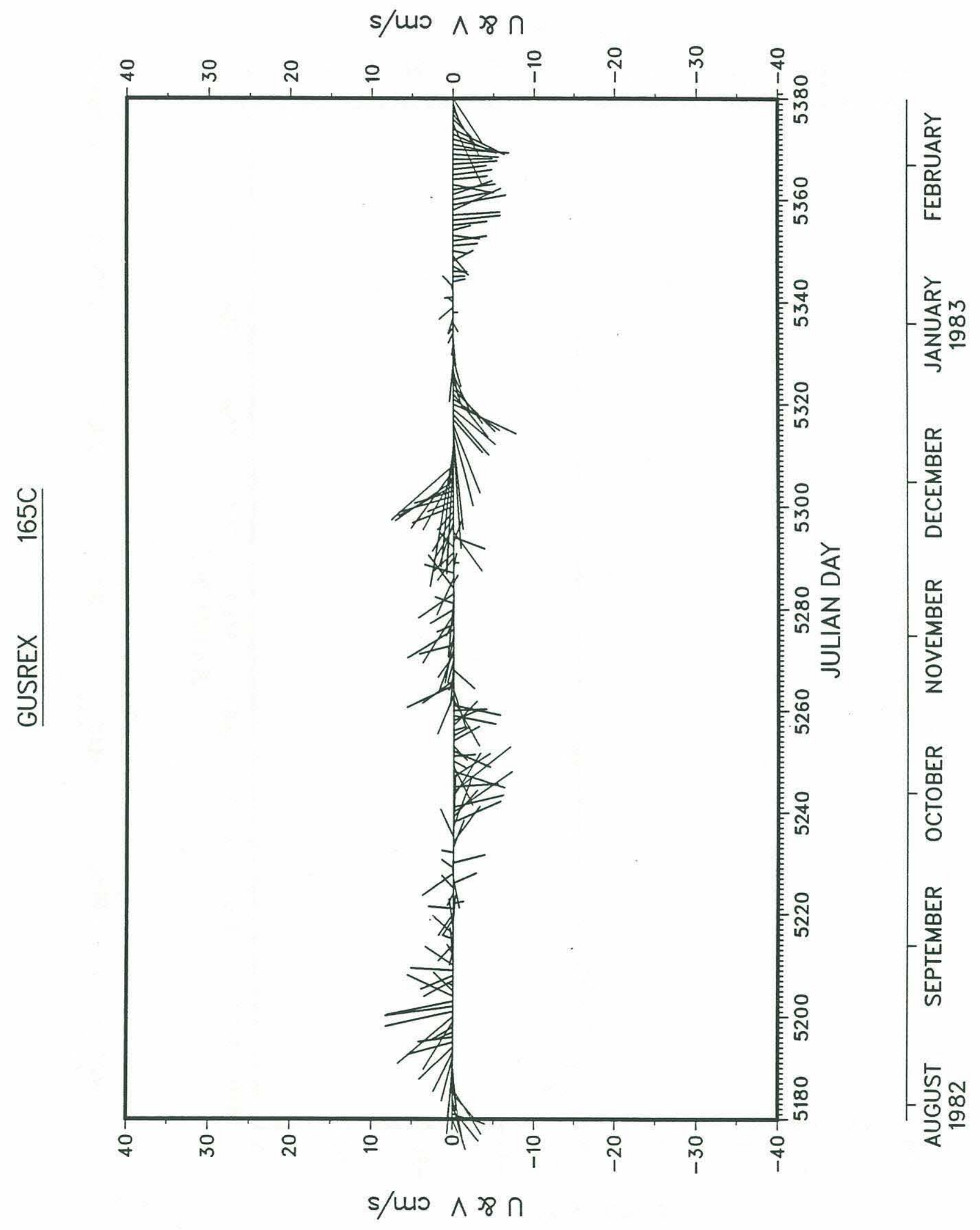




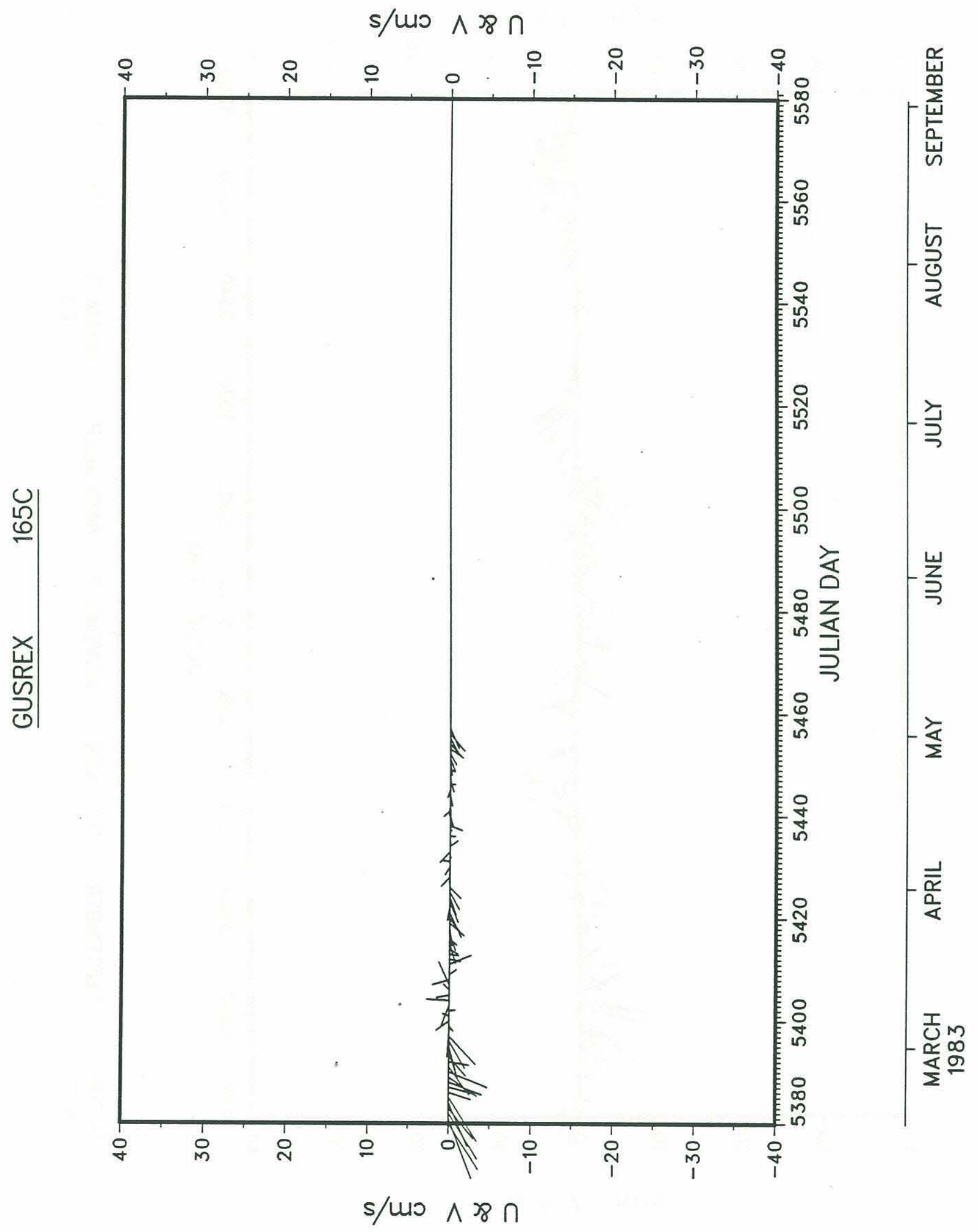




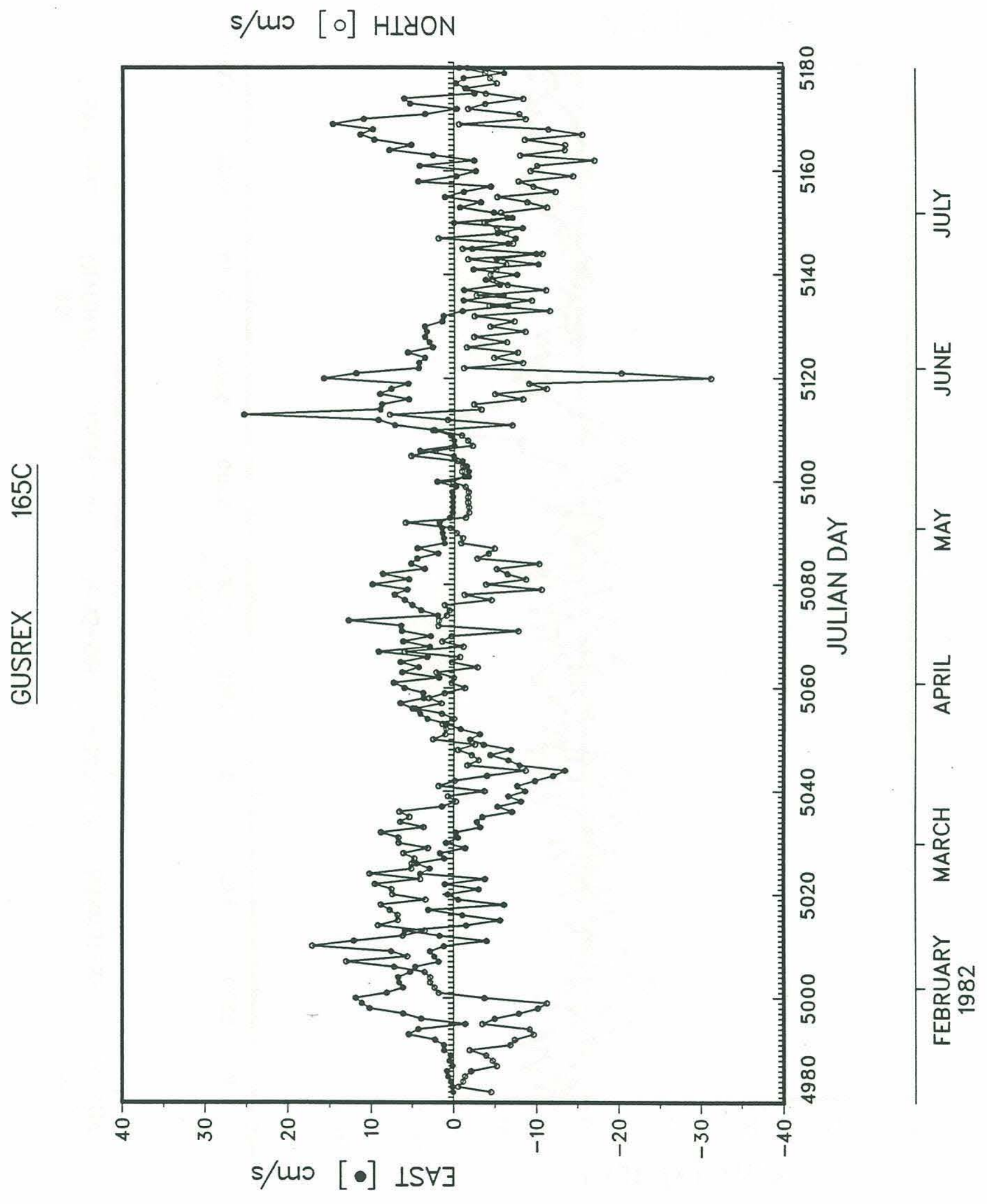




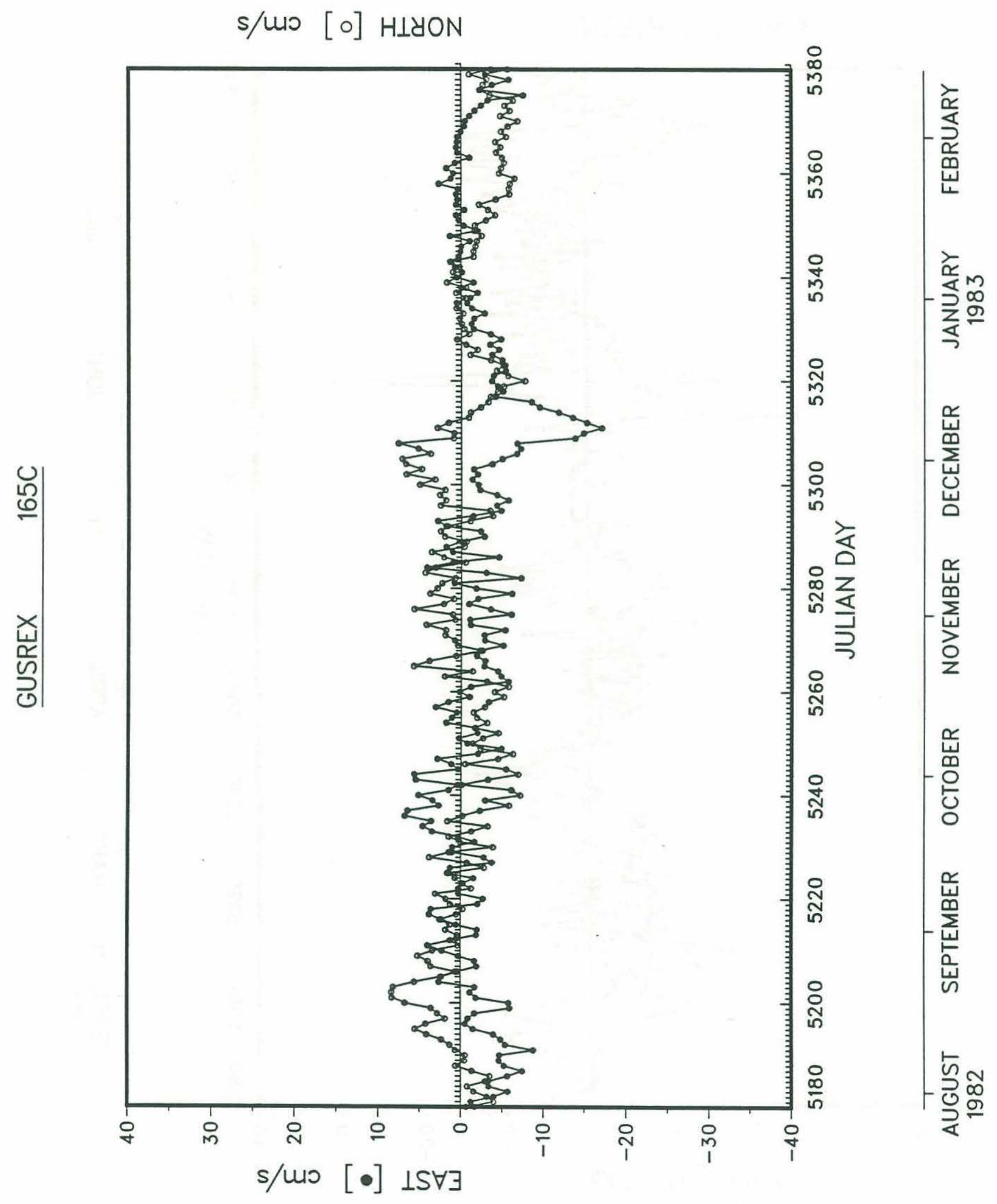




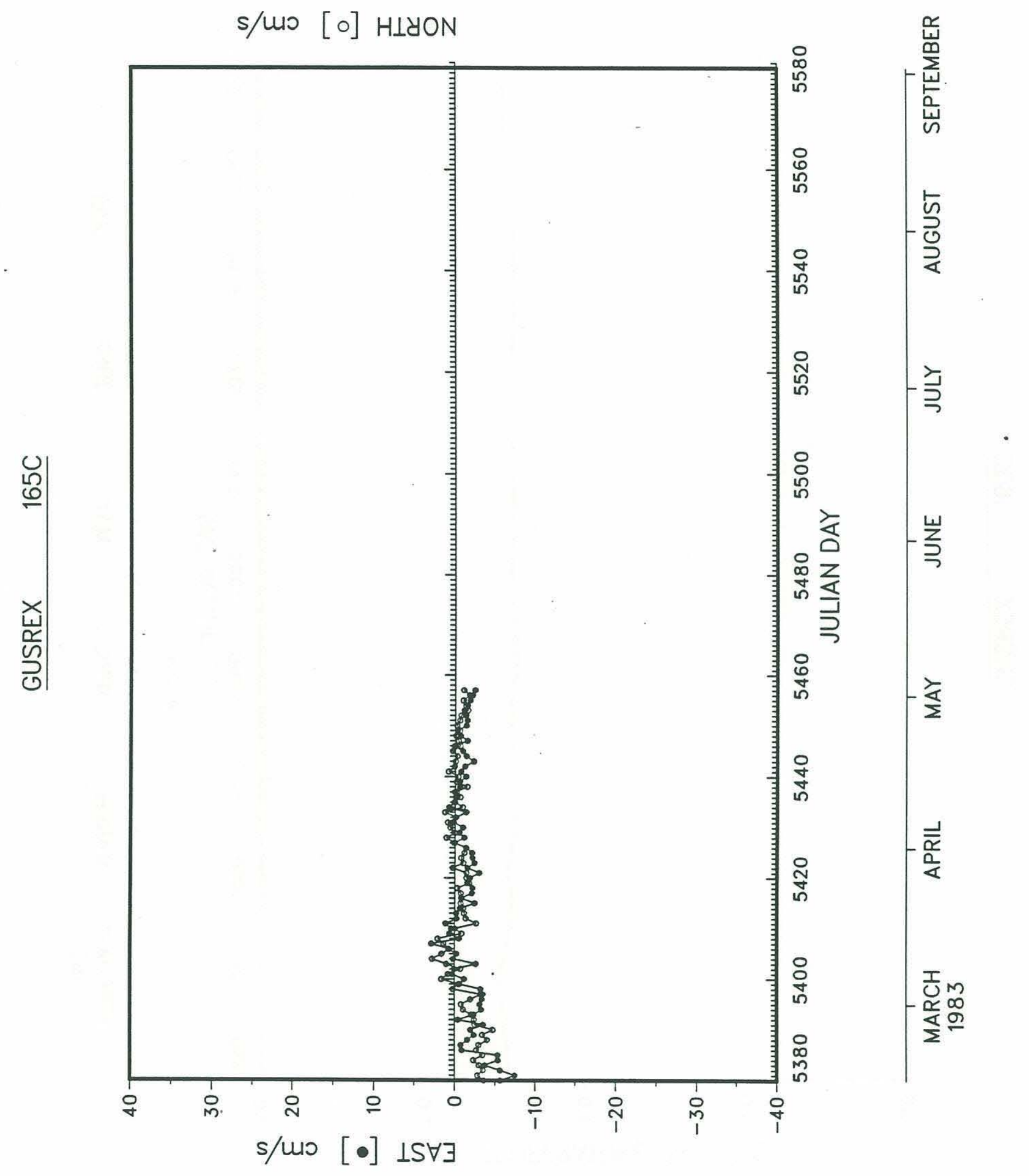



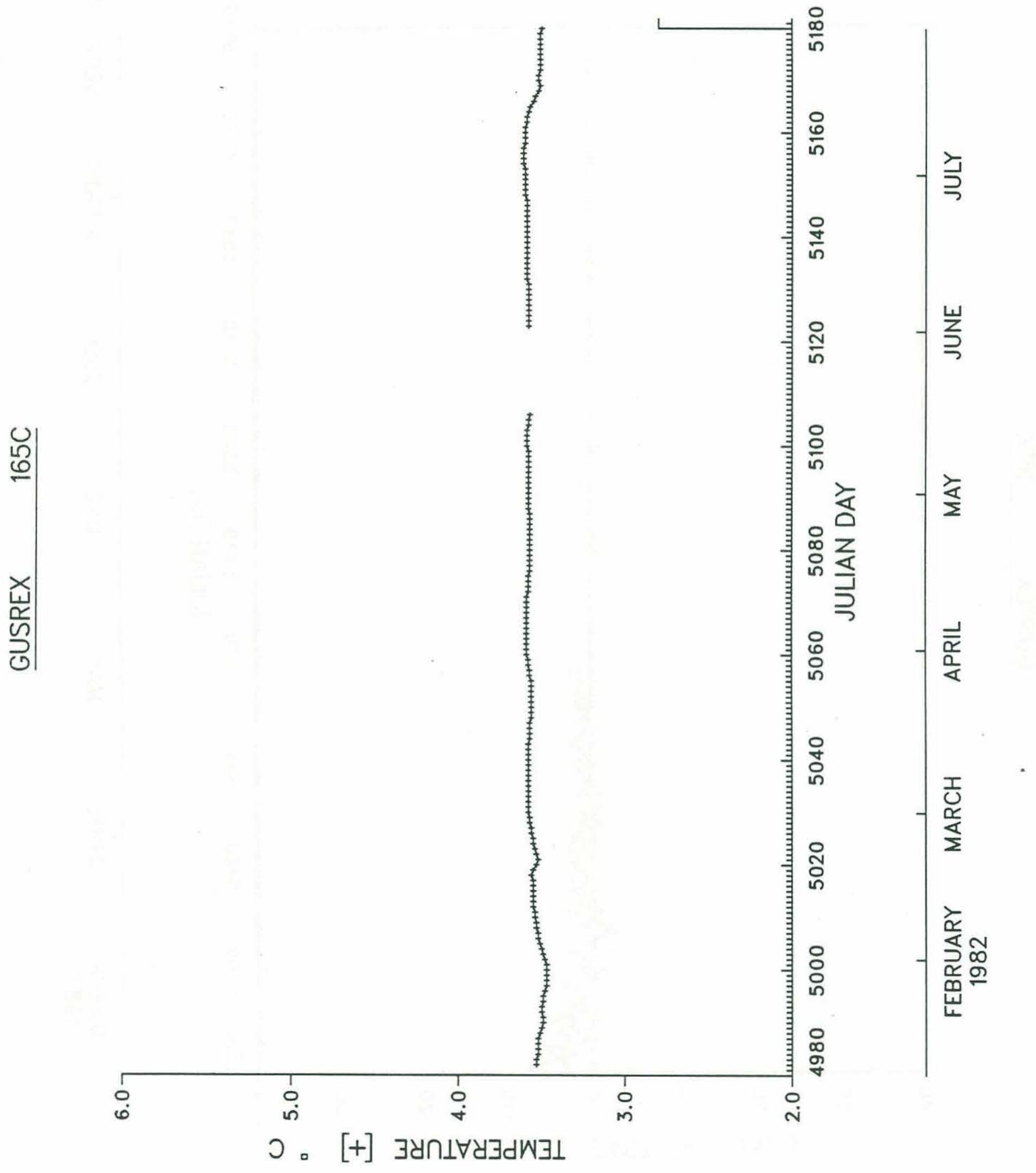

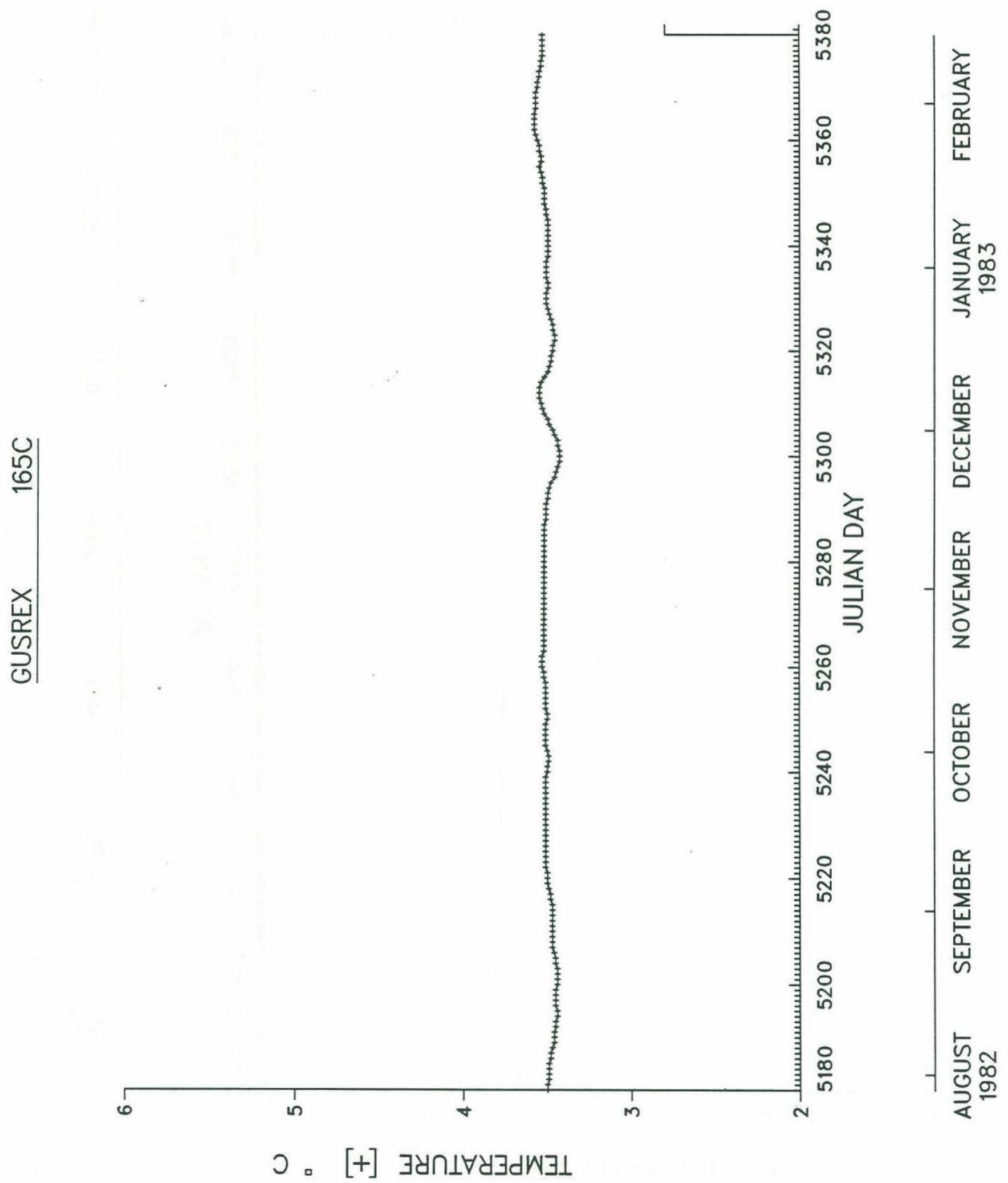


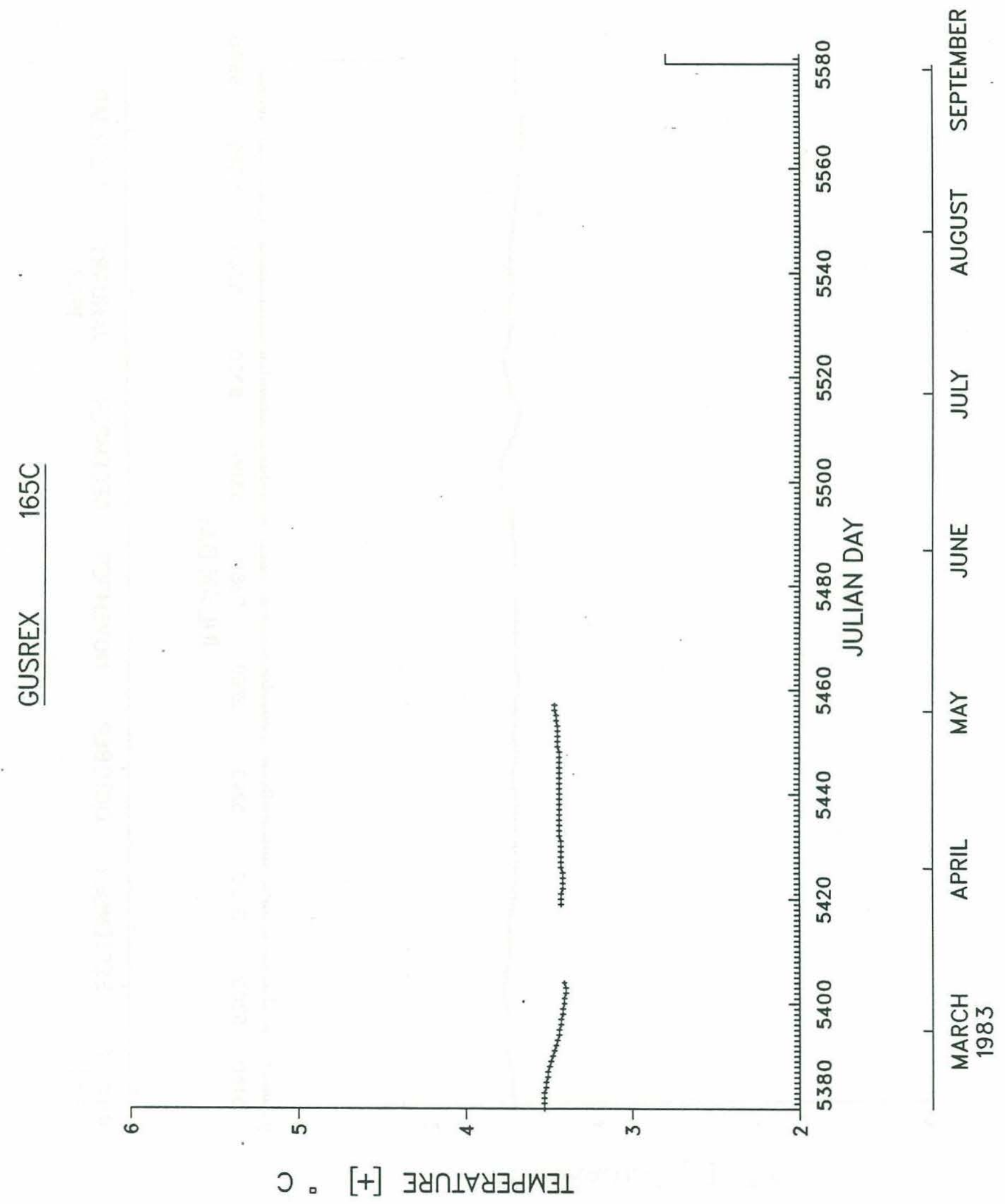




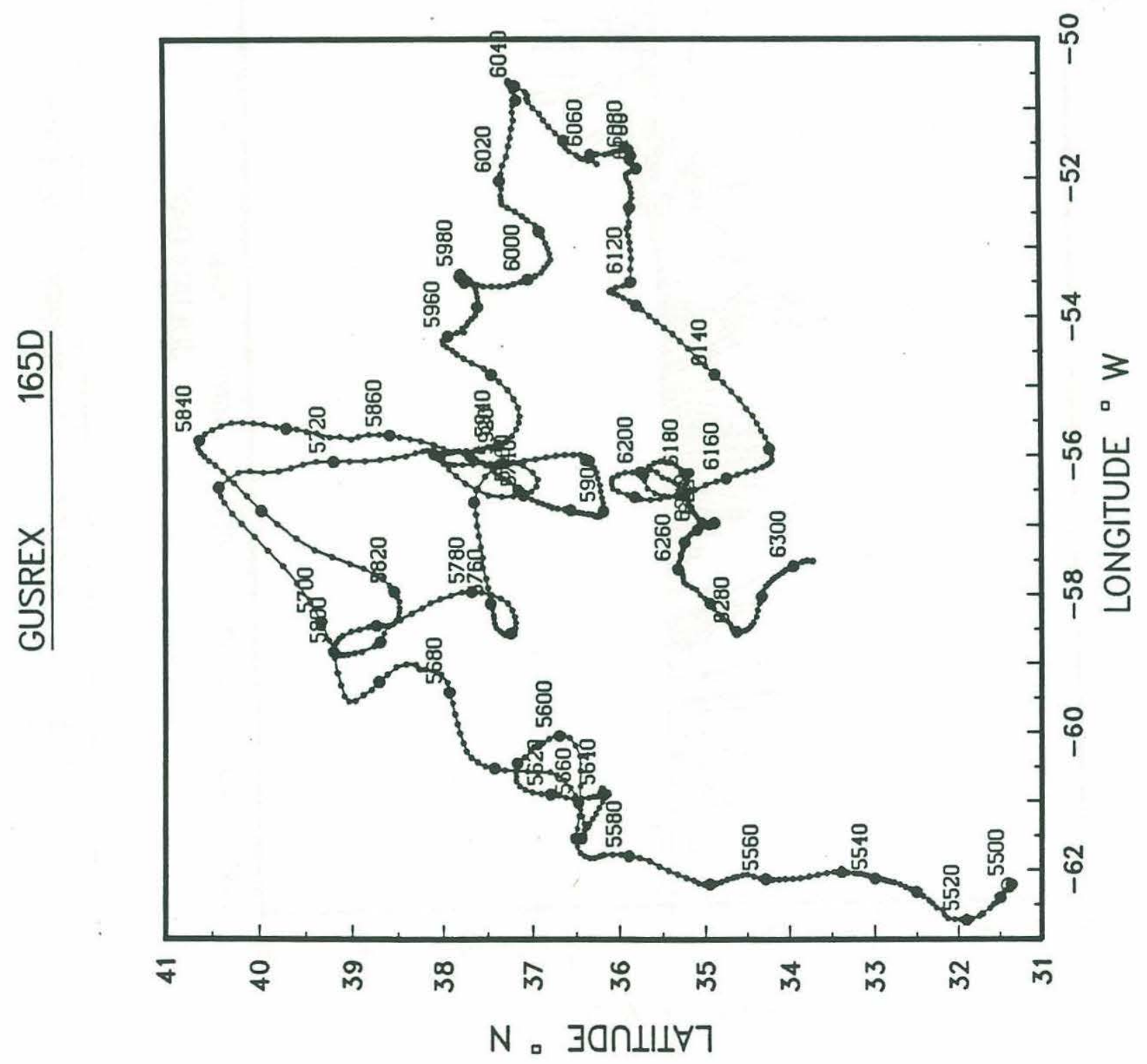




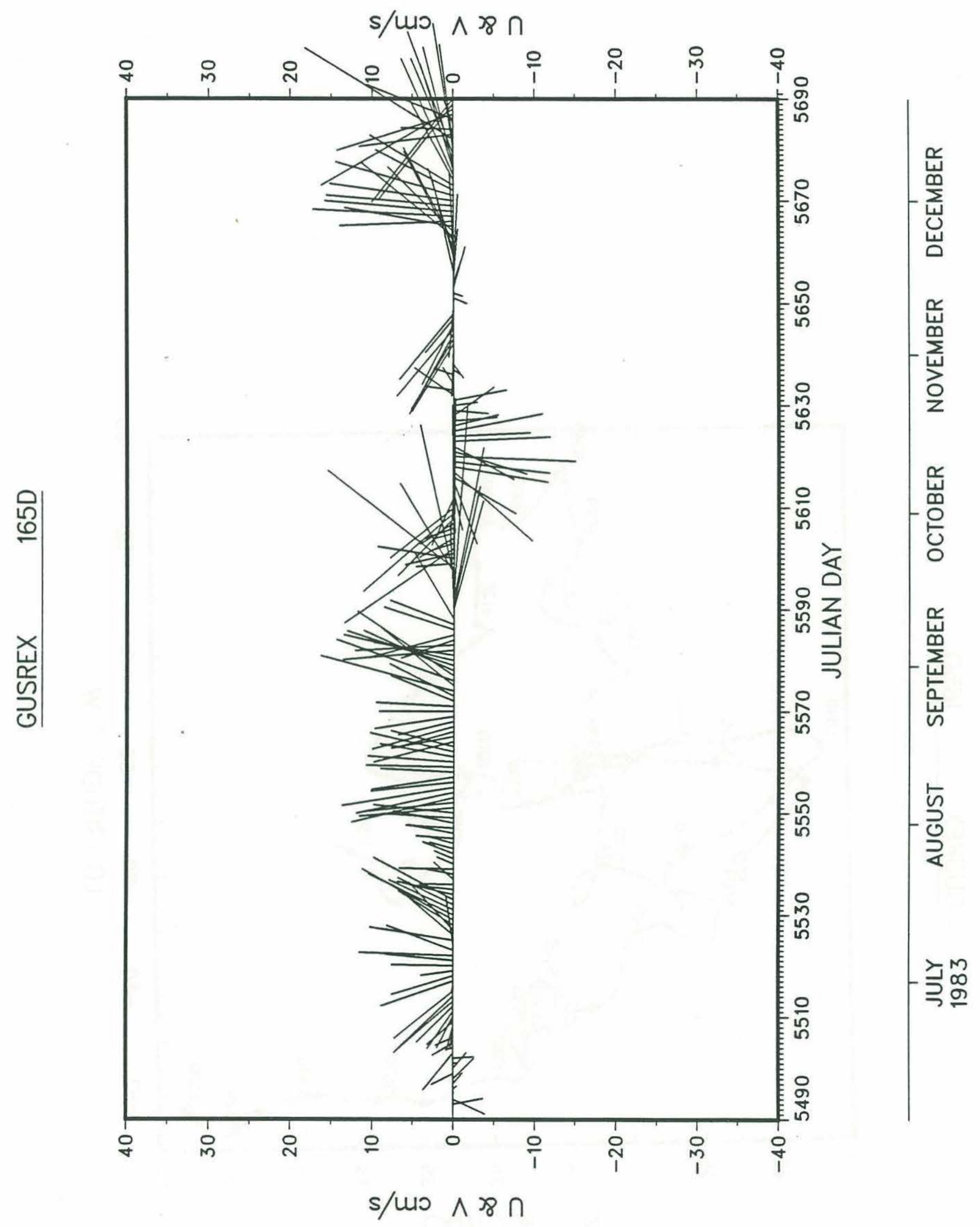




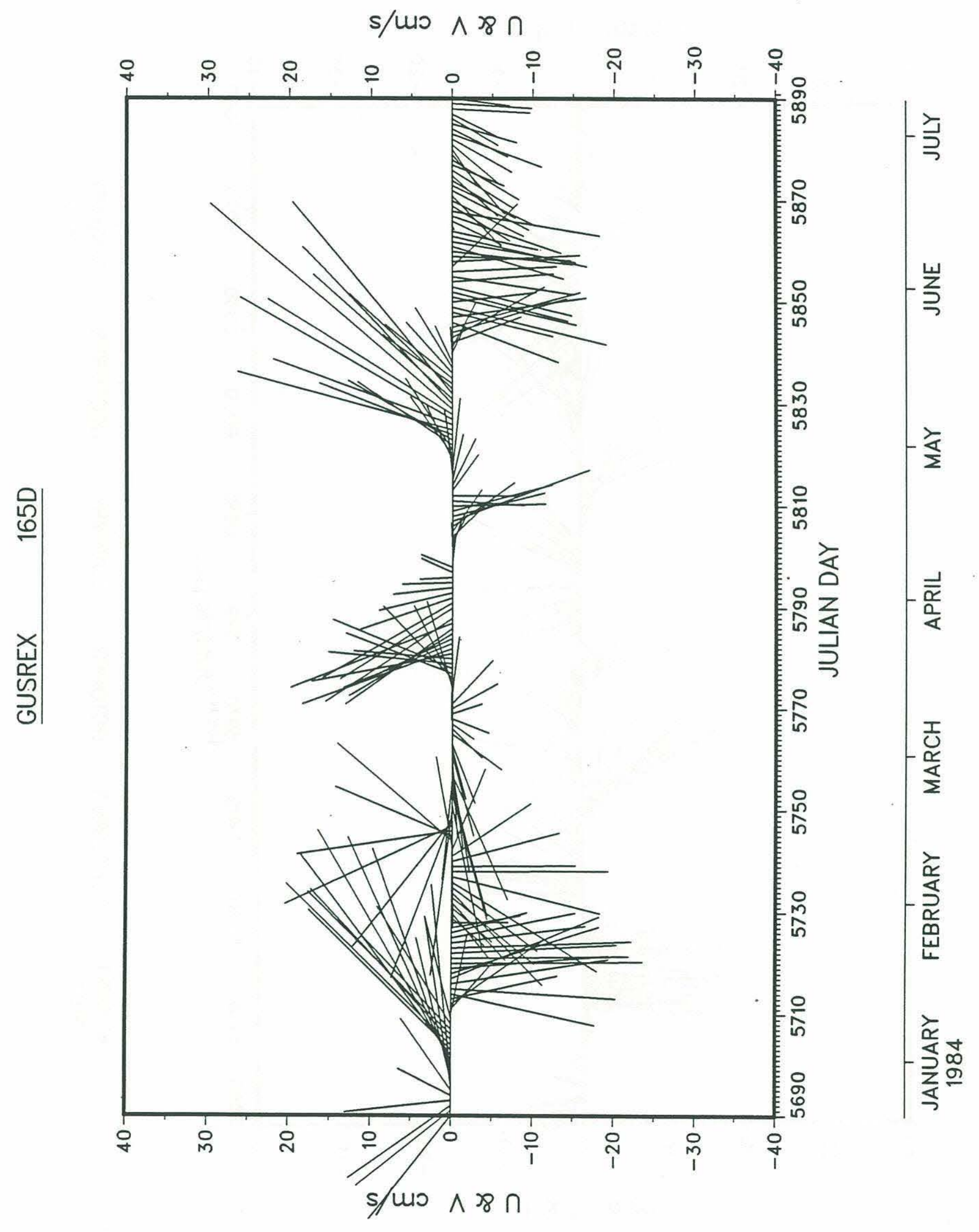




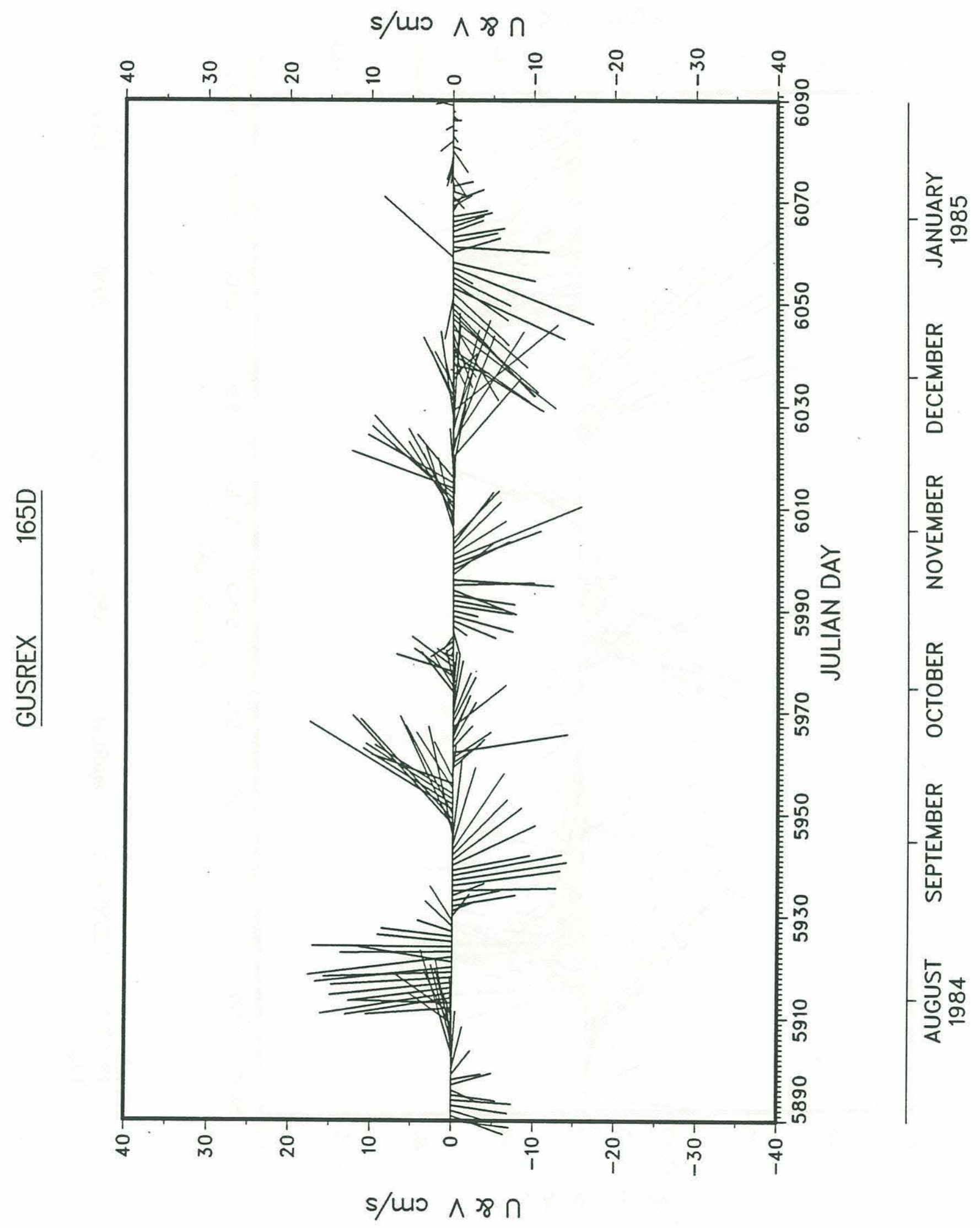




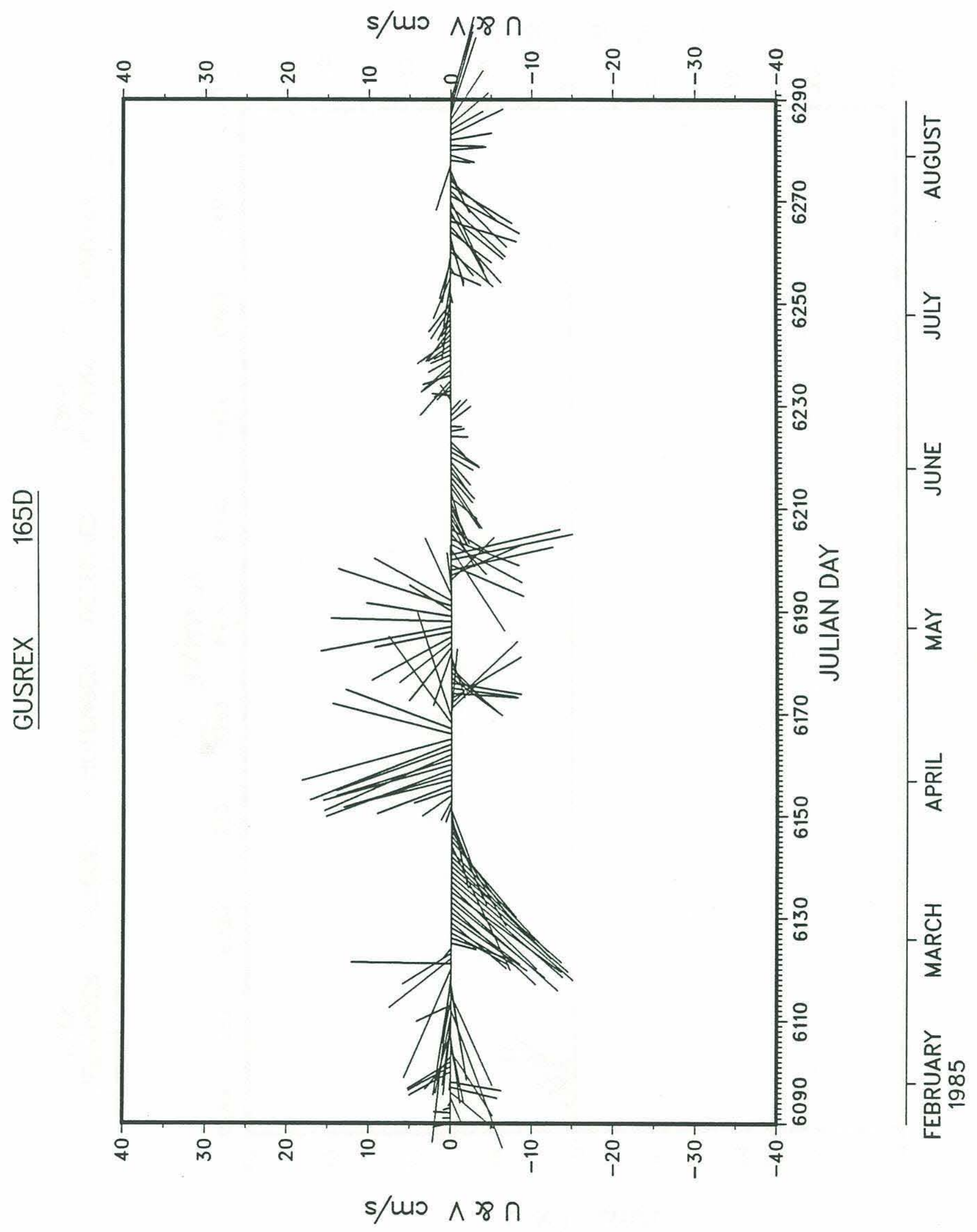




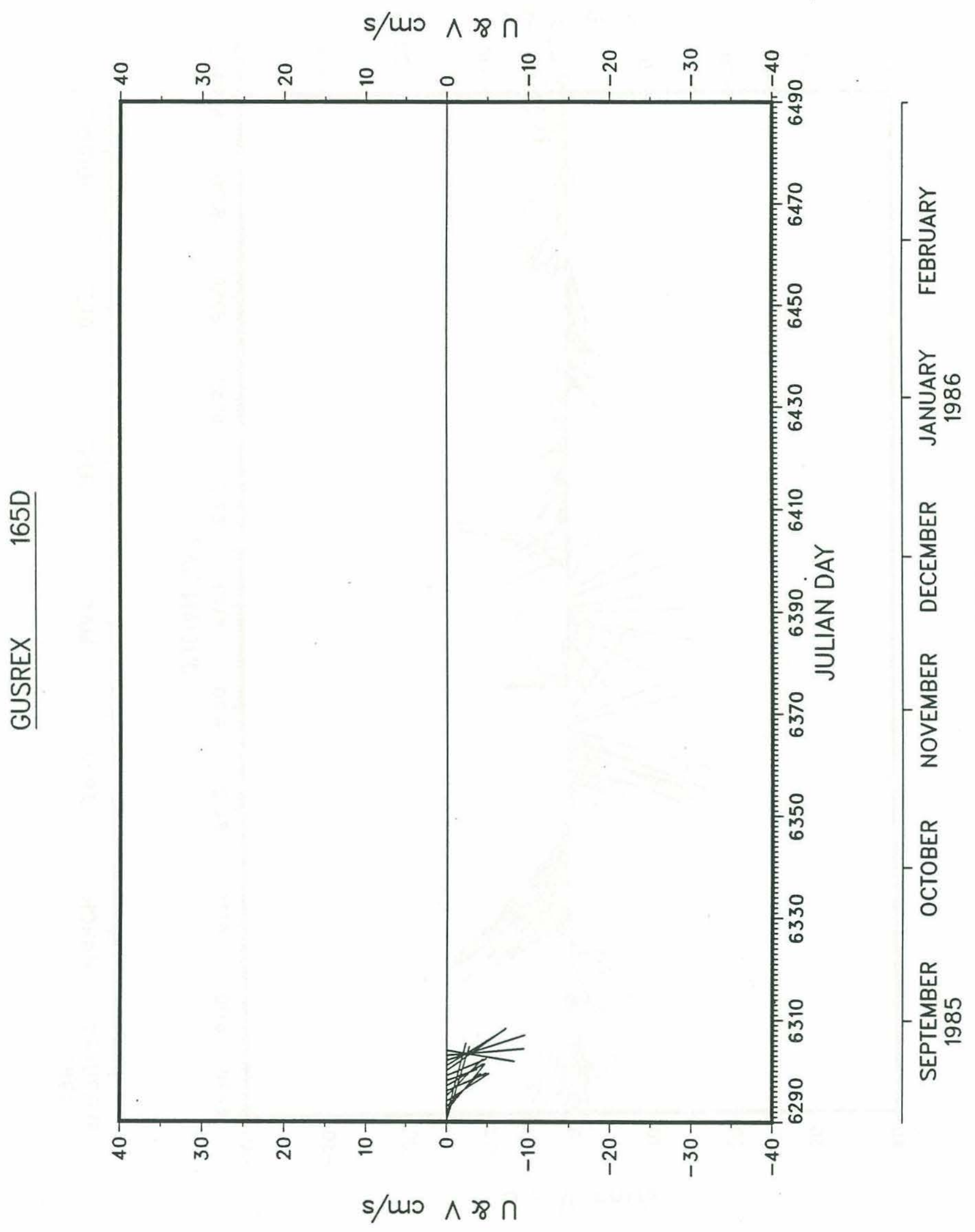




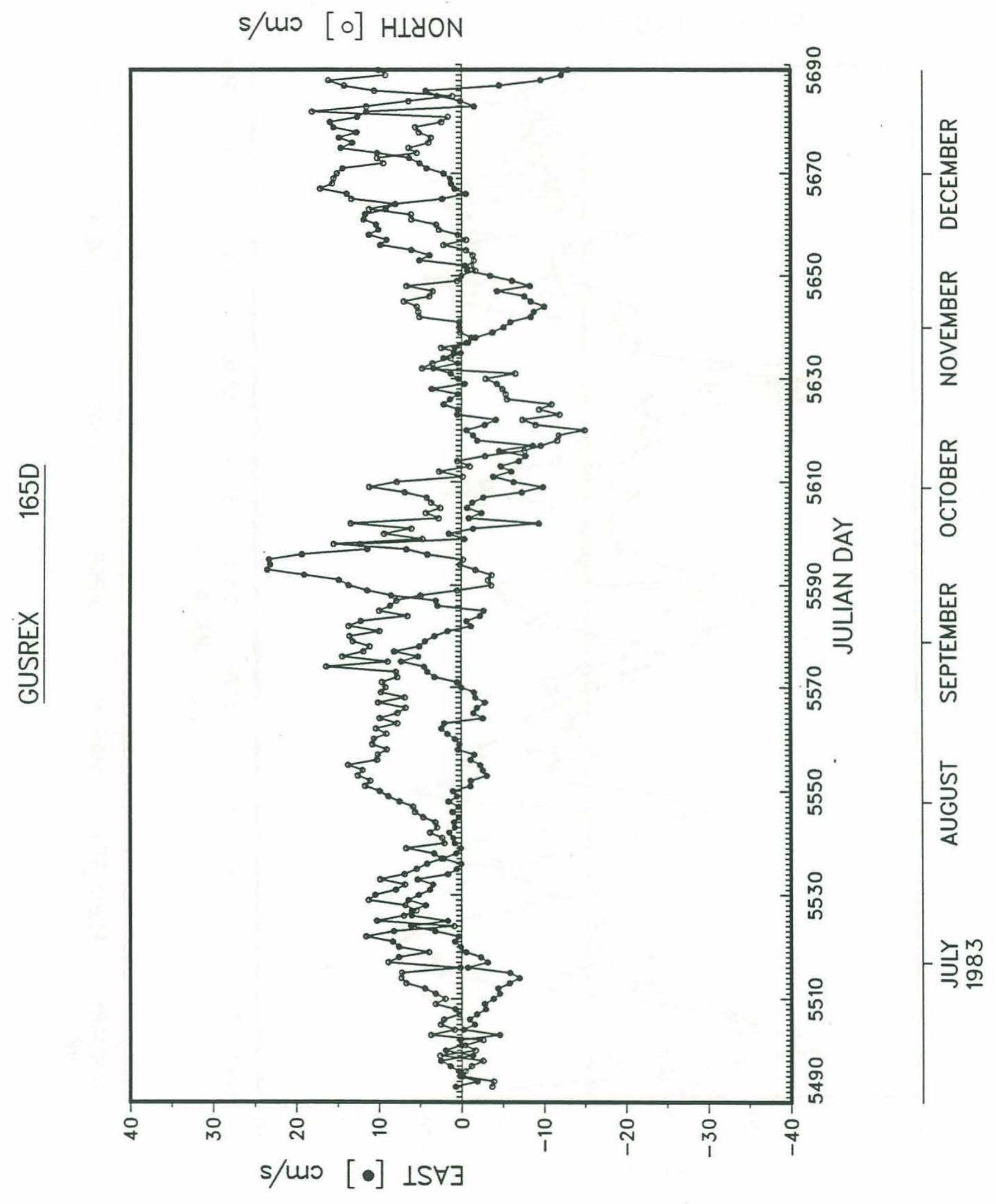




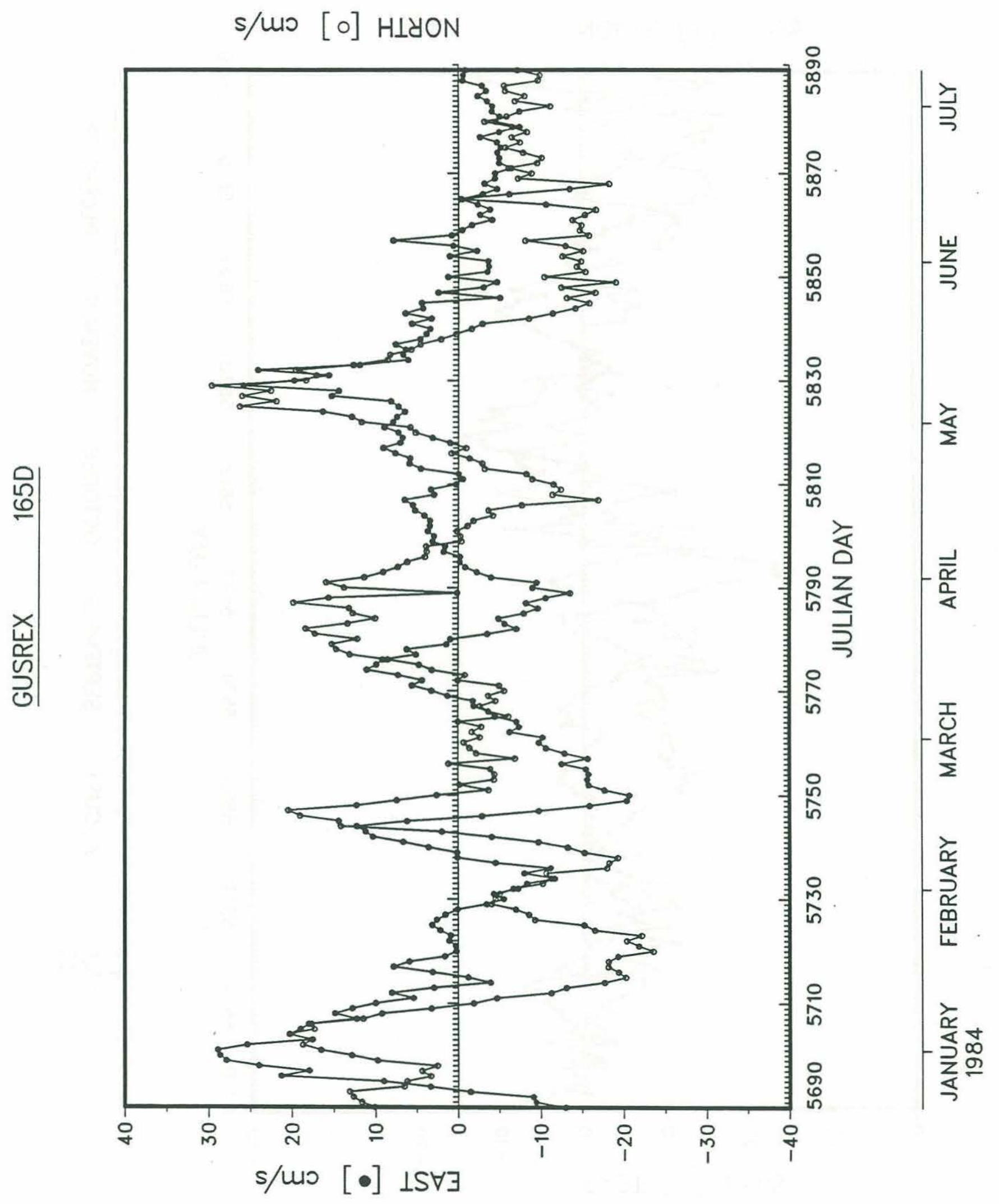




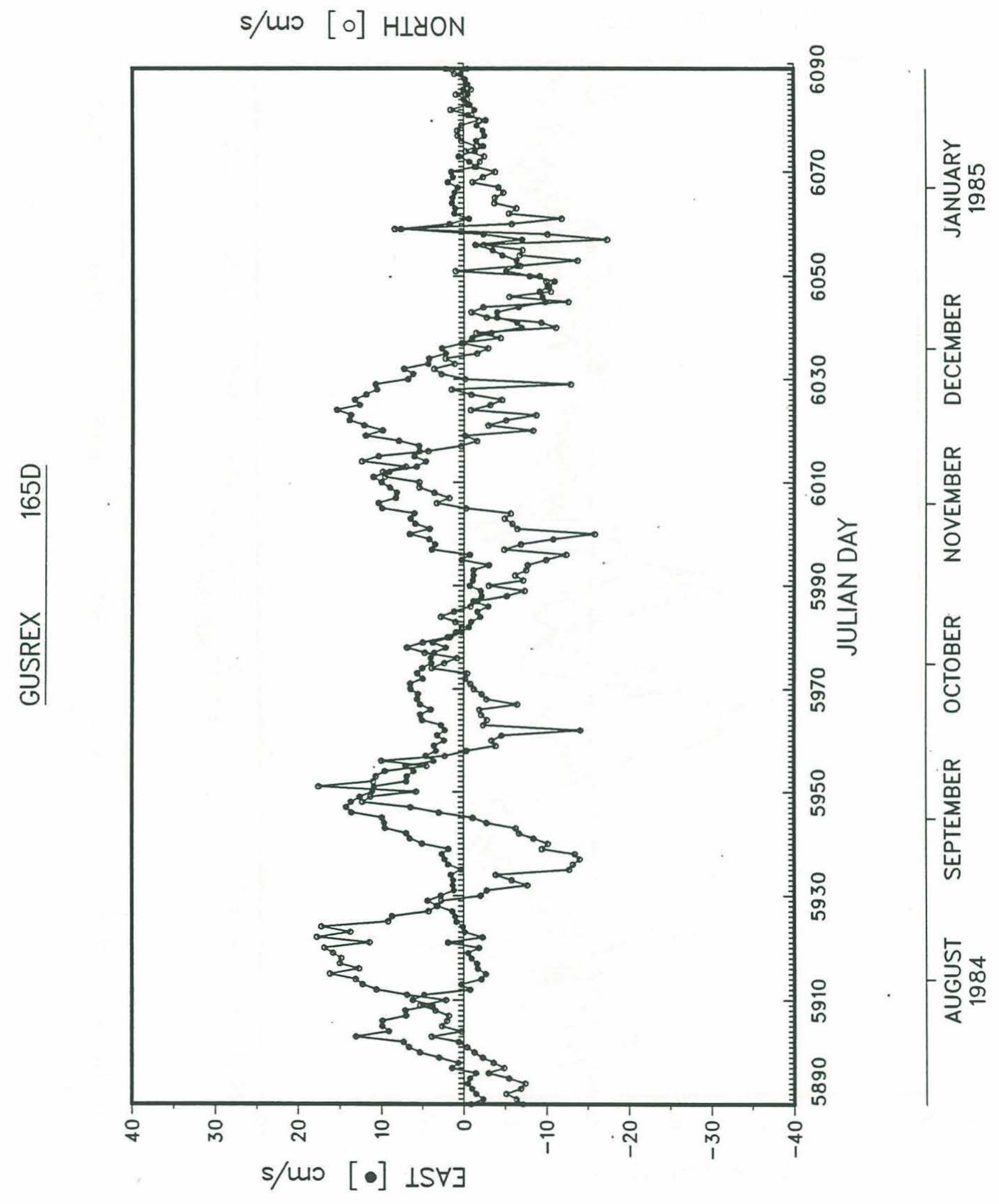




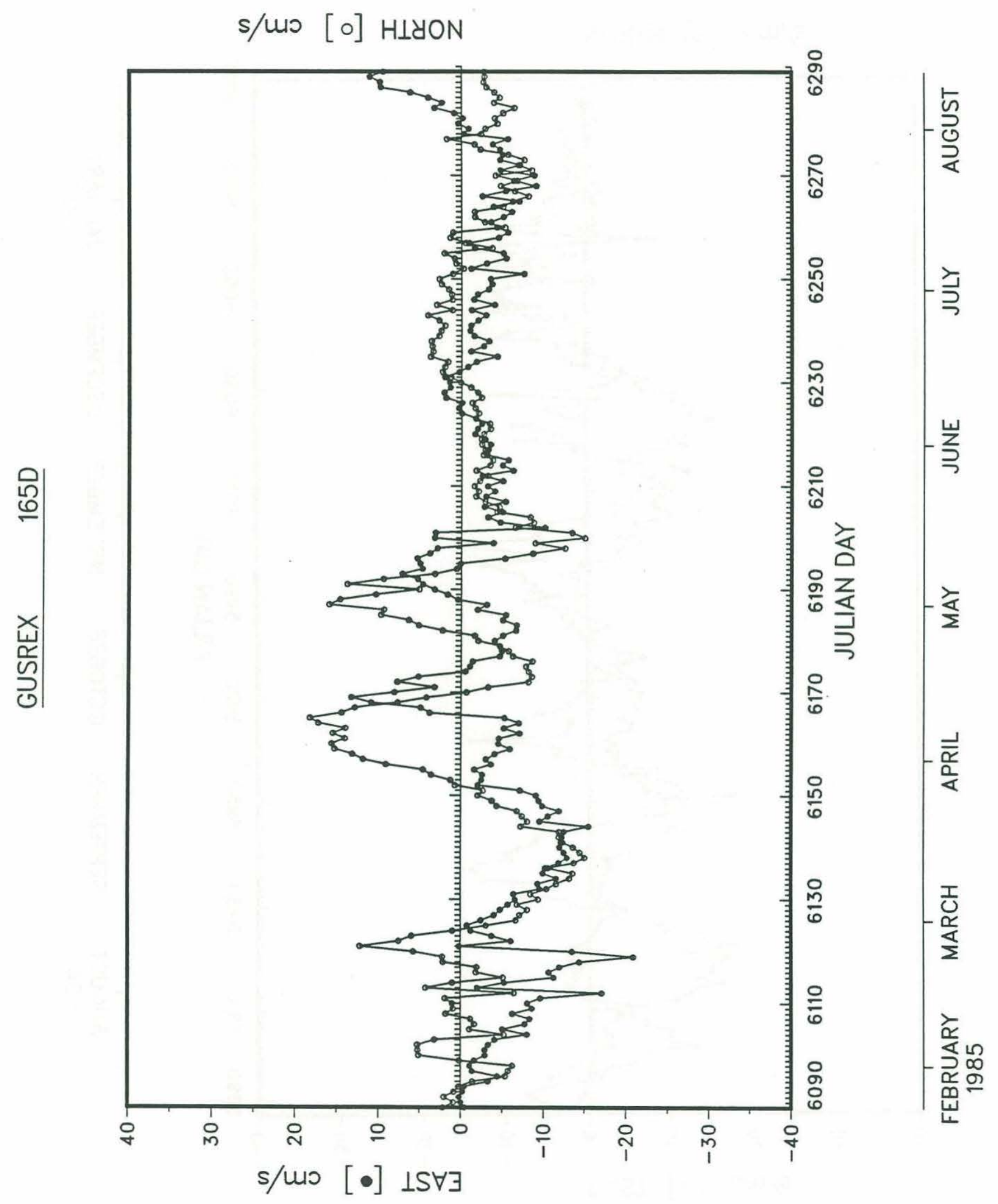




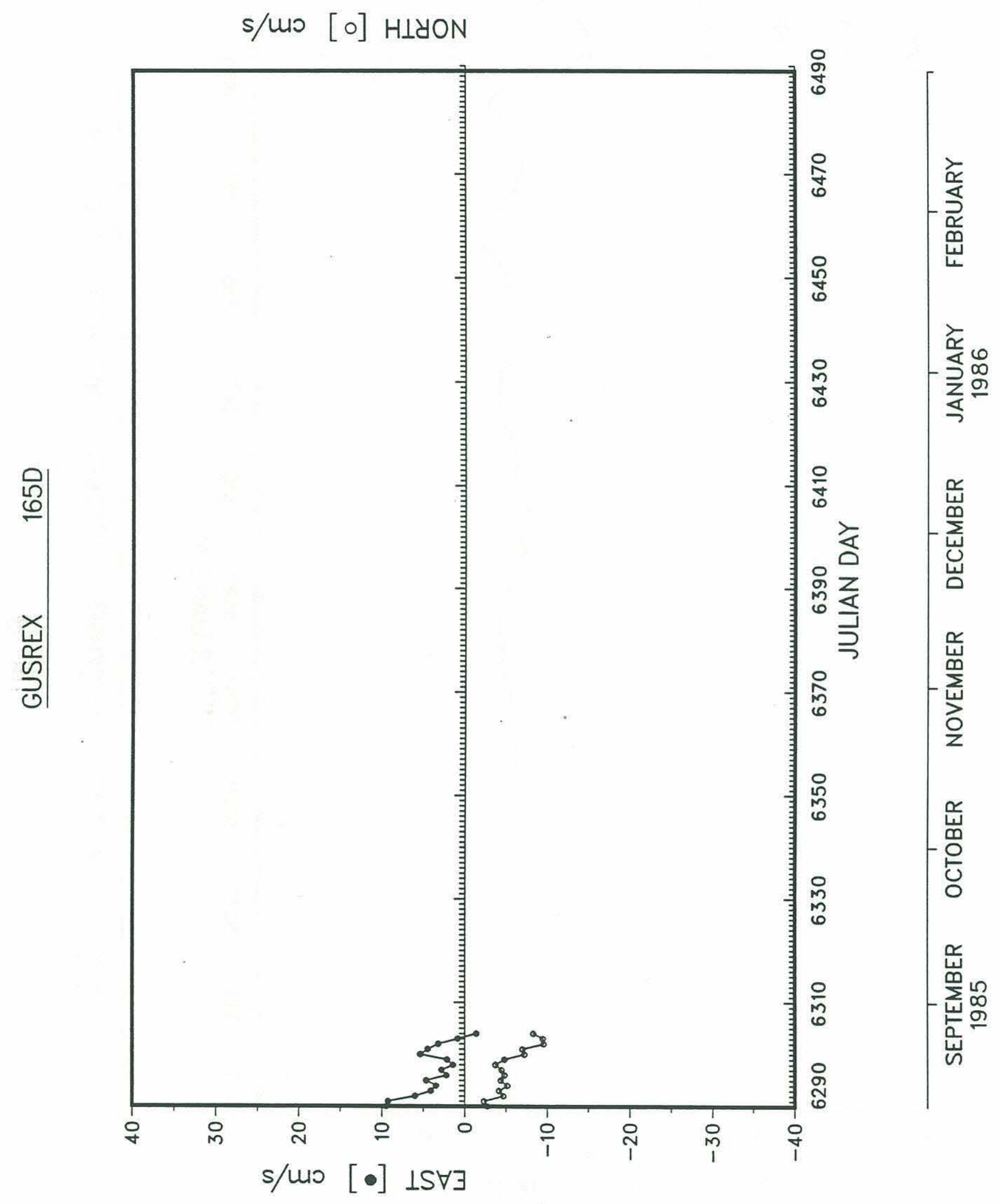




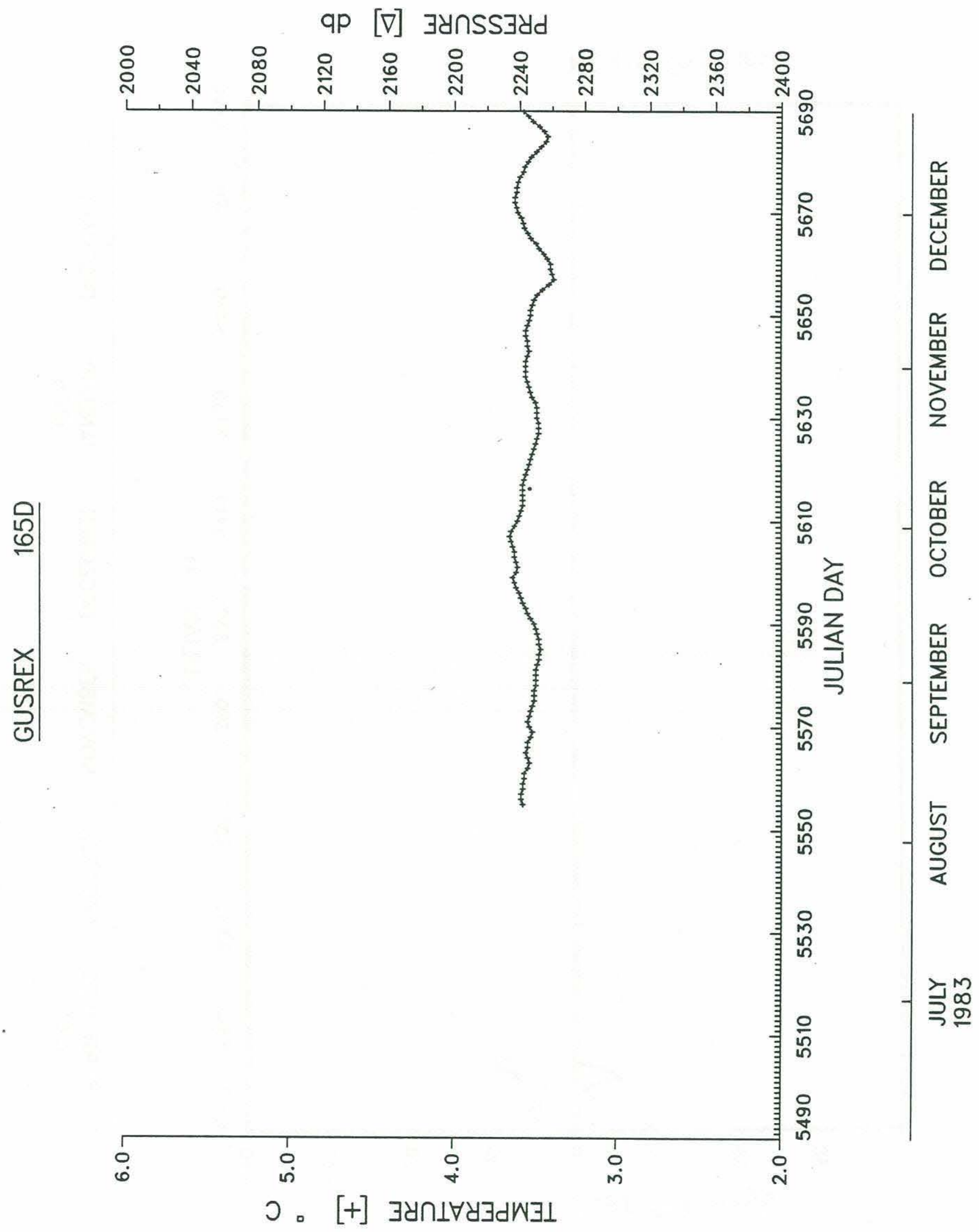




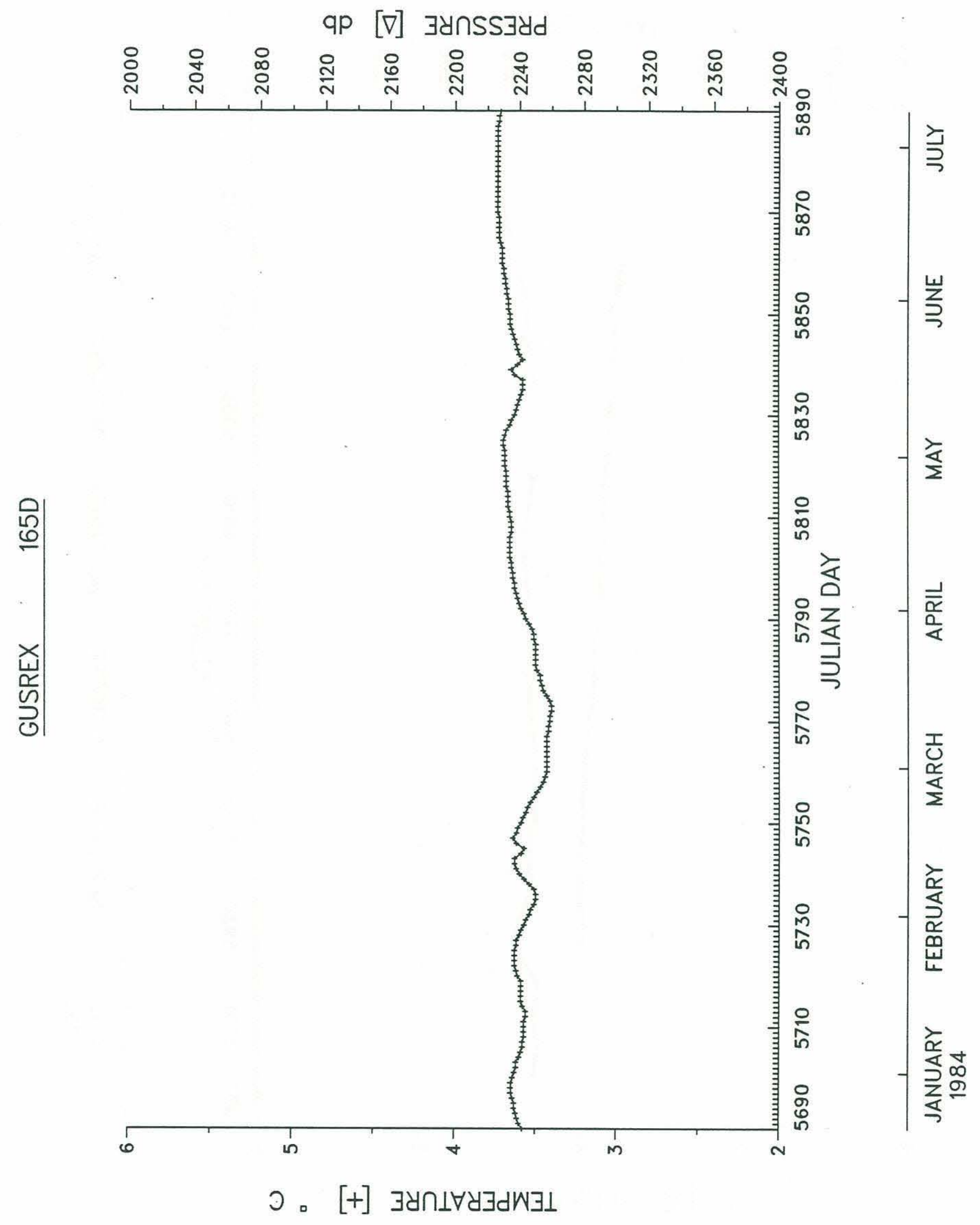




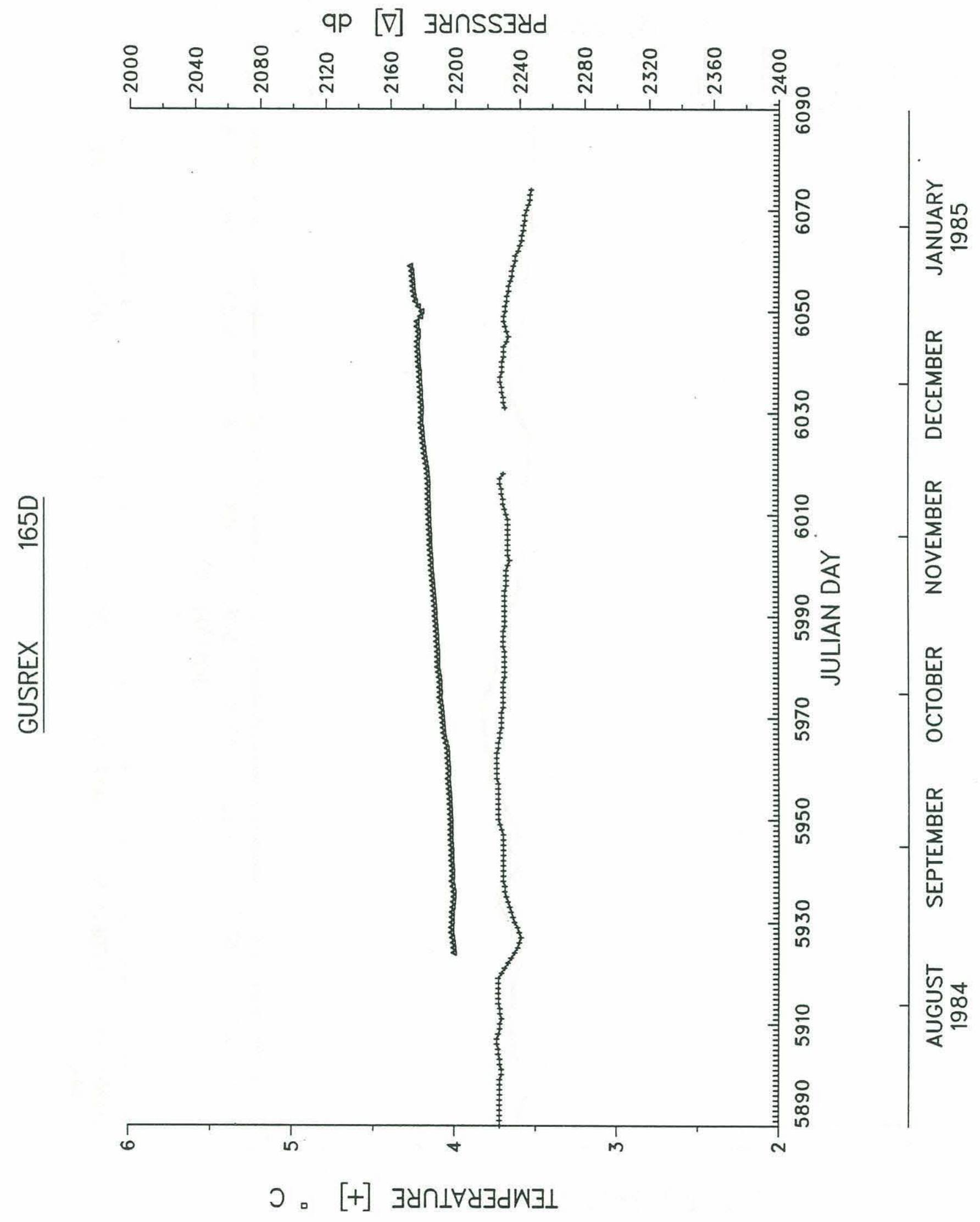




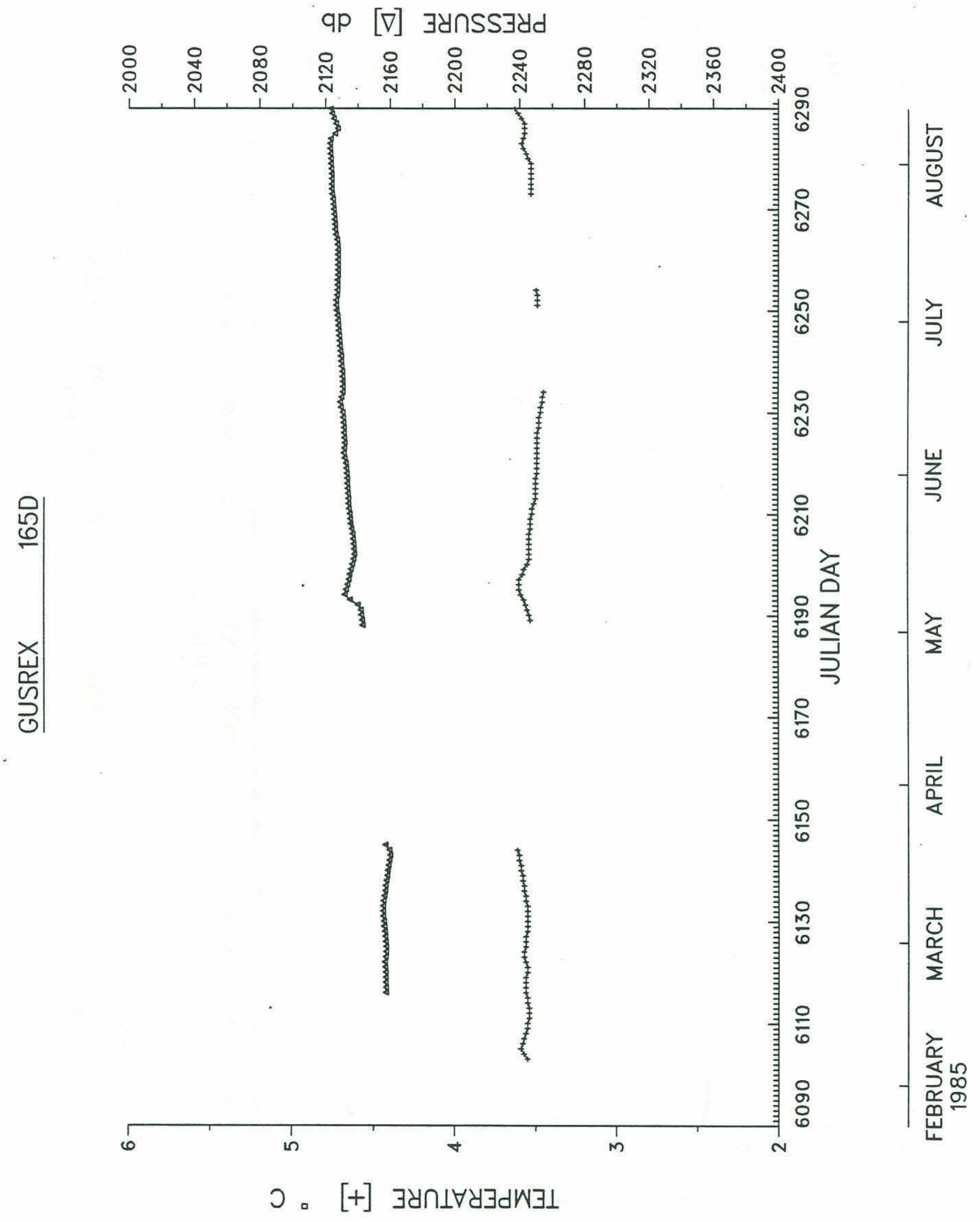




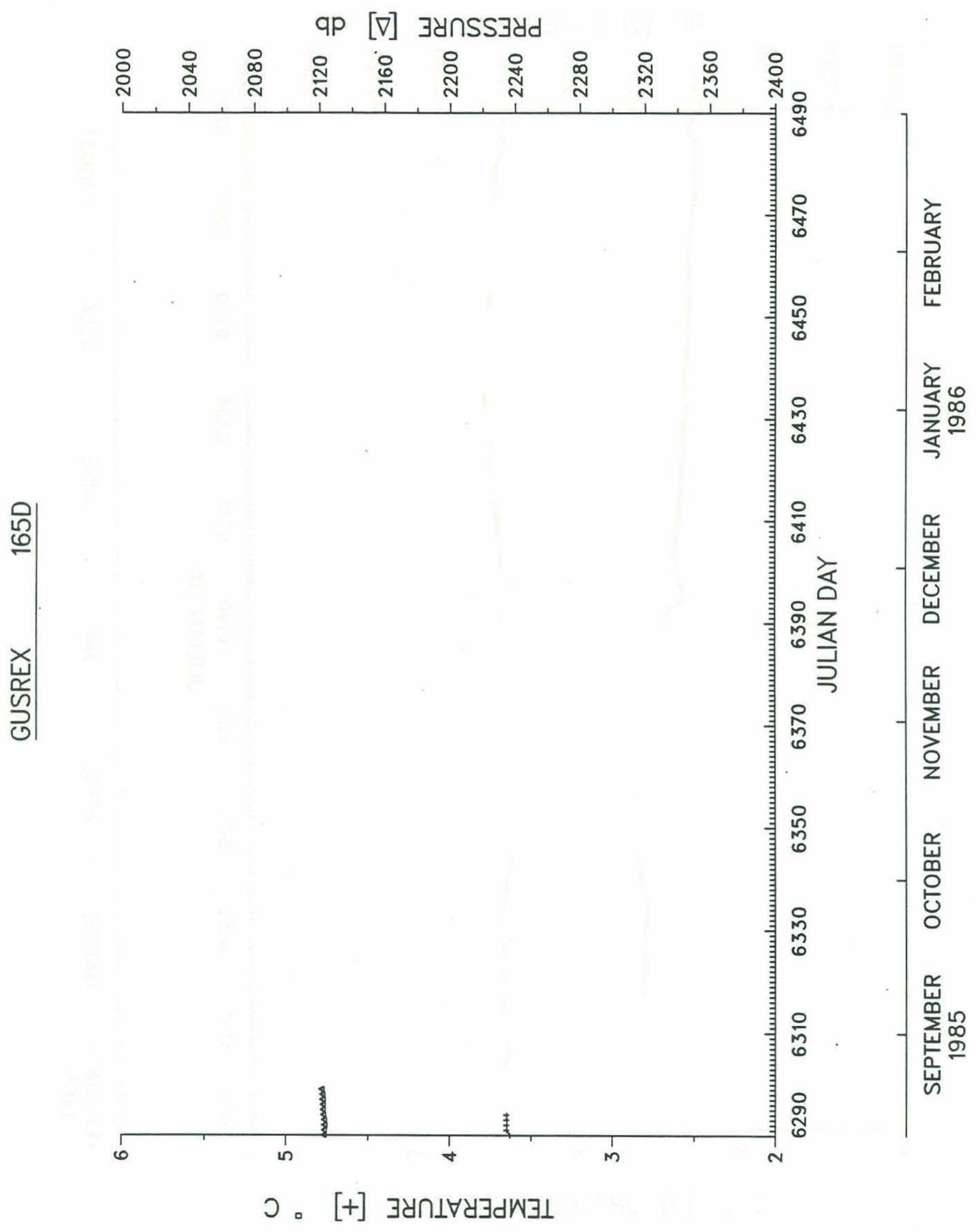




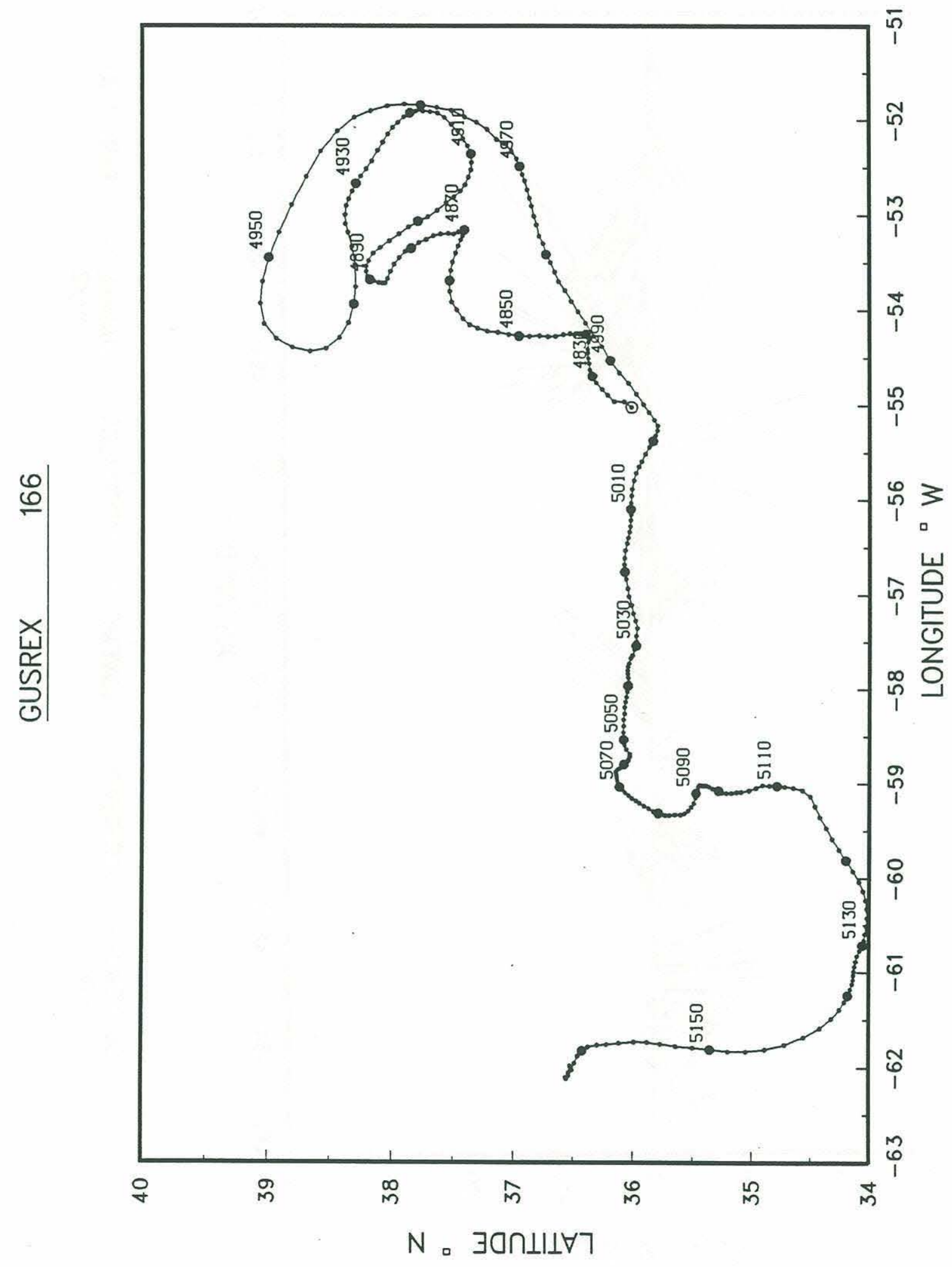




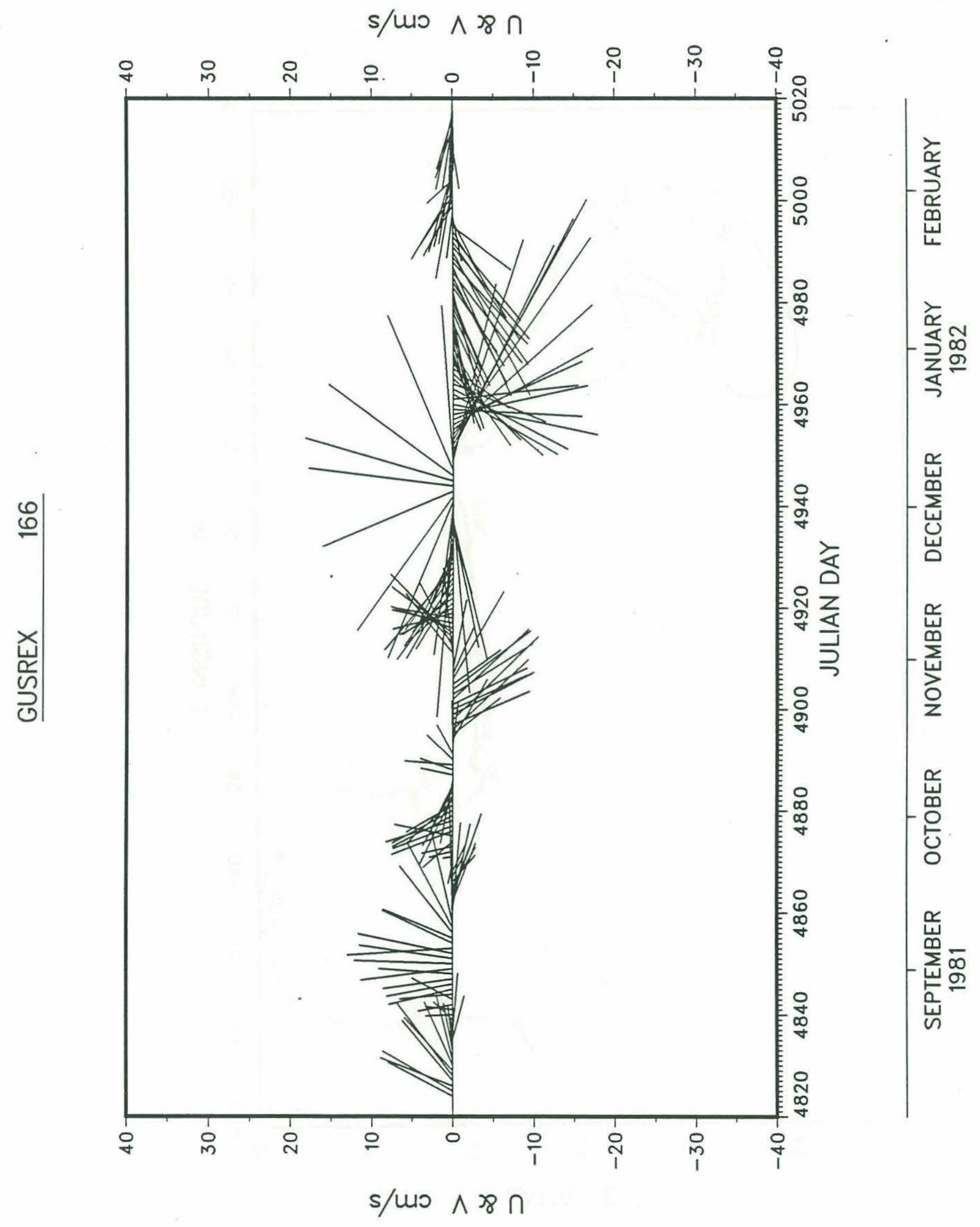




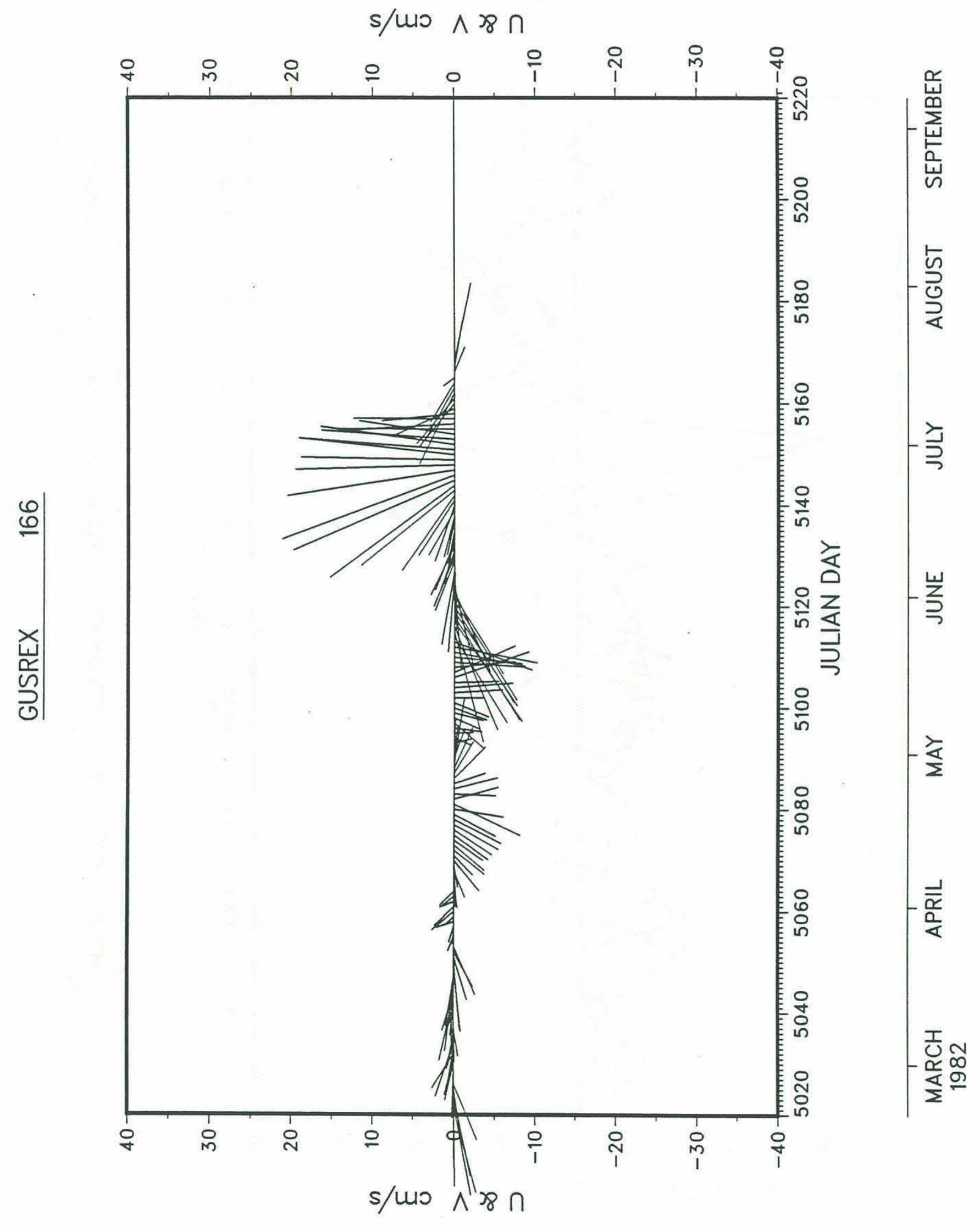




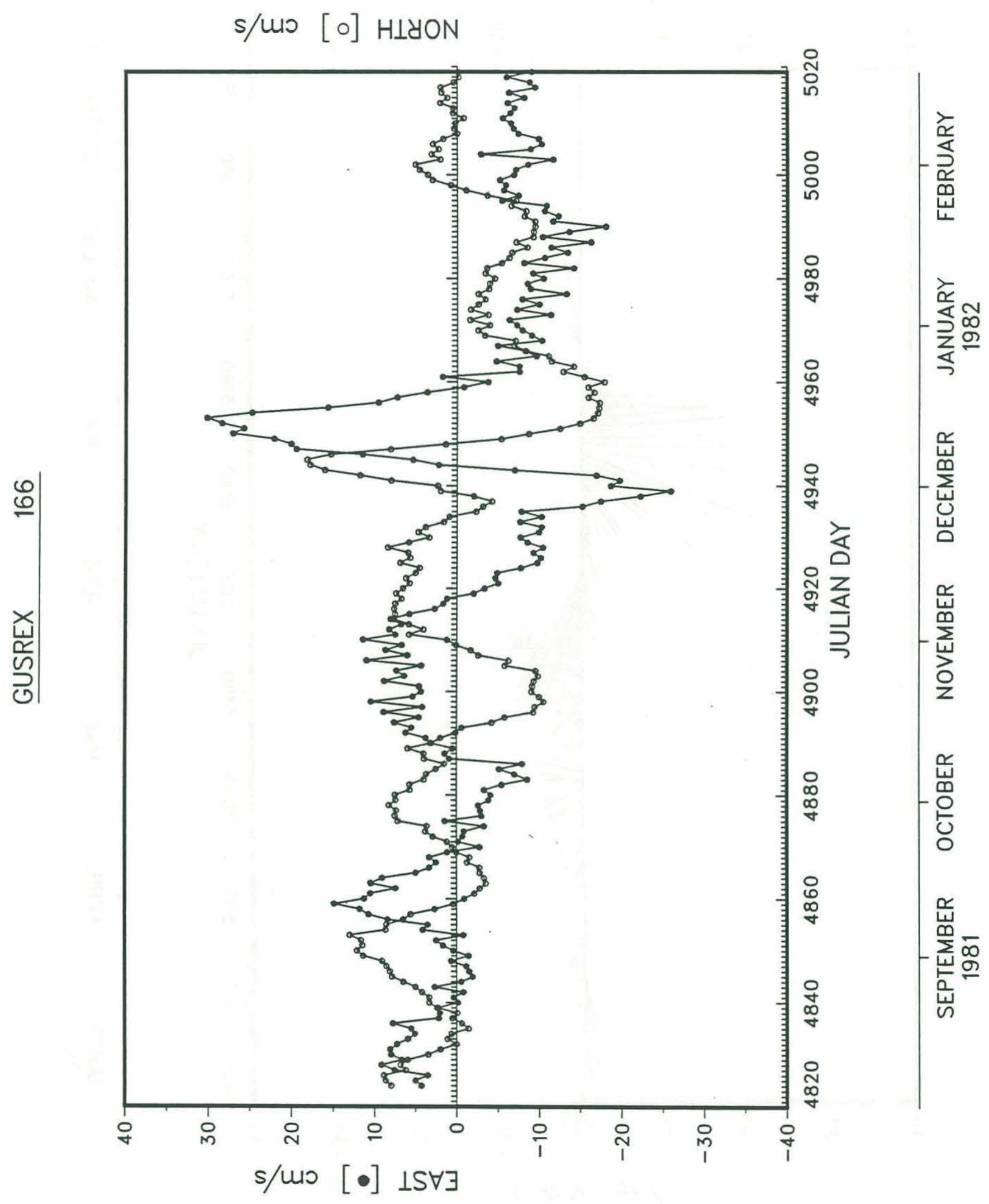


s/uo [o] HLYON

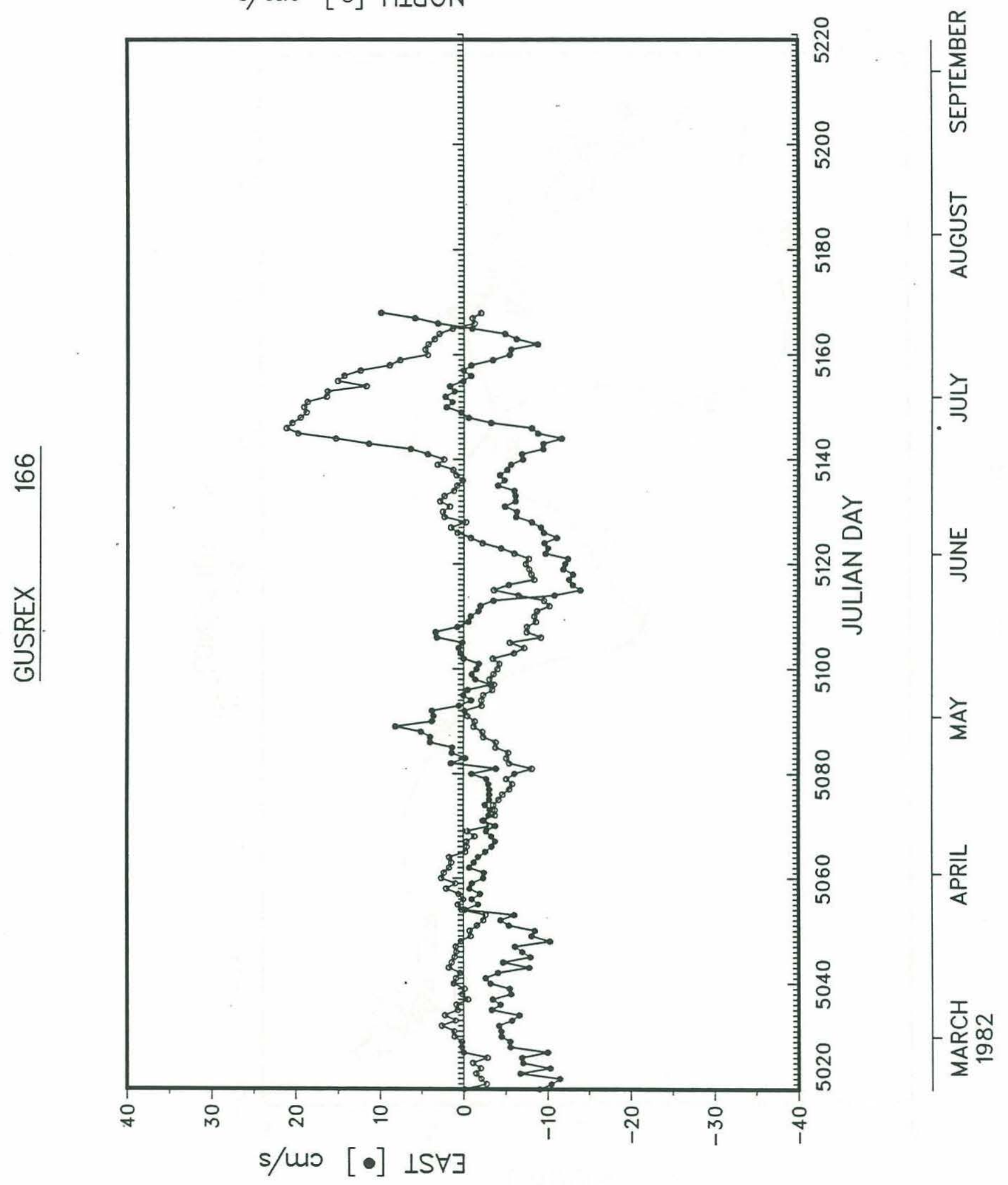




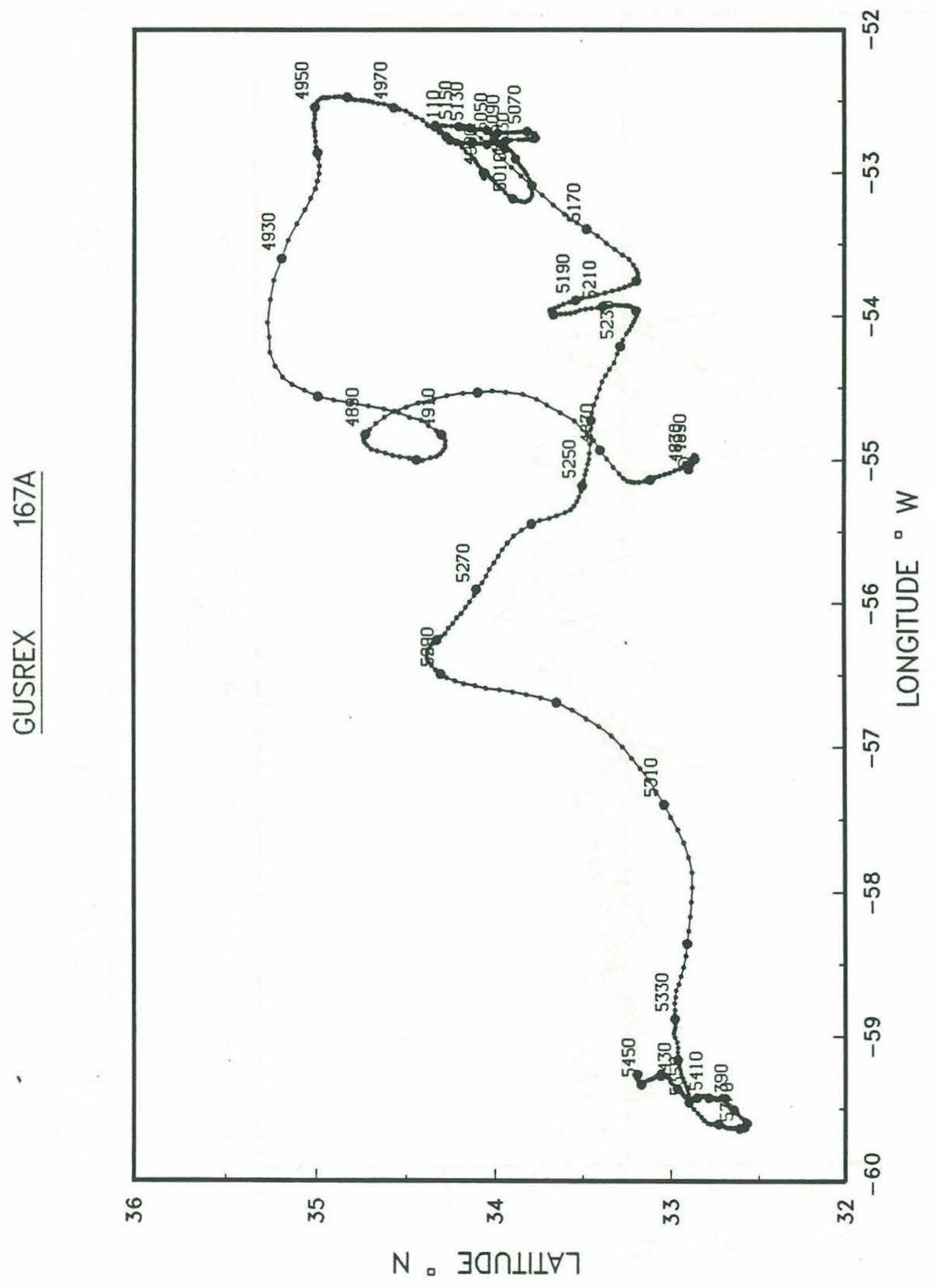




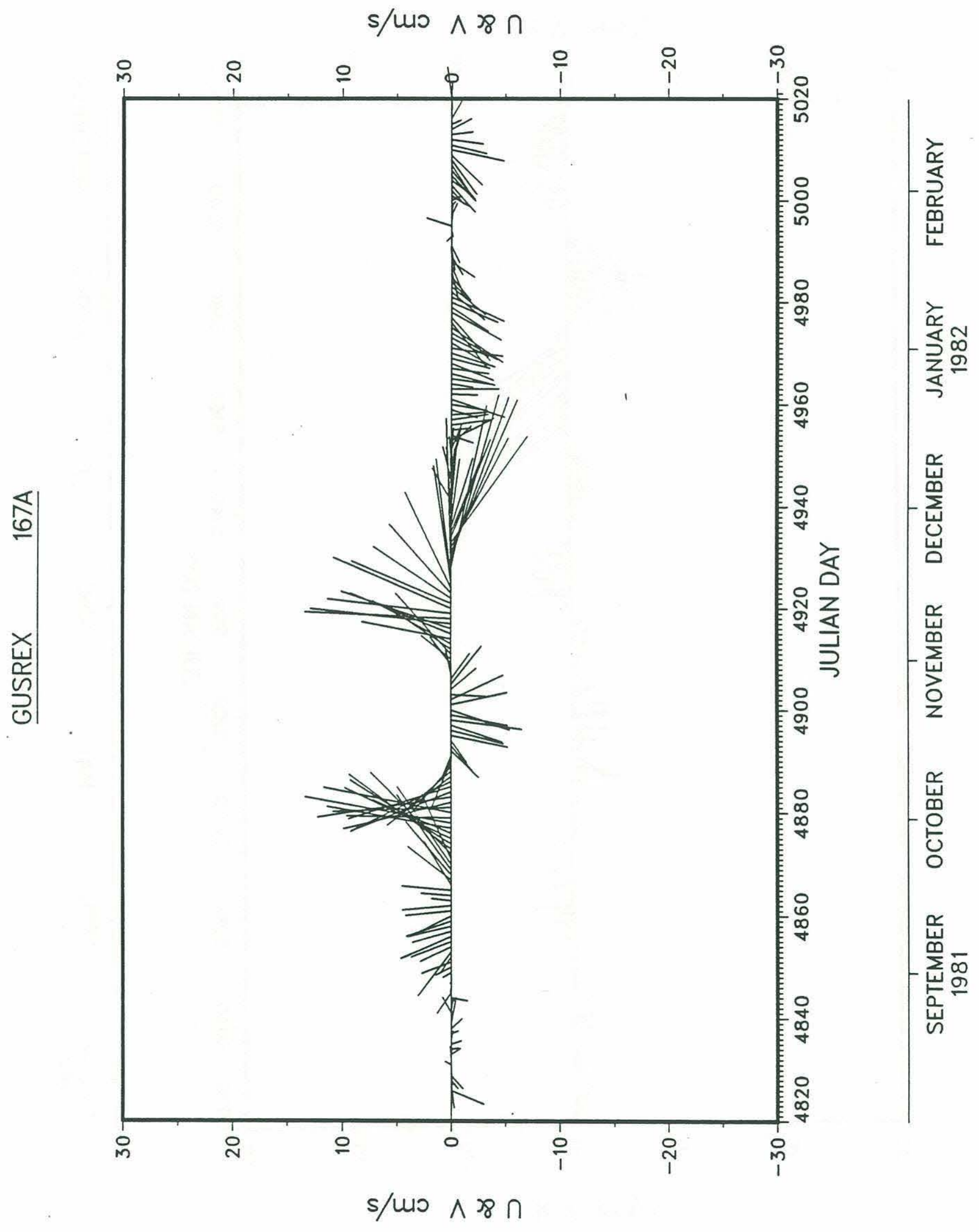




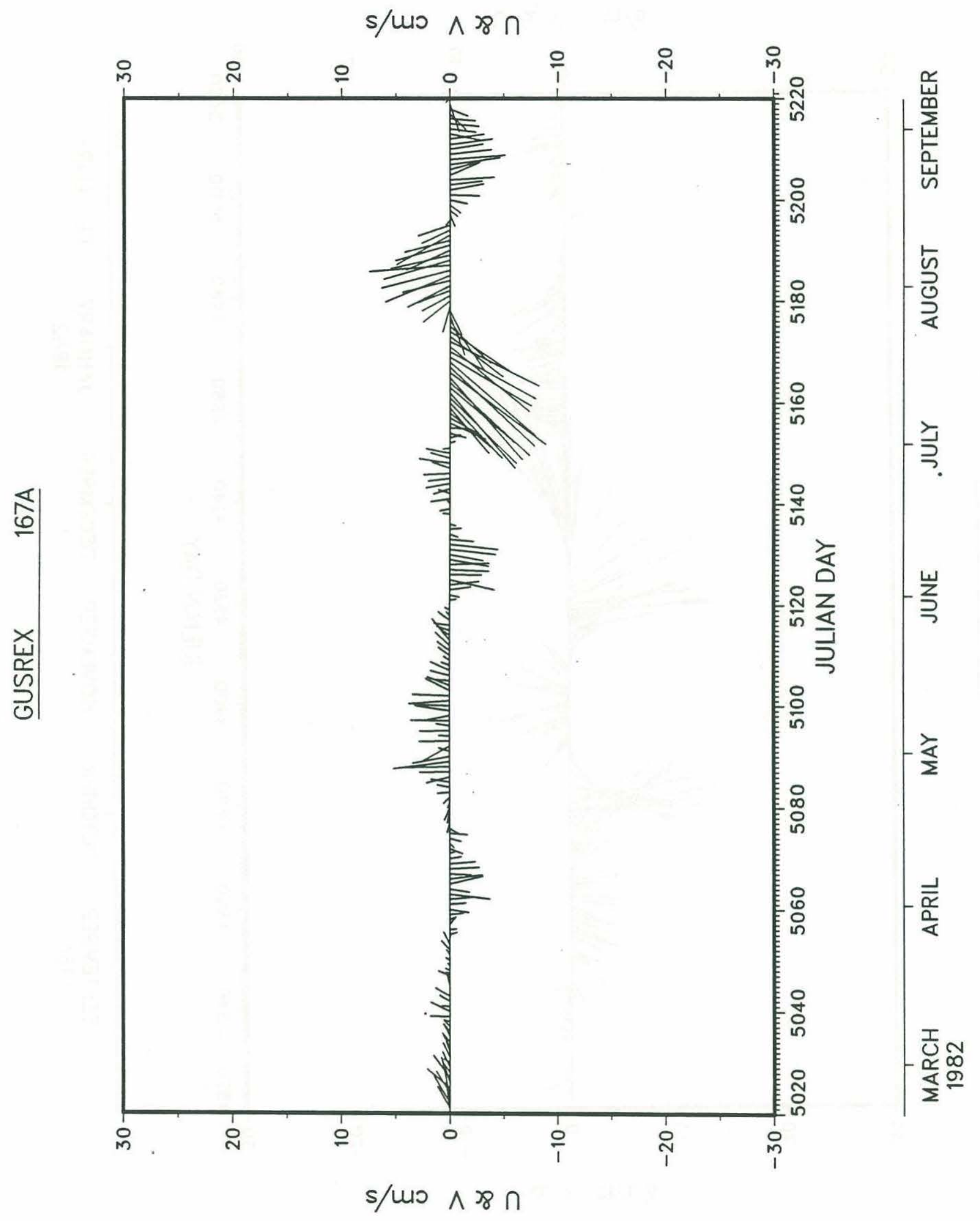




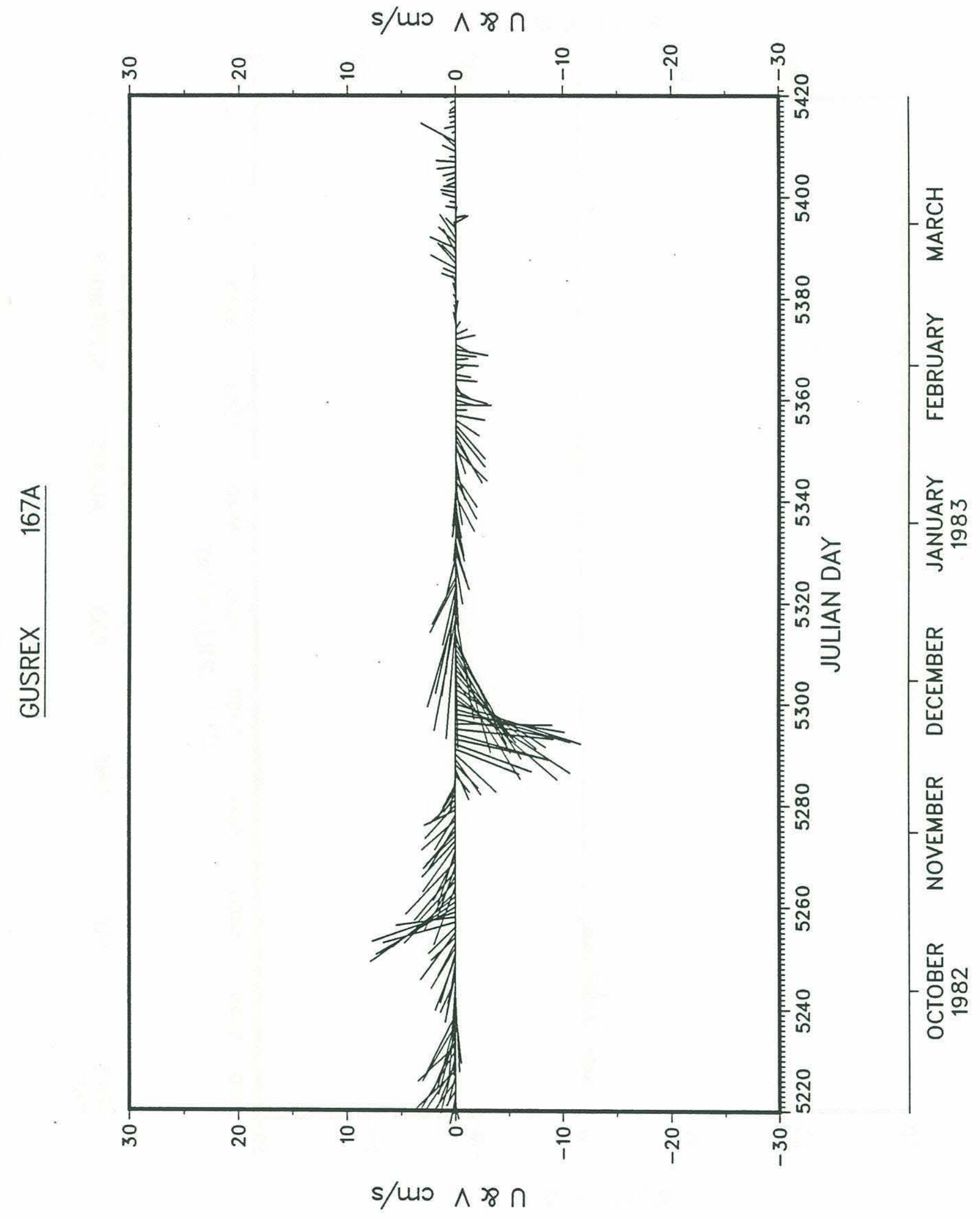




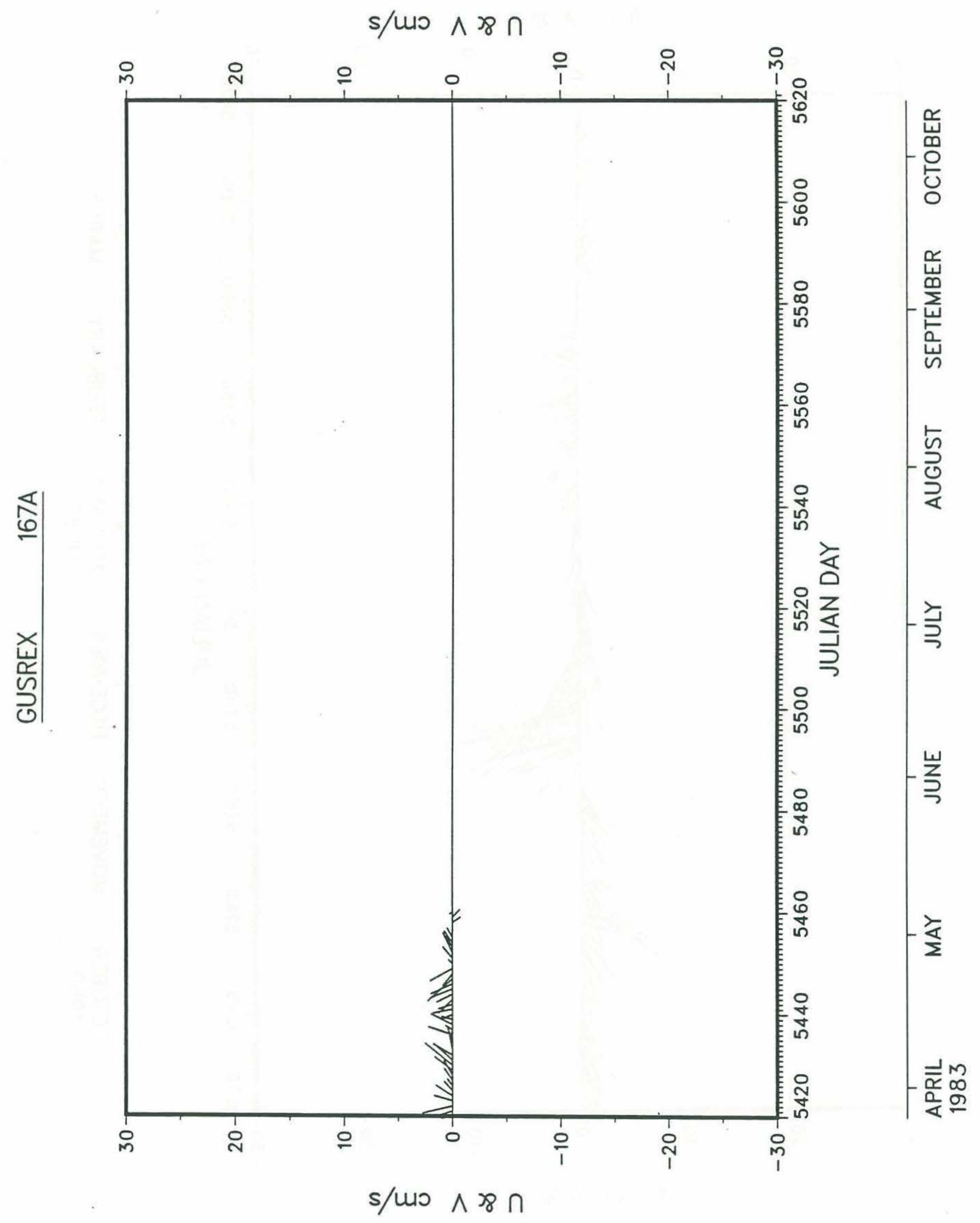




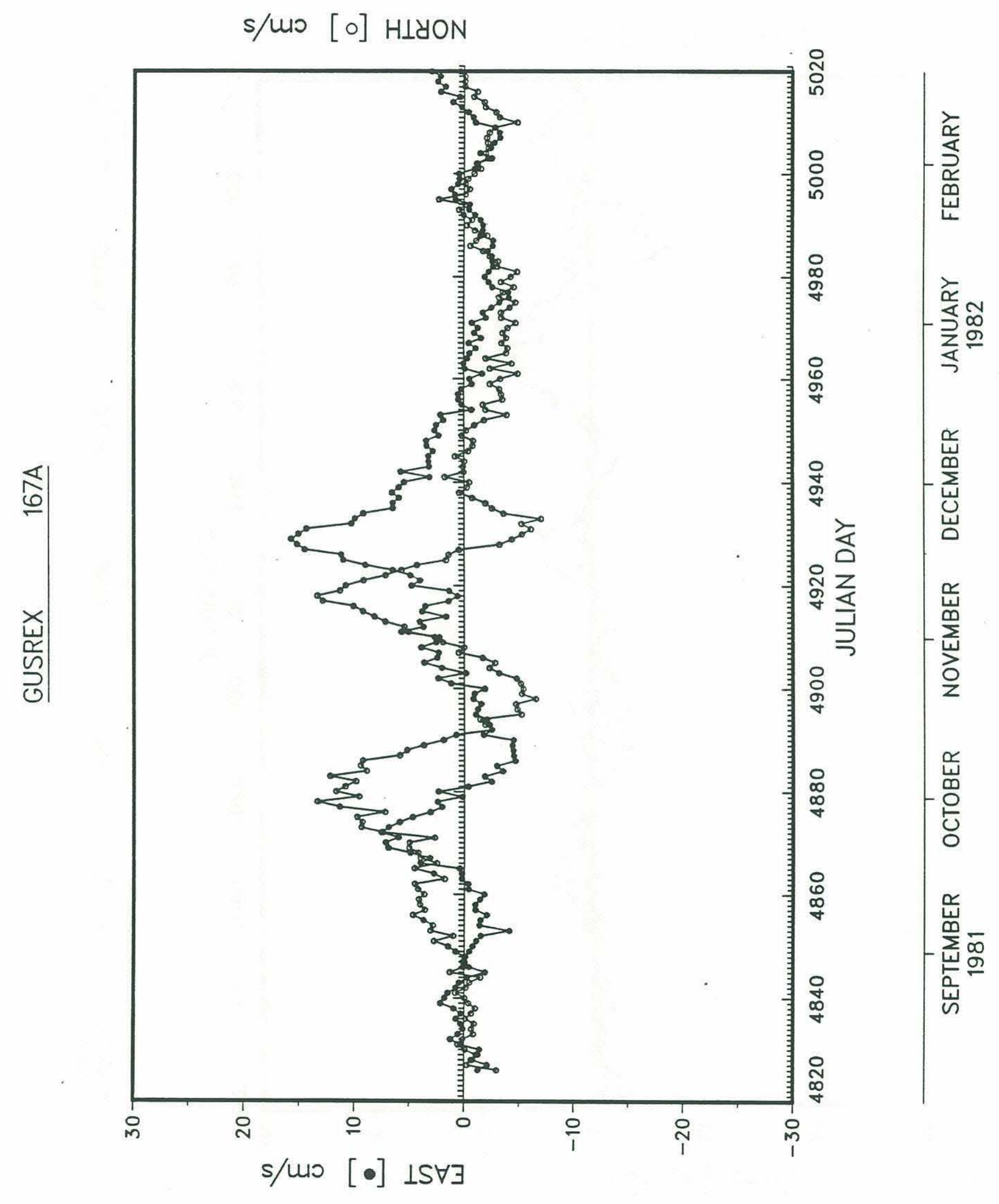




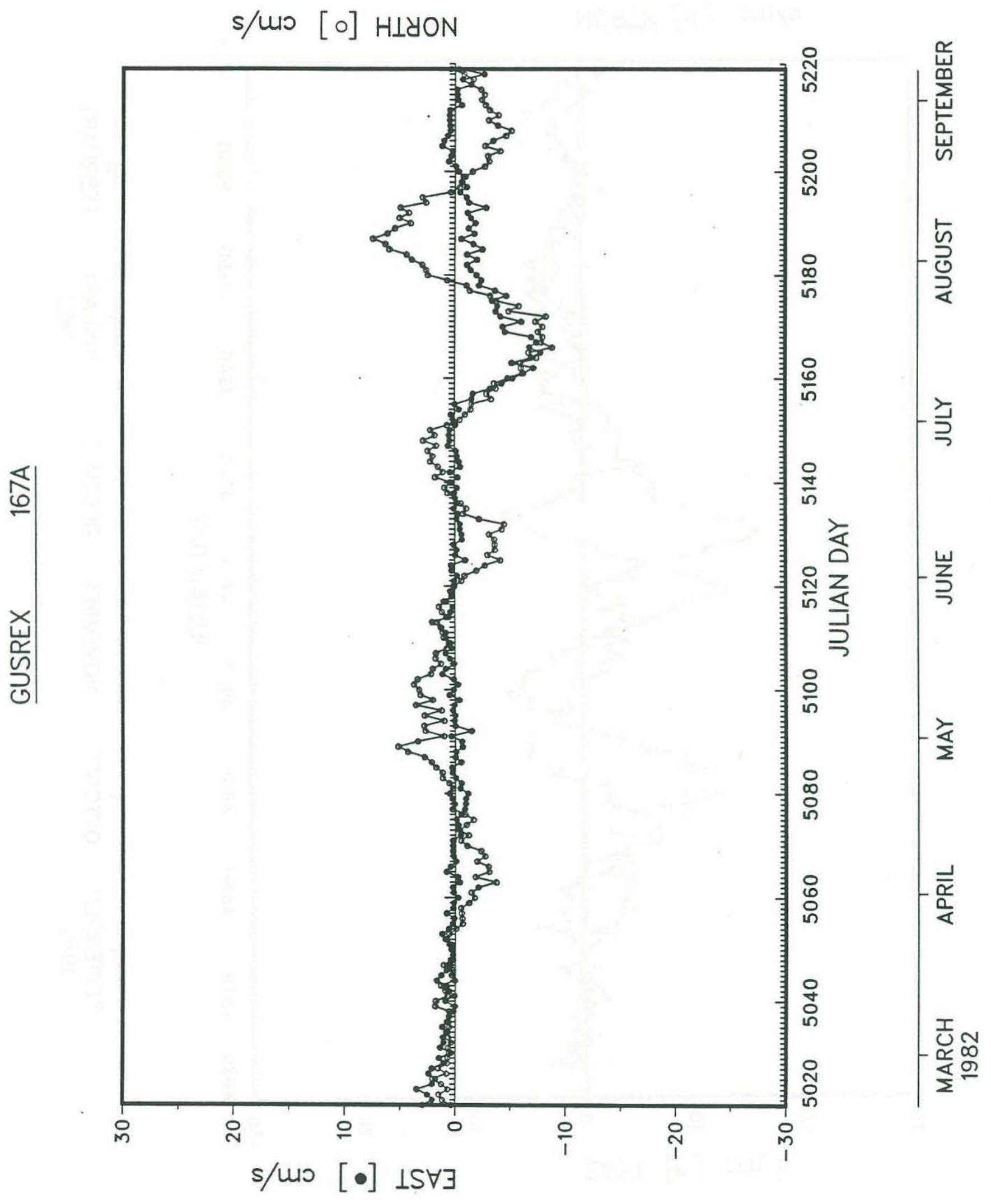




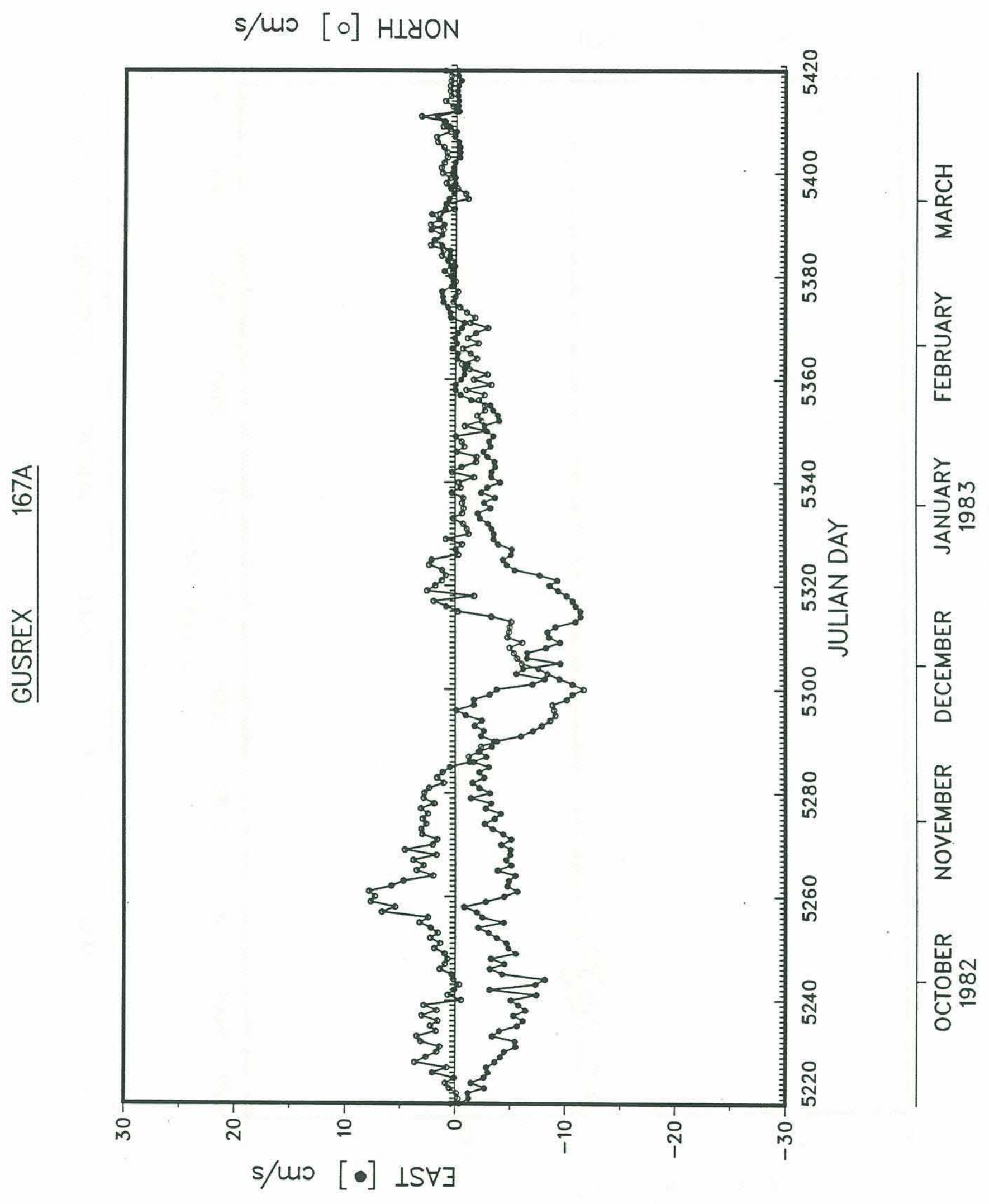




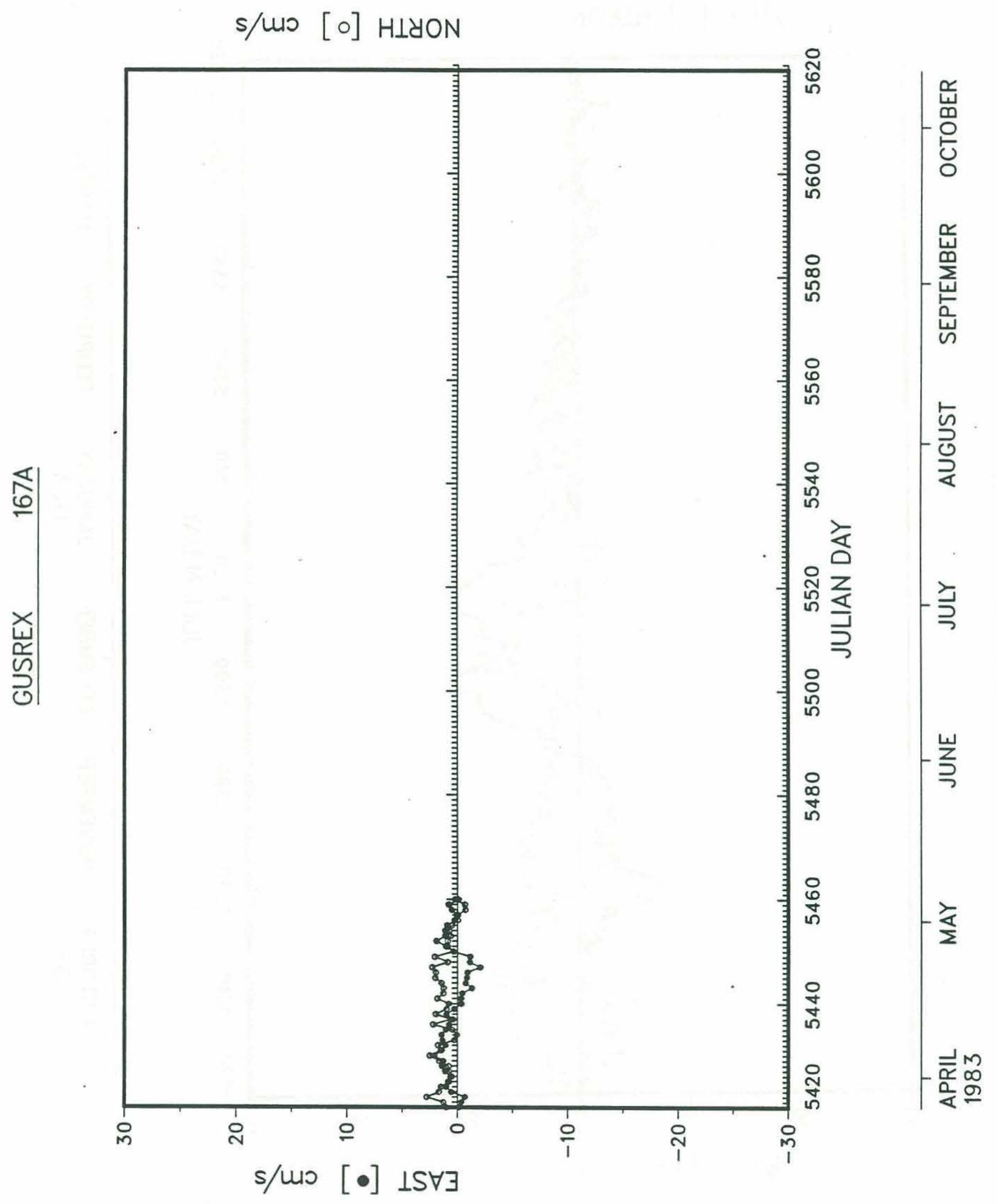




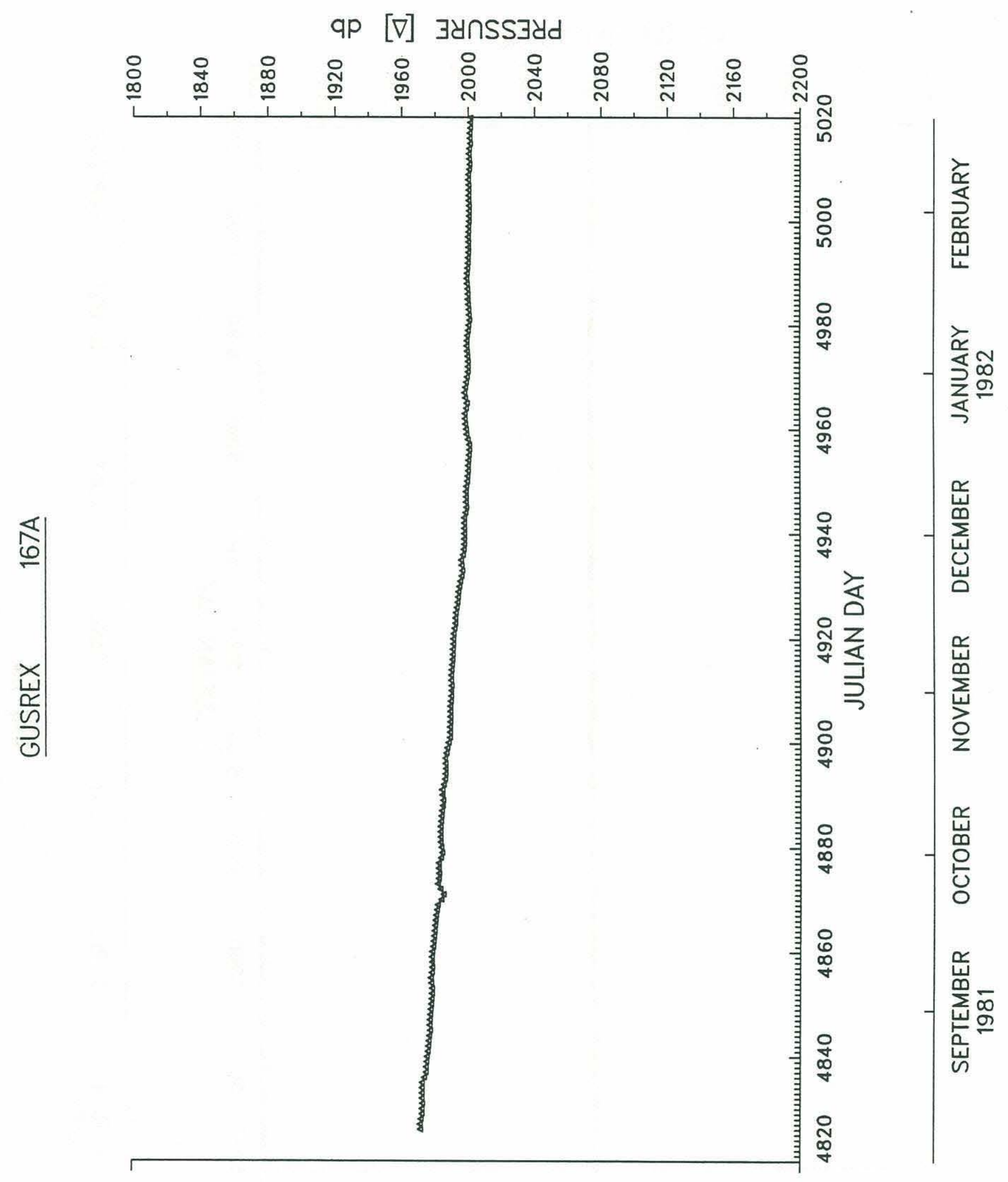




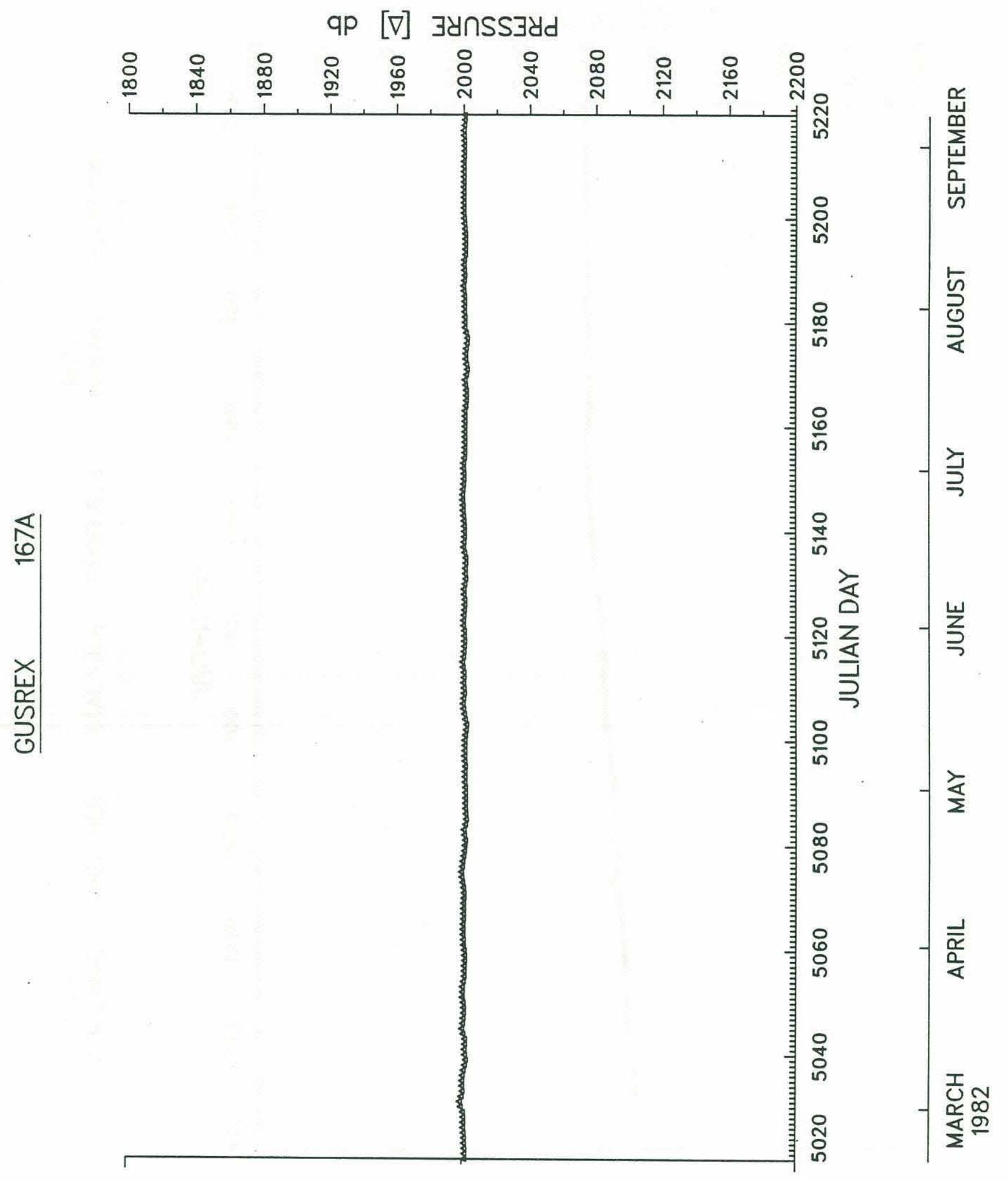




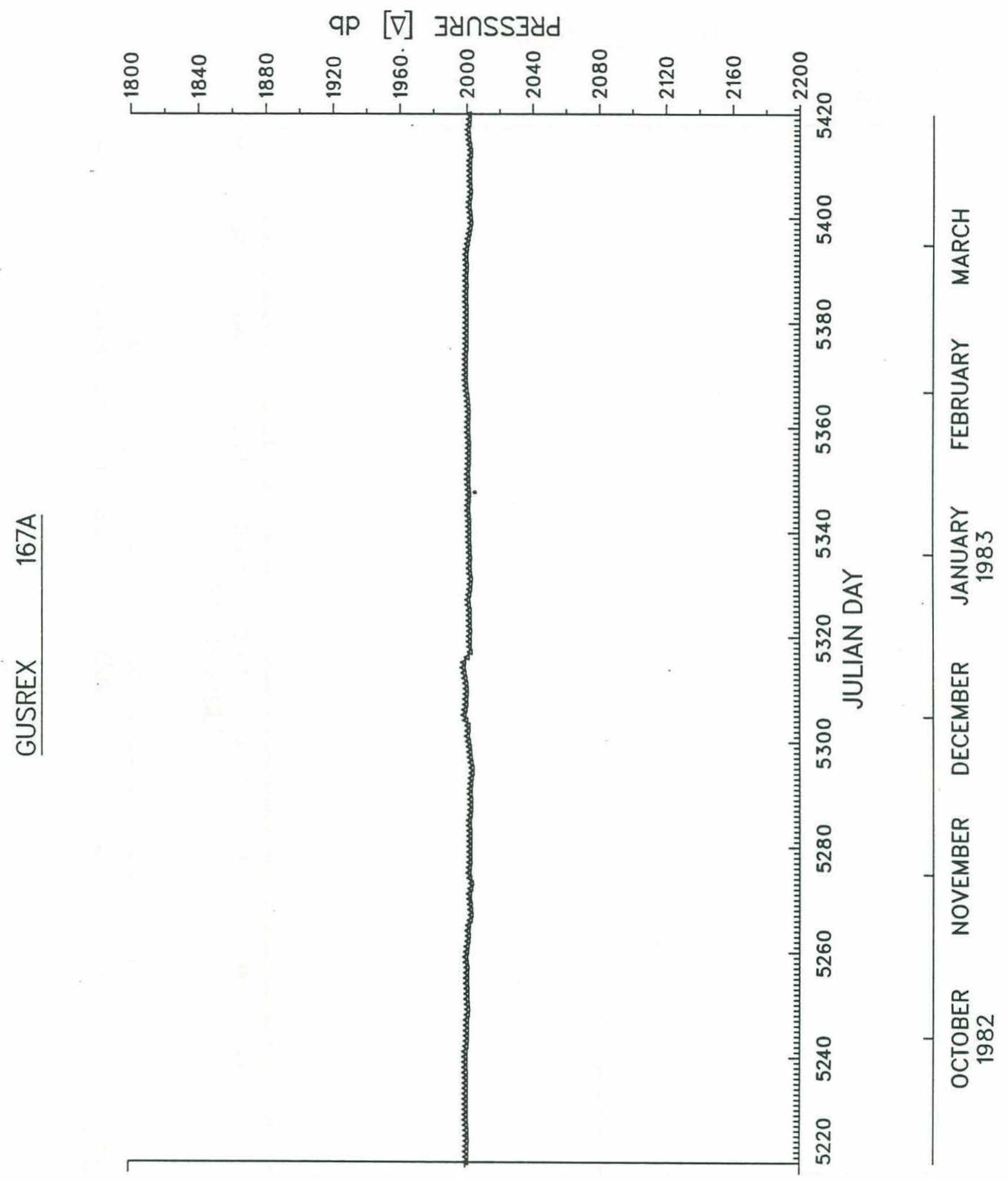




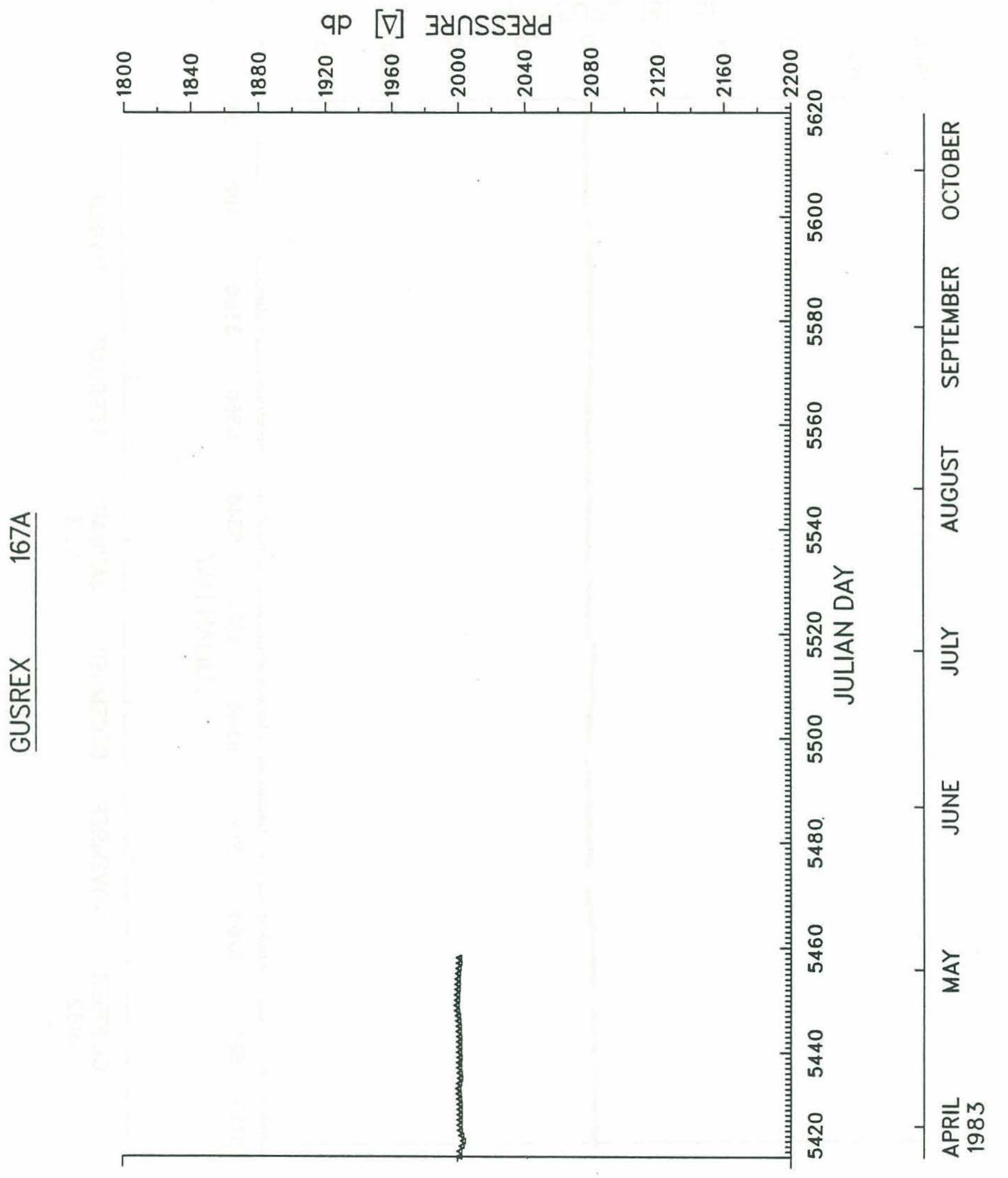




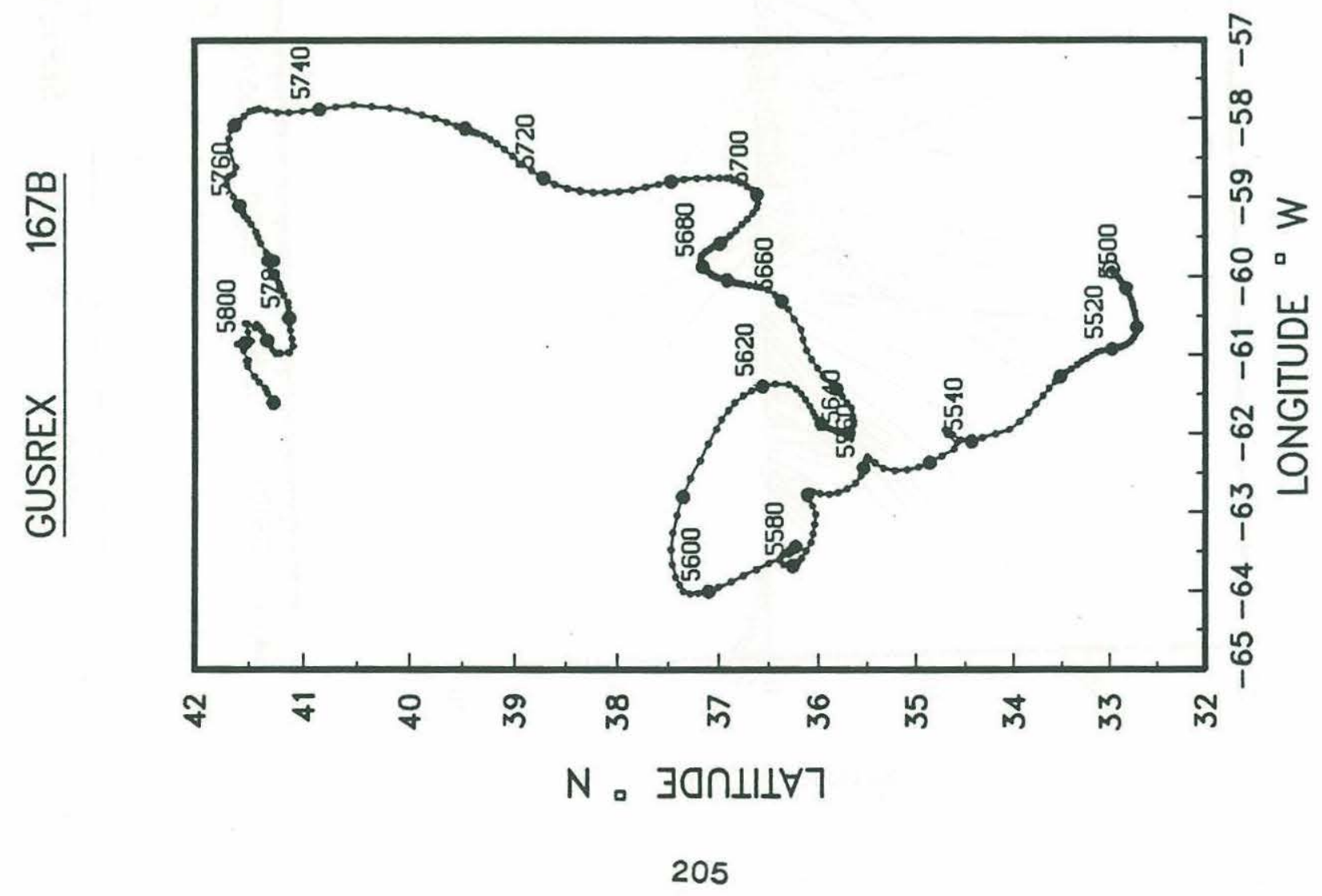




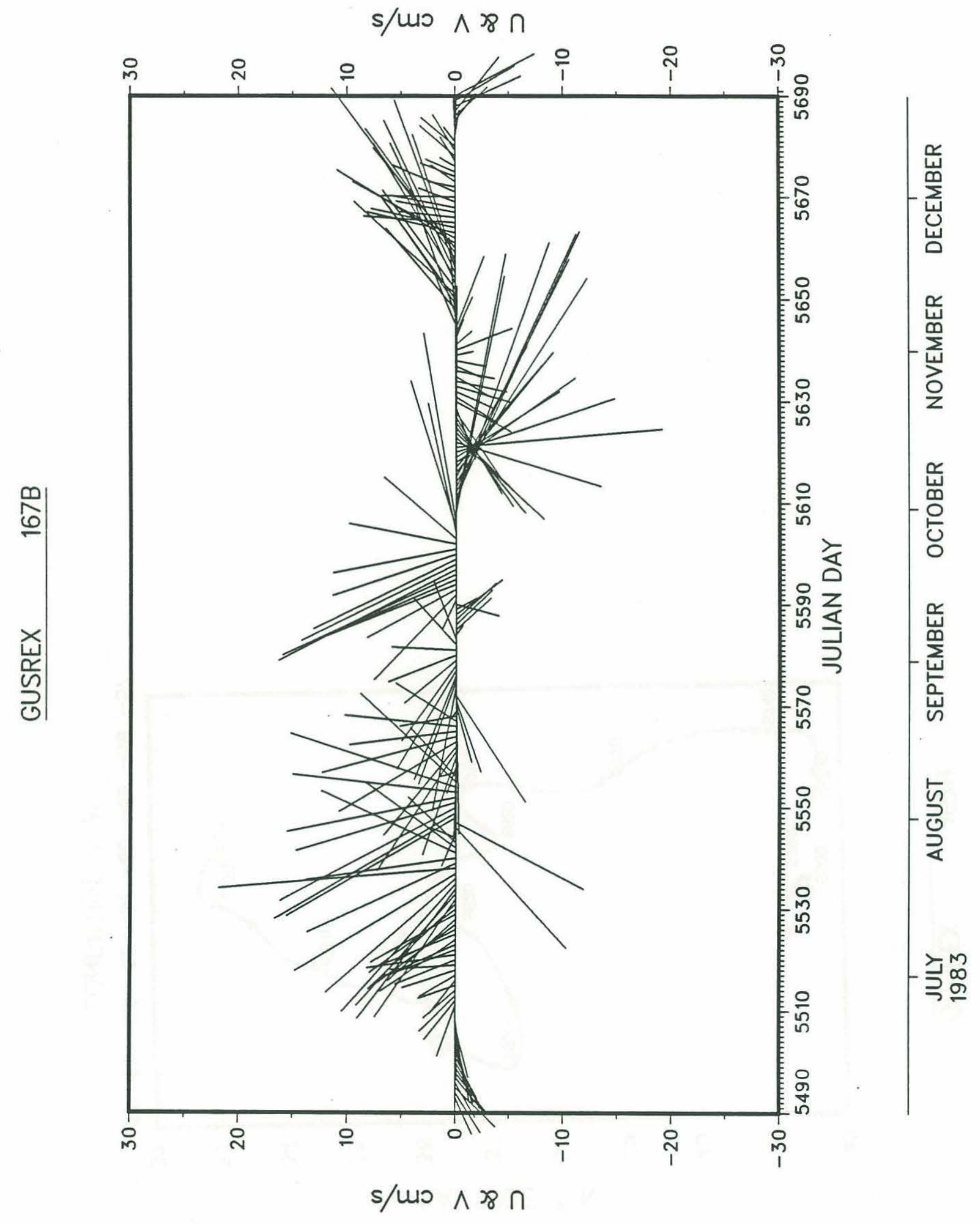




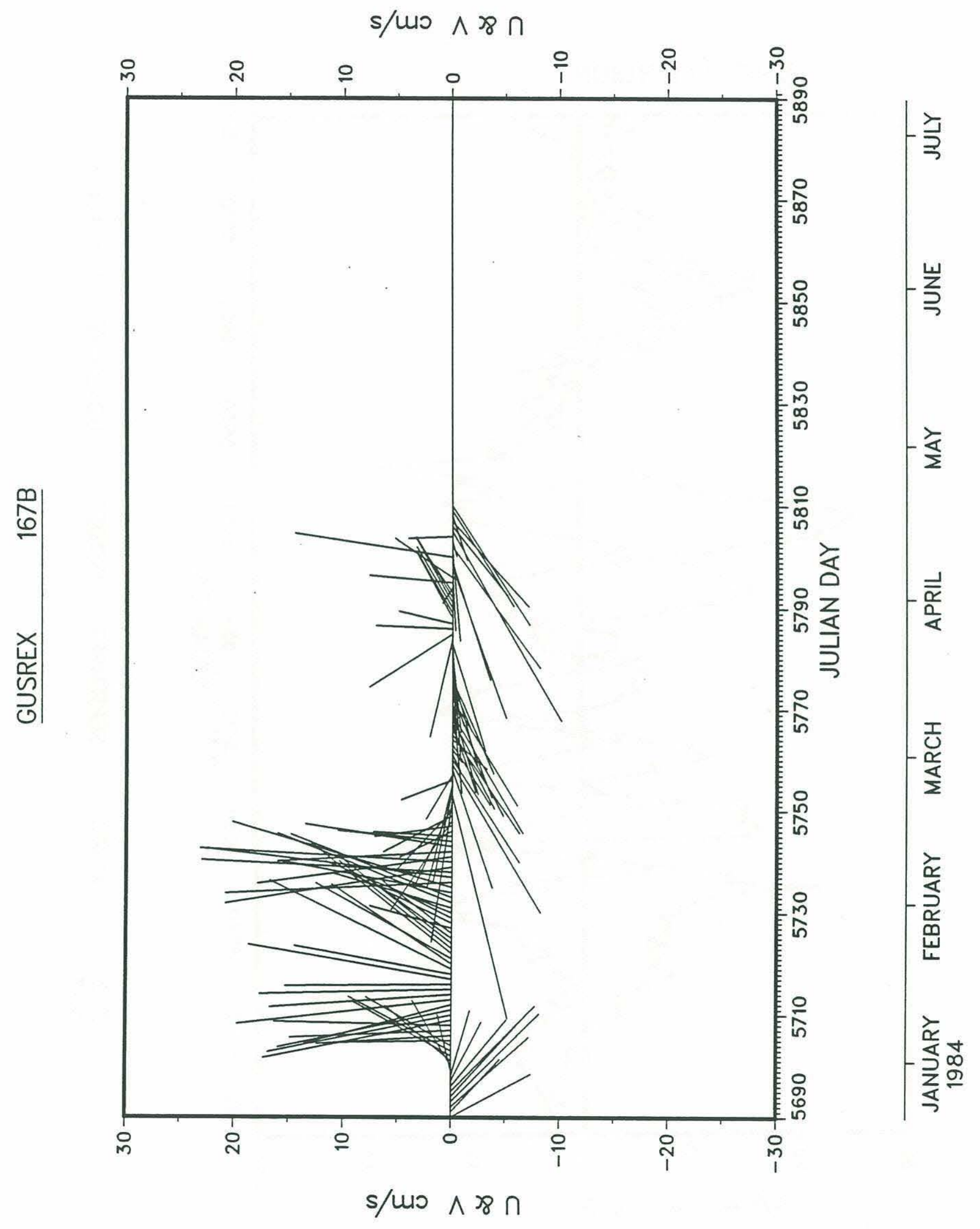




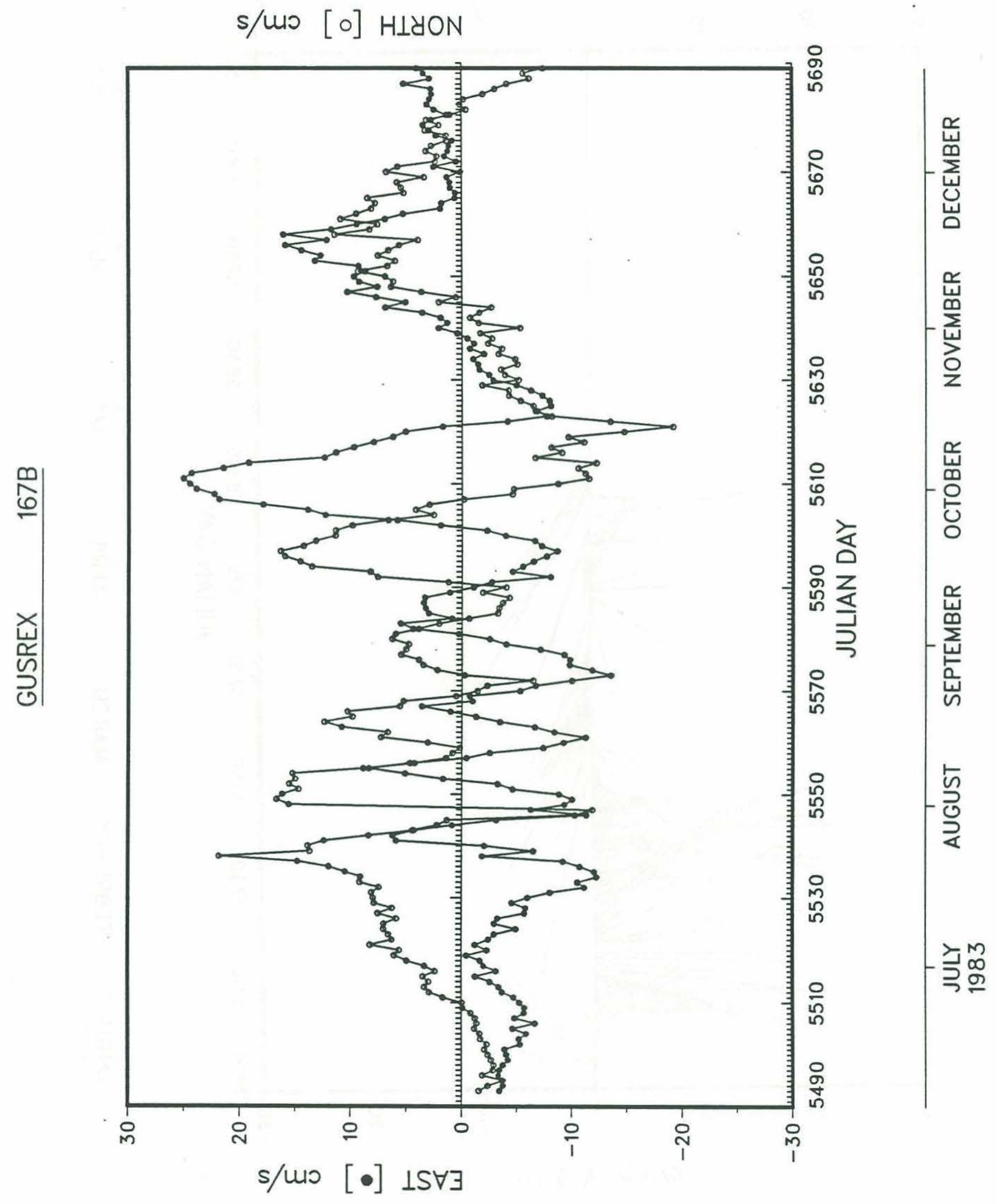




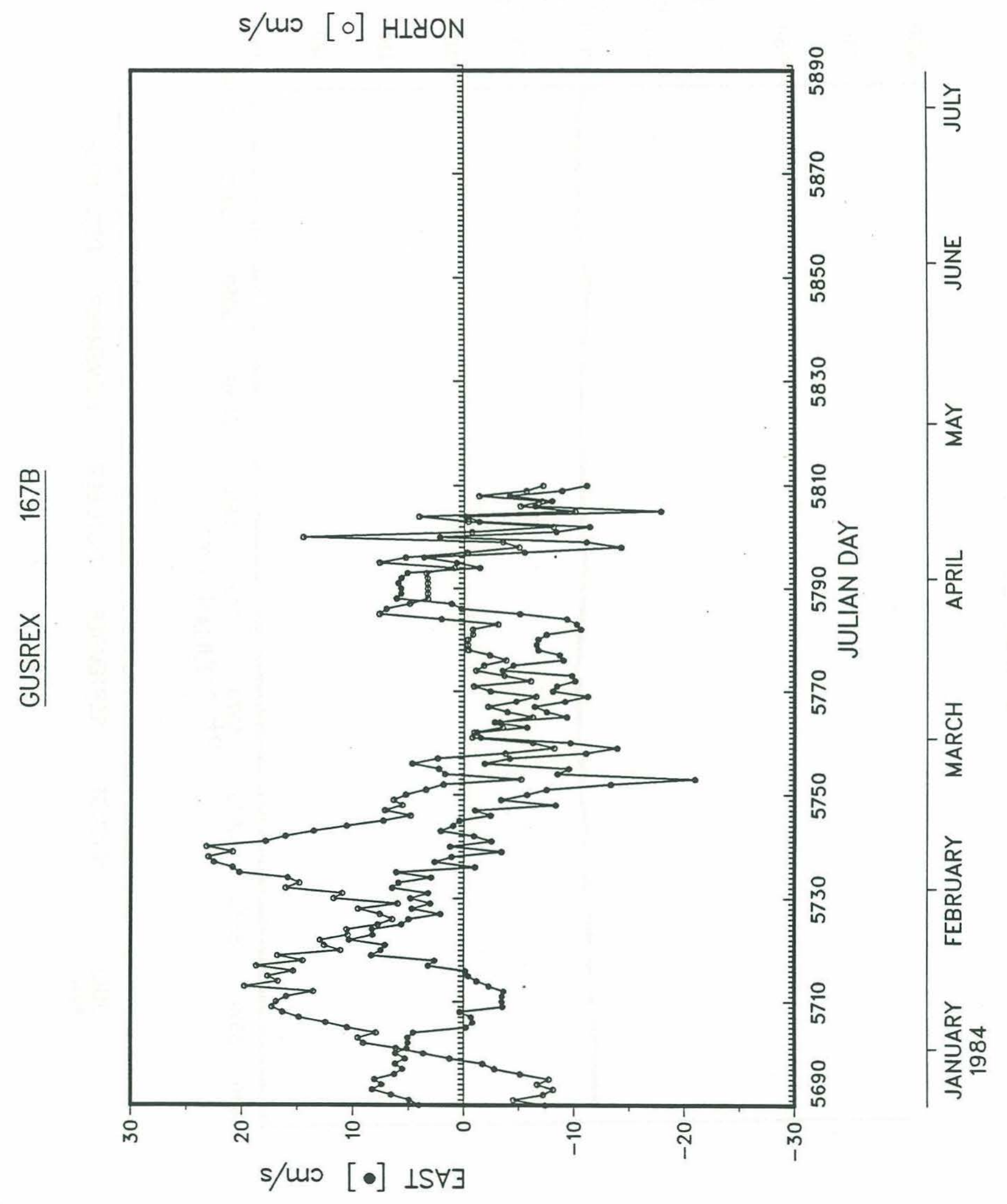




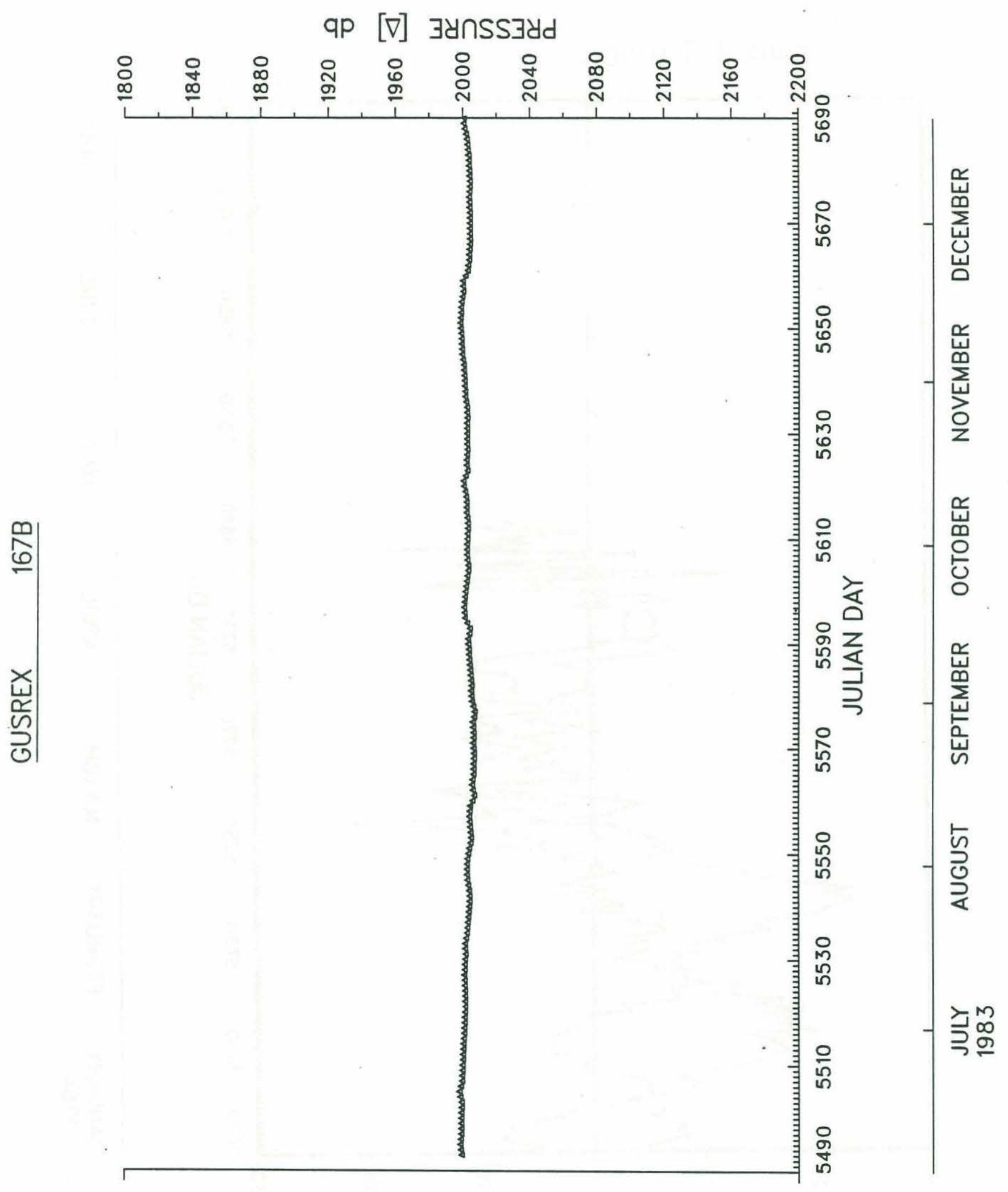




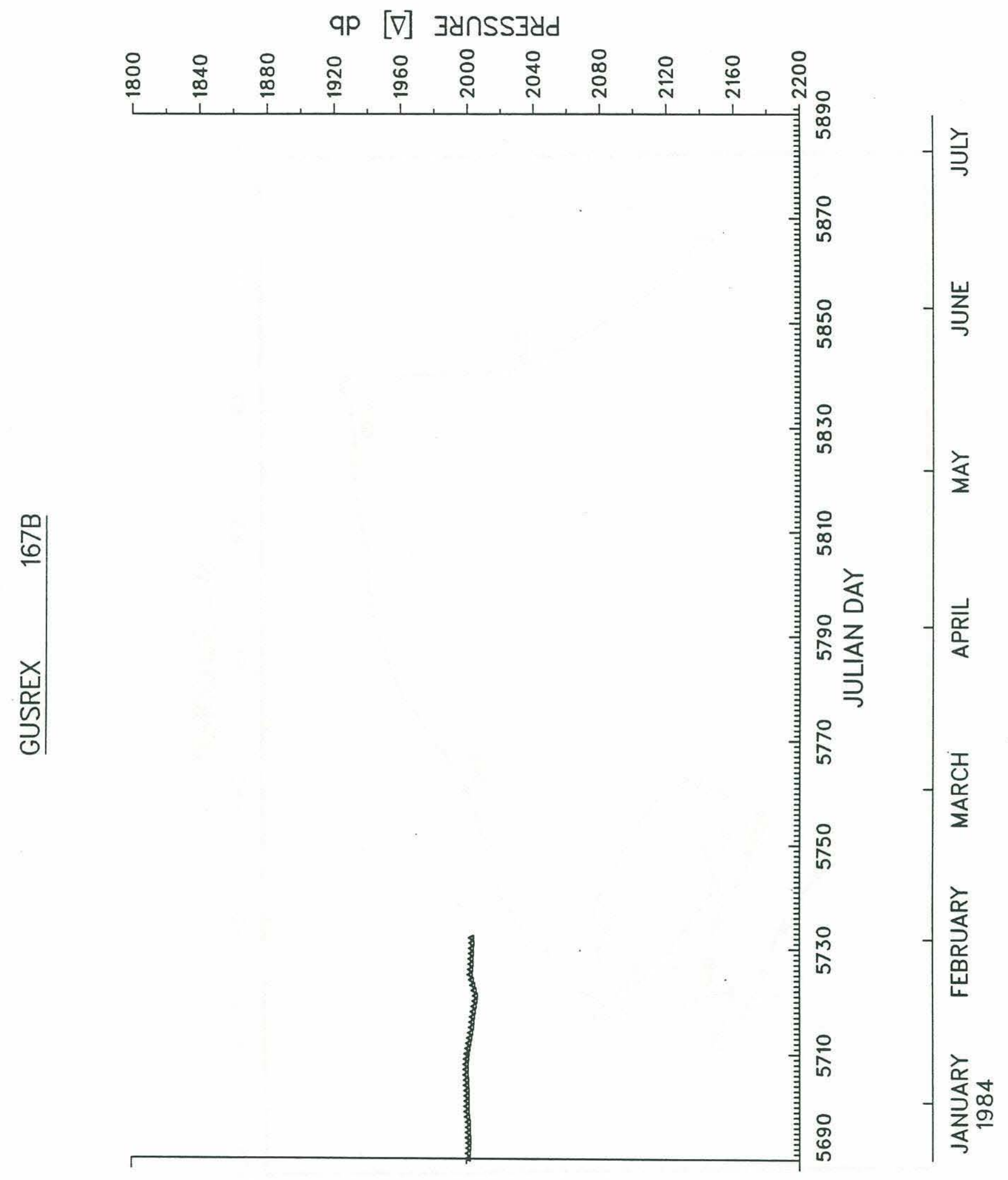




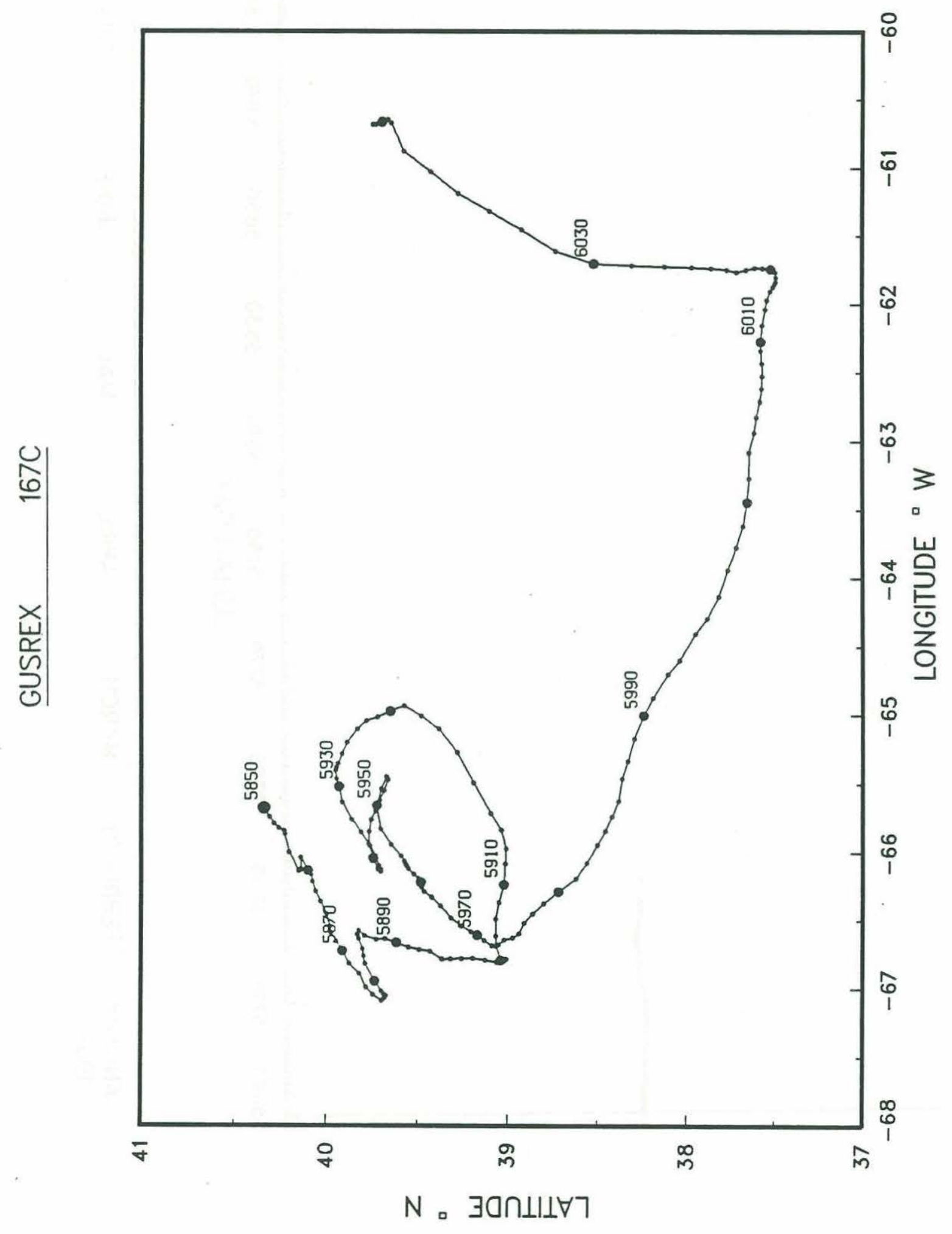




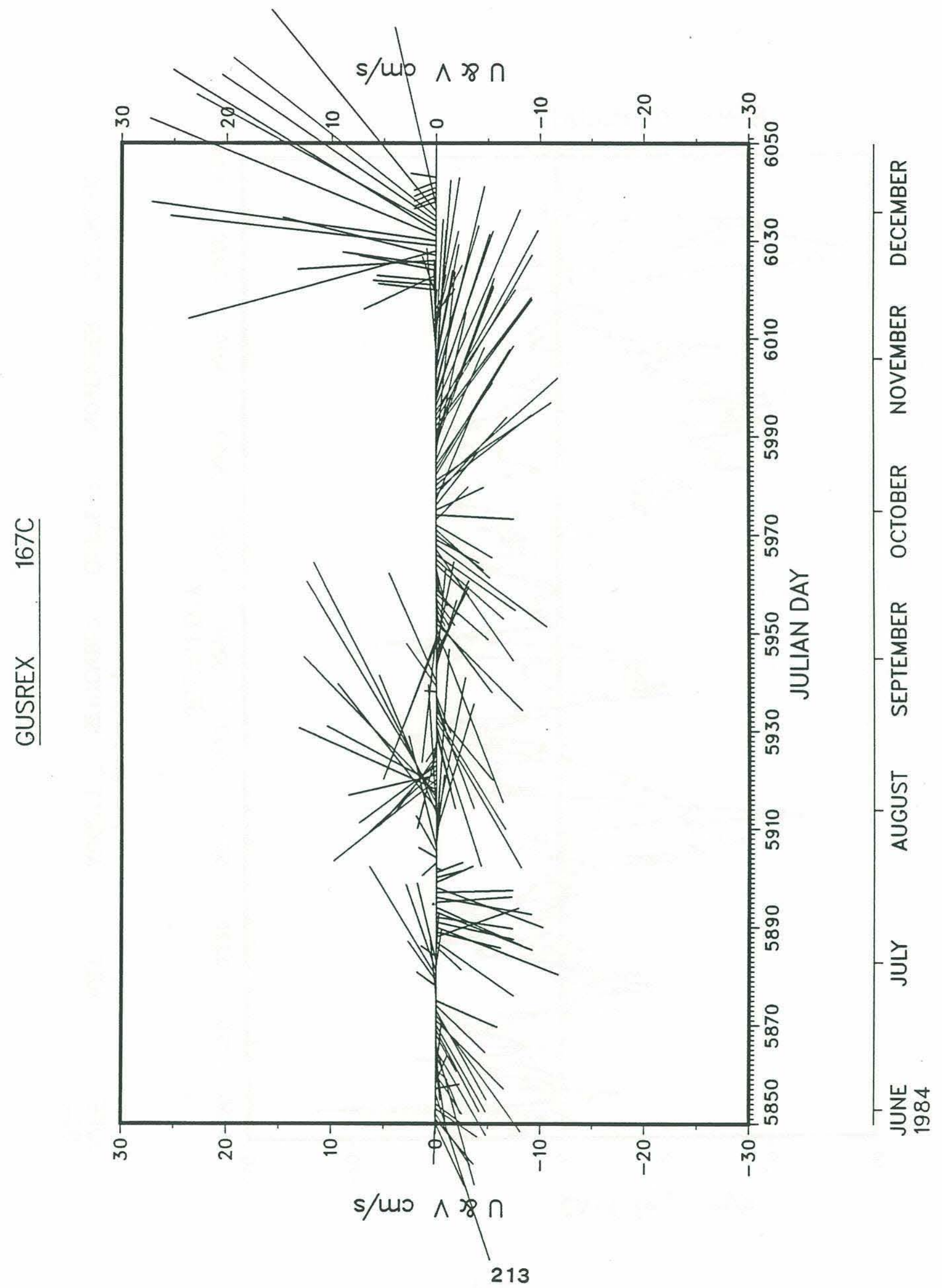




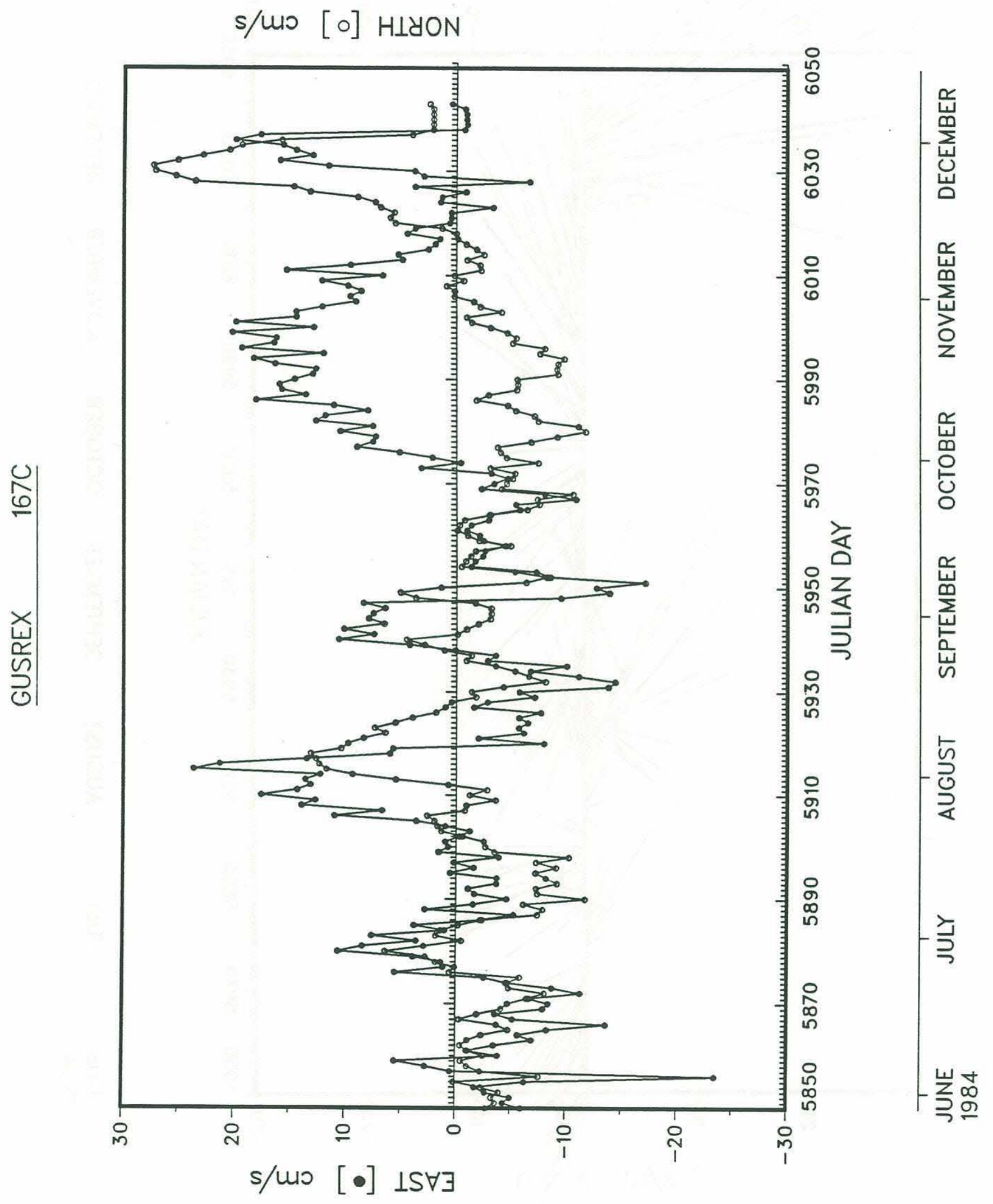




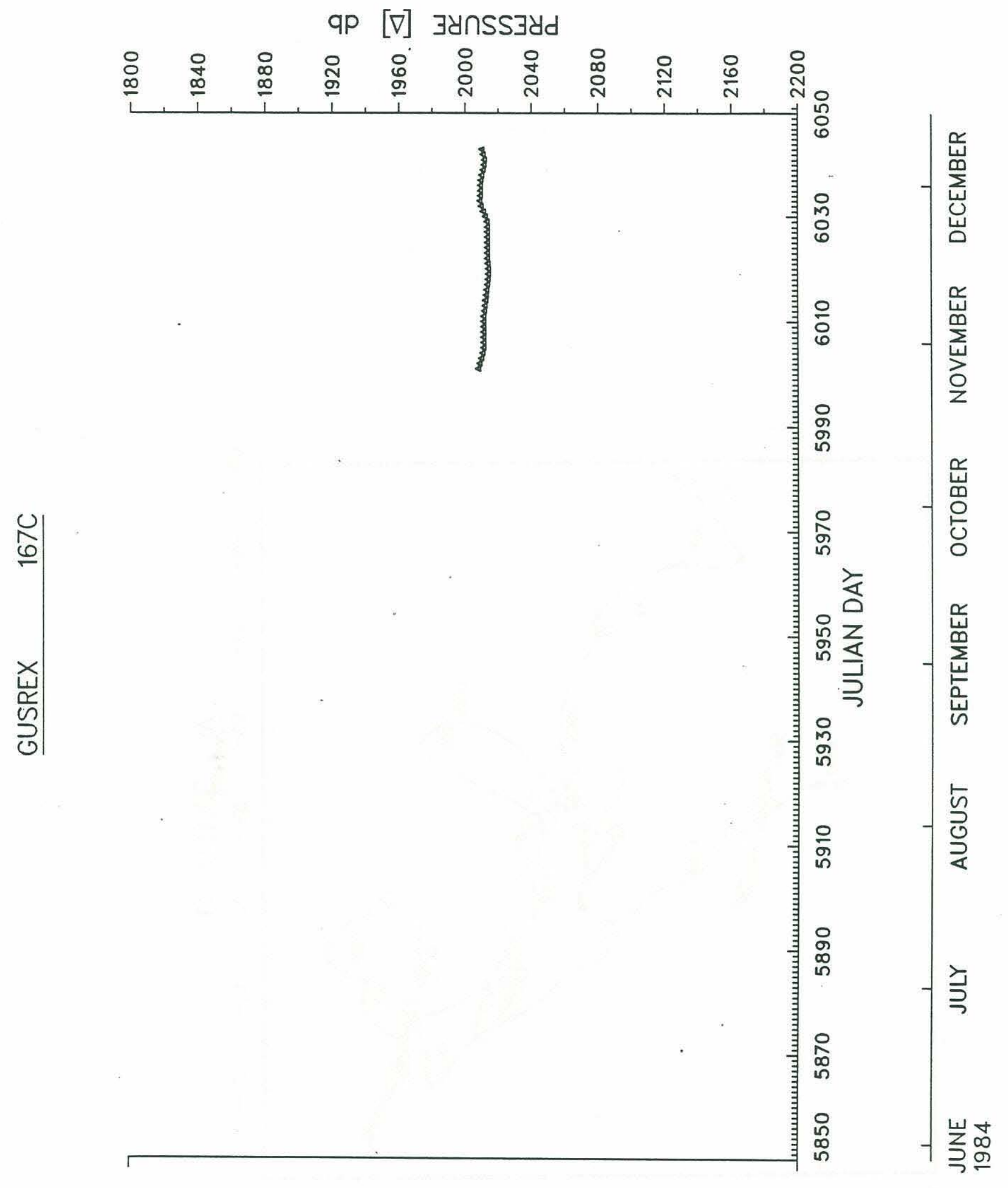




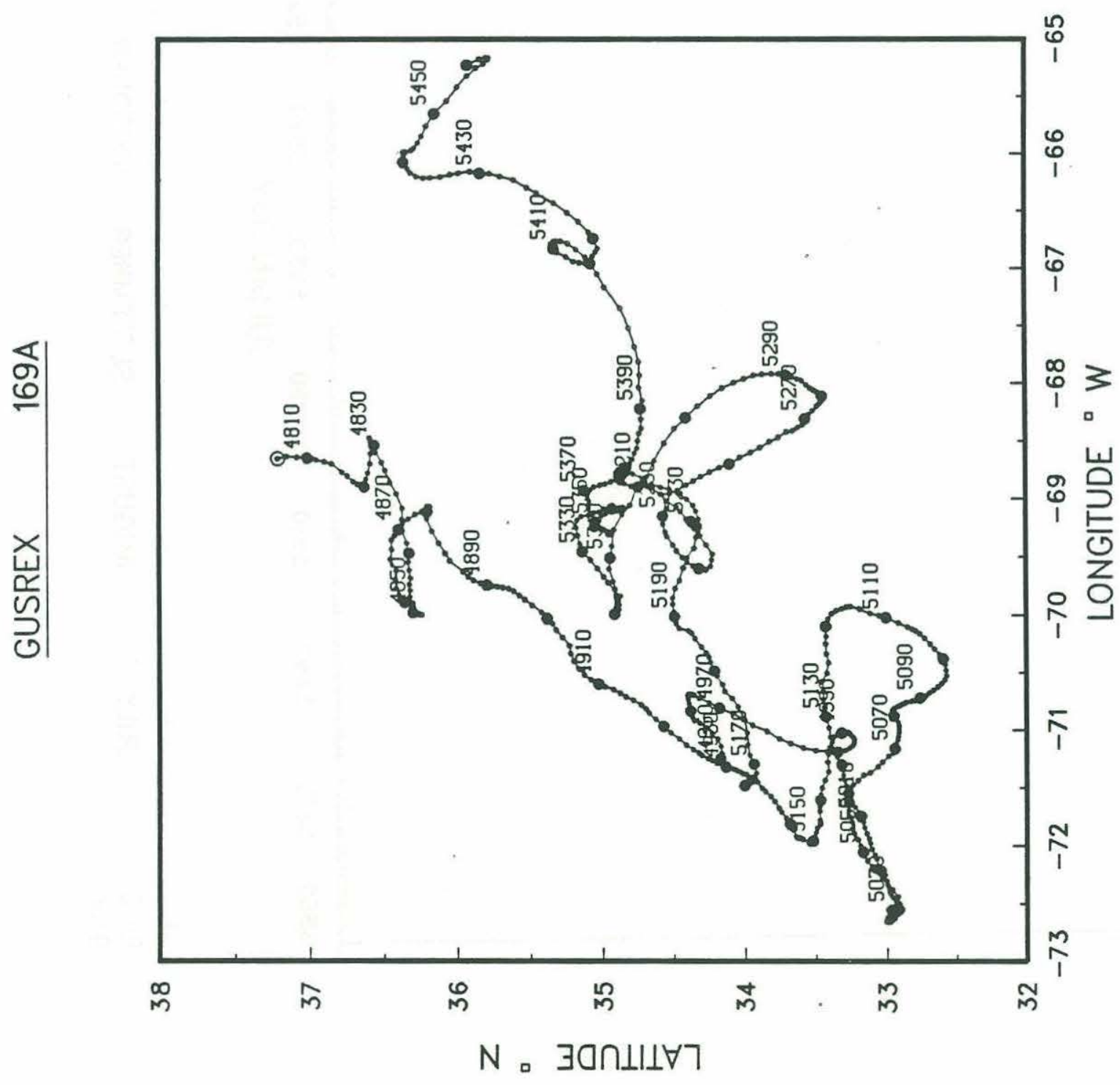




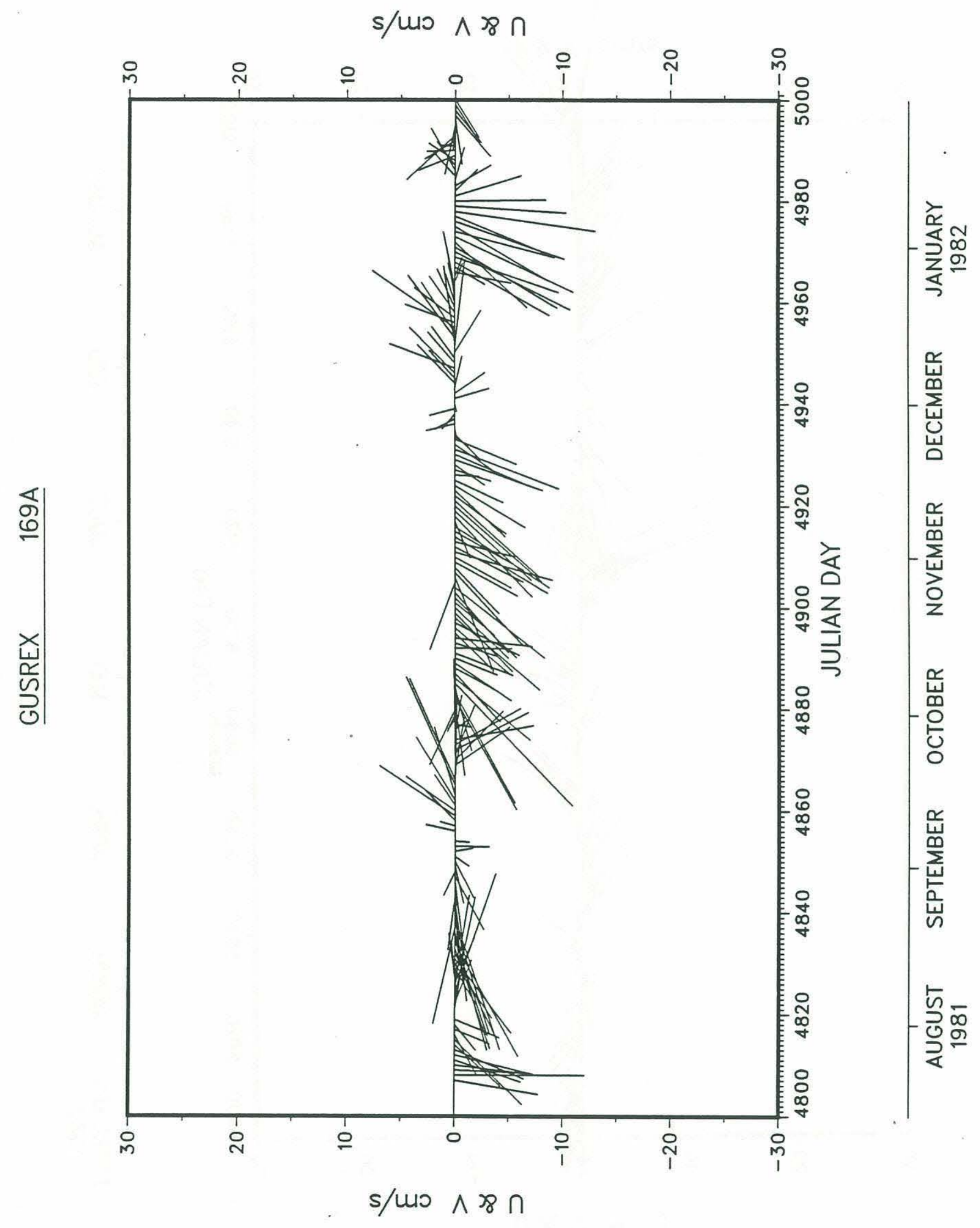




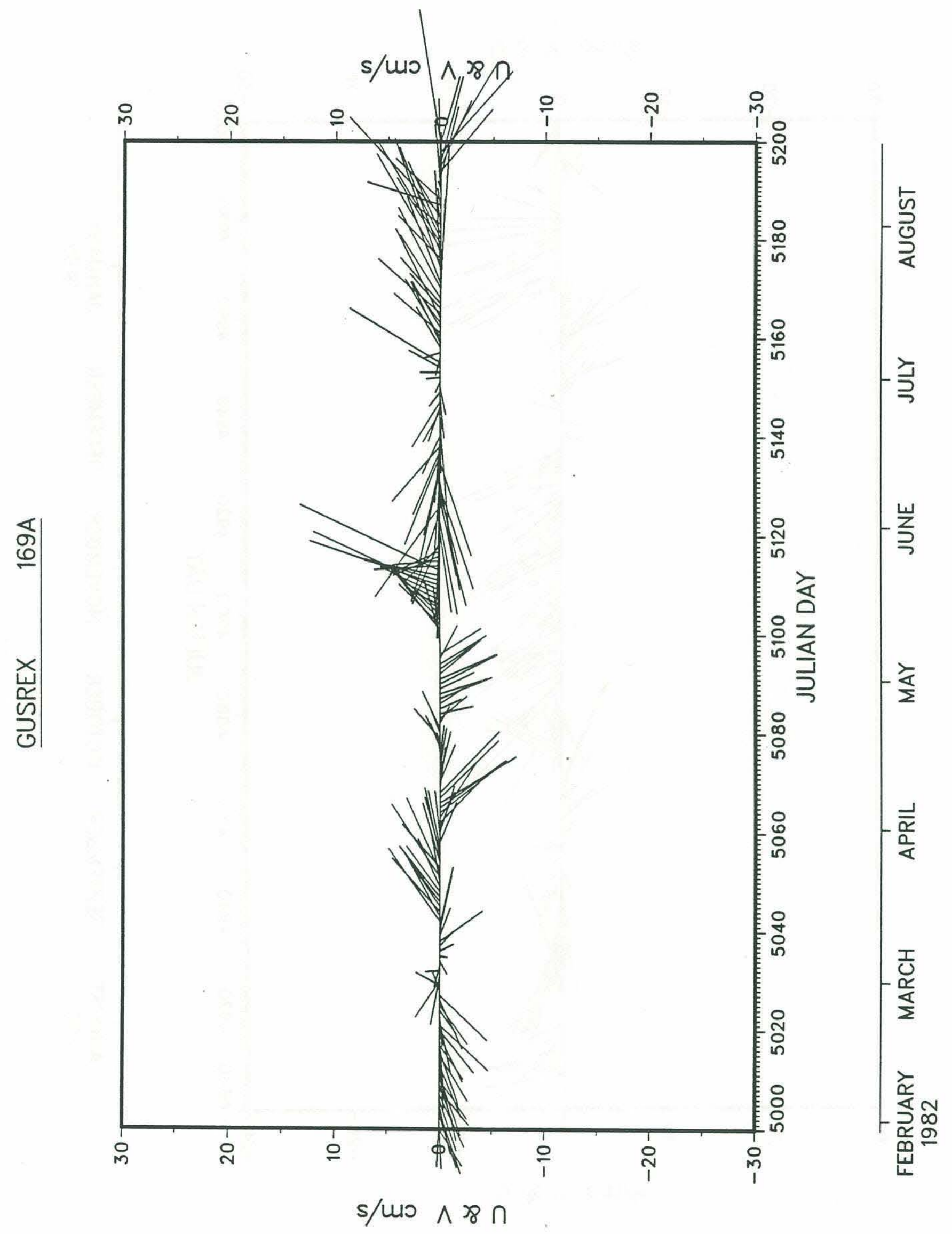




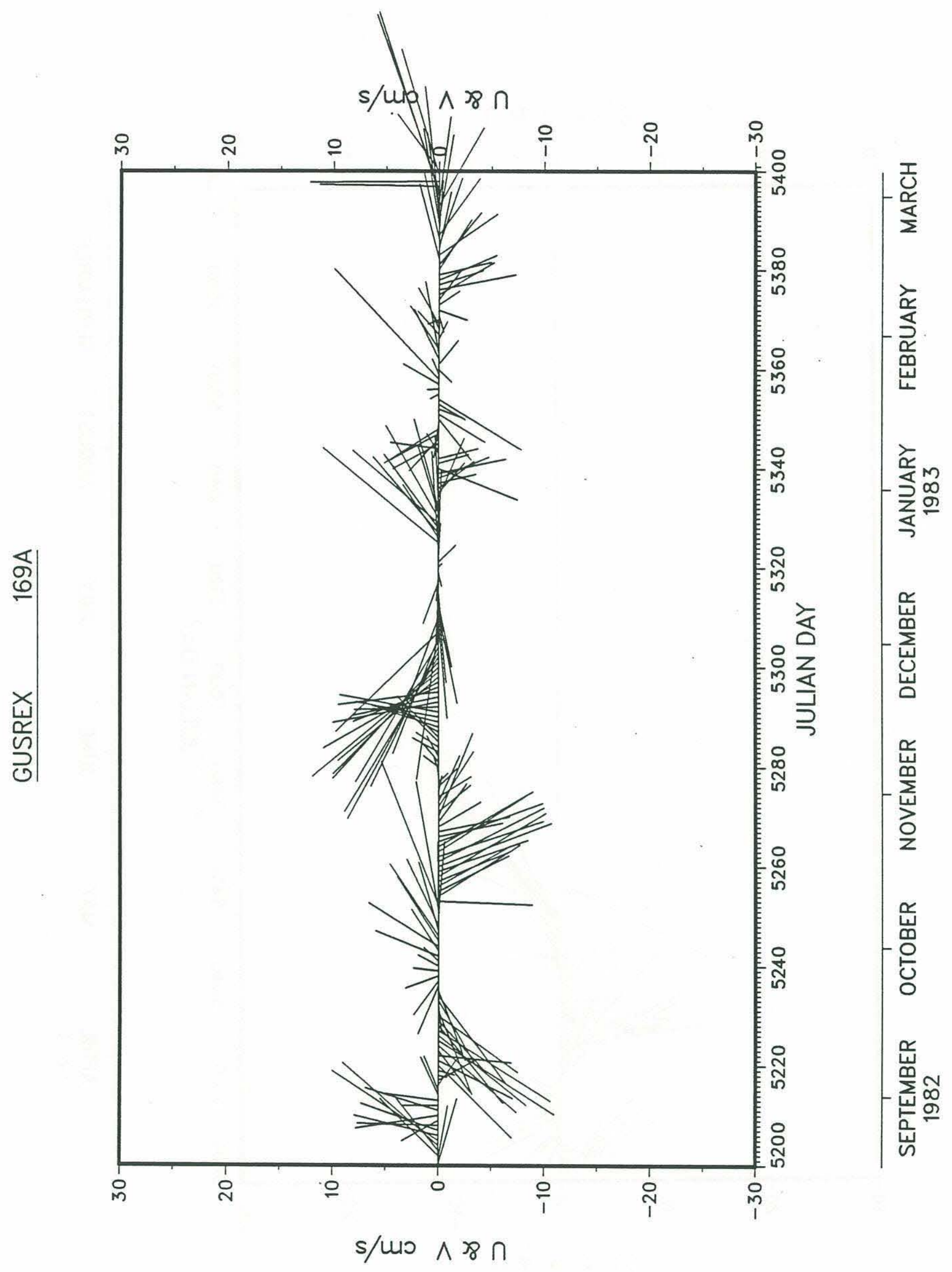




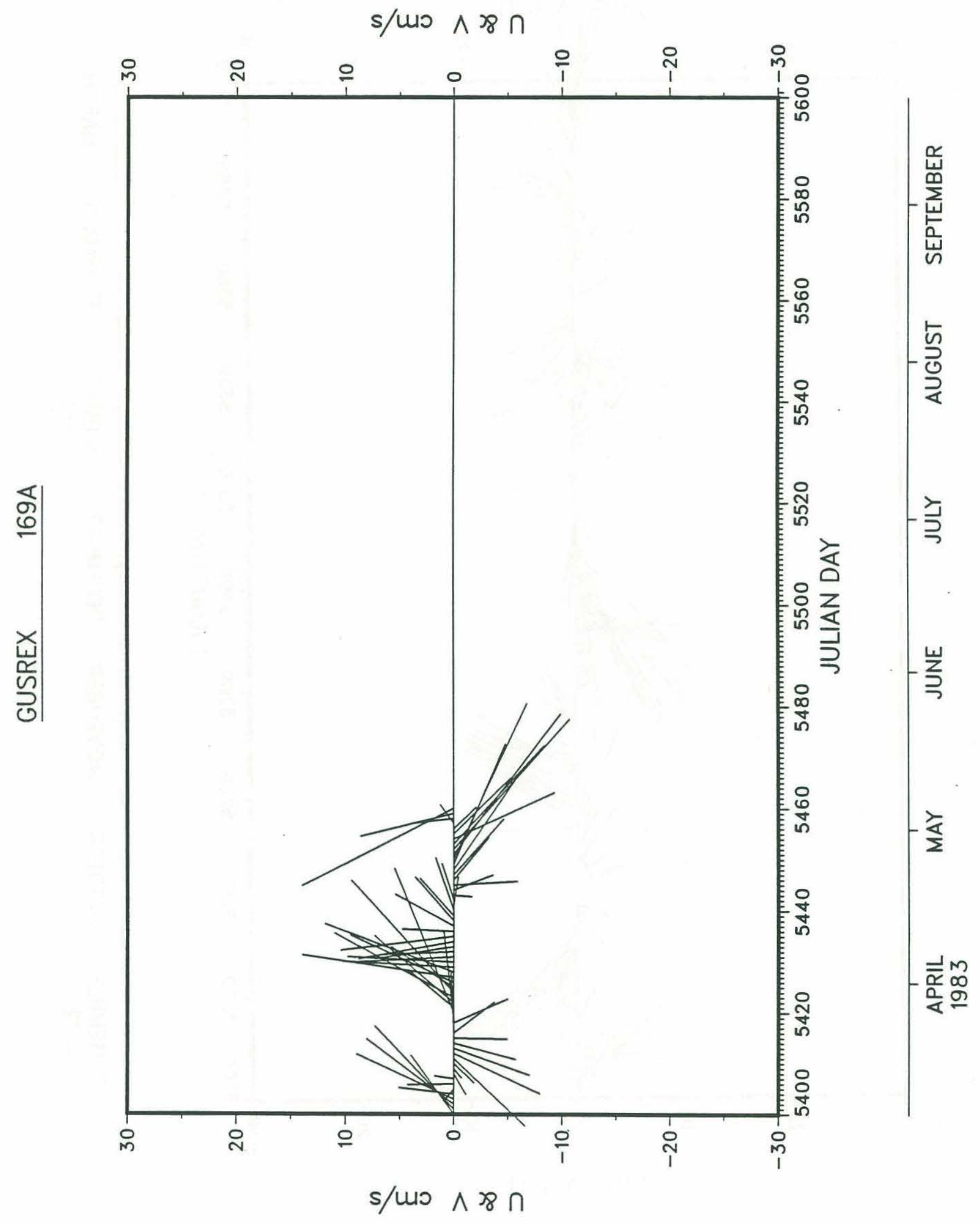


s/Mo [0] HLYYON

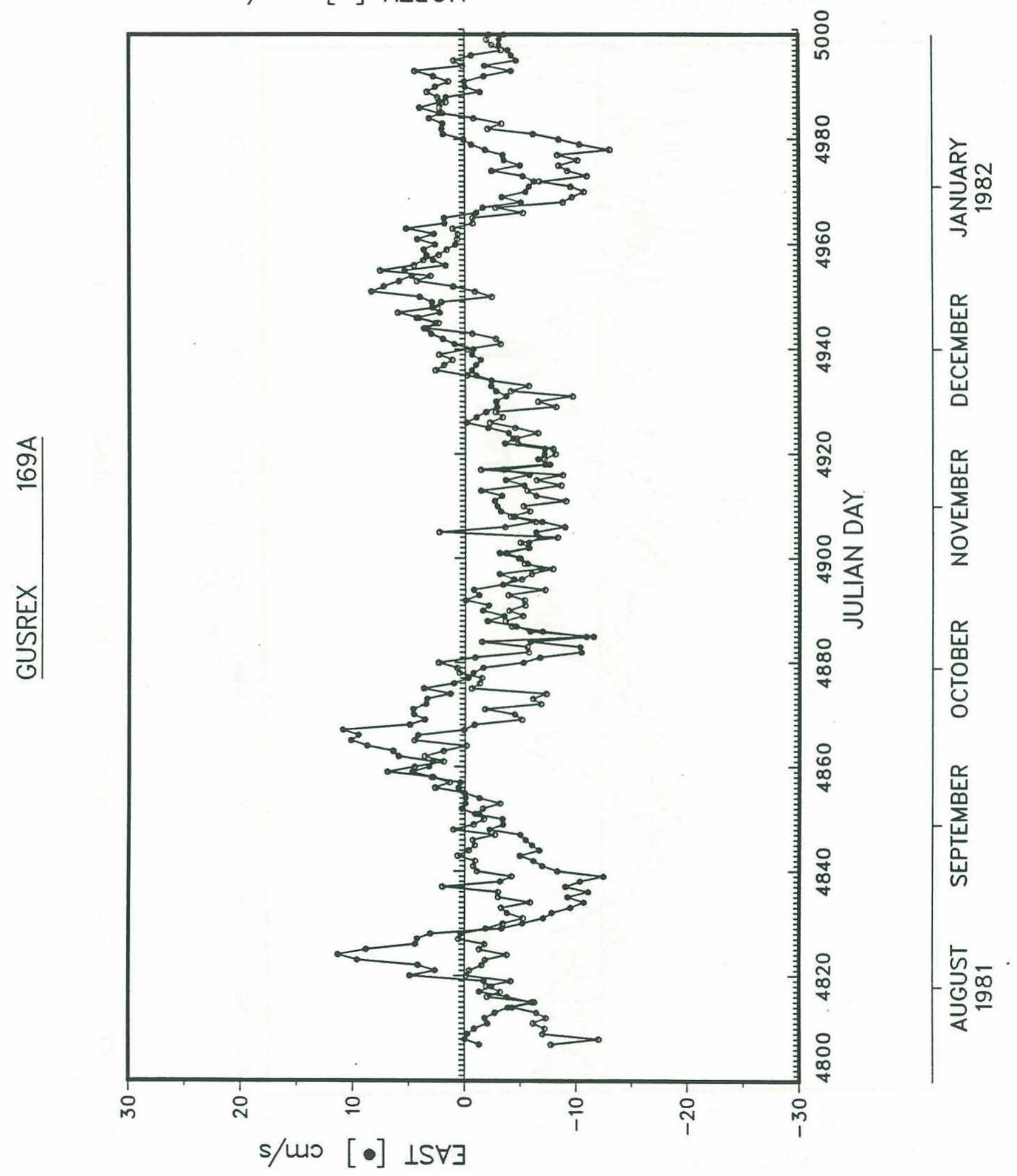




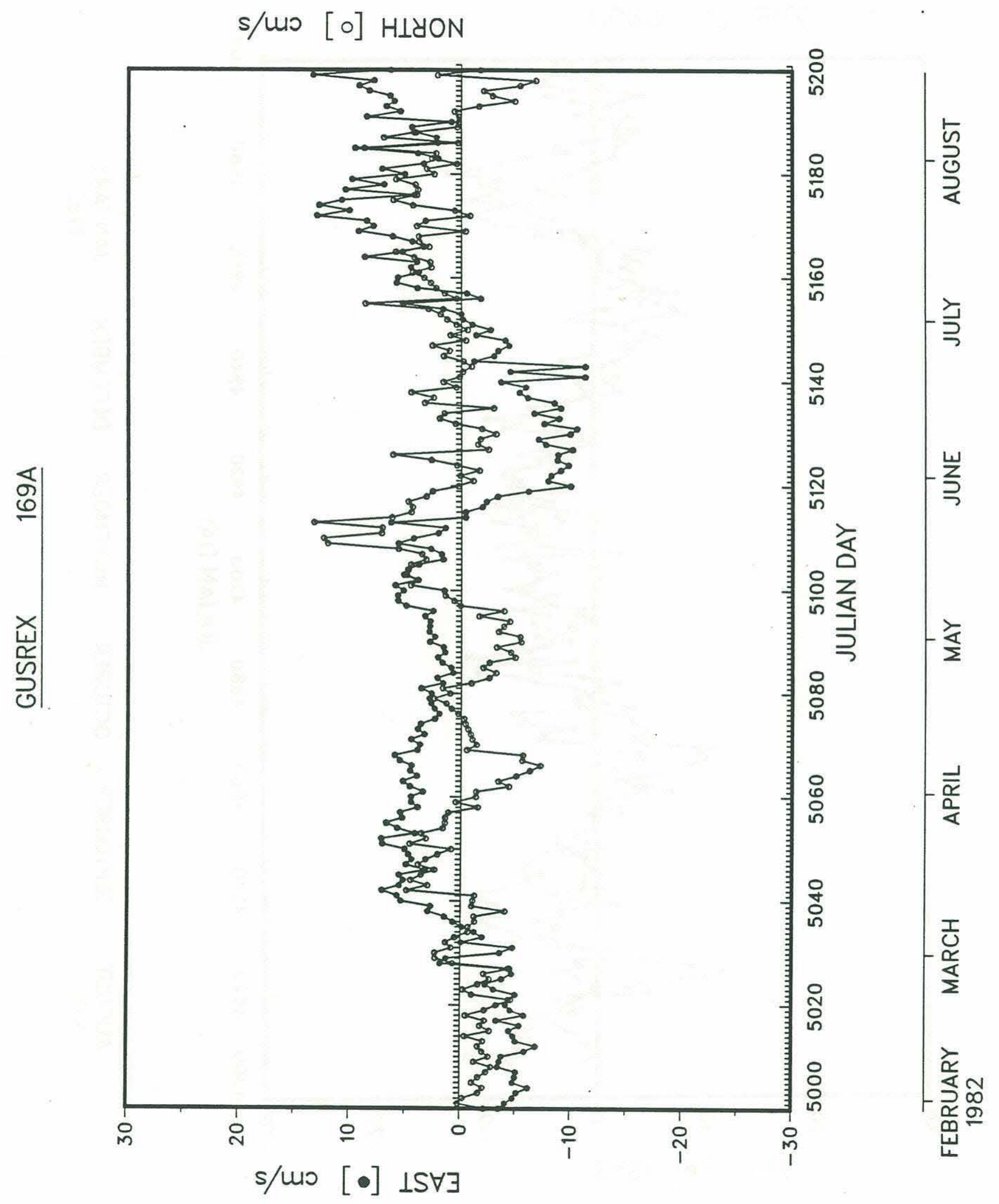




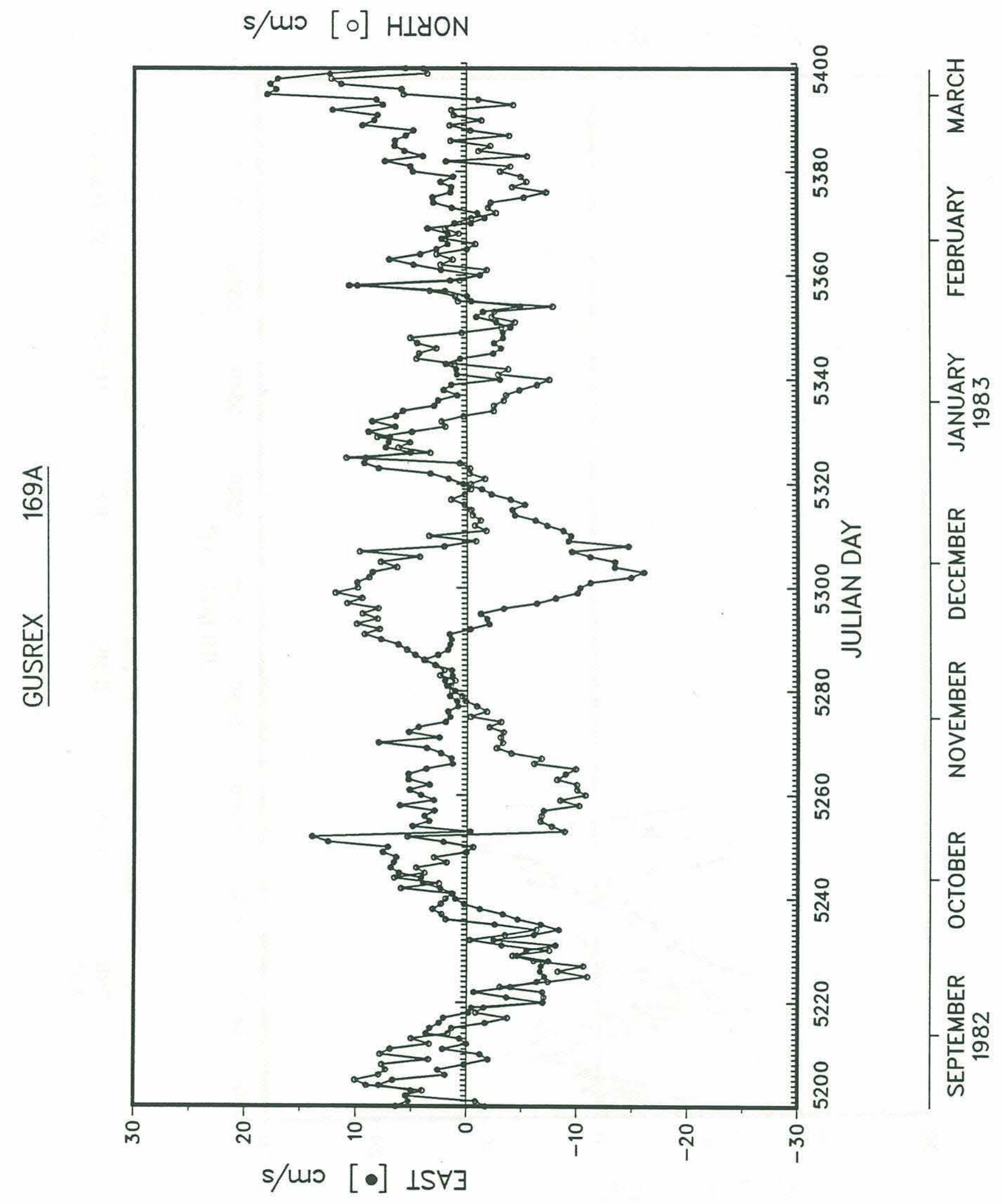




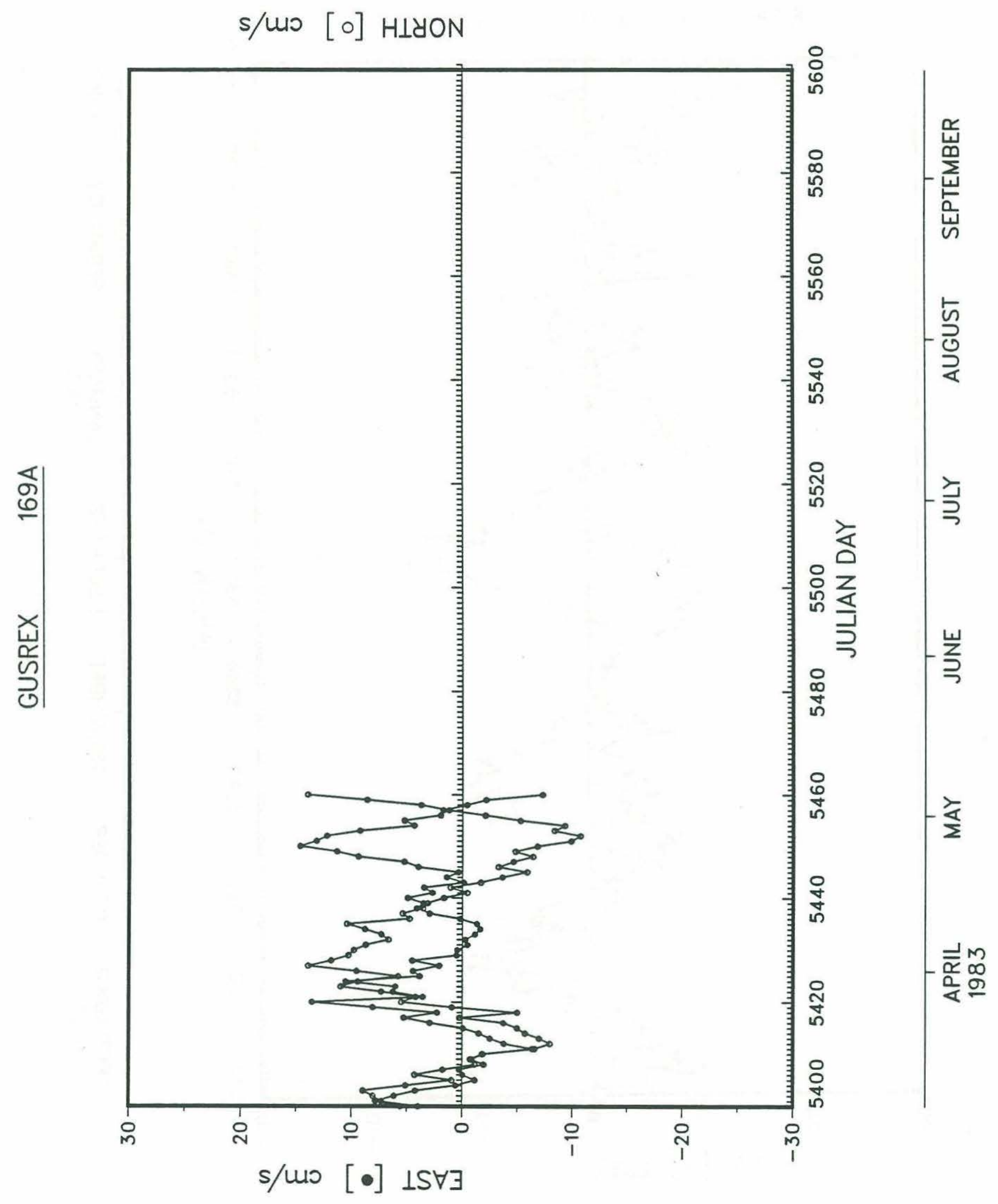




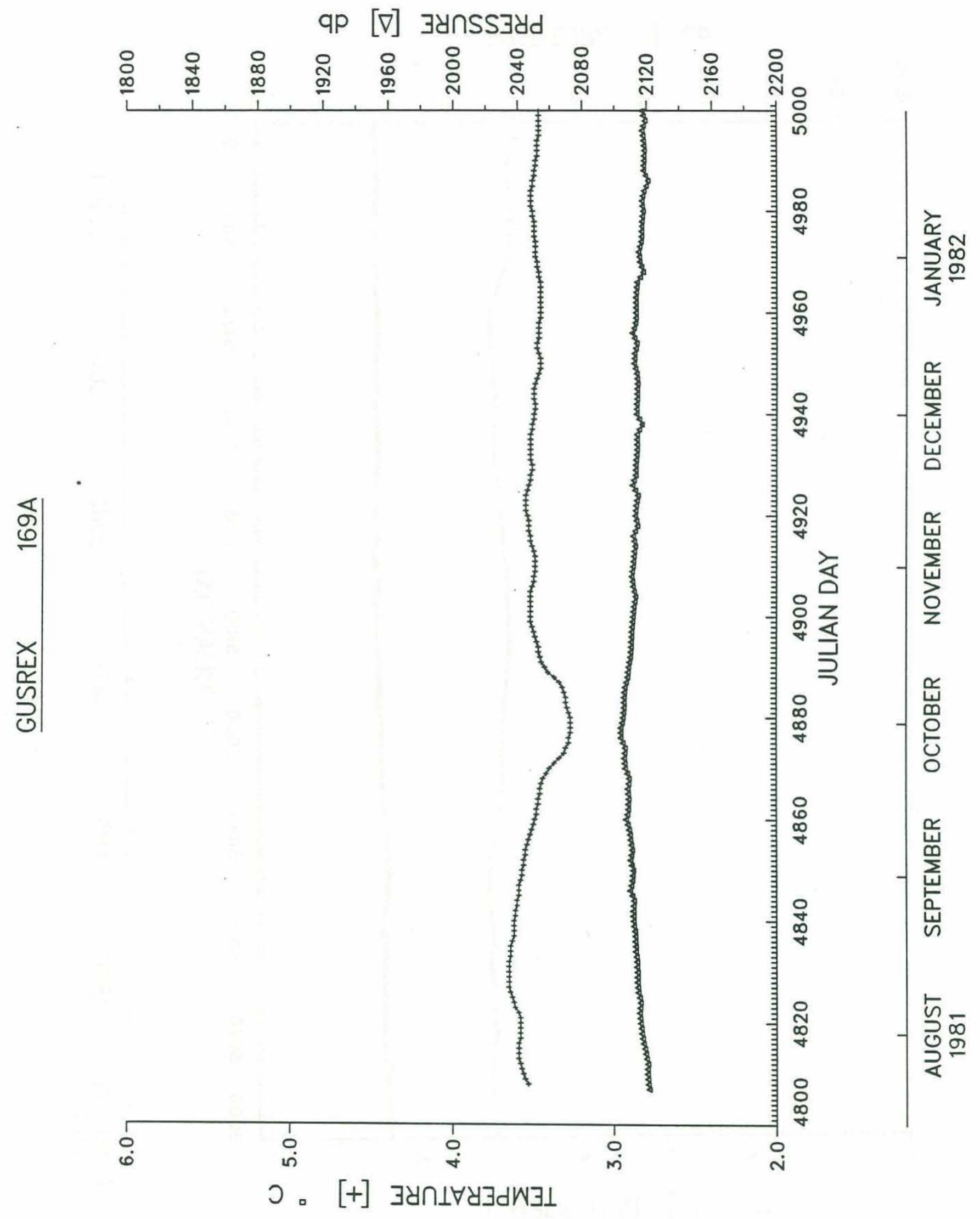




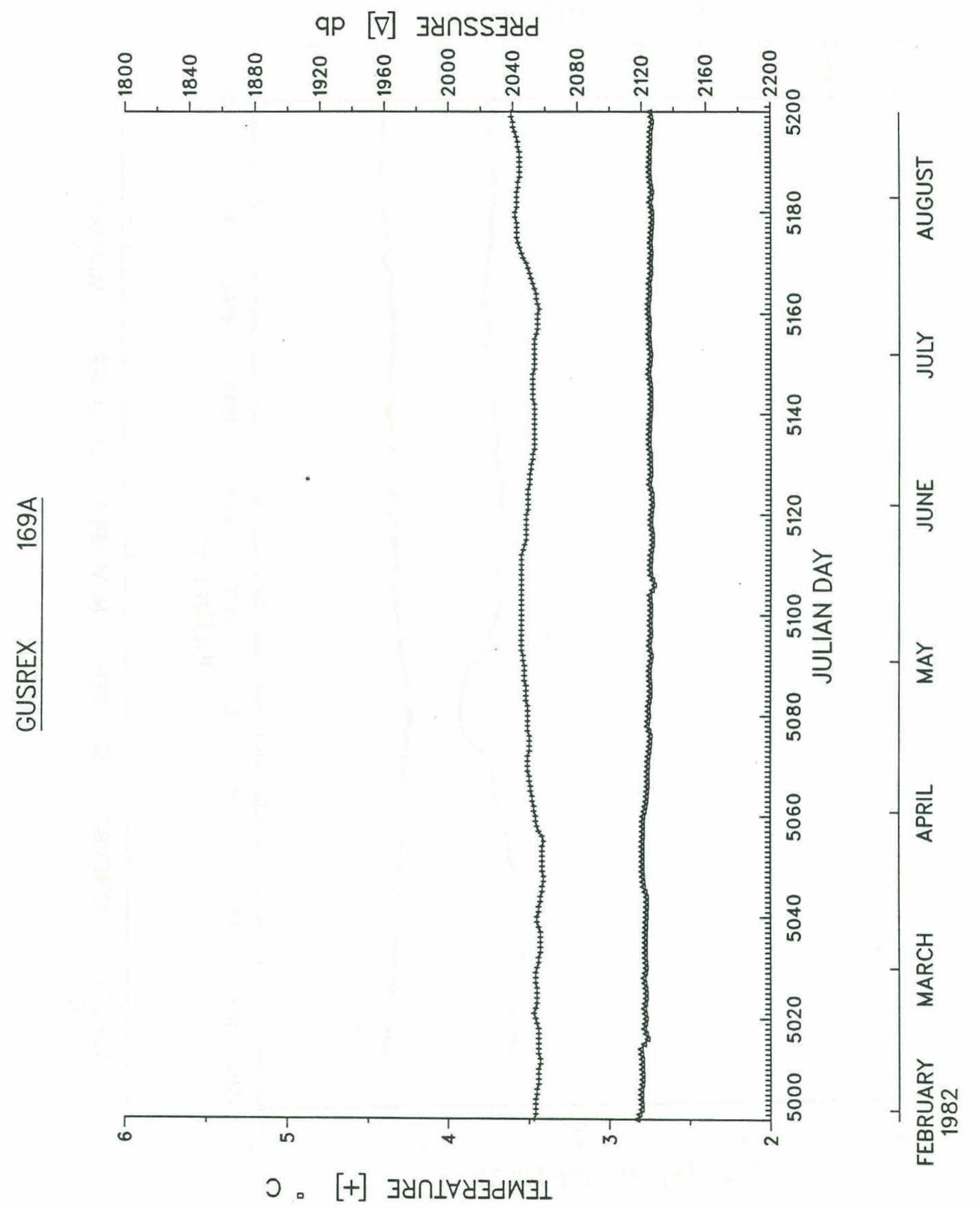




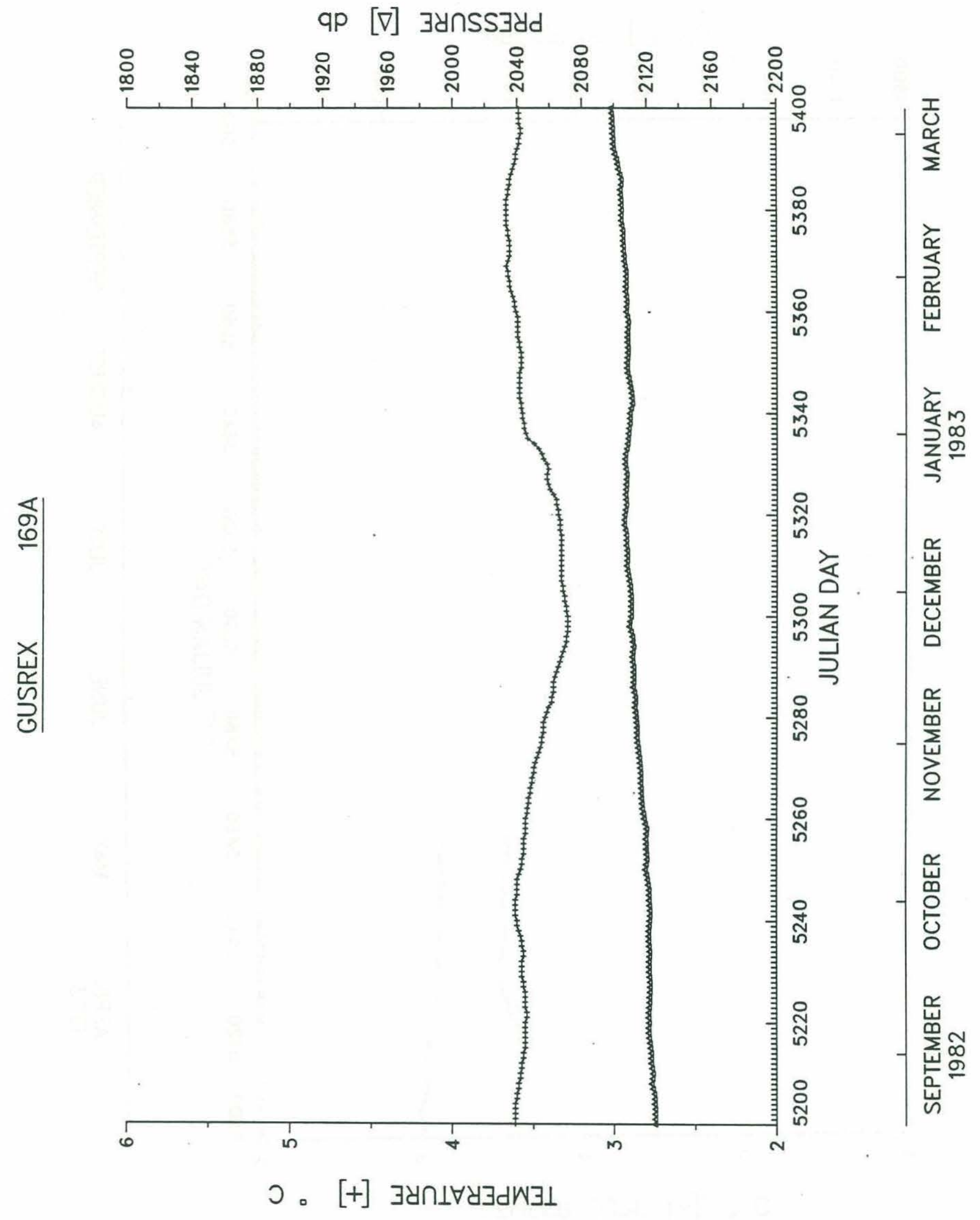




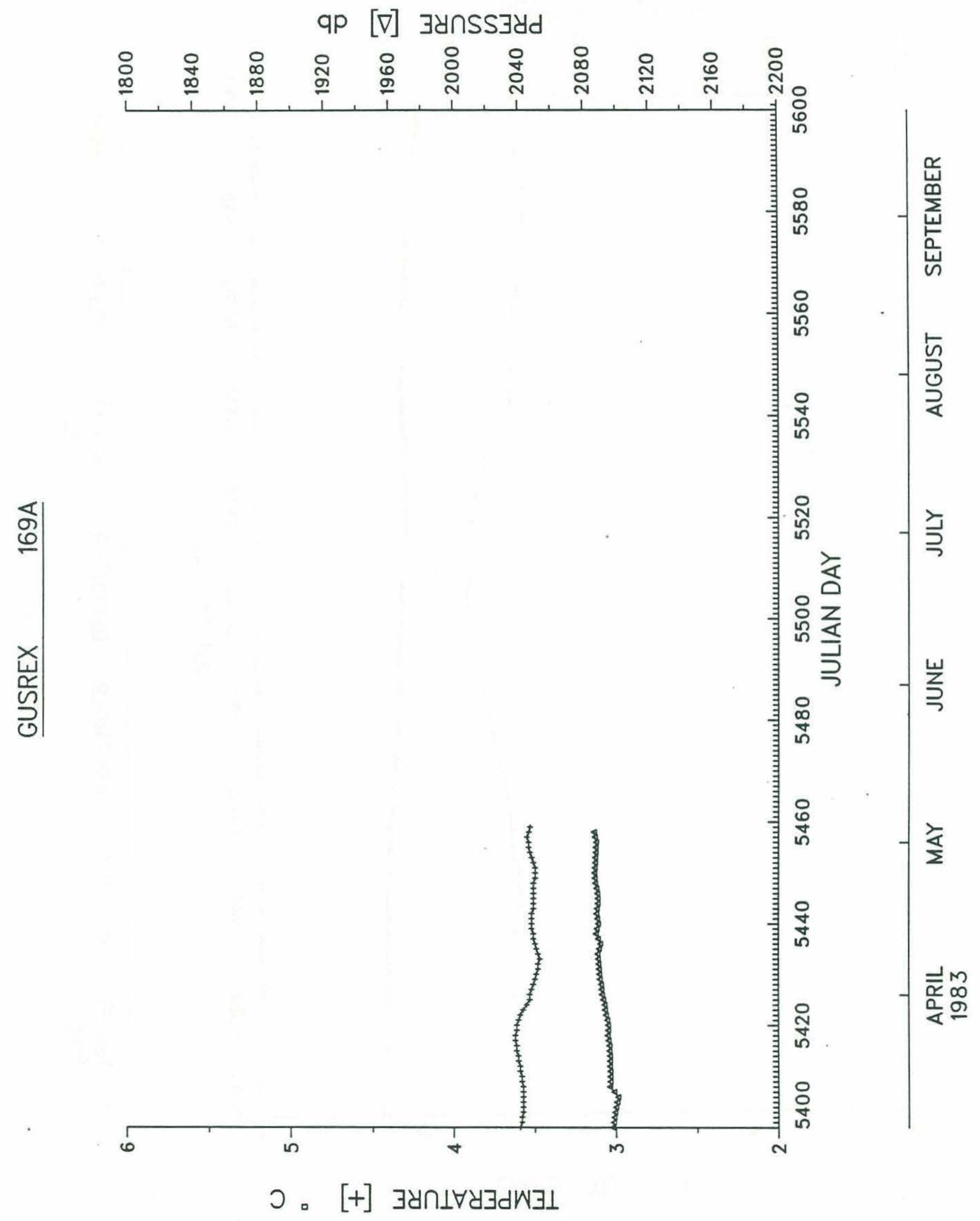




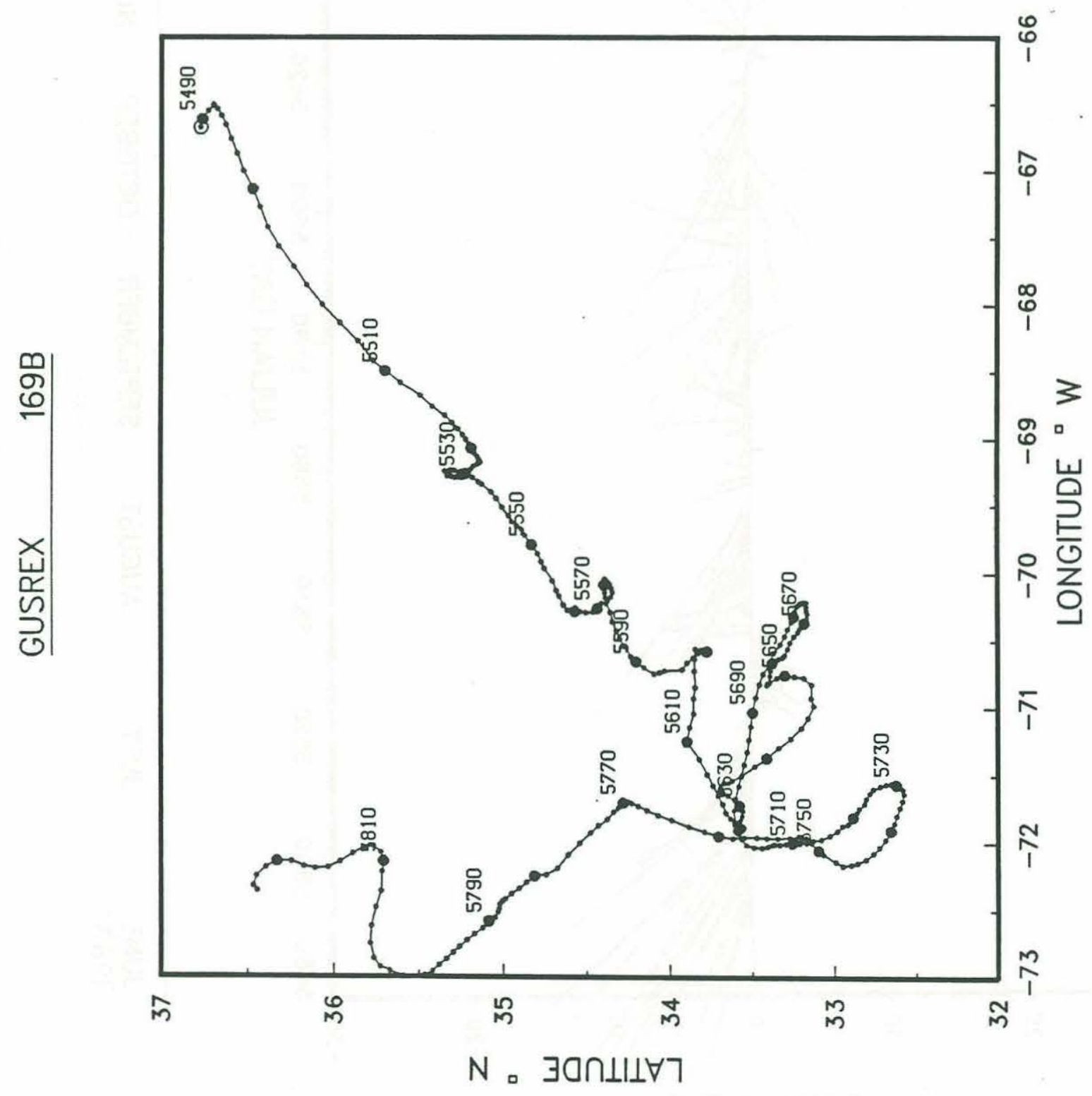




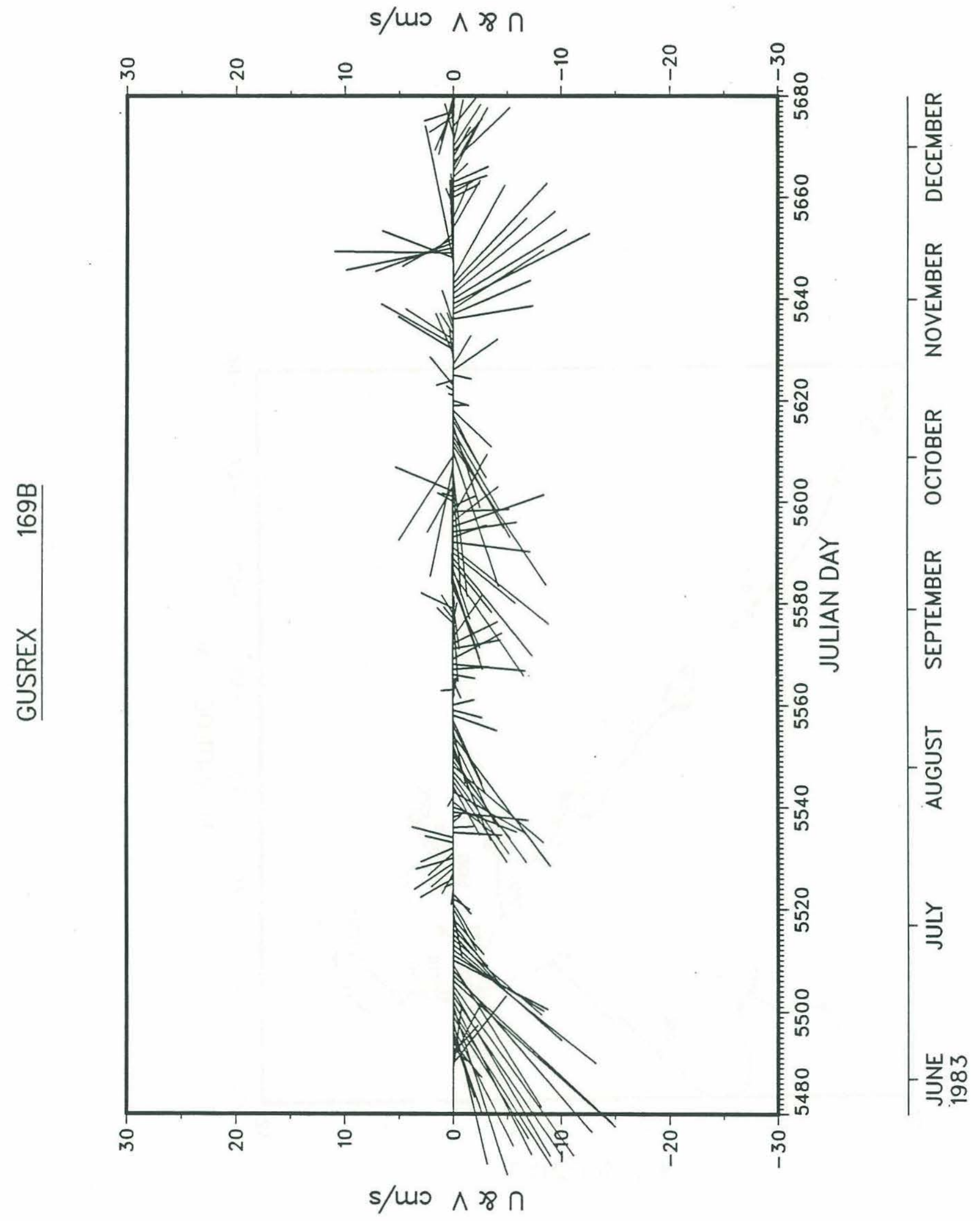




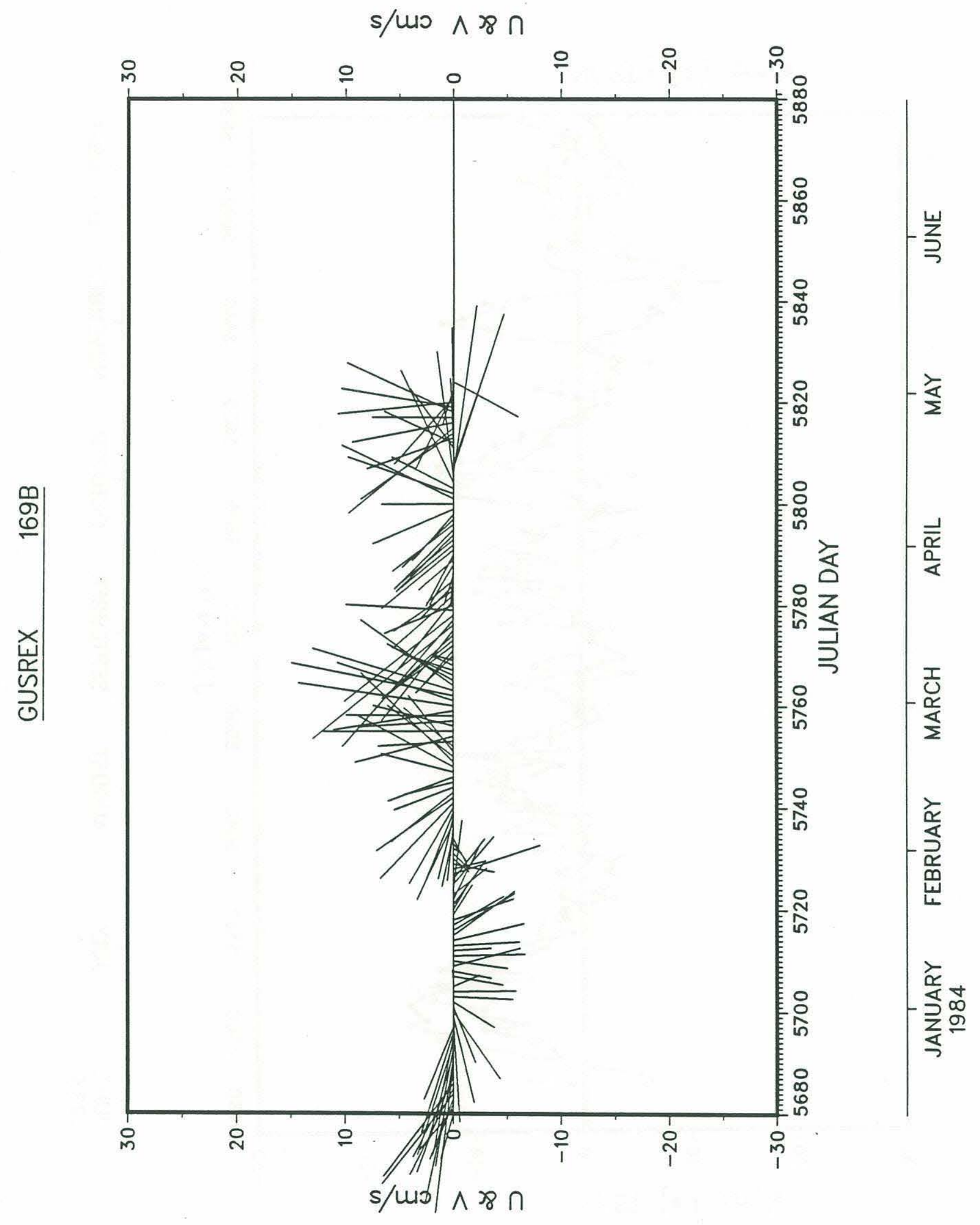




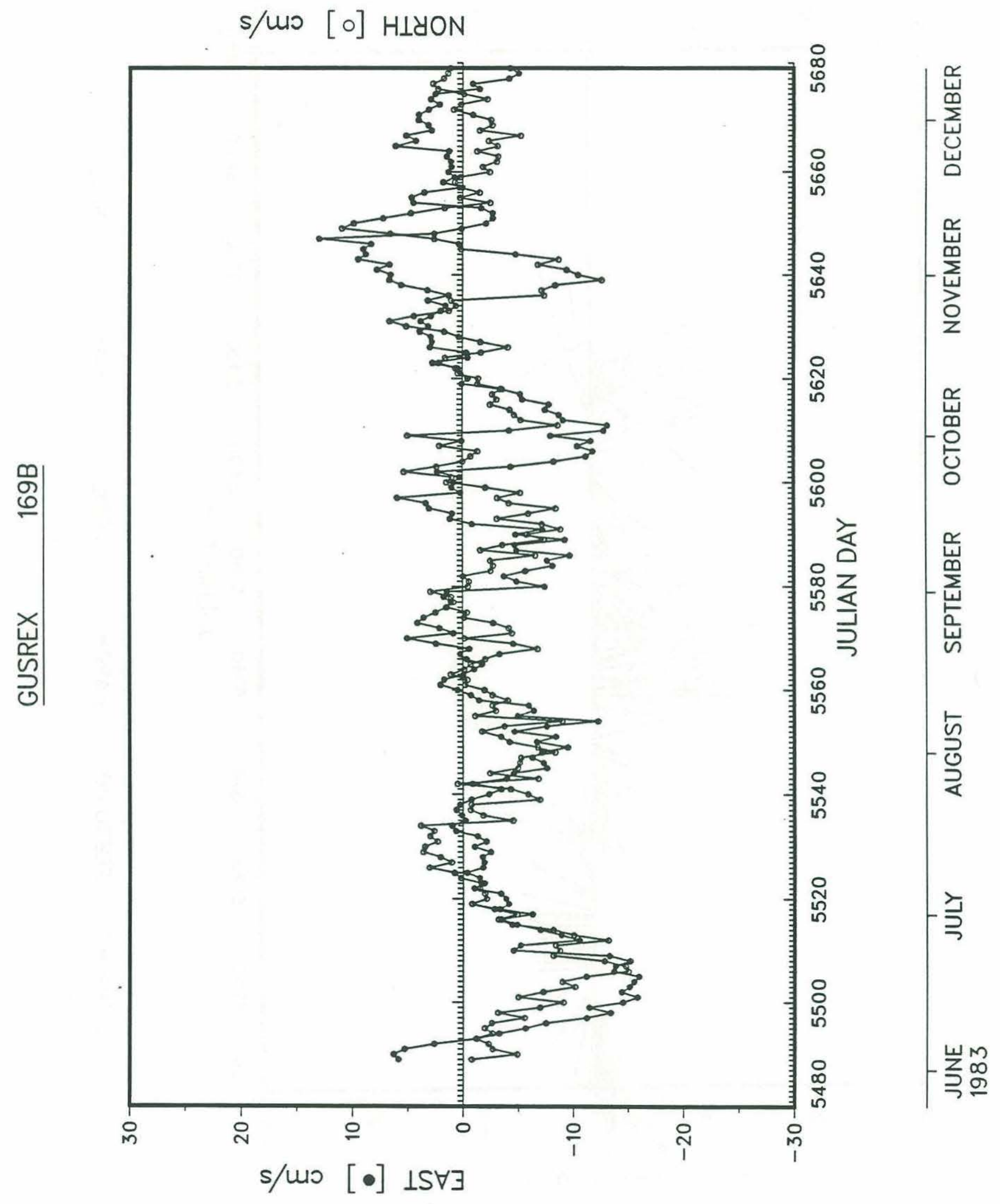




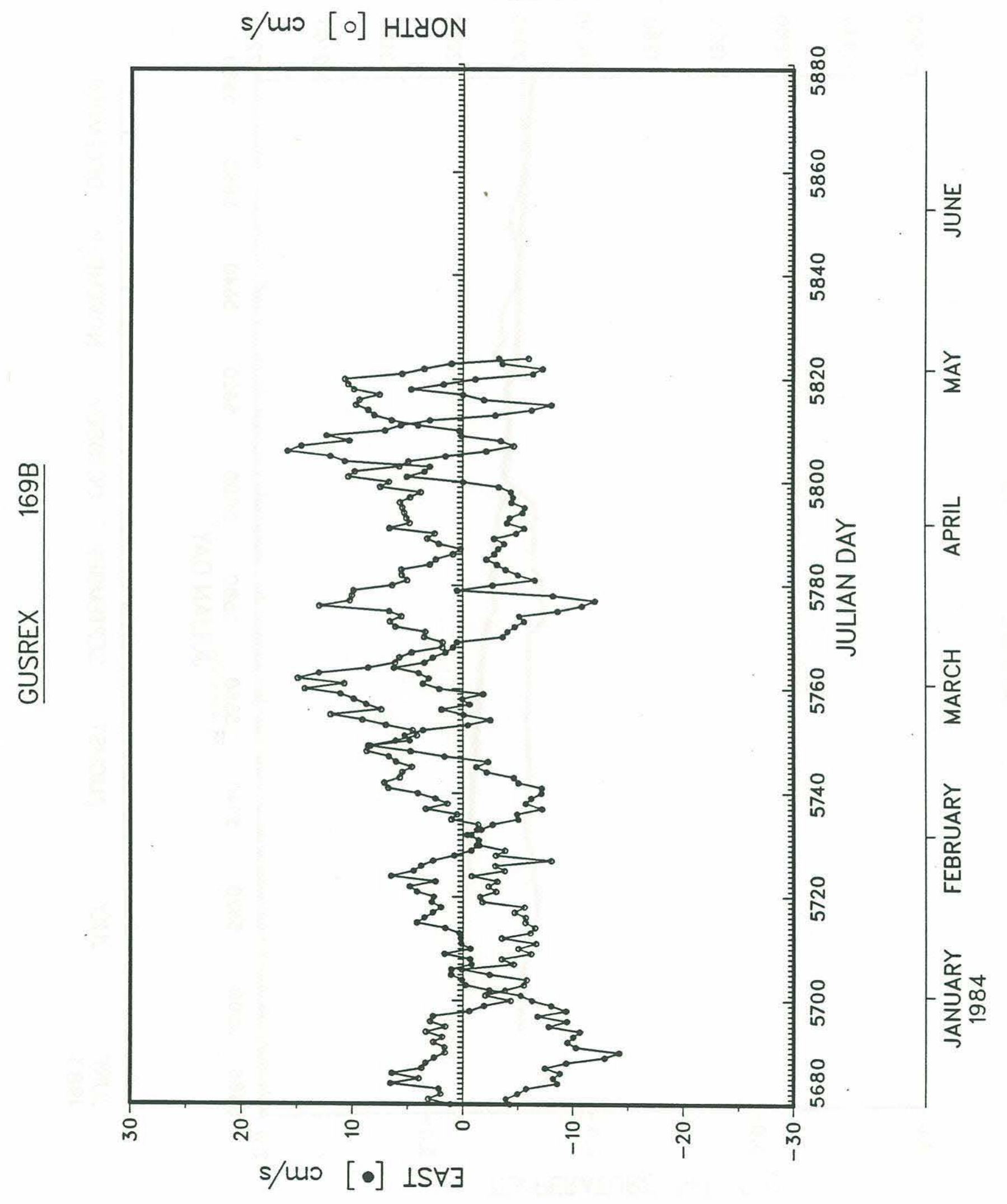




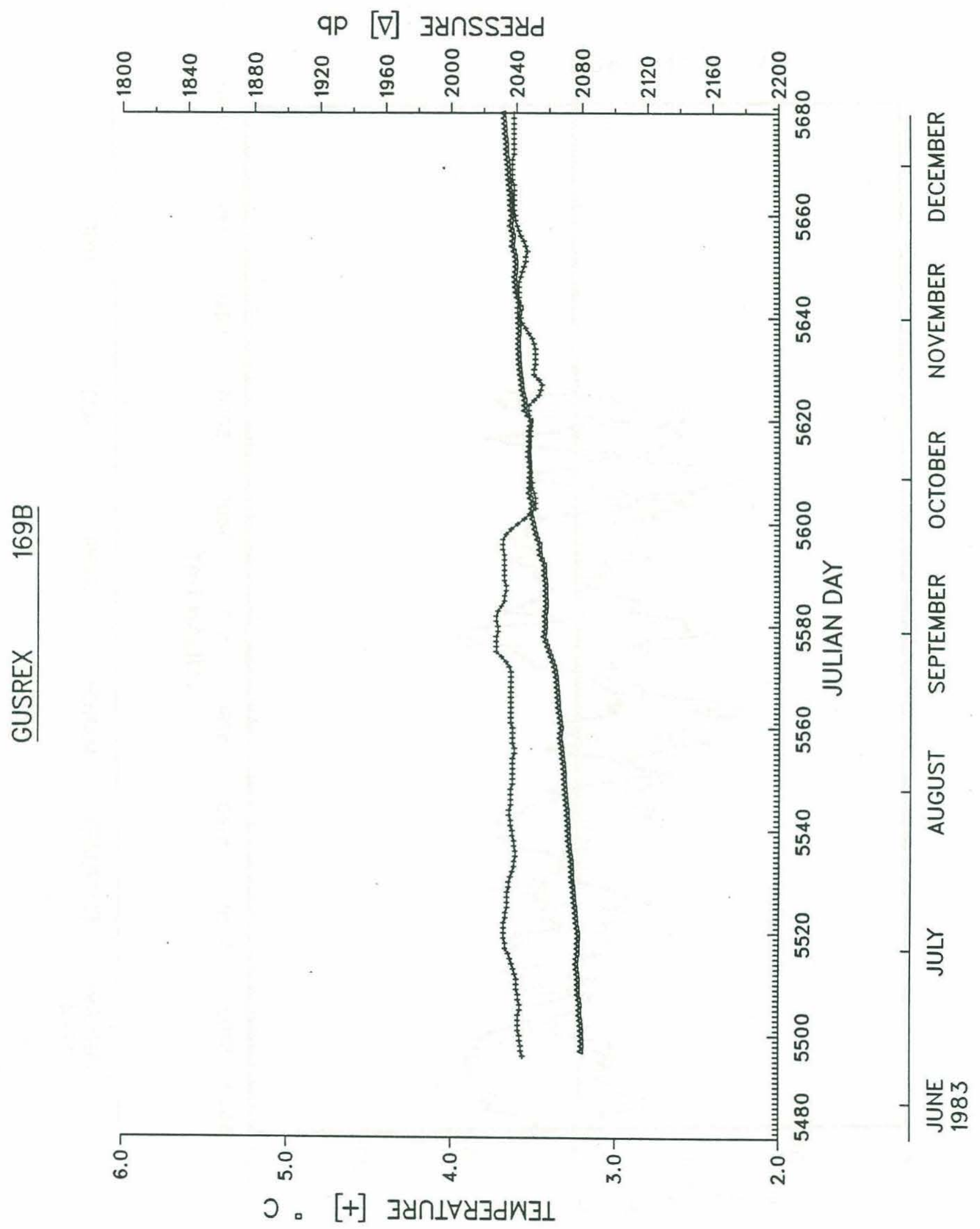



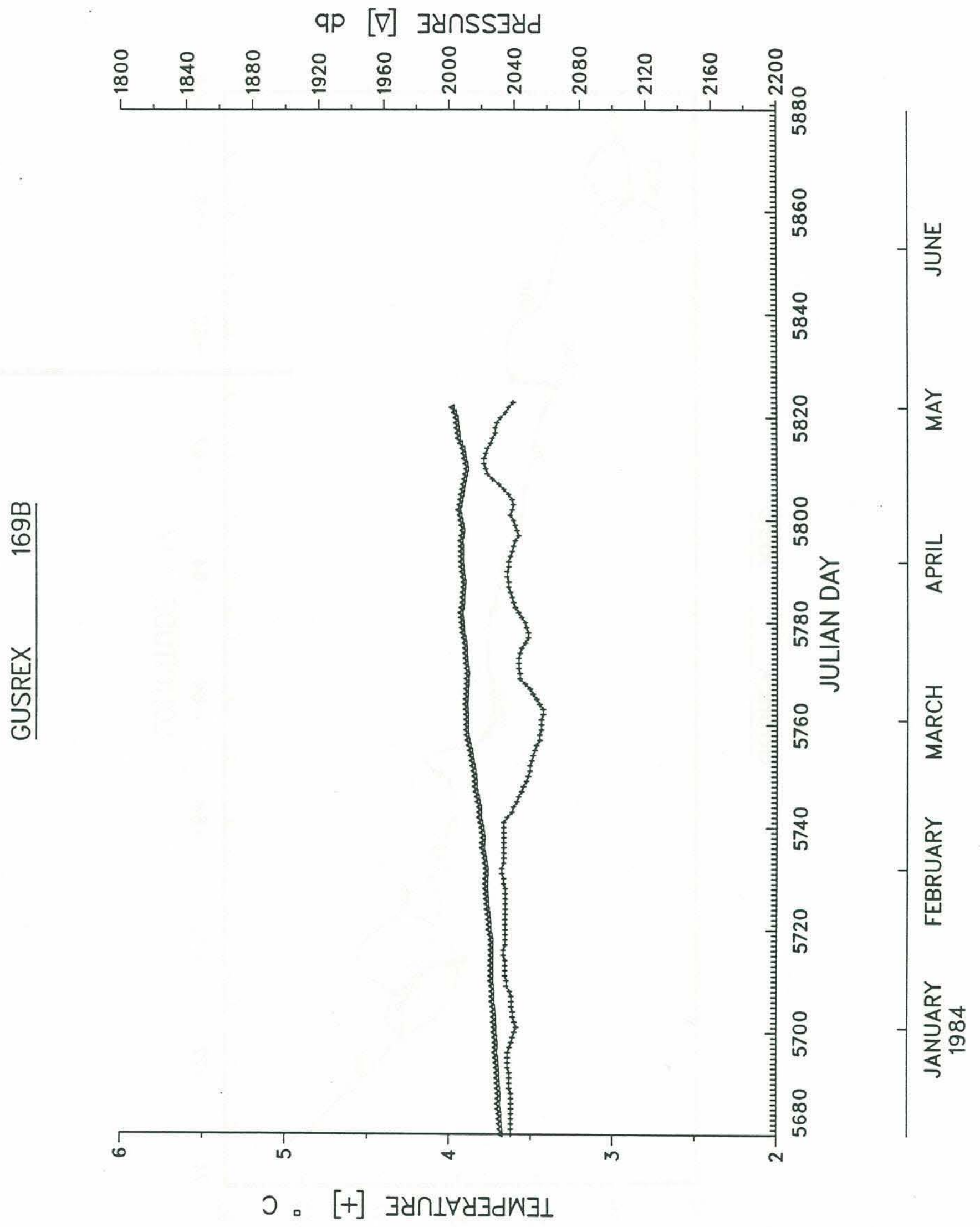


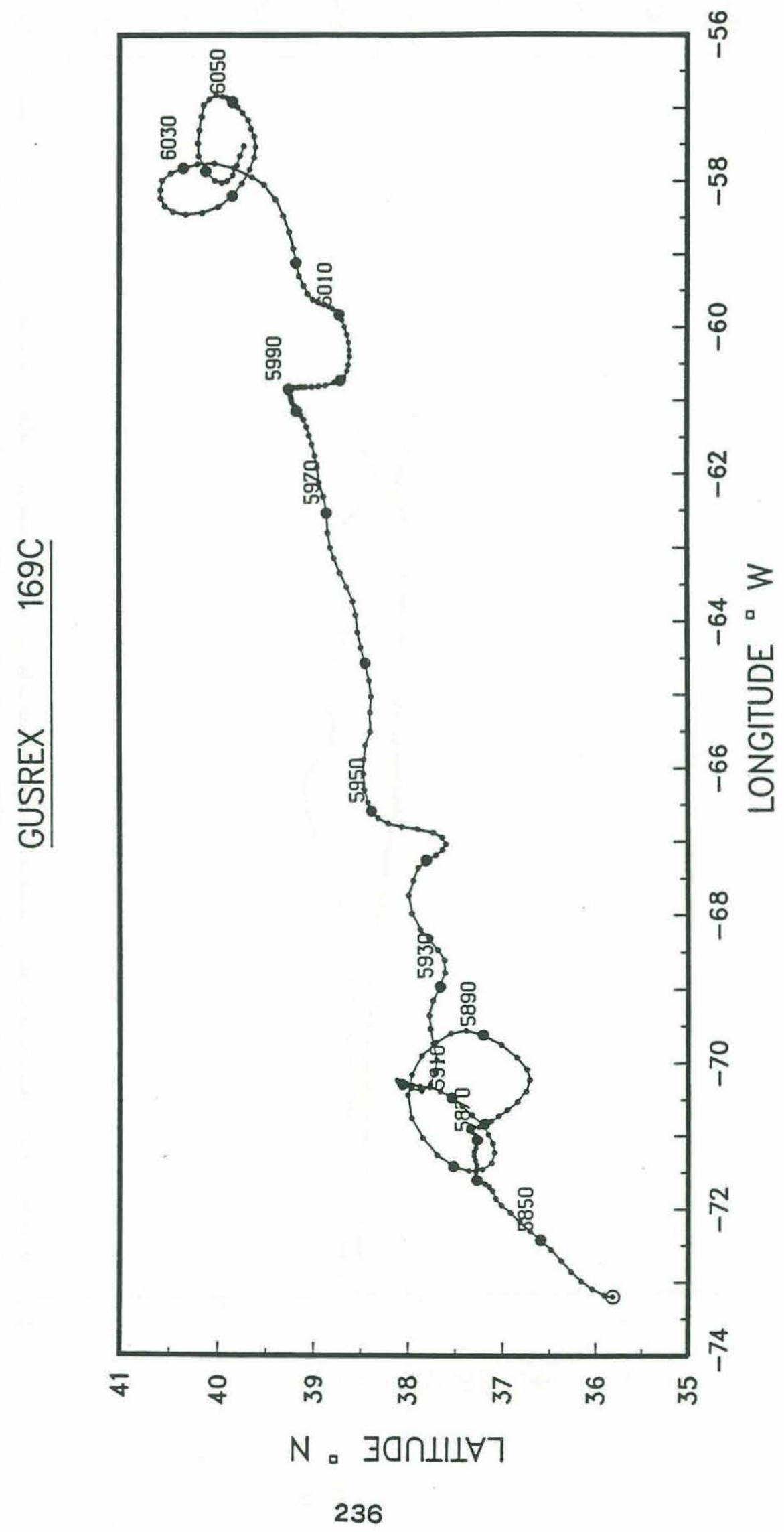




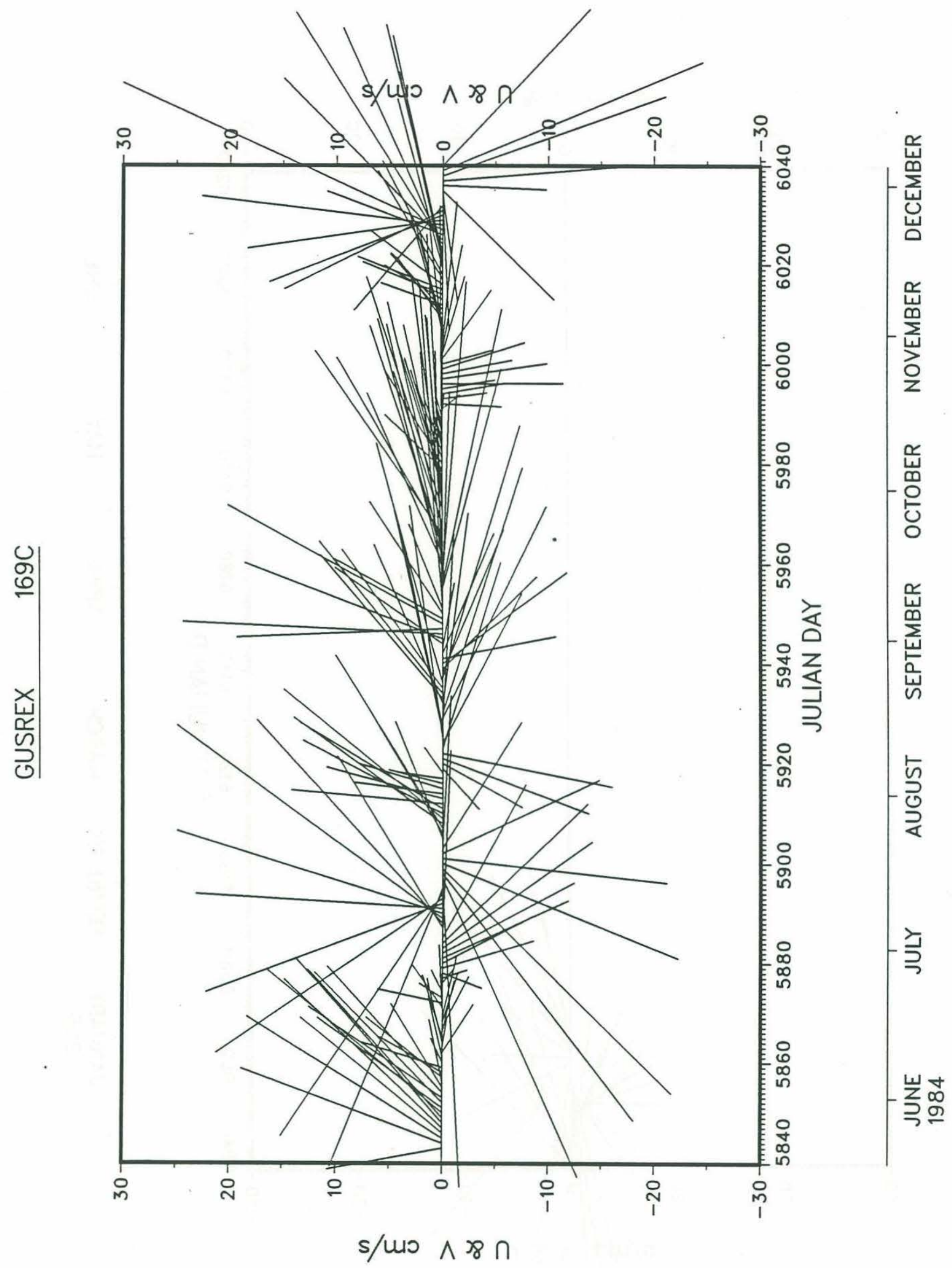




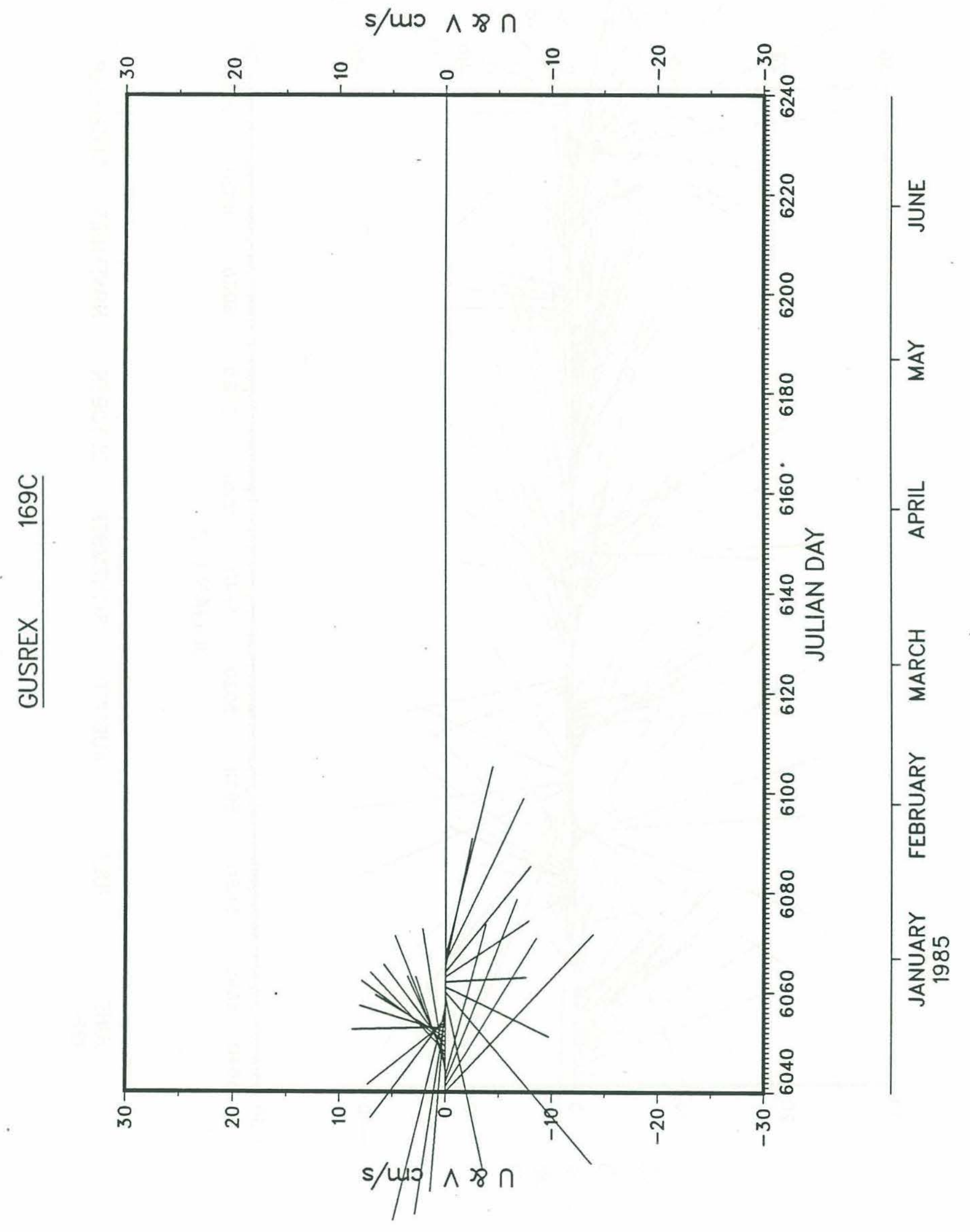




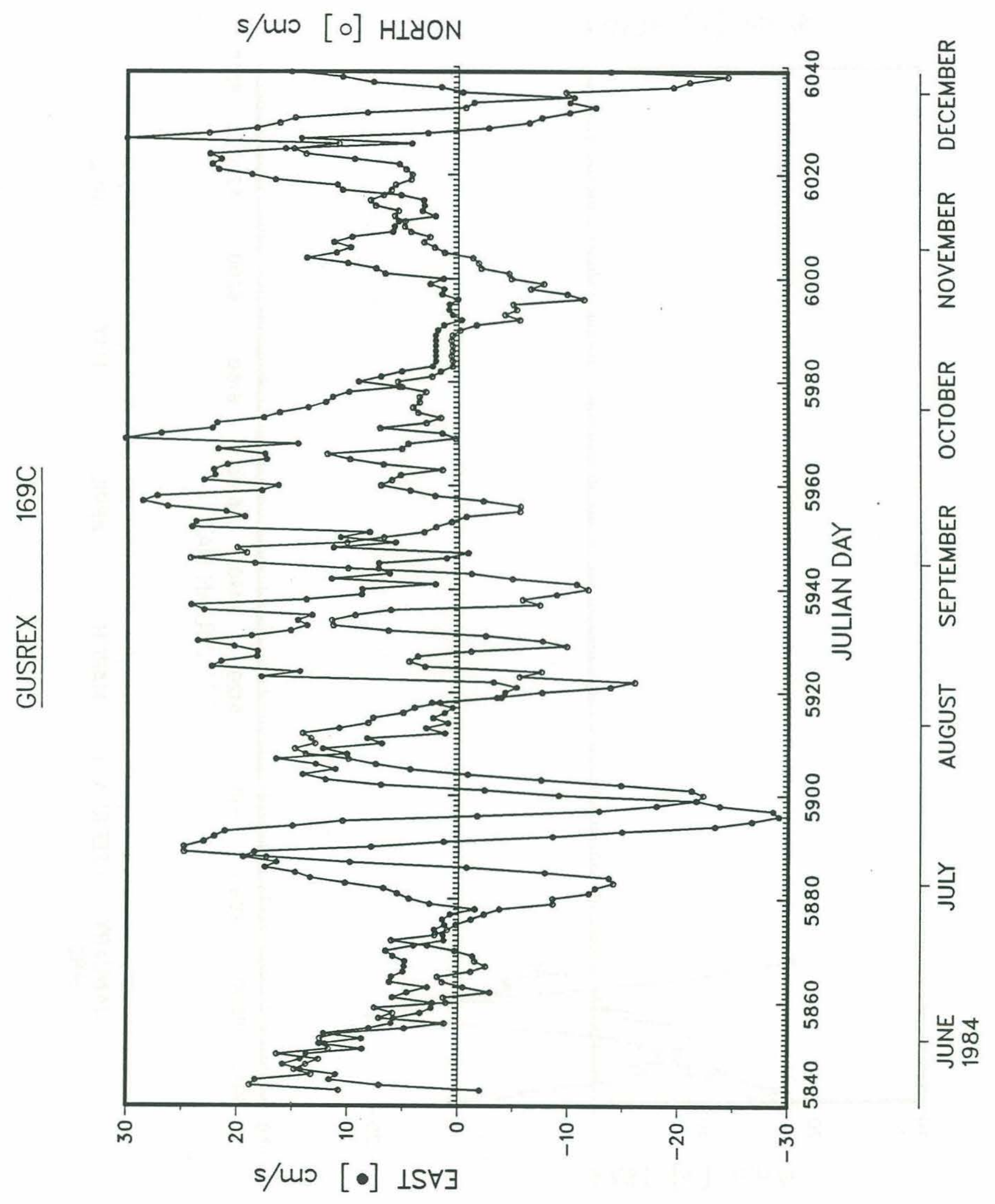




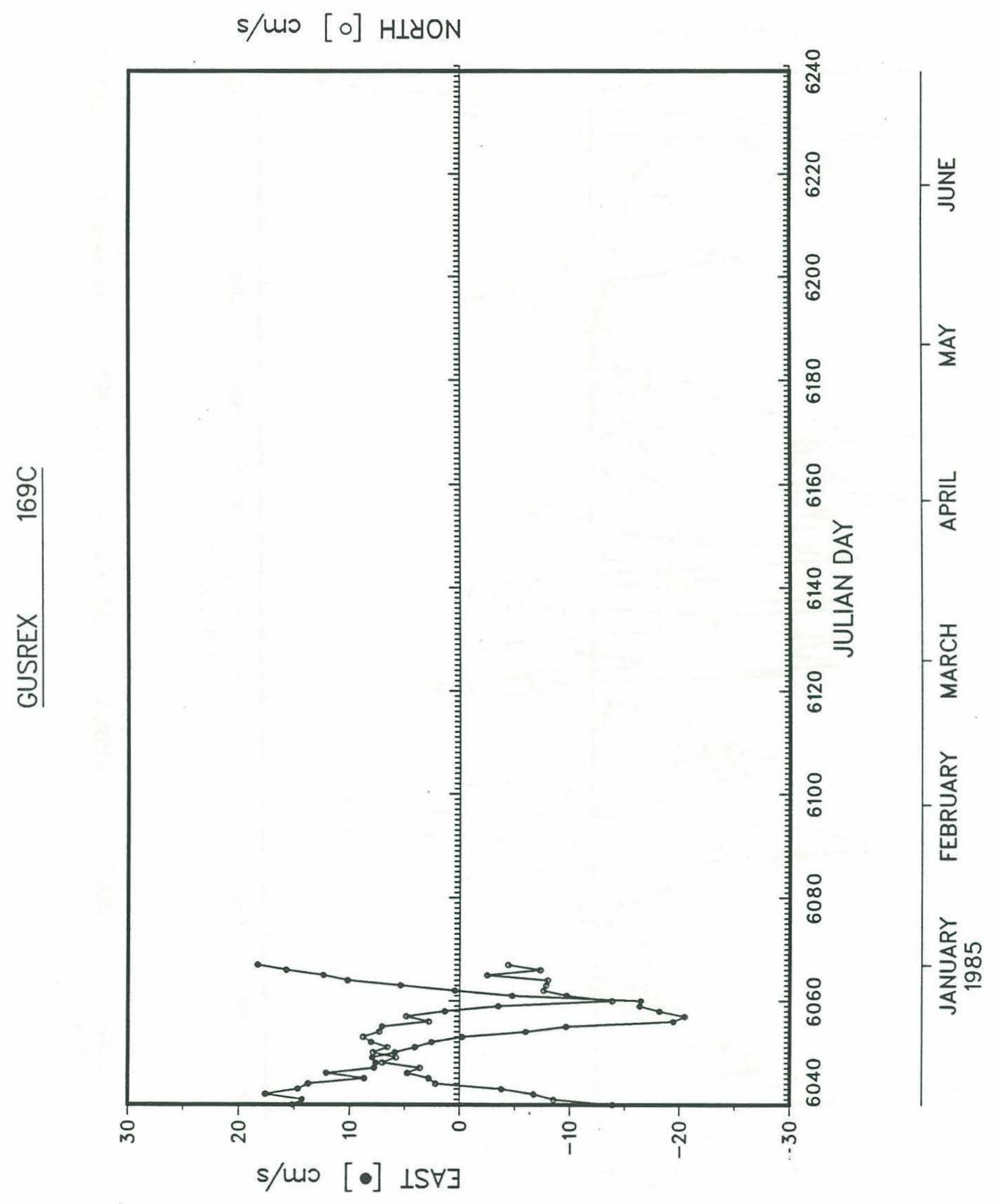




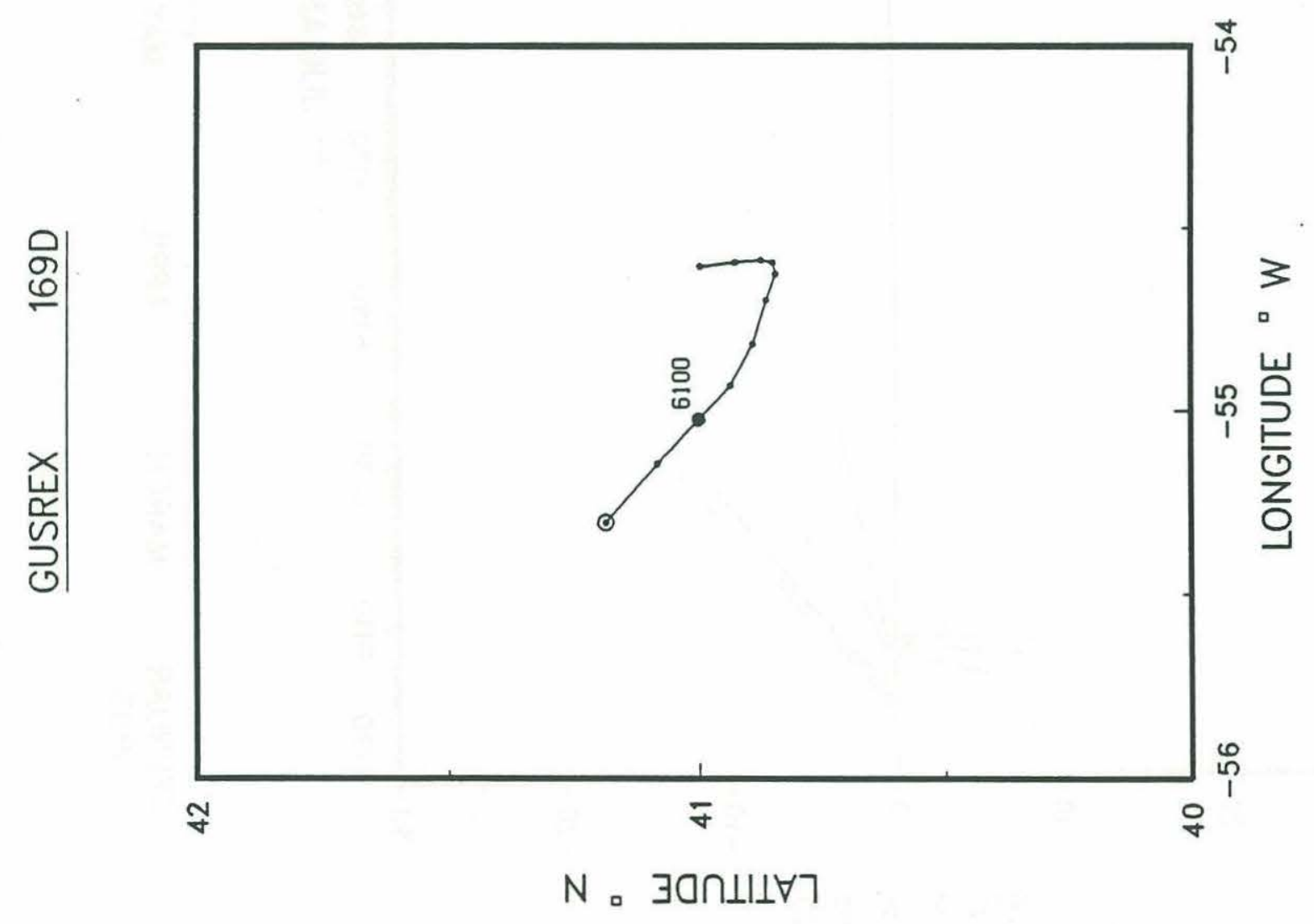




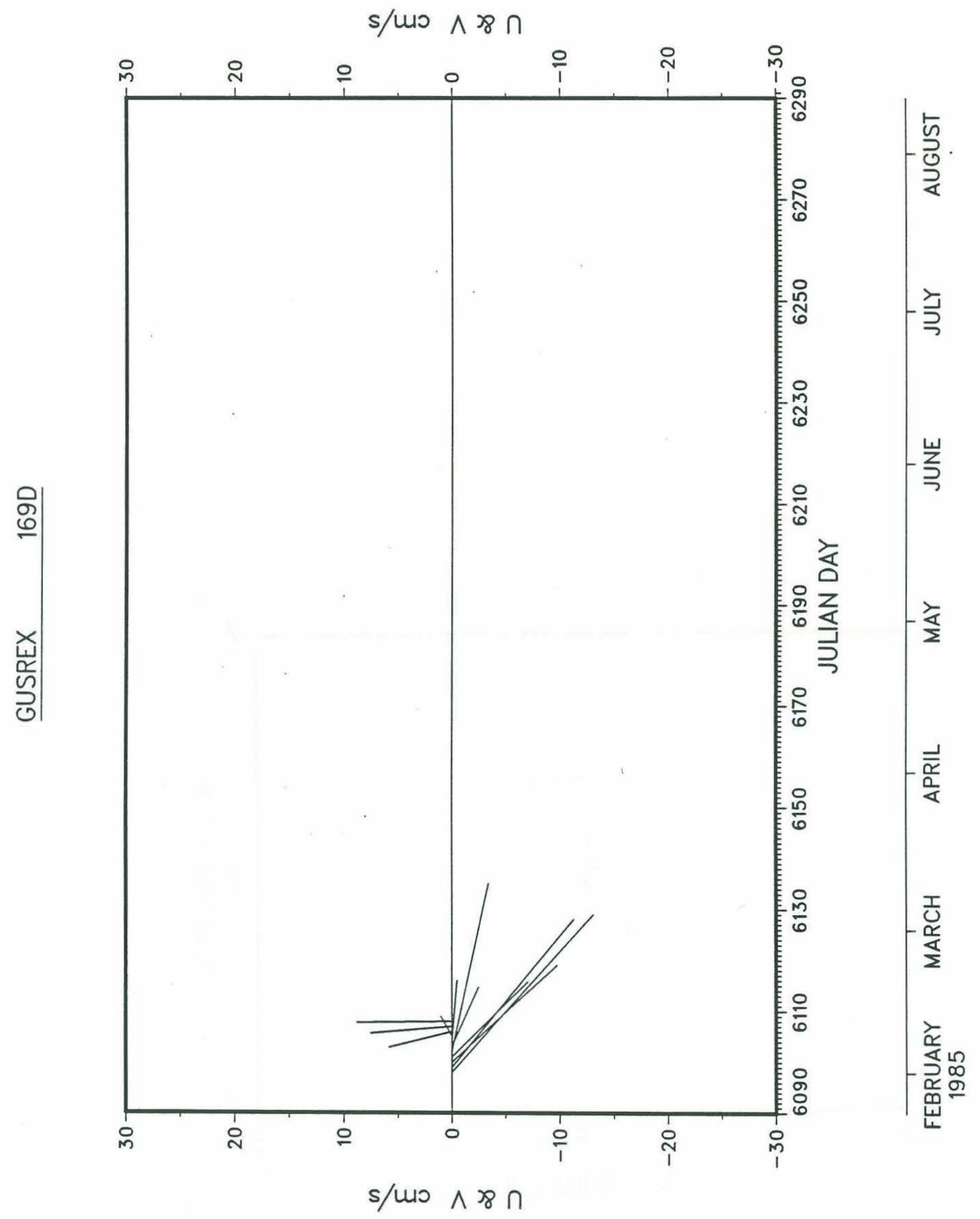




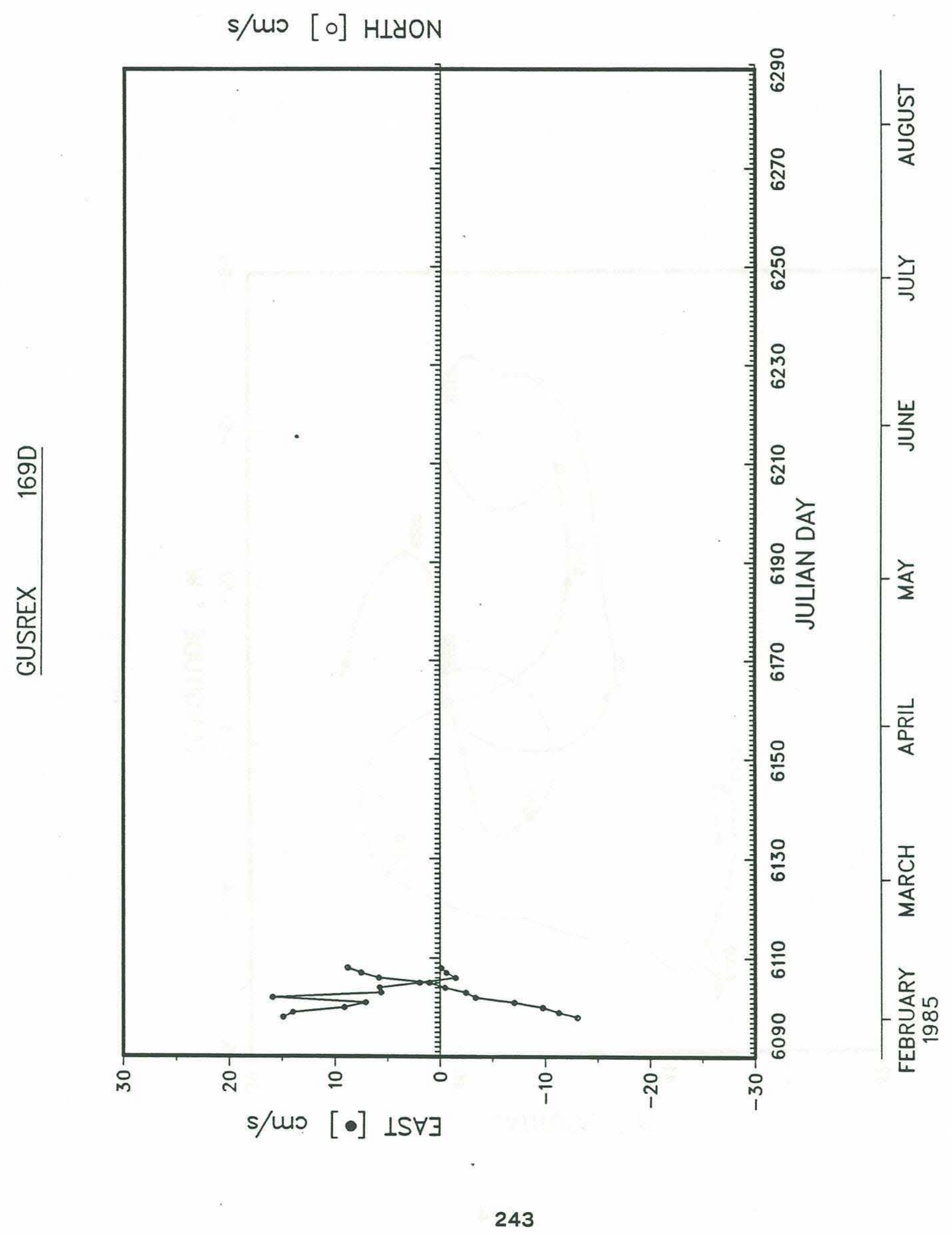




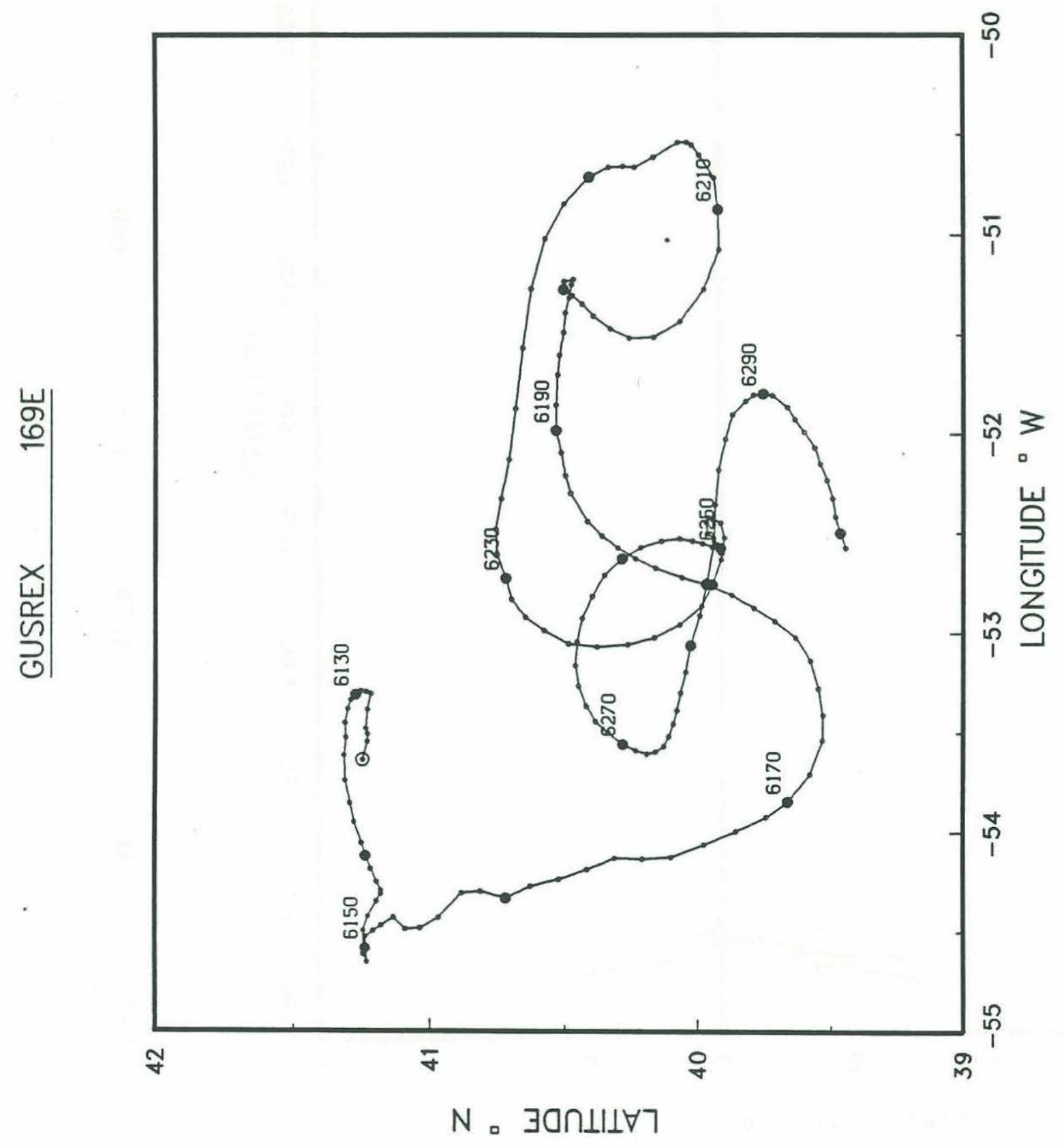




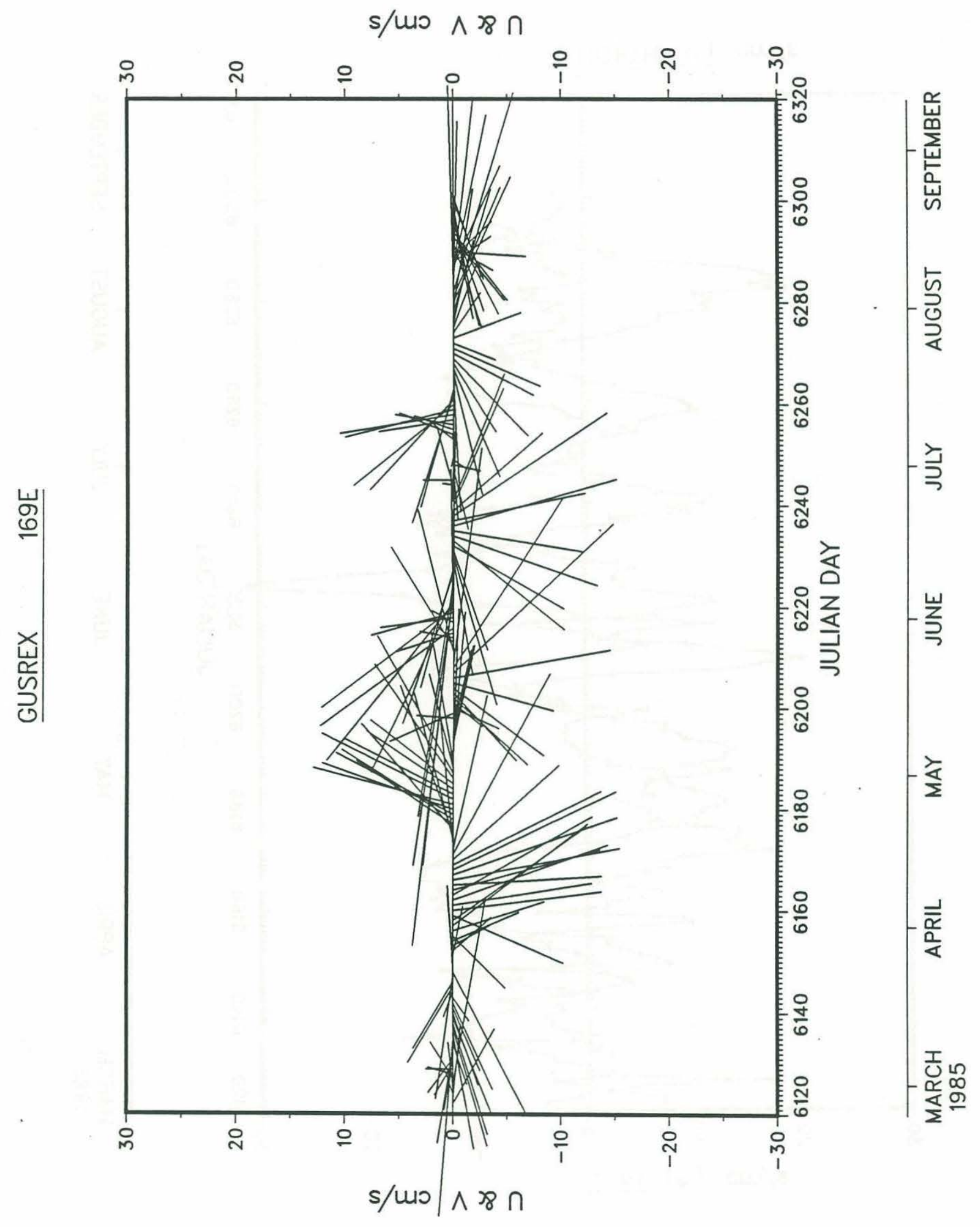




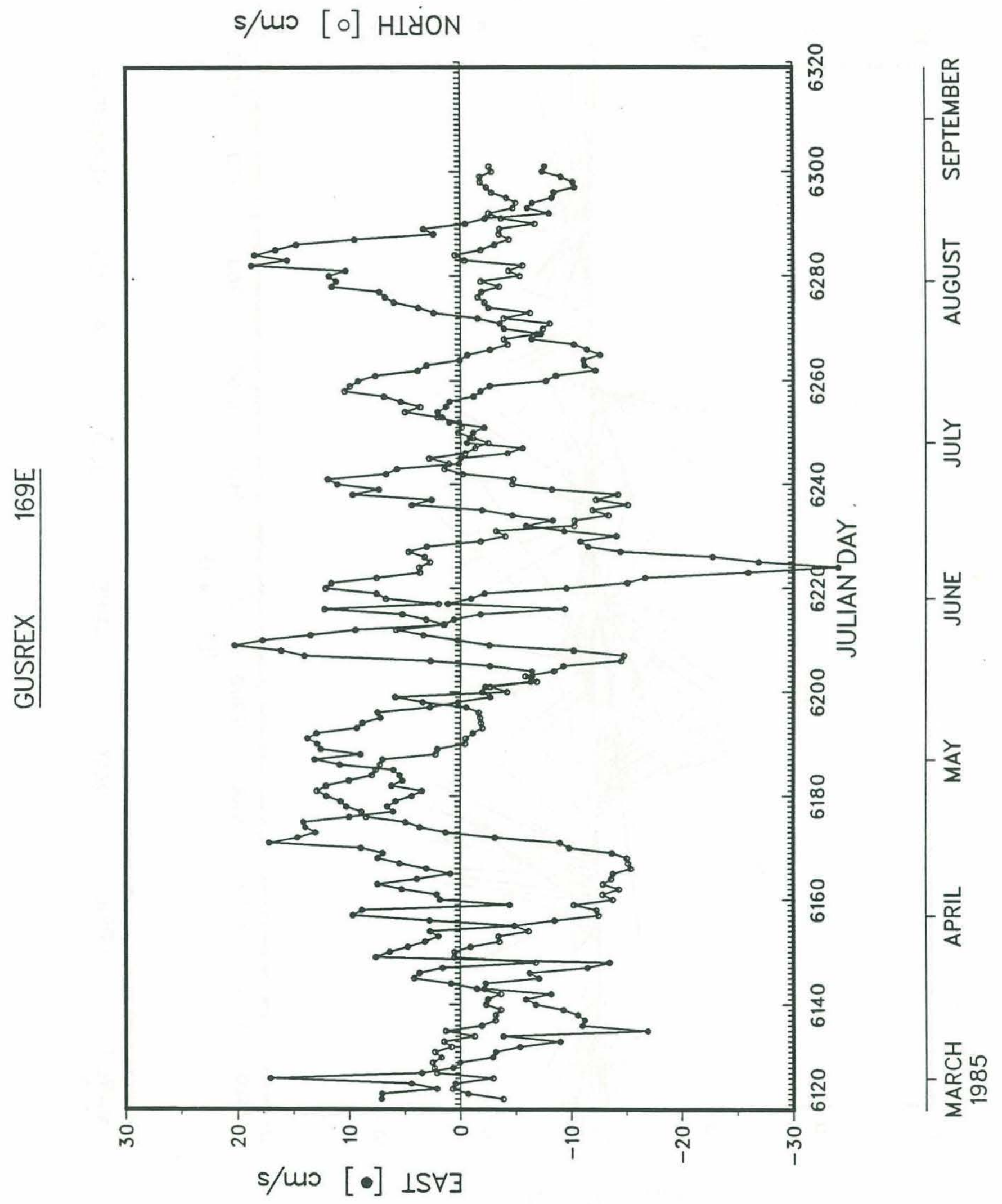




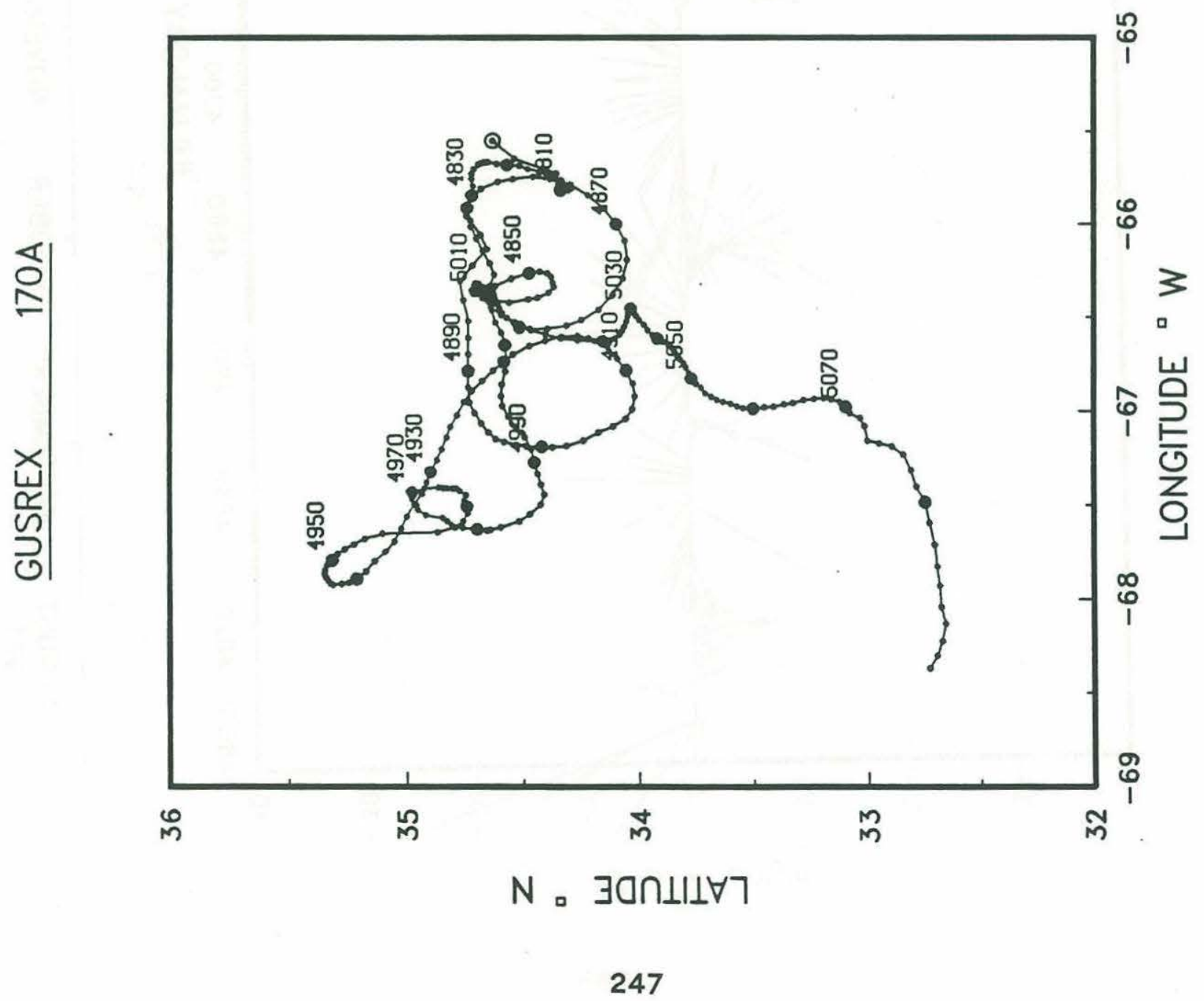




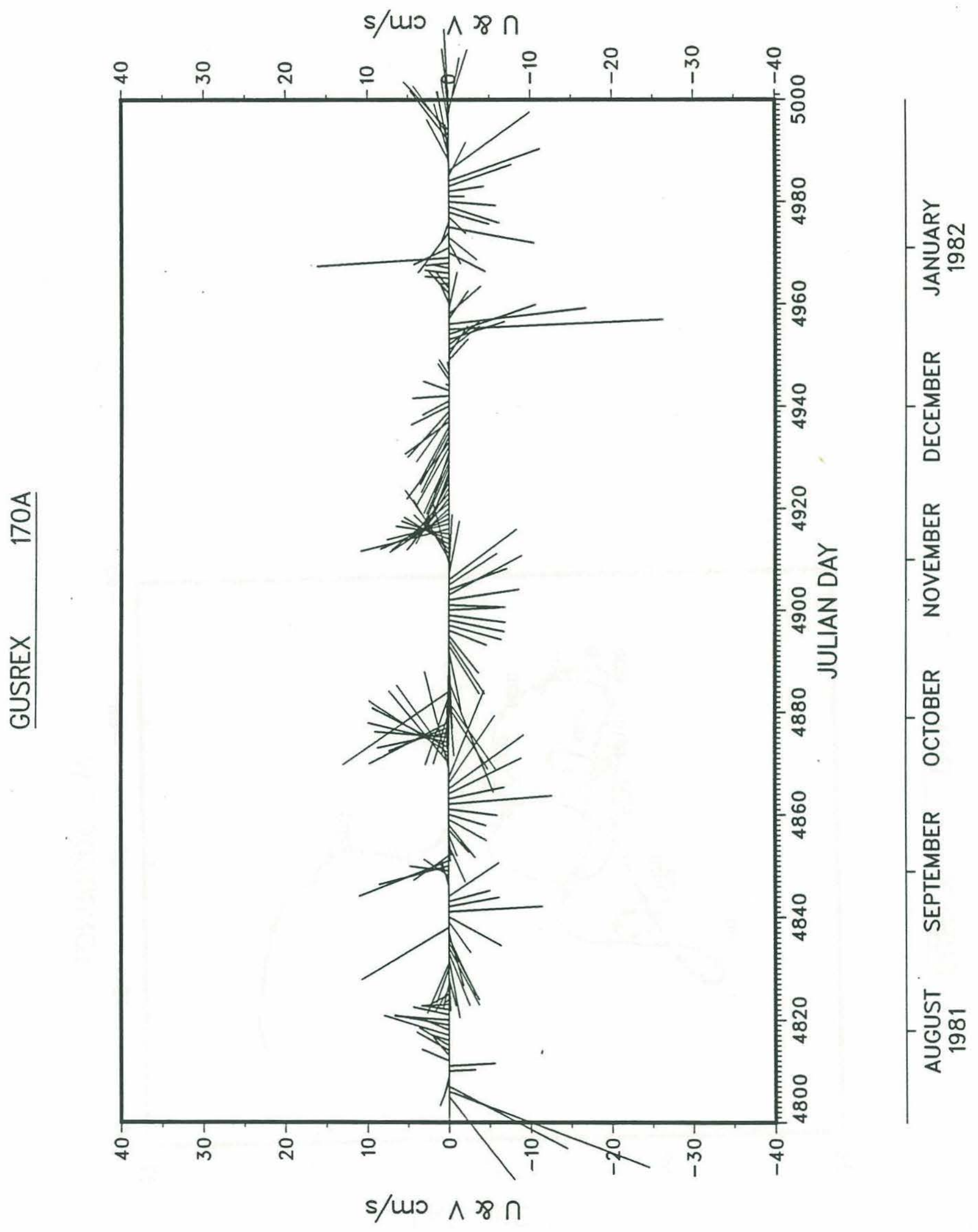




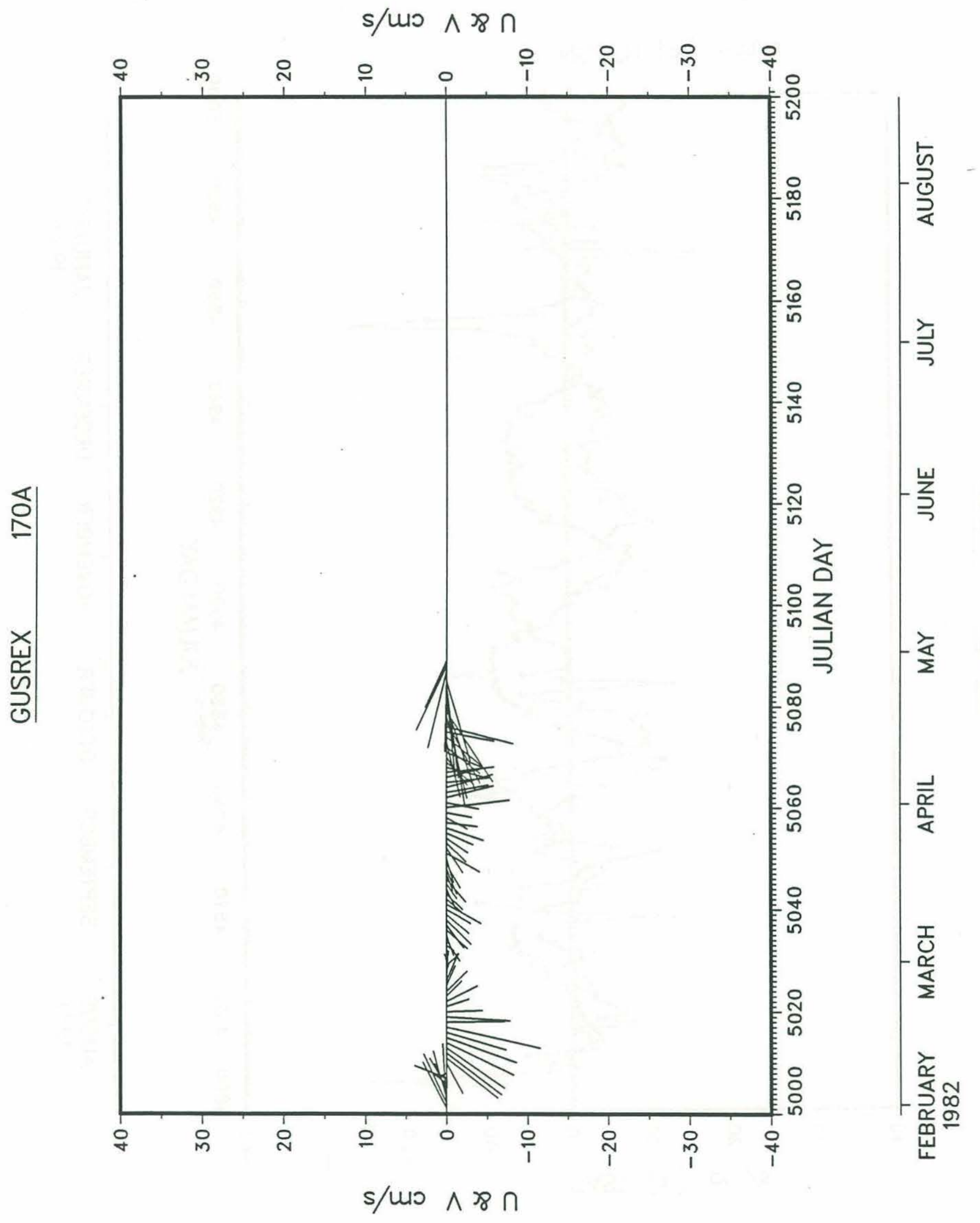




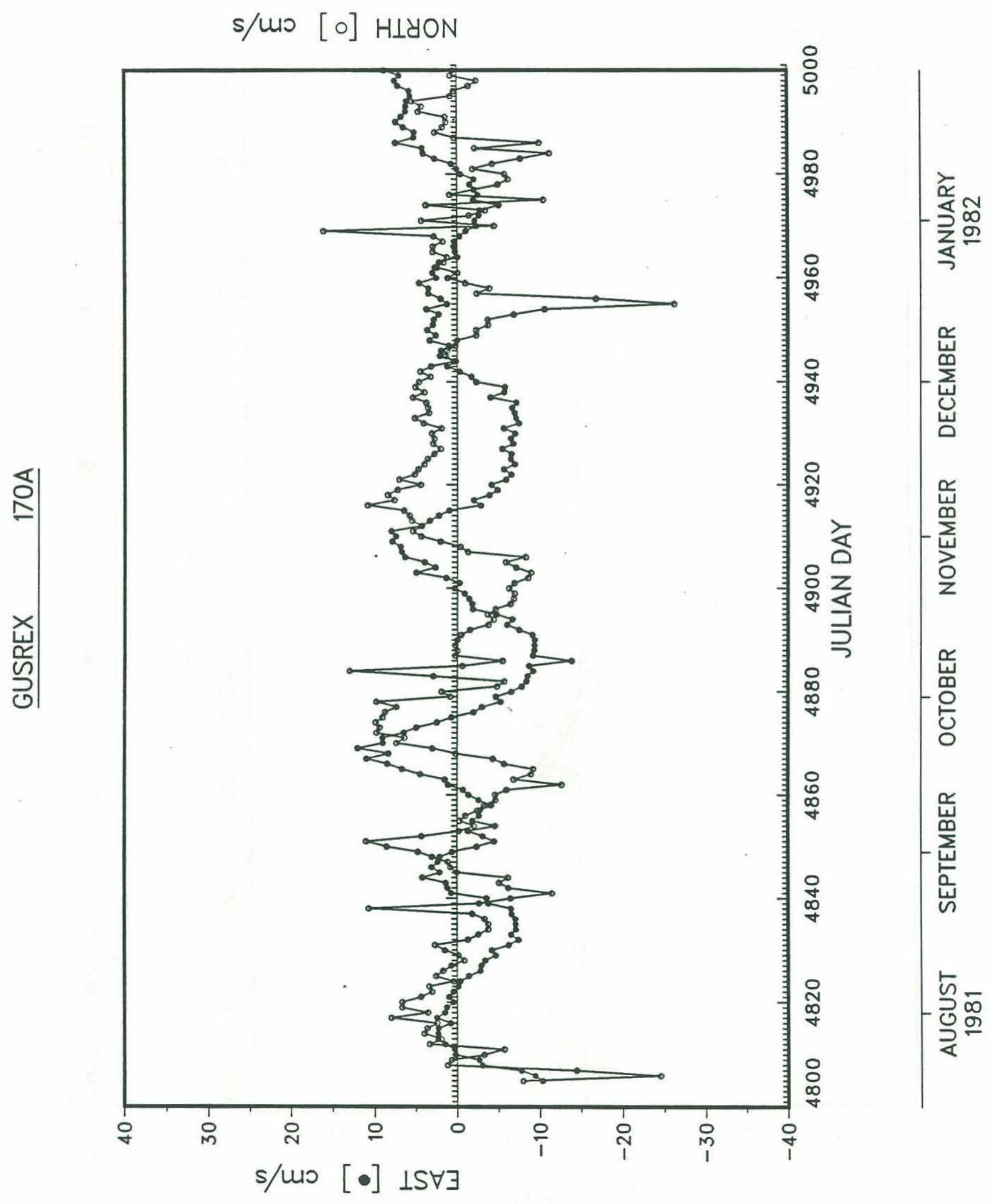




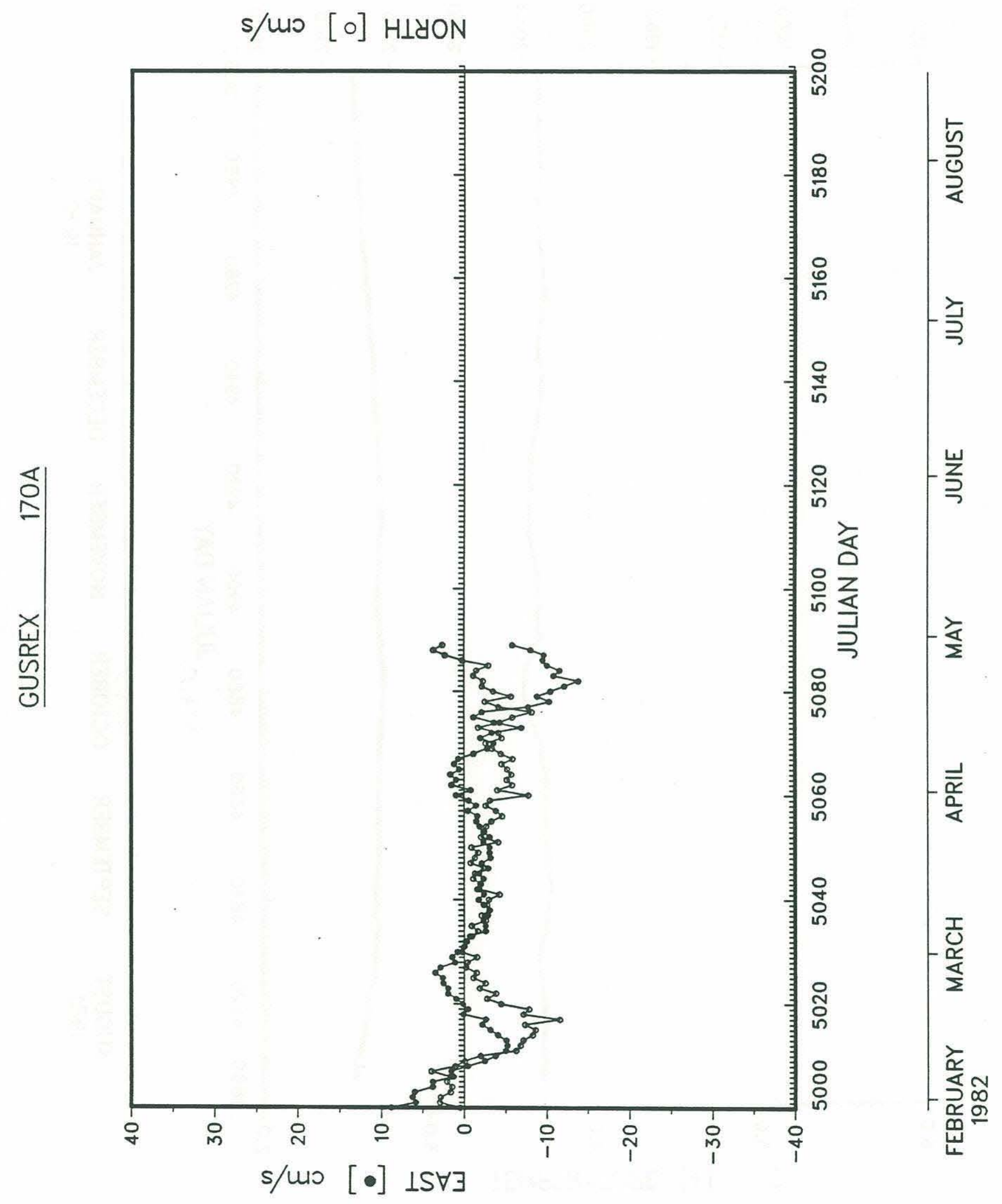




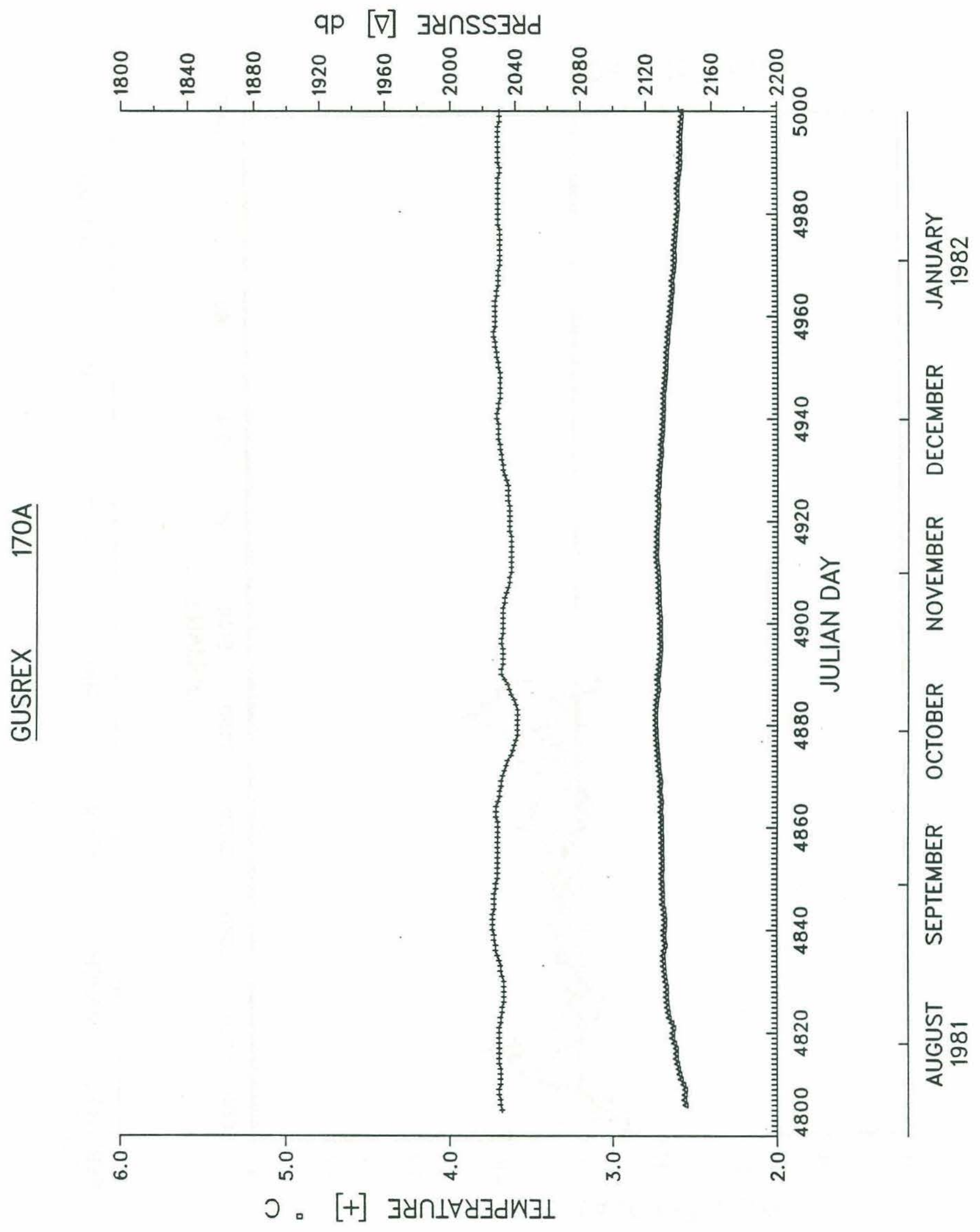




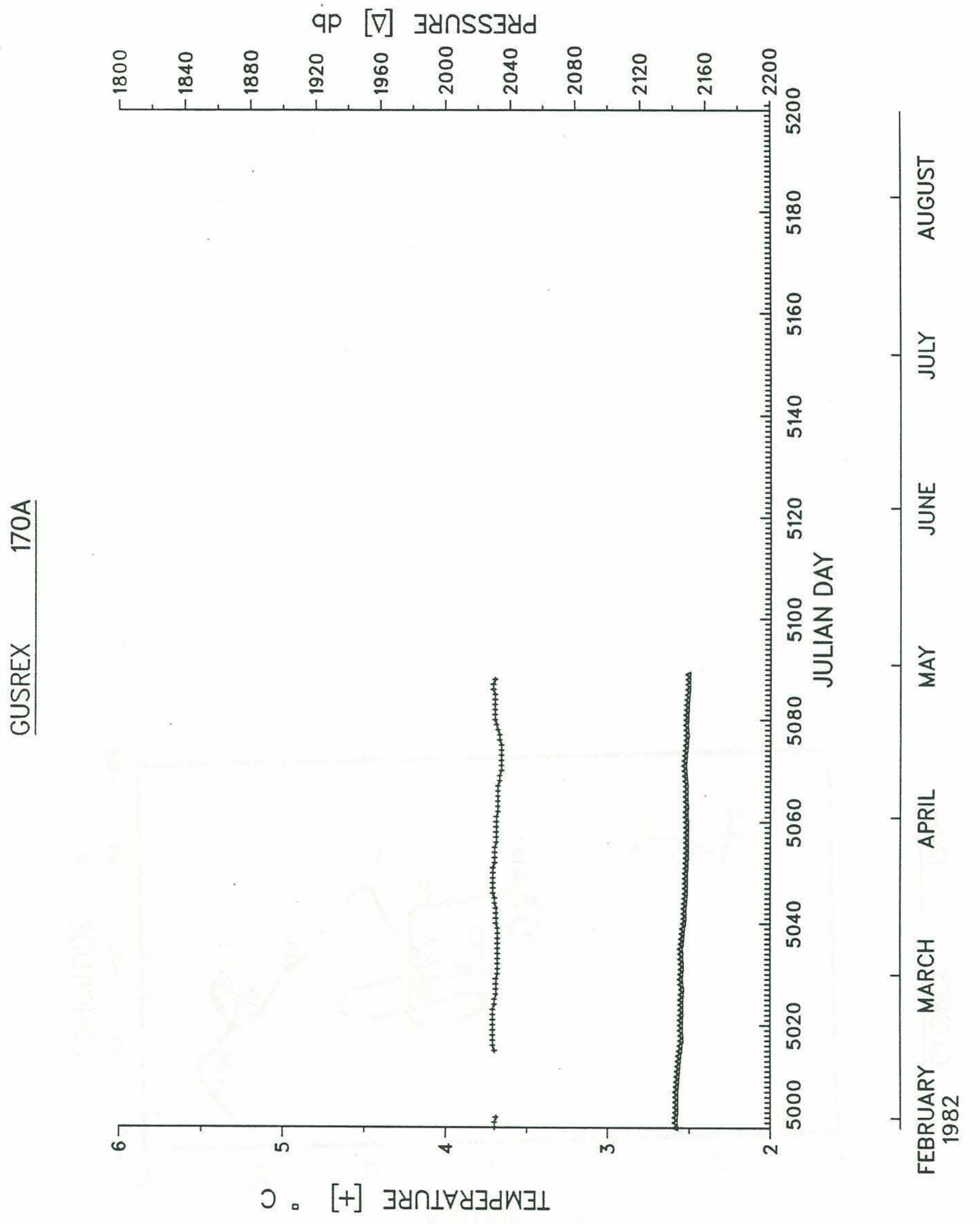




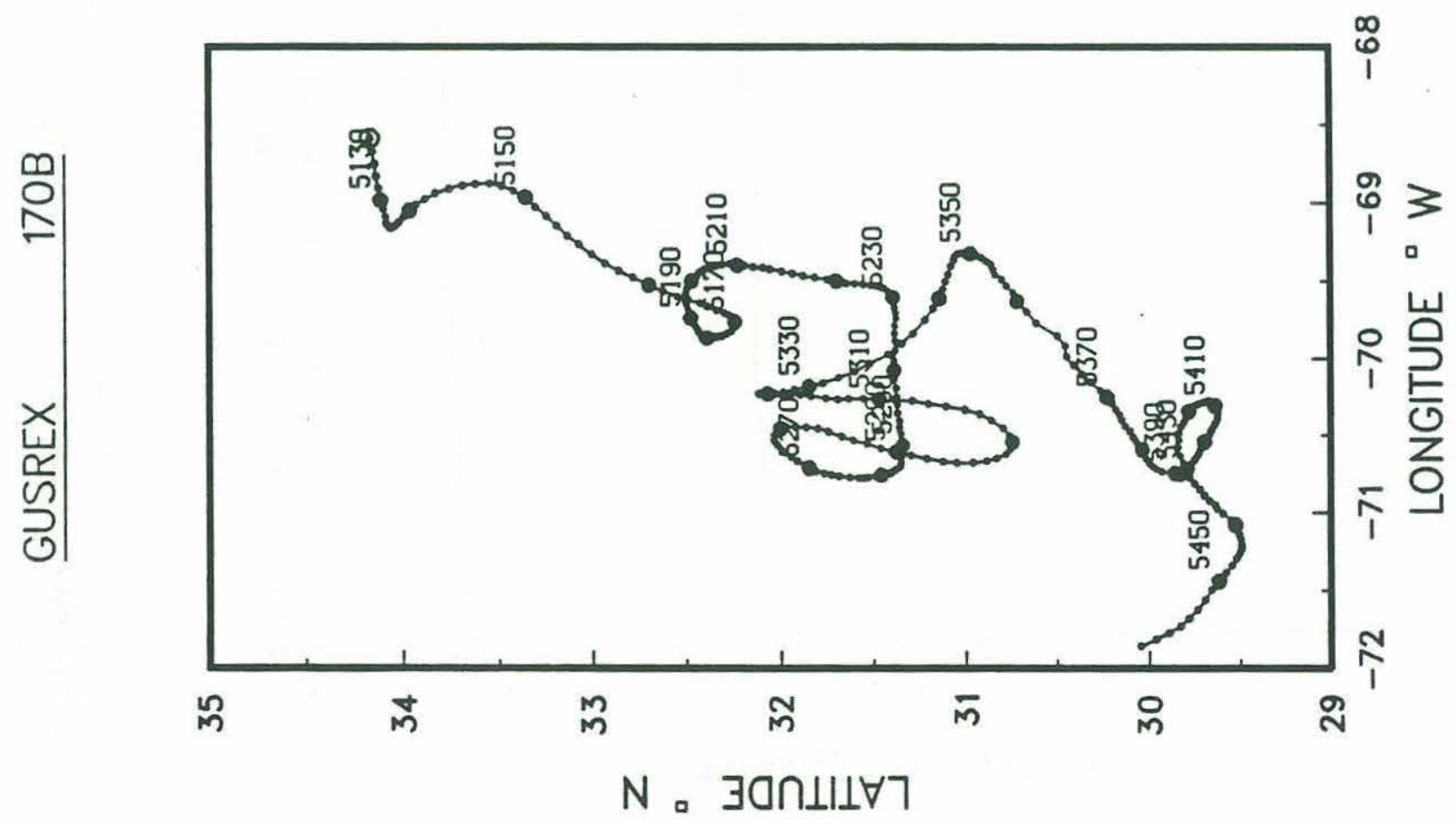




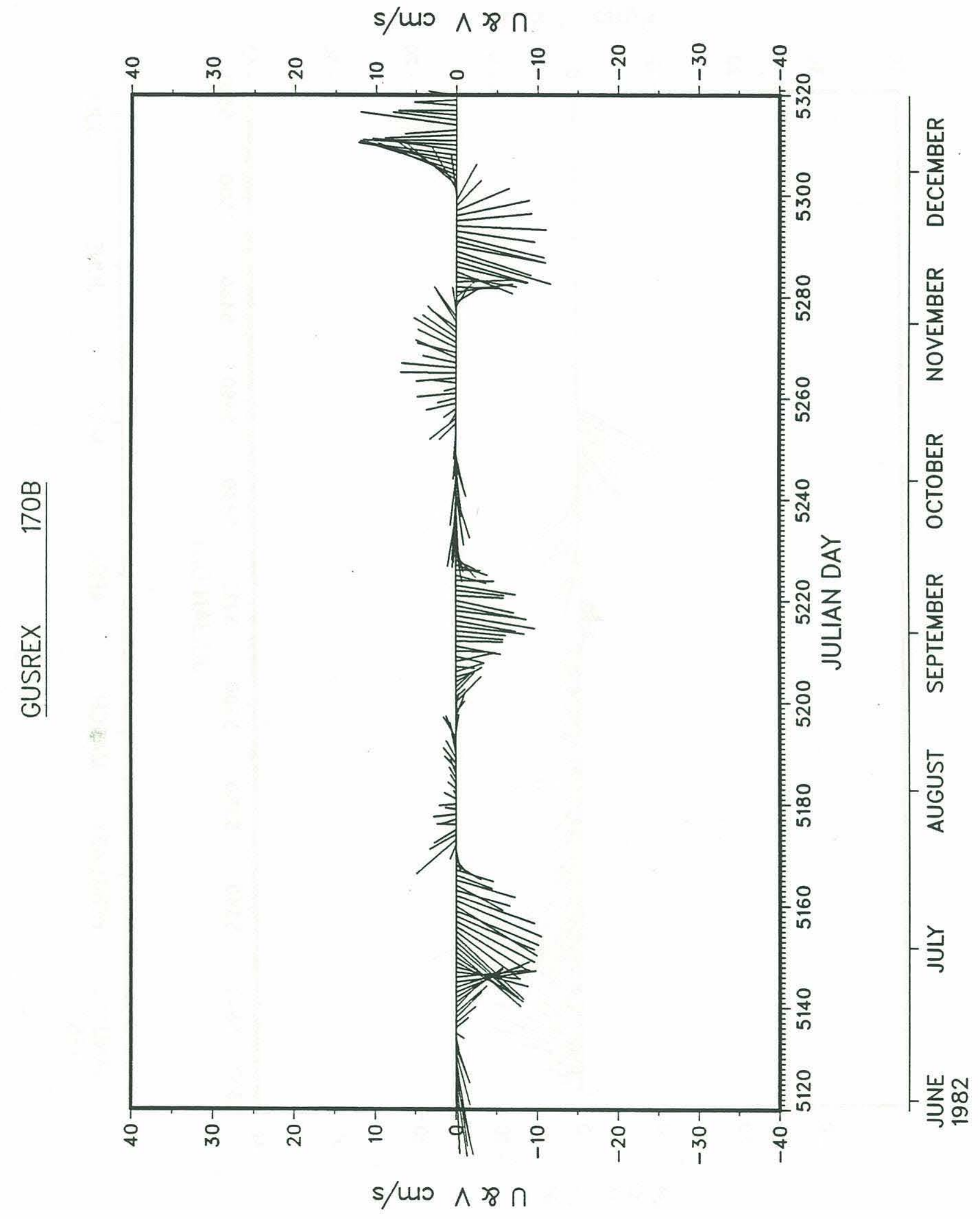




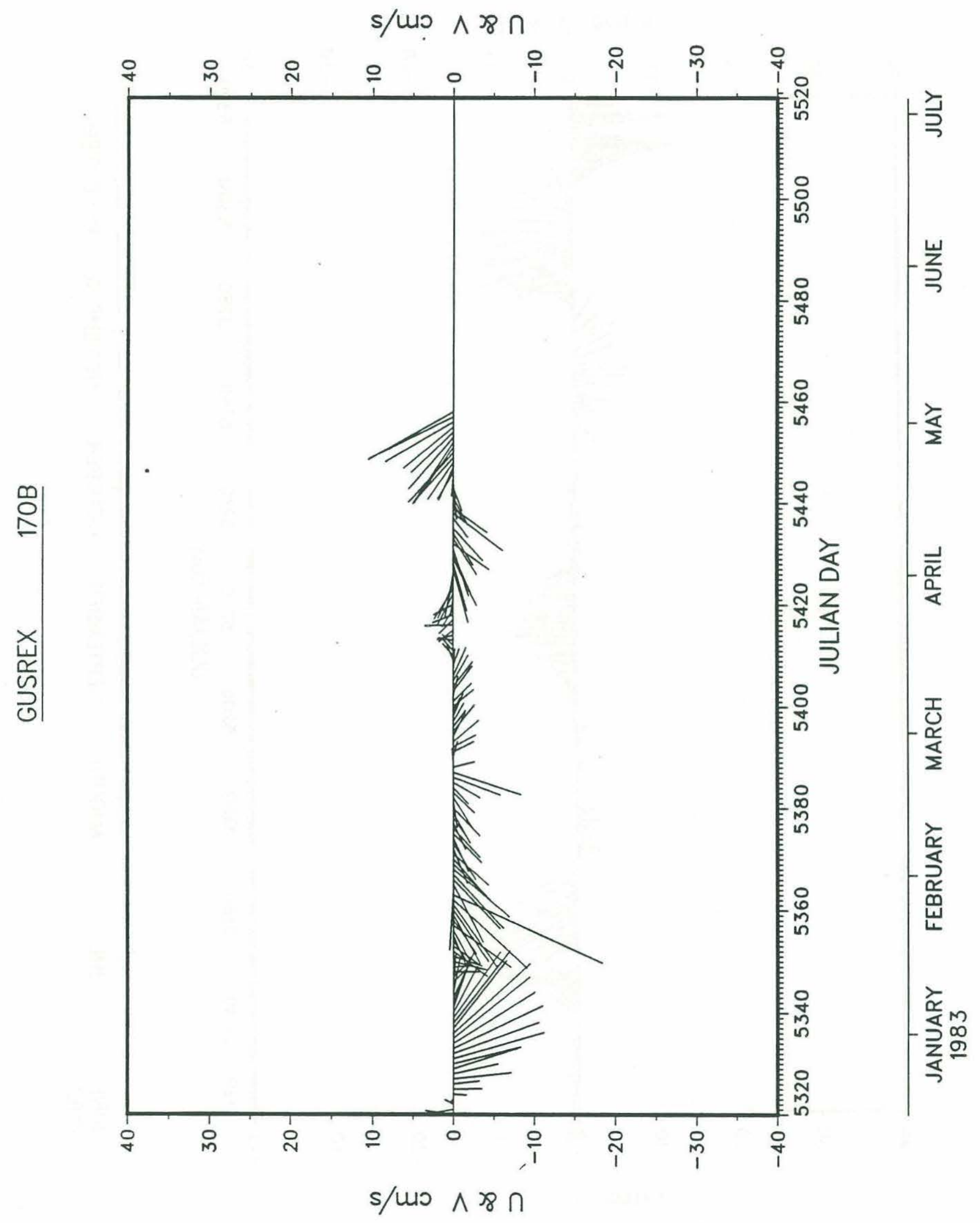




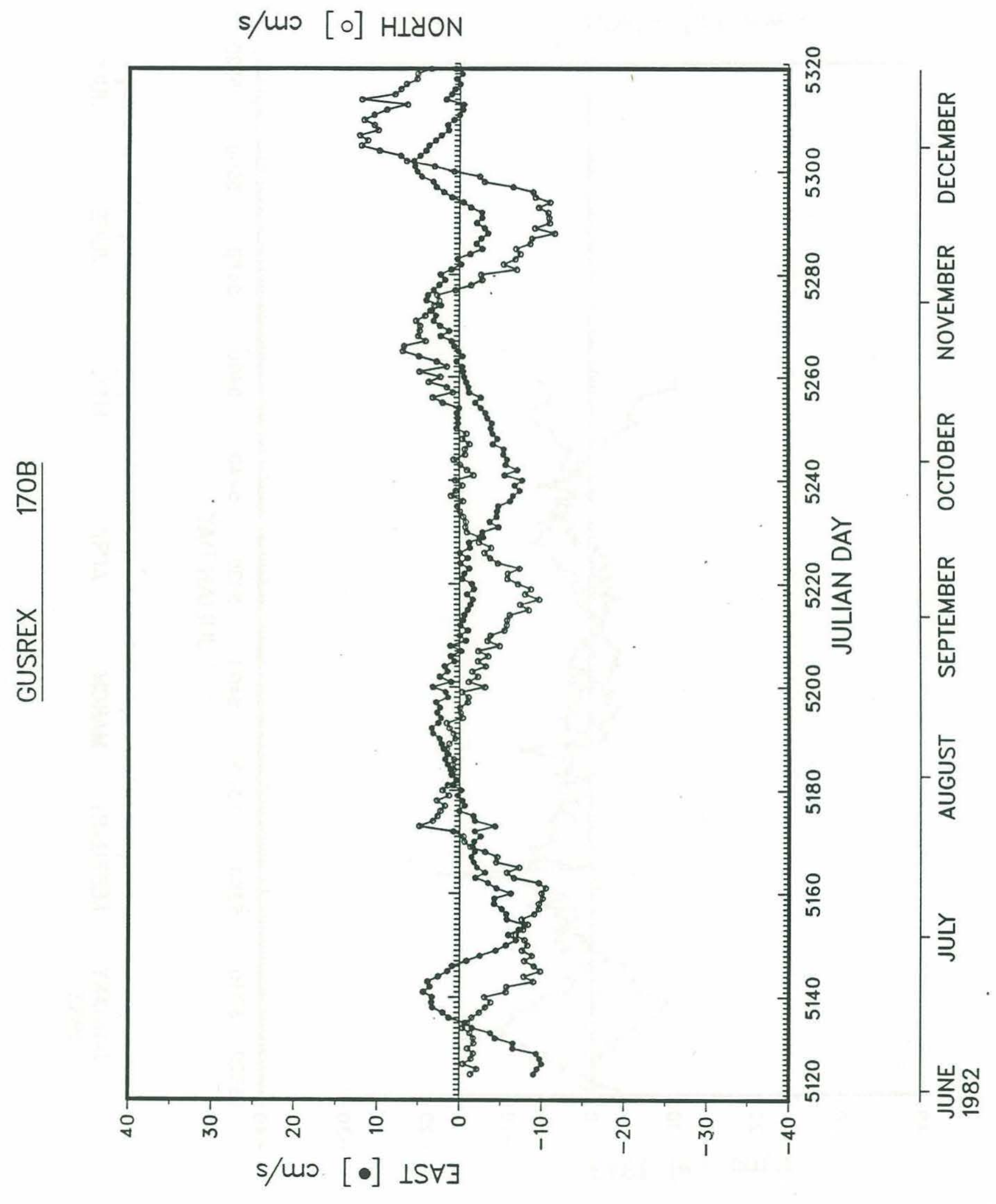




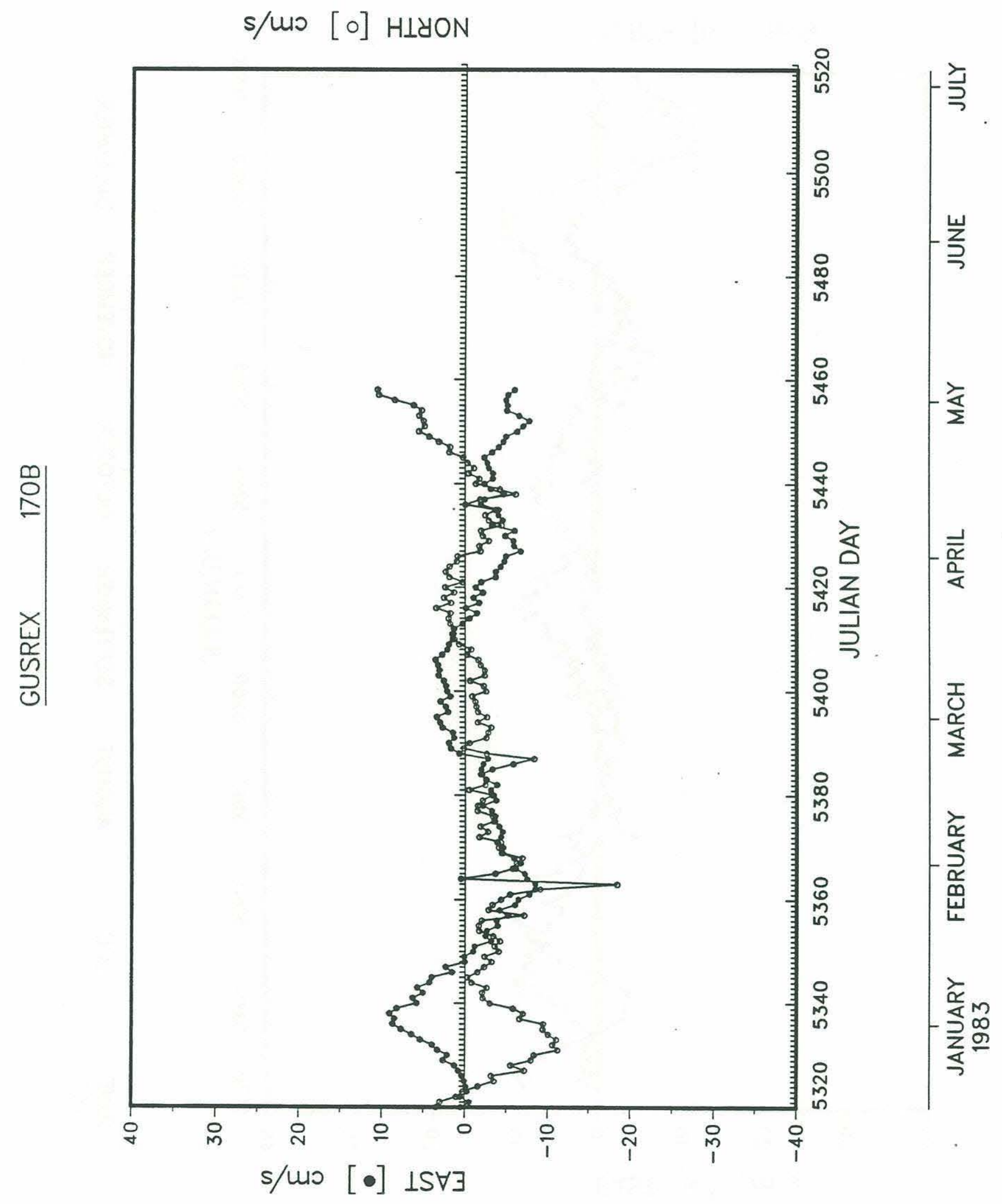




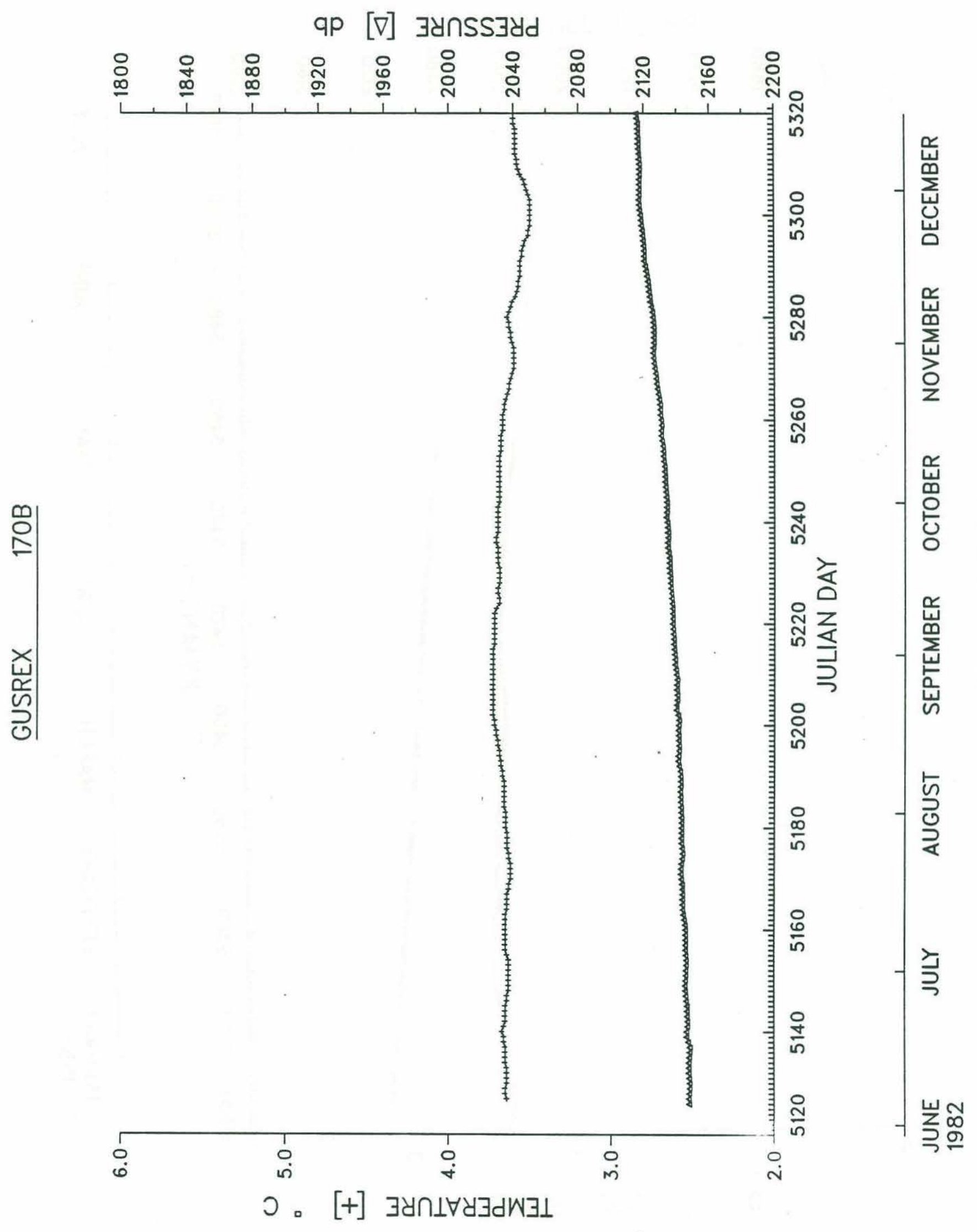




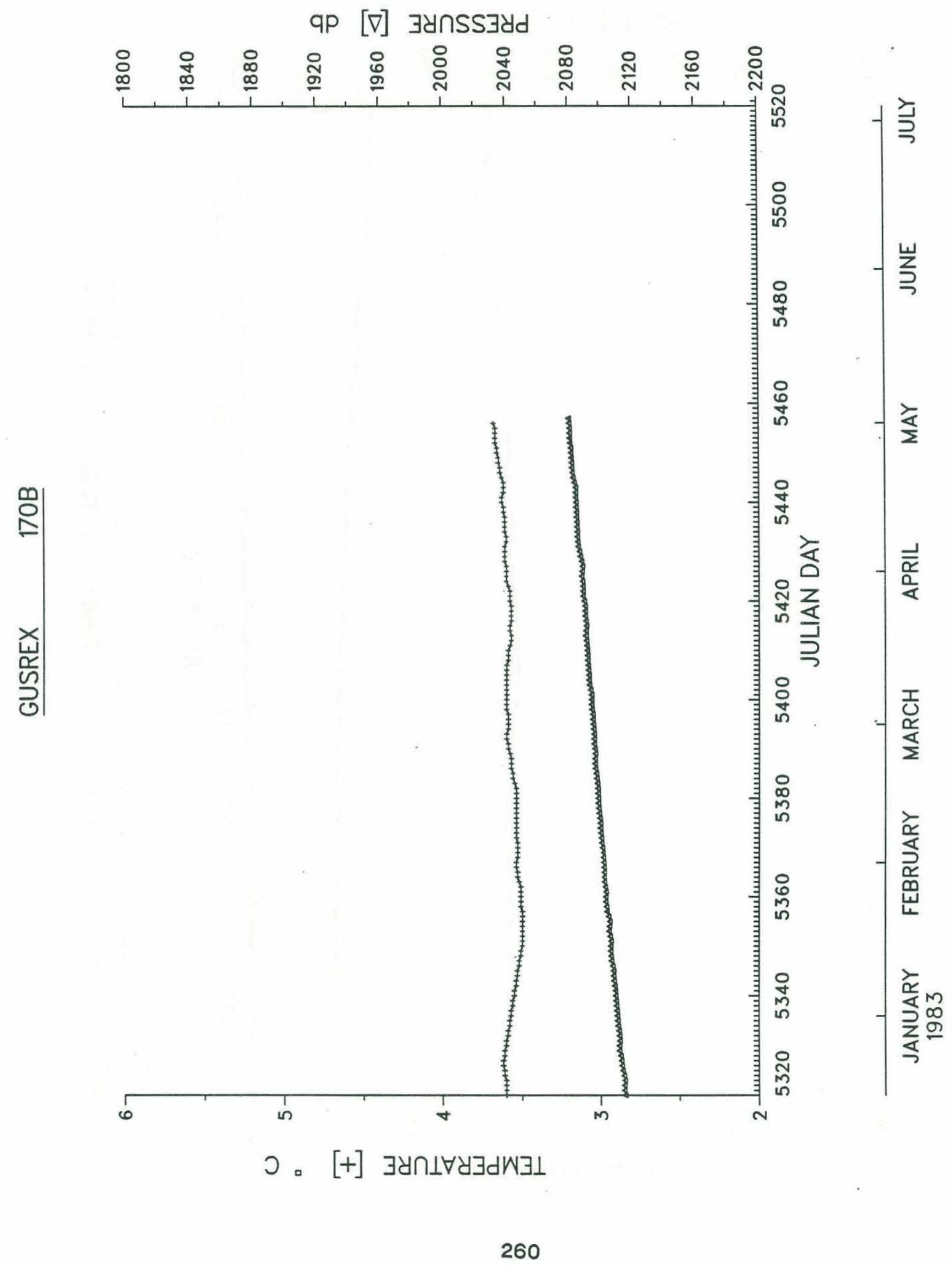




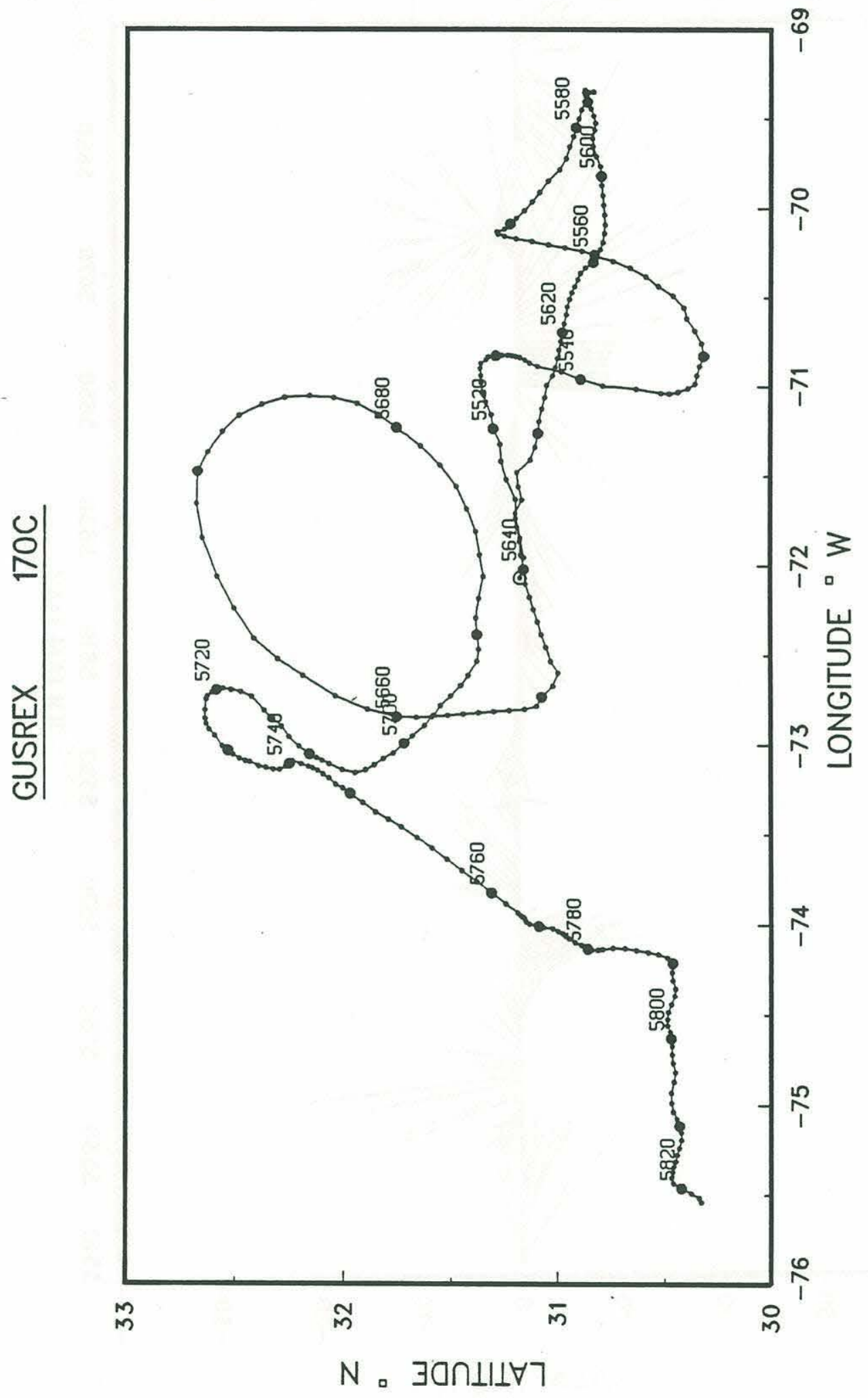




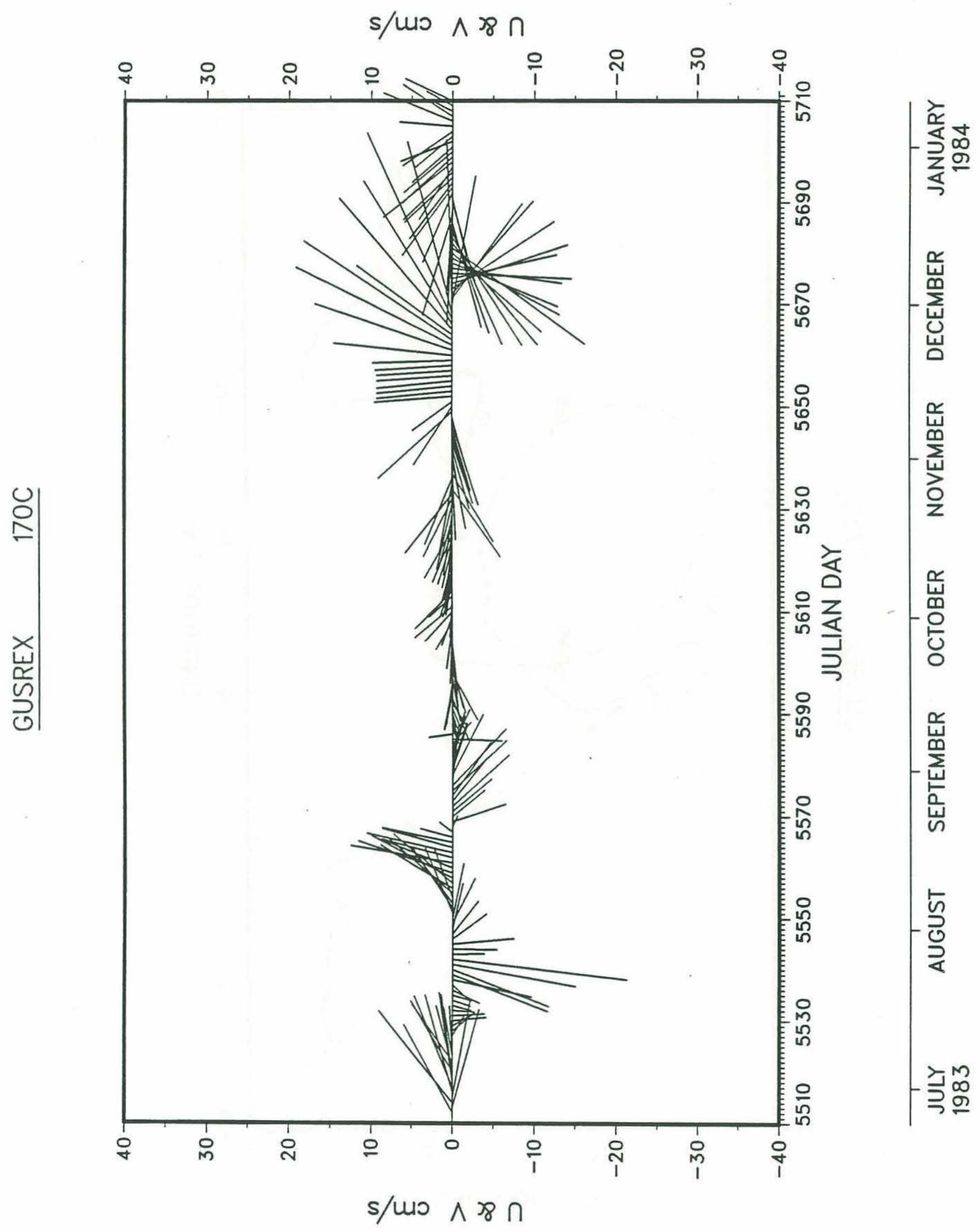




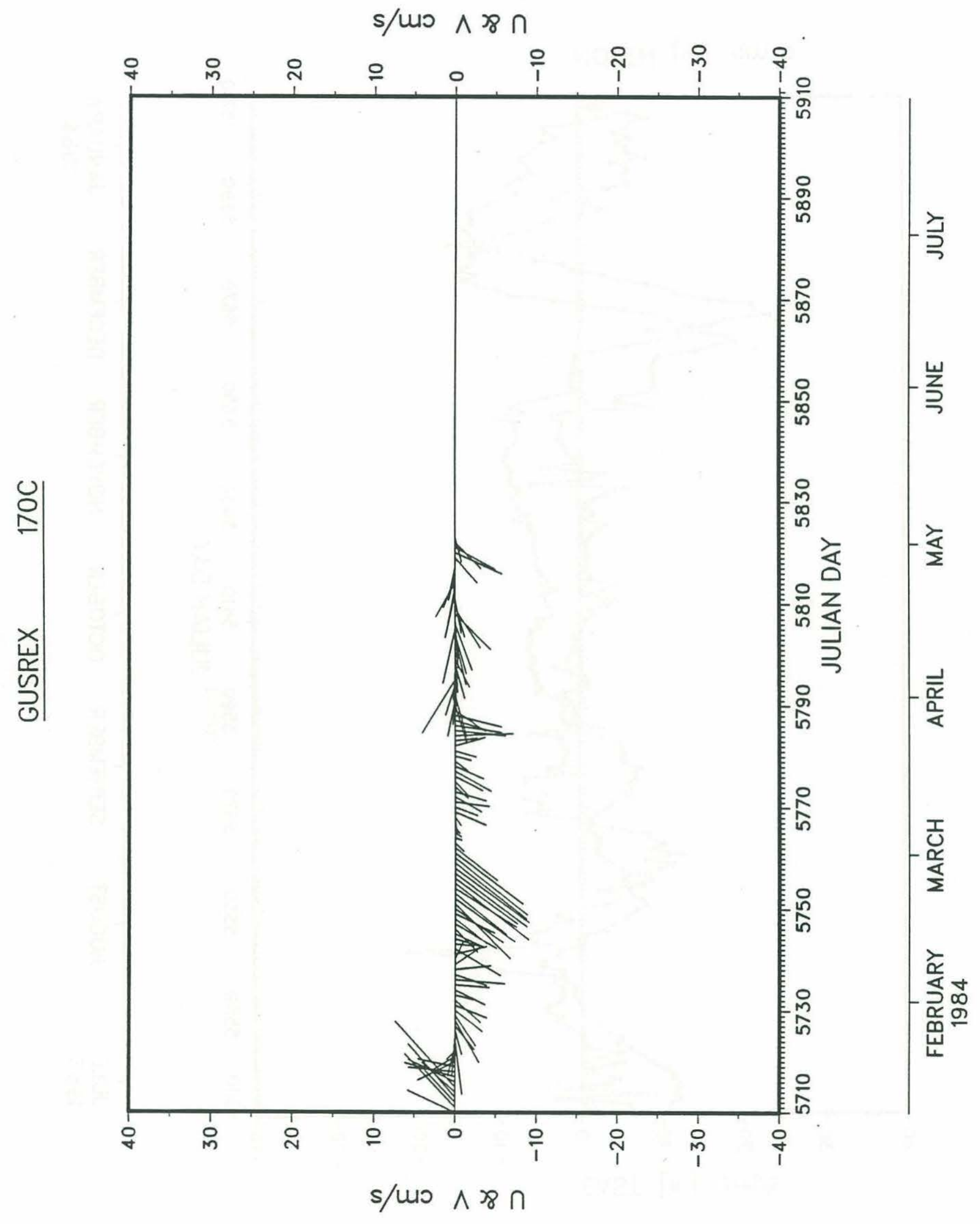




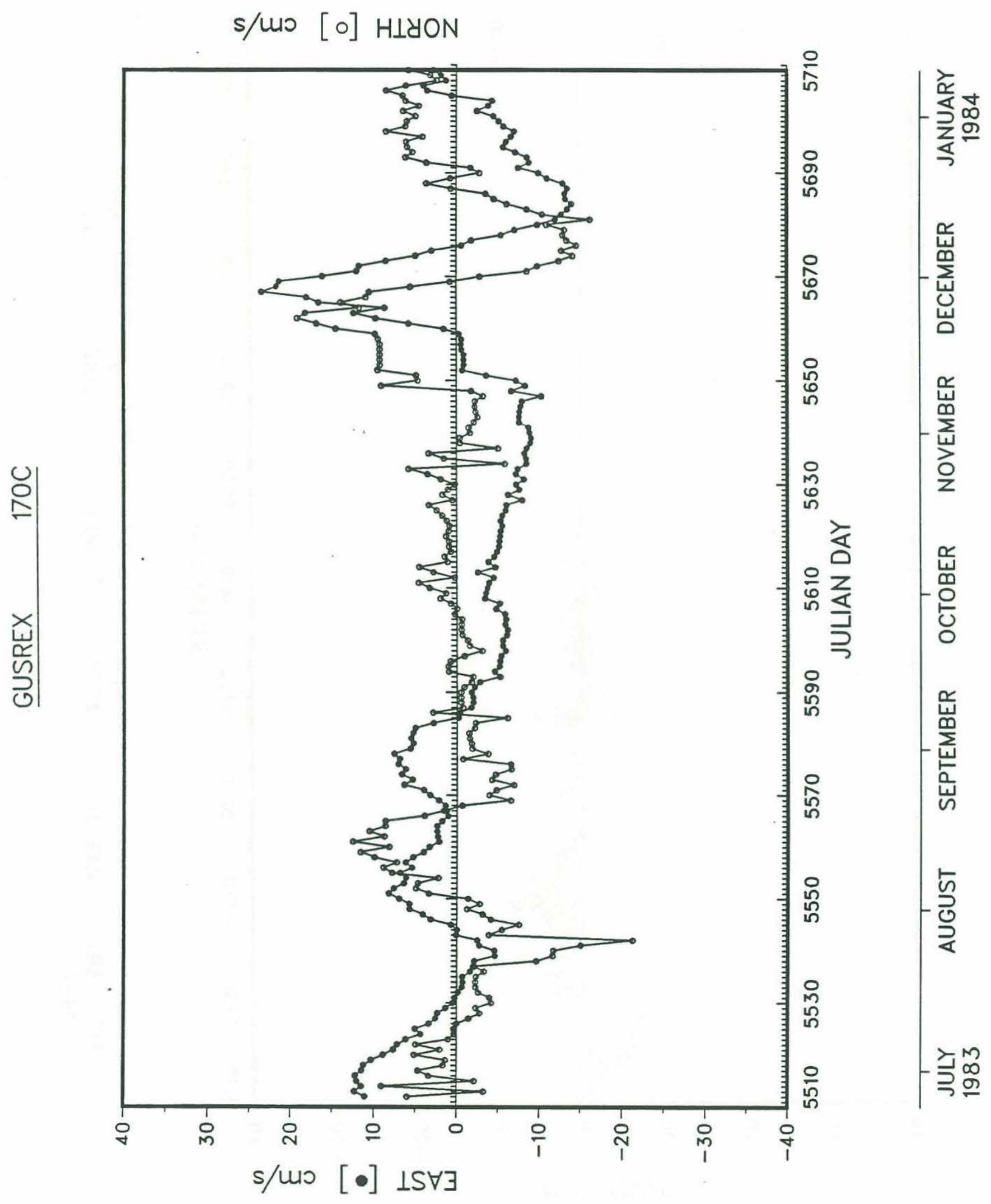




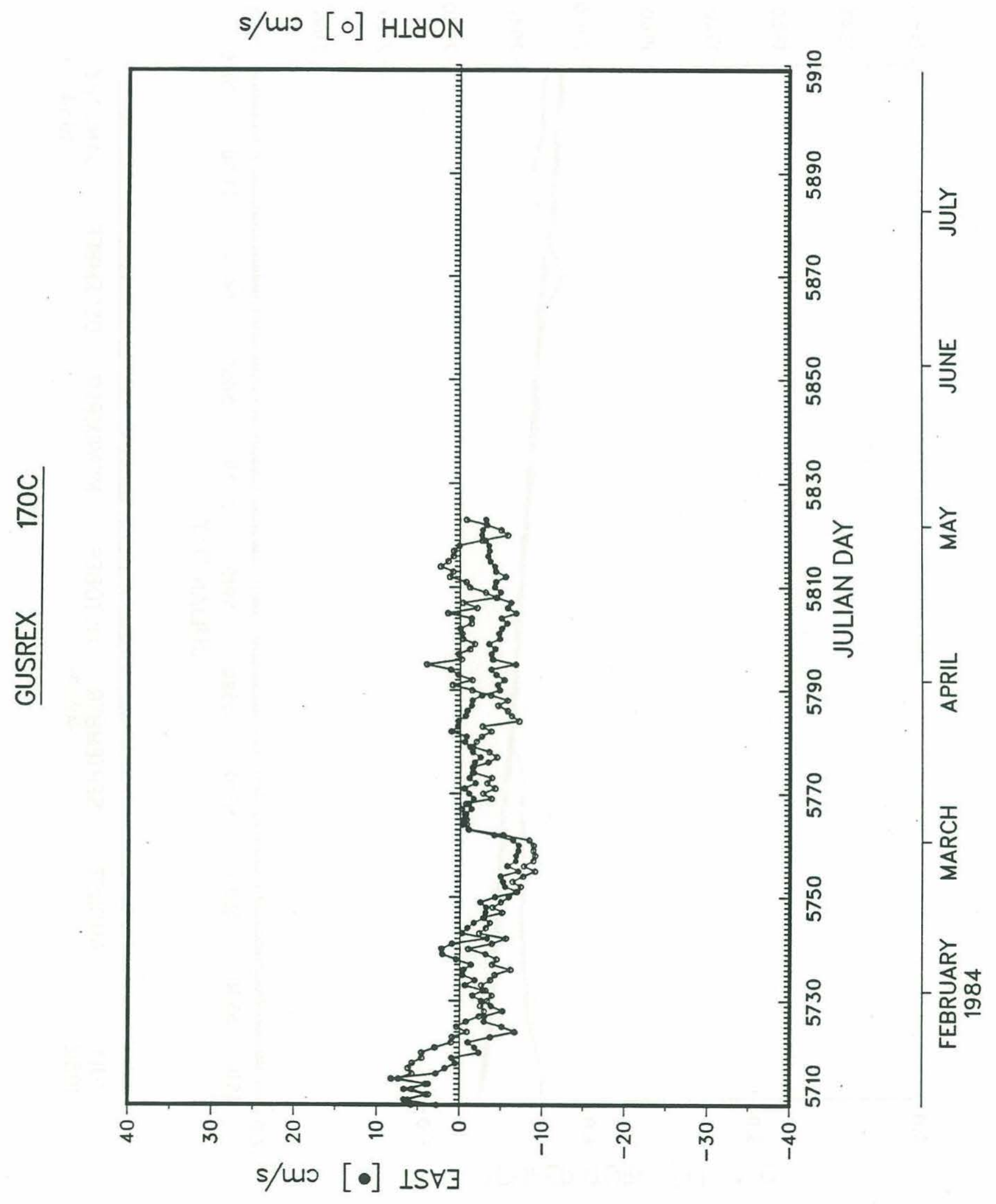




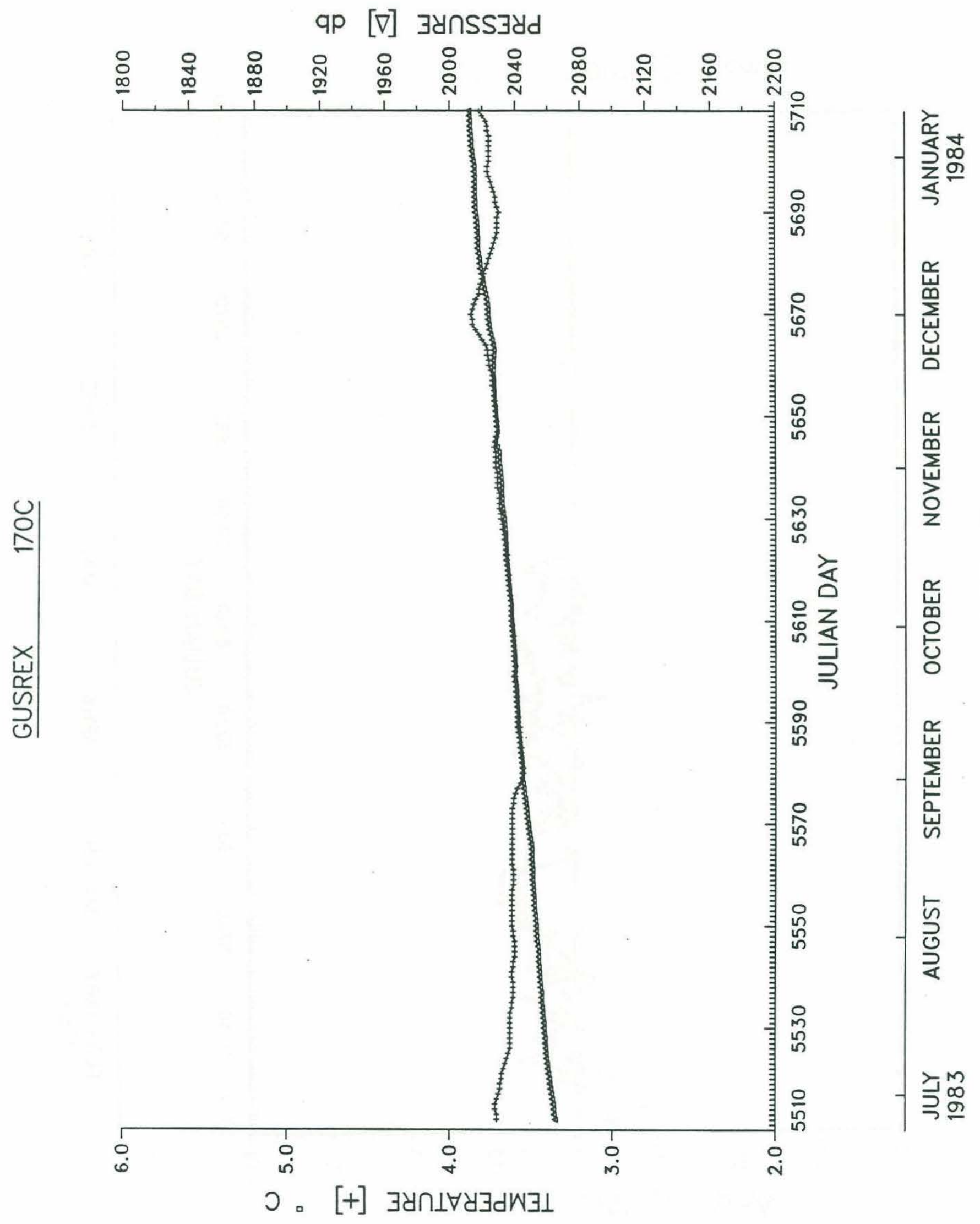




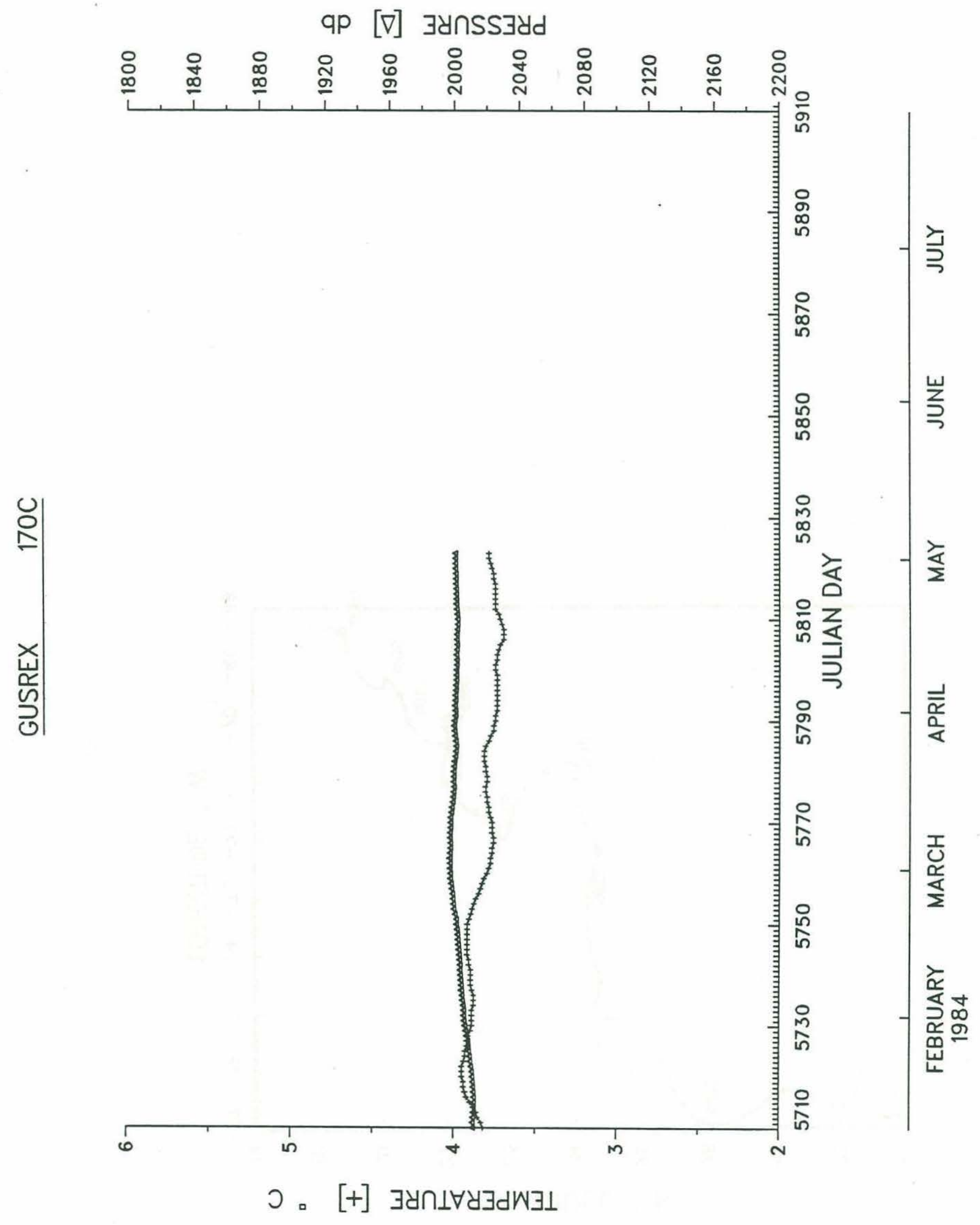




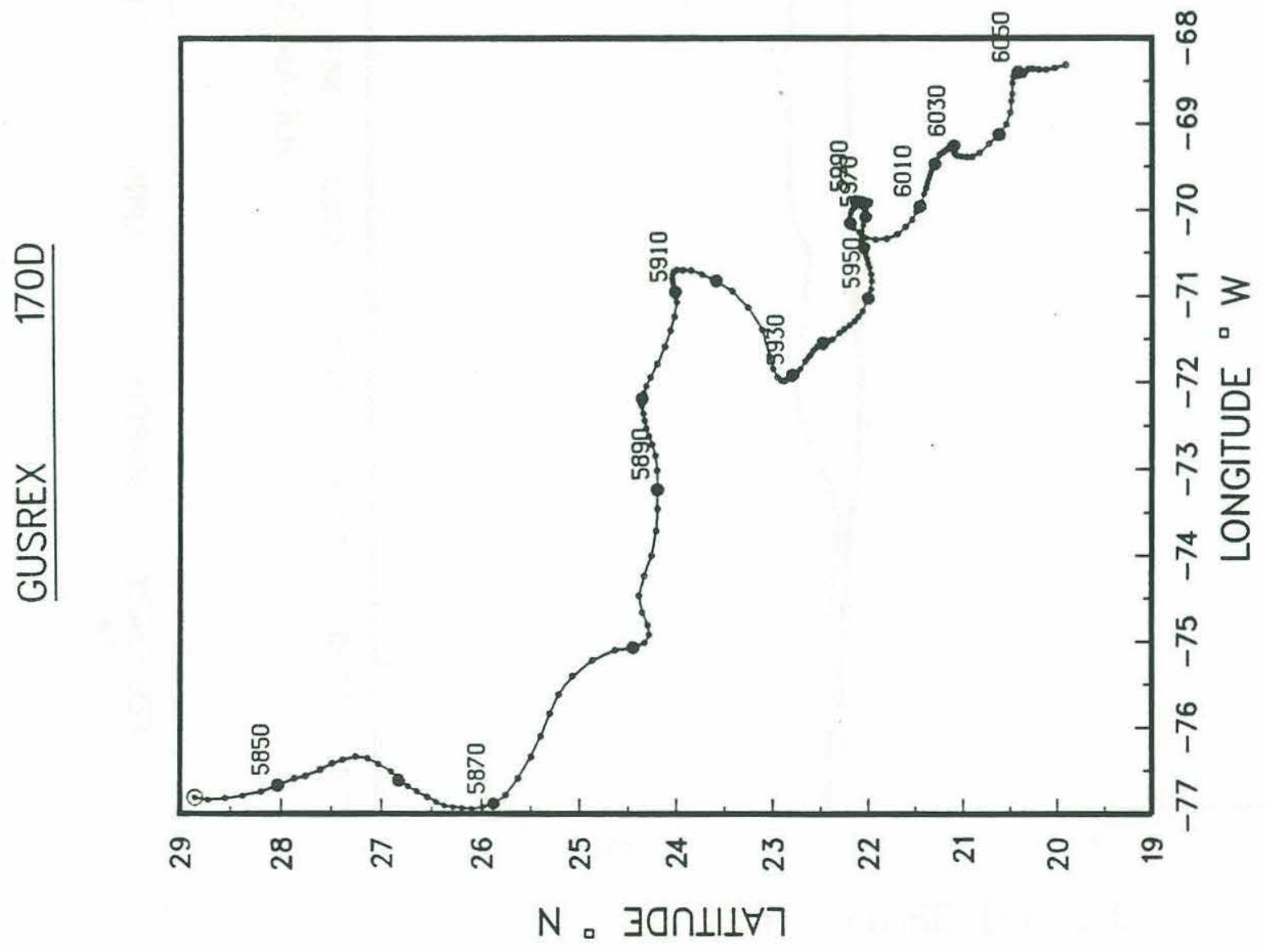




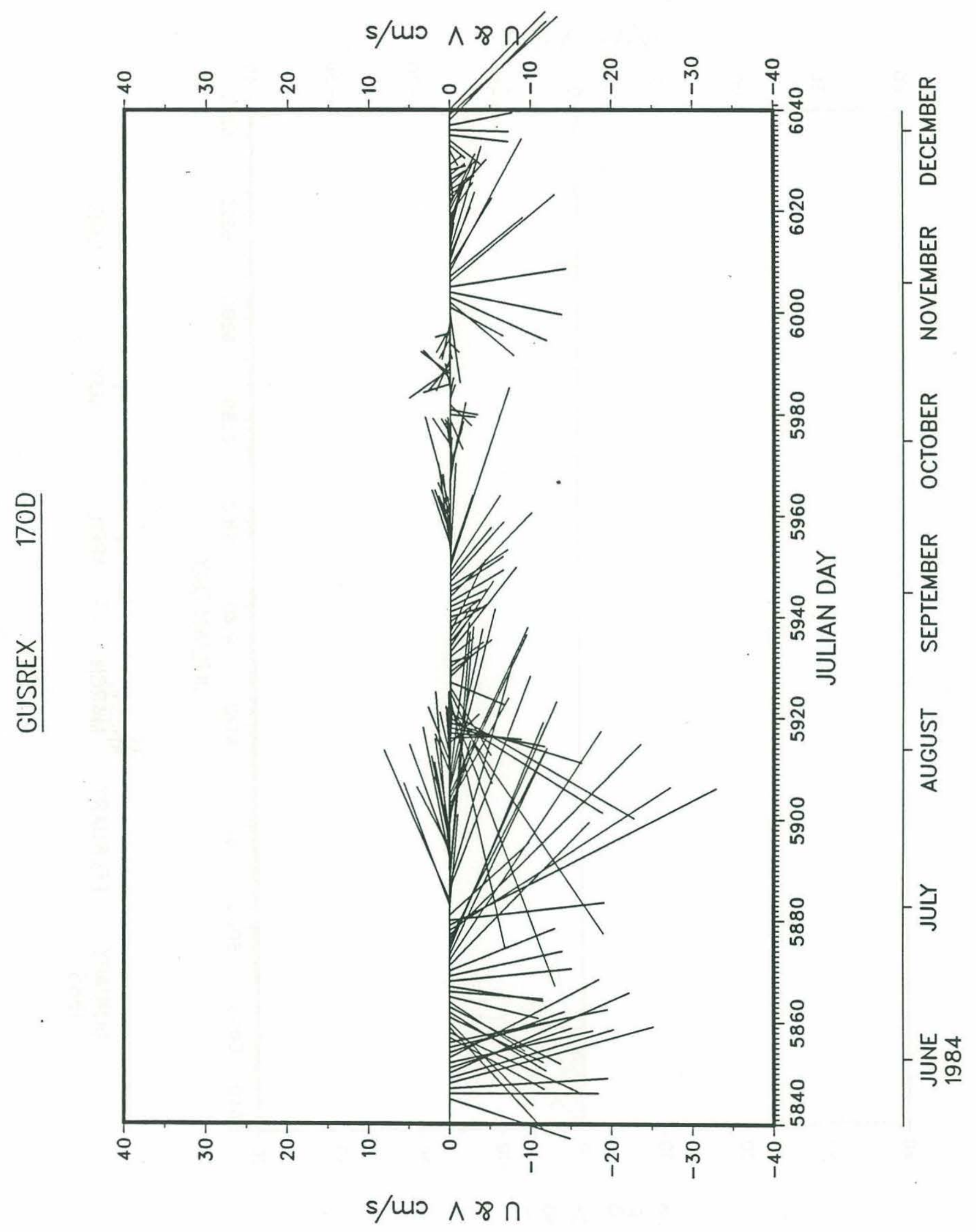




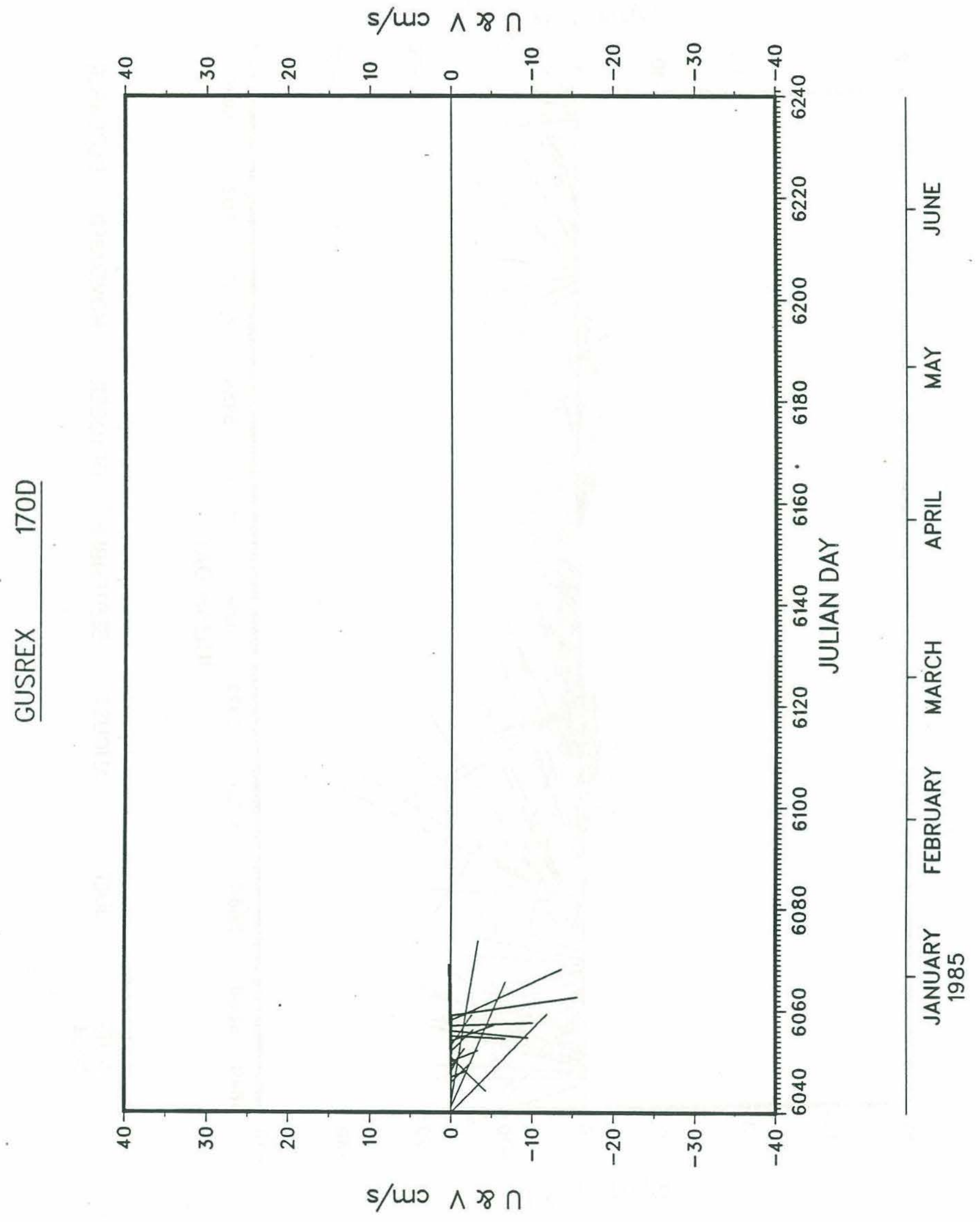




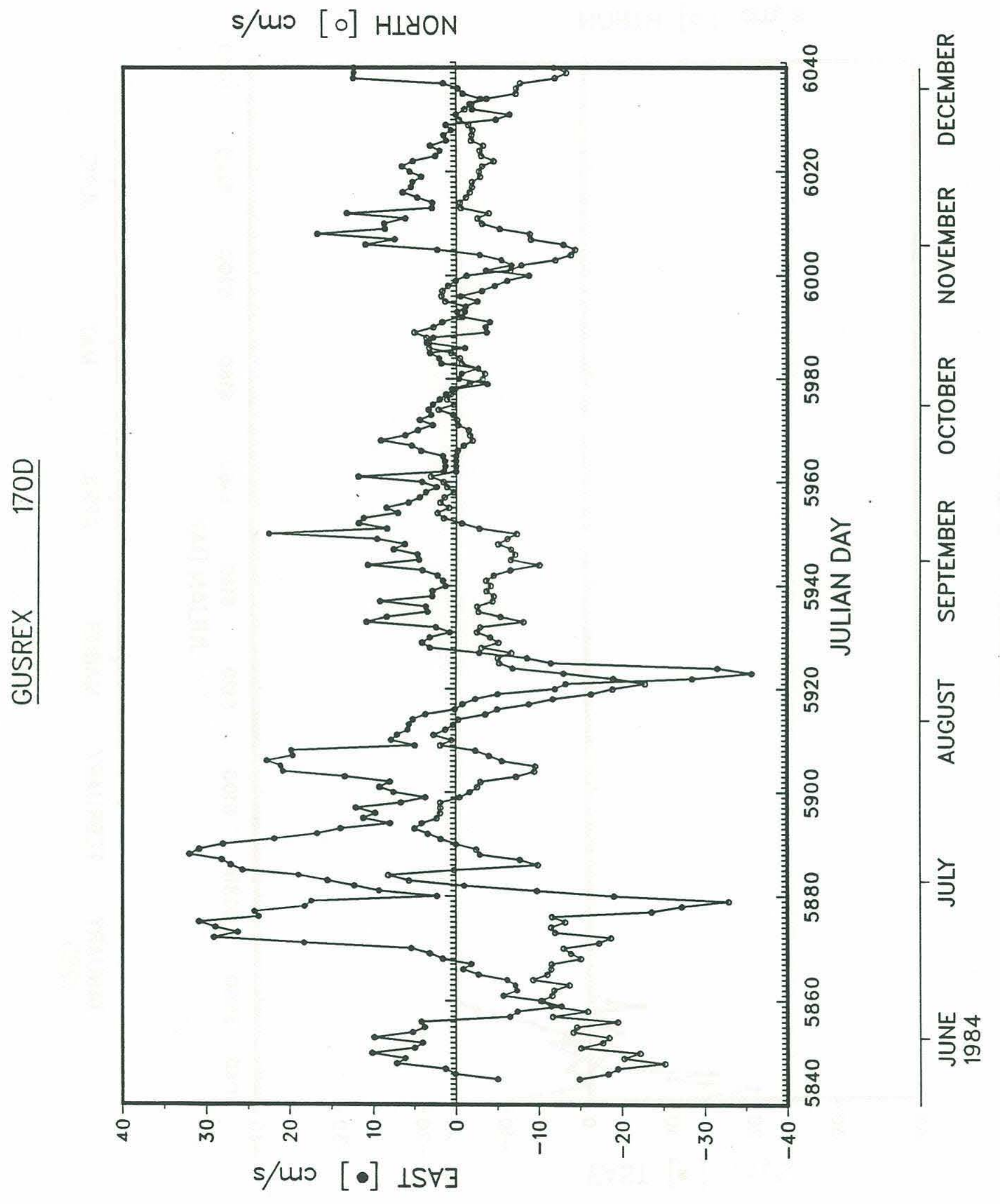




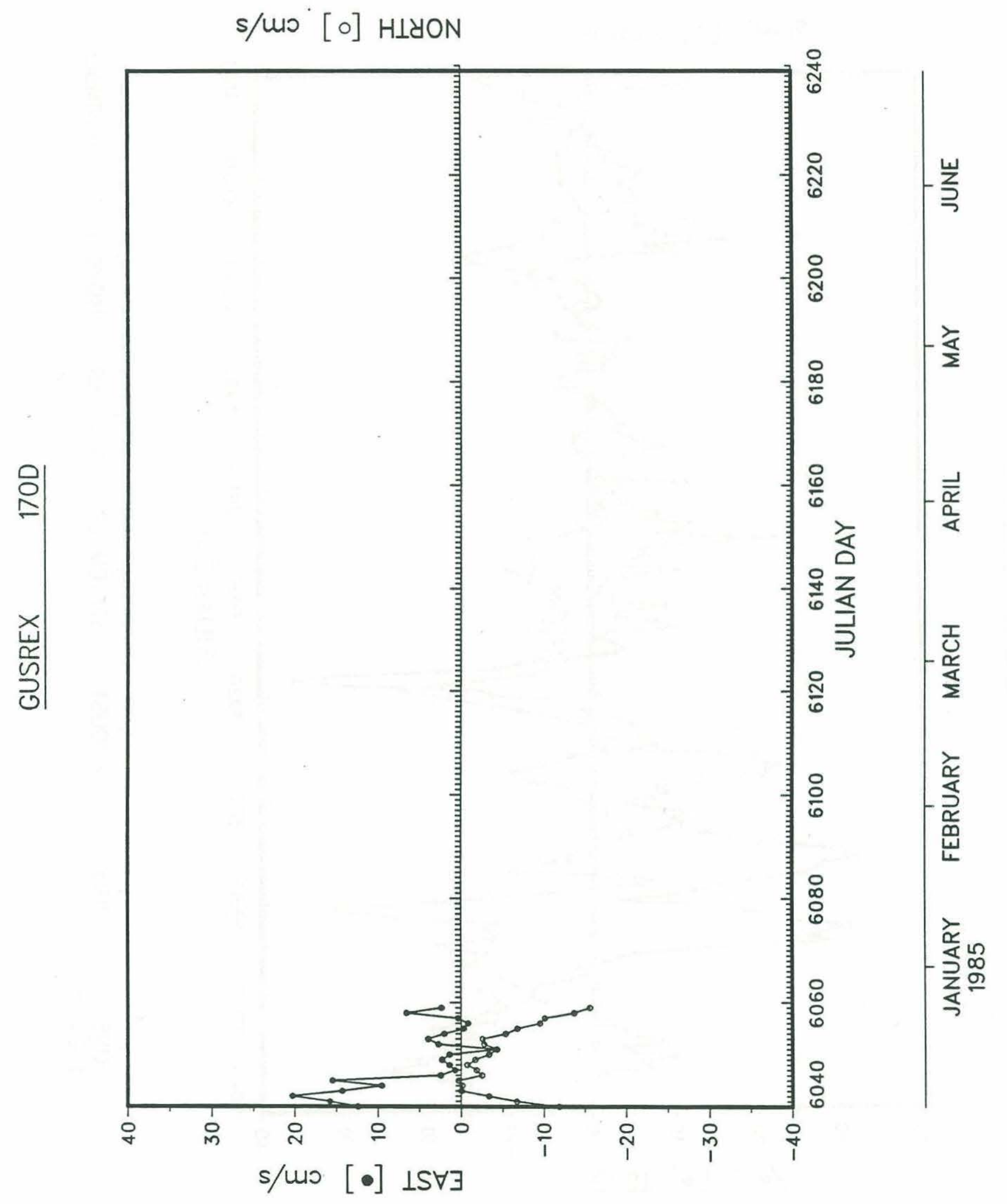




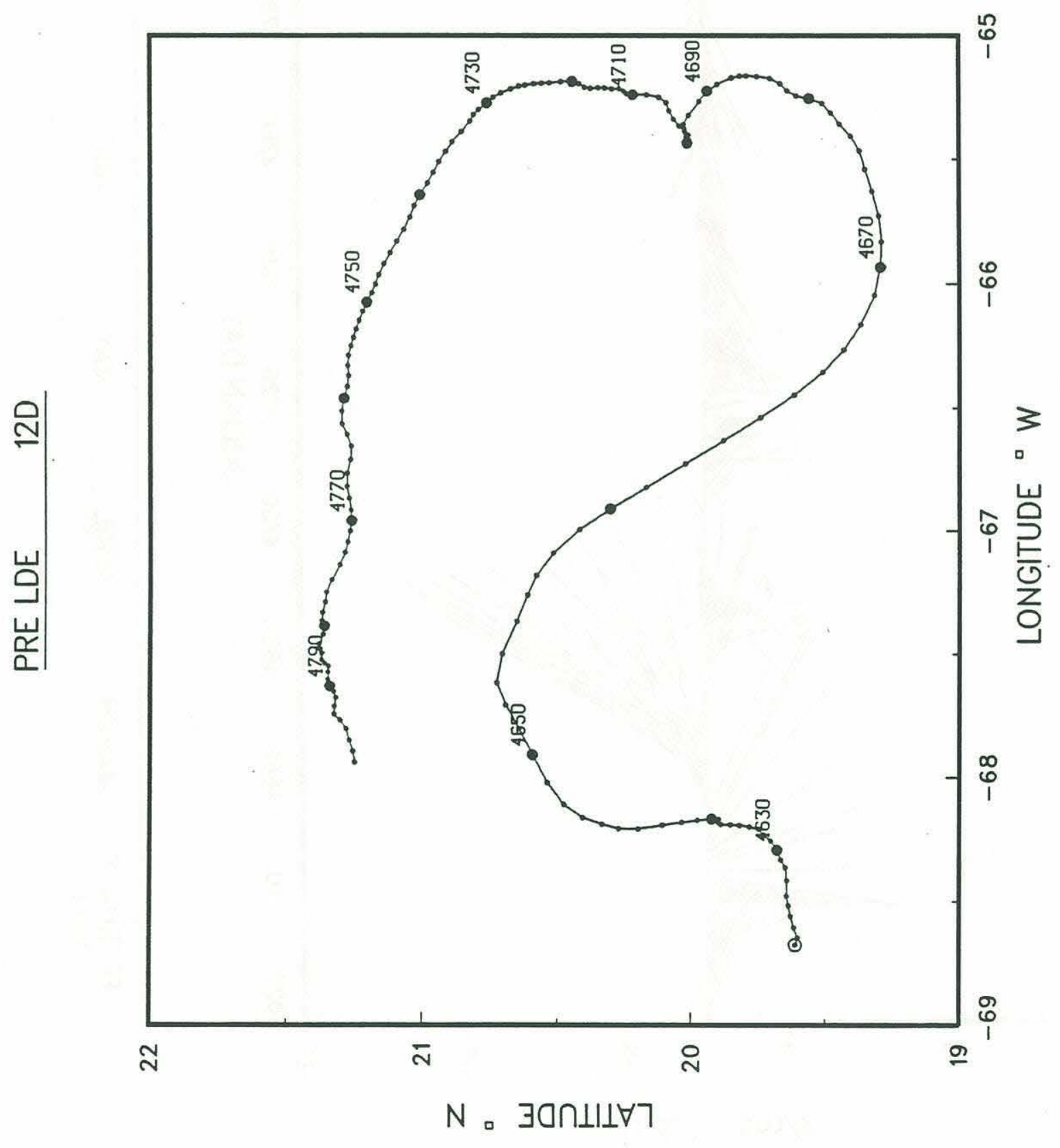




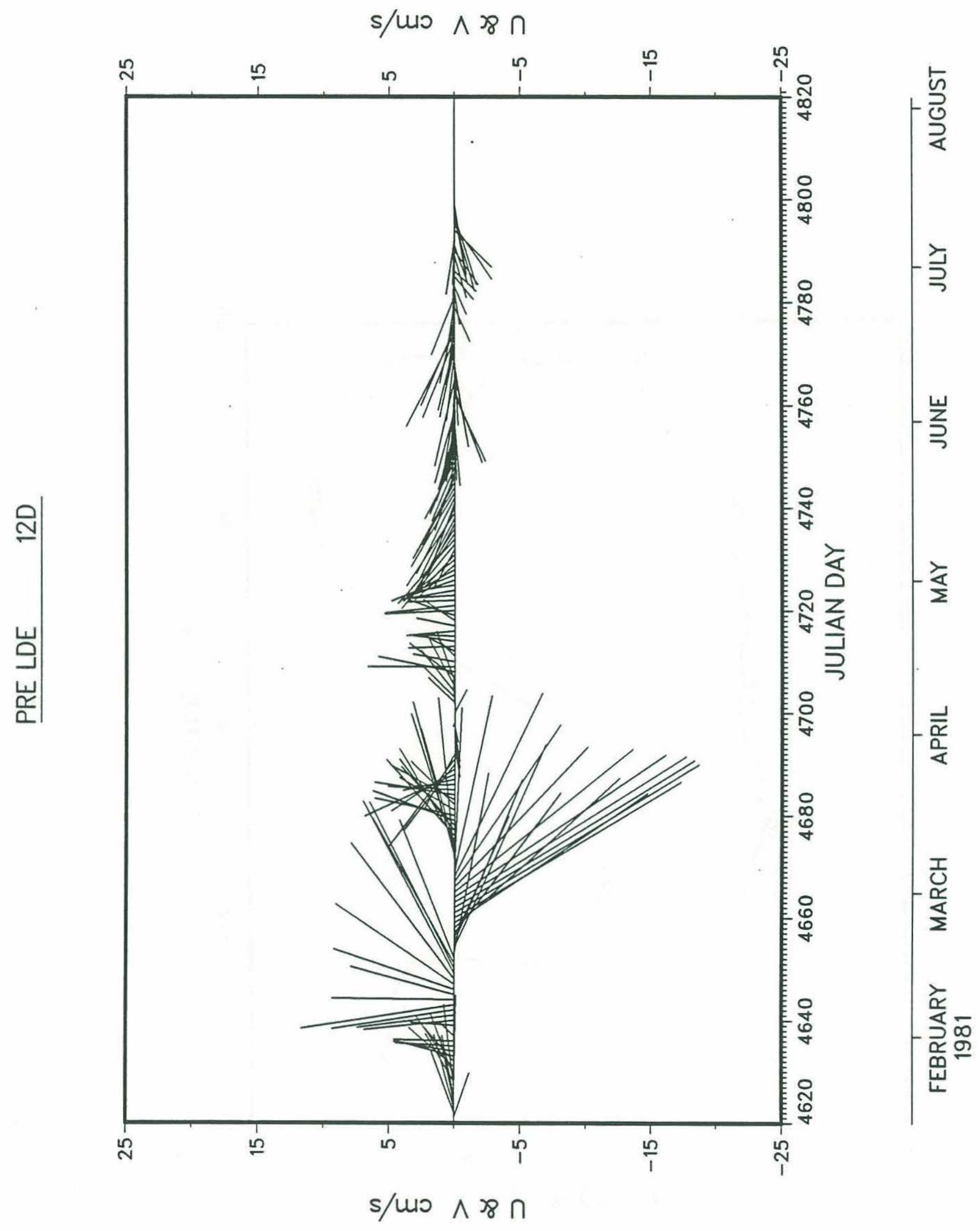




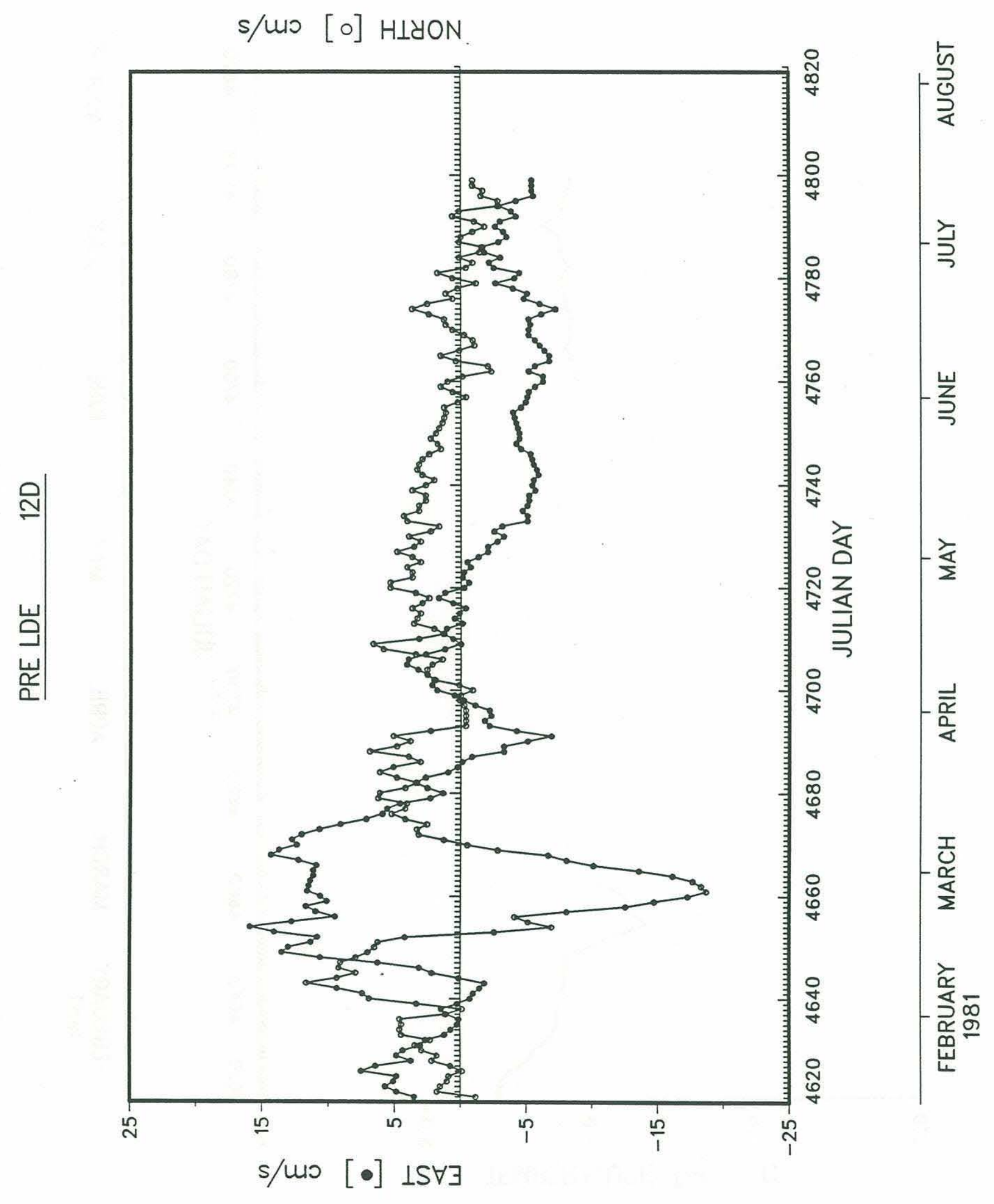




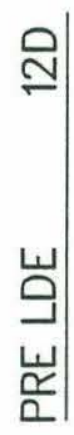

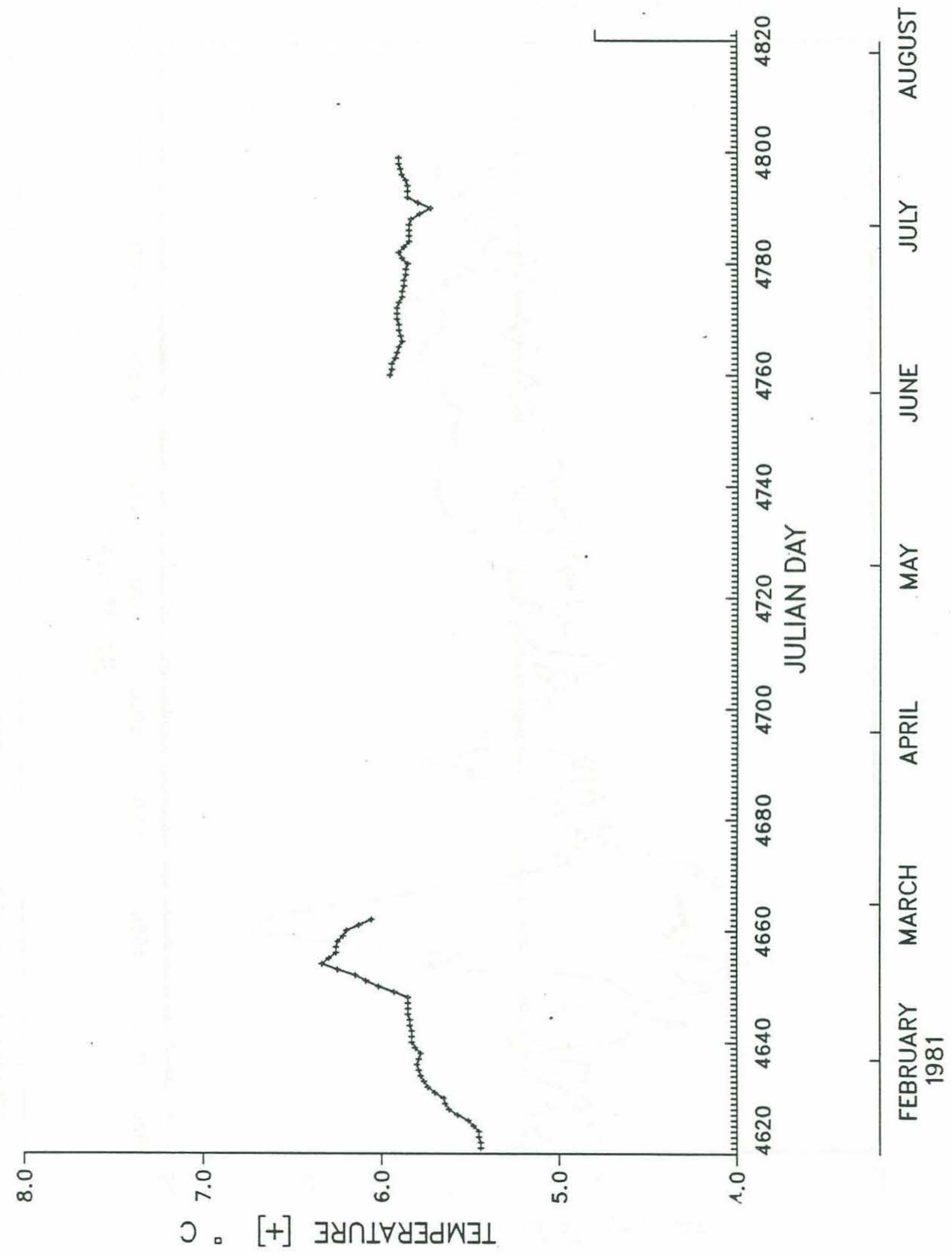




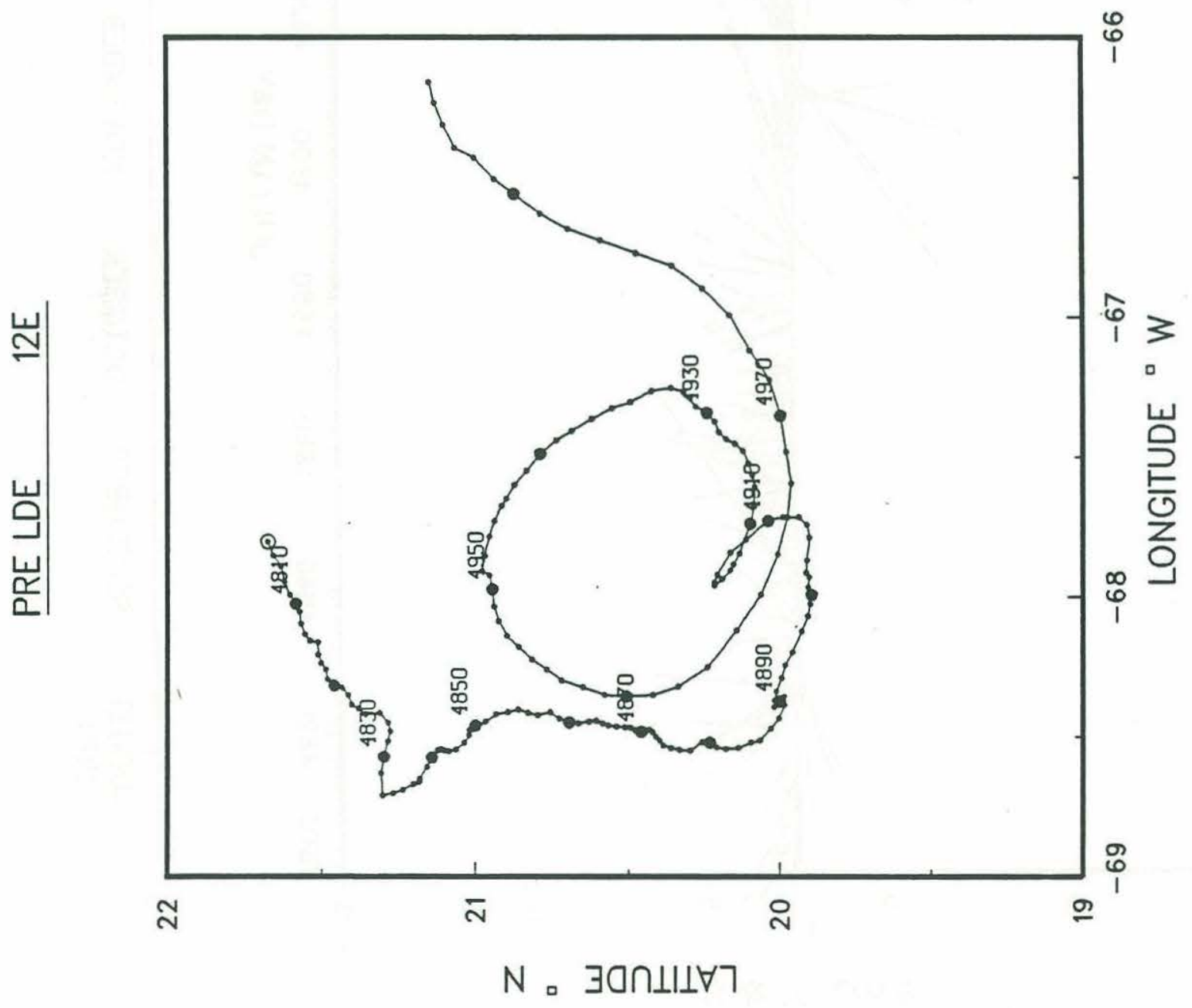




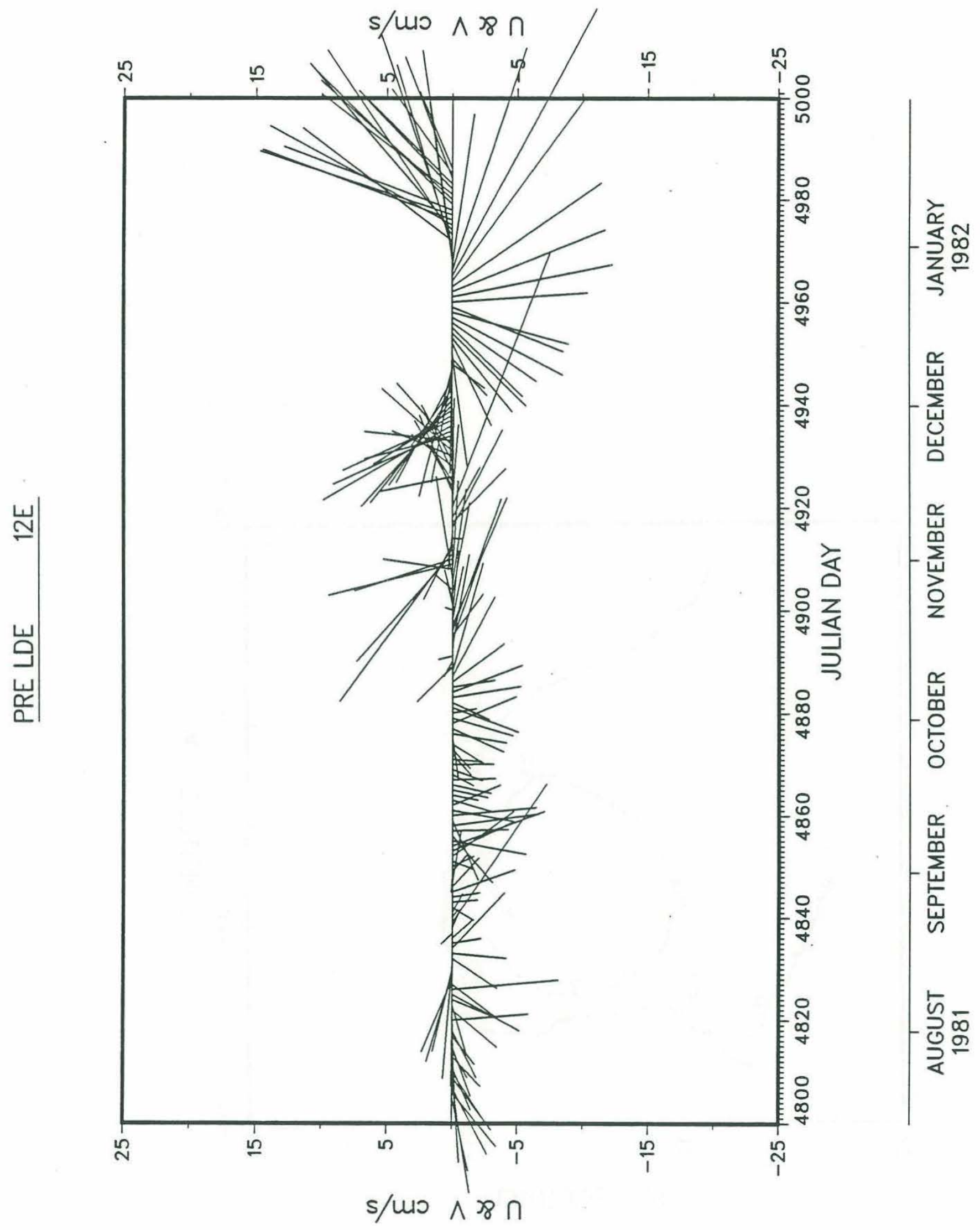




\section{s/Mo [o] HLYON}

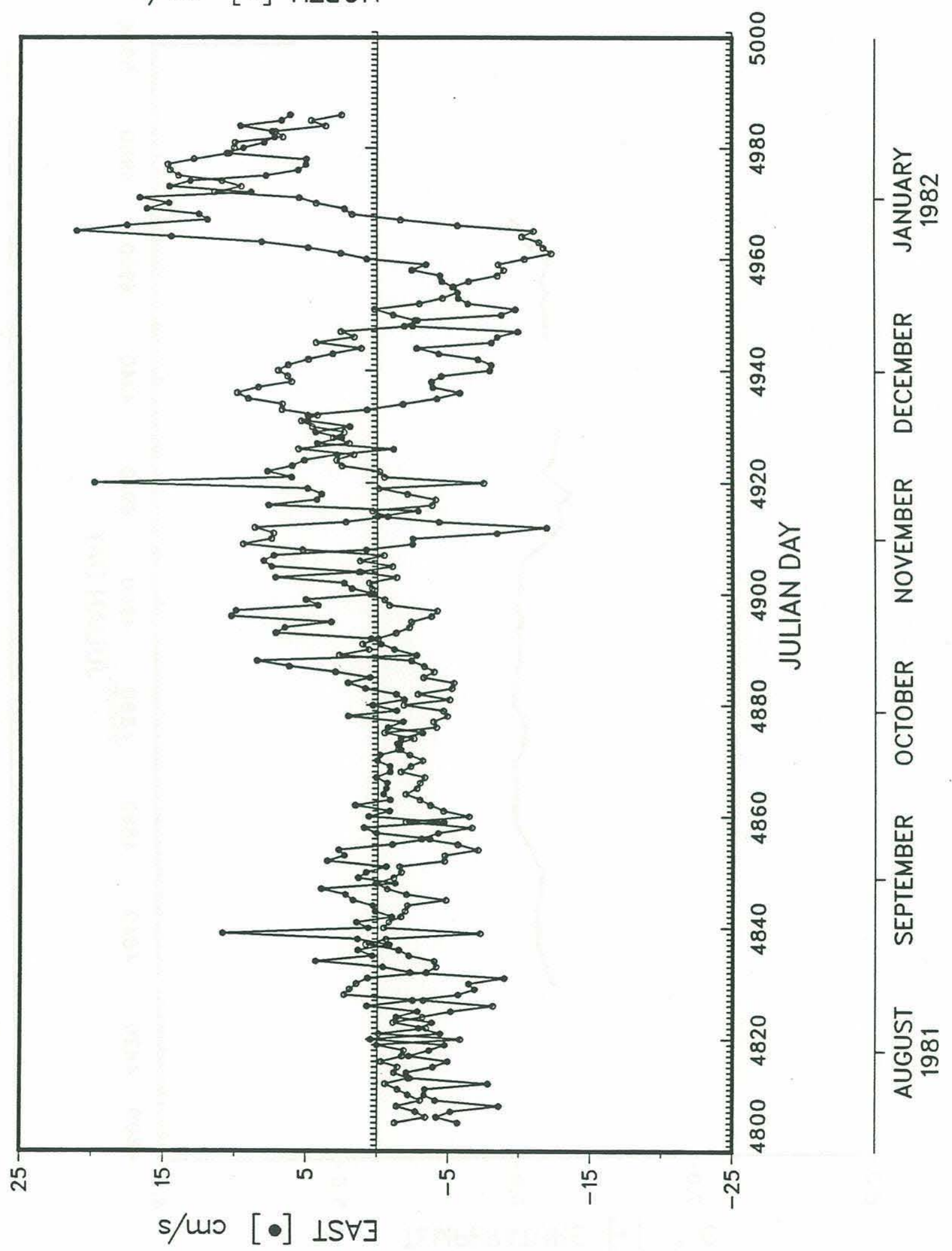



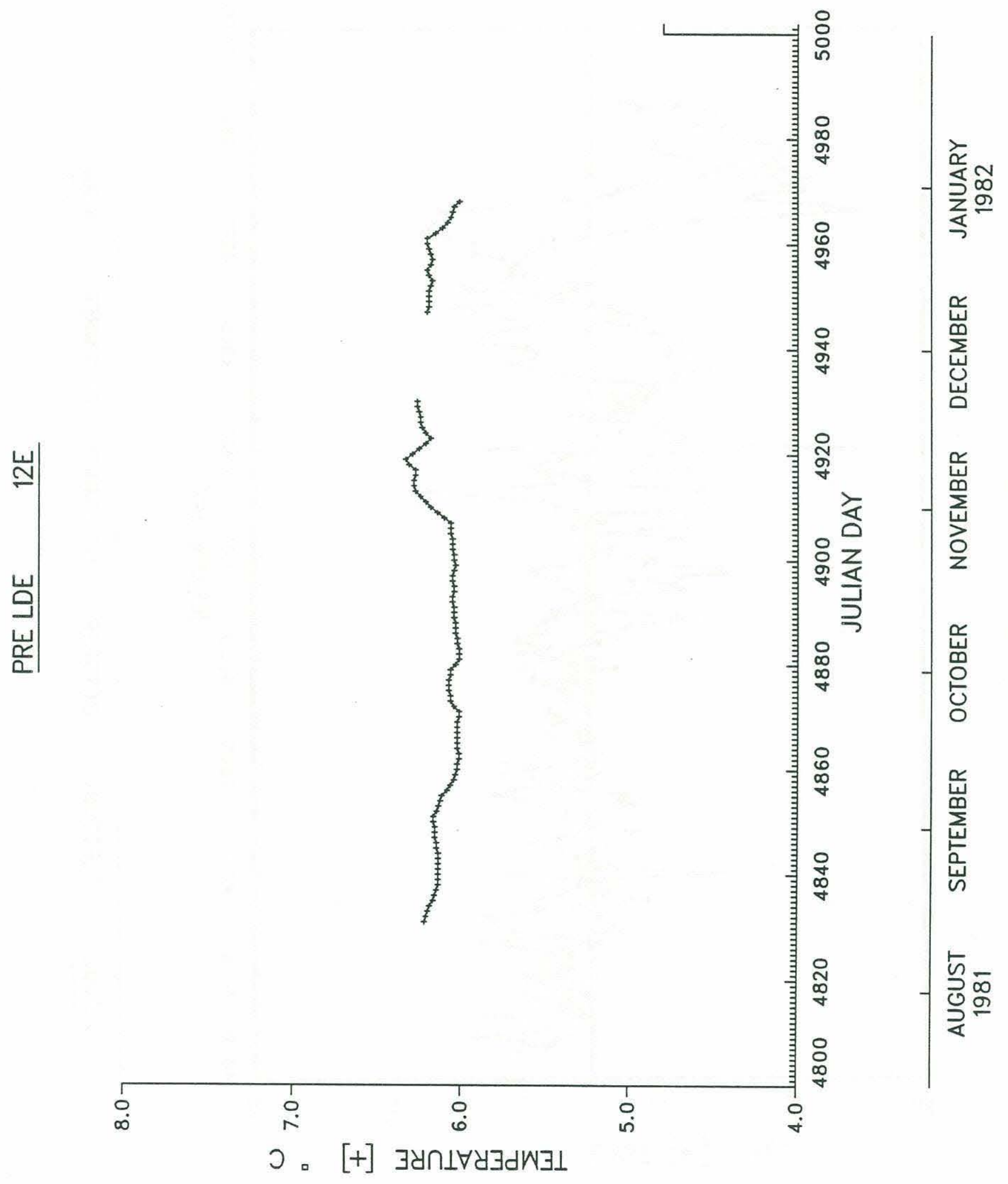


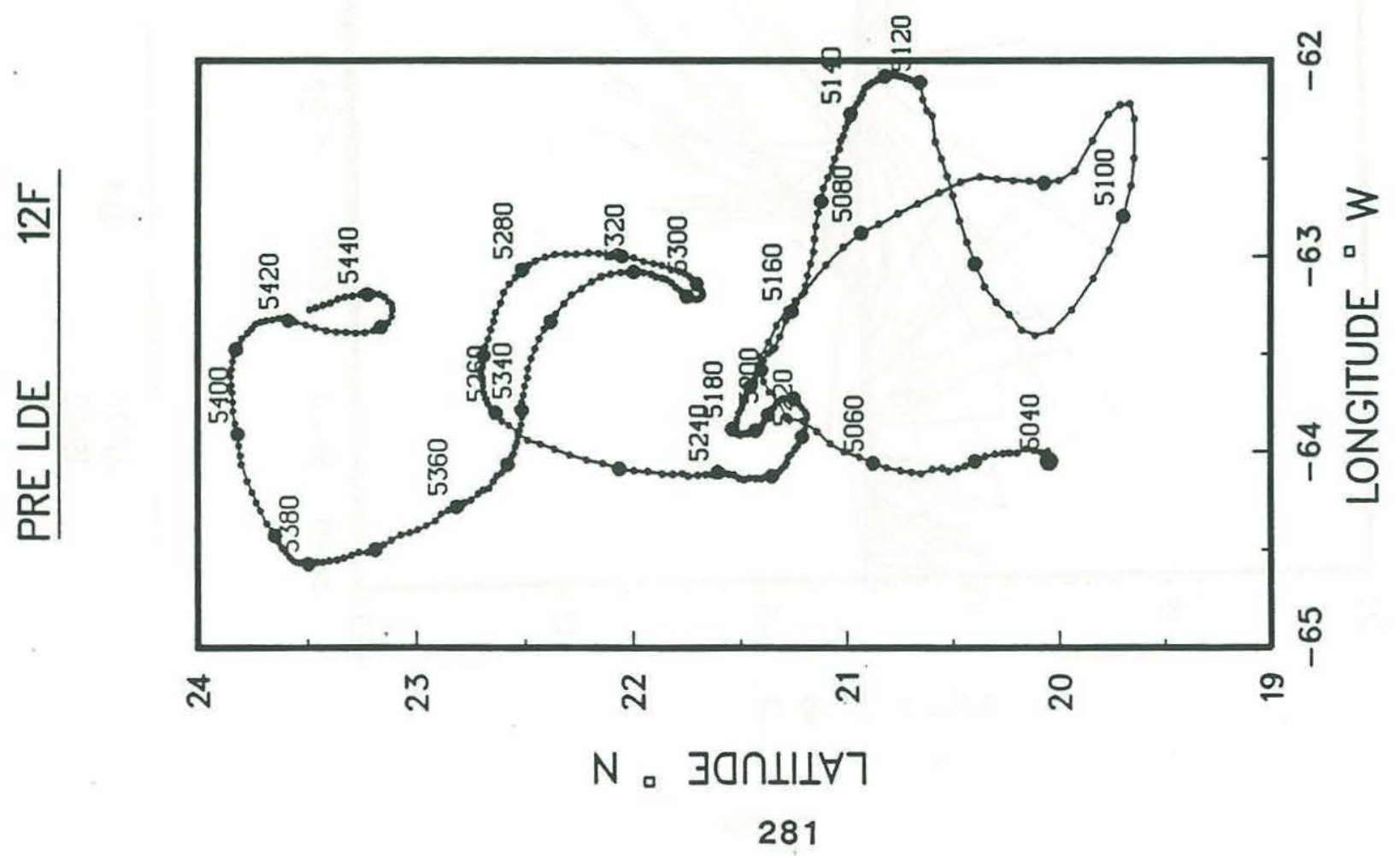




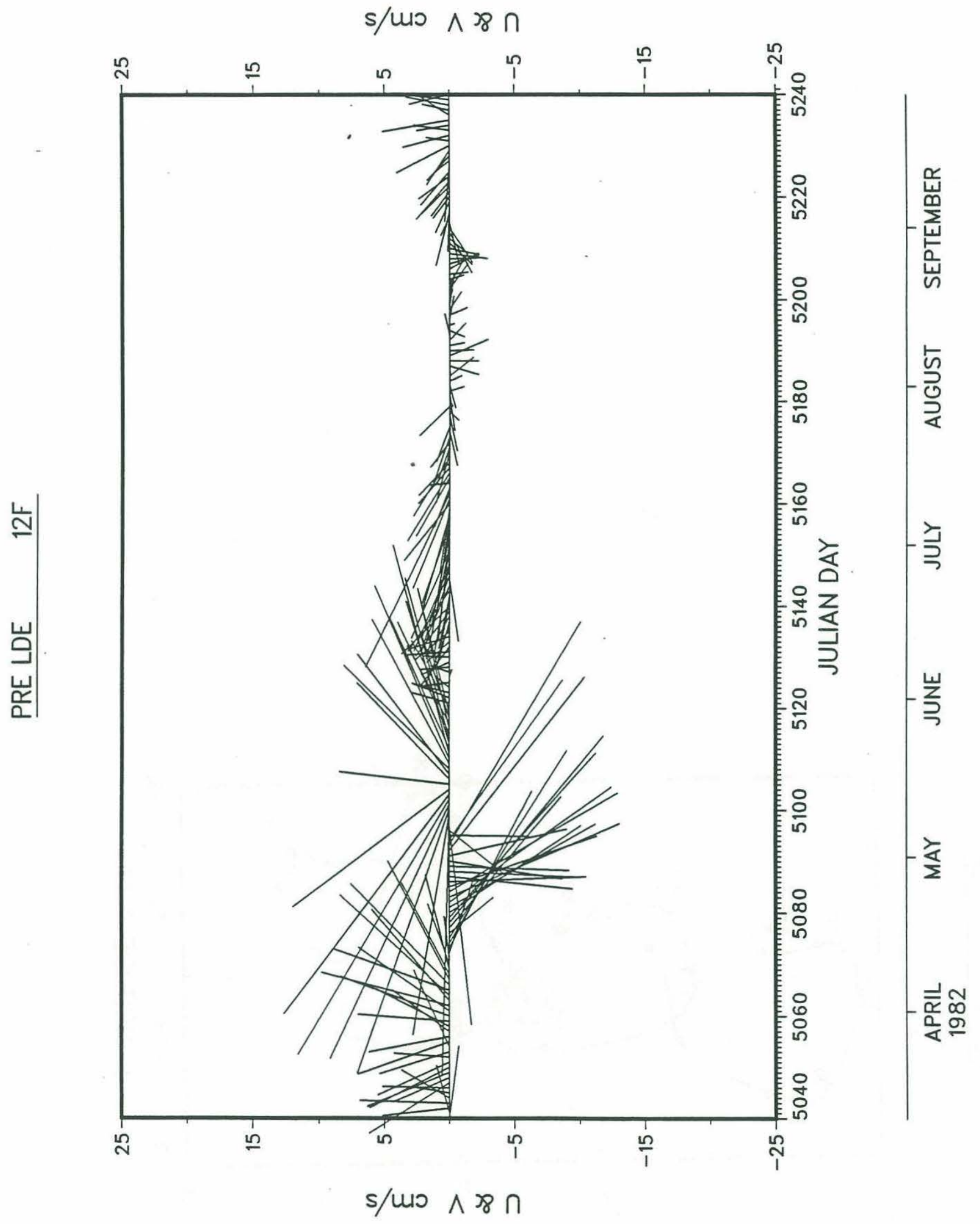




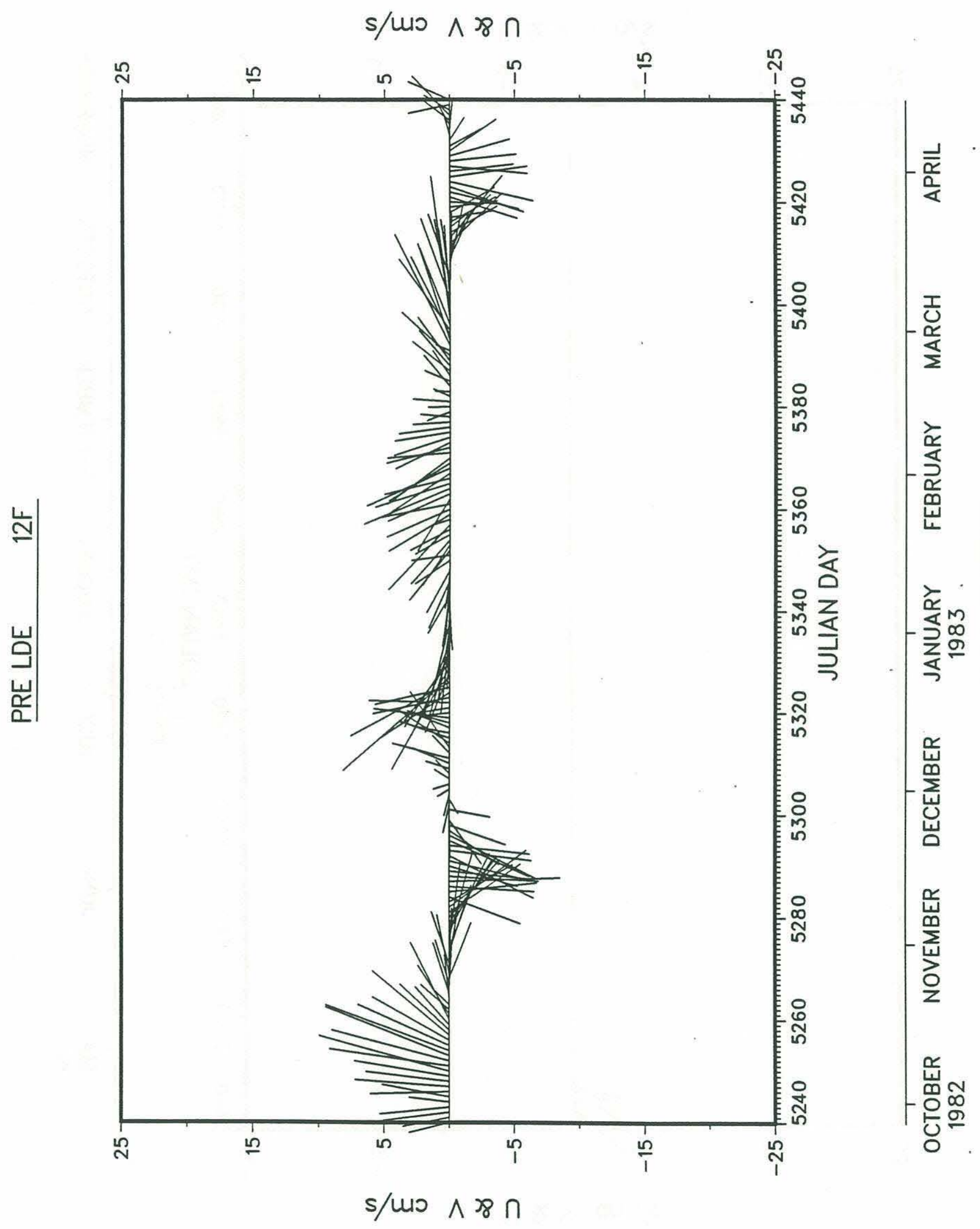




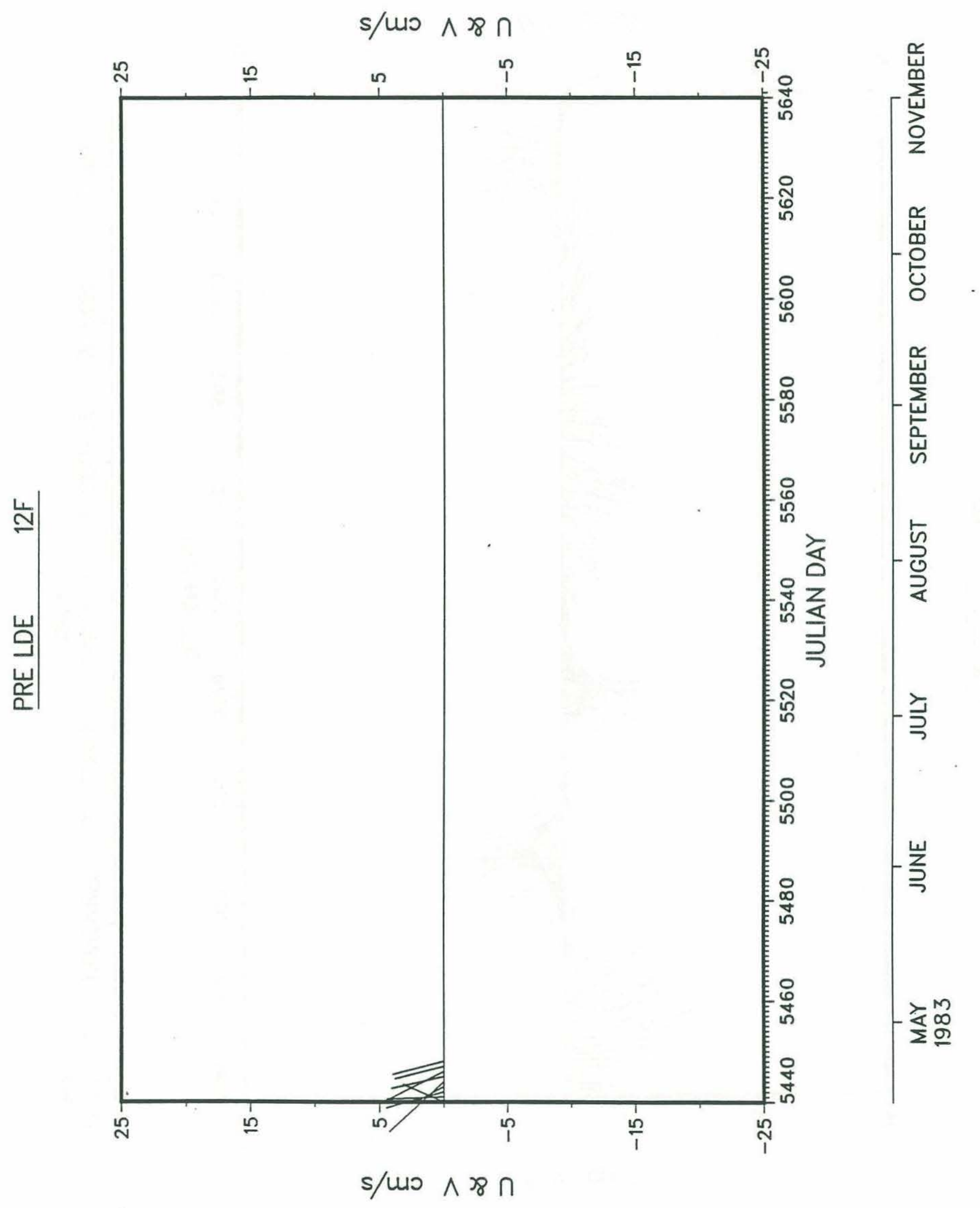




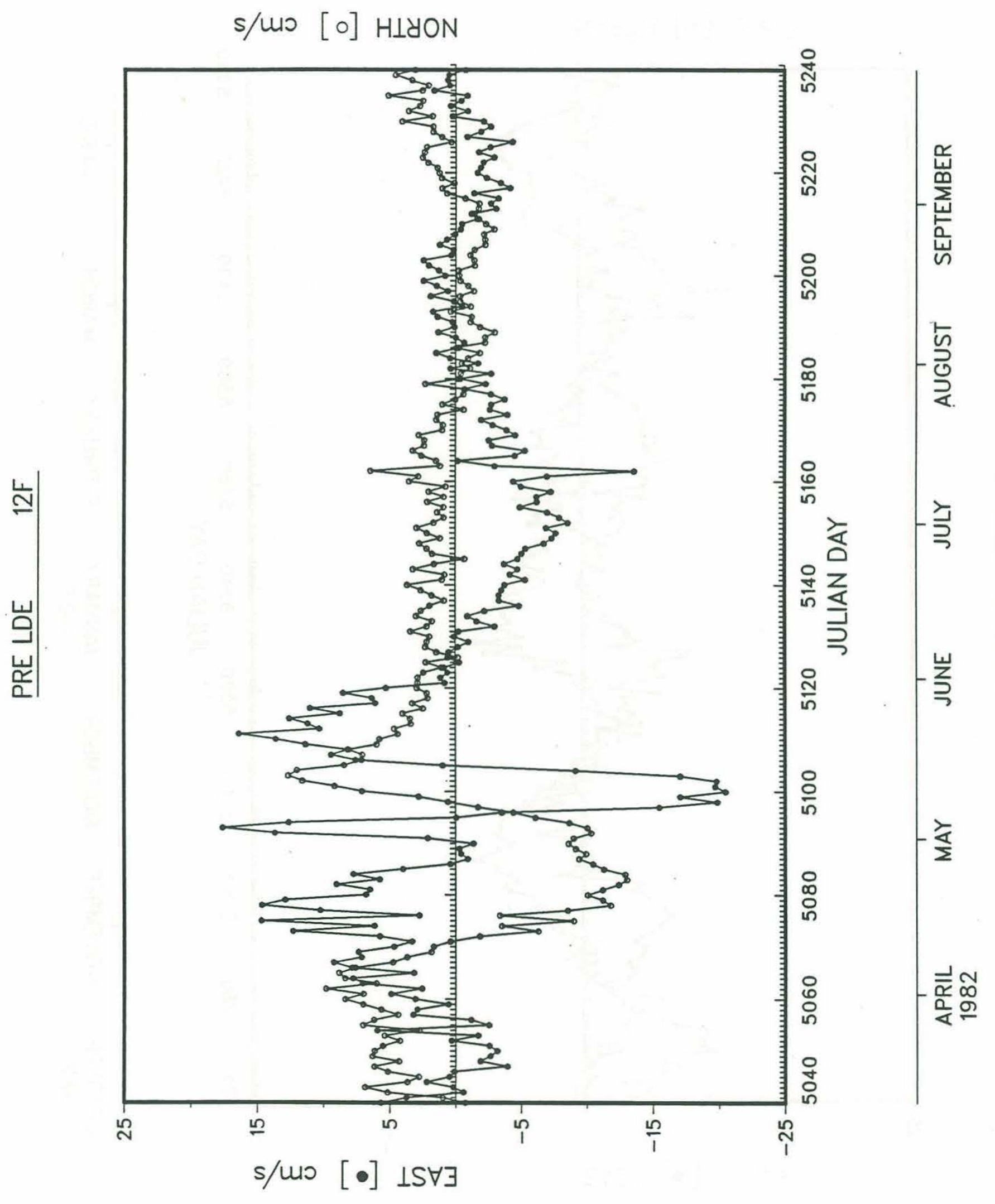




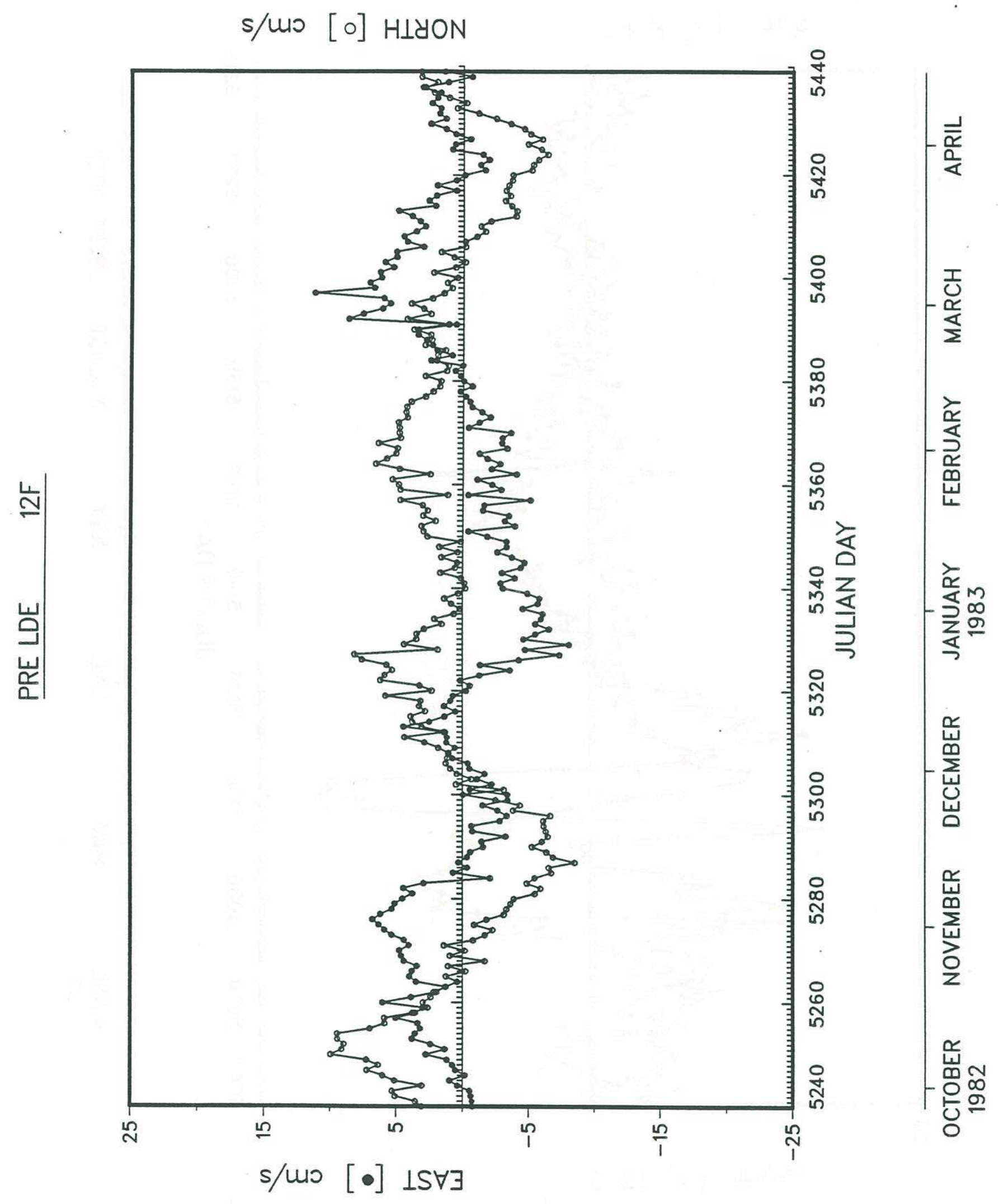




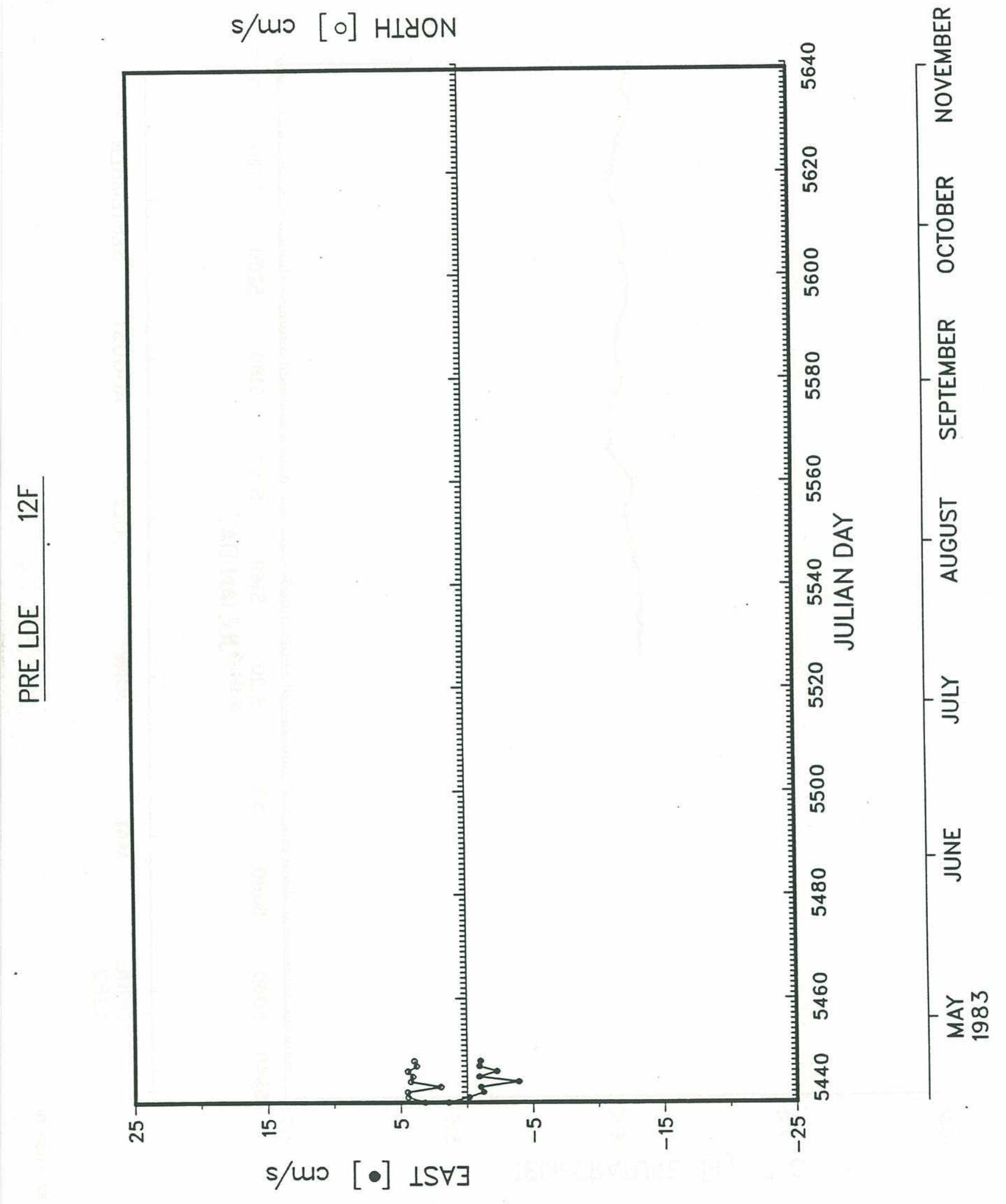



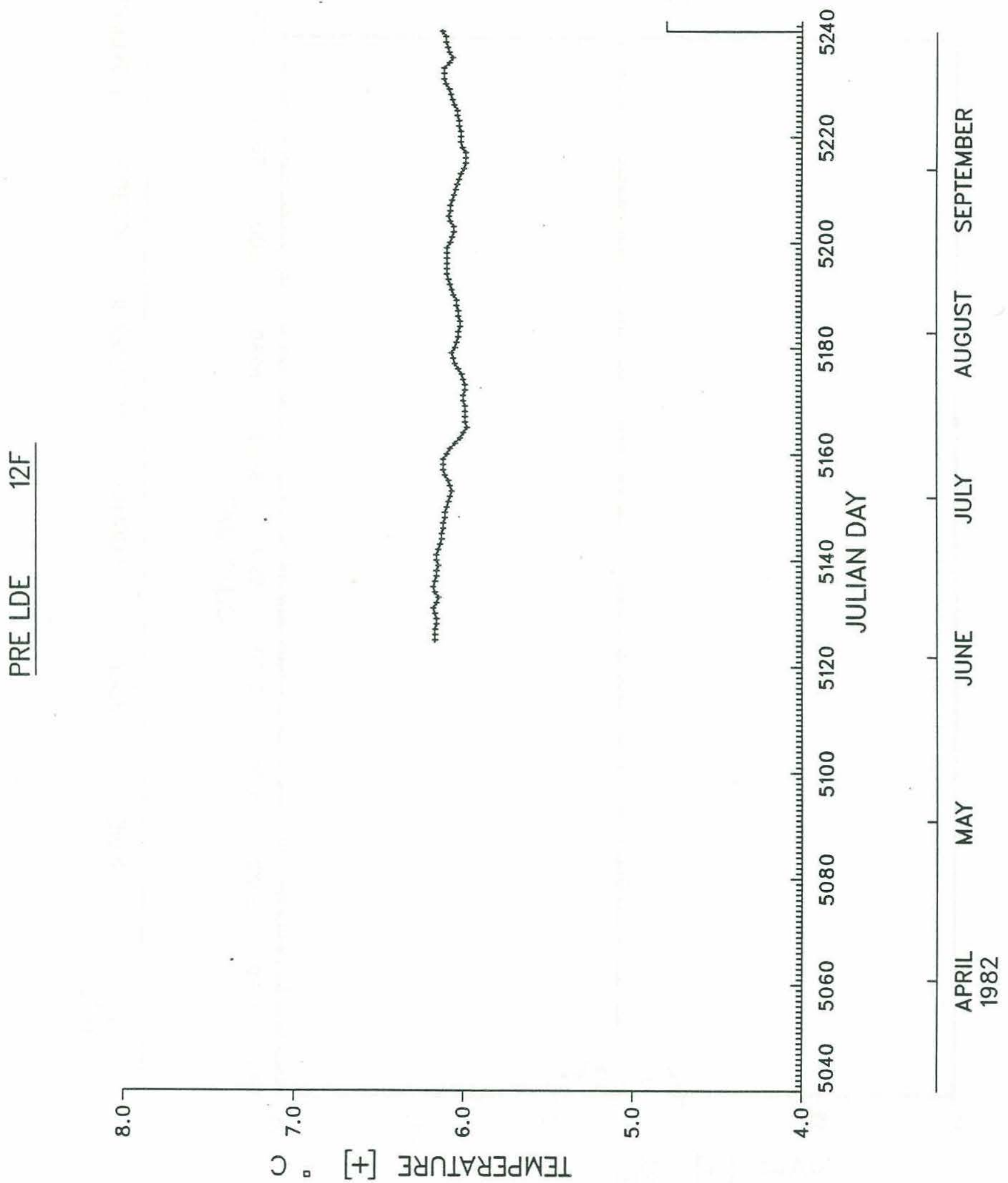

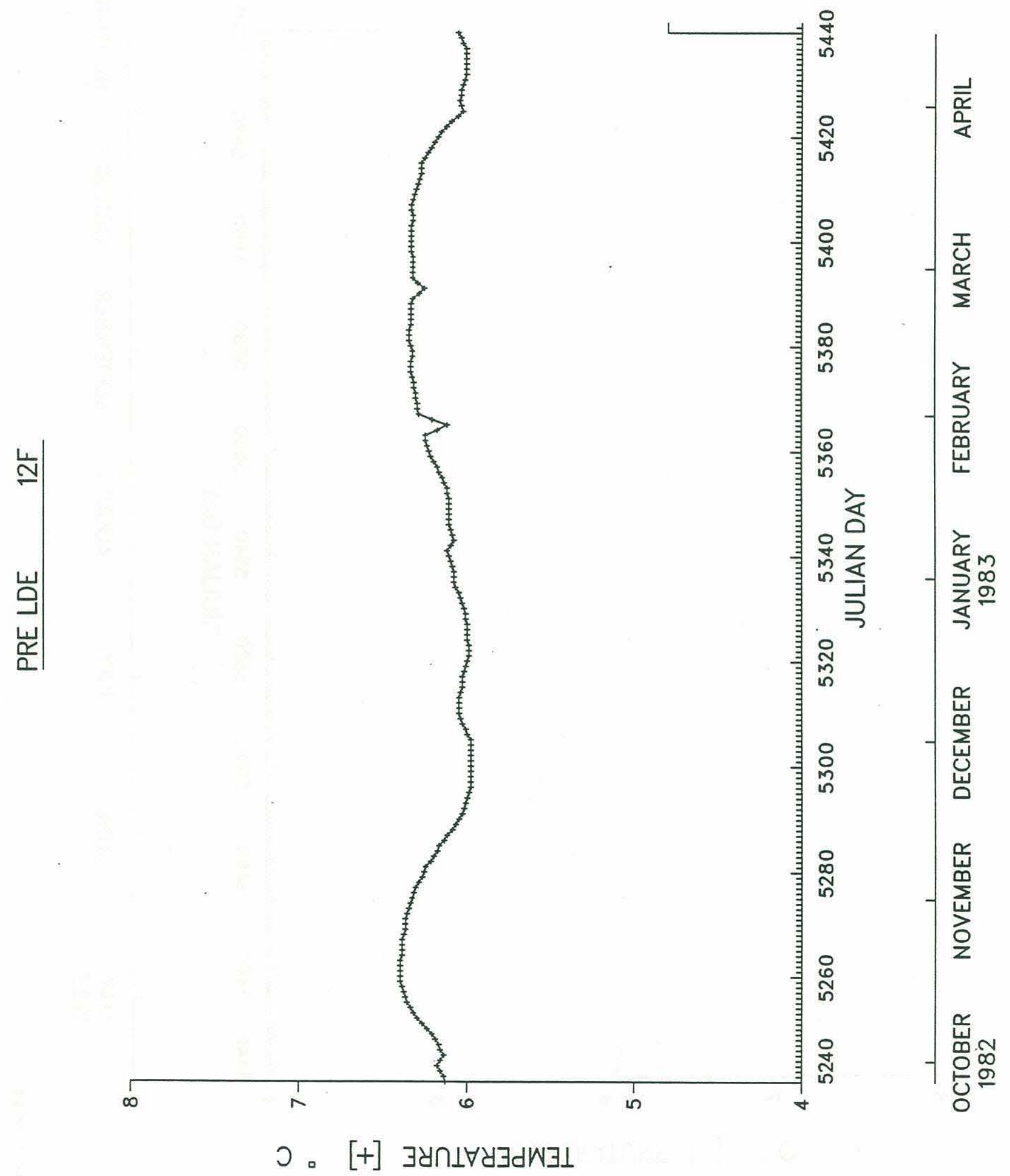


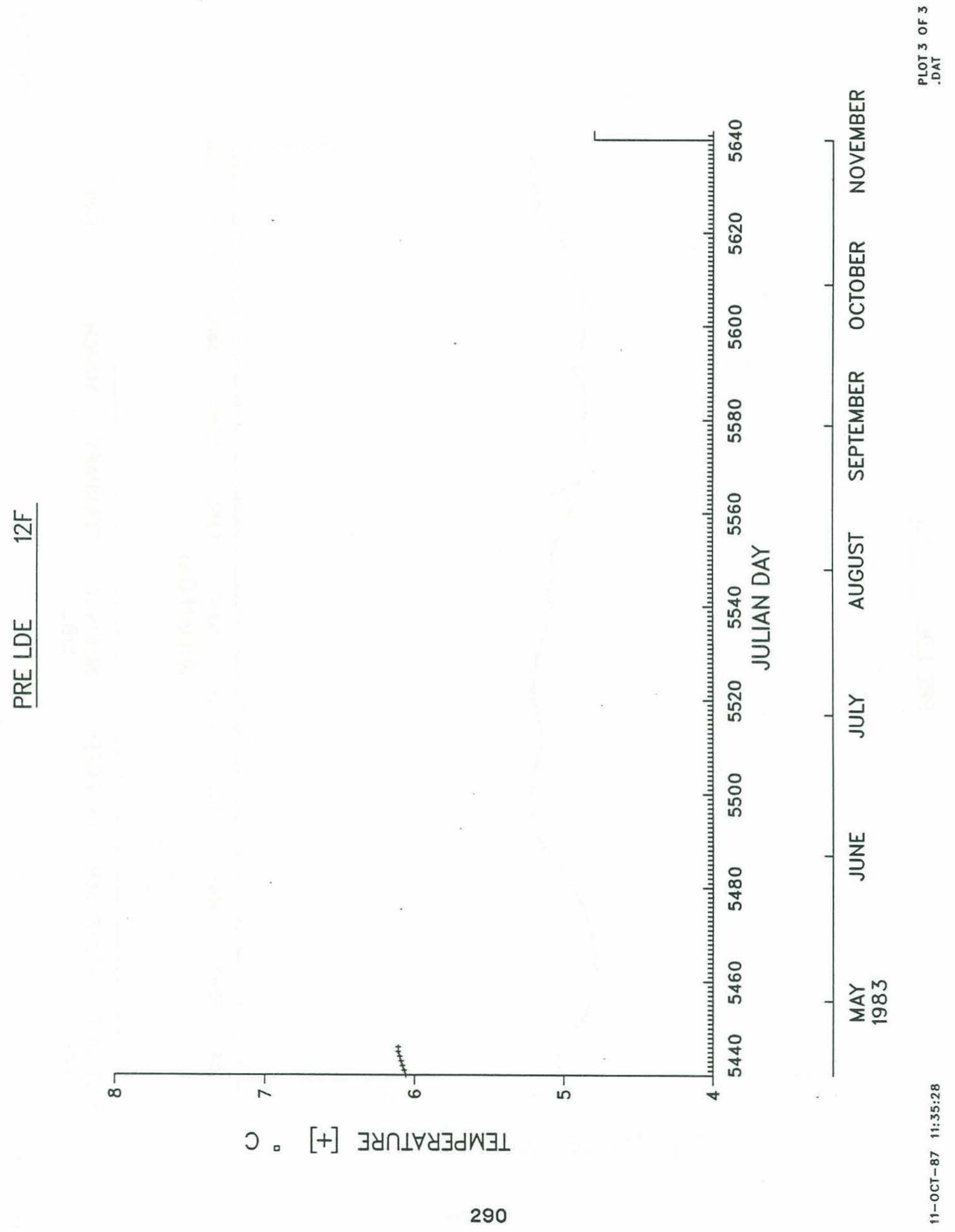




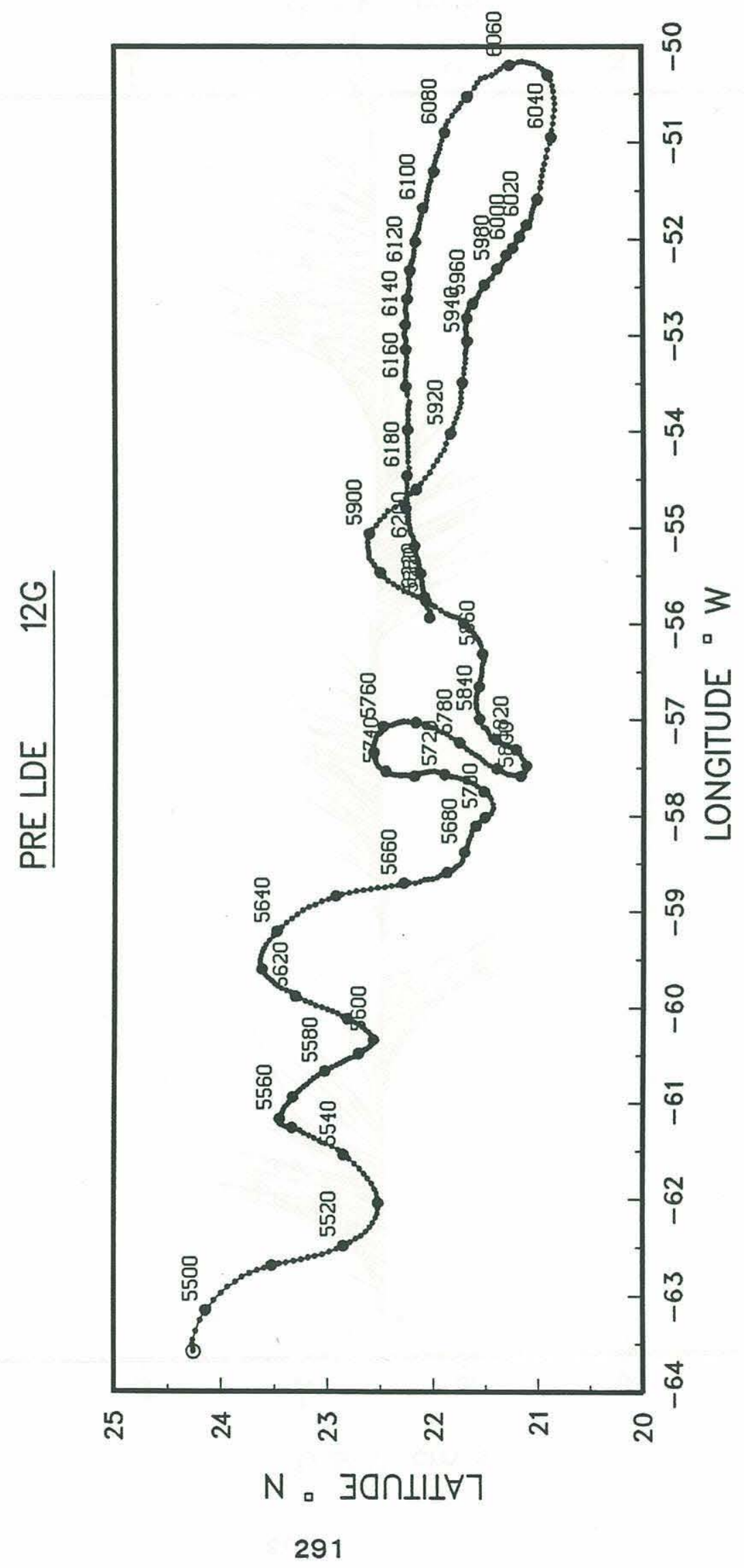




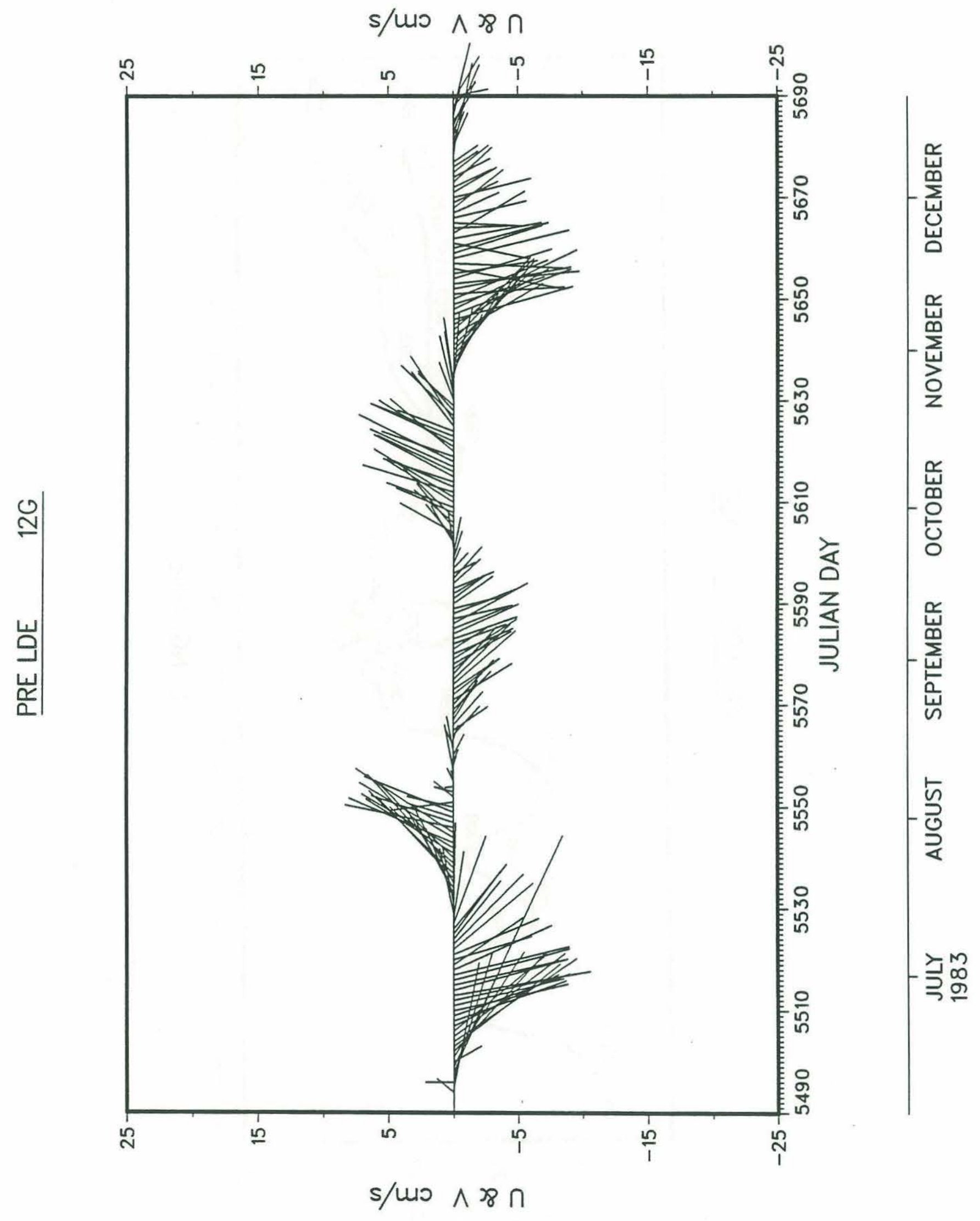




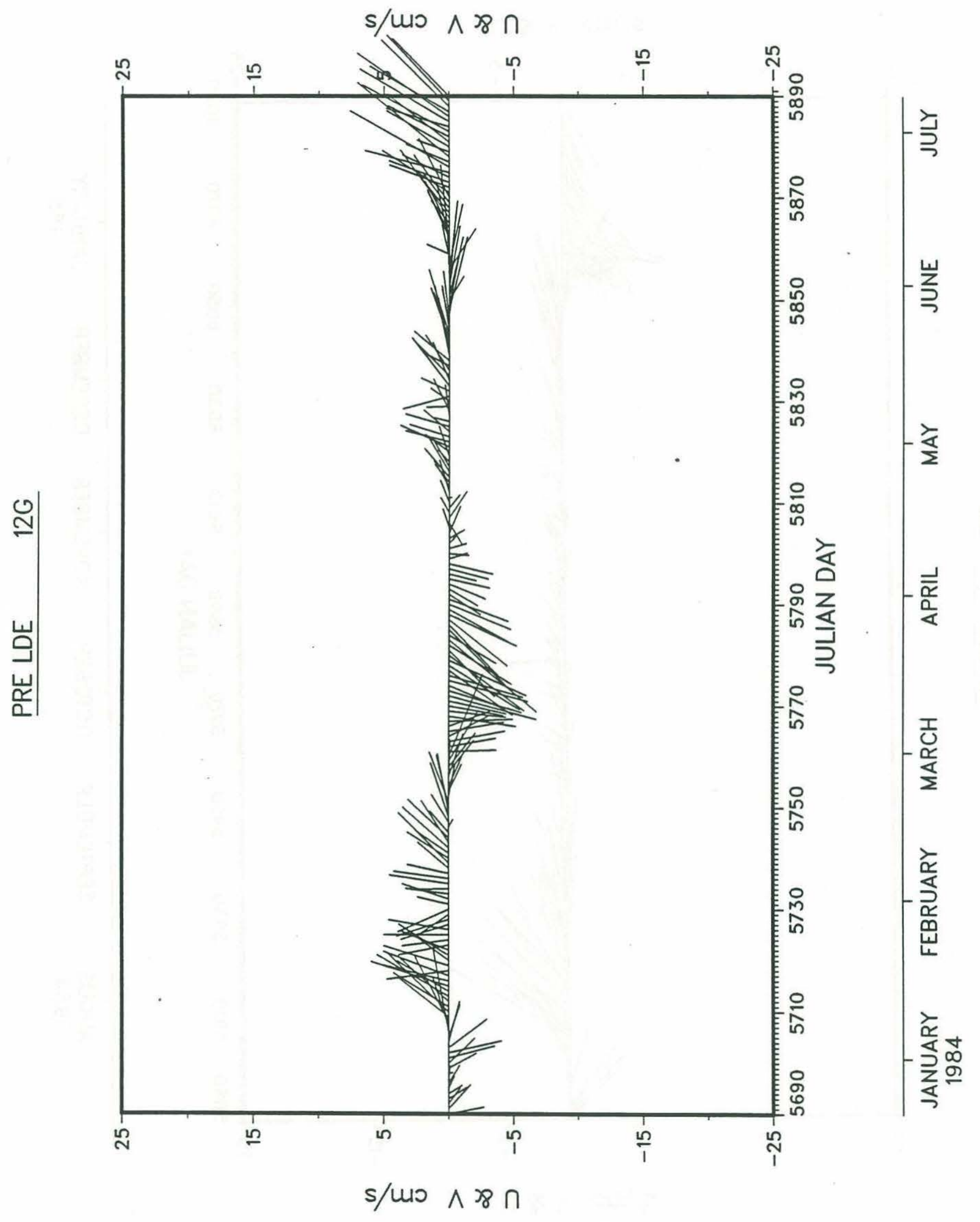




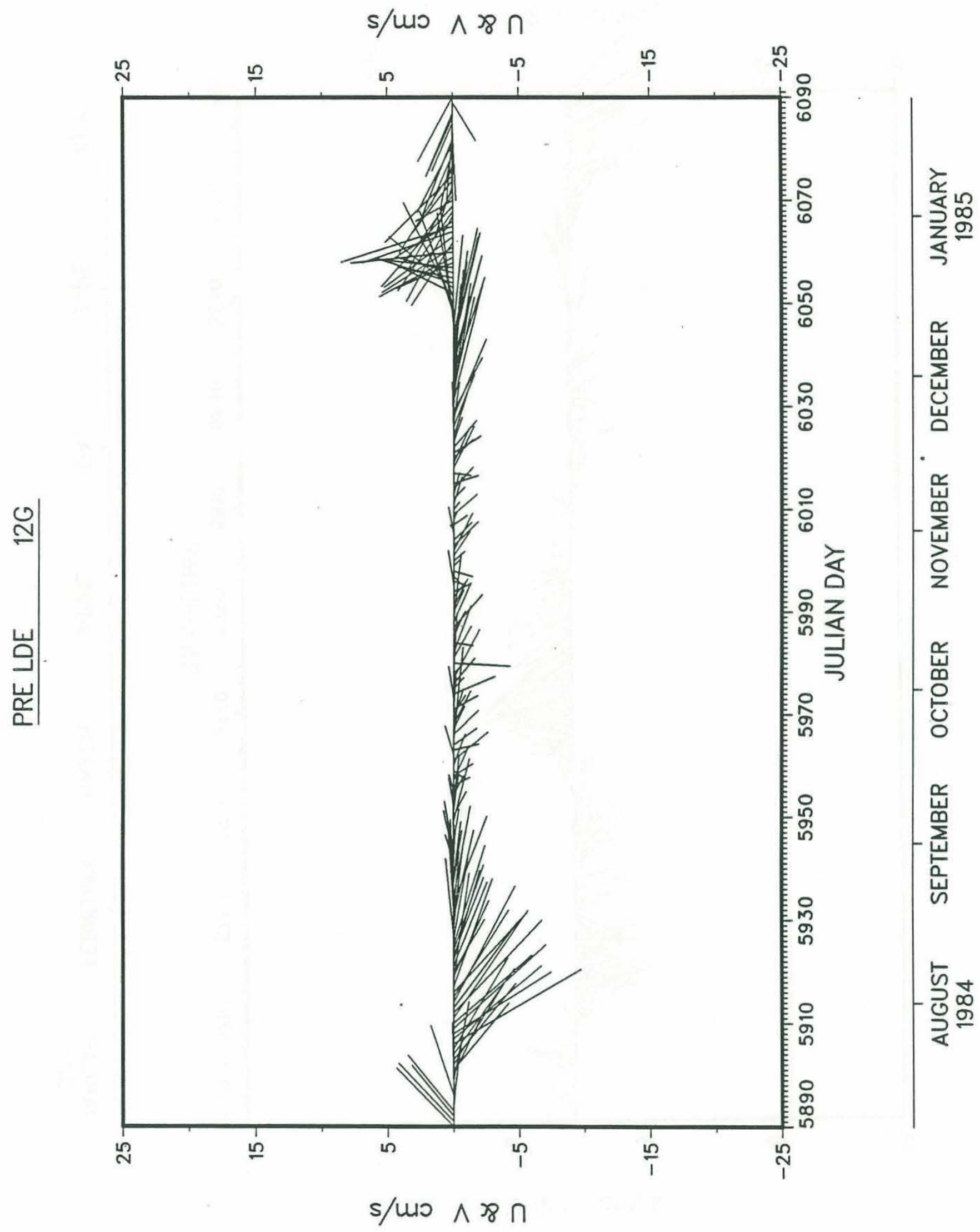




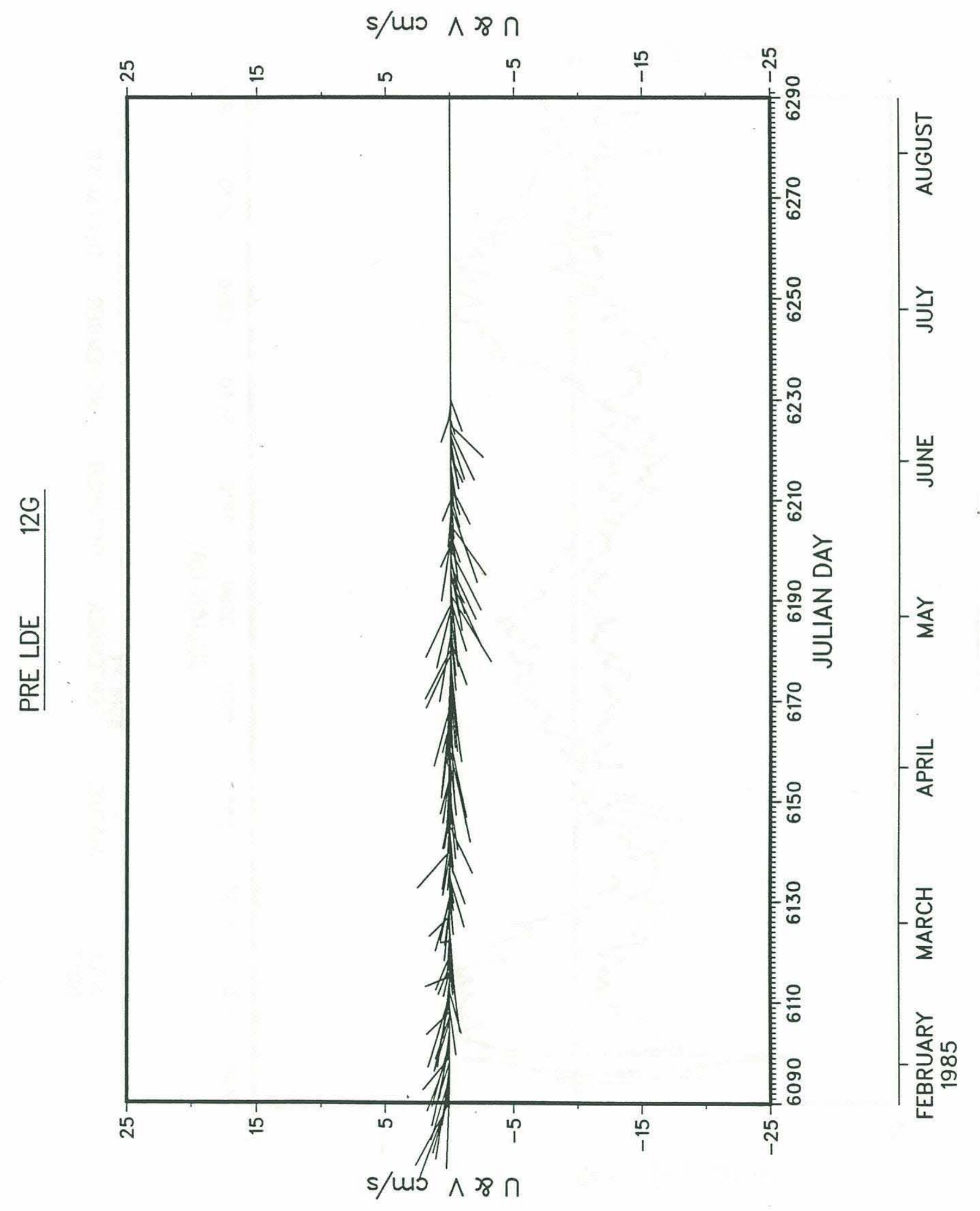




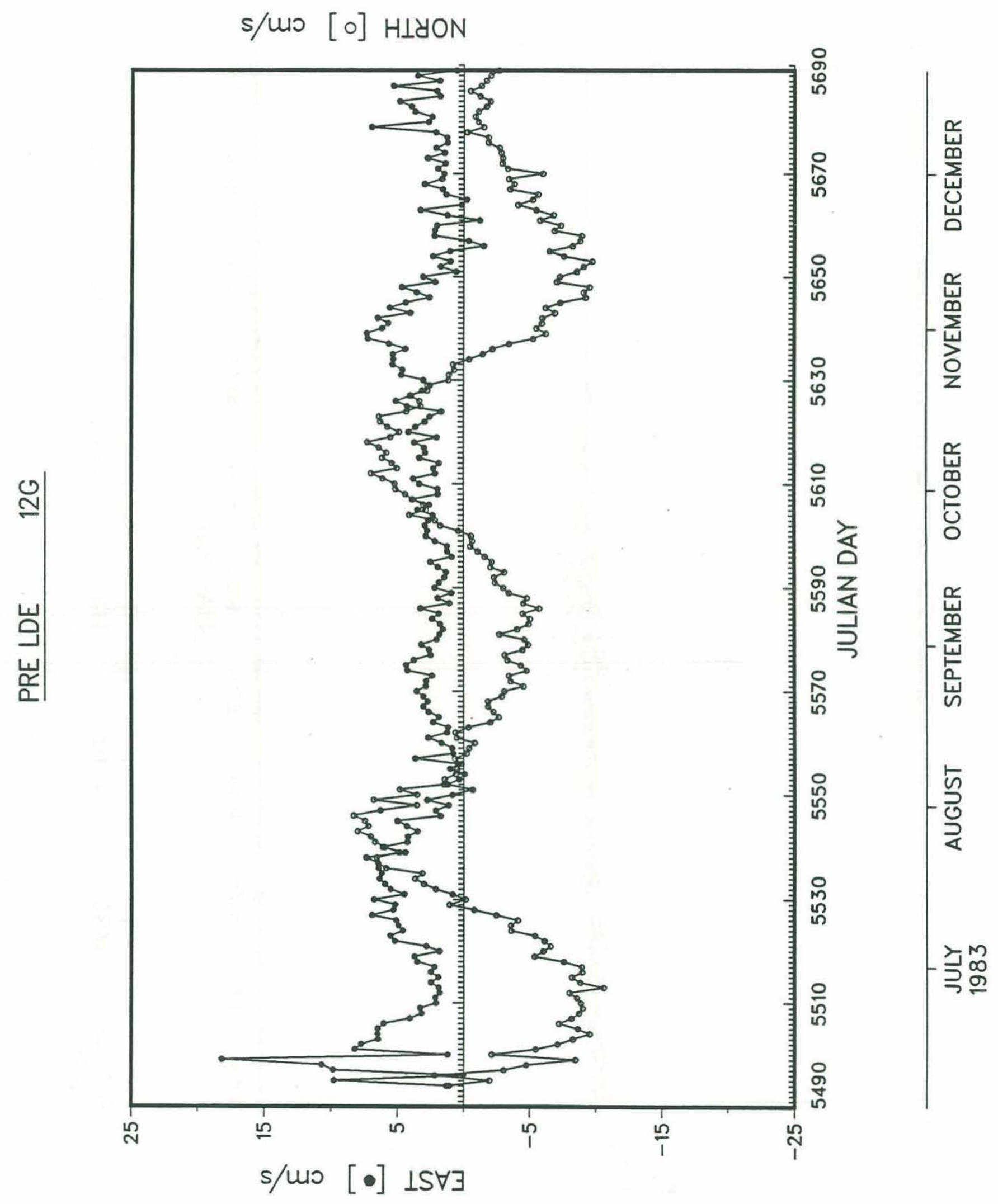




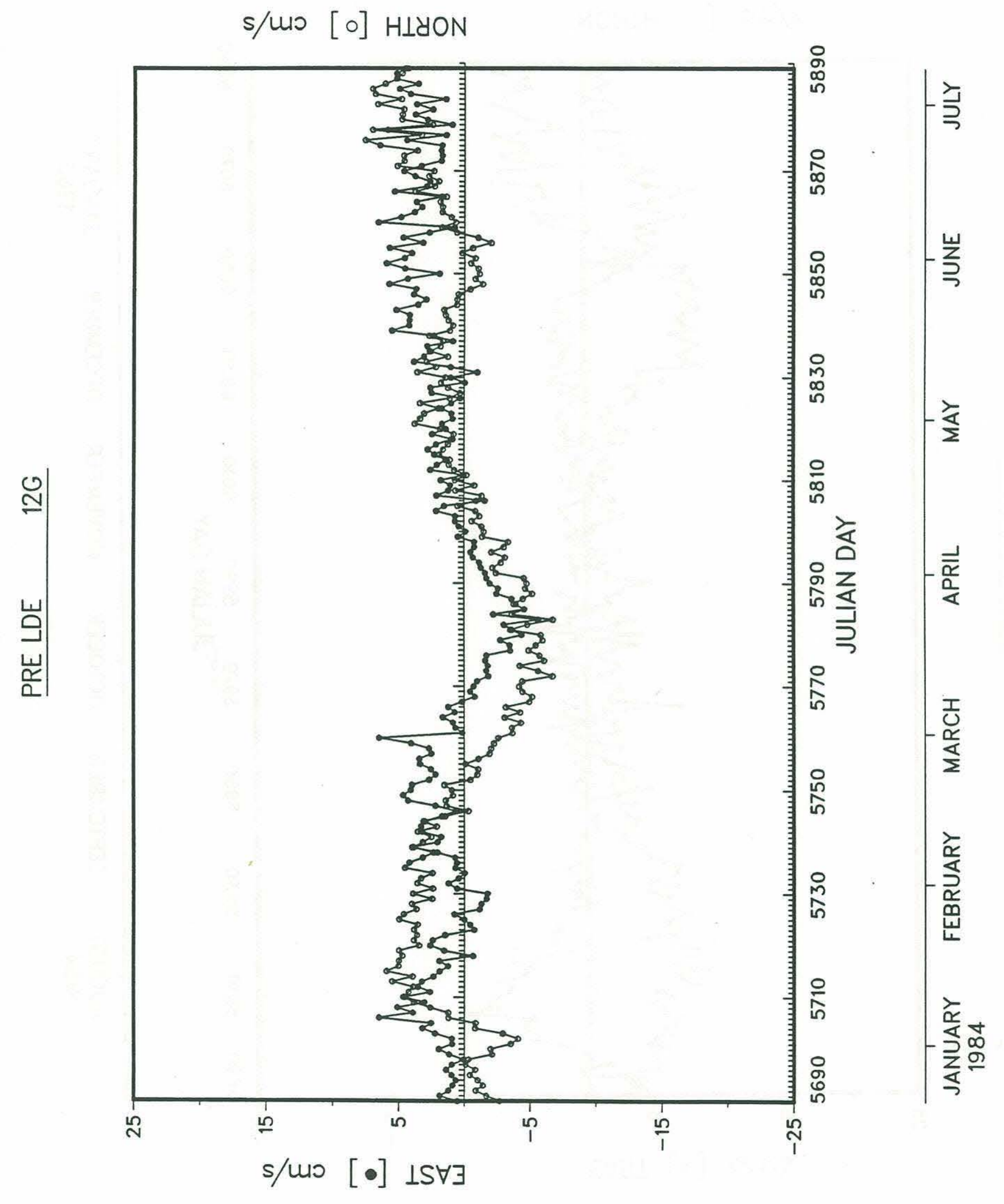




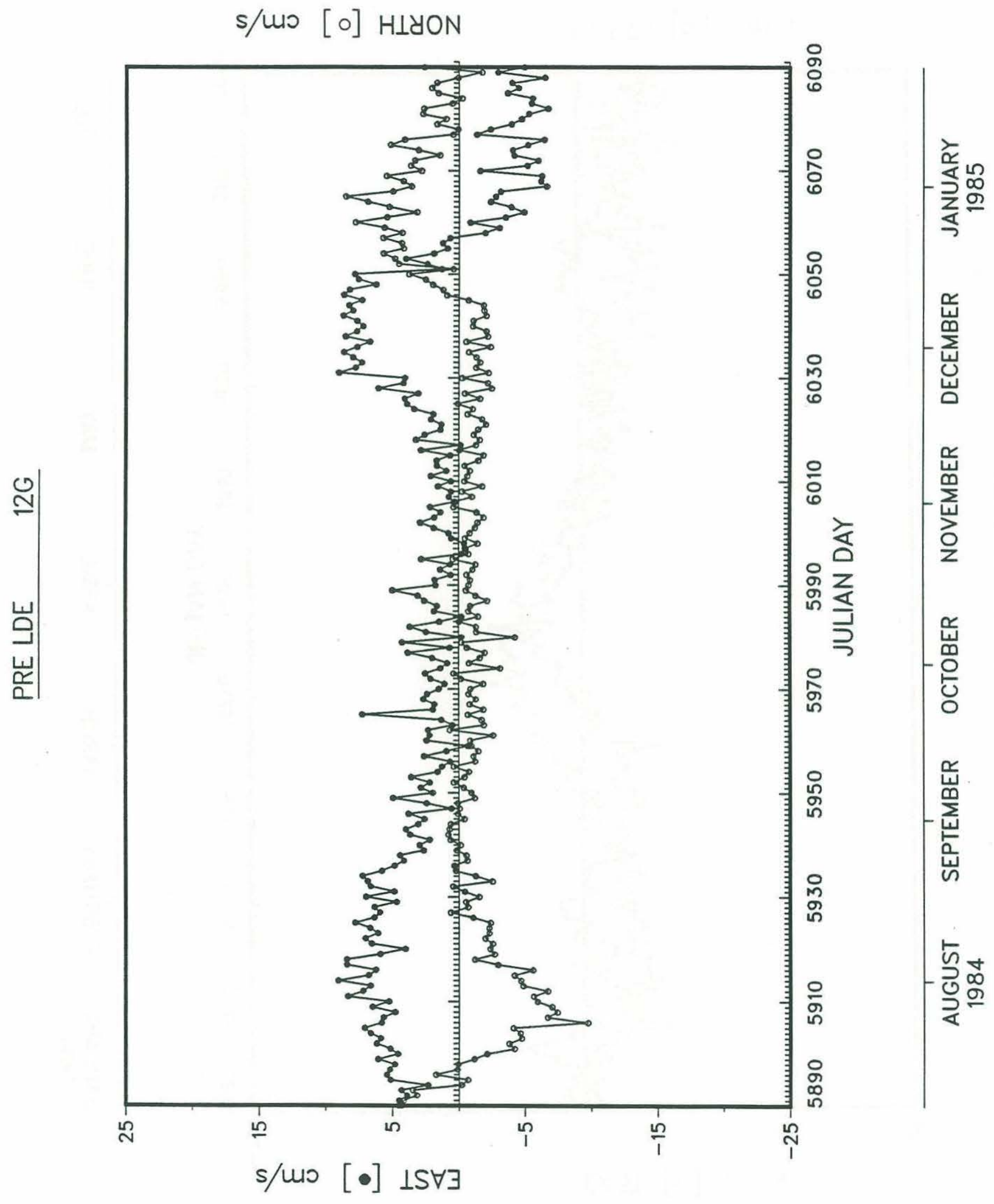




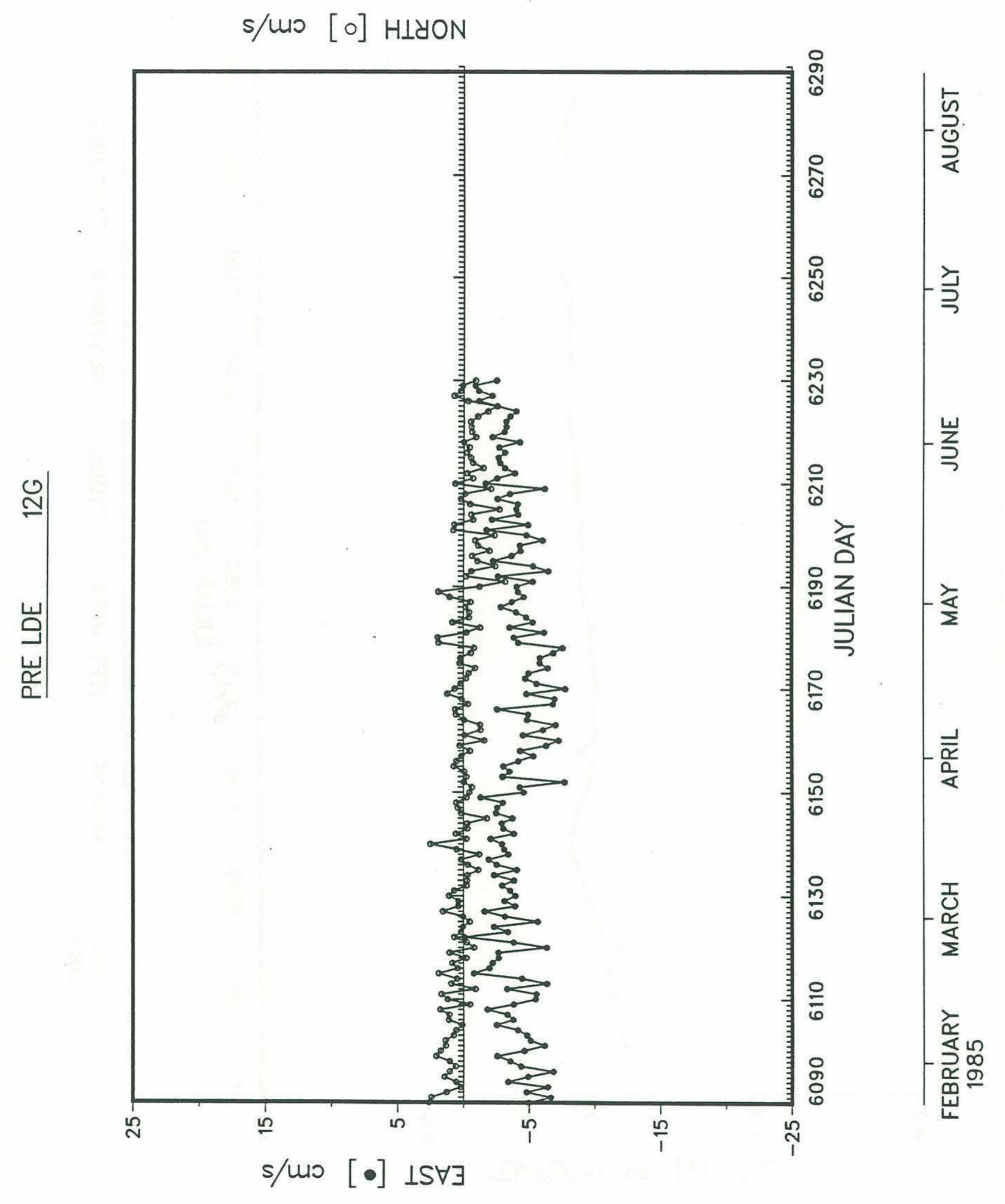




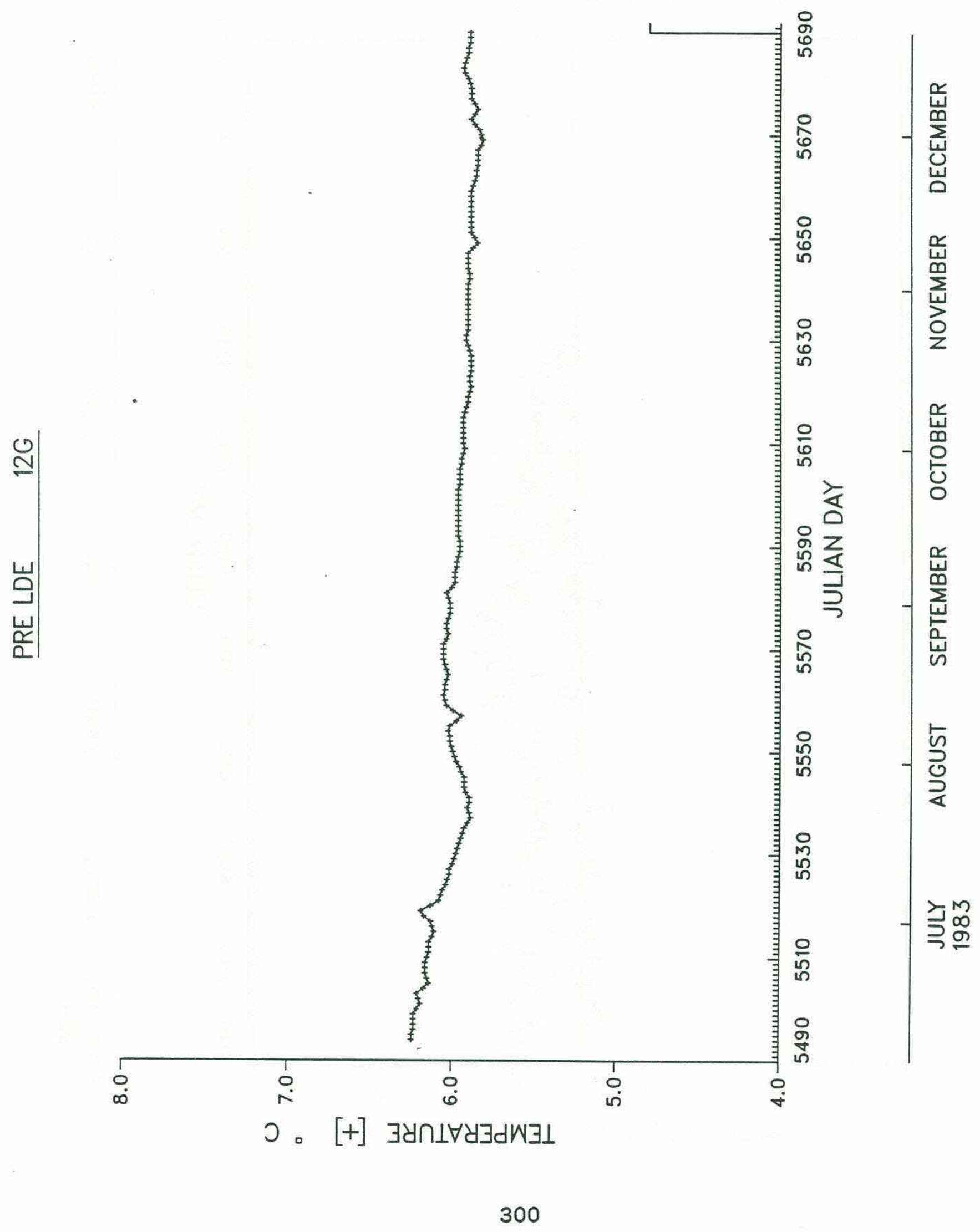



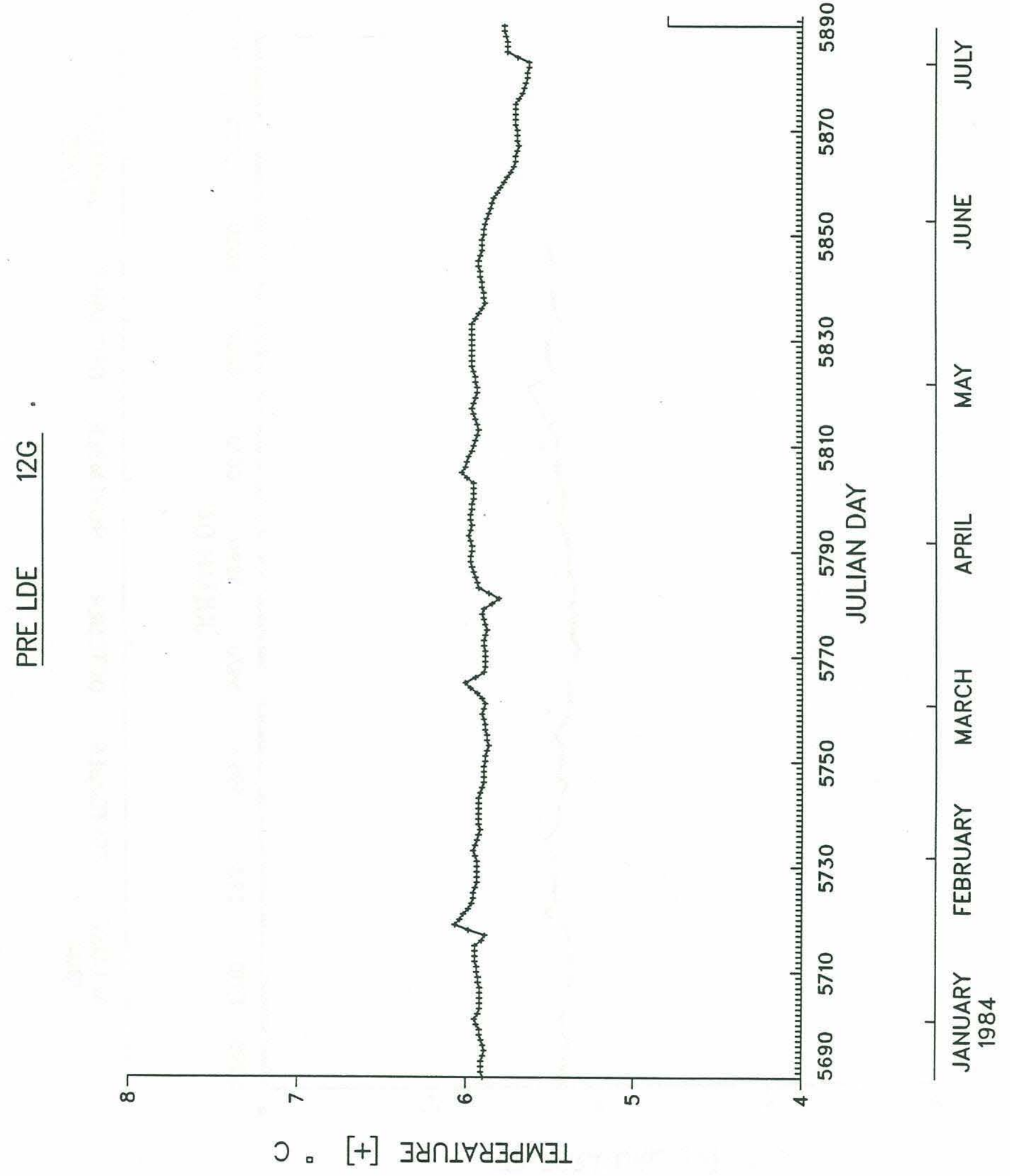
웜

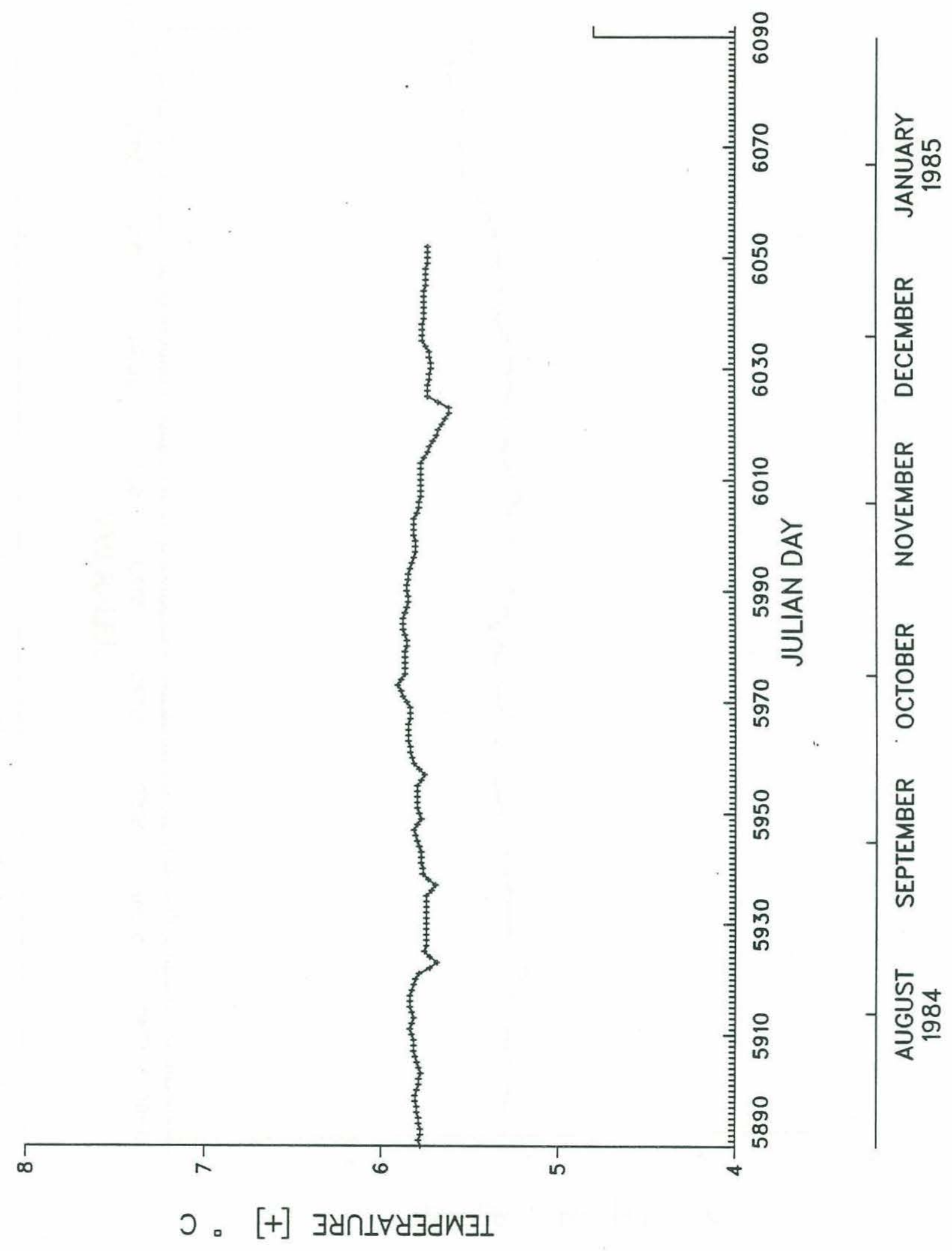




\section{APPENDIX B: Publications Using GUSREX Float Data}

Owens, W. B., 1984. A synoptic and statistical description of the Gulf Stream and subtropical gyre using SOFAR floats. Journal of Physical Oceanography, 14, 104-113.

Owens, W. B., P. L. Richardson, W. J. Schmitz, Jr., H. T. Rossby, and D. C. Webb, 1988. Nine-year trajectory of a SOFAR float in the southwestern North Atlantic. Deep-Sea Research, 35, 1851-1857.

Price, J. F., 1985. Particle dispersion in the western North Atlantic. Unpublished manuscript.

Price, J. F., and H. T. Rossby, 1982. Observations of a barotropic planetary wave in the western North Atlantic. Journal of Marine Research, 40(Suppl.), 543-558.

Richardson, P. L., 1983. A vertical section of eddy kinetic energy through the Gulf Stream system. Journal of Geophysical Research, 88, 2705-2709.

Richardson, P. L., 1985. Average velocity and transport of the Gulf Stream near $55^{\circ} \mathrm{W}$. Journal of Marine Research, 43, 83-111.

Schmitz, W. J., Jr., 1985. SOFAR float trajectories associated with the Newfoundland Basin. Journal of Marine Research, 43, 761-778.

Schmitz, W. J., J. F. Price, and P. L. Richardson, 1988. Recent moored current meter and SOFAR float observations in the eastern Atlantic near 32N. Journal of Marine Research, 46, 301-319.

Shaw, P., and H. T. Rossby, 1984. Towards a Lagrangian description of the Gulf Stream. Journal of Physical Oceanography, 14, 528-540. 


\section{APPENDIX C: Calendar Conversion Tables - 1981 to 1986}

These tables give the year-day and truncated Julian day for each calendar date for the years 1981 through 1986. The truncated Julian days range from 4606 to 6796 . To convert to true Julian day, add 2440000.5 to these numbers. 


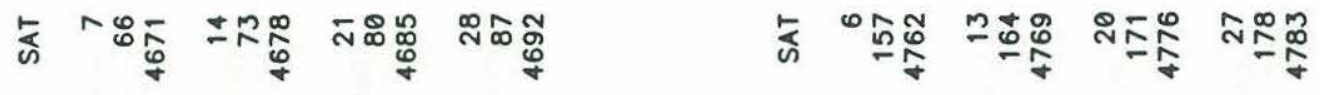

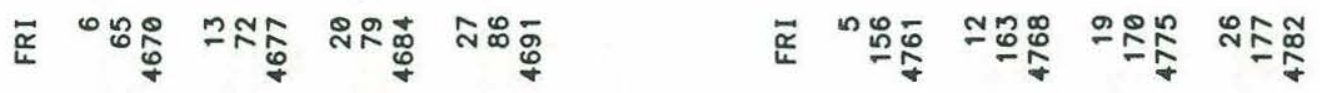

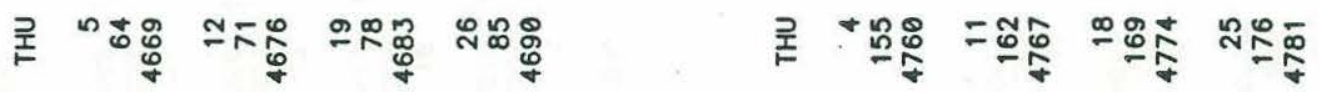

$$
\begin{aligned}
& \text { 䨔 崖 }
\end{aligned}
$$

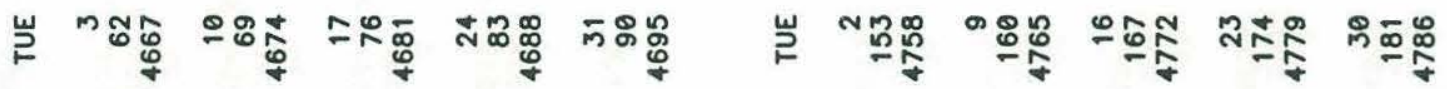

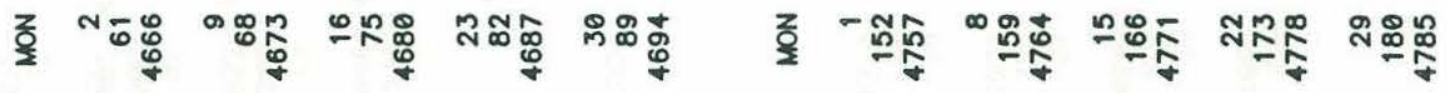

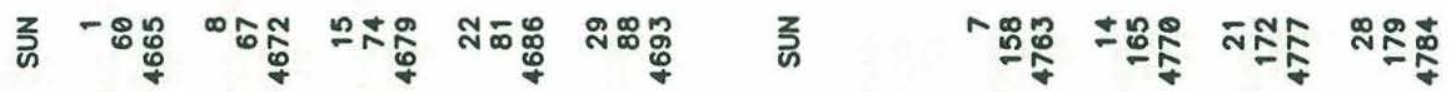

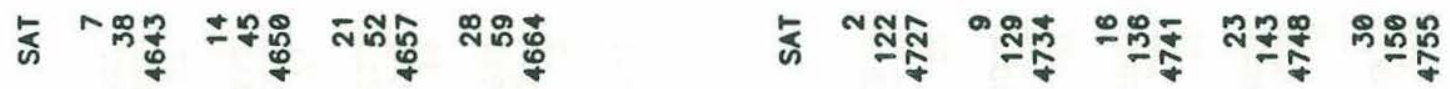

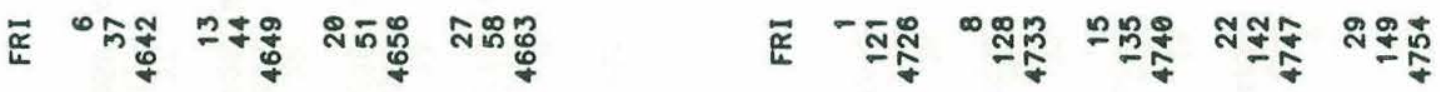

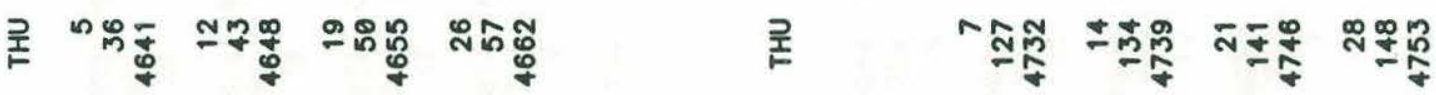

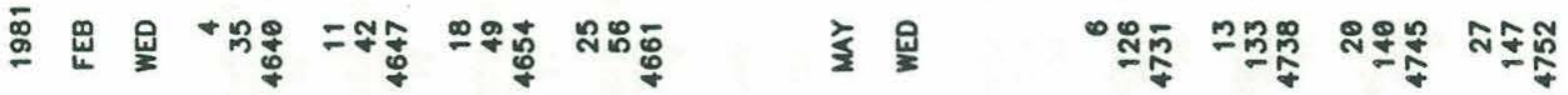

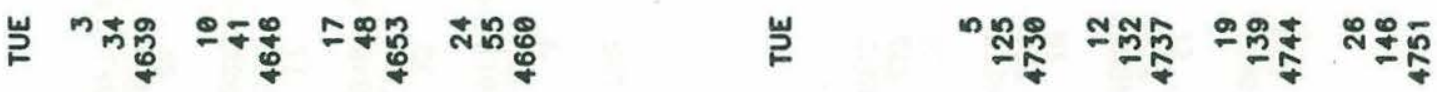

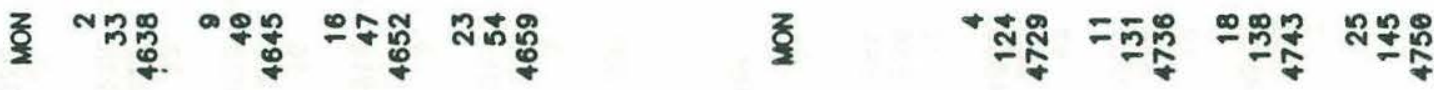

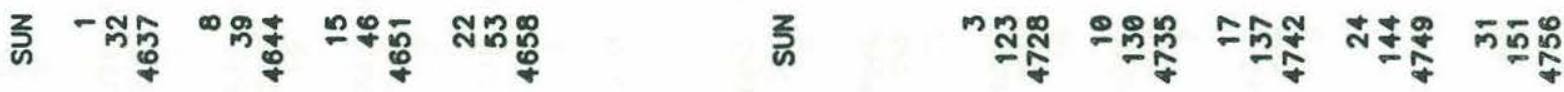

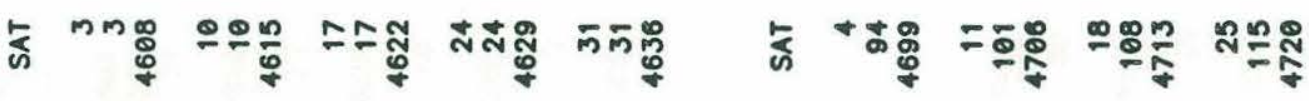

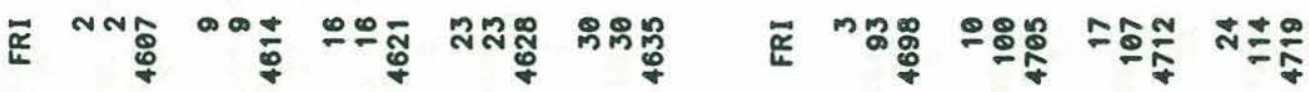

$$
\begin{aligned}
& \text { 곤 - - }
\end{aligned}
$$

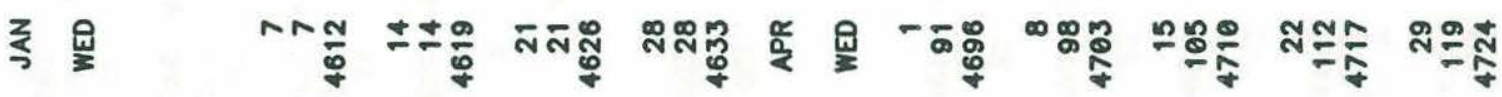

$$
\begin{aligned}
& \text { 岁 }
\end{aligned}
$$

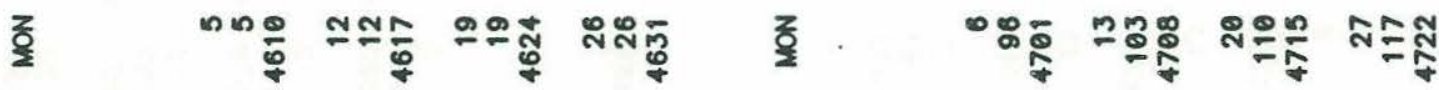

$$
\begin{aligned}
& \text { 蛋 +每 }
\end{aligned}
$$




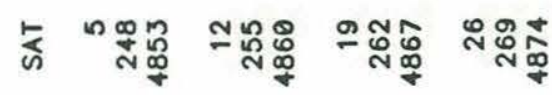

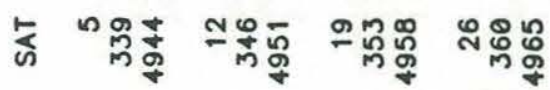

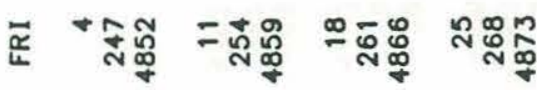

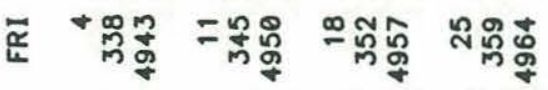

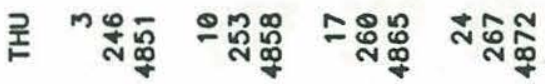

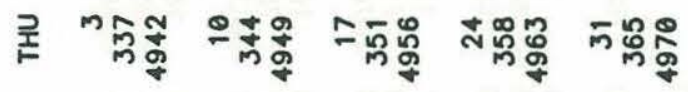

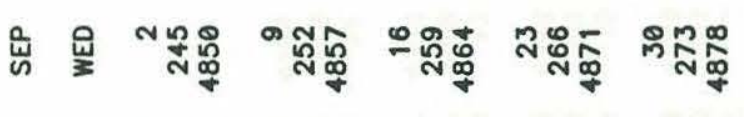

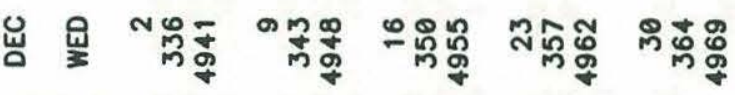

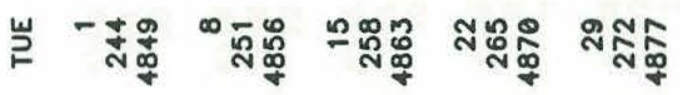

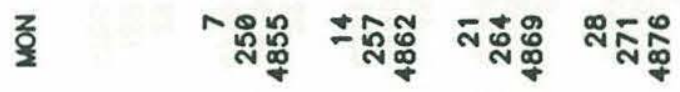

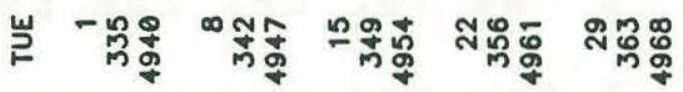

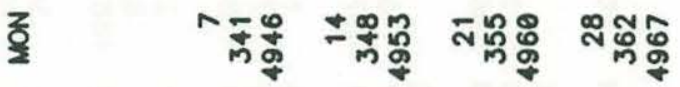

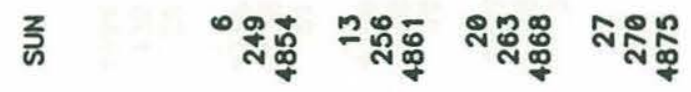

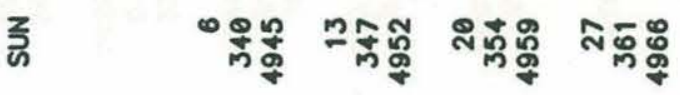

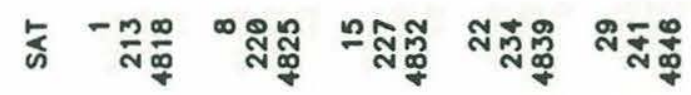

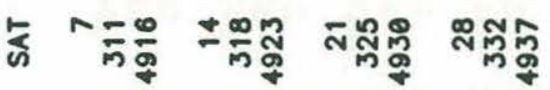

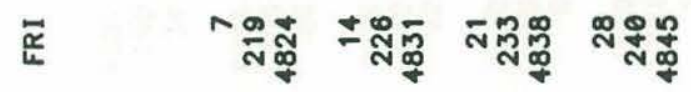

$$
\begin{aligned}
& \text { ะ }
\end{aligned}
$$

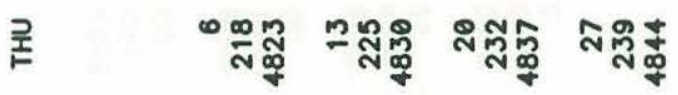

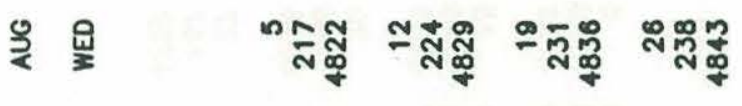

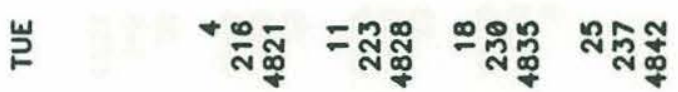

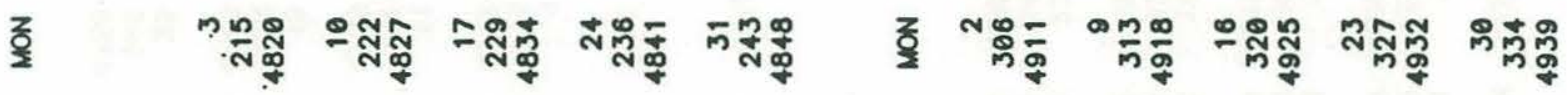

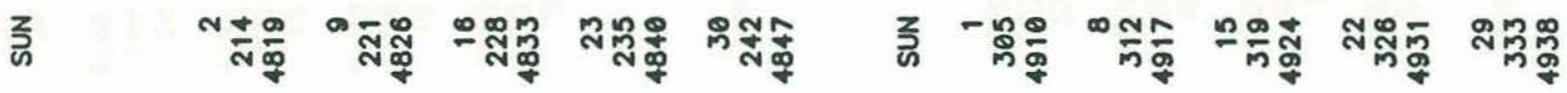

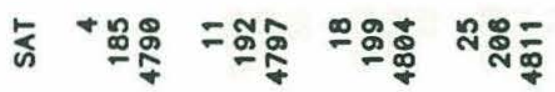

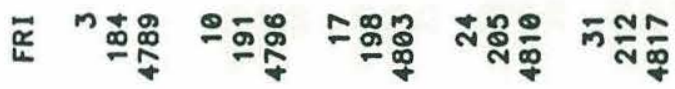

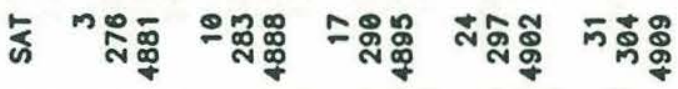

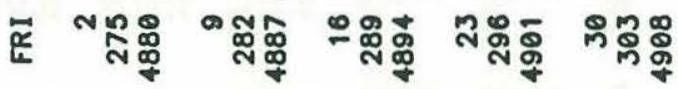

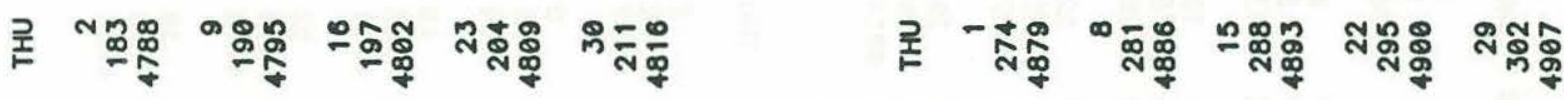

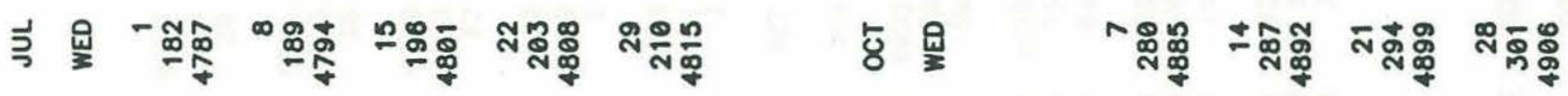

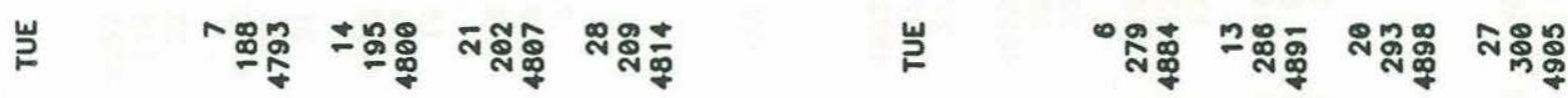

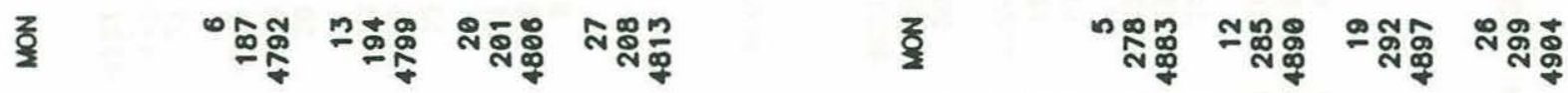

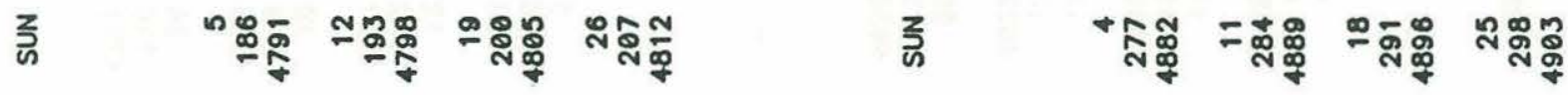




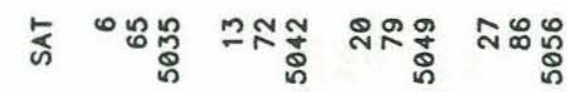

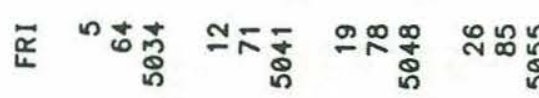

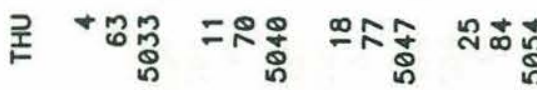

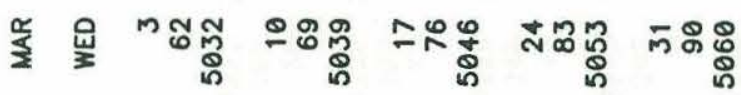

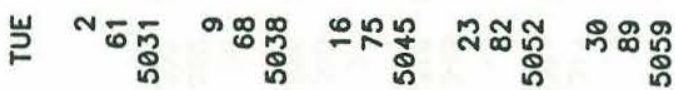

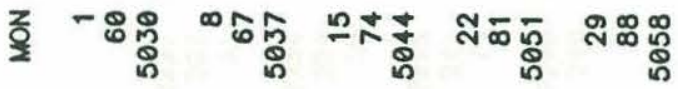

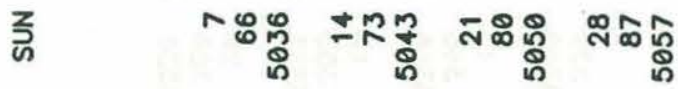

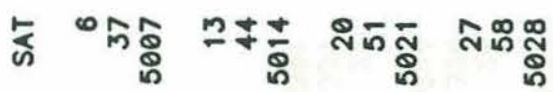

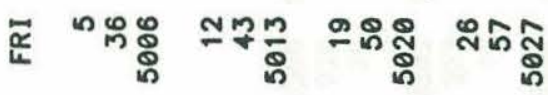

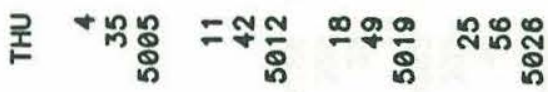

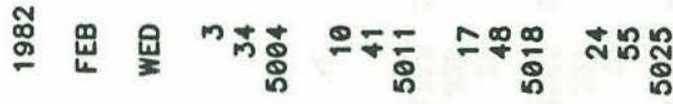

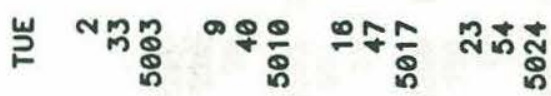

$$
\begin{aligned}
& \text { ช พก๊ }
\end{aligned}
$$

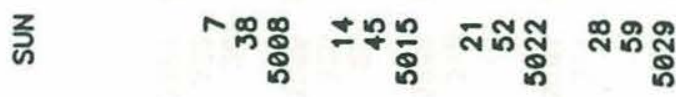

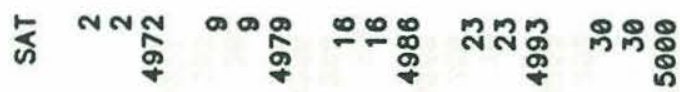

$$
\begin{aligned}
& \text { 荙 - } \\
& \text { 곤 MN帝 }
\end{aligned}
$$

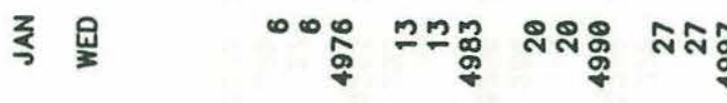

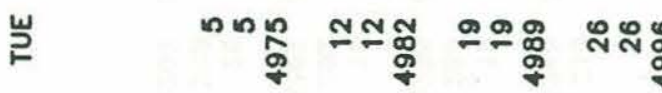

$$
\begin{aligned}
& \text { J. } \\
& \text { 蛋 的昘 }
\end{aligned}
$$

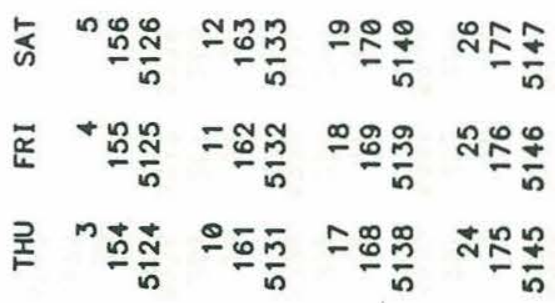

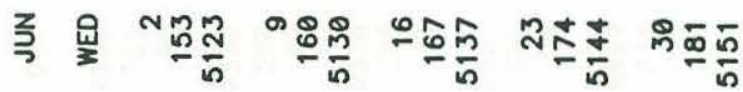

$$
\begin{aligned}
& \text { 岂 一ำสำ } \\
& \text { ว }
\end{aligned}
$$

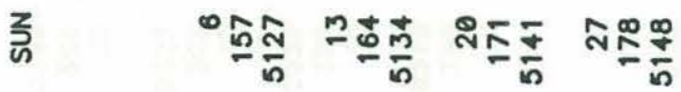

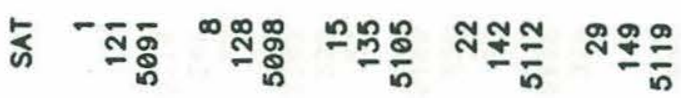

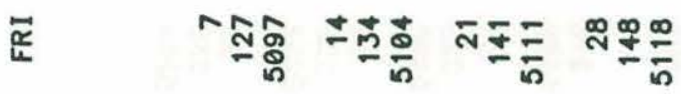

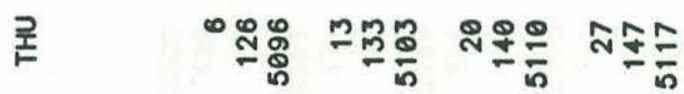

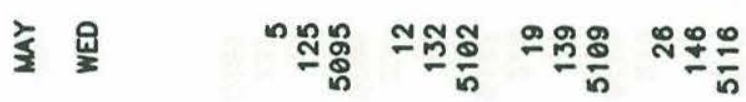

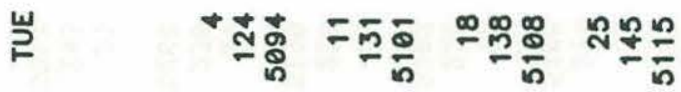

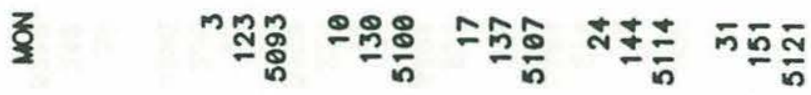

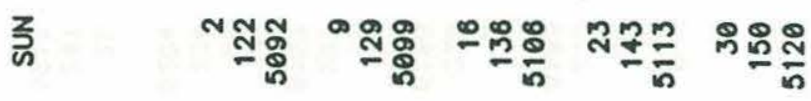

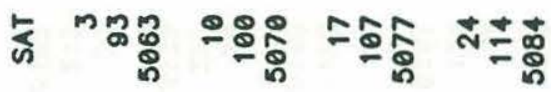

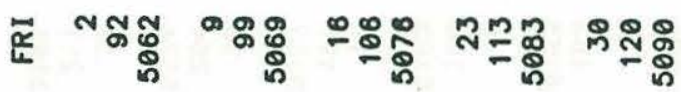

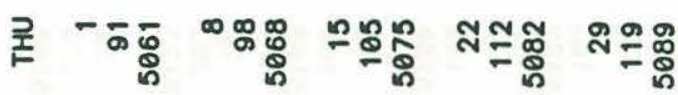

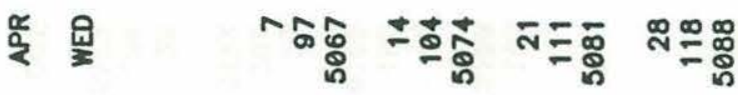

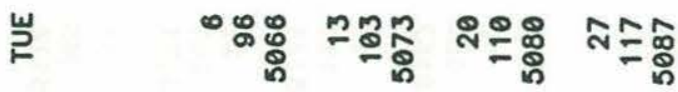

$$
\begin{aligned}
& \text { ช }
\end{aligned}
$$

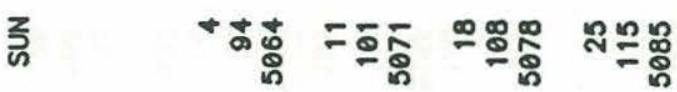




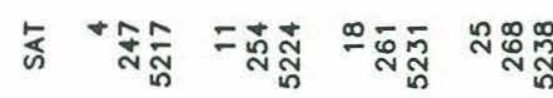

区ี

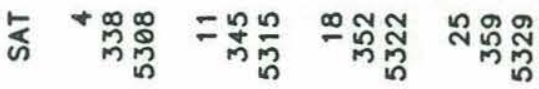

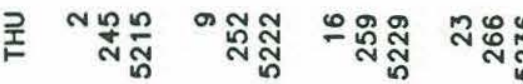

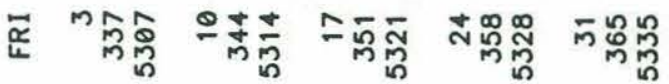

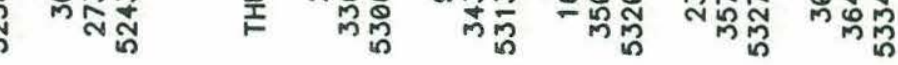

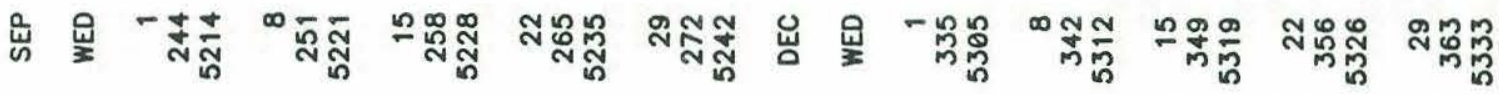

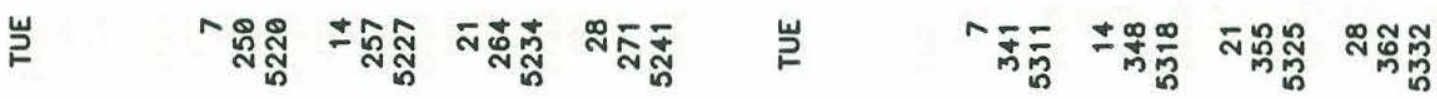

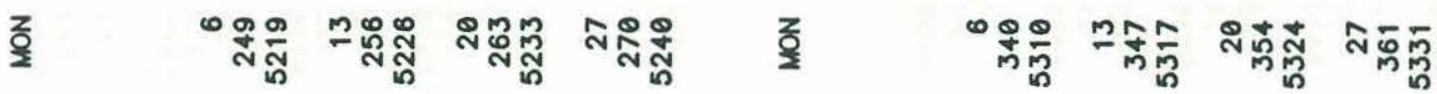

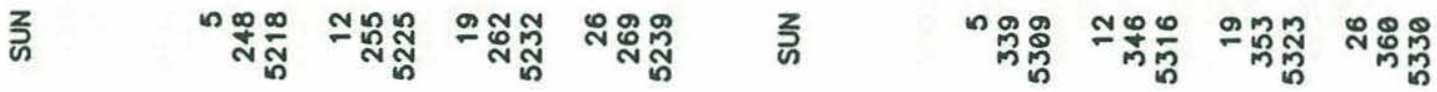

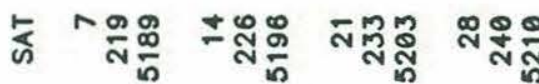

ฉี

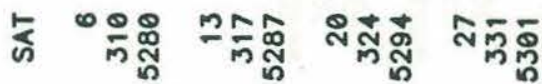

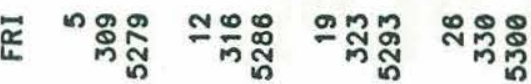

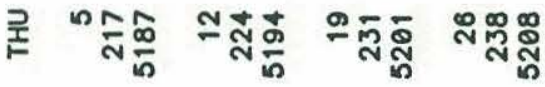

곤 +

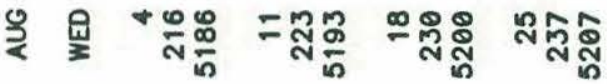

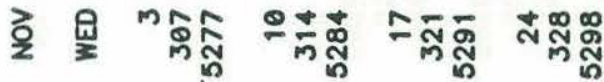

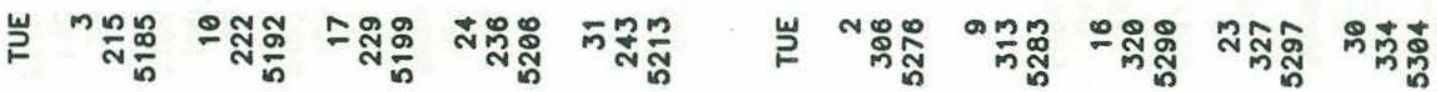

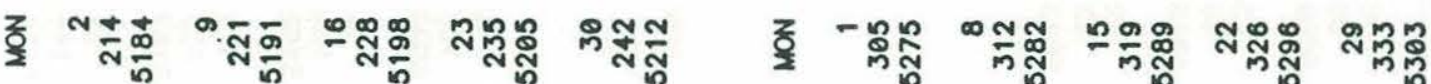

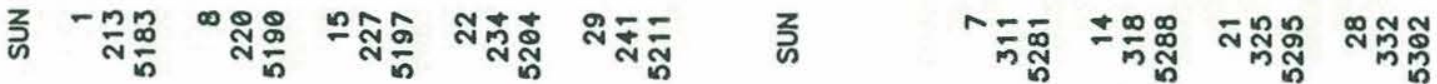

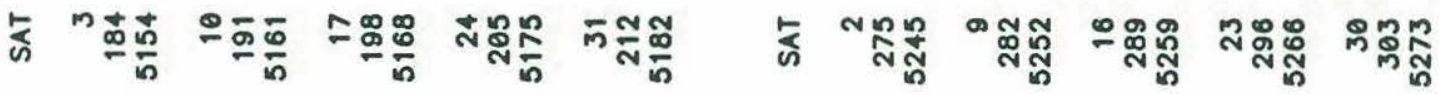

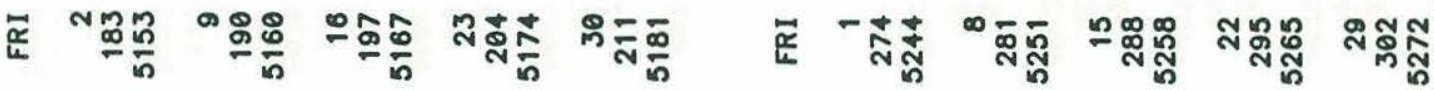

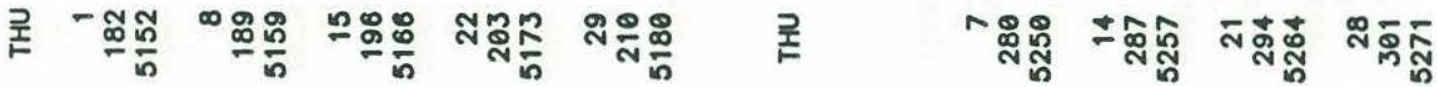

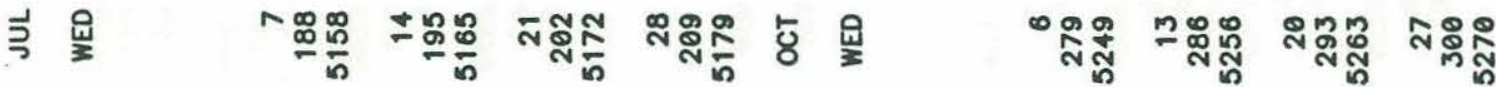

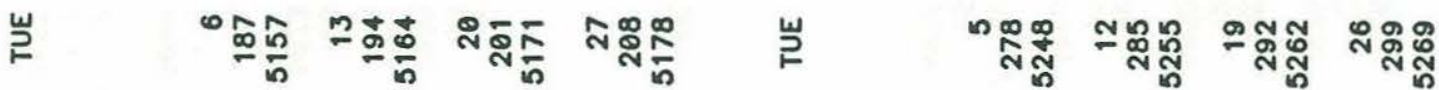

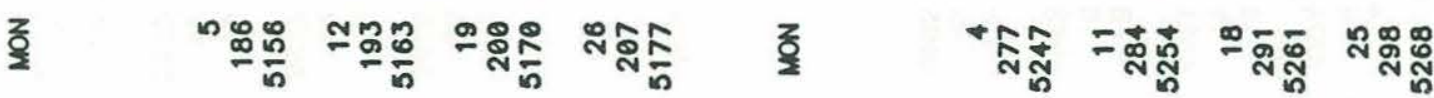

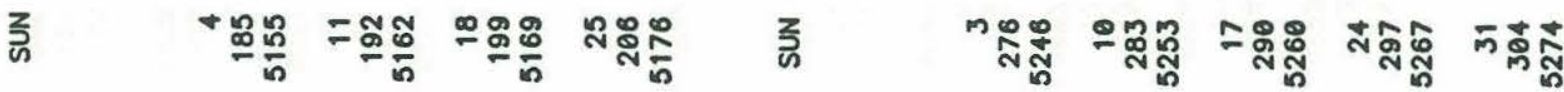




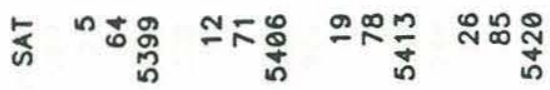

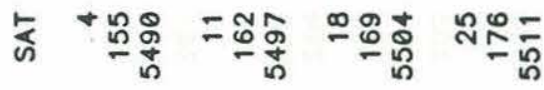

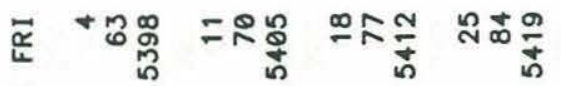

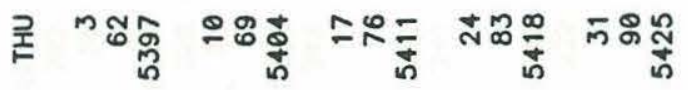

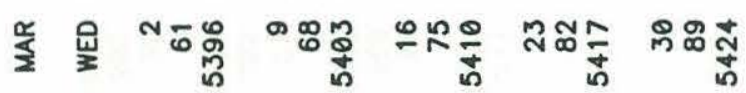

$$
\begin{aligned}
& \text { 岁 一绐, } \\
& \text { ว } \\
& \text { ว }
\end{aligned}
$$

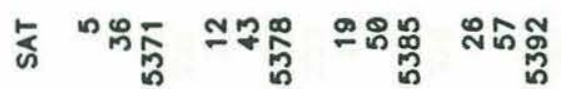

$$
\begin{aligned}
& \text { 采 +品命 }
\end{aligned}
$$

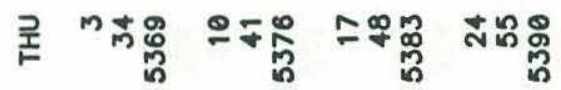

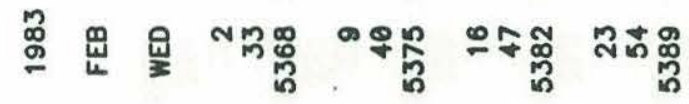

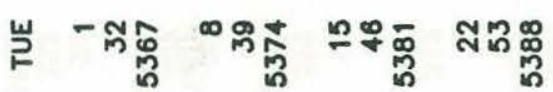

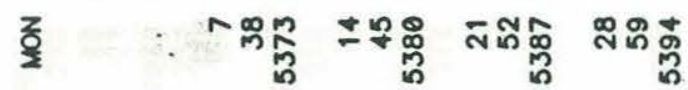

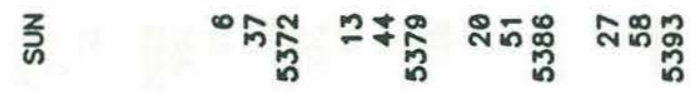

$$
\begin{aligned}
& \text { เ }
\end{aligned}
$$

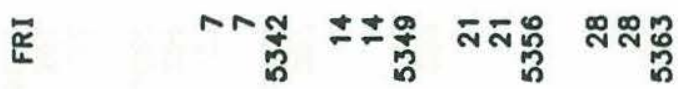

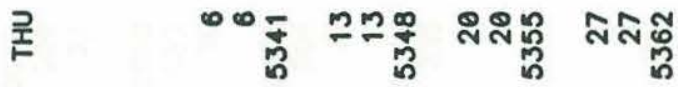

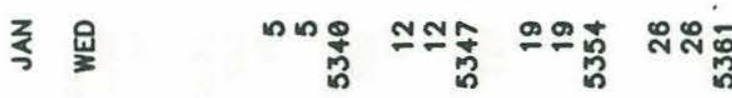

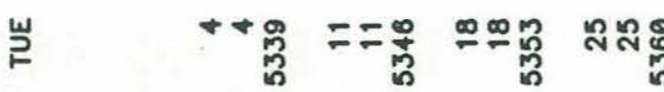

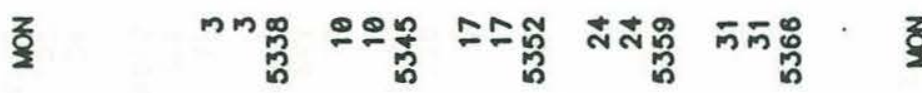

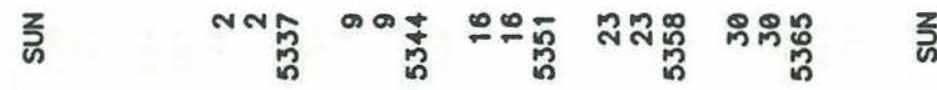

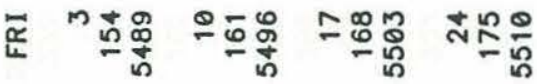

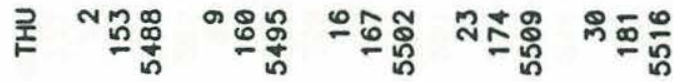

$$
\begin{aligned}
& \text { ว }
\end{aligned}
$$

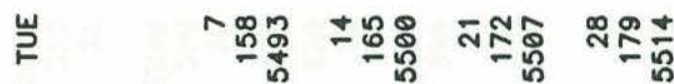

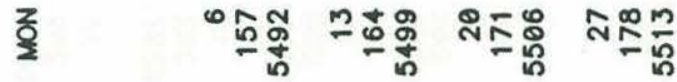

$$
\begin{aligned}
& \text { แ }
\end{aligned}
$$

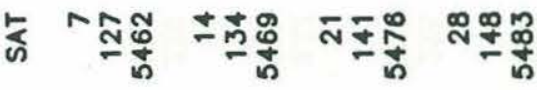

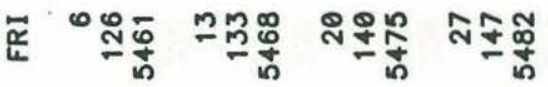

$$
\begin{aligned}
& \text { 곤 ๓ }
\end{aligned}
$$

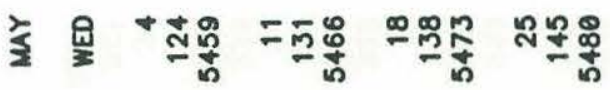

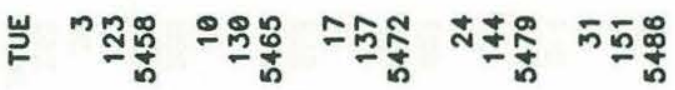

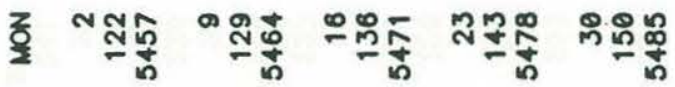

$$
\begin{aligned}
& \text { ไ క }
\end{aligned}
$$

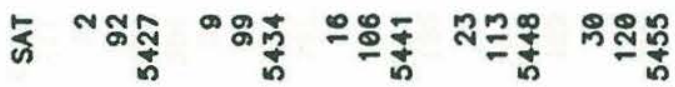

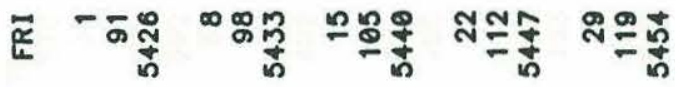

$$
\begin{aligned}
& \text { 곤 গ旅 } \\
& \text { 㧔 产 }
\end{aligned}
$$

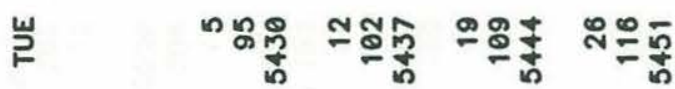

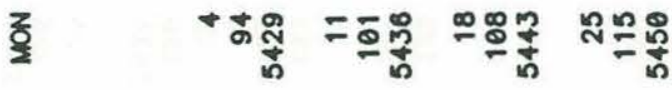

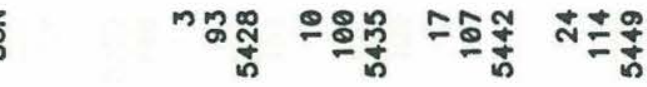




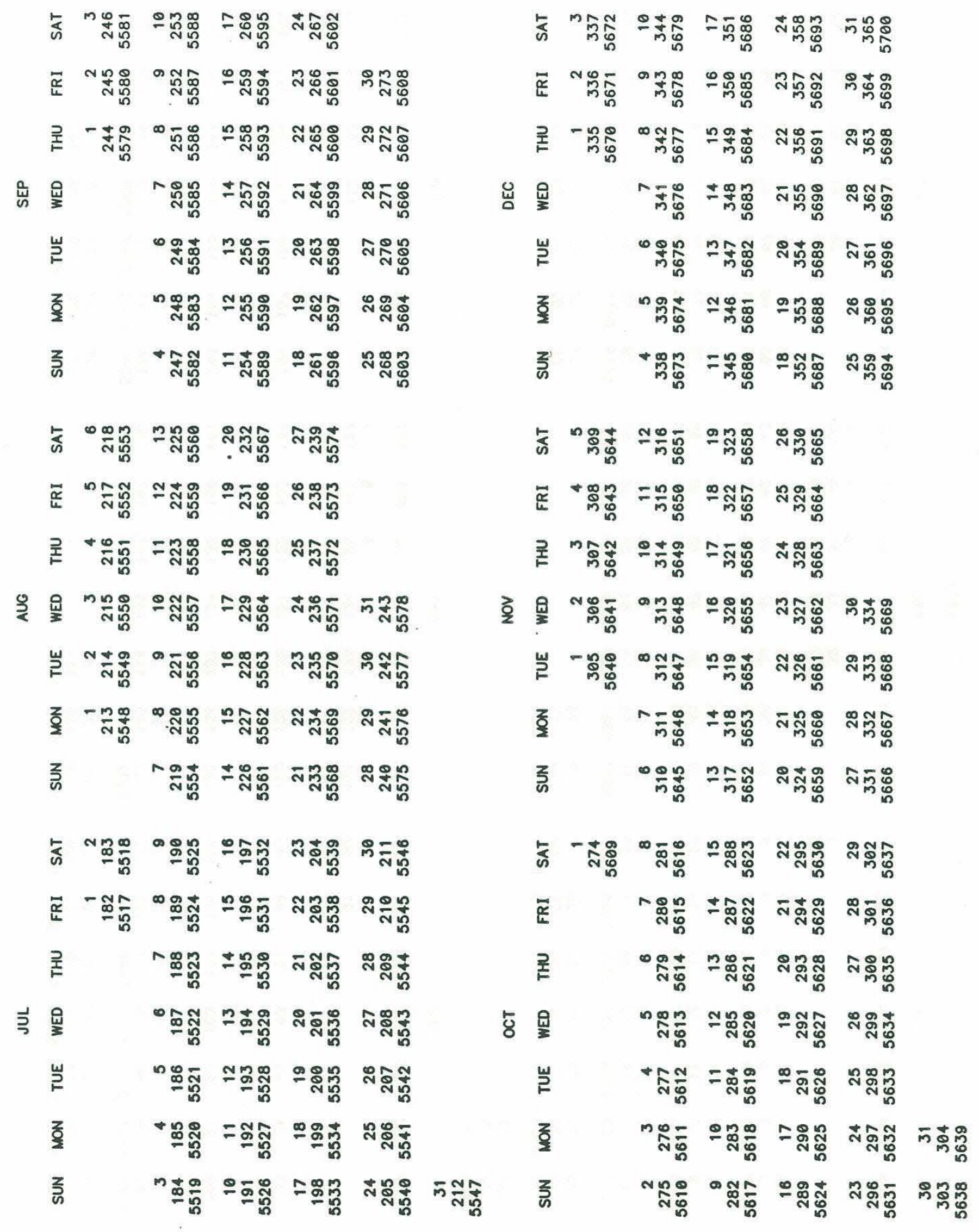




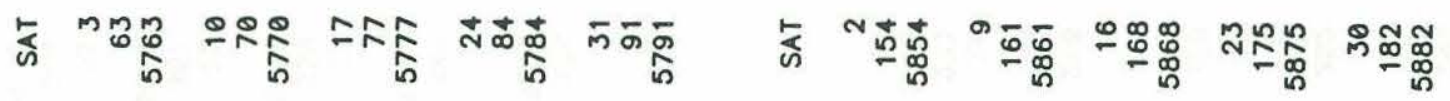

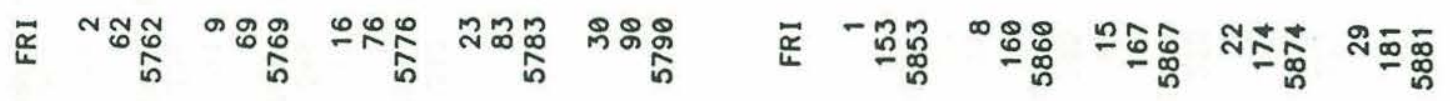

$$
\begin{aligned}
& \text { 寺 一б }
\end{aligned}
$$

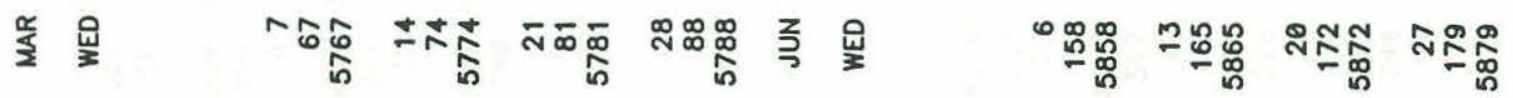

$$
\begin{aligned}
& \text { 岂 }
\end{aligned}
$$

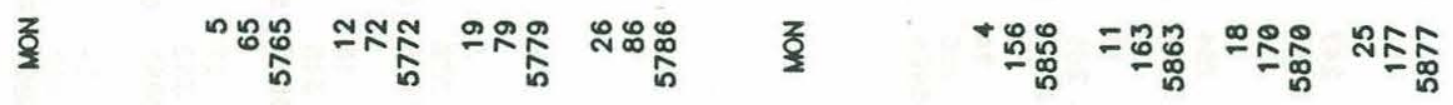

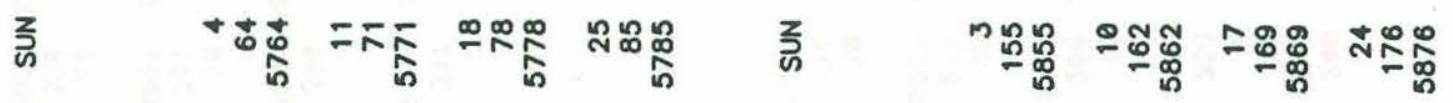

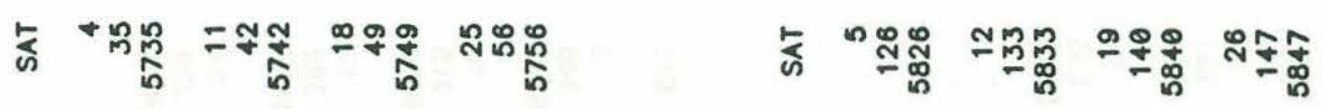

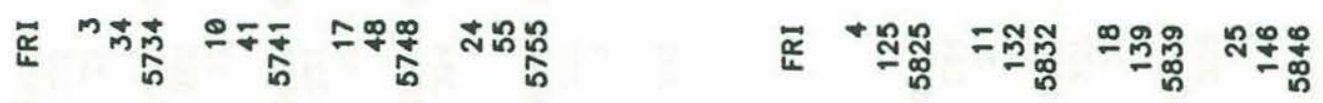

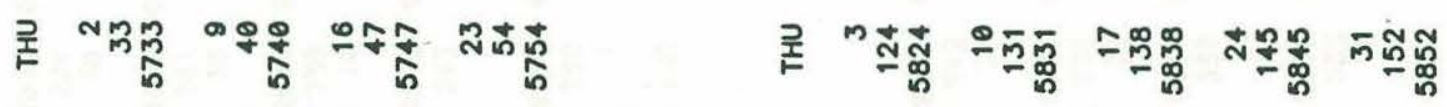

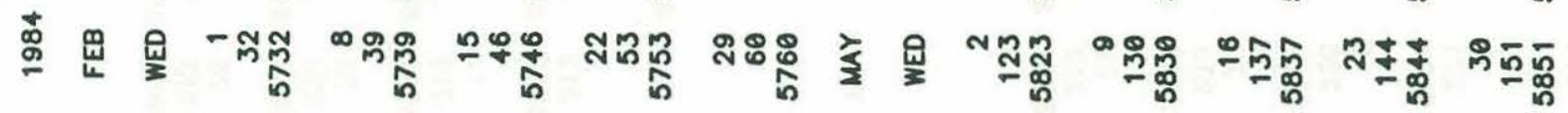

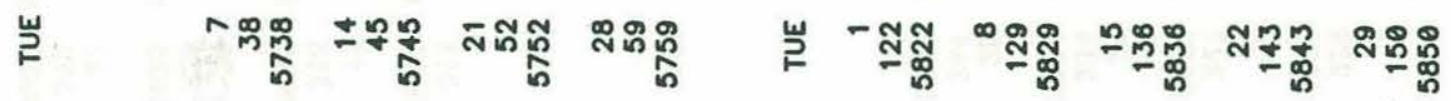

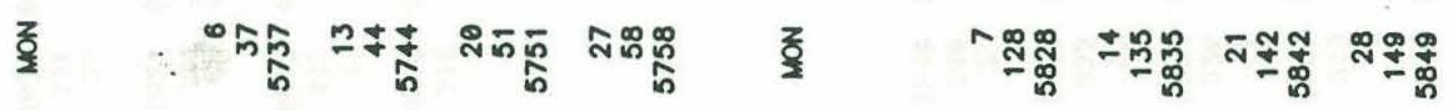

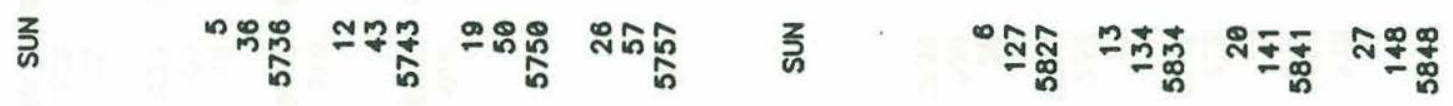

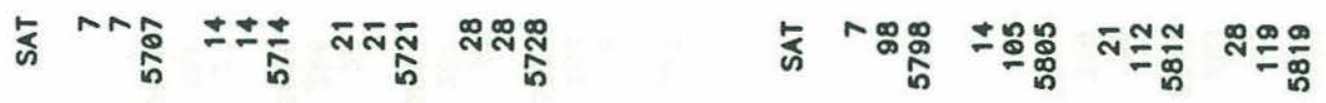

$$
\begin{aligned}
& \text { ธับ } \\
& \text { 곤 以ா }
\end{aligned}
$$

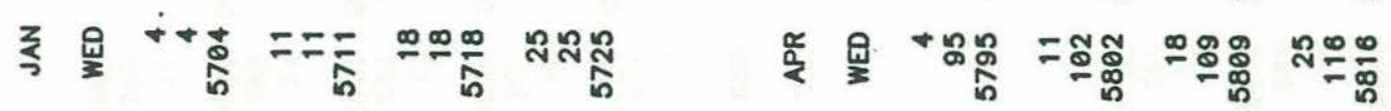

$$
\begin{aligned}
& \text { 岂的范 }
\end{aligned}
$$

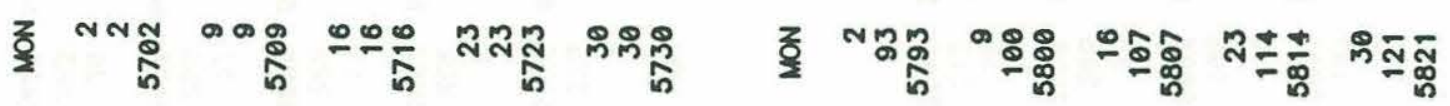

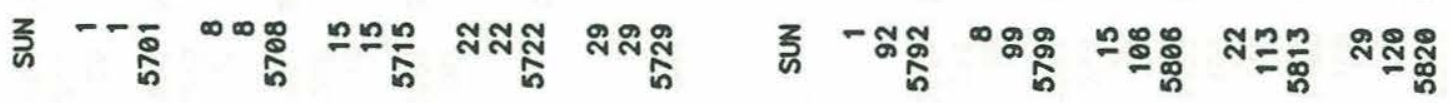




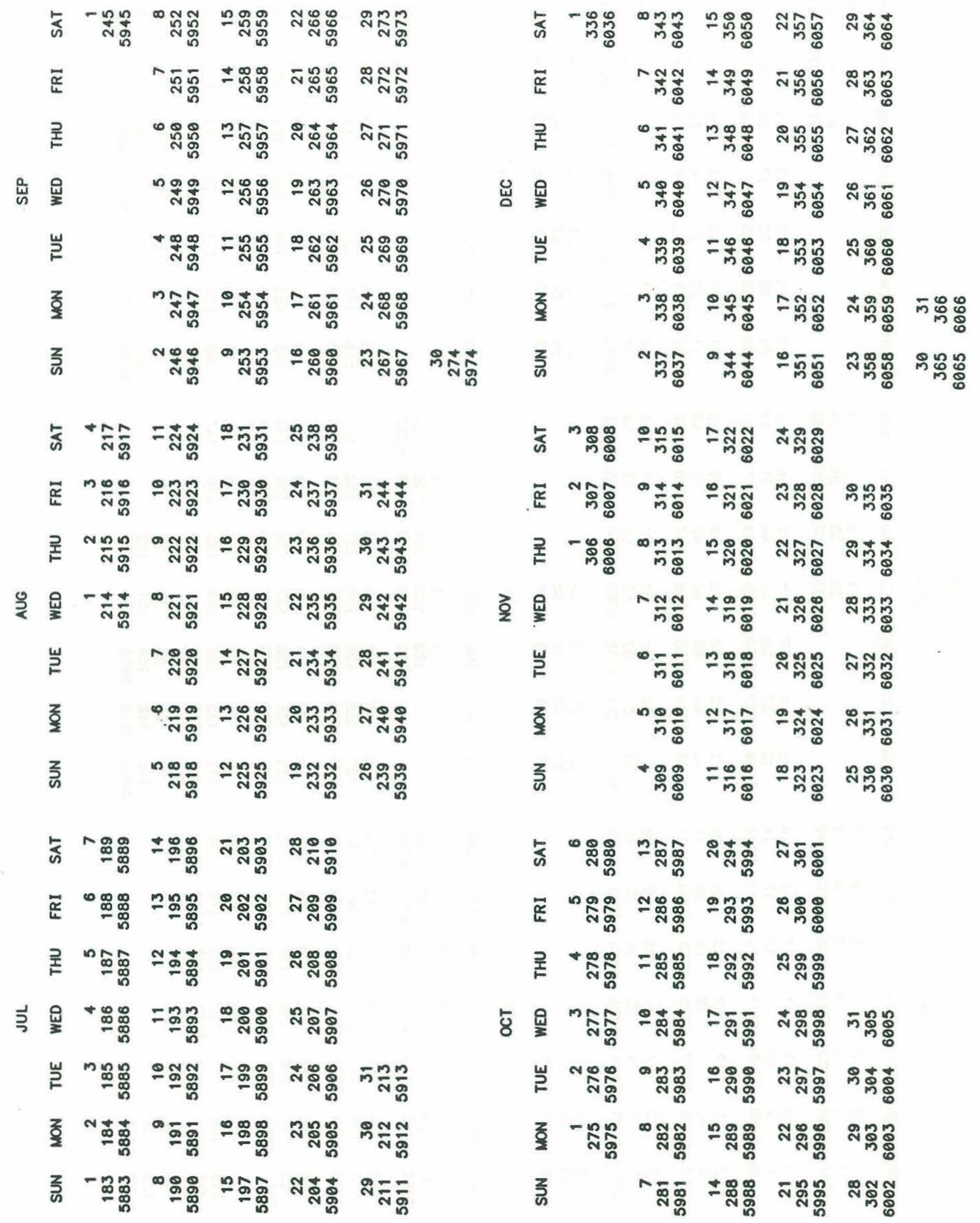




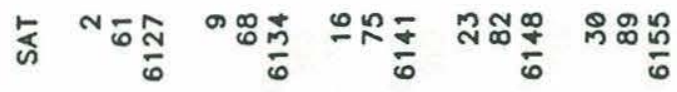

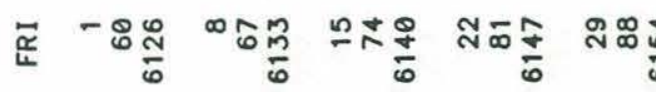

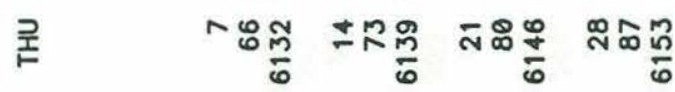

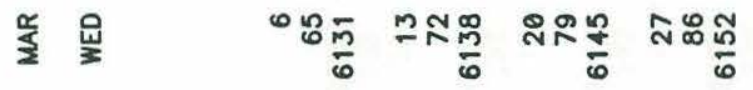

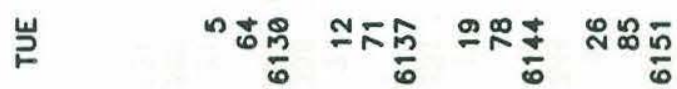

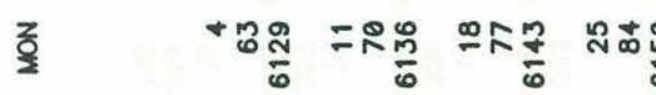

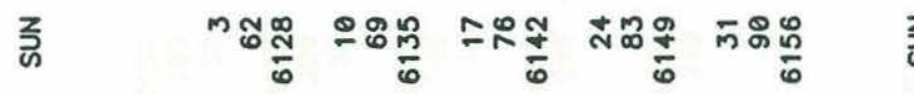

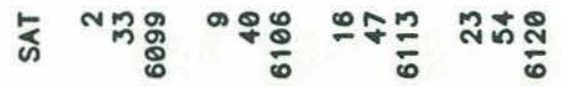

$$
\begin{aligned}
& \text { ช - }
\end{aligned}
$$

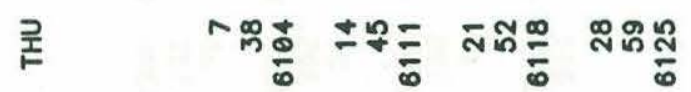

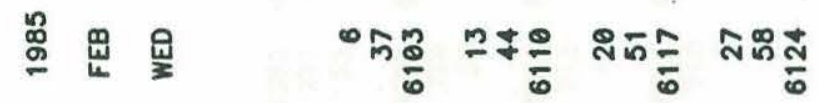

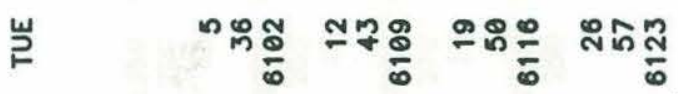

$$
\begin{aligned}
& \text { ร. } \\
& \text { 蛋 m芭 } \\
& \text { 点 }
\end{aligned}
$$

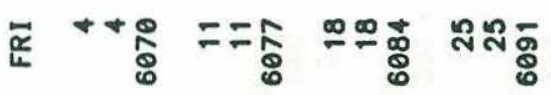

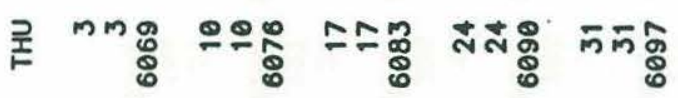

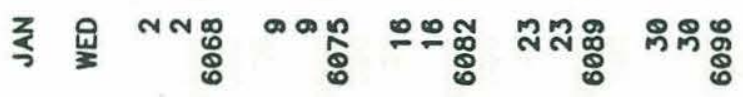

$$
\begin{aligned}
& \text { 岁 - } \\
& \text { ว } \\
& \text { ว } \\
& \text { 衣 一్ำ }
\end{aligned}
$$

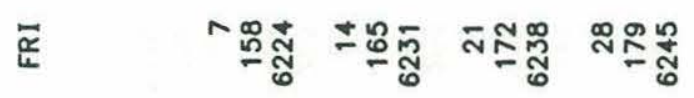

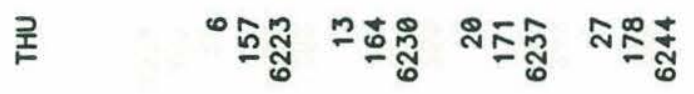

$$
\begin{aligned}
& \text { ว } \\
& \text { 岁 + }
\end{aligned}
$$

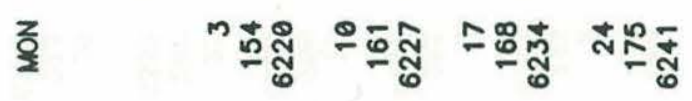

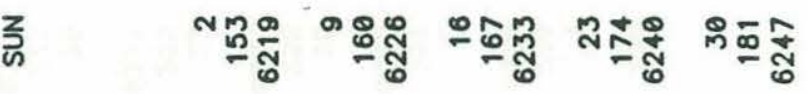

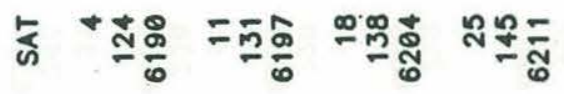

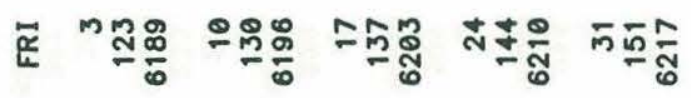

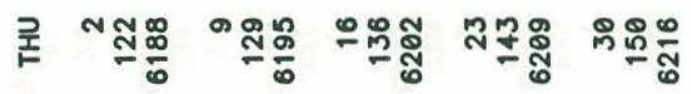

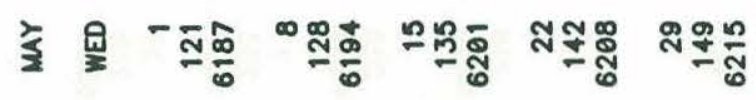

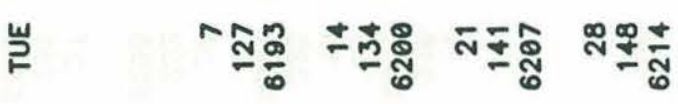

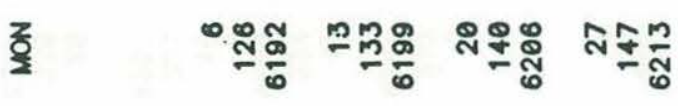

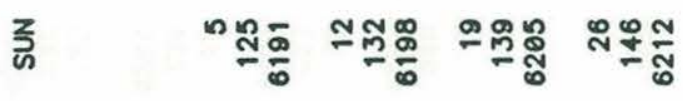

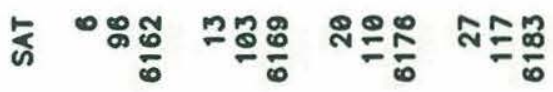

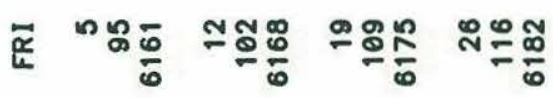

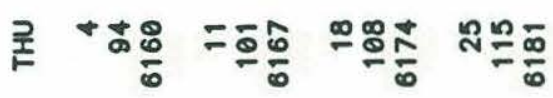

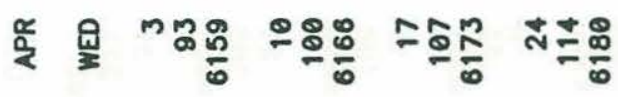

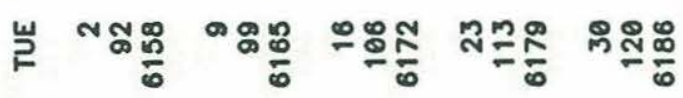

$$
\begin{aligned}
& \text { ช - }
\end{aligned}
$$

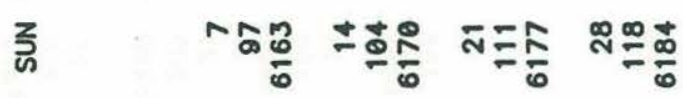




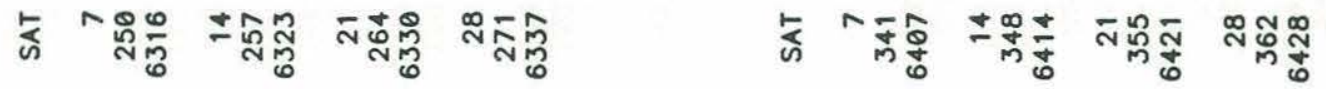

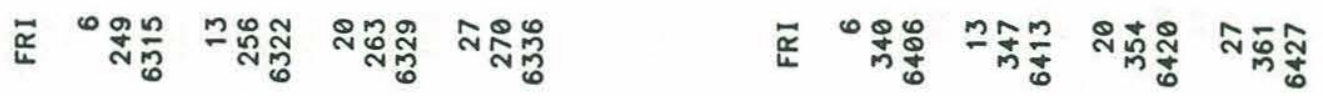

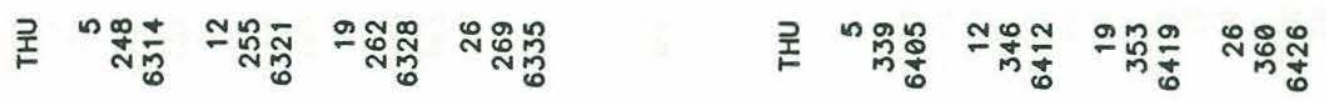

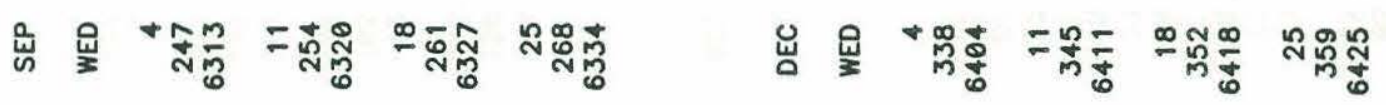

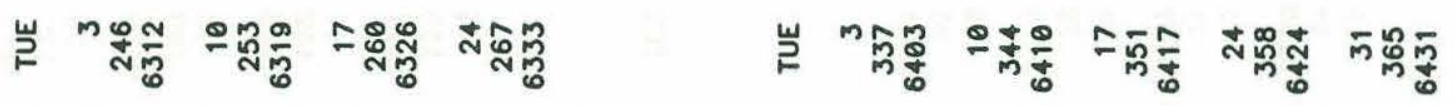

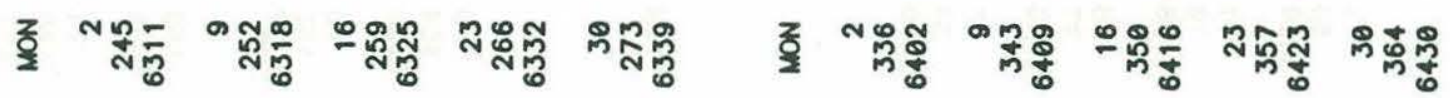

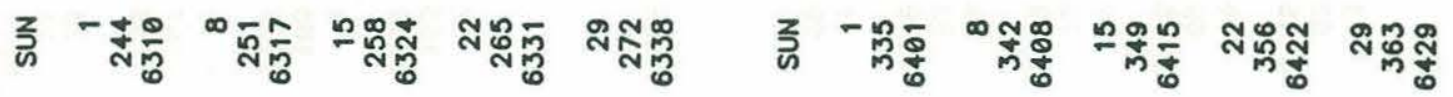

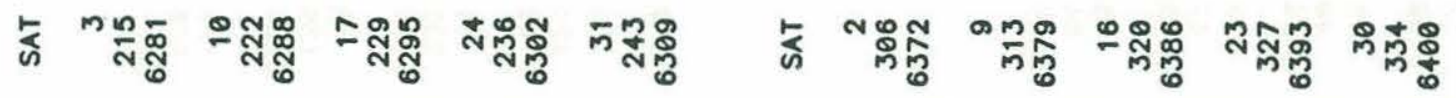

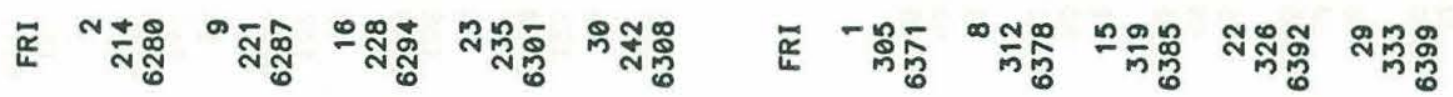

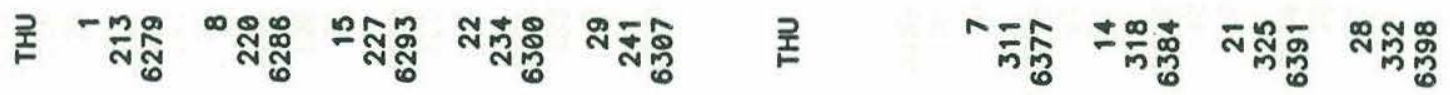

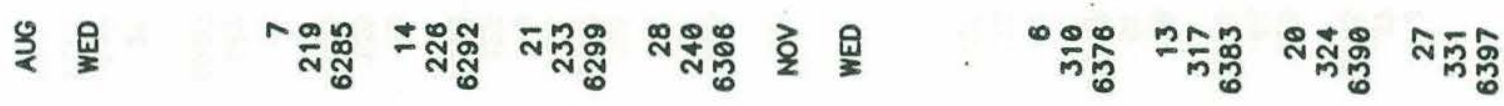

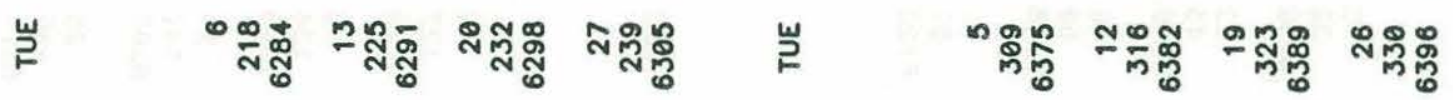

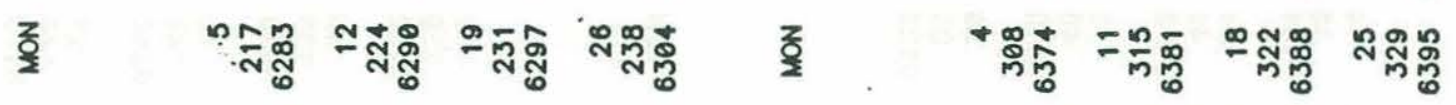

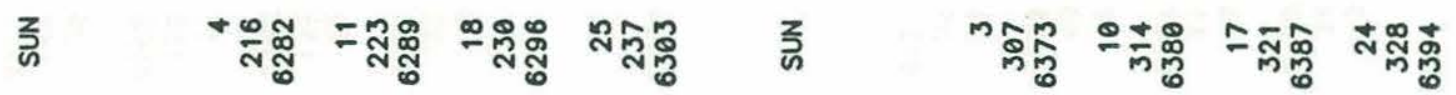

$$
\begin{aligned}
& \text { ๒ }
\end{aligned}
$$

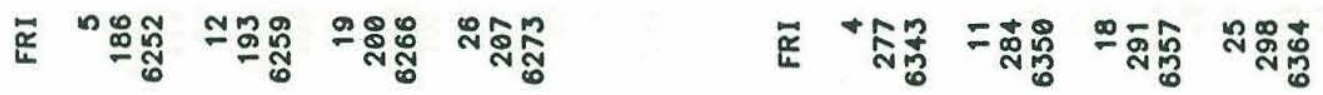

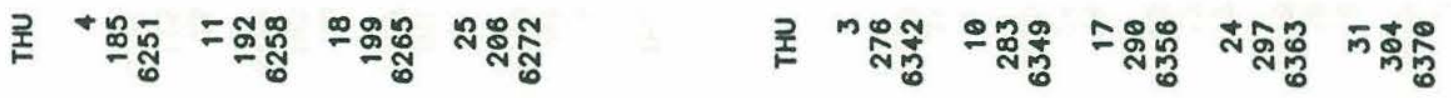

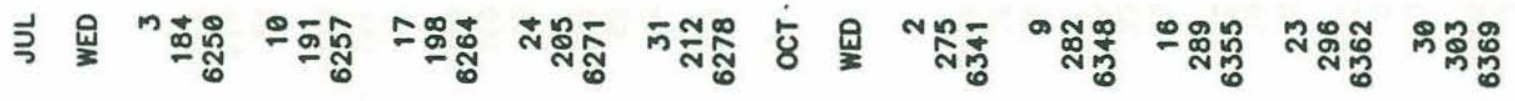

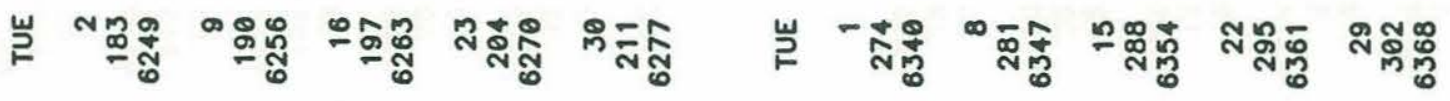

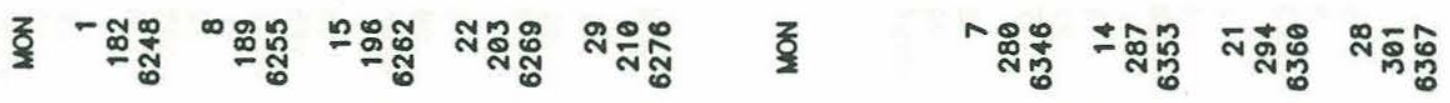

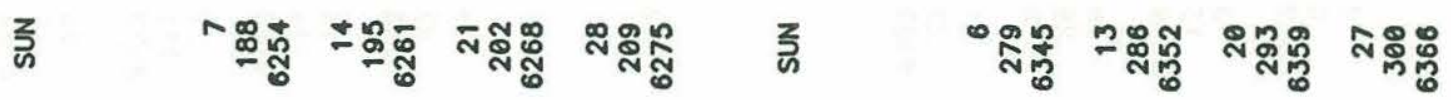




$$
\begin{aligned}
& \text { เ }
\end{aligned}
$$

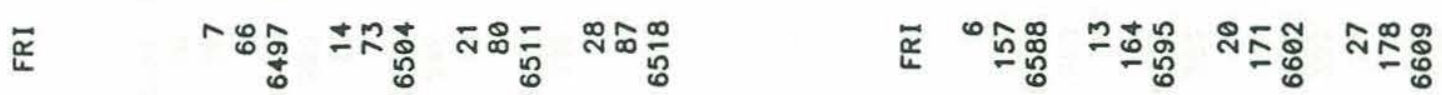

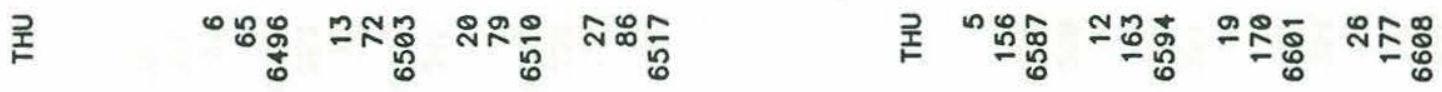

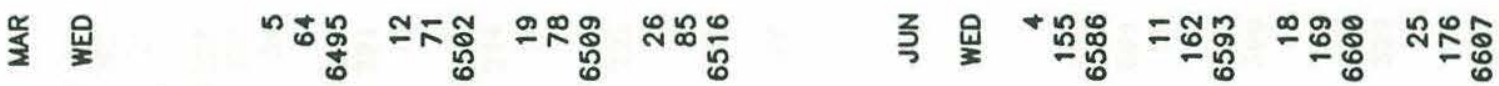

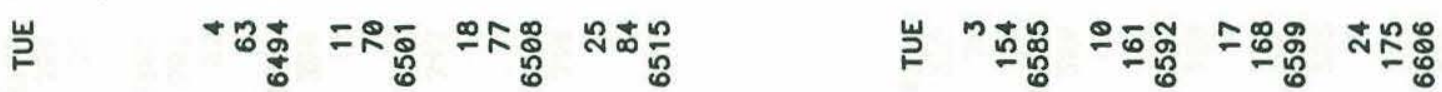

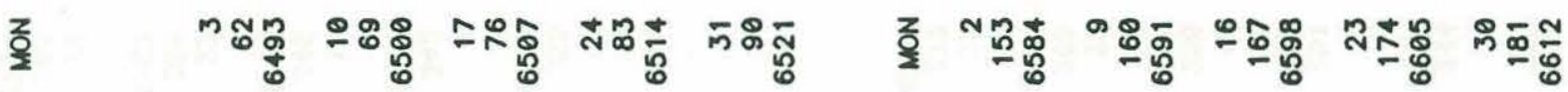

$$
\begin{aligned}
& \text { ว }
\end{aligned}
$$

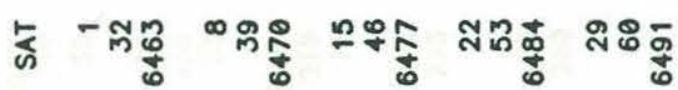

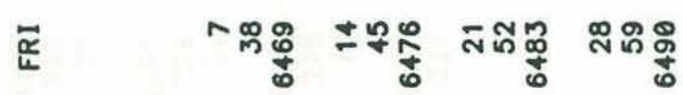

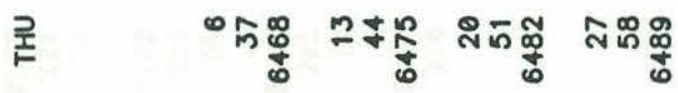

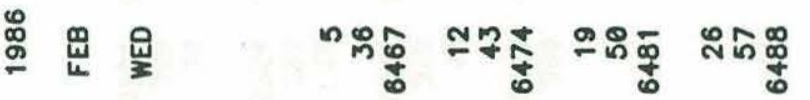

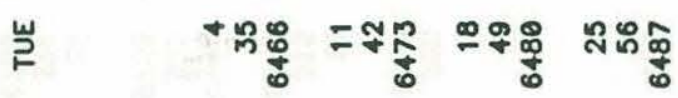

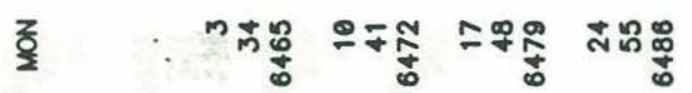

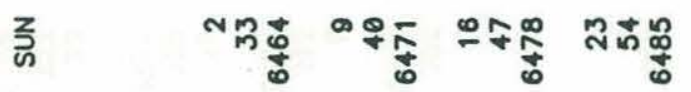

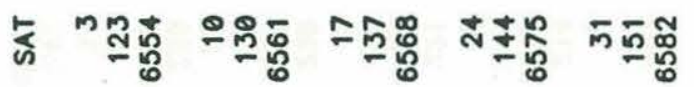

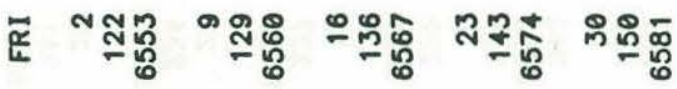

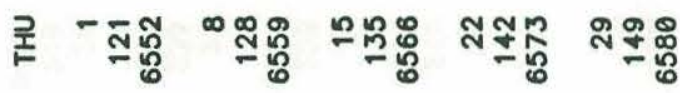

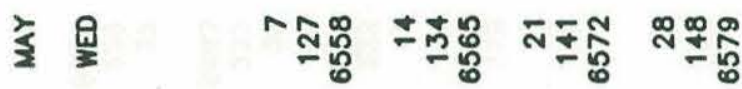

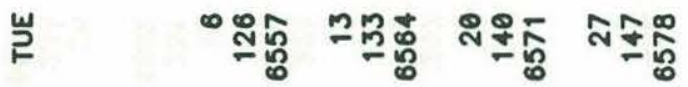

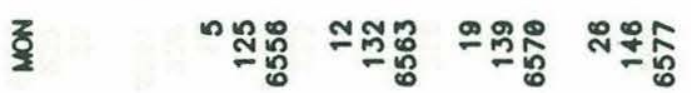

$$
\begin{aligned}
& \text { క }
\end{aligned}
$$

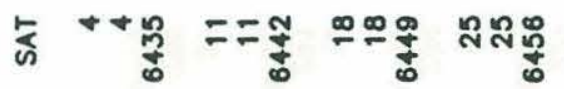

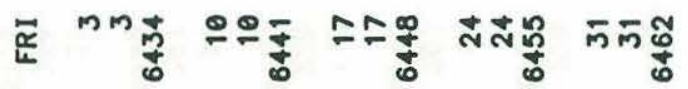

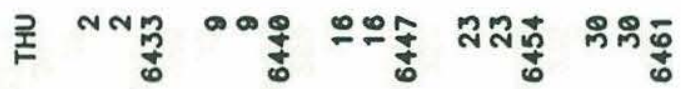

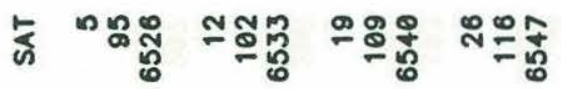

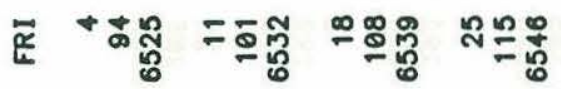

$$
\begin{aligned}
& \text { 곡 以ू⿺辶ู }
\end{aligned}
$$

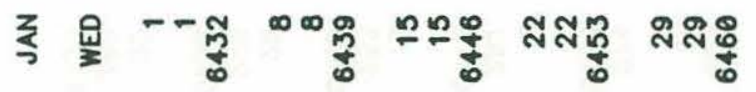

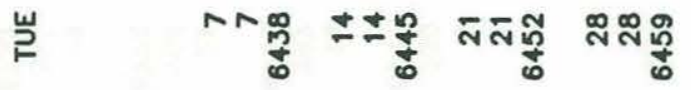

$$
\begin{aligned}
& \text { ริ }
\end{aligned}
$$

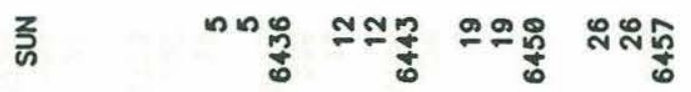

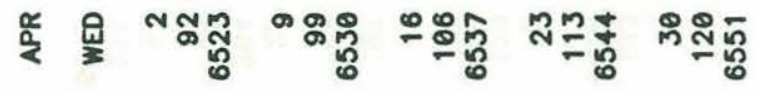

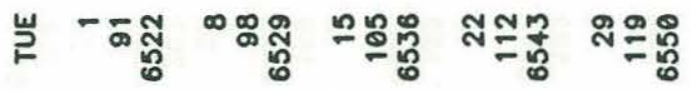

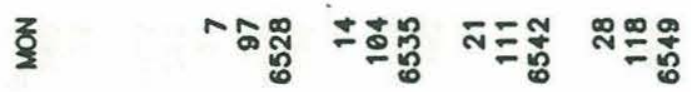

$$
\begin{aligned}
& \text { ๒ }
\end{aligned}
$$




$$
\begin{aligned}
& \text { ๒ } \\
& \text { に }
\end{aligned}
$$

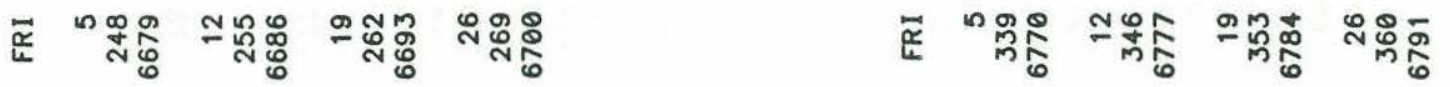

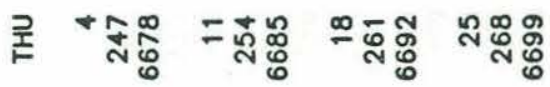

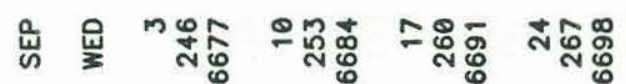

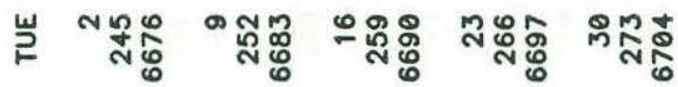

$$
\begin{aligned}
& \text { そ }
\end{aligned}
$$

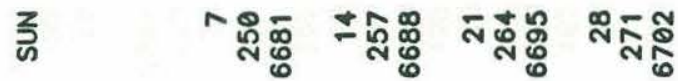

$$
\begin{aligned}
& \text { 곤 + }
\end{aligned}
$$

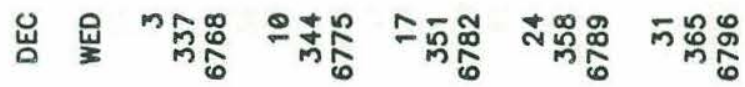

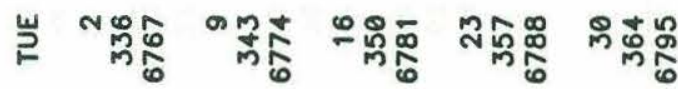

$$
\begin{aligned}
& \text { 을 - }
\end{aligned}
$$

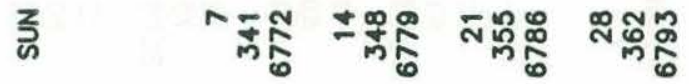

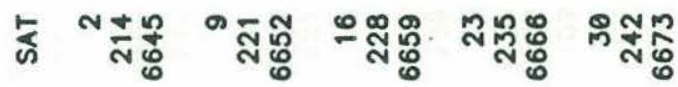

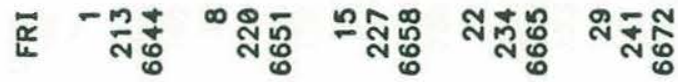

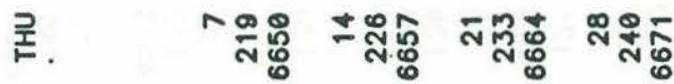

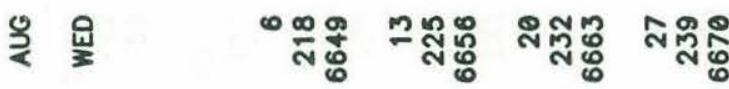

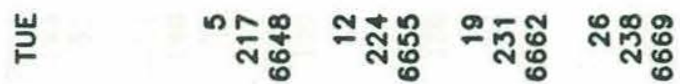

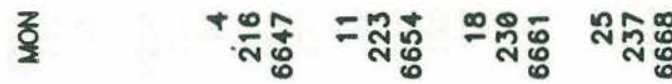

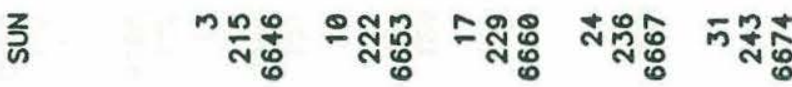

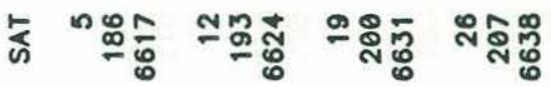

$$
\begin{aligned}
& \text { జ }
\end{aligned}
$$

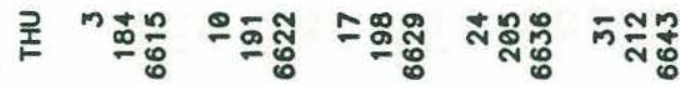

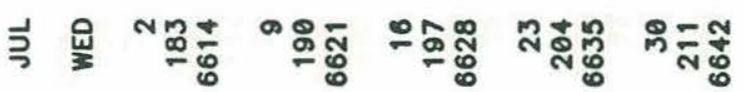

$$
\begin{aligned}
& \text { แ } \\
& \text { ว } \\
& \text { ฺ }
\end{aligned}
$$

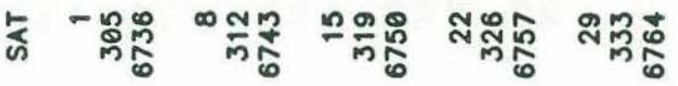

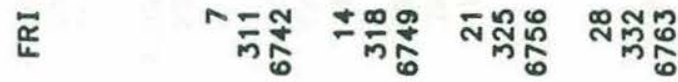

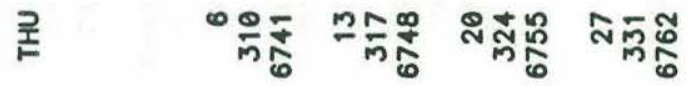

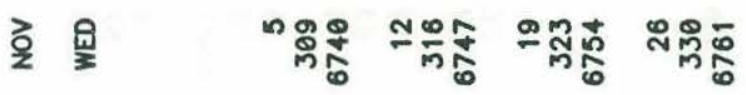

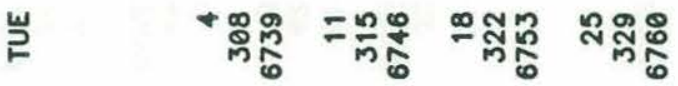

$$
\begin{aligned}
& \text { ₹ }
\end{aligned}
$$

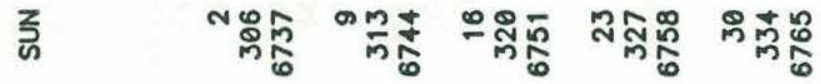

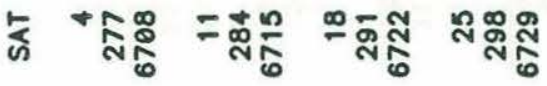

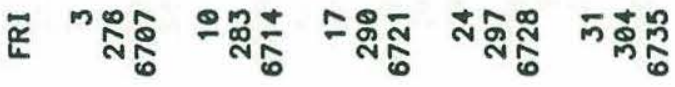

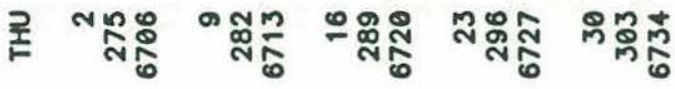

$$
\begin{aligned}
& \text { ไ }
\end{aligned}
$$

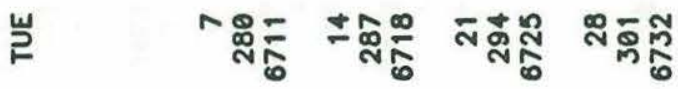

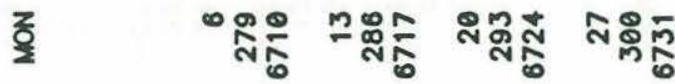

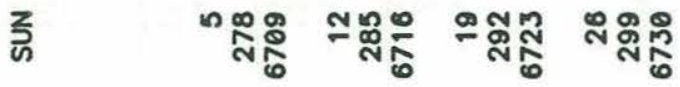




\section{DOCUMENT LIBRARY}

July 5, 1989

Distribution List for Technical Report Exchange

Attn: Stella Sanchez-Wade

Documents Section

Scripps Institution of Oceanography

Library, Mail Code C-075C

La Jolla, CA 92093

Hancock Library of Biology \& Oceanography

Alan Hancock Laboratory

University of Southern California

University Park

Los Angeles, CA 90089-0371

Gifts \& Exchanges

Library

Bedford Institute of Oceanography

P.O. Box 1006

Dartmouth, NS, B2Y 4A2, CANADA

Office of the International

Ice Patrol

c/o Coast Guard R \& D Center

Avery Point

Groton, CT 06340

Library

Physical Oceanographic Laboratory

Nova University

8000 N. Ocean Drive

Dania, FL 33304

NOAA/EDIS Miami Library Center

4301 Rickenbacker Causeway

Miami, FL 33149

Library

Skidaway Institute of Oceanography

P.O. Box 13687

Savannah, GA 31416

Institute of Geophysics

University of Hawaii

Library Room 252

2525 Correa Road

Honolulu, HI 96822

Library

Chesapeake Bay Institute

4800 Atwell Road

Shady Side, MD 20876

MIT Libraries

Serial Journal Room 14E-210

Cambridge, MA 02139
Director, Ralph M. Parsons Laboratory

Room 48-311

MIT

Cambridge, MA 02139

Marine Resources Information Center

Building E38-320

MIT

Cambridge, MA 02139

Library

Lamont-Doherty Geological Observatory

Colombia University

Palisades, NY 10964

Library

Serials Department

Oregon State University

Corvallis, OR 97331

Pell Marine Science Library

University of Rhode Island

Narragansett Bay Campus

Narragansett, RI 02882

Working Collection

Texas A\&M University

Dept. of Oceanography

College Station, TX 77843

Library

Virginia Institute of Marine Science

Gloucester Point, VA 23062

Fisheries-Oceanography Library

151 Oceanography Teaching Bldg.

University of Washington

Seattle, WA 98195

Library

R.S.M.A.S.

University of Miami

4600 Rickenbacker Causeway

Miami, FL 33149

Maury Oceanographic Library

Naval Oceanographic Office

Bay St. Louis

NSTL, MS 39522-5001

Marine Sciences Collection

Mayaguez Campus Library

University of Puerto Rico

Mayagues, Puerto Rico 00708 


\begin{tabular}{|c|c|c|c|}
\hline $\begin{array}{l}\text { REPORT DOCUMENTATION } \\
\text { PAGE }\end{array}$ & $\begin{array}{l}\text { 1. REPORT NO. } \\
\text { WHOI-89-37 }\end{array}$ & 2. & 3. Recipient's Accession No. \\
\hline \multirow{2}{*}{\multicolumn{2}{|c|}{$\begin{array}{l}\text { 4. Title and Subtitle } \\
\text { Gulf Stream Recirculation Experiment - Part } \mathbf{~}\end{array}$}} & & $\begin{array}{l}\text { 5. Report Date } \\
\text { September } 1989\end{array}$ \\
\hline & & & 6. \\
\hline \multicolumn{3}{|c|}{$\begin{array}{l}\text { 7. Author(s) } \\
\text { C Wooding, W. B. Owens, M. E. Zemanovic, and J.R. Valdes }\end{array}$} & $\begin{array}{l}\text { 8. Performing Organization Rept. No. } \\
\text { WHOI-89-37 }\end{array}$ \\
\hline \multirow{2}{*}{\multicolumn{2}{|c|}{$\begin{array}{l}\text { 9. Performing Organization Name and Address } \\
\text { The Woods Hole Oceanographic Institution } \\
\text { Woods Hole, Massachusetts } 02543\end{array}$}} & & 10. Project/Task/Work Unit No. \\
\hline & & & $\begin{array}{l}\text { 11. Contract(C) or Grant(G) No. } \\
\text { (C) OCE 81-09145 } \\
\text { (G) OCE 81-17467 }\end{array}$ \\
\hline \multirow{3}{*}{\multicolumn{2}{|c|}{$\begin{array}{l}\text { 12. Sponsoring Organization Name and Address } \\
\text { The National Science Foundation }\end{array}$}} & & 13. Type of Report \& Period Covered \\
\hline & & & Technical Report \\
\hline & & & 14. \\
\hline
\end{tabular}

15. Supplementary Notes

This report should be cited as: Woods Hole Oceanog. Inst. Tech. Rept., WHOI-89-37.

16. Abstract (Limit: 200 words)

This report presents trajectories and time series of velocity, pressure, and temperature for twelve neutrally-buoyant floats launched during the Gulf Stream Recirculation EXperiment (GUSREX) and two from earlier experiments, that continued to operate after May 1982. These float data were obtained from Autonomous Listening Stations (ALSs) deployed from May 1982 to August 1985.

\section{Document Analysis a. Descriptors}

1. Gulf Stream

2. recirculation

3. Lagrangian measurements

b. Identifiers/Open-Ended Terms

c. COSATI Field/Group

\begin{tabular}{|c|c|c|}
\hline \multirow{2}{*}{ Approved for publication; distribution unlimited. } & $\begin{array}{c}\text { 19. Security Class (This Report) } \\
\text { UNCLASSIFIED } \\
\end{array}$ & $\begin{array}{l}\text { 21. No. of Pages } \\
316\end{array}$ \\
\hline & 20. Security Class (This Page) & 22. Price \\
\hline
\end{tabular}

